BIOCHEMICAL CHANGES ASSOCIATED WITH

EMBRYONIC AND LARVAL DEVELOPMENT

IN THE AMERICAN LOBSTER

Homarus americanus Milne Edwards

\author{
by \\ Glenn Craig Sasaki \\ A.B., University of California at Berkeley \\ 1977 \\ Submitted in partial fulfillment \\ of the requirements for the degree of \\ Doctor of Philosophy \\ at the \\ MASSACHUSETTS INSTITUTE OF TECHNOLOGY
and the \\ WOODS HOLE OCEANOGRAPHIC INSTITUTION
}

January 1984

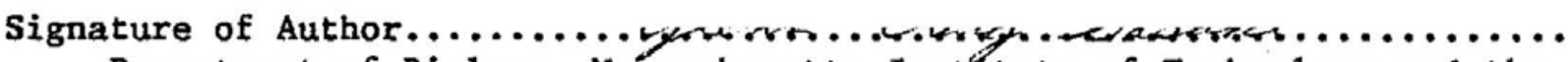
Department of Biology, Massachusetts Institute of Technology and the Joint Program in Oceanography, Massachusetts Institute of Technology/ Woods Hole-Oceanographic Institution, January 1984.

Certified by..$\ldots \ldots \ldots$... Judith M. Capuzto Thesis Supervisor

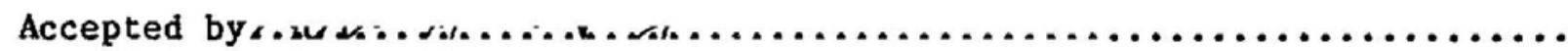
John 1./Stegemath

Chairman, Joint Committee for Biological Oceanography, Massachusetts Institute of Technology/Woods Hole Oceanographic Institution. 


\title{
BIOCHEMICAL CHANGES ASSOCIATED WITH \\ EMBRYONIC AND LARVAL DEVELOPMENT \\ IN THE AMERICAN LOBSTER \\ Homarus americanus Milne Edwards
}

\author{
by \\ Glenn Craig Sasaki \\ Submitted in partial fulfillment \\ of the requirements for the degree of \\ Doctor of Philosophy
}

\begin{abstract}
Proximate analysis was carried out on developing Homarus americanus Milne Edwards larvae and eggs. Lipids were further analyzed for class and fatty acid composition. During embryogenesis, triacylglycerol supplied most of the energy and the fatty acid 20:5 was selectively utilized. Absence of prolonged cold incubation temperatures resulted in increased yolk reserves in newly hatched larvae. Weight, ash, protein, carbohydrate and 1 ipid varied in association with the larval molt cycle. Scant metabolic reserves were accumulated in premetamorphic larvae while large triacylglycerol reserves were stored in postmetamorphic (fourth stage) animals. Absolute levels of metabolic reserves limited the extent of their utilization. Triacylglycerol was the preferred (first used) energy substrate but was usually limited in amount; hence protein was the major source of metabolic energy during starvation in premetamorphic larvae. Dietary intake of carbohydrate and possibly protein based gluconeogenesis were responsible for most chitin synthesis as established carbohydrate reserves were not sufficient.

Triacylglycerol served mainly as an energy storage form. P. choline appeared to exist in two metabolic pools; one of which could be catabolized without concomitant tissue loss. P. ethanolamine and sterols were primarily associated with cellular membranes. During tissue catabolism (starvation), P. ethanolamine was catabolized but sterol was conserved. On average, P. ethanolamine fatty acids were the most unsaturated, followed by the fatty acids of $\mathrm{P}$. choline and then triacylglycerol.

The type of fatty acid may influence food consumption and rates of absorption. Compared to a diet containing 16:0, one containing 20:5 was related to greater consumption and faster 1 ipid absorption. The fatty acid pattern of the larvae was heavily influenced by diet. The fatty acids 16:0, 18:0, 20:4, 20:5, and 22:6 were conserved under starvation. Conservation of the saturated fatty acids was probably related to control of cellular membrane fluidity. 16:0 and 18:0 were probably used to compensate for the increased membrane unsaturation from the conserved, potentially essential 20:4, 20:5, and 22:6 fatty acids.
\end{abstract}

Thesis supervisor: Dr. Judith M. Capuzzo 
ACKNOWLEDGEMENTS

This work could never have been done without the help of many, many people ( $I$ believe the phrase is "those to numerous to mention"). Well it's true, however I would like to mention a special few.

My deepest thanks to two friends, Drs. Judith Capuzzo and Roger Mann. Judy was the perfect advisor. She guided me with firm direction while giving me freedom and assistence to explore. She never demanded but let me know what should be done; and she pulled my tail out of the fire more times than I'11 probably ever know. Roger supported and encouraged me through the stressful initiation of becoming a "Graduate Student" and made me feel at home. His good advice throughout was helpful and welcomed.

In addition, I would like to thank the other members of my thesis committee: Drs. John Farrington, Robert Langer, and John Castell for contributing their support and expertise during the course of this work. Thanks also to Drs. Nancy Marcus and Marvin Freadman for chairing my general examination and thesis defense, respectively. Both made these otherwise harrowing experiences relaxed and (almost) enjoyable. Special thanks to Dr. Penny Chisolm for finding a space for me in her laboratory at MIT. I am also grateful to Dr. Stuart Wakeham and Al Davis for putting up with me while I learned the basics of chromatography, and to Jake Pierson and Abbie Alvin of the Education Department for their behind-the-scenes support.

The Environmental Systems Laboratory was my home away from home during this work and I'd like to thank the people that made it such a special home: Patricia Biesiot, Ka Chu, Scott Gallager, Bruce Lancaster, Anne McElroy, Steve McCormick and especially Dale Leavitt, all of whom helped in innumerable ways on innumerable occasions, who served as sources and sinks of good-natured grief and fun, and who are very good friends.

Last1y, I thank my parents and the rest of my family for all the encouragement, understanding, and love that they have always provided.

This research was supported by the Massachusetts Institute of Technology/ Woods Hole Oceanographic Institution Joint Program in Oceanography, the WHOI Ocean Industry Program, the U.S. Department of Interior, Bureau of Land Management under Contract No. AA551-CT9-5 and the U.S. Department of Commerce, National Oceanic and Atmospheric Administration, Office of Sea Grant under Grant No. NA80-AA-D-00077 (R/P-5 and R/P-11). 
TABLE of CONTENTS

$\underline{\text { TEXT }}$

Page number

TITLE

1

$\begin{array}{ll}\text { ABSTRACT } & 3\end{array}$

ACKNOWLEDGEMENTS

$\begin{array}{ll}\text { TABLE } & 7\end{array}$

LIST of APPENDICES 11

LIST of TABLES 13

LIST of GRAPHS $\quad 15$

$\begin{array}{ll}\text { INTRODUCTION } & 21\end{array}$

Background Information 25

$\begin{array}{ll}\text { Mo1t Cycle } & 28\end{array}$

Larval Energetics $\quad 31$

Crustacean Lipids $\quad 32$

Crustacean Lipid Physiology 46

Feeding, Transport and Starvation 46

$\begin{array}{ll}\text { Mo1ting } & 49\end{array}$

Early Development $\quad 50$

$\begin{array}{ll}\text { Summary } & 52\end{array}$

MATERIALS and METHODS 53

Culture and Sampling: Eggs 53

Culture and Sampling: Larvae 55

Experiments: Eggs $\quad 57$ 


\section{TEXT continued}

$\underline{\text { Page Number }}$

Experiments: Larvae

Normal Development

58

Starvation

60

Pulse Fed

61

Fatty Acid Supplement

61

Labelled Fatty Acid Feeding

62

LSC-TLC Counting

68

Proximate Analysis

68

Lipid Analysis

71

Extraction and Storage

71

Total Lipid

74

Lipid Class/ Iatroscan

75

Fatty Acid

82

87

87

Embryogenesis and Hatching

87

Proximate Analysis

91

Lipid Class

93

Fatty Acid

95

Pulse Fed and Immediate Posthatch Development

Proximate Analysis

95

Lipid Class 


\section{TEXT continued}

Page Number

Larval Development

Proximate Analysis

98

$\begin{array}{ll}\text { Lipid Class } & 102\end{array}$

Fatty Acid 104

$\begin{array}{ll}\text { Starvation } & 107\end{array}$

$\begin{array}{ll}\text { Proximate Analysis } & 107\end{array}$

$\begin{array}{ll}\text { Lipid Class } & 109\end{array}$

$\begin{array}{ll}\text { Fatty Acid } & 110\end{array}$

Fatty Acid Supplementation 113

Labelled Fatty Acid Feeding 115

$\begin{array}{ll}\text { DISCUSSION } & 121\end{array}$

$\begin{array}{ll}\text { Embryogenesis and Hatching } & 121\end{array}$

$\begin{array}{ll}\text { Proximate Analysis } & 121\end{array}$

Lipid Class 131

$\begin{array}{ll}\text { Fatty Acid } & 135\end{array}$

$\begin{array}{ll}\text { Summary } & 139\end{array}$

Pulse Fed and Immediate Posthatch Development 141

$\begin{array}{ll}\text { Summary } & 144\end{array}$

$\begin{array}{ll}\text { Larval Molt } & 145\end{array}$

$\begin{array}{ll}\text { Proximate Analysis } & 146\end{array}$

Lipids 154

$\begin{array}{ll}\text { Summary } & 158\end{array}$ 
TEXT continued

Page Number

Larval Development and Metamorphosis

160

$\begin{array}{ll}\text { Proximate Analysis } & 160\end{array}$

$\begin{array}{ll}\text { Lipid Class } & 170\end{array}$

Fatty Acid and CLO Feed Supplement 174

$\begin{array}{ll}\text { Summary } & 181\end{array}$

$\begin{array}{ll}\text { Starvation } & 183\end{array}$

$\begin{array}{ll}\text { Proximate Analysis } & 185\end{array}$

$\begin{array}{ll}\text { Lipid Class } & 190\end{array}$

$\begin{array}{ll}\text { Fatty Acid } & 194\end{array}$

$\begin{array}{ll}\text { Summary } & 196\end{array}$

Labelled Fatty Acid Feeding 198

$\begin{array}{ll}\text { Summary } & 204\end{array}$

Overview \& Epilogue 205

$\begin{array}{ll}\text { APPENDICES } & 209\end{array}$

$\begin{array}{ll}\text { TABLES } & 259\end{array}$

$\begin{array}{ll}\text { GRAPHS } & 313\end{array}$

LITERATURE CITED 
TABLE of CONTENTS

\section{APPENDICES}

Appendix

Number

Page Number

1. Molt staging technique. 210

2. Formulation of fatty acid supplement, 251 and ${ }^{14} \mathrm{C}$ fatty acid labelled feed.

3. Sample analysis flowchart. 255

4. Lipid nomenclature: abbreviations 257 and common names. 
TABLE of CONTENTS

TABLES

Table

Number

Description

Page Number

Master Key for Tables

260

1. Artemia and CLO supplement; FAME profiles

261

2. Embryogenesis; egg dry and wet weights, egg and eye dimensions

3. Embryogenesis; 1ipid classes as $\mu \mathrm{g}$ per individual, with standard deviations

4. Hatching Period; 1ipid classes as $\mu \mathrm{g}$ per individual, with standard deviations

5. Embryogenesis; neutral lipid fatty acids as FAME, $\mu \mathrm{g}$ per individual

6. Embryogenesis; P. ethanolamine fatty acids as FAME, $\mu \mathrm{g}$ per individual

7. Embryogenesis; P. choline fatty acids as FAME, $\mu \mathrm{g}$ per individual

8. Hatching Period; neutral 1ipid, P. ethanolamine and P. choline fatty acids as FAME, $\mu \mathrm{g}$ per individual

9. Pulse Fed, immediate post hatch development; proximate analysis data, mg per individual with stand. dev.

10. Pulse Fed, immediate post hatch develop.; lipid class data, $\mu$ g per individual with standard deviations

11. Larva1 Development; Hatch I-3, tota1 \& class 1ipid data, $\mu \mathrm{g}$ per individual with standard deviations

12. Larval Development; Hatch I-5, total \& class lipid

13. Larval Development; Hatch II-4, total \& class 1ipid

14. Larva1 Development; Hatch II-7, total \& class 1ipid

15. Larval Development; Hatch $I-5$, neutral 1ipid fatty acids as FAME, $\mu \mathrm{g}$ per individual

16. Larval Development; Hatch $I-5, \underline{P}$. ethanol. fatty acids as FAME, $\mu \mathrm{g}$ per individual 


\section{TABLES continued}

Table

Number

Description

Page Number

17. Larva1 Development; Hatch I-5, P. choline fatty acids

as FAME, $\mu \mathrm{g}$ per individual

18. Larval Development; Hatch II-4, neutral lipid fatty

289 acids as FAME, $\mu \mathrm{g}$ per individual

19. Larva1 Development; Hatch II-4, P. ethanolamine fatty 290 acids as FAME, $\mu \mathrm{g}$ per individual

20. Larva1 Development; Hatch II-4, P. choline fatty acids as FAME, $\mu \mathrm{g}$ per individual

291

21. Starvation; total and class Iipid data, $\mu$ ger individual with standard deviations

22. Starvation; neutral lipid fatty acids as FAME, $\mu \mathrm{g}$ per individual

23. Starvation; P. ethanolamine fatty acids as FAME, $\mu \mathrm{g}$ per individual

24. Starvation; P. choline fatty acids as FAME, $\mu \mathrm{g}$ per individual

25. Control vs. CLO supplement; proximate analysis data, mg per individual and \% dry wt. with stand. dev.

26. Contro1 vs. CLO supplement; 1ipid class data, $\mu g$ per individual and \% dry weight with stand. dev.

27. Control vs. CLO supplement; neutral 1ipid, P. ethano1., 304 P. choline fatty acids as FAME, $\mu \mathrm{g}$ per individual

28. Contro1 vs. CLO supplement; neutral lipid, P. ethanol., 306 P. choline fatty acids as FAME, \% of class FAME

29. ${ }^{14} \mathrm{C}$ Fatty Acids; recovered label in each class, DPM 308

30. ${ }^{14} \mathrm{C}$ Fatty Acids; control, lipid classes, $\mu \mathrm{g}$ per 309

31. ${ }^{14} \mathrm{C}$ Fatty Acids; DPM per 1ipid class as percent of 310

32. ${ }^{14} \mathrm{C}$ Fatty Acids; pellet weights and DPM, ingested DPM, recovered versus ingested DPM 
TABLE of CONTENTS

GRAPHS

Graph

Number

Master Key for Graphs

1 A,B,C. Temperature profile during each experiment

2 A. Embryogenesis; wet, dry and \% dry/wet weights,

2 B. Embryogenesis; $\underline{\text { ash, }}$ mg per individual, \% dry weight

3 A. Hatching Period; wet, dry and \% dry/wet weights,

3 B. Hatching Period; ash, mg per individua1, \% dry weight

4 A. Embryogenesis; carbohydrate, mg per individ., \% dry wt.

4 B. Embryogenesis; chitin, mg per individual, \% dry weight

5 A. Hatching Period; carbohydrate, mg per individ., \% dry wt. 325

5 B. Hatching Period; chitin, mg per individual, \% dry weight 325

6 A. Embryogenesis; protein, mg per individual, \% dry wt. 327

6 B. Embryogenesis; lipid, mg per individual, \% dry weight 327

7 A. Hatching Period; protein, mg per individual, \% dry wt. 329

7 B. Hatching Period; 1ipid, mg per individual, \% dry weight 329

8. Embryogenesis; caloric equivalents for protein, lipid, 331 carbohydrate, calories per individual

9 A. Embryogenesis; triacylglycerol, $\mu$ g per ind., \% dry wt.

9 B. Embryogenesis; sterols, $\mu \mathrm{g}$ per individual, \% dry weight 333

10 A. Embryogenesis; P. ethanolamine; $\mu g$ per indiv., \% dry wt. 335

10 B. Embryogenesis; P. choline, $\mu$ g. per individua1, \% dry wt. 335

11 A. Embryogenesis; neutra1 lipids, NEFA as FAME, $\mu$ ger ind. 337

11 B. Embryogenesis; neutral 1ipids, NEFA as FAME, \% of tota1 337 FAME in neutra1 lipids 
GRAPHS continued

Number

Description

Page Number

12 A. Embryogenesis; neutral lipids, PUFA as FAME, $\mu \mathrm{g}$ per ind. 339

12 B. Embryogenesis; neutral 1ipids, PUFA as FAME, \% of total 339 FAME in neutral lipids

13 A. Embryogenesis; P. ethanolamine, NEFA as FAME, $\mu$ g per ind. 341

13 B. Embryogenesis; P. ethanolamine, NEFA as FAME, \% of total 341 FAME in $P$. ethanolamine

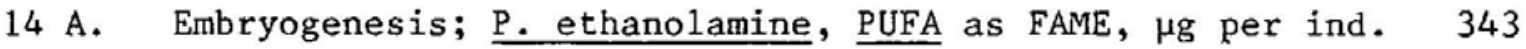

14 B. Embryogenesis; P. ethanolamine, PUFA as FAME, \% of total 343 FAME in $P$. ethanolamine

15 A. Embryogenesis; P. choline, NEFA as FAME, $\mu \mathrm{g}$ per ind. 345

15 B. Embryogenesis; P. choline, NEFA as FAME, \% of total 345 FAME in $P$. choline

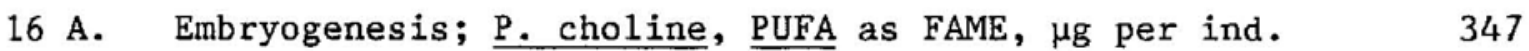

16 B. Embryogenesis; P. choline, PUFA as FAME, \% of total 347 FAME in P. choline

17 A. Pulse Fed, immediate posthatch development; 349 carbohydrate, $\mu \mathrm{g}$ per individual, \% dry weight

17 B. Pulse Fed, immediate posthatch development; ash, $\mu \mathrm{g}$ per individual, \% dry weight

18 A. Pulse Fed, immediate posthatch development; protein, $\mu \mathrm{g}$ per individual, \% dry weight

18 B. Pulse Fed, immediate posthatch development; 1ipid, $\mu \mathrm{g}$ per individual, \% dry weight

19 A. Larval Development; wet weight, mg per individual

19 B. Larva1 Development; ash free dry wt., mg per individual 353

20 A. Larval Development; dry weight, mg per individual 355

20 B. Larva1 Development; \% dry/wet weight 355

21 A. Larval Development; ash, mg per individual 357

21 B. Larval Development; ash, \% dry weight 357 


\section{GRAPHS continued}

Number

22 A. Larval Development; chitin, mg per individual

22 B. Larval Development; chitin, \% dry weight

23 A. Larval Development; carbohydrate, mg per individual

23 B. Larval Development; carbohydrate, \% dry weight

24 A. Larval Development; protein, mg per individual

24 B. Larva1 Development; protein, \% dry weight

25 A. Larval Development; 1ipid, mg per individual

25 B. Larval Development; 1ipid, \% dry weight

26 A. Larval Development; caloric equivalents of protein, lipid and carbohydrate, calories per individual

26 B. Larval Development; caloric equivalents of protein, lipid and carbohydrate, as \% of total calories

27 A. Larval Development; triacylglycerol, $\mu \mathrm{g}$ per individual

27 B. Larval Development; triacylglycerol, \% vs. protein

28 A. Larval Development; sterols, $\mu$ ger individual

371

28 B. Larval Development; stero1s, \% vs. protein

29 A. Larval Development; P. ethanolamine, $\mu g$ per individual 373

29 B. Larval Development; P. ethanolamine, \% vs. protein 373

30 A. Larval Development; P. choline, $\mu$ g per individual 375

30 B. Larval Development; P. choline, \% vs. protein 375

31 A. Larval Development; neutral 1ipid, NEFA as FAME, 377 $\mu \mathrm{g}$ per individual

31 B. Larval Development; neutral lipid, NEFA as FAME, 


\section{GRAPHS continued}

Number

Description

Page Number

32 A. Larva1 Development; neutra1 1ipid, PUFA as FAME, $\mu \mathrm{g}$ per individual

32 B. Larval Development; neutral 1ipid, PUFA as FAME,

$\%$ of total FAME in neutral lipids

33 A. Larval Development; P. ethanolamine, NEFA as FAME, $\mu \mathrm{g}$ per individual

33 B. Larval Development; P. ethanolamine, NEFA as FAME, $\%$ of total FAME in P. ethanolamine

34 A. Larval Development; P. ethanolamine, PUFA as FAME, $\mu \mathrm{g}$ per individual

34 B. Larval Development; P. ethanolamine, PUFA as FAME, $\%$ of tatal FAME in P. ethanolamine

35 A. Larva1 Development; P. choline, NEFA as FAME, $\mu \mathrm{g}$ per individual

35 B. Larval Development; P. choline, NEFA as FAME, $\%$ of total FAME in P. choline

36 A. Larval Development; P. choline, PUFA as FAME, $\mu g$ per individual

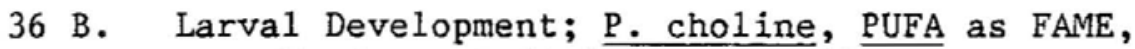
$\%$ of total FAME in P. choline

37 A. Starvation; wet weight, $\mathrm{mg}$ per individual

37 B. Starvation; ash free dry weight, $\mathrm{mg}$ per individual

38 A. Starvation; dry weight, mg per individual

38 B. Starvation; \% dry/wet weight

39 A. Starvation; ash, mg per individual 393

39 B. Starvation; ash, \% dry weight 393

40 A. Starvation; chitin, mg per individual 395

40 B. Starvation: chitin, \% dry weight 
GRAPHS continued

$\underline{\text { Number }}$

Description

Page Number

41 A. Starvation; carbohydrate, mg per individual

41 B. Starvation; carbohydrate, \% dry weight

42 A. Starvation; protein, mg per individual

42 B. Starvation; protein, \% dry weight

43 A. Starvation; lipid, mg per individual

401

43 B. Starvation; 1ipid, \% dry weight 401

44 A. Starvation; percent of protein reserve utilization 403

44 B. Starvation; percent of 1ipid reserve utilization 403

44 C. Starvation; percent of carbohydrate reserve utilization 403

45. Starvation; caloric equivalents, protein, lipid and 405 carbohydrate, calories per individual

46 A. Starvation; triacylglycerol, $\mu$ g per individual 407

46 B. Starvation; triacylglycerol, \% vs protein 407

47 A. Starvation; sterols, $\mu$ g per individual 409

47 B. Starvation; sterols, \% vs protein 409

48 A. Starvation; P. ethanolamine, $\mu g$ per individual 411

48 B. Starvation; P. ethanolamine, \% vs protein 411

49 A. Starvation; P. choline, $\mu$ ger individual 413

49 B. Starvation; P. choline, \% vs protein 413

50 A. Starvation; neutral 1ipids, NEFA as FAME, \% of reserve 415

50 B. Starvation; neutral 1ipids, NEFA as FAME, ratio of 415 relative concentrations.

51 A. Starvation; neutral 1ipids, PUFA as FAME, \% of reserve 417

51 B. Starvation; neutral lipids, PUFA as FAME, ratio of 417 relative concentrations 
GRAPHS continued

Number

52 A. Starvation; P. ethanolamine, NEFA as FAME, \% of reserve 419

52 B. Starvation; P. ethanolamine, NEFA as FAME, ratio of 419 relative concentrations

53 A. Starvation; P. ethanolamine, PUFA as FAME, \% of reserve 421

53 B. Starvation; P. ethanolamine, PUFA as FAME, ratio of 421 relative concentrations

54 A. Starvation; P. choline, NEFA as FAME, \% of reserve 423

54 B. Starvation; P. choline, NEFA as FAME, ratio of 423 relative concentrations

55 A. Starvation; P. choline, PUFA as FAME, \% of reserve 425

55 B. Starvation; P. choline, PUFA as FAME, ratio of 425 relative concentrations

56 A. ${ }^{14} \mathrm{C}$ Fatty Acids; $\underline{\mathrm{DPM}}$ as \% of recovered total in 427 triacylglycerols

56 B. ${ }^{14} \mathrm{C}$ Fatty Acids; DPM as \% of recovered tota1 in 427 free fatty acids

57 A. ${ }^{14} \mathrm{C}$ Fatty Acids; $\underline{\text { DPM }}$ as \% of recovered total in 429 steryl esters

57 B. ${ }^{14}$ C Fatty Acids; DPM as \% of recovered total in 429 1-2 diacylglycero1s

58 A. ${ }^{14} \mathrm{C}$ Fatty Acids; $\underline{D P M}$ as $\%$ of recovered total in P. ethanolamine

58 B. ${ }^{14} \mathrm{C}$ Fatty Acids; $\underline{\mathrm{DPM}}$ as \% of recovered total in P. choline 


\section{INTRODUCTION}

Successful larval development and metamorphosis of planktotrophic larvae, including those of decapod crustaceans, requires the efficient and coordinated utilization of available metabolic resources. Of the many factors affecting resource utilization during this larval period, nutrition is of prime importance. As nutrition involves both endogenous and exogenous dietary sources, the yolk reserves from the embryonic period can play a crucial role. Consequently, the nutritional aspects of embryogenesis are also important for a complete understanding of larval development.

The available biochemical and physiological data on crustacean embryonic and larval periods in terms of normal development and the response to environmental and nutritional stress are notably scarce. A goal of this research was to fill in some of these gaps in our knowledge of the early developmental period in crustaceans.

The research focused on the embryonic and larval development of the American lobster Homarus americanus Milne Edwards. Biochemica1 analyses, with an emphasis on 1ipids, were done to ascertain and correlate any biochemical changes with the morphological and known physiological events that occurred during these periods. In addition, controlled nutritional stresses were imposed upon larvae to determine biochemical responses to these perturbations.

The American lobster was chosen for the study animal as it possesses several characteristics that make it ideal for this type of research. Partly because of its fisheries importance, there is a vast sum of literature on this species. Ecologically, much research has been, and is 
being, conducted on the American lobster concerning its habitats, ranges and community interactions. The gross morphology and life history have been worked out in detail (Bumpus, 1891; Herrick, 1896, 1909). Current and recent works have focused on physiology and nutrition (Castell and Covey, 1976; Capuzzo and Lancaster, 1979a-d). The next logical steps are in the fields of endocrinology, enzymology and biochemistry. Certainly, there is already substantial information from all three of these fields (Brockerhoff et al., 1970; Gilgan and Burns, 1979; Phillips et al., 1980). Most of it has, however, concentrated on adult or sub-adult animals. The American lobster then is one of a handful of marine crustaceans about which sufficient background information exists to allow interpretation of basic biochemical data on the larval stages.

A further benefit of the American lobster's commercial importance is the accessibility of laboratory animals. For example, in order to study embryogenesis in its entirety, females that would extrude eggs in the laboratory were required. Predictive methods for this existed (Aiken and Waddy, 1982) but nonetheless required examination of numerous individuals. Re-stocking programs of lobsters in the past and present (Hughes et a1., 1962, 1974) and aquacultural concerns have been the impetus for the development of successful culturing techniques (Hughes, 1974). Experience in the implementation of these techniques was available at the E.S.L. (Environmenta1 Systems Laboratory) of WHOI from Dr. Judith Capuzzo and Bruce Lancaster.

The characteristics of Homarus ameicanus eggs and larvae were also important. A single egg brood is composed of numerous, large individuals. The eggs are carried externally by the female lobster for the entire 9-11 month incubation period (Cobb, 1976). For embryogenic 
studies, this allows repeated samplings of eggs from the same brood throughout the developmental period. Due to a synchronous pattern of pulsed hatching in lobsters, numerous larvae are released simultaneously. This allows one hatch to supply all the animals needed for an experiment. Ameican lobster larvae are also comparatively large (1 mg dry weight at hatch) which eases individual manipulation and allows some analyses to be done on single animals.

Early 1arval development in American lobsters is synchronous which makes it possible to treat a single hatch as a population of near identical individuals. This is important as sampling for biochemical parameters is of a destructive nature. Usually, time series data with destructive sampling necessitate presenting the data as a ratio (e.g. percent dry weight) to neutralize the variability in absolute size between individuals. Percent data, however, preclude certain interpretations. By having a population of near identical larvae the data can also be expressed as an absolute amount per individual.

Physiologically, the response to nutritional stress may be more rapid in larvae than adults. This would occur if the metabolic reserves are at comparatively lower levels in the larvae. Growth is proportionally more rapid in larvae so that any limiting growth factors would quickly exert an effect. Ecdysis or molting also occurs at a greater frequency in the larvae, allowing for more molt cycles to be studied within a shorter period of time.

In addition, there are two major transitions that occur during early development, namely hatching and metamorphosis. At hatching many complex processes are occurring, with one of them being the switch from an endogenous to exogenous food supply. At metamorphosis, a characteristic 
morphological change occurs, but little is known of the physiological changes that may also occurr.

of the major biochemical classes of compounds, that is, protein, carbohydrate, nucleic acids, and lipids, the latter was chosen for a more detailed examination. Lipids were selected for several reasons. Many, if not most, organisms use some form of 1ipid for energy storage. This is true for crustaceans and one type of 1ipid used has generally been assumed to be triacylglycerol (Holland, 1978). A confirmation of triacylglycerol's function, the possible roles of other 1ipid classes, and the energetic importance of each class were then topics to be addressed. A further avenue of study is the lipid nutritional requirements of crustaceans (Teshima, 1972; Holland, 1978; D'Abramo et al., 1981). These requirements are few and therefore allow for the biochemical characterization of the organisms response to selected lipid deficiencies. A final basis for specialization in 1ipids is the recognition that many organic xenobiotics are preferentially deposited in the lipid material of an organism, and as such, can potentially affect lipid metabolism. Prior to elucidating the effects of such xenobiotics, it is necessary to know the normal pattern of lipid metabolism in the organism and how it relates to normal developmental processes. In summary, the questions that were addressed are: What are the biochemical correlates, with an emphasis on lipids, associated with embryogenesis and larval development in the American lobster? How do these changes relate to the physiology and nutritional requirements of the organism? What are the responses of the organism when placed under nutritional stress? 
Background Information

This section will cover embryology, larval development, molting, and energetics in Homarus americanus and will be followed by a brief review of lipids in crustacea.

Homarus americanus

In Homarus americanus, the most common period of the year for egg extrusion is June through September (cf Aiken and Waddy, 1980). Egg extrusion can be predicted in individual females by the condition of the cement gland on the pleopods or swimmerets beneath the tail (Aiken and Waddy, 1982). Fertilization occurs upon extrusion of the eggs which can number from between 3000 to over 100,000. for a single brood. (Herrick, 1909; Chaikelis, 1949; Saila et al., 1969; Perkins, 1971). The eggs attach to the pleopods and the female lobster broods them for 9-11 months until they hatch in May to June of the following year (Cobb, 1976). The length of embryogenesis is high1y temperature dependent (Templeman, 1940; Perkins, 1972; Branford, 1978).

When extruded the individual eggs are dark green in color due to the yolk protein- carotenoid complex ovoverdin (Stern and Saloman, 1937; Goodwin, 1951); though as a mass, the eggs appear black. During embryogenesis, the initial dark green color lightens and then shifts into a brown, then gold color. This is due to the break up of ovoverdin into its constituent proteins and astaxanthin (Goodwin, 1951). Just prior to hatching, the egg color shifts again to a bluish tinge (Davis, 1964).

The most complete morphological description of lobster embryogenesis was completed by Bumpus (1891). The eggs are initially centrolecitha1 but become telolecithal with development. Cell division is somewhat 
independent of the yolk so that a central mass of undivided yolk always exists. At $21^{\circ} \mathrm{C}$ the morula stage is reached in 2 to 3 days and the blastopore forms by about the fourth day. By the tenth day after fertilization the nauplius stage appendages can be seen as buds. Shortly thereafter the first embryonic molt occurs. By the fourth week the eye pigmentation becomes visible (Bumpus, 1891; Herrick, 1909; Templeman, 1940). Development continues and by hatching the embryo has become a pre-larval zoea.

In a closely related species, Homarus gammarus (Linnaeus), hatching occurs in a pulsed, regular rhythm. Under a natural light regime, hatching usually occurred shortly after darkness each day of the two to six week hatching period (Ennis, 1973). Ennis (1975) did not find such a consistent pattern in Homarus americanus, since minor hatches occurred throughout the day; however, if only the major hatches (greater than 100 1arvae) are considered, then personal observation shows that $\underline{H}$. americanus often does have a rhythm of one major hatch per day within the same 1-2 hour period.

The length of the hatching period is dependent on the temperature history of the developing eggs. An exposure of several months to cold (less than $5^{\circ} \mathrm{C}$ ) water compresses the hatching period to $2-3$ days (Aiken, 1980) or less than a week (personal observation), whereas continuous exposure to $15-21^{\circ} \mathrm{C}$ water causes hatching periods of 2 to 3 weeks (Aiken, 1980 and persona1 observation).

Part of the hatching behavior of the female lobster consists of straightening of the abdomen and rapid movement of the pleopods. This 
lasts about a minute and pushes the newly hatched larvae posteriorly out (Templeman, 1937), however, the actual hatching time for these released larvae may have been up to $24 \mathrm{~h}$ previously (Ennis, 1975).

Upon release from the egg, a molt rapidly occurs and the animal becomes a first stage mysis larvae (Davis, 1964). After large hatches, the exuvia or molted cuticle from this first molt can be seen floating on the surface of the holding tanks.

This following section on larval development was based on Herrick (1909). There are four freeswimming stages in the American lobster. The first three are premetamorphic larvae of the mysis form; the fourth is postmetamorphic and has the adult form.

After the hatch molt, the first stage larvae are capable of swimming and remain near the surface. They may or may not still have remnants of yolk in their masticatory stomach. They are capable of feeding within the first day and are cannabilistic if insufficient food is available. A visible characteristic of this stage is the lack of swimmerets which are still in bud form. The range of total length for this stage is $7.5-8.0$ $\mathrm{mm}$; the duration is $1-5$ days.

The second and third larval stages have much the same lifestyle as the first stage, though their swimming abilities have improved. The second stage can be distinguished visually from the first by the presence of the swimmerets, though these do not become fully functional until the fourth stage. The third stage can be distinguished visually from the second by the presence of uropods. The range of total length and development times are 8.3-10.2 $\mathrm{mm}$ and 2-5 days and 10-12 $\mathrm{mm}$ and 2-5 days for the second and third stage larvae, respectively. 
The metamorphosis from the third to fourth stage changes not only the morphology but the behavior as well. Fourth stage animals (these were termed lobsterling by Herrick (1909), but in the literature and this text, the term "larvae" also includes this stage) adopt a forward swimming style using the pleopods and show avoidance and hiding behavior. Later in this stage they begin to explore the substrate and either settle as fourth stage larvae or soon after as fifth stage juveniles. The total length range of the fourth stage larvae is $11-14 \mathrm{~mm}$; the duration of this stage is $10-19$ days.

of the many environmental factors affecting the development of the larvae, temperature and nutrition exert the greatest effects. In general, the lower either the temperature or quantity of food, the slower the developmental rate (Templeman, 1936; Aiken, 1980).

\section{Molt Cycle}

Development within a single larval stage can be followed with the molt cycle. This molt cycle was first fully characterized by Drach (1939) for decapods and later refined (Drach, 1944; Drach and Tchernigovtzeff, 1967). Their method to characterize the molt cycle has been adapted for use by several researchers (Hepper, 1965; Barlow and Ridgeway, 1969; Gilgan and Zinck, 1975), and most notably by Aiken (1973, 1980), for the lobster.

The term "molt cycle" covers the cyclic change in the animal that is correlated with the process of ecdysis or molting. Drach (1939, 1944, 1967) gave each phase of this cycle, determined by certain morphological changes, a letter designation. Aiken (1973) refined this method, and based a staging technique on setal tissue characteristics. 
In brief, characterization of the molt cycle uses the letters A to E. A and B are the stages immediately after ecdysis and constitute the periods of 'molt recovery'. Stage C, of ten termed the intermolt, is a period of indefinite length during which molt recovery is completed, the new exoskeleton becomes fully formed, and tissue and organic reserves are built up. The D stages classically constitute the premolt period when the animal is preparing for the upcoming ecdysis; tissue and reserves continue to accumulate in the earlier D stages. In lobster the first D stage, Do, is considered a transition period since the animal has not yet committed itself to molting and can pause indefinitely at this stage (Aiken, 1973). The last stage, E, is the ecdysis itself when the animal sheds its old exoskeleton.

The classical hypothesis for decapod molting (cf. Aiken, 1980) has the process of molting being mediated by two hormones -- a molt inhibiting hormone (MIH) which is a peptide (Freeman and Barte11, 1976; Cooke and Sullivan, 1982), and a molting hormone (MH) which is a steroid. The MIH is produced by the $\mathrm{X}$-organ and stored in the sinus gland, both of which reside in the eyestalk (Pyle, 1943). In larval Homarus americanus the X organ is present in late egg development and increases in size during the first four larval stages (Aiken, 1980). The sinus gland in larval $\underline{\mathrm{H}}$. americanus does not appear until the third larval stage and may not be active in the larval stages (Aiken, 1980). The MH is produced by the Y-organ as the precursor ecdysone ( $\alpha$-ecdysone) and is converted to the active form ecdysterone ( $\beta$-ecdysone) by other tissues (Chang and 0'Connor, 1978). This conversion to ecdysterone appears to occur quite rapidly in larval H. americanus (Chang and Bruce, 1981). Another source 
of MH may be the cephalic gland in the antennal segment (Gersch, 1979).

The exact hormonal processes controlling the molt cycle in crustaceans are unknown though the concensus appears to be converging towards hyphotheses derived from insect based research (Aiken, 1980). Aiken (1980) reviewed selected crustacean and insect molt literature and summarized a hypothetical scenario for decapod molt control. His scenario is as follows: The hormone ecdysone, produced by the molt gland or glands, initiates gastrolith formation and activates an enzyme system which hydroxylates ecdysone to ecdysterone. The consequent rise in ecdysterone is responsible for cuticle formation, influences the mid to late premolt events, and acts as a negative feedback for ecdysone hydroxylation. Insects and decapods can inactivate and excrete ecdysterone when its presence is inappropriate. MIH appears to modify tissue response to ecdysterone but does not appear to prevent ecdysterone synthesis or accelerate ecdysterone inactivation.

Ecdysis is necessary in order for a crustacean to increase in physical size. After shedding the old exoskeleton and prior to hardening of the new one, the animal can absorb water and expand. Later in the molt cycle, new tissue growth displaces this absorbed water. The period of ecdysis is, however, also when the animal is most vulnerable to predation and when, without a hard shell or mouthparts, it usually cannot feed. A rapid transition and recovery from an ecdysis would be beneficial, yet, because feeding is impossible, such a recovery must rely on endogenous reserves. The coordinated pattern of metabolism that is required for the molt has been the subject of numerous physiological and biochemical studies. The contents of these studies and what they imply for this work 
will be covered in detail in the Discussion section.

\section{Larval Energetics}

The energetics of Homarus americanus have been found to change through 1arval development (Logan and Epifanio, 1978; Capuzzo and Lancaster, 1979a,c). Oxygen consumption or respiration is a quantitative indicator of energy catabolism. Various factors, such as food assimilation, stress, activity, and oxygen levels, can affect respiration rates. If care is taken to minimize the effects of these factors, oxygen consumption can be used to determine the basal or routine (at rest) metabolic rate of an animal under aerobic conditions. Using Gilson respirometers, Logan and Epifanio (1978) measured oxygen consumption in the larval stages of $\underline{H}$. americanus and determined that the weight specific respiration rates were higher in early larval stages compared to later larval stages. Using a more accurate technique (microrespirometers), Capuzzo and Lancaster (1979a) found the opposite trend, where younger larvae had lower weight specific respiration rates compared to older larvae and they suggested that the higher rates observed among older larvae were a result of the increased energy demands of metamorphosis. The oxygen consumption values can also be compared against excreted nitrogen. This $0: \mathrm{N}$ ratio (atoms oxygen consumed versus atoms nitrogen excreted) can be used to determine the relative quantities of catabolized substrates (Corner and Cowey, 1968; Ikeda, 1974). Catabolism of protein produces nitrogen. In contrast, catabolism of lipid or carbohydrate (these two cannot be distinguished with this method) results in very little or no excreted nitrogen. The $0: N$ ratios of larval Homarus americanus indicate that lipid, carbohydrate, and protein were all 
important energy substrates in the first three larval stages. In the fourth larval stage, however, protein catabolism increased in importance relative to lipid and carbohydrate catabolism (Capuzzo and Lancaster, $1979 a, c)$.

The assimilation rates of $\underline{\text { Homarus }}$ americanus did not appear to change through larval development, but the gross growth efficiency (100 $\mathrm{X}$ growth/consumption, expressed as calories) increased in the fourth larval stage (Logan and Epifanio, 1978).

\section{Crustacean Lipids}

A variety of abbreviations will be used when discussing lipids in this text. Upon introducing a new lipid, both the common name and its abbreviation will be 1isted. The IUPAC-IUB (1976)(International Union of Pure and Applied Chemistry - International Union of Biochemistry) recommended format for lipid nomenclature will not be followed in full unless there is a possibility for misinterpretation. For convenience, a compilation of the abbreviations are listed as Appendix 4.

The operational definition of a lipid is any biocompound that is insoluble in water but soluble in a range of non-polar solvents. The term lipid covers a heterogeneous group of biochemicals that are responsible for a suite of different actions. Many hormones, vitamins, structural components, energy stores, and neurocrine products are lipids. The quantitatively major types or classes of lipids do have one other feature in common, that is, with the exception of free sterols, they all have fatty acids as components. In this text only the quantitatively major, fatty acid containing lipids (plus sterol) are considered.

The lipids covered in this text can be operationally split into the 
neutral and polar lipids depending on the solubility of the lipid in solvents of different polarities. The neutral lipids (NL) contain the acylglycerols (triacylglycerol (TRI), diacylglycerol (DI) and monoacylglycerol (MONO)) which consist of three to one fatty acids esterified to a common glycerol backbone. NL also contains the sterols (Ster) and stery1 esters (SE). Sterols share a common condensed ring structure (cyclopentanoperhydrophenanthrene) with at least one hydroxyl group capable of forming an ester. Steryl esters are sterols with a fatty acid esterified to carbon number 3 of the parent cyclic ring structure. The polar lipids (PL) usually contain a phosphate moiety so that a synonomous name for this group is the phospholipids. The two major PL in animals are phosphatidyl (P.) choline (PC) and P. ethanolamine (PE). Both lipids have a phosphate group attached to the third carbon of a glycerol backbone. The other two carbons of the glycerol are esterified with fatty acids. (This structure alone is called phosphatidic acid (PAD).) Attached to the phosphate are choline in P. choline and ethanolamine in P. ethanolamine. The lyso form of these lipids occurs if one fatty acid is removed. P. inositol (PI) and $P$. serine (PS) are two other, quantitatively minor PL. Both have the fatty acid-glycerol-phosphate (PAD) structure as described above with inositol or serine attached to the phosphate.

The last PL considered in this study was sphingomyelin. This differs from the phosphatidyl types by having, sphingosine instead of glycerol as the alcoholic component. Choline is attached to sphingosine via a phosphate linkage and the fatty acid is attached to sphingosine's amino group. 
Fatty acids are the common 1 ink between these lipid classes and will be discussed first.

\section{Fatty Acids}

In this text only the aliphatic, straight chain, unsubstituted fatty acids with a single carboxyl group will be considered. A variety of branched, cyclic and substituted fatty acids exist but they are quantitatively minor. In marine animals, fatty acids composed of 14 to 24 carbons are common.

There is no evidence that the primary de novo synthesis of fatty acids is any different in crustacea than in other higher animals. The process uses acetyl-CoA molecules and sequentially links them to form the end product palmitic acid (16:0)(16 carbons, no double bonds: refer to Appendix 4). A multi-enzyme system, fatty acid synthetase, is responsible for this de novo fatty acid synthesis and resides in the cytosol as a dimer where each identical monomer. is composed of seven different enzymes (unless specified, this section is from Gurr and James, 1980 and Mayes, 1983). Acetyl-CoA functions as a primer for the fatty acid synthetase system and malonyl-CoA, derived from acetyl-CoA and bicarbonate, provides the two carbon units which are added to the carboxyl end of the growing molecule. Once a 16 carbon chain has been formed, the product is released as palmitate.

Further elongation of this palmitate, or of nutritionally derived fatty acids, usually takes place in the membrane of the endoplasmic reticulum. This microsomal reaction requires acy1-CoA derivatives of fatty acids of 10 carbons or more. Malonyl-CoA is the donor of the two carbon units. Another elongation pathway, not as quantitatively important 
as the microsomal reaction, occurs in the mitochondria. This reaction is basically the reverse of the $B$-oxidation pathway.

The B-oxidation pathway is the primary catabolic reaction for fatty acids. A fatty acid is activated in the cytosol by conversion to its CoA form. Carnitine provides a transfer mechanism for this acyl-CoA to pass into the mitochondria where two carbon units (as acetyl-CoA) are sequentially cleaved off. A single palmitate (16:0) molecule, when catabolized, yields enough energy to form 129 ATP molecules from ADP and phosphate, resulting in a $48 \%$ conversion efficiency of energy. This high conversion efficiency of lipid to ATP, plus the hygrophobic nature of some lipids, make lipids an excellent energy storage form. (Being hygrophobic allows dense packing of material without accompanying water, unlike glycogen which requires relative large amounts of water.)

Fatty acids also occur in unsaturated forms where one or more carboncarbon double bonds exist. In animals, when two or more double bonds are present, the double bonds are almost always separated by one methylene group and are hence described as being methylene interrupted. Synthesis of monounsaturated fatty acids in animals is done with a $\Delta-9$ desaturase. The $\Delta$ (de1ta) number indicates the number of carbons from the carboxylic carbon of the fatty acid. An alternate scheme uses an $\omega$ (omega) number which indicates the number of carbons from the methyl end ( $\omega$ carbon) of the fatty acid.

The $\Delta-9$ desaturase in animals has a maximum affinity for 18 carbon

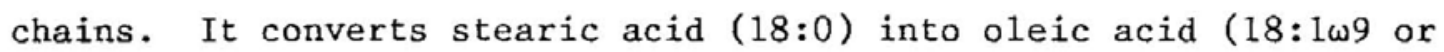

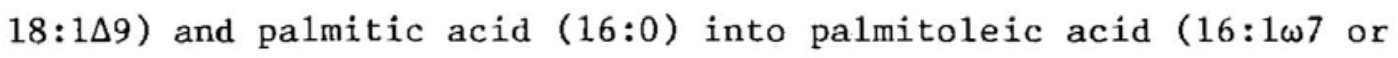
$16: 1 \Delta 9)$. 
When polyunsaturated fatty acids (PUFA) are formed by animals, additional double bonds can only be introduced between the carboxyl group and the nearest existing double bond. Therefore, de novo animal fatty acids can not have an $\omega$ number less than 7 ; though more of ten, because 18:0 is the preferred substrate, the $\omega$ number is 9.

Most animals have requirements for $\omega 3$ and/or $\omega 6$ fatty acids and therefore must obtain them via their diet. Plants are ultimately the source of these fatty acids as they are capable of introducing double bonds between existing double bonds and the $\omega$ carbon on fatty acids.

In 1930 , Burr and Burr proved that rats had certain dietary lipid requirements. It was shown that linoleic acid $(18: 2 \omega 6)$ and later arachidonic acid $(20: 4 \omega 6)$ fulfilled these requirements, though their mode of action was uncertain. The discovery of prostaglandins and the fact that arachidonic acid was the direct precursor of prostaglandins offered one mode of action for these essential fatty acids (EFA). Prostaglandins are the most characterized of the eicosanoids, a group of physiologically potent compounds derived from arachidonic and other 20 carbon PUFA (Mayes, 1983a). Other modes of action for EFA are 1ikely to be present, however, as the characteristic EFA deficiency symptoms are not cured with infusion of prostaglandins (Mayes, 1983a) (though prostaglandins are rapidly catabolized and so may never reach the required sites for their actions).

Animals other than the mammals on which the above work was based also require certain fatty acids. Castell (1979) reviewed the EFA requirements of finfish and their possible modes of action. Tinoco (1982) reviewed the requirements and functions of $18: 3 \omega 3$ in all animals. Several works (cf. 
Castell, 1979) have shown that fish require fatty acids of the w3 family. In trout, the EFA deficiency symptoms are similar to those seen in rats (Caste11, 1972a,b; Burr and Burr, 1929) which suggests that the $\omega 3$ fatty acids may be fulfilling some of the roles in trout that $\omega 6$ fatty acids fulfilled in rats. Other evidence comes from Richardson et al. (1961) with respect to mitochondrial functions. Certainly, prostaglandins exist that use eicosapentanoic $(20: 5 \omega 3)$ (the $\omega 3$ equivalent to arachidonic acid) as their precursor (Gurr and James, 1980). Since fish and invertebrates are poikilotherms, environmental temperature becomes an influencing factor. There has long been a recognized relationship between decreased temperature and increased fatty acid unsaturation (Lewis, 1962; Kayama et al., 1963; Knippeth and Mead, 1965; Kemp and Smith, 1970; DeTorrengo and Brenner, 1976; Farkas et a1., 1976, 1981a,b; Chape11e, 1978; Haze1, 1979; Hi11-Manning and Blanquet, 1980; Sellner and Hazel, 1982). This relationship is due to the required maintenance of cellular membrane fluidity. The greater the degree of unsaturation, the more fluid the cell membrane is at any given temperature. The connection to EFA is that all EFA are highly unsaturated, even more so if they are of the $\omega 3$ family. This does not, however, directly imply that membrane fluidity regulation is an EFA function.

In rats and man the EFA requirements can be fulfilled by $18: 2 \omega 6$ or

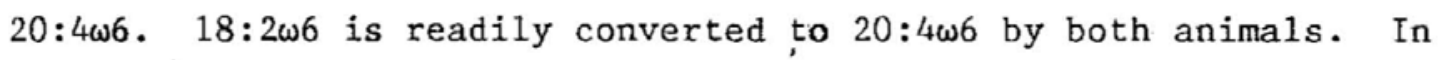
trout the EFA requirements can be met with $18: 3 \omega 3$ or $20: 5 \omega 3$ or 22:6w3. The latter two yield better growth (Takeuchi and Watanabe, 1977) but $18: 3 \omega 3$ can stil1 be readily converted to $22: 6 \omega 3$ (Owen et 
a1., 1975). In some marine fish, however, the ability to convert 18:3w3 to $20: 5 \omega 3$ or $22: 6 \omega 3$ is limited. In marine fish $20: 5 \omega 3$ or $22: 6 \omega 3$ are the essential fatty acids and 18:303 has on1y a slight sparing effect (Owen et a1., 1975; Fujii and Yone, 1976). The limited effect of $18: 3 \omega 3$ also applies to marine crustaceans where some are able to

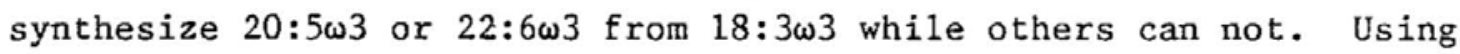
radiolabelled lipids or defined diets, the mysid Neomysis integer. (Leach)(Morris et al., 1973), the copepod Paracalanus parvus (Claus)(Moreno et al., 1979), the brine shrimp Artemia (Kayama et al., 1963) and the prawn Penaeus japonicus Bate (Kanazawa et a1., 1979c; Kayama et al., 1980) were shown to be able to elongate and desaturate $\cdot 18: 3 \omega 3$ to $20: 5 \omega 3$ and 22:6w3. In contrast, labelling studies with other crustaceans established that neither the prawns Penaeus monodon Fabricius and $\underline{P}$. merguiensis de Man (Kanazawa et al., 1979d) nor P. setiferus Linnaeus (Lilly and Bottino, 1981) were able to synthesize appreciable amounts of $20: 5 \omega 3,22: 6 \omega 3$ (Kanazawa et a1., 1979d) or 20:4w6 (Lilly and Bottino, 1981). Dietary studies, where growth was measured, demonstrated that $20: 5 \omega 3$ and $22: 6 \omega 3$ were nutritionally superior to 18:3w3 for P. japonicus (Kanazawa et a1., 1978, 1979a,h). This may indicate that there is a definite metabolic cost in converting $18: 3 \omega 3$ to $20: 5 \omega 3$ and $22: 6 \omega 3$, or that the rate of this conversion is not sufficient for rapid growth in this animal.

Studies with Homarus americanus have shown that they also grow better if $\omega 3$ fatty acids are present in the diet (Castell and Covey, 1976). One further point with $\underline{H}$ americanus is that there may also be some degree 
of requirement for $\omega 6$ fatty acids (Castell and Boghen, 1979).

When fatty acids occur unesterified to any other molecule they are termed free fatty acids (FFA) and fall within the neutral lipid category. In general, fatty acids do not exist in this carboxylic acid form because of their marked affinity for proteins which inhibits most enzymes. An exception to this is the mammalian transport form for lipids of FFA-albumen complexes. This exception does not apply for crustacea where the transport form for lipids generally appears to be phospholipids (Gilbert and O'Connor, 1970; Lee and Puppione, 1978; Teshima and Kanazawa, 1979, 1980). Instead, in crustacea, the presence of FFA is probably due to its active intermediary metabolic role resulting in a small, rapidly turning over FFA pool. Where FFA have been reported as major constituents, the high levels of FFA are usually artifacts due to enzyme degradation of other lipids.

Acylglycerols

The acylg1ycerols include the tri-, di- and mono- forms consisting of 3, 2 or 1 (respective1y) fatty acids esterified to the same glycerol molecule. The di- and mono- forms, though quantitatively small, are important in intermediary metabolism. Triacylglycerol, on the other hand, is a primary energy storage form and can account for the majority of lipids in an organism.

Diacylglycerols occur in three isomeric forms where the two fatty acids are esterified to either the 非, and 非2, 非 2 and 非3, or 非 1 and 非 carbons of g1ycerol. In early studies the 非1 and 非 3 carbons were not distinguishable and were thought to be metabolically equivalent; therefore, these studies recognized only the 1,2 and 1,3 isomers of 
diacylglycerol. Though similar levels of 1,2 and 1,3 isomers were found in the dungeness crab Cancer magister Dana (A1len, 1972), only the 1,2 isomer is thought to be metabolically important. 1,2-diacylglycerol functions as a direct precursor for triacylglycerols, P. choline and P. ethanolamine.

Monoacylglycerol also occurs in three isomeric forms depending on the position of the fatty acid on the glycerol backbone. Monoacylglycerol can serve as a precursor for diacylglycerol which then becomes transformed to triacylglycerol. While there is some evidence for a regulatory nature in adipose tissue this monoacylglycerol pathway is mainly evident in the absorption of lipids. In this process, triacylglycerols are rebuilt from the absorbed, partially broken down, 1ipids. Brockerhoff and his coworkers studied this process in Homarus americanus. They found that in H. americanus there appeared to be no preference between the 非 1 and 非 3 fatty acids of triacylglycerols and that prior to absorption, one or both were sp1it off (Brockerhoff et a1., 1963). Both the free fatty acids and monoacylglycerols that were formed could then act as emulsifiers to aid digestion prior to their absorption. Reformation of triacylglycerols occurred after absorption, but the original fatty acid pattern was not conserved (Brockerhoff et al., 1967).

In many crustacea, triacylglycerol appears to serve as a primary energy store. This can be inferred from a variety of studies (Dawson and Barnes, 1966; Guary et al., 1974; Clarke, 1977, 1979; Holland, 1978; Teshima and Kanazawa, 1983). This is not the case with all crustacea, however, as certain species predominantly store wax esters. 


\section{Wax Esters}

Only a brief overview will be given on wax esters as only minor quantities have ever been reported in Homarus americanus.

A wax ester consists of a fatty acid esterified to a fatty alcohol, resulting in a total of 26-42 carbons for most marine waxes (Sargent et al., 1976). The fatty acid moiety often reflects dietary intake while the fatty alcohol, of ten monounsaturated 20 or 22 carbons, does not (Lee et al., 1971).

Al1 of the crustaceans with significant stores of wax ester that have been examined have the capacity for de novo wax ester synthesis (Kayama and Nevenze1, 1974; Sargent et al., 1976). They function, of ten in conjunction with triacylglycerols, as energy stores (Lee et al., 1970; Bensen et a1., 1972; Morris and Sargent, 1973; Lee and Barnes 1975); however, many additional possible explanations for their presence have been offered. These range from buoyancy control (Bensen et al., 1972; Lewis, 1970) to optimizing energy storage by maintaining cellular redox balances (Sargent, 1978). Wax esters are not transferred through the food chain since most organisms hydrolyze ingested wax esters and immediately oxidize the fatty alcohols to fatty acids (Patton and Benson, 1975; Sargent et al., 1979). $\underline{\text { Sterols and Stery } 1 \text { Esters }}$

Mammals synthesize sterols from acetyl-CoA. It is first converted to mevalonate, then isoprenoid units and, then squalene. Squalene is then turned into the parent steroid compound lanosterol (Mayes, 1983b). Cholesterol, which is the most abundent animal sterol (Lehninger, 1975) is formed from lanosterol via several additional steps. 
Crustaceans, in contrast, appear not to be able to synthesize sterols from either acetate or mevalonate (species studied include the crabs Callinectes sapidus Rathbun (Whitney, 1970), Cancer pagurus Linnaeus (van Den Ord, 1964), the crab Portunus trituberculatus (Miers), the prawn Penaeus japonicus, the lobster Panulirus japonica (Siebold)(Teshima and Kanazawa, 1971a); the crayfish Astacus astacus Linnaeus (Zandee, 1966) and the lobsters Ibacus ciliatus (Siebold)(Teshima and Kanazawa, 1976) and Homarus gammarus (Zandee, 1967)).

Conversion of other sterols to cholesterol is possible, however. Ergosterol was shown to be converted to cholesterol by a number of crustacean species (Kanazawa et a1., 1971b; Teshima and Kanazawa, 1971b; Teshima 1971a, 1972), as was ß-sitosterol (Kanazawa et al., 1971b; Teshima 1971b, 1972), 24-methylcholestero1 (Teshima, 1971b, 1972), stigmastero1 (Kanazawa, et al., 1971b) and brassicastero1 (Teshima, 1972). Since de novo synthesis is not possible, crustacea must obtain cholesterol via their diet. For normal growth, there is a confirmed nutritional requirement in several crustacea (Kanazawa et a1., 1971a; Teshima, 1972), including Homarus americanus (Castel1 et a1., 1975). As in other animals, cholesterol is the predominant sterol in crustaceans, though many other types are present (Teshima, 1972; Goad, 1978). In H. americanus cholesterol accounts for $97.8 \%$ of the sterols, with 24-methylcholestero1, 24-ethylcholesterol, desmosterol, 24-methylenecholesterol and 22-dehydrochọlesterol also present (Gagosian, 1975).

Metabolically, cholesterol is essential for modulating cell membrane fluidity though other regulatory roles seem Iikely (Bloch, 1983). In 
mammals it is also used as the precursor for bile salts (Lehninger, 1975). Crustaceans, however, do not form bile salts. Instead they use fatty acids as a base to form fatty acyl-sarcosy1-taurines, which are detergent-like emulsifiers that are analogues to the vertebrate bile salts (Vonk, 1969; Lester et a1., 1975).

Cholesterol can also be metabolically transformed into steroid hormones. In crustacea, one such hormone is ecdysterone (Kater and Spaziani, 1971) which is generally acknowledged as the molting hormone (Gagosian and Bourbonniere, 1976; Gilgan and Burns, 1979; Chang and Bruce, 1981; Soumoff and Skinner, 1983).

Studies on steryl esters with crustacea are scarce and, as yet, their functions are unknown. In mammals, cholesterol can only be absorbed as free sterol. Steryl esters are first hydrolyzed to remove the fatty acid and after absorption most of the sterol is re-esterified. The proportion of sterols to steryl esters in mammals is tissue dependent, with no stery1 esters in the brain and nervous tissue where cholesterol is a component of myelin, and high levels in the adrenals and blood plasma.

In the spiny lobster Panulirus interruptus (Randa11) hemolymph lipoproteins, free sterols account for $4 \%$ of the 1 ipid, while only trace amounts occur in the esterified form (Lee and Puppione, 1978). When radiolabelled cholesterol was injected into the prawn Penaeus japonicus, less than $2 \%$ of the labelled sterols in the hemolymph were in the esterified form (Guary and Kanazawa, 1973). In contrast to mammals then, crustaceans may transport cholesterol in the unesterified form (Ceccaldi and Martin, 1969).

One hypothesis for steryl esters is that they function as storage 
forms for sterols (cf Whitney, 1969). Whitney examined the sterol content of developing crab (Callinectes sapidus) eggs and found a decreasing level of steryl esters with development, while total sterols remained constant. She hypothesized that steryl esters may be acting as a storage form, releasing free sterol for new tissue growth during development. Phospholipids - phosphoglycerides

The phosphoglycerides (glycerol is the alcohol) are the dominant form of phospholipids. In animals phosphatidyl (P.) choline (PC) is predominant followed by $\mathrm{P}$. ethanolamine $(\mathrm{PE})$.

Both PC and PE are synthesized from diacylglycerol and cytidine phosphate activated choline or ethanolamine. An alternative pathway for PC synthesis is the methylation of the ethanolamine nitrogen of $\mathrm{PE}$ (Lehninger, 1975); although this was found to be limited in the crab Eriochier sinensis (Milne Edwards)(Chape11e et a1., 1981). A requirement for choline is found in the prawn Penaeus japonicus (Kanazawa et a1., 1976b). The primary function for these 1ipids is in the cell membrane where they serve as structural components and possibly perform as aids to enzyme activity (Gurr and James, 1980).

As mentioned earlier, the phosphoglycerides in crustacea also appear to be the transport form for lipids (Gilbert and $0^{\prime}$ Connor, 1970; Lee and Puppione, 1978; Teshima and Kanazawa, 1979, 1980). In several species approximately $65 \%$ of the hemolymph lipids were phospholipids (B1igh and Scott, 1966; Gilbert and O'Connor, 1970); When the prawn Penaeus japonicus was fed labelled triacylglycerol (tripalmitin), greater than $80 \%$ of the label in the hemolymph was in the phospholipids (Teshima and Kanazawa, 1980). 
The type of 1ipoprotein phospholipid in marine decapods seems to be limited to PC as $82 \%$ of the 1ipoprotein 1ipid was PC in the 1obster Panulirus interruptus, versus $2 \% \mathrm{PE}, 3 \%$ sphingomye 1 in and $1 \% 1$ yso PC (Lee and Puppione, 1978).

Another possible function for PC is related to nutrition. Homarus americanus have exhibited better growth when certain types of PC (the type characterized by the source, i.e. fatty acid composition) are present in significant levels of their diet (Boghen and Caste11, 1980; Conk1in et a1., 1980; D'Abramo et al., 1981). Without PC in their formulated diets, juvenile $\underline{H}$. americanus of ten failed to molt properly (Conklin et al., 1980). The mechanism responsible for $P$. choline's effects is unknown though there was a correlation between hemolymph levels of cholesterol and phospholipid with dietary soy PC (D'Abramo et a1., 1982). PC did not appear to aid in nutrient absorption ( $D^{\prime}$ Abramo et al., 1982) nor was the choline moiety the important factor (Conklin et al., 1980).

The fatty acid composition of the phospholipids in crustacea is general1y more unsaturated than the neutral lipids (DeKoning, 1966, 1970; Chapelle and Pequeux, 1982). Within the phospholipids, PE is more unsaturated than PC (DeKoning, 1966; Addison, 1972; Mahr1a, 1975; Lee and Consoulin, 1979; Chape11e and Pequeux, 1982). The isomeric position of the fatty acid within the phospholipid also correlates with the degree of unsaturation, as the fatty acid on carbon 2 of the glycerol is more unsaturated (Brockerhof $\mathrm{f}$ et al., 1963).

\section{Phosphosphingolipid}

Phosphosphingolipid (sphingosine is the alcohol) is the minor group of phospholipids. Sphingomyelin', which contains choline, is the most 
common phosphosphingolipid. In relation to the phosphoglycerides in the whole animal, sphingomyelin is a relatively minor component; however, it is present in proportionately much greater amounts in some tissues where it may be the dominant PL. Examples are the erythrocyte membranes of mammals (Gurr and James, 1980) and the myelin sheaths of nerves (Deue1, 1955). In lobsters, the nerves, which are not myelinated, sti11 have relatively high levels of sphingomyelin (Sheltawy and Dawson, 1966; Condrea et a1., 1967; Chako et a1., 1977). Crustacean Lipids: Physiology

The physiological events of molting, development, feeding, and starvation all affect an organism's lipid metabolism and composition. Since only subadult stages of Homarus americanus were utilized in this study, reproduction and sex, both important factors affecting lipid regulation (Adiyodi and Adiyodi, 1970, 1971; Middleditch et a1., 1979; Read and Caulton, 1980; Teshima and Kanazawa, 1983) are not reviewed. Feeding, Transport and Starvation

In many crustaceans, including Homarus americanus, preliminary digestion of ingested lipids occurs in the pyloric region of the stomach via the action of lipases secreted by the hepatopancreas ( $0^{\prime}$ Connor and Gilbert, 1968; Brockerhoff et al., 1970); the level of 1ipase activity being influenced by the recent dietary history of the animal (Hoyle, 1973). With triacylglycerols the lipases preferentially hydrolyze the $\alpha$ fatty acids (on carbons 1 and 3)(Brockerhoff et al., 1964, 1967). This same preference may also app1y to phosphog1ycerides, forming FFA and 1yso derivatives (Brockerhoff, 1964; Turunen, 1979). The ingested material is peristaltically moved into the midgut or hepatopancreas tubules where 
digestion continues (Barker and Gibson, 1977; Leavitt et a1., 1980) and where the ingested material comes into contact with the brush borders of the R-ce11s of the hepatopancreas. The brush borders possess intense phosphatase activity (Barker and Gibson, 1977) and, in the crab Scylla serrata (Forskal), is the main site of lipase activity (Momin and Rangneker, 1975). The lipids are absorbed via these brush borders and accumulate in vacuoles in the R-cells. In the R-cells lipids may undergo further digestion, as low levels of phosphatases and lipases are present (Barker and Gibson, 1977). Since reformation and storage of TRI and PL occurs in these cells, however, these enzymes probably are present for transport and metabolism of these stored 1ipids.

The type of ingested 1ipid may affect digestion and absorption. PC, an apparently essential lipid for Homarus americanus (Boghen and Caste11, 1980; Conklin et al., 1980) was thought to aid in absorption of 1ipids, in particular cholesterol (Lester, 1975; Kanazawa et al., 1979g), since PC is an effective emulsifier. D'Abramo et al. (1981,1982) do not, however, believe PC aids in this manner; they speculate instead that PC is involved with lipid transport. In either case, the inhibitory effect that PC has on TRI digestion in mammals (Klein et al., 1967; Borgstrom, 1980) has not been established for crustaceans.

The principle storage site for 1ipids in crustacea is the hepatopancreas (Gilbert and O'Connor, 1970) with muscle tissue as a secondary site. In fed Homarus americanus, for example, the lipids in the hepatopancreas range from 59.4 to $65.1 \%$ (dry weight)(Stewart et al., 1972 ), or 13.6 to $29.2 \%$ (wet weight) (D'Abramo et al., 1982). The 1ipids in the hepatopancreas are mainly neutral (mostly TRI), with a neutral to 
polar ratio greater than 3.4 . In $\underline{\mathrm{H}}$ americanus muscle, the $\mathrm{PL}$ were the dominant type (D'Abramo et al., 1981).

Lipid transport, from the hepatopancreas to the rest of the body, occurs via the hemolymph. The hemolymph contains varying amounts of lipids depending on dietary history, molting stage and so forth, with the lipids existing almost exclusively within a lipoprotein complex (Lee and Puppione, 1978). In Homarus americanus the average leve1 in captured adults was $2.3 \mathrm{mg}$ of 1ipids per $\mathrm{ml}$ of hemolymph (B1igh and Scott, 1966). Lipoproteins are categorized by density level; in the spiny lobster, Panulirus interruptus, the densest (HDL - high density 1ipoprotein) was responsible for more than $90 \%$ of the lipids (Lee and Puppione, 1978). The dominant lipid type in the 1ipoproteins was PL, with PC being the most common (B1igh and Scott, 1966; Allen, 1972; Lee and Puppione, 1978; Teshima and Kanazawa, 1980).

The actual process of lipid mobilization from reserves and the formation of a lipoprotein complex is unknown in crustacea, although the lipoprotein itself may facilitate the lipid release from the hepatopancreas (Teshima and Kanazawa, 1978b).

During periods of starvation the energy reserves must be utilized. In a number of crustacea, Iipid has been implicated as the major source of energy during starvation (Heath and Barnes, 1970; Armitage, 1972), while in other studies protein was found to be just as, or more, important than lipids (Neiland and Scheer, 1953; Schafer,, 1968; Spech and Urich, 1969; Hazlett et a1., 1975; Dall, 1974, 1981; Regnault, 1981).

The type of lipid that is utilized during starvation is predominanty a neutral lipid. In a number of copepod species NL was utilized prior to 
the use of PL (Lee et a1., 1974; Mayzaud, 1976). In the crab Carcinus maenas (Linnaeus), a large drop of hepatopancreatic NL occurred with starvation while the PL increased (Heath and Barnes, 1970). Mo1ting

The process of molting has been correlated with changes in the lipid composition and metabolism of the organism. In general, the absolute levels of lipid increase in the body and hepatopancreas during the early and mid premolt stages, followed by a decline during the late premolt, molt and recovery stages (Paul and Sharpe, 1919; Renaud, 1949; Travis, 1955a; Kanazawa et al., 1976a; Spindler-Barth, 1976; Teshima and Kanazawa, 1976; Ando et al., 1977; Teshima et al., 1977; Galois, 1980; Read and Caulton, 1980; Barclay et a1., 1983). This fluctuation of lipid is apparently a reflection of its function as a major energy reserve responsible for maintaining the animal through its molt. An increasing level of lipids is also observed after eyestalk ablation (0'Connor and Gilbert, 1968; Bollenbacher et al., 1972), though not after ecdysterone injection (Bollenbacher et a1., 1972).

The source of the increased levels of lipids during the molt cycle is an increase in lipid synthesis (0'Connor and Gilbert, 1968, 1969; Bollenbacher et a1., 1972; Patrois et a1., 1978) that is possib1y supplemented with an increased rate of dietary 1 ipid absorption (0'Connor and Gilbert, 1969).

Few studies have correlated fatty acid metabolism with molting. 0'Connor and Gilbert (1969) examined the freshwater crayfish Orconectes virilis (Hagen) and found practically no change in the fatty acid composition of NL and PL between intermolt and premolt animals. They also 
examined the land crab Gecarcinus lateralis (Freminville) and found an increase in $18: 1 \omega 9$ and a decrease in $18: 2 \omega 6$ in the hepatopancreatic PL after eyestalk ablation. The intermolt stage of the prawn Penaeus japonicus was found to have more $18: 1 \omega 9$ and less polyunsaturated acids

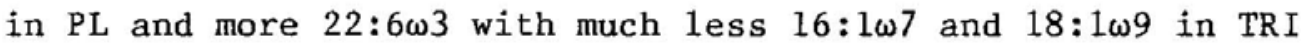
(Teshima et al., 1977). In a shrimp the TRI fatty acids did not change with the molt cycle, while the PL showed higher levels of saturated fatty acids during intermo1t (Teshima and Kanazawa, 1976).

\section{Early Development}

of the studies considering lipids during the developmental periods most have measured only total lipids and then usually for an energetic analysis. In the shrimp Crangon crangon (Linnaeus)(Pandian, 1967), the hermit crab Eupagurus bernhardus (Linnaeus)(Pandian and Schumann, 1967), and the barnacles Balanus balanoides (Linnaeus) and B. balanus (Linnaeus)(Barnes, 1965), a significant decrease in the lipid content occurred during embryogenesis that reflected the role of lipids as the main, or a major, energy source. A comparable decrease in 1ipids was seen in the lobsters Homarus gammarus (53\%, Pandian, 1970b; 60\%, Saudray, 1954) and $\underline{\mathrm{H} .}$ americanus (\% not stated, Pandian, 1970a). In $\underline{\mathrm{H}}$. gammarus 1ipids were the main energy source for embryogenesis and were responsible for as much as $87.7 \%$ of the expended energy (Pandian, 1970b). A similar, less extreme, interpretation was also presented for $\underline{H}$. americanus (Pandian, 1970a). In H. gammarus the decrease in lipids was greater than the decrease in dry weight, a phenomenon interpreted to indicate a metabolic use for 1ipids other than strictly for energy (Saudray, 1954). It should be noted that 1 ipids may not always be the main energy source for 
embryogenesis, since in the prawn Macrobrachium idella (Hilgendrof), protein catabolism was thought to supply most of the energy (Vijayaraghavan and Easterson, 1974).

In the few studies available on crustacean larvae no general trend is apparent when total lipids are considered. In the larval development of the crab Rhithropanopeus harrisii (Gould)(Frank et al., 1975) the lipid levels, expressed as gross dry weight, increased in total amount but fluctuated if expressed as a percentage of the animal's wet weight. In the prawn Macrobrachium ide11a (Vijayaraghavan and Easterson, 1974) the lipid levels remained the same as gross dry weight but increased if expressed as a percent of dry weight. In the crab Hyas araneus (Linnaeus), lipid as absolute levels increased, but as percent dry weight tended to decrease, from hatching to metamorphosis (Anger et a1., 1983). In Homarus americanus the lipid levels, as gross dry weight, increased for the first four larval stages and declined for the fifth (Capuzzo and Lancaster, 1979a).

Few studies have examined fatty acid changes in developing crustacean 1arvae. Ward et a1. (1979) studied the egg, nauplius, protozoea, mysis and early postlarva of the shrimp Penaeus setiferus. A shift in the major fatty acids was seen from the egg to postlarva. Their data suggest an energy dependence for lipids during the egg and early larval stages and a buildup of lipid reserves in the older stages. During development, the abundant, non-essential fatty acids were utilized, while $22: 6 \omega 3$ increased. This suggests a selective use of fatty acids in the larvae, though the effects of diet were not determined. Waldock and Holland (1978) examined the fatty acids of triacylglycerols in developing 
non-feeding barnacle ( extensive triacylglycerol utilization, but no change in fatty acid composition, which indicates non-selective fatty acid utilization. $\underline{\text { Summary }}$

The heterogeneous nature of lipid, with its wide range of metabolic uses from structural components to energy substrates to hormone precursors, results in its many integral functions in the development of crustaceans. Yet, little is known about the relative importance of the different lipids through development and the extent to which the 1ipid metabolic system in crustaceans responds to stress. The early developmental period of crustaceans, though lacking as a subject of physiological and biochemical studies, is especially well suited for the elucidation of lipid functions due to the rapid relative growth and limited or defined metabolic reserves of the developing animal. These gaps in our knowledge of the physiology and biochemistry of ear1y development and lipids in crustaceans were the subjects of this work. This dissertation focused on the biochemical changes associated with early development in $\underline{H o m a r u s}$ americanus with an emphasis on the metabolism and biochemistry of 1ipid. 
MATERIALS and METHODS

The ear1y 1ife history of the American 1obster, from fertilization of the egg to the end of the larval stages, was studied in this work. Samples taken during this period were analyzed for their major biochemical constituents, with an emphasis on the lipid class and fatty acid patterns. The general culturing and sampling techniques will be covered first, followed by descriptions of each experiment and lastly, the details of each analytical method.

The early life history stages can be conveniently divided into two groups; the egg -- covering the period from egg extrusion and fertilization to hatch, and the larvae -- covering the period from hatch to the end of the fourth larval stage.

\section{Culture and Sampling: Egg}

Female lobsters normally extrude their eggs between June and September (Aiken and Waddy, 1980). Fertilization occurs at this time and the eggs are held under the tail, attached to the pleopods. In order to sample the entire period from fertilization to hatch, it was necessary to obtain lobsters that would extrude eggs while in the laboratory. This was accomplished by examining the cement gland structures of the pleopods according to the method of Aiken and Waddy (1982). Lobsters from local fish markets (collected locally nearshore) were examined and potential egg producers brought into the laboratory (E.S.L. -- Environmental Systems Laboratory, Woods Hole Oceanographic Institution) and held in individual 951 aquaria with flowing seawater at'ambient temperatures (initially $23^{\circ} \mathrm{C}$ ). They were fed squid and fish. Within one month approximately $50 \%$ of the lobsters had extruded, eggs, though three of these four egg bearing lobsters dropped their eggs within the first three weeks. Though 
the stress of the culturing conditions may have caused this egg loss, identical culture conditions have successfully been used to raise numerous other lobsters (caught with eggs) for months. One possibility is that these females had not successfully mated since the previous molt so that the eggs were not fertile (Aiken and Waddy, 1980). The single female lobster with viable eggs had extruded on August 23, 1981 and was kept in the aquaria with flowing seawater at ambient temperatures (see Graph 1A) through June 1982 when the larvae hatched. During this period she was fed squid, clams and fish. Samples of eggs were taken periodically (weekly or bi-week1y), with a last sample of hatched larvae taken on June 14, 1982 . The eggs were detached from their surrounding cement matrix, measured with an ocular micrometer in a dissecting scope for the greatest egg diameter and, if present, the embryonic eye width and length (Perkins, 1972). They were then dipped quickly into distilled water to remove external salt and blotted dry on a paper towel. Three pooled samples of two eggs each were taken for ash analysis, four pooled samples of two eggs each were taken for protein and carbohydrate (both determined from the same sample) and two pooled samples of 10 eggs each were taken for 1ipid analyses. Each sample was measured for wet weight to the nearest $0.1 \mathrm{mg}$ on a Mettler H15 balance. The non-lipid samples were then frozen and 1yophilized. After 1yophilization, they were weighed to the nearest 0.01 mg on a Mettler M5 microbalance and stored at $-20^{\circ} \mathrm{C}$ until ana1ysis. These provided the wet to dry weight conyersions for the samples for 1ipid and chitin analyses. After wet weighing, the samples for lipid analysis were immediately extracted for lipids with the procedure listed later (under Lipid Analysis). 
A second set of egg samples was taken from a lobster which had extruded her eggs in the wild (offshore, Buzzards Bay, MA) prior to being brought into the laboratory in mid-September 1982. This lobster was held in a 951 aquarium with flowing heated seawater at a constant temperature of $22-24^{\circ} \mathrm{C}$ to accelerate embryogenesis. Hatching began in early January 1983 and samples were taken at this time. The time until hatch for each of these samples was estimated and adjusted according to the time of sampling and the time of the major hatches. Five samples were taken, consisting of: (1.) eggs 3 to 7 days from hatch, gold in color; (2.) eggs $12 \mathrm{~h}$ to 3 days from hatch, gold in color; (3.) eggs 0 to 1 day from hatch, blue in color (Davis, 1964); (4.) larvae, hatched but not yet swimming; (5.) 1arvae, swimming, within $36 \mathrm{~h}$ of hatching (this represents the maximum time range, swimming larvae were collected within $6 \mathrm{~h}$ of their appearence in the water). The egg samples were treated in the same manner as the first series of egg samples; the larval samples were treated as described below.

\section{Culture and Sampling: Larvae}

Lobsters with eggs were collected offshore near Buzzards Bay, MA by Fred Stowe11, Jr. in 1980-82. They were transported to the laboratory (E.S.L.) and individually held until hatching in 951 aquaria with flowing seawater at ambient temperatures $\left(12-19^{\circ} \mathrm{C}\right)$. The hatching period lasted from one to two weeks, with a major hatch (or larval release) occurring approximately every day. The majority of the larvae were released during a peak hatching period which lasted from two to four days. The major hatch times were approximately synchronous from day to day for a single lobster, though the time of day for this hatch varied between lobsters. 
There was no preference for night hatching in the present study, although such a preference was seen in another study (Ennis, 1973). This lack of preference may be due to the fluctuating ambient light levels and lack of dark periods. Ennis (1973, 1975) observed synchronous hatching in Homarus gammarus but not in $\underline{H}$ americanus since the latter showed erratic larval release times; however, he was counting the numerous small hatches as 'well which may have disrupted any pattern. The synchronous major hatches seen in $\underline{H}$. americanus in this work allowed the prediction of upcoming hatch times so that all the hatches that were used in these experiments were collected within 30 minutes of their release from the female. Only the major hatches during the peak hatching periods were used.

To minimize individual variation and provide baseline animals as nearly identical as possible, only larvae from a single hatch from a single female were used for each experiment. Each hatch was placed in a separate kriesel according to the method of Hughes (1974). Water was filtered to less than $100 \mu \mathrm{m}$ and flowed through the kriesel only once. Flow rate was sufficient to suspend the larvae. The tank inner filters and tank bottoms were cleaned daily. Water temperature varied between and within experiments within the range of $15-23^{\circ} \mathrm{C}$. The exact temperatures for each experiment are shown in graphs $1 \mathrm{~A}, \mathrm{~B}, \mathrm{C}$.

The larvae were sampled periodically, with the time between samples dependent on the experiment. To ensure that all the larvae were in the same stage of development, they were molt staged under a microscope and only those larvae that were in the most common molt stage were used. The remainder were replaced into their kriesels. The molt staging procedure 
was adapted from Aiken's $(1973,1980)$ method for adult Homarus americanus and is detailed in Appendix 1. The photoperiod, which does not seem to influence the molt cycle in the lobster (Aiken, 1969), was approximately a 14 h light, 10 h subdued light cycle.

The sampled larvae were kept in seawater until immediately prior to their wet weighing. To prepare for weighing, the larvae were placed on a nylon mesh which was quickly dipped in distilled water. The larvae were then blotted dry on a paper towel and individually weighed on a Mettler H15 balance to the nearest $0.1 \mathrm{mg}$. Only a few larvae were prepared at one time so that the drying time could be kept as consistent as possible. Three individuals were used for ash values, four for protein and carbohydrate (both analyzed from the same sample) and two pooled groups of 2-5 individuals for lipid analysis. (Five first and second stage larvae, three or four third stage larvae and two or three fourth stage larvae were used per sample.)

Once weighed, the larvae not to be used for lipid analysis were frozen, lyophilized and weighed to the nearest $0.01 \mathrm{mg}$ on a Mettler M5 to provide the wet to dry weight conversions for the larvae for the lipid and chitin analyses. These lyophilized larvae were then stored at $-20^{\circ} \mathrm{C}$ until analysis. After the wet weighing, the two pooled groups were immediately extracted for lipids as described later (under Lipid Analysis). Experiments: Eggs

The culture conditions for the experiment following egg development were kept as natural as possible as the intent of this study was to determine the biochemical changes associated with normal embryogenesis. A later study required the use of temperature accelerated embryogenesis to 
allow close examination of the period surrounding the hatching or eclosion event.

\section{Experiments: Larvae}

\section{Baseline, normal development}

A major goal was the determination of the biochemical changes associated with normal larval development. The term "normal development", however, implies natural development, i.e. what occurs in the wild. Unfortunately, such a study is unfeasible as it would be impossible to obtain a time series of samples throughout the developmental period from the same population. Obtaining samples of each larval stage from one sampling expedition, though possible, would not yield much information either as each of the different larval stages would have experienced completely different environmental conditions during their respective developments. In fact, any feasible field study would involve too much individual variation to be able to allow examination of subtle, or even not so subtle, changes.

The only alternative is to culture the organisms in the laboratory. While it is possible in the laboratory to closely mimic the natural environment as to food type and availability, such an attempt would only introduce unaccountable variation. Instead, only one or two types of food, at constant or timed availability, should be used so that correlations between diet and larval composition can be made. For larval Homarus americanus, an accepted total diẹt has been the brine shrimp, Artemia (Hughes et al., 1974). (The binomen Artemia salina Leach is no longer valid and until speciation in brine shrimp is better understood, only the genus designation should be used (cf. Persoone, et a1., 1980)). 
Artemia, however, lack or have low levels of certain fatty acids (Watanabe et al., 1978; Fujita et a1., 1980; Schauer et a1., 1980) which are thought to be essential for many crustaceans, including the lobster (Kanazawa et a1., 1978, 1979b,1979c,1979f; Caste11 and Covey, 1976). Therefore, a dietary supplement containing cod liver oil was formulated (see Appendix 2) and fed daily to supplement the three to four daily feedings of Artemia. The Artemia used were frozen subadults and adults from San Francisco Bay. Prior to each feeding the excess food from the previous feeding was removed. An automatic feeding device was used for the night-early morning feeding. For all the experiments, a total of 11 lots of brine shrimp were used where each lot consisted of a two pound frozen package (Table 1).

This diet and ad libitum feeding levels (3-4 times daily) were meant to enhance the culturing conditions so that the larval development, used as the baseline and as representative of a "normal development", was as optimal (i.e. healthy) as possible.

Samples were taken at least once daily, with the exception of the fourth stage larvae where the period between samples was sometimes extended to two or three days. Two hatches were followed from the moment of hatch to near the end of the fourth larval stage. (Each hatch or series was given a code identification; e.g. I-3, where the roman numeral identified the female lobster and the end number was the day of that hatch as counted from the first day where hatching occurred.) For the baseline development series, hatches I-3 and I-5 (both from the same lobster, hatch days 3 and 5) were used. They were fed from brine shrimp lots 非's 5-10. 


\section{Baseline vs Starvation}

Hatch I-5 was used to determine the biochemical effects of prolonged starvation on the developing larvae. It was a large hatch $(>1000)$ which allowed portions of it to be separated and the larvae individually isolated without food. The remaining bulk of the hatch, which was used as the fed control, also served as a baseline normal development series.

Four separate groups were isolated from the parent hatch for these starvation trials. Using the time of hatch as day zero the times that these groups were isolated, and hence began their starvation, were day 0 (time $0 \mathrm{~h})$ (at hatch), day $3(70 \mathrm{~h})$, day $4(107 \mathrm{~h})$, and day $7(167 \mathrm{~h})$.

The larvae were first molt staged and only the most common stage found at that time was used. For the four isolated groups, their initial molt stages were $1 \mathrm{~A}$ (day 0$), 1 \mathrm{D}_{1} "$ (day 3 ), $1 \mathrm{D}_{2-3}$ (day 4 ), and $2 \mathrm{D}_{0}$ (day 7). The number refers to the larval stage (first through fourth) and the letter and numbers after the hyphen are the molt stage (see Appendix 1). The larvae were then individually isolated in fine plastic mesh containers approximately $4 \mathrm{~cm}$ in diameter and approximately $12 \mathrm{~cm}$ high. These were held in a larger plastic chamber sectioned off into an $8 \times 8$ grid. Each square of the grid was $8 \times 8 \mathrm{~cm}$ with an open mesh bottom to allow for water flow. Two plastic mesh containers were placed in each grid square, giving a total of 128 larvae as the starting number of each starvation group. The plastic grid chambers sat in flowing $1 \mu \mathrm{m}$ filtered seawater inside a shallow trough, with airstones providing adequate water flow past each mesh container. The depth of the water which the larvae experienced was approximately $9 \mathrm{~cm}$. Prior to the experiment, every container had been thoroughly cleaned of any growth. 
Samples were taken from these starved groups each day at the same time that the fed control group was sampled. Starved samples were molt staged and treated in the same manner as the control samples. Pulse Fed and Immediate Posthatch Development

To elucidate the gross flow of nutrients through the larvae upon feeding and the biochemical changes immediately after hatching, a newly hatched group of larvae was fed and sampled on a closely regulated schedule. For this experiment, a lobster which had been kept at elevated temperatures of $22-24^{\circ} \mathrm{C}$ was used. The resulting accelerated embryogenesis produced larvae in January.

Upon hatching, a group of approximately 250 larvae was placed in a kriesel at an elevated water flow rate. The flow rate and low larval density minimized the chances of cannibilism. Between $12-18$ hours after hatching the larvae were allowed to feed on Artemia ( 1 ot $\equiv_{111}$ ). The next period of feeding occurred between 36 - 40 hours after hatching. During the first 36 hours the larvae were sampled every six hours, followed by sampling every four hours until 60 hours after hatching. These larval samples were taken and analyzed with the same procedures used for the baseline normal development samples.

\section{Fatty Acid Supplement}

The fatty acid feed supplement (see Appendix 2) used for the baseline studies was formulated to add the fatty acids 20:5 and 22:6 to the diet of the larvae. Prior to its use, two hatches (II-4 and II-7) were fed solely with Artemia (lot 非s 1-4) and their samples provided the data on larval development under an essential fatty acid (EFA) deficient (low levels of EFA were present, see Table 1) diet. 
To provide a comparison between these EFA deficient induced results and the baseline normal development results, Hatch $I-3$ was split into equal groups at hatch and placed in separate kriesels. Both groups were cultured as described for the baseline normal development trials, with the exception that for one group the feed supplement did not contain cod liver oil. Sampling of this group was intermittent, with $3-6$ days between samples.

\section{Labelled Fatty Acids}

In an attempt to determine the metabolic fate of ingested fatty acids, larvae were isolated and fed diets containing ${ }^{14} \mathrm{C}$ labelled fatty acids. Two fatty acids were used -- $1-{ }^{14} \mathrm{C}$ palmitic acid (16:0), a non-essential fatty acid and $1-{ }^{14} \mathrm{C}$ eicosapentaenoic acid $(20: 5 \omega 3)$, an essentia1 fatty acid. After being fed the label, half the larvae were maintained on Artemia (lot 非1) and the other half starved. Samples were taken periodically and analyzed for their 1ipid class structure and ${ }^{14} \mathrm{C}$ incorporation.

To minimize the influence of the molt cycle, only larvae initially at stage $\mathrm{C}$ were used. These larvae were hatched in January from a female kept at $22-24^{\circ} \mathrm{C}$ since the previous September. Second stage larvae were used as prior work had shown that 1C (1st larval stage, molt stage C) larvae did not always ingest significant amounts of the EFA feed supplement .

Ninety six molt staged larvae were placed in individual $150 \mathrm{ml}$ plastic cups which were capable of being sealed with airtight plastic screwtop 1ids. Each cup contained $20 \mathrm{ml}$ of $1 \mu \mathrm{m}$ filtered seawater $(1 \mu \mathrm{m}$ filtered seawater was used throughout) which was changed every $12 \mathrm{~h}$. The . 
cups were kept in a constant temperature environment of $22^{\circ} \mathrm{C}$.

Three groups of larvae were formed -- two groups of 30 that were each fed a labelled diet and one control group of 36 that was fed a non-labelled diet. Each of these groups was subdivided again into a fed group and a starved group. The labelled diet was made in a similar fashion as the EFA enhanced feed supplement except for additional procedural steps. As 1isted in Appendix 2 (preparation of 1abelled feed), the feed was divided into 33 approximately equal sized pieces and each piece weighed to the nearest microgram with a CAHN mode1 29 electrobalance. Three random pieces from each labelled feed were counted for radioactivity by dissolving them in one $\mathrm{m} 1$ of water in a scintillation vial and then mixing in $10 \mathrm{ml}$ of Aquasol (New England Nuclear) (Aquasol contained acetic acid at $7 \mathrm{ml}$ per liter as an anti-chemiluminescent agent). Counting, which was done with a Beckman Mode1 100 LSC using the ${ }^{14} \mathrm{C}$ wide channel, showed a mean and standard deviation for the 'DPM/ $\mu \mathrm{g}$ feed' ratio of $3220 \pm 169$ and $3053 \pm 117$ for the ${ }^{14} \mathrm{C} 16: 0$ and ${ }^{14} \mathrm{C}$ 20:5w3 labelled feeds respectively. ${ }^{14} \mathrm{C}$ toluene was used as an internal standard.

Once the larvae were transferred to the plastic cups, food was withheld for $24 \mathrm{~h}$ prior to the introduction of the nonlabelled or 1abelled feeds. Two hours prior to the feeding, the water was changed. Feeding was done by using a flexible fine tip forceps (one per feed) to individually pick up each particle of food to present it to the larva. The larva responded by holding onto the particle and feeding. The normal response would be to drop the particle after 5-15 minutes, so after all the larvae were initially fed, each was examined and if the particle had. 
been dropped it was picked up with the forceps and re-introduced. The larvae would always grab onto the food particle again and resume feeding for 5-15 minutes. This cycle was repeated for the 90 minute feeding period.

After the feeding period, each larvae was transferred to new cups containing $20 \mathrm{ml}$ of seawater. The cups in which they were fed, and which now contained the orts of their feeding, were extracted with $20 \mathrm{~m} 1$ of chloroform- methanol at $1: 1$. If particles of the feed were visible, they were first dispersed in the seawater with a glass stirring rod before the solvent was added. After solvent addition, the cups were loosely capped and allowed to sit for 30 minutes, and then firmly capped, shaken, pressure carefully released, and shaken again. They were then allowed to sit for one hour with additional shakings every 10 minutes. A two m1 aliquot of the bottom chloroform layer was then placed in a scintillation vial, the chloroform evaporated off, and $10 \mathrm{ml}$ of Aquasol was added. Counting was done on a Beckman model $100 \mathrm{LSC}$ with the ${ }^{14} \mathrm{C}$ wide channel. ${ }^{14} \mathrm{C}$ toluene was used as an internal standard.

This procedure gives an estimate of the label remaining after feeding, while the weight of the food particle gives an estimate of the original amount of label presented to the larvae. This allows the estimatation of both the amount of DPM ingested and the absolute quantity of feed ingested.

The schedule for the feeding and sampling of this experiment was as fo1lows:

$-24 \mathrm{~h}$ Larvae molt staged, placed in individual cups in $20 \mathrm{ml}$ seawater. $-2 \mathrm{~h}$. Water changed.

0-90 min Larvae fed labelled or non-1abelled feed. 
91 min 1st larval sample taken. Controls molt staged, each sample placed in first extraction solvent in homogenizing tubes in freezer.

100 min Remaining larvae switched to new cups containing $20 \mathrm{ml}$ seawater.

110 min Half of each group fed Artemia, the other half starved.

$2 \mathrm{~h} \quad$ 1st larval sample extraction started.

3-6 h Feeding cups extracted as described above.

$6 \mathrm{~h} \quad$ 2nd sample taken and extracted.

$12 \mathrm{~h} 3 \mathrm{rd}$ sample taken, controls molt staged and samples stored in first extraction solvent in homogenizing tubes in freezer.

$12.2 \mathrm{~h}$ Water changed and fed groups refed.

12.5 h 3 rd sample extraction started.

$24 \mathrm{~h} \quad 4$ th sample taken, (see $12 \mathrm{~h}$ ).

$24.2 \mathrm{~h}$ Water changed and fed groups refed.

$24.5 \mathrm{~h} \quad 4$ th sample extraction started.

$36 \mathrm{~h} \quad 5$ th sample taken (see $12 \mathrm{~h}$ ).

$36.2 \mathrm{~h}$ Water changed and fed groups refed.

$36.5 \mathrm{~h} \quad 5$ th sample extraction started.

$48 \mathrm{~h} \quad 6 \mathrm{th}$ and final sample taken and extracted, controls molt staged.

Sample Procedures

Each larval sample was given a code identification. For example, EF6A

-- the first letter indicates the type of label fed, either (E)

eicosapentaenoic acid $(20: 5 \omega 3)$, or (P) palmitic acid (16:0), or (C)

control non-labelled; the second letter indicates whether it had been (F)

fed or (S) starved; the number indicates the hours after feeding of the

label that the sample was taken and the last letter ( $A$ or $B$ )

differentiates the two larvae that were sampled at that time from each

group. The control samples do not have an A or B code as a11 the larvae 
in each sample ( 3 vs 2 for the labelled samples) were pooled for analysis. The control samples were molt staged, weighed for wet weight and then pooled and extracted for 1ipids in the same manner as the labelled samples. This extraction procedure is a simplified version (method 2) of the full method (method 1), both of which are described later. The control samples' 1ipids were analyzed for total lipids and for their lipid class patterns; both procedures are described later in the Lipid Analysis section.

The label fed larvae were individually extracted for lipids without prior weighing or molt staging. The chloroform extracts were placed in one dram teflon capped vials, flushed with nitrogen, sealed and stored at $-20^{\circ} \mathrm{C}$ until analysis.

The lipids from the label fed larvae were class separated with thin-layer chromatography (TLC) and the amount of label in each class determined by the methods given below. The lipid classes that were ana1yzed were: neutral 1ipids -- stery1 esters, triacylglycerols, free fatty acids, 1-2 diacylglycerols and total neutral lipids (taken from the plate used to separate the polar 1ipids); polar 1ipids -- phosphatidy1 (P.) ethanolamine, P. choline (which also contained P. serine, P. inositol and sphingomyelin) and total polar lipids (taken from the plate used to separate the neutral lipids).

Whatman LK5D 20x20 $\mathrm{cm}$ glass backed plates were used for the separations. These silica plates are pre-laned and have a pre-absorbent spotting layer. Prior to their use, chloroform:methanol at $1: 1$ was allowed to migrate up the plates overnight to clean them of any solvent soluble contaminants. 
Each sample was split in half and each half spotted onto the center lane of a five lane TLC strip (cut from a whole plate). The outer four lanes on each TLC plate had previously been spotted, with a 1ipid extract from an adult lobster, to provide standard reference points. Spotting of the labelled sample and its subsequent drying before development was done under flowing nitrogen to retard degradation.

Development of the TLC plates was done in standard all glass development chambers ( $\mathrm{h} \times \mathrm{w} \times \mathrm{d}$ of $26 \times 29 \times 9 \mathrm{~cm}$ ) lined along the back inner wall with absorbent paper. Neutral 1ipids were separated with hexane: diethy1 ether: acetic acid at 80:20:1 (Mangold, 1969) and the polar lipids were separated with chloroform: methanol: water at 65:25:4 (cf. Kirchner, 1978). The solvents were allowed to thoroughly saturate the chamber atmosphere but no pre-conditioning of the TLC plates with this atmosphere was done. It was found that a quick placement of the TLC plate into the solvent (allowing 1ittle time for the solvent equilibrated tank atmosphere to escape) resulted in an even solvent front and spot migration through the width of the plate. The solvent front was allowed to migrate to within $3 \mathrm{~cm}$ of the plate top, whereupon the plate was removed and dried in the open atmosphere.

The lipid spots were detected with iodine vapor (cf. Kirchner, 1978) under a hood. This was accomplished by blocking the inner end of a pasteur pipet with glass wool, filling the pipet with iodine crystals, blocking the rear end with more glass wool and connecting it to a. compressed air line via silicone tubing. This allowed a steady stream of iodine vapor to be directed at the TLC plates. The center lane was protected by pressing a glass plate vertically down the middle of the lane 
adjacent to the center lane. Once one side was iodine exposed, the protective glass plate was switched to the other side of the center lane and that side exposed. The positions of each lipid spot were noted and then the center lane very lightly exposed to the iodine for confirmatin of even spot migration. The corresponding areas in the center lane were then scraped into scintillation vials.

\section{Liquid Scintillation Counting from TLC plates}

The following method is an adaptation of the methods reported by Webb and Mettrick (1972) and Kobayashi and Harris (1978).

In order to scrape the lipid-silica spots into scintillation vials, the center lane was first moistened with distilled water from a pipet. This allowed the silica to be lifted off in a single clean, dustfree layer using a flat front edged razor blade (X-Actor blade $\left.{ }^{r} 17\right)$. After placing the silica into the vial, one ml of distilled water was added, the vial capped and placed into an ultrasonic cleaner (Bransonic 220) for one minute. This completely dispersed the silica. $10 \mathrm{ml}$ of Aquasol (with 7 ml per liter of acetic acid) was then added, the vial capped, mixed, and placed back into the sonicator for an additional minute.

Counting was done with a Beckman model $100 \mathrm{LSC}$ set on the ${ }^{14} \mathrm{C}$ wide channel. ${ }^{14} \mathrm{C}$ toluene was used as an internal standard. With this procedure, counting recovery of ${ }^{14} \mathrm{C}$ free fatty acids (usually the lowest of all the classes (Kobayashi and Harris, 1978)) was $100 \%$ versus the 88-93\% of the above cited methods.

\section{Proximate Analysis Procedures}

A flow chart outlining the sampling and analysis procedures is given as Appendix 3. 
$\underline{\text { Ash }}$

Ash was determined gravimetrically to the nearest microgram on a CAHN model 25 or 29 electrobalance after combustion at $475^{\circ} \mathrm{C}$ overnight (12-15 h). Each sample (individual larvae or two pooled eggs) was placed in a pre-ashed, pre-weighed aluminum thimble which was then loosely crimped closed and placed upright in the bottom of a pyrex $10 \times 75 \mathrm{~mm}$ test tube. The test tubes were held in a metal rack and each tube was covered with a loose fitting aluminum thimble to prevent contamination. The sample thimbles were made using a cork borer to punch out $22 \mathrm{~mm}$ diameter circles from aluminum foil which were then wrapped around a glass rod tip.

\section{$\underline{\text { Chitin }}$}

Chitin was determined from the lipid extraction residue using an acid-base digest method (Austin, P.R.; Univ. of Delaware, personnal communication). As priority was given to the lipid sample, some particulates were always lost during lipid extraction. The residue, upon drying with a stream of air was exposed to $2 \mathrm{ml}$ of one percent HCl $(\mathrm{v} / \mathrm{v})$ for 10-20 minutes. A thin glass rod was used to scrape the adhering residue off the test tube walls. After centrifuging and removal of the supernatant, $2 \mathrm{ml}$ of two percent $\mathrm{NaOH}$ was added for 10-15 minutes. The test tubes were centrifuged again, the supernatant discarded and $2 \mathrm{ml}$ of two percent $\mathrm{NaOH}$ added for 10-15 minutes. At this point only clear, transparent material was left. This was filtered out onto pre-rinsed, pre-weighed $25 \mathrm{~mm}$ diam. glass fiber filters, which were then lyophilized and weighed to the nearest $.01 \mathrm{mg}$ on a Mettler M5 balance.

\section{Carbohydrate}

Carbohydrate was determined using the phenol-sulfuric acid method of 
Dubois et al. (1956), using D-glucose as the standard. The sample was prepared by homogenizing it in $400 \mu 1$ of distilled water in a $5 \mathrm{ml}$ homogenizing, centrifugation tube (Kontes 非85250). (These volumes were doubled or trebled for the larger samples.) The pestle (which had been wetted with distilled water prior to homogenization) was rinsed off during the addition of $1.6 \mathrm{~m} 1$ of distilled water into the tube. From this two $\mathrm{m} 1$ homogenate, two aliquots of $800 \mu 1$ (or less depending on the sample size) were pipetted into separate tubes for duplicate carbohydrate ana1yses.

\section{Protein}

Protein was determined with one of two methods using the homogenate remaining from the carbohydrate analyses. The preferred method, used for the experiments based on Hatches I-3 and I-5 and the eggs, was Hartree's (1972) adaptation of Lowry's et al. (1951) protein assay. Bovine serum albumen was used as the standard.

For this assay, done in duplicate, two aliquots of $150 \mu 1$, or less, were placed in separate test tubes and brought up to one $\mathrm{ml}$ with distilled water. Thereafter, Hartree's method was followed exactly.

The other method, which was used for the experiments based on Hatches II-4 and II-7, was based on the Coomassie blue dye binding technique (Bradford, 1976) marketed in kit form by BioRad (BioRad Laboratories, 1979). Bovine gamma globulin was used as the standard.

For this assay, $400 \mu 1$ of $0.2 \mathrm{~N} \mathrm{NaOH}$ was added to the $400 \mu 1$ of homogenate left over from the carbohydrate assay. The contents was stirred, sonicated in an ultrasonic cleaner for 5 minutes and heated at $80^{\circ} \mathrm{C}$ for 30 minutes. Triplicate, identical aliquots were each placed in 
separate test tubes and $0.1 \mathrm{~N} \mathrm{NaOH}$ used to bring the volume of each tube to one $\mathrm{ml}$. One $\mathrm{ml}$ of $0.15 \mathrm{~N} \mathrm{HCl}$ was then stirred in, followed by $0.5 \mathrm{ml}$ of BioRads Coomassie blue solution (added with rapid mixing). After five minutes the contents of each tube was read at $595 \mathrm{~nm}$ against a control blank tube containing everything but protein.

The $\mathrm{NaOH}$ and $\mathrm{HCl}$ additions (not part of the BioRad method) were found to be necessary to solubilize the proteins as the BioRad reagent alone was not sufficient; however, this method still underestimated the total protein.

\section{Lipid Analysis}

\section{Extractions}

This lipid extraction procedure was modified from the methods of Folch et al. (1957) and B1igh and Dyer (1959). It involves sequential extractions with chloroform: methanol, where the polarity of the extraction solvents become progressively more non-polar.

Al1 glassware (only glass and teflon were used) was washed or rinsed with (in order): soap, water $(10 \mathrm{x}), 10 \% \mathrm{HC} 1(2 \mathrm{X})$, distilled water ( $3 \mathrm{X})$, methanol $(2 \mathrm{X})$, acetone $(2 \mathrm{X})$, and hexane (2x). The only exception was the disposable pasteur pipets which were not soap or acid washed. The wash methanol and acetone were reagent grade, all other solvents were glass distilled from Burdick and Jackson. The chloroform used was the type preserved with ethanol.

The samples were extracted immediately after their wet weighing. Storage of samples prior to their extractions was not done except for short term storage times for the pulse fed experiment samples (less than 
$24 \mathrm{~h}$ ) and the label fed animals (less than $1 \mathrm{~h}$ ). Storage for these samples was done at $-20^{\circ} \mathrm{C}$ in one $\mathrm{ml}$ of chloroform: methanol $(1: 2$, the first extraction solvent) in the teflon capped, $5 \mathrm{ml}$ homogenizing tubes. Similar, but longer storage, of another crustacean Artemia (Sasaki, in preparation), did not result in spontaneous fatty acid methyl formation (Lough, et al., 1962), nor hydrolysis to form free fatty acids (Christie, 1973), nor oxidation of fatty acids (Sasaki, in preparation).

The listed extraction procedure is a slight modification of the one used for most of the samples in the present work. The modification involves only steps 2 to 4 and is given here because it makes the extraction easier. There are, however, no differences between either extraction procedure in terms of quantitative or qualitative recoveries. For the original extraction procedure (used for the present work) substitute the following for steps 2 to 4 in the succeeding full 1 isting.

2. Add sufficient distilled water to bring the total water volume (sample included) to $400 \mu \mathrm{l}$.

3. Thoroughly homogenize the sample with the glass pestle.

4. Add $1.5 \mathrm{ml}$ of chloroform: methanol (ratio 1:2)(hereafter written as $\mathrm{C}: \mathrm{M}, 1: 2$ ) to the homogenizing tube, using it to rinse off the pestle as it is removed.

\section{Extraction Procedure Method 1}

1. Place the sample in a $5 \mathrm{ml}$ teflon capped, homogenizing, centrifugation tube (Kontes 非885201).

2. Add $1.0 \mathrm{ml}$ of chloroform: methanol (ratio $1: 2$ ) (hereafter written as $C: M, 1: 2$ ) and thoroughly homogenize the sample with the glass pestle.

3. Add 0.5 of $C: M, 1: 2$, while using it to rinse off the pestle as it is removed from the homogenizing tube.

4. Add sufficient distilled water so that the total water content of the homogenizing tube, sample included, is $400 \mu 1$. 
5. Flush with nitrogen, cap and shake thorough1y.

6. Let stand for 10-15 minutes with intermittent mixing.

7. Centrifuge (use extra cushions in the tube holders so that the test tube caps are not supporting the test tube in the holder).

8. Transfer the supernatant to a $10 \mathrm{~m} 1$ teflon capped, calibrated graduated centrifuge tube.

9. Add $1.5 \mathrm{ml}$ of $\mathrm{C}: \mathrm{M}, 1: 1$ to the homogenizing tube

10. Flush with nitrogen, cap and mix thoroughly.

11. Let stand for 10-15 minutes with intermittent mixing.

12. Centrifuge and transfer supernatant to same graduated centrifuge tube.

13. Add $1.0 \mathrm{ml}$ of $\mathrm{C}: \mathrm{M}, 3: 1$ to the homogenizing tube.

14. Flush with nitrogen, cap and mix thoroughly.

15. Let stand for 5-10 minutes with intermittent mixing.

16. Centrifuge and transfer supernatant to same graduated centrifuge tube.

17. Add $1.4 \mathrm{ml}$ of $0.7 \% \mathrm{NaCl}$ solution to the graduated centrifuge tube.

18. Flush with nitrogen and cap.

19. Shake the tube once and crack open the cap to relieve pressure.

20. Repeat step 19.

21. Shake thoroughly and place in refrigerator for at least 30 minutes.

22. Centrifuge 1-2 minutes (tube must remain cold so don't overcentrifuge).

23. Record the volume of the bottom lipid-chloroform layer.

24. Use a pasteur pipet to remove the top layer and any interfacial fluff that exists between the two layers.

25. Remove a measured amount of the chloroform layer for storage. Leave sufficient extract if total lipid analysis is to be done immediately.

To reduce handling, a simplified version (method 2) of the above method was used for extraction of the labelled animals. With smaller 
samples (e.g. single larvae) the efficiency of this simplified procedure is equivalent to the full method.

- For step 非, use $1.5 \mathrm{~m} 1$ of $\mathrm{C}: \mathrm{M}, 2: 1$ instead of $\mathrm{C}: \mathrm{M}, 1: 1$.

- Omit steps 13 to 16.

- For step 117 , add $.95 \mathrm{~m} 1$ instead of $1.4 \mathrm{ml}$ of the $0.7 \%$ NaC1 solution.

\section{Storage of Lipid Extracts}

After extraction, the chloroform-1ipid extract was stored with one of two methods. For lipid class analysis, the extracts were placed in one dram glass vials with teflon lined caps. The extracts were bubbled with nitrogen and the vials sealed and stored at $-70^{\circ} \mathrm{C}$.

For fatty acid analysis, the extracts were placed in freeze drying ampules and evaporated to dryness with a stream of nitrogen. The ampule was then connected to a high vacuum pump, cycled through at least six vacuum-nitrogen cycles and then flame sealed under vacuum. The ampules were then stored at $-70^{\circ} \mathrm{C}$.

\section{Total Lipid Analysis}

Total 1ipids were determined gravimetrically, either after extraction or after storage. After extraction, 50, 100 or $200 \mu 1$ of the original lipid- chloroform solution was used per determination. If after storage, the sample was evaporated to dryness (if necessary) with a stream of nitrogen, brought up to 100 or $200 \mu 1$ with $\mathrm{C}: \mathrm{M}, 1: 1$ and $8 \mu 1$ of this solution used for each determination.

Manipulation of 50 and $100 \mu 1$ amounts was done with high quality Lang Levy pipets. The $8 \mu 1$ volumes were measured with a $10 \mu 1$ Hamilton syringe with the 非 style needle $\left(90^{\circ}\right.$ bevel) using a (in sequential order), plunger, air, $1.5 \mu 1 \mathrm{C}: \mathrm{M}, 1: 1$ solvent block, air, 8 
$\mu 1$ sample, technique to assure complete transfer of the sample.

$12 \mathrm{~mm}$ diameter, numbered aluminum pans were pre-weighed to $0.1 \mathrm{\mu g}$ on a CAHN model 25 or 29 electrobalance. The pans were placed on a glass petri dish on a hot plate set at $80-90^{\circ} \mathrm{C}$ and the chloroform-lipid samples pipetted or syringed onto these pans. After the solvent evaporated, each aluminum pan was placed in a separate covered petri dish to cool and then re-weighed. The minimum sample size was $20 \mu \mathrm{g}$; duplicate analyses were done and these never varied from each other by more than $3 \%$.

\section{Lipid Class Analysis}

\section{Sample Preparation}

The 1ipid extracts stored in chloroform were used for the lipid class determinations. After analysis for total lipids, an internal standard of palmitic acid propyl ester (PAPE) was added to give a ratio of PAPE/1ipid sample of $9-14 \%$. The solution was then evaporated to dryness with a stream of nitrogen and 50 or $100 \mu 1$ of $\mathrm{C}: \mathrm{M}, 1: 1$ added. Trip1icate analyses were done, with each analysis using 25-35 $\mu \mathrm{g}$ of the 1ipid sample. Typically, this involved $1-4 \mu 1$ of the lipid solution, though small samples required up to $8 \mu 1$.

\section{Iatroscan -- Introduction}

The samples were analyzed with an Iatroscan TH-10 Mark III coupled with an HP 3390A integrator. As this is a relatively new analytical instrument, many of the following methods were developed for this work and are therefore given in full detail.

The separation of the lipid classes was done on Chromarods (type S-II) which separate compounds in the same manner as silica thin-layer 
chromatography. A chromarod (S-II) is a thin quartz rod covered with a layer of fused glass and $5 \mu \mathrm{m}$ silica particles. 10 chromarods make up a set which is run and analyzed as a unit. Once the lipid classes are separated along the length of the chromarods, the set of rods is placed in the Iatroscan which sequentially carries each rod through the flame of a flame ionization detector (FID). The flame burns off each separated spot (1ipid class) and the resulting signal from the FID is integrated.

\section{Rod Matching}

Since rod to rod variation exists, the rods in a set must be matched. This was done in two steps, first for solvent flow characteristics and then for separation and quantification. 20 new rods were placed in one of two metal rack containers (these containers normally hold a set of 10 rods throughout development and analysis). Each set was then scanned twice through the Iatroscan flame to clean off accumulated contaminants. The Iatroscan was at its normal settings of: scan speed 4 , hydrogen 8 $\mathrm{kg} / \mathrm{cm}^{2}$, air $2000 \mathrm{ml} / \mathrm{min}$.

Both racks were then simultaneously placed in the solvent system used for neutral lipid separation ( 1 isted below). The average solvent front, seen with an oblique high intensity 1ight, was allowed to rise up the rods approximately $12 \mathrm{~cm}$. Any rod whose solvent front was greater than $1 \mathrm{~cm}$ off from the average was taken out of the rack and placed back in upside down. This usually corrected the difference, if not, the rod was rejected.

After this procedure, the rods were transferred to a glass holder for overnight immersion in a chromic-sulfuric acid cleaning solution. The next day the rods were rinsed of $\mathrm{fith}$ distilled water and soaked in 
distilled, deionized water for at least 30 minutes.

After a blank scan through the Iatroscan to dry and prepare the rods, equal amounts of a sample solution was spotted onto each rod (spotting procedure covered below). The spotting location was determined with the spotting guide provided with the instrument. The sample solution was identical to the one used to generate the neutral lipid standard curves (1isted below). The rods were then developed for neutral 1ipid separation (1isted below) and scanned through the Iatroscan. The integrated values for each class were compared between rods and the 10 rods with the most similar responses as to spot location and quantification were pooled into one set.

A final check was made with this matched set which consisted of a second run using the neutral lipid sample solution to verify the previous results.

\section{$\underline{\text { Standard Curves }}$}

A standard curve was required for each lipid class as each class had a different FID response. The compounds used to generate these standard curves were, for neutral lipids -- cholesteryl palmitate, palmitic acid propyl ester (internal standard), palmitic acid, tripalmitin, cholesterol and 1,2-dipalmitin; for polar 1ipids -- monopalmitin, phosphatidyl (P.) ethanolamine dipalmitoyl, P. choline dipalmitoyl and sphingomyelin (all obtained from Sigma). Monopalmitin is a neutral lipid but was separated out during the polar lipid runs. P. serine and P. inositol, when discernible, were quantified with the P. ethanolamine standard curve. P. serine, phosphatidic acid and monopalmitin would of ten occur as a single broad peak, in which case the monopalmitin standard curve was used. 
Two standard solutions were used, one contained the neutral lipids as listed above, the other contained the polar classes. Concentrations of each compound in the $\mathrm{C}: \mathrm{M}, 1: 1$ solvent were approximately 5 or 10 $\mu \mathrm{g} / \mu \mathrm{l}$. To generate standard curves, 0.2 to $2.0 \mu \mathrm{l}$ of the standard solutions were spotted onto each rod with a $1.0 \mu 1$ positive displacement syringe. This yielded absolute amounts in the ranges of 1-10 or $0.5-5$ $\mu \mathrm{g}$ for each compound. Development and scanning of the rods after spotting was identical to the procedure used for sample analysis and is listed below. The relationship between the known spotted amount $(x)$ and the integrator readings (y) was a power function $\left(y=a x^{b}\right)$.

\section{Sample Spotting Procedure}

This is covered in detail as much of the error in this method can be attributed to spotting of the sample. A 5 or $10 \mu 1$ Hamilton syringe, with a septum piercing needle, was used for all sample spotting volumes greater than $1.0 \mu 1$. Typical spotting volumes ranged between $2-4 \mu 1$.

To measure out a sample, the syringe was wetted with blank solvent (C:M, 1:1) and then $0.5 \mu 1$ of the blank solvent was drawn up (an air gap existed between the plunger and this $0.5 \mu 1$ solvent block). $0.5 \mu 1$ of air was then drawn in, followed by the sample. The sample volume was verified by drawing it completely into the barrel for examination of the meniscus to meniscus distance.

To spot the sample, the syringe was positioned so that the concave portion of the needle tip faced downwards towards the rod. Approximately $1 / 6-1 / 10$ of a microliter of sample was slowly ejected to form a hanging drop contained within this concave portion and the needle lowered to gently touch the drop to the rod. This was repeated until the entire 
sample and solvent block was transferred to the rod.

When the $1.0 \mu 1$ syringe was used, as for the standard curves, no solvent block was necessary due to the positive displacement mechanism. The needle was also placed more towards a vertical position as this syringe needle has a $90^{\circ}$ bevel tip.

\section{$\underline{\text { Rod Development }}$}

The overall procedure to develop the rods for separation of the 1 ipid classes was based on the methods outlined by Newman-Howells, Associates (1982). After the rods were spotted, the rack of 10 rods was suspended in a constant humidity chamber for 10 minutes. This humidity chamber consisted of a TLC development tank containing a saturated NaCl solution which maintained the humidity at $75.7 \%$ (cf. Neuman-Howe11s, Assoc., 1982).

The rack was then quickly transferred and suspended in the solvent saturated atmosphere of the development tank containing the neutral lipid solvent system. The development tanks were lined on one inner side with solvent soaked absorbent paper to aid in saturating the tank atmosphere. After 10 minutes, the rack was lowered into the solvent system for development. When the solvent front reached a mark $10 \mathrm{~cm}$ above the sample spotting origin (typically in 30 minutes), the rack was removed, placed in a brass, hinged lid carrying case and placed on a hot plate $\left(110^{\circ} \mathrm{C}\right)$ for five minutes to evaporate the solvent.

The rods were then scanned with the Iatroscan set at a scanning speed of 4 , hydrogen pressure of $8 \mathrm{~kg} / \mathrm{cm}^{2}$ and air flow of $2000 \mathrm{ml} / \mathrm{min}$. The integrator was set at an attenuation of 5 , chart speed of 5 , peak width of 0.01 , threshold of 3 , area reject of zero and an integer function 非 set at time 0.01 . This first scan covered the separated neutral lipid 
classes. The polar classes and monoacylglycerols stayed at the spotting origin and were not burned off with the FID because the instrument was set to scan only partially down the rods -- just past the 1,2-diacylglycerol spot. This allowed analysis of the polar lipids of the same applied sample used for the neutral lipid analysis.

After the neutral lipid scan the rack was again suspended in the humidity chamber for 10 minutes and then transferred to the polar lipid solvent saturated atmosphere of the development tank. After another 10 minutes, the rods were lowered into the solvent and developed until the solvent front reached a spot $10 \mathrm{~cm}$ above the spotting origin (typically 45 min). After solvent evaporation in the brass case on the hot plate ( 5 $\mathrm{min}$ ), the rods were fully scanned with the Iatroscan (at the same settings used for the neutral lipids):

After scanning the polar lipids, the rods were ready to accept another set of samples. After each day's analyses, the rods were cleaned by transferring them to their glass rack holder and immersing them overnight in chromic-sulfuric acid. Prior to their use the next day, the rods were rinsed with distilled, deionized water and soaked in distilled, deionized water for at least 30 minutes.

The development and scanning procedure for the standard curves was identical to the above except that the neutral and polar runs were completely separate. That is, the polar standards were spotted on the rods after the neutral lipids were scanned.

\section{Solvent Systems}

The solvent system used for separation of the neutral lipid classes was a modification of the system of Christie and Hunter (1979). It was 
comprised of dichloroethane: chloroform: acetic acid: isopropanol at the ratio of 92: 8: 0.1: 0.03. The amount of isopropano1 was varied from 0.03 to 0.3 parts for each set of rods or for the same set as it aged. The more isopropanol, the more the triacylglycerol peak moved in relation to the rest. $100 \mathrm{ml}$ of the total solvent mix was used at a time and would remain adequate for up to $6 \mathrm{~h}$ or three developments. The solvent mix was prepared fresh just prior to its use by adding in acetic acid and isopropanol to a premixed batch of dichloroethane and chloroform. The solvent was placed in a smaller TLC tank within the larger development chamber so that the solvent depth was between $15-20 \mathrm{~mm}$.

The solvent system for the polar lipids was developed by Innis and Clandinin (1981) and consisted of chloroform: methanol: water at the ratio of $80: 35: 3$. Approximately $80 \mathrm{ml}$ of this solvent system were used, which was placed in a metal trough within the larger TLC development tank, to a depth of 15-20 mm. The solvent was placed into the trough 10 minutes prior to the introduction of the rods to the chamber atmosphere and was disposed of after each development.

\section{Data Interpretation}

Triplicate analyses were done, with each analysis using the same amount of sample material. The integrator readings were interpreted with the standard curves and the resulting values averaged. The standard deviations, therefore, represent analytical variation rather than sample variation.

The mean values for each class were also summed and compared against the spotted sample size as determined from the internal standard. This estimate of recovery of the technique usually fell within 85-95\%. 
For each sample, the results were expressed as percent of the recovered total (i.e. were normalized to $100 \%$ ); these percent values were also used with the individual total lipid values to give estimates of the absolute amounts of each lipid class per individual.

\section{Plasmalogen Analysis}

The Iatroscan was used for qualitative plasmalogen analysis by adapting Schmid and Mangold's (1966) thin-1ayer chromatographic acid hydrolysis technique. After analysis of the neutral lipids, the chromarods were transferred to their glass rack holder and suspended for 5 minutes in a closed jar above a layer of concentrated HC1 at room temperature. The chromarods were then transferred back to the metal rack and processed in the normal manner for polar lipid analysis.

Plasmalogen P. ethanolamine (obtained from Sigma) yielded lyso-P. ethanolamine and aldehyde with this procedure.

\section{Fatty Acid Ana1ysis}

The fatty acid patterns of the major 1ipid classes were determined with thin-layer chromatography (TLC) separation of the major 1ipid classes, followed by gas-1iquid chromatographic (GLC) analysis of the fatty acids.

\section{TLC Class Separation}

Lipid class separation was on a gross leve1, with the total lipid sample being fractioned into a neutral lipid pool (containing all the neutral lipids), a P. ethanolamine pool (predominantly P. ethanolamine) and a P. choline pool (predominantly P. choline, with P. serine, P. inositol, and sphingomyelin).

This separation was done on Whatman LK5D TLC plates that had been 
previously run overnight with $C: M, 1: 1$ to wash out contaminants. The plates were cut into 5 lane strips, with the outer four lanes used for the standards -- tripalmitin, P. ethanolamine dipalmitoyl and P. choline dipalmitoyl. The center lane was used for the sample which was applied last under a stream of nitrogen. When the carrier solvent evaporated, the plate was quickly transferred into the developing solvent without prior equilibration. The developing solvent was chloroform: methanol: water at the ratio of 65:25:4 (cf. Kirchner, 1978). The solvent had been placed in the developing tank and swirled over the absorbent paper lining one inner side of the tank to insure complete saturation of the tank atmosphere.

The plate was removed from the developing tank when the solvent front reached a point $3 \mathrm{~cm}$ from the plate top. It was transferred to a closed chamber under flowing nitrogen and the detection agent (20 ppm primuline in acetone (Wright, 1971)) was applied to the standard containing lanes with a pastuer pipet. After the solvents were dry the center lane was covered with a strip of aluminum foil and the lipid standards were visualized with longwave UV light. The corresponding sample areas (adjusted for the presence of plasmalogens) were scraped into teflon capped $16 \times 100 \mathrm{~mm}$ pyrex test tubes. Fatty Acid Methyl Ester (FAME) Production

The above test tubes were washed with the procedure 1 isted under the Lipid Extraction section with the added step of a $10 \%$ HC1 in methano1 rinse prior to the methanol rinses (three instead of two).

Prior to the introduction of the lipid-silica from the TLC plate, 100 $\mu 1$ of methanol containing the internal standard of heptadecanoic acid (17:0) (Hatches II-4 and II-7) or heneicosanoic acid (21:0) (Hatches I-3 
and I-5) was added. This kept to a minimum the period in which the dry lipid-silica was exposed to air and therefore highly susceptible to oxidative degradation.

The following is adapted from Metcalfe et a1. (1966), van Wijngaarden (1967) and J. Farrington (personal communication). All solvents were glass distilled from Burdick and Jackson. The $0.5 \mathrm{~N} \mathrm{NaOH}$ in methanol used below was prepared by partially dissolving $\mathrm{NaOH}$ pellets in methanol and then using the undissolved pellets to prepare the $0.5 \mathrm{~N}$ solution. This eliminated most of the contaminants from the $\mathrm{NaOH}$ which were found to reside on the outer surfaces of the $\mathrm{NaOH}$ pellets.

To a 1ipid-silica containing test tube, $0.8 \mathrm{ml}$ of $0.5 \mathrm{~N} \mathrm{NaOH}$ in methano1 was added and, as throughout the procedure prior to each sealing, the tubes flushed with nitrogen. The tube was then placed in boiling water for 10 minutes and cooled to room temperature in water. A jet of air was directed between the cap and the tube to blow out condensed water, the tube opened and $1.0 \mathrm{ml}$ of $14 \% \mathrm{BF}_{3}$ in methanol (Sigma) was added. After three minutes in boiling water, the tube was cooled and $1.5 \mathrm{~m} 1$ of pentane added. After sealing and shaking (after an initial mixing, the tube was unsealed to relieve pressure, then resealed and thoroughly mixed), $2.0 \mathrm{ml}$ of a hexane extracted saturated aqueous $\mathrm{NaCl}$ solution was added. After capping, shaking and centrifuging, the pentane layer was pipetted off and $1.0 \mathrm{ml}$ of fresh pentane added, mixed and the tube recentrifuged. The second pentane layer, was removed and the extraction repeated with a third $1.0 \mathrm{~m} 1$ of pentane. The three combined pentane extractions were evaporated to dryness with a stream of nitrogen and the FAME redissolved in hexane for injection into a GLC. 
GLC Analysis

FAME standards from Sigma and Supelco were used for peak identification. For Hatches II-4 and II-7, a $183 \mathrm{~cm}(6 \mathrm{ft}), 2.1 \mathrm{~mm}$ I.D. stainless steel column packed with GP $10 \% 2330$ on $100 / 120$ Chromosorb W AW (Supelco, Inc.) was run isothermally at $200^{\circ} \mathrm{C}$ in an $\mathrm{HP} 5830 \mathrm{~A}$ gas chromatograph. Attached to the column was a $15 \mathrm{~cm}$ ( 6 inch) stainless steel pre-column packed with the same material. For Hatches I-3 and I -5 , a $183 \mathrm{~cm}$ ( $6 \mathrm{ft}), 2.1 \mathrm{~mm}$ I.D. stainless steel column packed with GP $3 \% \mathrm{SP}$ $2310 / 2 \%$ SP 2300 on $100 / 120$ Chromosorb W AW (Supe1co, Inc.), was run on the HP 5830A. A $15 \mathrm{~cm}$ precolumn, packed with the same material was also used. Temperature was programmed from $190-210^{\circ} \mathrm{C}$ at $2^{\circ} \mathrm{C} / \mathrm{min}$ with an initial two minute hold. For both columns, the injection and FID temperatures were $200^{\circ}$ and $250^{\circ} \mathrm{C}$ respectively. Nitrogen flow was 20 $\mathrm{ml} / \mathrm{min}$. The polarity of these two columns was different and selected samples were run on both columns to confirm fatty acid identification.

This procedure does not always differentiate between the various isomers (different $\omega$ numbers) of the fatty acids; however, the major isomer of each fatty acid, as deduced from numerous studies, were assumed to be the ones present in this study and were the ones used as qualitative and quantitative standards.

\section{Statistical Analysis}

Student's T-test (cf. Zar, 1974) was used for data analysis. When percent values were compared, an arcsin transformation was used (cf. Zar, 1974) $\left(\arcsin \left(\mathrm{X}^{0.5}\right)\right)$. 
$-86-$ 


\section{RESULTS}

This section is organized into discrete parts by experiment. These parts, in order, are; (1.) embryogenesis and hatching, (2.) pulse feeding and immediated post hatch development, (3.) 1arval development, (4.) starvation, (5.) control versus fatty acid supplementation, and (6.) ${ }^{14} \mathrm{C}$ fatty acid feeding.

The graphic representations of the data have been organized by experiment though to facilitate inter-experiment comparisons they have been kept together as one group. A master key has been provided at the beginning of the graph section and should be referred to prior to the graphs. Some data have also been presented as tables. A key for these tables is located at the beginning of the Table section.

\section{Embryogenesis and Hatching}

This section discusses the results for two studies - the embryogenic study which covered a single batch of eggs during their approximately 10 month development at ambient temperatures and the hatching period study which focussed on the changes occurring around eclosion or the hatching event. In this latter study, the eggs previously had experienced accelerated development by being kept at elevated temperatures during the fall and winter.

\section{Proximate Analysis}

The wet weight of the egg increased for the first 10 weeks and then leveled off and remained constant until a second increase occurred approximately three weeks prior to hatch (Graph 2A). This wet weight increase, due to water absorption, was also reflected in an increase in egg size. Table 2 lists the egg, length in $\mathrm{mm}$ as well as the eye width and 
length measurements of the developing embryo. The wet and dry weight measurements and their standard deviations $(n=7)$ are also listed and showed a steady but gradual decline in egg dry weight.

In the graphs depicting data for the complete embryogenic period, the last point was derived from larvae which hatched the day after the last egg sampling (next to last graph point) took place. Because the hatching process was extended over more than a week, the distance in metabolic time between these last two points may actually be up to seven days instead of the single day as graphically presented.

Losses of both wet and dry weight occurred at this hatching point which, when coupled, produced a large decline in the dry to wet weight ratio. Closer examination of this hatching period (Graph $3 A$ ) showed that most of the dry weight loss occurred at hatch and not during the seven day period immediately preceding it.

The absolute amount of ash rapidly increased during the first month. Ash then leveled off until it again quickly rose approximately one week prior to hatch (Graph 2B). Closer examination (Graph 3B), however, somewhat contradicted this pattern as the ash increase prior to hatch appeared to have occurred only within a couple of days of hatch. Ash definitely rapidly increased immediately after hatching.

The results for chitin (Graph 4B) were not clear except that, judging from the variation within and between samples, the assay used barely had the resolution to handle such small samples. There did appear to be an increase in chitin approximately one week before hatching. Using an outlier test (Grubbs, 1950), the two chitin values for the last egg sample were significantly different $(p<.01)$ from either the rest of the egg 
sample chitin values $(n=50)$ or from the preceding 9 samples $(n=18)$. This latter comparison was done to rule out any influence from earlier, and potentially lower, values. Another approach is that the mean and standard deviation for the rest of the egg samples was $23.9 \pm 10 \mu \mathrm{g}$ in comparison to the 68 and $74 \mu \mathrm{g}$ values for the last egg samples. These latter values were more than 4.5 standard deviations distant.

A closer look at the hatching period (Graph 5B) did not show as large a chitin increase prior to hatching. It is likely, however, that a chitin increase had already occurred prior to the first sample. The chitin percent dry weight values for the first hatching period sample were approximately $8 \%$, which was equivalent to the value for the last egg sample in the embryogenesis study.

The absolute levels of carbohydrate fluctuated in the first two to three months, though the trend was for a slight decline (Graph 4A). Thereafter, carbohydrate levels remained the same until hatch. The slight rise in carbohydrate as percent dry weight through embryogenesis was due to the decline in dry weight during this period. A sharp decline occurred at hatch and Graph 5A shows that this dec1ine began immediately (0-2 days) prior to hatching.

Absolute levels of protein fluctuated throughout embryogenesis (Graph $6 \mathrm{~A})$, though a consistent decline did occur over the first three months. Thereafter, protein fluctuations appeared random with the exception of a sharp, large decrease in protein at hatching. Graph 7A shows that this protein decline began 1-2 days prior to hatching.

Total lipid showed a steady decline throughout embryogenesis, though a slight leveling of this. decline may have occurred during the central 
months of this period. A large drop also occurred at hatching (Graph 6B), though unlike protein and carbohydrate this drop appears to be an accentuation of the prevalent decline rather than a sudden change (Graph 7B).

Protein, 1ipid and carbohydrate were also expressed as their caloric equivalents using the heat of combustion conversion factors of $5.7 \mathrm{cal} . / \mathrm{mg}$ (protein), $9.5 \mathrm{ca} 1 . / \mathrm{mg}$ (1ipid), and $4.0 \mathrm{cal} . / \mathrm{mg}$ (carbohydrate) (Kleiber, 1975). These data are shown in Graph 8. It can be seen that 1 ittle or no change occurred for carbohydrate while protein showed an initial gradual decline which leveled off until hatching. Lipid, on the other hand, showed a steady decline throughout which was accentuated at hatch. The magnitude of change during embryogenesis (excluding post-hatch effects) was greatest for 1ipids followed by protein and then carbohydrate. Had the catabolizable energy conversion factors been used ( 4.8 instead of 5.7 cal./mg for protein, Kleiber, 1975) then the difference between protein and 1ipid would have been even greater. (The catabolizable energy conversion factor for protein was not used because this value takes into account the metabolic cost of converting protein nitrogen into urea. For the most part, lobsters excrete nitrogenous wastes as ammonia. Protein energy conversion in lobsters is probably between the two values listed above.)

Using the heat of combustion conversion factors, protein declined by 0.90 cal. per egg and lipid declined by $1.57 \mathrm{cal}$. per egg during embryogenesis (fertilization to the last egg sample). Using the losses of protein, lipid and carbohydrate to estimate the caloric cost of embryogenesis, one derives the value of $2.53 \mathrm{cal}$. (31:6\% of the original 
tota1). Protein and 1ipid account for $36 \%$ and $62 \%$ of this total respectively.

\section{Lipid Class}

The lipid class data for the embryogenic and hatching period studies are given as Tables 3 and 4 , respectively. As a reminder, the listed standard deviations represent the variation which occurred in the triplicate analysis of identical samples and therefore do not directly represent variation within the sampled population. Only the four major classes, triacylglycerols, sterols, phosphatidyl (P.) ethanolamine, and P. choline, from the embryogenic study were graphed (Graphs 9 and 10). Particular attention should be paid to the Y-axis scales on these graphs as they differ widely between classes.

Triacylglycerol (Graph 9A) showed a steady decline throughout embryogenesis and, in terms of absolute levels, showed the greatest decline of any class. A sharp decrease occurred at hatch which almost depleted the store of this class. A closer examination of the hatch period (Table 4) also showed a decrease which in absolute terms was equivalent to the triacylglcerol decrease at hatching in the embryogenesis study. In the hatching period study, however, a greater store of triacylglycerol was present prior to the hatching period and so the hatched larvae still had substantial levels of triacylglycerol. It was also evident from the hatching period study that most of the triacylg1ycerol decrease seen near the hatch occurred between the transition from a gold to blue egg. A final note is that while triacylglycerol levels were elevated in the eggs of the hatching period study versus the embryogenic study, the absolute levels of sterols, P. 
ethanolamine and P. choline were equivalent.

Sterols showed no decline in levels throughout embryogenesis (Graph 9B). Sterols also did not decrease during hatching in either the embryogenesis or hatching period study (Table 4).

The sudden appearance of steryl esters after a month of development appears to be a real phenomenon (Table 3), though prior to this point the levels may have been just below detection and so the actual magnitude of the steryl ester increase is uncertain.

The minor classes of 1-2 diacylglycerol and sphingomyelin followed the pattern of the sterols with no definite change through embryogenesis. The appearance of monoacylglycerols and P. inositol at hatching may have been an artifact and will be discussed 1ater (Table 3 ).

The phospholipid P. ethanolamine showed an erratic, but overa11, slight decline through embryogenesis (Graph 10A). In the embryogenic study a slight decrease in P. ethanolamine was seen at hatching while in the hatching period study no such decline occurred.

P. choline showed an initial decline followed by a level period and then a second decrease prior to the hatch (Graph 10B). In the embryogenic study P. choline showed a sharp decline at hatch, similar to the decline seen for triacylglycerols. In the hatching period study (Table 3), however, this P. choline decrease at hatch was proportiona1ly much less.

The decreases of triacylglycerol, P. ethanolamine, and P. choline through embryogenesis (fertilization to the last egg sample in the embryogenic study) accounted for $74 \%, 9 \%$, and $23 \%$, respectively, of the total 1ipid loss. (These sum to greater than $100 \%$ due to increases in other lipid classes over this period.) 


\section{Fatty Acids}

The complete set of fatty acid data for the embryogenic study and the hatching period study are given as Tables $5,6,7$, and 8 . The data were expressed as $\mu \mathrm{g}$ per individual for each fatty acid in the form of fatty acid methyl ester (FAME) in the neutral 1ipid, P. ethanolamine, and $P$. choline pools. Portions of these data for the embryogenic study were also illustrated as Graphs 11, 12, 13, 14, 15, and 16. Graphs 11, 13, and 15 covered selected non-essential fatty acids (as FAME) for the neutral 1ipid, P. ethanolamine, and P. choline pools, respectively. The upper graph (A) shows them as $\mu \mathrm{g}$ per individual; the lower graph shows them as a percent of the recovered total FAME found in that pool. The selected non-essential fatty acids (NEFA) were palmitic acid (16:0), palmitoleic acid $(16: 1 \omega 7)$, stearic acid $(18: 0)$, and oleic acid $(18: 1 \omega 9)$.

Graphs 12, 14, and 16 cover selected polyunsaturated fatty acids (as FAME) in a format similar to that used for the NEFA. The selected

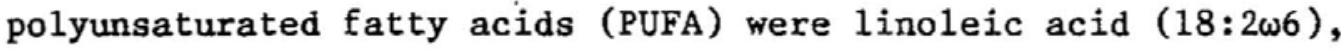
linolenic acid $(18: 3 \omega 3)$, arachidonic acid $(20: 4 \omega 6)$, eicosapentaenoic acid $(20: 5 \omega 3)$, and docosahexaenoic acid plus docosapentaenoic acid (1isted as $22: 6 \omega 3)$.

The NEFA of the neutral lipids for the embryogenic study showed an even decline in absolute amounts through development (Graph 11A) with a possible brief leveling during the middle period. At hatch a large drop in all quantities occurred. As a percent of the recovered total (Graph 11B) no change was seen throughout development except at hatch. This change at hatch was probably due to the near depletion of the normally dominant triacylglycerols so that the fatty acid pattern of the other 
neutral lipids became influential. The same pattern was seen for the neutra1 lipid PUFA -- a steady decline in absolute amounts (Graph 12A) but no change in the fatty acid composition (Graph 12B). An exception to this was 20:5w3 which decreased proportionately slightly faster than the rest of the fatty acids.

A closer look at the hatching period also showed that a large drop in absolute amounts occurred at this time (Table 8) with most of it occurring between the gold to blue egg transformation. As a percent of the total, however, there was no sudden shift in proportions of the fatty acids.

The NEFA in the $P$. ethanolamine fraction showed an erratic, but overall slight, decline in absolute amounts during embryogenesis which was accentuated at hatch (Graph 13A). As a percent of the total no apparent changes occurred for these fatty acids (Graph 13B). The PUFA also showed a general decline in absolute amounts over this period and a sharper decrease at hatch (Graph 14A). The one exception to this was 20:5 33 . which increased for the first six months before eventually decreasing. As a percent of the total (Graph 14B), no change was seen for the PUFA over this period with the exception of $20: 5 \omega 3$ which increased for the first 5 months and then leveled off. While the embryogenic study showed faster declines in $\mathrm{P}$. ethanolamine fatty acids at hatch, the hatching study, in contrast, showed only slight declines (Table 8).

In the $P$. choline fraction for the embryogenic study most of the NEFA showed a very slight decline until approximately two months before hatching when this decline increased (Graph 15A). The exception was 18:0 which in absolute terms appeared to remain constant. As a percent of the tota1 (Graph 15B), these NEFA underwent 1itt1e change throughout this 
period. The drop in absolute NEFA levels seen at hatch was again not as strong in the hatching period study (Table 8). The PUFA either very gradually declined or remained level for a period and then declined (Graph 16A). 20:503 was an exception to this as it appeared to rise slightly, in absolute terms, until about two months prior to the hatch. As a percent of total FAME, there were no changes except for 20:5 03 again, which showed a slight increase (Graph 16B).

Throughout, the general patterns of declines seen in the fatty acids during embryogenesis support the patterns of the lipid class data.

\section{Pulsed Feeding and Immediate Post-Hatch Development}

This section covers the results from a study which involved frequent sampling of larvae within $60 \mathrm{~h}$ of their hatching. Feeding was 1imited to two periods - 12-18 $\mathrm{h}$ and $36-40 \mathrm{~h}$ from hatch. The larvae used had undergone accelerated embryogenesis by being kept at elevated temperatures during the fall and winter.

\section{Proximate Analysis}

The ash, carbohydrate, protein and 1ipid values are presented as graphs 17 and 18. Ash rose immediately after hatching so that within $6 \mathrm{~h}$, rough1y $75 \%$ of the total ash increase for that larval stage had occurred (Graph 17B). The availability of food had no affect. Carbohydrate showed a continuous drop from hatching until, the second feeding period when it showed a general but erratic increase (Graph 17A). The levels of protein (Graph 18A) showed a constant decline until the second feeding period when it rose markedly and then again declined. The fluctuations in absolute 
levels of protein and carbohydrate after the second feeding are partly due to a variation in animal size (as dry weight) in these samples (Table 9). The percent dry weight 1 ine for both protein and carbohydrate compensated for this dry weight variation and showed a smoother pattern for both components. Lipid declined throughout the studied period and was not greatly affected by either feeding period (Graph 18B).

\section{Lipid Class}

Table 10 lists the lipid class data and reveals that most of the lipid classes remained relatively stable in quantity throughout the study period. The two exceptions were triacylglycerol and $P$. choline which decreased by approximately 80 and $40 \%$, respectively. In absolute amounts, triacylglycerol and P. choline respectively accounted for 55 and $35 \%$ of the total lipid loss seen during this period. 
Larval Development

This section covers larval development as it was followed in four separate hatches. Hatches I-3 and I-5, taken in 1982, were the most complete and used a cod liver oil dietary supplement during the raising of the 1arvae. Hatches II-4 and II-7, taken in 1981, used Artemia as the sole feed.

It should be noted at this point that the first sample of third stage larvae in Hatch I-5 was spurious. The molt stage of this sample was $D_{0}$, when at most it should have been $C$. These larvae were obviously more advanced than their siblings and were the result of unintentional sampling bias. The data from this sample have been included in all the graphs. The resulting exceptions to trends that resulted from this inclusion have been ignored in the following interpretations.

The duration of each molt cycle can be seen on the larval development graphs (e.g., Graph 19). The vertical lines represent the idealized times of each molt. They are the exact molt times found in Hatch I-5. Since Hatch I-5 was the most complete in terms of the total period covered, the data from the other hatches were normalized to the Hatch I-5 pattern. Normalization was done within each larval stage based on the known sampling and molting times for each hatch. Two separate broods from two different years were represented. The molt times within each brood were very similar. For Hatches II-4 and II-7, the average time spent as first, second, and third larvae were 98, 108, and $144 \mathrm{~h}$, respectively. For Hatches I-3 and I-5 these times (plus the fourth larvae) were 135, 139, 128 , and $365 \mathrm{~h}$, respectively. 
Proximate Analysis

The proximate analysis data were presented pictorially as graphs 19 through 26. Graphically, each component was shown as an absolute amount per individual and as a percent of dry weight. The following will also discuss each component as a percent of ash free dry weight (\% AFDW) though this data was not presented.

The wet weight, shown in Graph 19A, rapidly increased immediately at each molt and at hatch. (Since a molt occurs as part of the hatching process, genera1 references to molt related events will include the hatch molt as we11 as the molts between larval stages.) After this initial increase, the weight remained relatively stable until the next molt. The increase in wet weight from one larval stage to the next ranged from between $70-80 \%$ (compared to premolt weight).

Dry weight as mg per individual (Graph 20A) showed a smooth increase through larval development which was not strongly affected by the molt cycle. Using the mean values for hatches II-4 and II-7, an exponential regression against time in hours $\left(y=a e^{b x}\right)(y=d r t$ wt. in $m$; $x=t$ ime in $h$; $\mathrm{n}=39$ ) gave $\mathrm{a}$ and $\mathrm{b}$ values of 0.8154 and $4.523 \times 10^{-3}$, respectively, with a coefficient of determination $\left(R^{2}\right)$ of .967 . For hatches $I-3$ and I-5, using the data up to, but not including, the development stage of $4 D_{0}(n=52)$, the exponential regression gave $a, b$ and $R^{2}$ values of $.9851,4.452 \times 10^{-3}$, and .982 , respectively. For the (I) hatches, the dry weight began leveling off after the $4 D_{0}$ stage.

Based on the wet and dry weight data, the larvae from the (II) hatches were noticeably smaller than the larvae from the (I) hatches. When the wet and dry weight data were expressed as a ratio (Graph 20B), 
however, a consistent relationship, regardless of absolute weight, was seen for the first three larval stages. This ratio, which was at a minimum immediately after each molt, rose throughout the molt cycle to a maximum immediately prior to the next mo1t. During the fourth larval stage this ratio increased past the maximum levels attained in the previous larva1 stages ( $28 \%$ vs $21 \%$ ).

Ash as mg per individual (Graph 21A) showed an immediate increase after each molt which peaked during the molt stage $C$ of each cycle. Once the peak was attained there was a slight, but consistent trend for a small decline in absolute levels prior to the next molt. Again the size difference between (I) and (II) hatch larvae was evident in the absolute levels of ash. As percent dry weight (Graph 21B), however, a consistent relationship was seen regardless of size. As a percent value the peak in ash occurred earlier, by late B - early C (molt stage), and then underwent a straight decline until the next molt. For the first three larval stages this cycle remained within the same range, though during the fourth stage ash was seen to make up a comparatively greater portion of the larvae.

Graph 22A, which depicts the chitin data as $\mathrm{mg}$ per individual shows that chitin increased relatively smoothly through larval development with no apparent affect from the molt cycle, though there was a trend for a slight leveling in absolute amounts toward the end of the molt cycle. The pre-stage $4 D_{0}$ exponential increase (hatches $I-3$ and $I-5 ; y=c h i t i n$ in $\mathrm{mg}$; $\mathrm{x}=$ time in $\mathrm{h} ; \mathrm{n}=52: \mathrm{a}=7.412, \mathrm{~b}=5.210 \mathrm{x} 10^{-3}, \mathrm{R}^{2}=.966$ ) began to level of $\mathrm{f}$ after the $4 D_{0}$ stage.

The apparent random variation of chitin as a percent of dry weight, or as \% AFDW, belies the resolution of the chitin assay (Graph 22B). 
Because of this variation, no correlation can be made against the molt cycle. What can be said is that for the first three larval stages, chitin made up to 6 to 10 percent of the larvae by dry weight (8-13\% AFDW) and that this level increased in the fourth stage to $14 \%$ (20\% AFDW).

Carbohydrate, in absolute terms, increased through larval development until the $4 D_{0}$ stage when it levieled off (Graph 23A). The effect of the molt cycle was not readily apparent, though there were some trends for molt stages $C$ to $D_{0}$ as being the period of greatest carbohydrate increase. Another trend was a slight drop in absolute amounts at the time of molt.

The size difference between (I) and (II) hatch larvae was also apparent for carbohydrate, though as percent dry weight the levels of carbohydrate remained, for the most part, within the range of $3-5 \%(4-7 \%$ AFDW) (Graph 23B). While the exact pattern was not identical between hatches, the overall general pattern for the percent dry weight (and $\%$ AFDW) data was consistent. After a molt in the first three larval stages, the percent level of carbohydrate immediately dropped to a low point during molt stage $C$. Carbohydrate then quickly increased to reach a peak in molt stage $D$, late in the molt cycle but early enough for subsequent samples to have of ten detected a drop prior to the upcoming molt. The fourth larval stage showed the postmolt decline but the premolt increase during this larval stage did not occur.

Protein as $\mathrm{mg}$ per individual is shown in Graph 24A. Hatch (II) larvae were not shown in this graph as the Coomassie blue (BioRad, 1979) technique, used for hatches II-4 and II-7, was found to underestimate protein in these samples. Through larval development the increase in 
protein was relatively steady with only a hint of a leveling off during the post $4 \mathrm{D}_{0}$ stages. The effect of the molt cycle was not clearly evident though the increases in protein appeared to occur more strongly during the late $C$ to early $D_{1}$ molt stages.

A pattern does emerge for protein when expressed as a percent of dry weight (Graph 24B). Overall, the percent level of protein declined from a high of $55-65 \%(70-80 \%$ AFDW) at hatch to a 1 ow of $40 \%$ ( $58 \%$ AFDW) during the fourth larval stage. In relation to the molt cycle, for the first three larval stages, protein was at a peak immediately prior to the molt followed by a decline at molt to a low during molt stage C. Protein percent levels then rose prior to the next molt. During the fourth larval stage protein appeared to rise after the stage $\mathrm{C}$ low; however, this rise was proportionately much less than the same rises in the previous molt cycles. If expressed as \% AFDW the above described cyclic pattern, while still present, was not as evident.

Lipid, depicted in Graph 25A in $\mathrm{mg}$ per individual, rose relatively smoothly through developement with a leveling off at stage $4 D_{1}$. The values for Hatches II-4 and II-7, which were not listed in these graphs, can be found in Tables 13 and 14. An effect of the molt cycle was not consistently evident in terms of absolute quantity, though in the (I) hatches a premolt leveling usually occurred. The smaller size of the larvae from the (II) hatches was initially evident as smaller quantities of lipid in the first and second larval stages. Increases in lipid in these two hatches, however, made them equal in quantity (mg per individual) by the end of the third larval stage. Expressed as a percent of dry weight, different patterns were seen 
for total lipid in the (I) and (II) hatches (Graph 25B: (I) hatches only). Both the (I) and (II) hatches showed declines after each molt with a minimum at stage $\mathrm{C}$ followed by an increase prior to the next molt. Beginning in the second larval stage, however, the (II) hatches showed a greater overall increase. In the fourth larval stage (hatches (I) only) lipid increased to a comparatively higher level than the previous larval stages. This molt related lipid level cycling was not always present if the data was expressed as \% AFDW. As \% AFDW a large drop did occur after hatch, but subsequently the levels merely rose slowly through development until the fourth stage when a rapid increase occurred.

Protein, 1ipid, and carbohydrate are graphed as their caloric equivalents using the heat of combustion conversion factors of Kleiber (1975) (see embryogenesis results). They are expressed both as calories per component per individual (Graph 26A) and as a percent of the summed caloric tota1 (Graph 26B). Calorically, protein was always the largest component followed by lipid, though these two began to merge during the fourth larval stage. Carbohydrate remained at a low, stable level throughout larva1 development.

\section{Lipid Class}

The 1ipid class data sets are presented as Tables $11,12,13$, and 14 for hatches I-3, I-5, II-4, and II-7 respectively. For hatches I-3 and I-5, the triacylglycerols, sterols, P. ethanolamine, and P. choline data subsets are also presented in $\mu \mathrm{g}$ per individual and as a ratio against. protein in graphs 27, 28, 29, and 30, respectively. Except where noted, the data for hatches II-4 and II-7 paralleled the I-3 and I-5 hatch results and thus were not included in the graphs. 
Triacylglycerol, depicted in Graph 27A in absolute amounts per individual, did not begin to replace the levels which were nearly depleted at hatch, until the middle of the second larval stage. Accumulation of triacylglycerol then occurred at a steady rate until stage $4 \mathrm{C}$ when it rapidly increased until stage $4 D_{1}$ when it began to level off. As a percent of the total 1ipid, triacylglycerol rose from less than $3 \%$ at hatch to over $50 \%$ at the end of the fourth larva1 stage and was mainly responsible for the total lipid pattern of accumulation. As a percent value against protein (Graph 27B) triacylglycerol rose throughout development which indicates that no constant relationship between triacylglycerol and protein existed.

Sterols showed a smooth increase through larval development when represented as $\mu \mathrm{g}$ per individual (Graph 28A). As a percent of dry weight, a slight decrease in sterols occurred with development. Graph 28B showed that the sterol versus protein ratio did not change over time. For the most part, this sterol/protein ratio remained between $1.5-2.0 \% \quad(n=57$, mean \pm S.D. $=1.67 \pm 0.19 \%)$. This tight ratio indicates that a constant relationship existed between these two throughout the normal larval developmental period.

P. ethanolamine, expressed as $\mu \mathrm{g}$ per individual in Graph 29A, showed an overall steady increase through larval development. As percent dry weight, P. ethanolamine declined through development. When compared against protein (Graph 29B) P. ethanolamine showed no molt related pattern, while through development it appeared to decline. This decline, however, was slight and doesn't discount the existence of a consistent 
relationship between $P$. ethanolamine and protein during larval development.

P. choline, as $\mu \mathrm{g}$ per individual (Graph 30A), showed a fairly

steady increase throughout development, and 1ike P. ethanolamine, did not appear to level off during the latter half of the fourth larval stage. There did appear to be a consistent decline in absolute amounts of $P$. choline after the hatch and first larval molt. As percent dry weight, P. choline declined through development ( $6 \%$ initially) until the fourth stage where it leveled off (to $2 \%$ ). A trend towards a decrease after each molt was partiy due to the increases in ash at this time. When $P$. choline was compared against protein (Graph 30B) a decrease was seen immediately after hatching. The $P$. choline/protein ratio fluctuated after this initial decrease, though generally declined. Fatty Acids

The complete data set of the fatty acid analyses for hatches $I-5$ and II-4 is given as Tables 15 through 17 and Tables 18 through 20, respectively. Hatch $I-5$ is also presented as Graphs 31 through 36 , with selected NEFA and PUFA for the neutral lipid, P. ethanolamine, and P. choline pools being separately graphed. Except where noted, hatch II-4 paralleled hatch I-5. The NEFA and PUFA chosen and the graphing formats were the same as detailed for the embryogenic study. The fatty acid profiles of the Artemia used in this study are given as Table 1.

The NEFA of the neutral lipids all showed a consistent increase in absolute amounts through larval development (Graph 31A). These increases were not proportionally equal between fatty acids however, as 18:1 and 16:1 showed a proportional increase over their initial hatch values, while 18:0 declined (Graph 31B). The PUFA of the neutral 1ipid poo1 showed 
several different patterns. As $\mu \mathrm{g}$ per individual (Graph 32A) the large increase of $18: 3$ was evident with the result that this fatty acid showed the greatest proportional increase (Graph 32B). 18:2 showed a proportional increase while 20:4 showed a decrease during the first two larval stages; both then leveled off. $20: 5$ and $22: 6$ both showed slight rises in absolute amounts during the second to early fourth larva1 stages. At other times the levels remained constant. As a percent of the total FAME, 20:5 and 22:6 sharply declined during the first larval stage followed by a continual but more gradual decline through development. In the P. ethanolamine fraction the NEFA all increased through larval development in a near proportional manner (Graphs 33A,B). 18:1 did show an increase in proportion from hatch while 16:0 experienced a s1ight decline. The PUFA in P. ethanolamine all increased through development though 18:3 showed a faster increase in comparison to the rest (Graph 34A). 20:5 also increased at a greater rate than 22:6. As a percent of the total FAME (Graph 34B) 18:3 showed a sharp rise and 18:2 a slow rise through development, while 20:4 remained proportionally constant. Both 20:5 and 22:6 decreased until the fourth larval stage where they fluctuated but stayed generally constant. A difference between hatch II-4 and I-5 showed up in some of these FAME as 20:5 and 22:6 in II-4 dropped faster and further than in hatch I-5 and 20:4 in II-4 proportiona11y increased instead of remaining constant as in hatch I-5.

Except for some random variation, the NEFA in P. choline (Graph 35A) all uniformly increased through development so that their percent levels (Graph 35B) remained fairly constant. In contrast, the PUFA of P. choline showed three different patterns. 18:3 rose at a much greater rate than 
the other FAME (Graph 36A) followed by 18:2 and 20:4 which showed slower, nearly identical rises. $20: 5$ and 22:6 showed the least and most variable increase. As a percent of the total P. choline FAME, 18:3 underwent an extremely sharp increase in the first larval stage which continued at a slower pace until a leveling off occurred in the fourth stage (Graph 36B). 18:2 showed a slight increase which leveled off in the fourth stage and 20:4 remained fairly constant. 20:5 and 22:6 both proportionally declined through development with the sharpest decrease immediately after the hatch (this reflected an actual decrease in absolute amounts for this period). In the fourth larval stage this decline leveled of $f$. Hatch II-4 differed from I-5 again for selected PUFA in this class with 18:3 showing a smaller absolute and proportional increase through development (Table 20). In addition, 20:5 showed only a minor absolute increase while 22:6 declined. This resulted in more extreme percent drops for these FAME in hatch II-4 as compared to hatch I-5. 


\section{$\underline{\text { Starvation }}$}

This section covers the starvation experiment which used subsets of the hatch I-5 population. These subsets, which were isolated from the main population on days 0 (hatch), 3, 4, and 7 , were initially at larval-molt stages $1 \mathrm{~A}, 1 \mathrm{D}_{0}, 1 \mathrm{D}_{2-3}$, and $2 \mathrm{C}$, respectively. The $\mathrm{d}-0$ (starvation commenced on day 0 ) subset stayed at stage 1C throughout and so did not undergo a molt. The larval stages for the d-3 subset or group, on a day to day basis (see Table 21 for the exact sample time) were $1 D_{1} ", 1 D_{2-3}, 2 C$, and $2 C$. For the $d-4$ group, the sequential larval sample molt stages were $1 D_{2-3}, 2 \mathrm{C}, 2 \mathrm{C}, 2 \mathrm{C}$, and $2 \mathrm{C}$; while for the $\mathrm{d}-7$ subset the larvae remained at $2 \mathrm{D}_{0}$ throughout. The molt which occurred in the $d-3, d-4$, and control groups occurred within the same time period as the subsequent sample from each group, taken at approximately the same time, were each at stage $2 \mathrm{C}$.

On the graphs depicting starvation data, the first asterisk on the $\mathrm{X}$-axis shows the time for the first molt in the controls and $d-3$ and $d-4$ groups. The second asterisk shows the time for the second molt which occurred in the controls only.

Proximate Analysis

The wet weight (Graph 37A) remained constant for the $d-0$ and $d-7$ groups. For the $d-3$ and $d-4$ groups the wet weight increased at molt with the $d-3$ increase smaller than the $d-4$ increase which appeared smaller than the control group increase.

The dry weight (Graph 38A) generally declined or remained stable from the onset of starvation, though if ash was discounted (Graph 37B) the dry weights of the s.tarved larvae ali declined. 
The control group line in the percent dry weight versus wet weight graph (38B) shows the molt related cyclic pattern. The large decrease in this ratio at molt, seen in the controls, was also evident in the d-3 and d-4 groups though in these latter cases it was preceded by a similar decrease due to starvation. The rise in this ratio which occurred in the controls after the molt was not seen in $d-3$ and $d-4$, instead the decline continued but at a slower rate. Both $d-0$ and $d-7$ showed a continuous decline through the experiment.

The control line in Graph 39A showed the molt related stepwise increase in ash. $d-3$ and $d-4$ also showed this increase after molt but the magnitude of these increases appeared less. As a percent of dry weight, a11 the starved groups showed an increase in the ash content above the levels found in the control group (Graph 39B).

Chitin in absolute amounts (Graph 40A) showed slight increases or stable levels with starvation, except for decreases at molt. As percent dry weight (Graph 40B) chitin usually increased, except at mo1t where a large drop occurred.

Carbohydrate in absolute amounts (Graph 41A) tended to dec1ine in each of the starved groups. As a percent of dry weight (Graph 41B) (and as \% AFDW) the pattern of a premolt increase-postmolt decrease was seen in the controls, while in the starved groups carbohydrate levels generally declined to lower-than-control levels.

Protein in absolute amounts (Graph 42A) showed a decline in each of the starved groups though this decline, except in d-0, did not begin immediately. As a percent of dry weight (Graph 42B) the trend was for protein to eventually decline to lower-than-contol levels. Because of the 
higher ash levels in the starved groups, however, protein as \% AFDW fluctuated around but stayed within the same range as the control levels. Lipid, in absolute amounts (Graph 43A), decreased in each starved group. As a percent of dry weight (Graph 43B) this decline was also evident and for the most part the presence of a molt did not perturb the rate of its loss. Unlike carbohydrate, no leveling of lipid to a minimum range was seen. As a $\%$ AFDW, lipid still declined but the range to which it eventual1y dropped ( $9 \%$ AFDW) was not much lower than the level attained during the post-molt decline seen in normal development for these early larva1 stages.

In Graph 44A,B,C, the comparative extent to which protein, 1ipid, and carbohydrate each decreased in the starved groups were depicted. This was determined by using the absolute levels of each component at the onset of starvation as the $100 \%$ level. The graphs for lipid and carbohydrate were quite similar as to the level of percent utilization (catabolism) of the available resources. Protein showed similar declines but, in general, it was not catabolized to as great an extent as 1 ipid or carbohydrate.

Using the heat of combustion caloric conversion factors (Kleiber, 1975) (see embryogenesis results), the caloric equivalents of protein, lipid, and carbohydrate were simultaneously graphed (Graph 45). Protein supplied the most metabolic energy during the starvation period, followed by 1 ipid and then carbohydrate.

\section{Lipid Classes}

The full lipid class data set for the starvation experiment is presented as Table 21. Triacylg1ycero1, stero1, P. ethanolamine and P. choline are also depicted as Graphs 46, 47, 48, and 49. 
In absolute amounts, triacylg1ycerol showed rapid declines within the first day of starvation (Graph 46A). These dec1ines continued and, except for d-0 which declined to low but measurable levels, all the other starved groups depleted their triacylglycerol stores. As a ratio versus protein, triacylglycerol declined in the starved groups (Graph 46B).

Sterols, shown in Graph 47A, remained stable through starvation, neither increasing nor declining in absolute amounts. The control line in Graph $47 \mathrm{~B}$ showed the relatively constant relationship between sterols and protein during normal development. In contrast, starvation caused the sterol versus protein levels to increase.

P. ethanolamine (Graph 48A) appeared to decline during starvation though in $d-3$ and $d-4$, both of which underwent molts, this decline did not occur immediately. Instead, both $d-3$ and $d-4$ showed an apparent increase prior to the eventual decline. The ratio of $P$. ethanolamine against protein (Graph 48B) showed that both the starved and control groups fluctuated but stayed within the same narrow, constant range.

P. choline showed relatively constant declines in absolute amounts in each of the starved groups (Graph 49A). Compared against protein (Graph 49B) al1 the starved groups declined to below the control, though in all cases this decline leveled off within the range of $5-6 \%$.

\section{Fatty Acids}

The full data set for fatty acids is given as Tables 22, 23, and 24 . To depict these data (Graphs 50 through 55) only the differences between the absolute levels of selected fatty acid at the onset and end of starvation were considered. The top graph on each page shows the percent of the fatty acid remaining at the end of the starvation period where the 
leve1 of that fatty acid at the onset of starvation was set at $100 \%$. The bottom graph on each page uses the fatty acid as a percent of the total recovered fatty acids (as FAME) in that class (i.e. the concentration of that fatty acid). This value for the larvae at the end of the starvation period was then compared against the same value obtained at the onset of starvation. The top graph then shows the degree to which each fatty acid was utilized. The bottom graph shows whether these fatty acids were non-selectively utilized (approximate ratio of 1 ), or selectively catabolized or conserved (ratios less than 1 and greater than 1 respectively).

As in the embryogenesis and development studies, on1y selected NEFA and PUFA were shown from the neutral lipid, P. ethanolamine and P. choline pools. The NEFA showed some consistent trends in each of the lipid pools. Except for the d-3 group in the neutral lipids, the monounsaturated fatty acids (16:1 and 18:1) were always utilized to a greater extent than their fully saturated analogues (16:0 and 18:0 respectively) (Graphs 50, 52, and 54). 16:0 was also always utilized more than 18:0 in al1 the groups for each 1ipid pool. Other trends for NEFA, based on the bottom graphs, were that 18:0 was usua11y conserved, while $16: 1$ appeared to be selectively utilized.

The greatest departures from non-selective utilization for the NEFA occurred in the neutral 1ipids. The data for the neutral 1ipid pool, however, may in fact be an artifact for this kind of comparison because the level of triacylglycerol was usually totally depleted. Therefore the fatty acid patterns that were seen may be the shift from a triacylglycerol. fatty acid dominated pattern to a sterol ester or diacylglycerol fatty. 
acid pattern.

For the two phospholipid pools (where the situation described above does not occur), P. ethanolamine showed definite departures, as previous $1 y$ described, from non-selective NEFA utilization. P. choline, on the other hand, tended more towards non-selective utilization of NEFA.

The PUFA also showed consistent trends through all the 1ipid pools. Except for the d-0 groups, 18:2 and 18:3 were always selectively utilized, while 20:4, 20:5 and 22:6 appeared to have been conserved. In the d-0 groups, the trend was always more towards non-selective usage (Graphs 51, 53 , and 55).

The NEFA and PUFA of P. ethanolamine taken together show that $16: 1$, 18:2 and 18:3 were all selectively utilized during starvation while 20:4, 20:5 and 22:6 a11 were conserved in relation to the rest of the fatty acids. 16:0 and 18:0 also tended to show evidence of conservation. The on1y exception to this occurred in the $d-0$ group where 18:2 and 22:6 did not follow the above pattern.

In general, the same patterns seen in the NEFA and PUFA of $P$. ethanolamine also occurred, but were not as definite, in P. choline. The exception was the $\mathrm{d}-0$ group which tended more towards non-selective usage. 


\section{Control versus Fatty Acid Supplementation}

This section covers the results from a single hatch (I-3) where half were fed Artemia plus the cod liver oil supplement (CLO) and half were fed only Artemia (contro1). The data were presented in Tables 25, 26, 27, and 28 .

Between the two treatments there was no difference in the larval or molt stages up to $4 \mathrm{C}$. No further samples were taken past $4 \mathrm{C}$ due to mortality in the control population. Up to the third larval stage mortality appeared equal between the two groups. In the late third to early fourth stages, however, the control group experienced heavy mortality while the CLO fed group experienced no noticeable increase in mortality. This heavy period of mortality in the controls was quite similar to the mortality seen in hatches II-4 and II-7 (both fed only Artemia) which also occurred during this developmental stage, and which also ended their sampling.

\section{Proximate Analysis}

These data are presented in Table 25. No differences existed between the control and CLO groups for most of these parameters, except 1ipid and possibly carbohydrate. Carbohydrate tended towards comparatively greater amounts in the control groups whereas 1ipid occurred in greater amounts in the CLO fed groups.

\section{Lipid Classes}

The 1ipid class data are given in terms of $\mu \mathrm{g}$ per individual and as percent dry weight (Table 26). By the end of the experiment, triacylglycerol had accumulated to a significantly higher level in the CLO fed animals. While triacylglycerol was responsible for much of the 
difference in total lipids between the two populations, the other 1ipid classes tended to be higher in the CLO fed groups as well.

Fatty Acids

The fatty acid data are presented as $\mu \mathrm{g}$ of fatty acid (as FAME) per individual (Table 27) and as a percent of the recovered FAME in its respective class (Table 28 ).

The $\mu$ galues all tended to be higher in the later samples for the CLO group. This supports the pattern seen in the lipid class data for higher levels of these classes in the CLO population. When presented as a percent of the recovered FAME, however, there was little difference between these values in the phospholipid pools. In the last sample $(6 / 28)$ 22:6 did tend to be higher in the CLO fed groups while 20:5 did not show any rea1 differences. 


\section{Labelled Fatty Acid Feeding}

This section covers the results for the pulse feeding experiment using $1-{ }^{14} \mathrm{C}$ labelled fatty acids.

The data are in Tables 29 through 32 and graphically as Graphs 56, 57, and 58. To review the sample code used in these tables: the first letter signifies the treatment used (C for control -- non-labelled diet, $P$ for $1-{ }^{14} \mathrm{C}$ palmitic acid fed, $\mathrm{E}$ for $1-{ }^{14} \mathrm{C}$ eicosapentaenoic acid fed); the second letter indicates if the animals were fed (F) or starved (S); the number denotes the hours between the sampling time and the onset of the label feeding period; and lastly, the final letter ( $A$ or $B$ ) separates the duplicate samples (if the last letter is not present, then the duplicates were averaged).

The larvae began the experiment in molt stage $C$. The starved larvae remained in molt stage $C$, while the fed larvae developed into stage $D_{0}$ by the end of the experiment.

Table 29, 1ists the recovered label in each class as DPM. The individual neutral lipid class DPM were summed and compared against the DPM value of the neutral 1ipid spot taken from the TLC plate used to separate the polar 1ipids. The DPM values of the polar lipid class spots were also summed and compared against the polar lipid spot from the neutral lipid separating TLC plate. This was done to verify the accuracy of the splitting procedure used to divide the sample for neutral and polar lipid TLC analysis. It also served as a check for any wildly spurious results (though none could be detected with this method).

The above ratios should have equaled one if the sample was split exactly in half and all the label was recovered. The mean \pm standard 
deviation for the neutral lipid ratio was $.98 \pm .08$, for the polar lipid ratio it was $.95 \pm .07$. Using Student's T-test (d.f.=82) the difference between the two ratios was significant $(p<.05)$ which indicates that overall a slight bias in favor of the neutral lipid class values may have occurred in either the original splitting of the sample or in recovery of the separated classes. However, no corrections were made based on this resu1t.

Table 30 shows the $\mu \mathrm{g}$ per individual amounts of the lipid classes as measured in the control animals. The total lipid values for the fed animals generally increased while the starved animal values continually declined. The erratic increase in lipid of the fed animals confirmed the visual observation that feeding on Artemia during the experiment was erratic and variable between animals. The fed animals did have elevated levels of triacylglycerol, P. ethanolamine, and P. choline in comparison to the starved animals.

Table 31 shows the amount of label found in each lipid class as a percent of the summed total recovered label. These data are a1so shown in graphs 56, 57, and 58. At the end of the 90 minute feeding period most of the labeled palmitic acid remained as fatty acid; triacylglycerols contained the next greatest amount of label, followed by $P$. choline.

At $6 \mathrm{~h}$, the fed animals had shifted most of the labeled 16:0 into triacylglycerol and P. choline followed by P. ethanolamine. In contrast, the starved animals, though erratic, showed more label in P. choline and as free fatty acid. A drop in the steryl ester levels also occurred in this time period.

From the $12 \mathrm{~h}$ sample onwards, most of the labeled 16:0 had stabilized 
into P. choline followed by $P$. ethanolamine, with the ratios in these two classes the same between the fed and starved larvae. The fed animals did consistently have a higher proportion of label in triacylglycerol while the starved animals, except for an unaccountably high value in PF48A, generally had higher label proportions as free fatty acid.

In the 20:5 fed animals, most of the label was in P. choline and triacylglycerol at the end of the 90 minute feeding period. $P$. ethanolamine contained the next greatest amount while the free fatty acids held only a small fraction.

At $6 \mathrm{~h}$ and onwards, the fed larvae held a larger fraction of the label in triacylglycerol as compared to the starved larvae. Both fed and starved animals held the same low fraction of label as free fatty acid. Both groups also had most of the label residing in P. choline followed closely by P. ethanolamine. The proportions in these latter two classes did not change from $12 \mathrm{~h}$ onwards. Between the fed and starved groups after $12 \mathrm{~h}$ the proportions of label in P. ethanolamine were equivalent; however, the starved larvae had a slightly higher percentage of label in P. choline than the fed larvae.

From left to right, Table 32 shows the dry weight of the food pellet given to the larvae and the estimated DPM in that pellet. The estimated amount of ingested label (as DPM) is then listed, followed by its equivalent as micrograms of food pellet (i.e. the amount of labelled pellet eaten to deliver the estimated amount of ingest DPM). Next, the total recovered label (sum of the lipid class DPM) is shown and lastly, the total recovered DPM is given as a percent of the ingested DPM.

In comparing the weights of the. labelled food pellet presented to the 
larvae, the ${ }^{14} \mathrm{C} 16: 0$ labeled pellets averaged ( $\left.\pm \mathrm{SD}\right) 189.7 \pm 40.5 \mu \mathrm{g}$, while the ${ }^{14} \mathrm{C} 20: 5$ labeled pellets averaged $172.3 \pm 34.7 \mu \mathrm{g}$. Using Students T-test there was no significant difference in pellet weights between the groups.

In comparing the amount of ingested pellet during the 90 minute feeding period, the high and low values from each set were ignored (this internal averaging was done to omit the value of PS36B). The mean ( \pm SD) values of the labeled 16:0 and 20:5 fed groups were $39.44 \pm 8.61 \mu \mathrm{g}$ and $52.89 \pm 5.18 \mu \mathrm{g}$, respective1y. Students T-test showed a significant difference $(p<.001)$ between these two groups.

In the last column of Table 32, the percent recovered versus ingested values always exceeded $100 \%$ for the samples taken immediately after the feeding period. Occurence of values greater than $100 \%$ indicate that an error had occurred. This error was most probably an underestimation of the amount ingested (derived by overestimation of the orts' DPM). The assumption can be made that such an error was a systematic one, in which case (since at this point little label had yet been lost via respiration) the values of 1.124 and 1.213 can be used as correction factors for the 16:0 and 20:5 data respectively. (These values are the averages of the DPM recovered/ingested ratios for the samples taken immediately after the feeding period. The $\mu \mathrm{g}$ of pellet eaten values were divided by these respective values. The result was a reduction in the pellet eaten values for both sets, with the reduction greater for the 20:5 fed group.) The end result of using these correction factors was a decrease in the difference between the two means of the 16:0 and 20:5 fed groups. The corrected means $( \pm S D)$ were $35.10 \pm 7.67 \mu \mathrm{g}$ and $43.60 \pm 4.27 \mu \mathrm{g}$ for 
the 16:0 and 20:5 fed groups respectively. Even with this correction, Students T-test shows there was still a significant difference between these groups $(p<.001)$.

The last column of Table 32 was also used to determine if there were any differences between the fed and starved groups within each of the label fed experiments. The data from the first samples taken immediately after the feeding period were not used, as the treatment between the fed and starved groups was identical up to this point. The following is based on arcsin transformed data where the high and low points were omitted and where the previously discussed correction factors (1.124 and 1.213) were used. The mean $( \pm S D)$ of the fed and starved groups in the 16:0 experiment were $41.16 \pm 16.43$ and $30.21 \pm 7.63$ respectively. For the $20: 5$ experiment these values were $70.25 \pm 3.68$ and $70.60 \pm 11.76$ respectively. The T-test showed no differences between the 20:5 labelled groups; the fed 16:0 labelled group had higher label recoveries over the starved groups at the $p<.063$ level. 
$-120-$ 


\section{DISCUSSION}

\section{Embryogenesis and Hatching}

Proximate Analysis: Graphs 2-8, Table 2.

The most active periods during embryogenesis, in terms of gross biochemical changes, were the month after fertilization and the month prior to hatching. During both periods ash showed proportionally large increases. A function of the ash increase may have been related to water intake. Salt may have been actively transported into the egg during these periods, causing water to move passively in to maintain osmotic balance. Other functions for ash may have existed, however, as the amount of water incorporated into the egg was not sufficient to osmotically balance the amount of incorporated ash. That is, at $35 \mathrm{ppt}$ salinity a $35 \mu \mathrm{g}$ increase in ash (the approximate increase seen after fertilization) should be accompanied by $1000 \mu \mathrm{g}$ of water. This assumes of course that the egg was initially iso-osmotic with seawater. Only $60 \%$ of this amount of water was actually incorporated into the embryos after extrusion, however, whereas the prehatch increase was only $50-60 \%$ of the ash extrapolated water uptake. Either the elastic egg membrane (Yonge, 1946) exerted sufficient pressure to prevent an iso-osmotic balance or the ash was incorporated into other structures, such as the embryonic shell, so that it was no longer osmotically active. Another possibility is that osmotically active organic compounds, such as amino acids, decreased during this period.

Other factors were also controlling water uptake, as the initial water content rose steadily for the first three months while the majority of the initial ash increase occurred within the first month. A more 
definitive statement about the relationship between ash and water uptake could be made if the ionic concentrations were measured in developing eggs.

Since there was a lag time between salt and water uptake, perhaps the permeability of the egg membrane was the controlling factor. This was suggested by Pandian (1970a,b) who also found a correlation between ash and water uptake in eggs of Homarus americanus and Homarus gammarus. In these studies he compared ash and water uptake against the caloric value of the egg. With this comparison, based on only four sample times, he found a continuous uptake of both salts and water. When time is used as the comparative axis, however, the present study shows that a latent period for water and ash existed during the mid portion of embryogenesis. The increase in ash prior to hatching likely served to increase the water content of the embryo; this may be a requirement of the eclosion event (Davis, 1964). The varying levels of ash buildup found with hatching time, which Pandian (1970a) called prehatch buildup, probably was an artifact of his sampling procedure and will be discussed fully in the Immediate Post Hatch Development section.

The chitin measurements were too variable to detect any small changes throughout embryogenesis. An increase in chitin would have been expected throughout most of embryogenesis due to the formation of the embryonic naupliar exoskeleton and the deposition of two chitinous inner egg membranes (Cheung, 1966; Pandian, 1970b). The increase in chitin just prior to hatch was sufficient to distinguish the prehatch chitin levels from previous levels. It appears as if this increase began about a week before hatching and that the levels attained immediately prior to hatching were comparable to the levels of chitin found in the early first stage 
larvae. As the hatching or eclosion process involves a molt, a decrease in chitin should have been seen immediately after hatching due to the lost exuvia. The fact that such a decrease was not detected was probably due to the assay used, but it may have also been an indication of low chitin content in the newly molted exuvia. A chitin analysis of the molt exuvia from the eclosion molt would provide further information. Although this analysis was not done, such exuvia can be easily collected after large hatches. It is also obvious that a better method for chitin quantification is necessary for critical studies. Several such methods are discussed by Muzzarel1i (1977).

The carbohydrate levels during embryogenesis showed an initial. decline followed by a stable period and then a precipitous decrease at hatch. In addition to its important role in intermediary metabolism (e.g., NADPH from the hexose monophosphate shunt (Eastman, 1968)), a primary use for carbohydrate in crustaceans is as a chitin precursor (Hohnke and Scheer, 1970). The importance of established carbohydrate reserves for chitin synthesis is not clear. Chitin is formed from glucosamine-6-P which is formed from fructose-6-P (Hohnke and Scheer, 1970). In a closed system, such as an egg, any synthesis of chitin can only draw upon established carbohydrate reserves or gluconeogenesis. In many crustaceans, carbohydrate is not stored in substantial amounts (Da11, 1964) thus established reserves simply cannot provide enough material to account for the amount of synthesized chitin (Scheer, 1960; Da11; 1965). In the present study the decrease in carbohydrate reserves and the increase in chitin around the hatch did not coincide exactly. Chitin levels began to rise before carbohydrate levels declined, which supports 
Barnes (1965) interpretation that other compounds, in addition to carbohydrate, can participate in chitin synthesis. If so, this may free the established carbohydrate reserves for other purposes.

The above does not imp1y that glucosamine for chitin synthesis was produced from anything other than carbohydrate (fructose). It does suggest that fructose came from gluconeogenesis as well as from the established carbohydrate reserves. The newly synthesized sugars must have come from protein as there is no evidence of a glyoxylate pathway in crustaceans (Benevides and Hammen, 1978; Cioni et al., 1981). Isocitrate lyase (the primary enzyme of the glyoxylate cycle) activity was apparent1y found in an insect (Carpenter and Jaworski, 1962) though this is open to debate (Hohnke and Scheer, 1970). Holland's (1978) interpretation of Hohnke and Scheer (1970) was in error as they never stated that lipid serves as a chitin precursor. There is recent evidence (Jones, 1980; Goodman et al., 1980; Ash and Atkinson, 1983), however, of possible glyoxylate cycle activity in higher animals and this pathway may exist in some animals during embryogenesis (though not in adults)(Kahn and McFadden, 1980). No attempts have been made to detect glyoxylate cycle activity in crustacean embryos. It would be interesting to determine if the glyoxylate cycle exists during embryogenesis since gluconeogenesis must be occurring.

In the embryogenic study, the carbohydrate decline at hatch was of a greater magnitude than the decline seen during the hatching period. Carbohydrate in the embryogenic study, but not in the hatching period study, was likely being used partially as an energy source or else chitin synthesis was more dependent on carbohydrate reserves. This difference 
between studies was the result of different nutritive states in the larvae. Evidence of these differences will be discussed below. Protein appeared to show an initial decline followed by a leveling off and then a sharp decrease at hatch. The cumulative efficiency (amount in last egg sample versus first egg sample) was $80 \%$ for protein which compares favorably with the $83 \%$ value found for the eggs of the shrimp Crangon crangon (Pandian, 1967b).

The percent levels of protein in the egg samples of the present study (65-74\% dry weight) are higher than values reported for eggs of other crustaceans and may be due to an artifact. The percent dry weight of protein was $58-72 \%$ in eggs of the shrimp C. crangon (Pandian, 1967b); $66 \%$ for the crab Eupagurus bernhardus (Pandian, 1967a); 56-62\% and 49-60\% for the barnacles Balanus balanoides and Balanus balanus (Barnes, 1965) and, most notab1y, 47-51\% in the lobster Homarus gammarus (Pandian, 1970b). Other evidence for a possible overestimation of protein came from comparing the sum of each of the measured components against the dry weight. When this was done, the summed values averaged $114 \pm 4 \%$ (S.D.) of the total dry weight, indicating an overestimation had occurred. The most probable candidate was protein. This overestimation was 1 imited to the eggs, however, as analysis of larvae (Hatch I-3 and I-5) yielded a comparable value of $101 \pm 3 \%$.

Since the overestimation occurred in the eggs and not the larvae, it was possible that with the Lowry method (Lowry et al., 1951; Hartree, 1972) for estimating protein there was either positive interference in the eggs or negative interference in the larvae. A positive interference would cause an overestimation, but if this occurred, the interfering 
source was unknown. Lipid, which was the next most abundant substance present in the whole homogenates, was we11 below tolerable limits for this protein assay (Peterson, 1979). Another possibility was that yolk protein, either because of its physical structure or content of tryptophan and tyrosine, reacted more strongly than tissue protein. Peptides can react more strongly than proteins with this assay (Choe and Goldstein, 1960) and eggs do contain more free amino acids than larvae (Costlow and Sastry, 1966).

If a negative interference had occurred in larval samples, it would have required that lobster protein react more strongly than bovine serum albumin. This is certainly possible as the assay has a recognized two-fold range of reactivity among various proteins (Peterson, 1979). A potential source of negative interference may have been chitin as it preferentially links with tyrosine (Muzzare11i, 1977), one of the two amino acids which the Lowry assay most strongly detects (Chou and Goldstein, 1960). The acidic nature of the Lowry assay, however, should have cleaved these chitin-tyrosine associations (Muzzare11i, 1977) and freed tyrosine for detection.

Total 1ipid content showed a more-or-less continuous dec1ine through embryogenesis. The slight increase in lipid seen in the first few weeks can not be considered significant though Pandian (1970b) also found a similar trend. The decrease in lipid around the hatch was roughly equivalent in the embryogenic and hatching period studies (approximately 0.1 versus $0.08 \mathrm{mg}$, respectively). This agreement and the patterns for the biochemical components between the embryogenic and hatching period study indicate that the last egg sample taken in the embryogenic study was 
at an approxmately equivalent metabolic state as the first egg sample of the hatching period study. That is, the embryos from the last egg samples in the embryogenic study would have actually hatched 3-7 days 1ater, rather than a day later as determined from the sampling procedure. Nevertheless, even if there was a seven day difference between the last two samples in the embryogenic study, the rate of lipid decline for this period would still have shown a sudden acceleration. This indicates that preparation for hatching required increased energy expenditures.

Lipid and protein had different patterns of utilization when expressed as caloric equivalents. Lipid clearly provided the majority of metabolic energy during embryogenesis as determined from absolute changes in amounts. This energy function for lipid is in agreement with previous studies with other crustaceans (Barnes, 1965; Pandian, 1967, 1970b; Pandian and Schumann, 1967). Under the assumption that 1ipid can provide nearly all the energy for embryogenesis, the loss of protein seen during embryogenesis may have been due to the inefficiency of conversion from yolk protein to tissue and not due to respiration for energy. A definite latent period existed during the middle of embryogenesis where no change in absolute protein content occurred. In contrast, lipid content showed a steady decline throughout embryogenesis.

As a poikilotherm, Homarus americanus embryo development is strongly linked to environmental temperature. A rapid exponential decrease in developmental rates occurs from $10^{\circ}$ to $5^{\circ} \mathrm{C}$ (Templeman, 1940; Perkins, 1972). In the present study, the mid-period for embryogenesis occurred at temperatures less than $6^{\circ} \mathrm{C}$, thus development during this mid-period was extremely slow. The latent period for protein was then a reflection of 
the temperature-induced slow development. If protein loss occurred during the conversion of yolk to tissue, there must have been very little tissue development during this period. Although tissue development was slow, maintenance of existing tissue was still required and was probably responsible for the continued decline in lipid at this time.

Embryogenesis can be accelerated by keeping the eggs at elevated temperatures (Templeman, 1940). If elevated incubation temperatures are used, there is no low temperature period and, consequent1y, no interval during which energy is being used primarily for maintenance. Larvae produced from accelerated embryogenesis should then have the energy reserves that would normally have been used during the low temperature maintenance period. The hatching and pulse fed studies used larvae that had undergone accelerated embryogenesis and there was evidence from both that at hatch the larvae possessed energy reserves in excess of those found in larvae that developed at ambient temperature conditions. The main difference was in the amount of lipid; the larvae resulting from accelerated embryogenesis had approximately $0.12 \mathrm{mg}$ more lipid per individual than larvae that developed at ambient temperatures. In the embryogenesis study, there was a decline in lipid of approximately $0.05 \mathrm{mg}$ during the interval in which the temperature was below $6^{\circ} \mathrm{C}$. This range of $0.05-0.12 \mathrm{mg}$ 1ipid (0.47-1.14 calories) thus provides an approximation of the metabolic cost of this maintenance period. The difference in the above lipid values also indicates a possible increased efficiency of development at higher temperatures in that more energy remained at the end of development at higher temperatures ( $1.14 \mathrm{cal}$ ) than could be accounted for by the cold temperature maintenance energy cost $(0.47 \mathrm{cal}$.) at ambient 
temperatures. An increased yolk conversion efficiency with temperature has been noted in fish (Hayes and Pelluet, 1945).

The total caloric loss during embryogenesis, estimated to be 1.57 calories, indicates a $68.4 \%$ efficient utilization of yolk. This value was determined from the difference between the newly extruded eggs and the last egg sample and so did not account for the changes just prior to hatch. As discussed, this period was energetically cost1y and can account for a major portion of expended energy. To obtain an estimate of this period the newly hatched larvae can be used instead of the last egg sample. This method will, however, overestimate the energy spent due to the loss of the egg membrane. The caloric equivalent of the larvae was 3.41 calories, resulting in a yolk utilization efficiency of $42.7 \%$. If Pandian's (1970b) caloric estimate of the egg membrane (0.33 calories per egg membrane) is used to compensate for the unmeasured egg membrane loss in the present study, then the larval caloric equivalent was $\cdot 3.74$ calories, resulting in a $46.8 \%$ yolk utilization efficiency.

The above values are considerably lower than the $73.3 \%$ and $64.7 \%$ yolk utilization efficiencies, respectively, found in Homarus gammarus and Homarus americanus (Pandian, 1970a). There are several possible reasons for these differences. The first explanation is the previously discussed overestimation of egg protein in the present study. The protein in the newly extruded egg can be estimated to be $0.52 \mathrm{mg}$, rather than the measured $0.77 \mathrm{mg}$ by adding a11 the other measured components and subtracting this value from the known dry weight. When this is done the yolk utilization efficiency becomes $52.0 \%$ (or $57.1 \%$ compensating for the egg membrane). The second reason is that Pandian $(1970 a, b)$ did not know 
the exact time of extrusion for the eggs in his studies. Consequently, he could not take into account the energy loss during the first portion of embryogenesis. The third possible explanation is the culturing conditions used by Pandian. Homarus gammarus were held at $16 \pm 1^{\circ} \mathrm{C}(1970 \mathrm{~b})$; Homarus americanus were held at $14 \pm 2^{\circ} \mathrm{C}$. Under these conditions, the embryos did not experience a low temperature maintenance period and may have developed more efficiently than in the field. The larvae may then have contained a laboratory induced artifact of excess energy.

Since incubation temperature apparently has an effect on yolk conversion efficiency, the same egg, incubated under different temperature profiles, should produce larvae with varying levels of energy reserves at hatch. Homarus americanus on the Atlantic coast occur at depths from near intertidal to at least $700 \mathrm{~m}$ (Cooper and Uzmann, 1980). Migration of deep water populations tends to keep a portion of the population within a temperature range of $8-14^{\circ} \mathrm{C}$ throughout the year (Uzmann et al., 1977). Inshore populations, in contrast, experience temperatures less than $8^{\circ} \mathrm{C}$ for at least half the year (Cooper and Uzmann, 1980).

The energetics of embryogenesis of inshore and offshore populations would be an interesting topic for further study. Two differing strategies may be utilized by these populations: either an egg is produced with just enough energy to produce a larva under the expected temperature regime, or an egg is produced with a potential surplus of energy to adapt to any temperature regime. In the former case, the female would be able to produce more eggs if the temperature was higher; in the latter case, larval hatching would be assured regardless of environmental temperature regimes. The duration of embryogenesis is another factor to be studied. 
Assuming there is an optimal period for larval hatching, what controls the timing of hatching? Possible methods would include time of egg extrusion, and migration to control the temperature regime. A final point to study would be whether the developmental rates of embryos were different between inshore and of fhore populations. Lipid Classes: Graphs 9-10, Tables 3-4.

Triacylglycerol was responsible for most of the total 1ipid pattern seen during embryogenesis. Triacylglycerol, or its analogues, was the energy storage form for this period. The use of triacylglycerol as the primary lipid energy store for embryogenesis has also been noted in the barnacles Balanus balanoides and Balanus balanus (Dawson and Barnes, 1966), the decapod Acanthephyra spp. (Herring and Morris, 1975) and inferred in the shrimp Pandalus montagui (Leach)(Clarke, 1979).

The term "analogue" used above refers to the possibility that the triacylglycerol peak on the chromarods (Iatroscan, see Methods) actually contained substantial amounts of alkyl derivatives of triacylglycerol. The most probable candidate was alkyldiacylglycerol, where the fatty acid on carbon 1 of the glycerol backbone was substituted with a fatty alcohol linked via an ether bond. The evidence for this analogue was circumstantial and it would be of interest to examine this possibility in more detail. The evidence consisted of a slight increase in the mobility of the triacylglycerol peak versus either the tripalmitin standard, a trilinolenin standard, or the triacylglycerol peak of larvae. The ether bond in alkyl forms causes such an increase (Christie, 1973). The plasmalogen analytical technique for chromarods, described in Materials and Methods, eliminated alk-1-eny1 diacylg1ycerol as a possibility, so 
alkyldiacylglycerol was the next candidate.

Alkyldiacylglycerol is relatively rare, except in marine animals (Malins and Varanasi, 1972); it has been reported in decapods (Addison et a1., 1972; Allen, 1972; Chapelle, 1977; Clarke, 1977). The loss in potential metabolic energy between alkyldiacylglycerols and triacylglycerols is minor, so there does not seem to be any energetic cost in using this form as an energy reserve. The advantages are unknown, though like wax ester synthesis, alkyldiacylglycerol synthesis may allow a more favorable redox balance in the cell during periods of rapid lipid synthesis (Sargent, 1976).

Analytical and sampling variations resulted in fluctuating levels of free sterol though the overall level remained constant. This indicates that sterols were not catabolized for energy, nor substantially lost in any metabolic transformations. Since crustaceans cannot synthesize sterols (Zandee, 1967) and the vast majority of sterol in lobsters is cholesterol (Gagosian, 1975), it is likely that the sterol in the egg and resulting larvae was also cholesterol. Therefore, it is probable that little or no conversion of sterol occurs during embryogenesis.

The appearance of steryl esters a month after fertilization is inexplicable. It was possible that the levels of steryl esters in the first month were below detection, so that the sudden appearance of stery1 ester in the fourth week does not necessitate a rapid conversion of free sterols. Whitney (1969) also found steryl esters in developing eggs of the blue crab and hypothesized that they serve as a storage form for free sterols. Steryl esters declined with development in blue crab eggs but did not in lobster. eggs; steryl esters may have an additional, unknown 
function.

There is the possibility that the chromatographic peak that was identified with standards as steryl esters also contained either hydrocarbons or wax esters. Both of these compounds co-migrate with steryl esters under the solvent system used. Low leve1s of natural hydrocarbons have been reported in crustaceans (B1igh and Scott, 1966; Addison et al., 1972), though at levels much lower than the "stery1 ester" quantities noted in the present study. Wax esters have never been reported in lobster except in trace amounts.

The appearance of monoacylglycerols and phosphatidy1 (P.) inosito1 at hatch was partly an artifact. The chromarod sets were switched at this point which resulted in increased resolution in the phospholipids, separating the P. inositol peak from the P. choline peak. Unfortunately, it must be assumed that P. inositol was incorporated into the P. choline peak for all the embryogenic samples. Therefore, any changes in $\mathrm{P}$. inosito1, if they occurred, were masked by P. choline. The appearance of the monoacylglycerol peak, which contained $P$. serine and phosphatidic acid as well, may also be due to the increased resolution, with $P$. serine becoming isolated from the P. ethanolamine peak.

The levels of $P$. ethanolamine appeared erratic throughout embryogenesis, though overall it showed a declining pattern. A portion of this variability was due to the solvent system used, which barely separated $P$. serine from $P$. ethanolamine in these samples. The result was of ten a shoulder on the P. ethanolamine peak that the integrator had difficulty interpreting. The analyses of $P$. ethanolamine fatty acids did not show as much variation which also suggests that the Iatroscan method 
was variable in quantifying this class with the present solvent system. One established method to quantify lipid classes has been the interpretation of fatty acid data. Basically, the fatty acid quantities are summed to yie1d a value that is an assumed percentage of the class (Christie et a1., 1970). This technique cannot be applied to the present study, however, as there is evidence that alkyl analogues of $\mathrm{P}$. ethanolamine and P. choline exist in Homarus americanus eggs. In both classes, as in triacylglycerol, the peaks had slightly greater mobilities than standards. Plasmalogen was not detected with the Iatroscan, although analysis with thin-layer chromatography (Schmid and Mangold, 1966), with primuline (Wright, 1971) and iodine vapor detection, indicated that trace amounts of the plasmalogen form (alk-1-eny1) were present, though not in sufficient quantities to have affected the peak mobility. It is suggested that alky1 acy1 forms of P. ethanolamine and P. choline were also present in the eggs of Homarus americanus.

In the embryogenesis study, the decline in P. choline occurred mainly at the beginning and end of embryogenesis, with a large decrease around the hatch. The decline in lipids up to the last egg sample was primarily due to triacylglycerol, P. choline, and P. ethanolamine (they accounted for $74 \%, 23 \%$, and $4 \%$ of the total 1ipid decline, respectively). When the larval sample is used as the endpoint, the comparative values are $61 \%$, $32 \%$, and $5 \%$, respectively. (P. inositol was added to P. choline; P. serine was added to $P$. ethanolamine). The relatively high leve1 of $P$. choline utilization indicates that it can supply a major portion of the energy required for embryogenesis.

Utilization of $P$. choline did not appear to be mandatory, however, as 
the hatching period study showed a much lower decline of $\mathrm{P}$. choline than the embryogenesis study for a comparable period surrounding the hatch (per individual, $18.0 \mu \mathrm{g}$ versus $53.5 \mu \mathrm{g}$, respectively). During this same period, triacylg1ycerol dec1ined $61.5 \mu \mathrm{g}$ versus $37.9 \mu \mathrm{g}$ for the hatching period and embryogenesis studies, respectively. The totals of these two classes in the two experiments were roughly equivalent at 79.5 $\mu \mathrm{g}$ and $91.4 \mu \mathrm{g}$ which indicates that comparable amounts of 1ipid were utilized.

The above data suggest that $P$. choline acts as a secondary energy reserve. If needed $P$. choline can be significantly catabolized without apparent detrimental effect in terms of subsequent larval survival. If other preferred energy sources (i.e. triacylglycerol) are available, however, then P. choline is spared. This could explain the much lower utilization of $P$. choline during the hatching period study, since abundant resources of triacylglycerol were present. During the embryogenesis study, however, triacylglycerol was depleted and P. choline catabolism became necessary. The same cannot be said for $P$. ethanolamine since it did not show a sudden decline near the hatch. P. ethanolamine is mainly found as a membrane component (Gurr and James, 1980), whereas P. choline, in crustaceans, serves other functions as well (Lee and Puppione, 1978). As cell membrane integrity is vital, it is likely that the P. choline utilized came from a non-membrane associated metabolic pool. Fatty Acids: Graphs 11-16, Tables 5-8.

The rise in lipid during the first month of embryogenesis, seen in both the total 1 ipid and 1ipid class data, was also reflected in the fatty acid results. It is doubtful that 1 ipid was actually being synthesized 
during this period, though a similar result was seen in the barnacle Balanus balanoides (Barnes, 1965). It may have been an artifact due to sampling variation. Since tritiated water is incorporated into newly synthesized fatty acid (Jungas, 1968; Lowenstein et al., 1975), 1ipid synthesis could be confirmed for this early embryonic period. The rapid influx of water by the egg after extrision would facilitate such a study since large amounts of label could be naturally introduced into what is otherwise an isolated system.

The presence of relatively large amounts of 20:5 and 22:6 in the neutral lipid fraction of the eggs was unexpected. These two fatty acids were present at levels between $15-20 \%$ of the total neutral lipid fatty acids, which made them the second and third most common neutral lipid fatty acids (after 18:1). Oogenesis must then act as a large drain of these presumed essential fatty acids from the female.

It is unknown whether the high levels of $20: 5$ and $22: 6$ were required for some metabolic function associated with neutral lipids or whether their presence was merely a reflection of the female's dietary history. The fatty acid profiles of triacylglycerols in some crustaceans are known to reflect their diet (cf. Holland, 1978) and, within limits, oogenesis may simply utilize whichever fatty acids are available.

One possible metabolic function of these two fatty acids is the maintenance of fluidity. Since $20: 5$ and 22:6 are both maximally unsaturated, they are the most fluid of the lobster's common fatty acids at any given temperature. Triacylglycerols are extremely hydrophobic and when present in large quantities, can occur as nearly homogeneous droplets (Lehninger, 1975). In marine crustacean eggs, storage 1ipids occur as 
vesicles (Holland, 1978). During winter, when the water temperature dropped to $0^{\circ} \mathrm{C}$ or less, triacylglycerol was still being metabolized for energy by the embryos. In this situation 20:5 and 22:6 may function to maintain a required degree of fluidity within the stored triacylglycerol vesicles so that they can be adequately metabolized. In the Antarctic benthic prawn Chorismus antarcticus (Pfeffer), egg triacylglycerols were also high1y unsaturated with large levels of 20:5 and 22:6 (Clarke, 1977). The pattern of utilization among the major saturated and monounsaturated fatty acids (termed non-essential fatty acids or NEFA in graphs 11,13 , and 15 ) show that these fatty acids were evenly catabolized. In other words, for the three lipid class pools, there was no selective metabolism of NEFA during embryogenesis.

At hatch, however, the percent composition patterns did change. The changes in the neutral lipid pools were due to the near depletion of the otherwise dominant triacylg1ycerols. The pattern that remained was therefore heavily influenced by the steryl esters, free fatty acids, and mono- and diacylg1ycerols.

The patterns seen in P. ethanolamine and P. choline did, however, indicate selective metabolism of NEFA at hatch. The ratios of $16: 1$ and 18:1 in both classes declined while 16:0 increased. Since in absolute terms all the NEFA declined at this point, the increase in 16:0 (and 18:0 in $\mathrm{P}$. choline) appears to be a result of conservation rather than synthesis.

The polyunsaturated fatty acid (PUFA) patterns in these classes indicate that 20:4, 20:5, and 22:6 all tended to increase proportionally at hatch. As these fatty acids cannot be synthesized de novo by the 
lobster, it appears that selective conservation of these PUFA was occurring. How conservation of any fatty acid occurred is unknown. Typically, P. choline and P. ethanolamine molecules contain PUFA on carbon 2 of the glycerol backbone (Brockerhoff et a1., 1963) and saturated fatty acids on carbon 1 (Hanahan et a1., 1960a,b). The molecules which contained these PUFA may have simply been preferentially conserved (and 16:0 and 18:0 were associated with these molecules), or the carbon 1 fatty acids may have been removed and the lyso derivatives of $P$. choline and $P$. ethanolamine that contained these PUFA were then selected, or all the fatty acids may have been cleaved and the PUFA conserved and re-esterified back into P. choline and P. ethanolamine. Whereas the former was probably the case, the latter two pathways cannot be ruled out.

The 1atter two pathways described above would require other fatty acids to reform $P$. choline and $P$. ethanolamine. The primary product of fatty acid synthesis is 16:0, followed by chain elongation to 18:0 (Mayes, 1983a). The conservation of PUFA may have then been linked with synthesis or selective retention of $16: 0$ and $18: 0$, possibly to maintain a certain degree of total unsaturation.

Obviously, the current data set cannot provide any clues to distinguish between the three possible mechanisms for PUFA conservation. The period of hatching, however, also involves a rapid intake of water which would make it conducive for tritiated water labelling. Such an experiment, coupled with GC-radiolabel detection and selective enzymatic hydrolysis of fatty acids (Hanahan et a1., 1960b; Mattson and Beck, 1956) would provide valuable information.

The fatty acid 20:5 appeared to be selectively metabolized during 
embryogenesis. This fatty acid was preferentially lost from the neutral lipids and conserved in P. choline and P. ethanolamine. As 20:5 cannot be synthesized by Homarus americanus its increased amounts in P. choline and P. ethanolamine must have originated from the neutral lipids. This indicates that turnover of P. choline and P. ethanolamine occurred during embryogenesis. Such turnovers occur in developed animals (Gurr and James, 1980) and, as this pattern did not begin to emerge until well into embryogenesis, it is likely that this turnover occurred in the embryonic tissues and not in the yolk.

\section{Summary: Embryogenesis and Hatching}

The most active periods during embryogenesis at ambient temperature conditions, in terms of biochemical changes, were the month immediately after fertilization and the month prior to hatch. Ash and water uptake appeared to be interrelated, with both increasing during these active periods. Resolution of the chitin measurements was insufficient to detect minor changes during embryogenesis, except for a major increase prior to the hatch. Carbohydrate reserves showed a slight initial decrease but otherwise were not utilized during embryogenesis until just prior to hatch. Carbohydrate reserves probably served as a precursor to chitin, though gluconeogenesis was also required. Protein was overestimated in eggs (but not larvae) and possible causes for this are discussed. Lipid was the primary source of energy during the embryonic period.

The difference in incubation temperatures between the embryogenesis study (seasonally fluctuating, ambient) and the hatching period study (constant, elevated) resulted in relatively greater energy reserves in the larvae of the latter study. This extra energy represented the energy 
required primarily for maintenance during the sustained mid-period cold temperatures that occur during normal nearshore field conditions.

Most of the catabolized 1ipid was triacylglycerol, followed by P. choline. P. choline can serve as a secondary energy source if triacylglycerol becomes depleted. There was evidence for alkyl analogues of triacylglycero1, P. choline, and P. ethanolamine in the lobster eggs. Unexpectedly large amounts of 20:5 and 22:6 fatty acids were found in the egg triacylglycerols. A possible function for these fatty acids was the maintenance of stored 1ipid fluidity. Utilization of triacylg1ycero1 fatty acids was non-selective with the exception of 20:5. This fatty acid appeared to be selectively transferred out of triacylglycerol and into P. choline and P. ethanolamine. Except for 20:5, utilization of P. choline and $P$. ethanolamine fatty acids were also non-selective throughout embryogenesis, though the fatty acids $16: 0,18: 0,20: 4,20: 5$, and $22: 6$ were slightly conserved during the hatching period. 
Pulse Fed and Immediate Post Hatch Development: Graphs 17-18, Tables 9-10

The feeding periods in this experiment were limited to $12-18 \mathrm{~h}$ and $36-40 \mathrm{~h}$ after hatching. Visual observation of the larvae indicated that they seized and manipulated food items (Artemia) during both feeding periods. The biochemical evidence, however, suggests that the amount of ingested food during the first period was minimal as there was no significant increase in any component. Even if assimilation was poor, as this was the larvae's first exposure to exogenous food, the gut contents of the larvae would have caused detectable increases in absolute levels of the assayed compounds.

Structurally the larvae were probably capable of feeding within $12 \mathrm{~h}$ of hatch. Ash and chitin levels and the molt stage at this point all indicated that the exoskeleton was hard enough to allow ingestion of food. The lack of feeding could have been an artifact, though, as the larger amounts of yolk reserves in these larvae (that developed at constant high temperatures) may have delayed the onset of first feeding. Lobster embryos maintained in the laboratory at ambient (field) temperatures hatch with a small amount of yolk visible in the hepatopancreas and the gut (P. Biesiot, personal communication). Normally, these larvae feed within $12 \mathrm{~h}$ of hatching. In such larvae, first feeding was biochemically detected in the present work (as increases in total protein) consistently within the first $12 \mathrm{~h}$ after hatch.

The increases in carbohydrate and protein indicate that feeding occurred during the second presentation of food. Lipid, in contrast, levelled off for a period and then continued to decline. As the food contained approximately $10 \%$ 1ipid by dry weight (Sasaki, in preparation), 
lipid catabolism was counterbalancing any incorporated lipid.

The caloric equivalents of the absolute losses of protein, lipid, and carbohydrate indicate that lipid supplied the majority of the energy during this post hatch period. The initial levels of protein appeared to be overestimated, as in the egg. This may have been due to the presence of yolk in the larva.

The extent to which the extra yolk affected these biochemical patterns is uncertain. Regulation between utilization of endogenous and exogenous food sources does occur in these larvae as sma11 yolk reserves are occasionally present in second stage larvae fed ad libitum ( $P$. Biesiot, personal communication). It appears as though the larvae in the present study heavily depended on their endogenous reserves for at least the first $60 \mathrm{~h}$.

The presence of yolk would appear to be an advantage for larvae as it would delay both the point-of-no-return (Anger and Dawirs, 1981) and the critical first feeding period (Kon, 1979; Paul and Paul, 1980). For starved larvae, the point-of-no-return is the stage when the ingestion of food no longer assures survival. The first feeding period is critical since too long a delay reduces the larvae's viability. The fact that at ambient temperature conditions the amount of yolk was apportioned to produce larvae with little yolk at hatching suggests that Homarus americanus larvae in the field probably can begin, and can benefit from, eating within $12 \mathrm{~h}$ of hatching.

The ash levels underwent a rapid increase just after the hatch. Intake of water also occurred during this period but most of the ash probably went into exoskeleton-production. The energetic cost of this. 
rapid ash intake and exoskeleton synthesis is unknown, though rapid decreases in protein, carbohydrate, and lipid simultaneously occurred.

Due to the rapid changes within the first six hours after hatching, sampling of sequential hatches from day to day could produce highly variable results unless the samples were all taken at exactly the same time after hatch. A six hour difference in sampling times in this study would have resulted in a change of $+98 \mu \mathrm{g}$ for ash and a change of -27 $\mu \mathrm{g},-24 \mu \mathrm{g}$, and $-4 \mu \mathrm{g}$ for protein, 1ipid and carbohydrate, respectively. The equivalent caloric change was -0.385 calories

Pandian (1970a) found ash and caloric changes in Homarus americanus larvae hatched on different days. He noted an increase in ash (and exoskeleton hardness) and a decrease in calories in succeeding days (three sample times; 1st, 2nd and 4th days of hatch). His interpretation was that these changes occurred prior to the hatch; resulting in less energetically fit larvae during the later hatches. This interpretation is in error. Since the hatching process involves a molt, any exoskeleton (and exoskeleton ash) formed prior to the hatch is lost. The magnitude of the ash and caloric changes that he noted over the three day span were $+113 \mu \mathrm{g}$ of ash and -0.31 calories. No such differences were noted in any of the analyses conducted on succeeding hatches in the present work. Therefore, it is probable that variances in sampling times among Pandian's three samples were responsible, as a six hour difference would have been sufficient to account for the noted disparities.

The decline in total lipid was dominated by triacylglycerol and P. choline (losses of $56 \mu \mathrm{g}$ and $36 \mu \mathrm{g}$, respectively, in the first $60 \mathrm{~h}$ ). The pattern of utilization in both classes was sufficiently linear $\left(R^{2}=\right.$ .95 and $R^{2}=.90$, respectively) that $P$. choline utilization was not the 
result of triacylglycerol depletion. That is, P. choline utilization began soon after the hatch when relatively large amounts of triacylglycerol were still present. The embryogenesis and hatching data suggested that $P$. choline served as a secondary energy source that was called upon when triacylglycerol levels became low. This hypothesis was not supported by the present data that suggest a certain portion of $P$. choline may be allotted for energy production regardless of other existing reserves. Utilization of this P. choline for energy, however, appears linked to growth rather than maintenance. Summary: Pulse Fed and Immediate Post Hatch Development

Greater than normal yolk reserves may delay initial feeding by larvae. This would also serve to delay both the point-of-no-return and the critical first feeding period. When extra yolk reserves were present, the larvae appeared to rely on yolk energy during the immediate post hatch period rather than on exogenous food. As in embryogenesis, lipid supplied most of the energy under high yolk conditions.

Ash underwent a rapid increase immediately after the hatch. A previous description of ash and caloric differences between successive hatches (Pandian, 1970a) was actually due to variances in sampling times. Triacylglycerol and P. choline were responsible for the total 1ipid pattern. A portion of P. choline may be allocated for energy production. 


\section{Larval Molt and Development}

This section will discuss the larval development period from hatch to the end of the fourth larval stage. It is organized into two parts--one discussing the molt cycle and the other encompassing general development through each larval stage and metamorphosis.

Mo1t Cycle

The larval molt cycle, in terms of duration and timing, can be affected by a variety of environmental factors (Aiken, 1980; Hartnoll, 1982). In this study, food quantity and water quality were kept as optimum as possible, so temperature was probably the major influencing factor (Hartnol1, 1982). The temperature during each set of experiments generally increased during the developmental periods, with the II hatches experiencing a smaller increase. With increasing temperatures, molt rate increases, though the size increment gained with each mo1t may dec1ine (cf. Hartno11, 1982). At a constant temperature, the duration of each larval stage in Homarus americanus larvae would have successively increased. The approximately equal durations for the first three larval stages seen in these graphs was due to the rising temperature regime that occurred. Since ambient water temperatures were used, this molt timing pattern was probably wel1 within the range of what natura11y occurs in the field.

Though temperature was higher for the fourth stage versus earlier stage larvae, the duration of the fourth stage was nearly equivalent to the sum of the previous three stages. As a postmetamorphic animal, the fourth stage larvae may have different physiological requirements. 
stages. The fourth stage larvae experienced a proportional1y longer $D_{1}$ ' stage interval (Appendix 1). Under certain conditions a lobster can delay the molt. When such a delay is in effect, the lobster remains in either molt stage C or D。(Aiken, 1973). The necessary conditions for such a delay, however, are rather drastic--temperatures less than $5^{\circ} \mathrm{C}$ is one example (Aiken, 1980). It would be interesting to determine if a longer $D_{1}$ ' period is also an indication of a slight molt delay. That is, given conditions that are not optimal, can the animal temporarily remain in $D_{1}$ ' to delay the molt.

The kriese1 system (Hughes et a1., 1974) used to raise the larvae in the present study has a bare plastic bottom. Cobb (1968) showed that a fourth stage larva (which must settle to the benthos after the mo1t) would delay molting by as much as two days if kept over a bare bottom versus gravel. In the present study, shortening the $D_{1}^{\prime}$ stage by one to two days would have resulted in a molt cycle pattern very similar to that found for stage III larvae. Proximate Analysis: Graphs 19-26.

The following discussions on the biochemical correlates of molting are generalized for the first three larval stages. The fourth larval stage, which of ten showed considerably different patterns, will be noted separate1y when appropriate.

The wet weight increased at each molt. In a few cases during the norma1 sampling procedure, larvae in early stage $\mathrm{E}$ were found and measured for wet weight. In these cases, the larvae had absorbed approximately $25-35 \%$ of the water that they would eventually absorb. This was comparable to the approximately equal proportions of pre- and postmolt 
water intake found in juvenile Homarus americanus (Mykles, 1980).

Dry weight, for the most part, did not fluctuate with the molt

cycle. Increases were constant from the start of each cycle to the end. This resulted in an oscillating pattern of dry versus wet weight through the molt with an approximate range between 14 to $21 \%$. As this is not a trivial difference, care must be taken to determine the proper wet to dry weight conversion factor for any work that requires processing of fresh tissue.

Water uptake in lobsters is not controlled by an osmotic gradient between the hemolymph and seawater (Da11 and Smith, 1978; Mykles, 1980). Therefore, salt was not transported into the hemolymph to form such a gradient during ecdysis when water was absorbed. This does not preclude an osmotic gradient in the intercellular space (Diamond, 1977), however, with the end result that water intake is accompanied by salt. This increase in salt, or ash, that was probably occurring at the time of ecdysis was not detectable, though, as stages $\mathrm{E}$ and $\mathrm{A}$ were too brief and any water related salt increase after the molt was swamped by salts in the newly forming exoskeleton.

The first three larval stages possess lightly calcified exoskeletons relative to older animals (Williams, 1907). The process of molting in these larvae is nonetheless facilitated by a reduction of exoskeleton calcium. The evidence for this was seen in the trend for an actual 1oss of ash during the latter half of the molt cycle. A similar trend is seen in prawns (Read and Caulton, 1980) whose exoskeltons are also 1ightly calcified. No gastroliths were present in the pre-metamorphic larvae (Williams, 1907), though it is possible that a small portion of 
exoskeleton salts were accumulated in the hemolymph or hepatopancreas (Paul and Sharpe, 1916; Travis, 1955b; Adegboye et a1., 1975). If so, these low levels of temporary stores may serve to quickly harden mouth and chelae parts after the molt to reduce the period of non-feeding by the larvae. Even if no such storage exists or there is non-selective calcification after a molt, the rapid intake of ash after a molt indicates an expeditious hardening of the new exoskeleton and presumably a quick resumption of feeding ability.

This swift return to feeding was apparent in the immediate decline in ash as a percent of dry weight. Since ash at this point remains steady in absolute amount, the increase in dry weight, that causes the ash as percent dry weight to decline, must be organic in nature, i.e. derived from food.

The resolution of the chitin assay was not fine enough to detect molt related patterns when expressed as a percent of dry weight. As an absolute amount, chitin continued to increase though the molt cycle though there was a tendency, most obvious in the fourth larval stages, for chitin to level off halfway through the cycle.

Chitin is synthesized throughout the molt cycle, with a sudden increase occurring at molt stage $D_{2}$. The rate of synthesis continues to increase after $D_{2}$, through ecdysis to stage $B$, when it declines (Hornung and Stevenson, 1971). The RNA responsible for the chitin synthesizing enzymes are apparently synthesized during stages $D_{1} \prime \prime$ and $D_{2}$ (Stevenson and Tung, 1971). Superimposed on this chitin buildup is chitin resorption which occurs during the end of the cycle and provides material for new chitin synthesis and energy (cf. Spindler-Barth, 1976). In the 
present study, chitin resorption was only evident in the fourth larval stage, though it probably occurred in the other stages as well. The decline of absolute chitin levels in the fourth stage was similar to the decline seen in the crab Carcinus maenas (Spindler-Barth, 1976).

Both ash and chitin are primary components of lobster exoskeleton. The fact that chitin buildup was seen to continue long past ash buildup was due to the multi-layered structure of the cuticle, with each layer being composed of different amounts of each component (Aiken, 1980). The outermost layers, the epicuticle and exocuticle, are formed prior to ecdysis during the burst of chitin synthesis seen at this point. After ecdysis, the outer layer of the endocuticle is formed. This layer is heavily calcified (Aiken, 1980) and is probably the primary sink for the newly absorbed ash. The inner endocuticle layer, which is proportionally quite thick in Homarus spp., forms next (Aiken, 1980). It is the formation of this inner, lightly calcified endocuticle layer that is probably responsible for the continued chitin synthesis after ash absorption has stopped.

In the first three larval stages, absolute carbohydrate content showed an early premolt rise followed by a late premolt and postmost decline. As percent dry weight, this pattern was more evident, with the same molt related rise and decline. This tends to support what has been found in several other crustaceans (Renaud, 1949; Te1ford, 1968; Adiyodi and Adiyodi, 1970) and has been interpreted to mean that carbohydrate serves as either an energy source to support the animal through the molt, and/or as a precursor for the rapid chitin synthesis which occurs at this time (Renaud, 1949; Travis, 1955a). This.type of pattern has not always 
been found (Scheer, 1960; Heath and Barnes, 1970), however, nor was it evident in the fourth stage larvae in the present study.

This diversity in patterns is the result of the various functions of carbohydrate in crustaceans. Carbohydrate is used as an oxidative substrate throughout the molt cycle (Bergreen et a1.,1961), it can function as an energy storage form (Renaud, 1949), and it is a chitin precursor (Hohnke and Scheer, 1970). While it serves these and other functions, the relative importance of each varies through the molt cycle and probably through development and from species to species.

The decreases in carbohydrate at molt in Homarus americanus larvae were not sufficient to account for the newly synthesized chitin, so $\underline{H}$. americanus larvae were dependent on other reserves or food. This does not, however, preclude the carbohydrate reserves from functioning as chitin precursors. To speculate, perhaps carbohydrate from reserves and gluconeogenesis in larval $\mathrm{H}$. americanus function as chitin precursors during the molt only to synthesize sufficient chitin for the protein-chitin matrix (Aiken, 1980) of the outer endocuticle layer of the exoskeleton. This outer layer is the primary calcified structure of the cuticle (Aiken, 1980) so once it is formed the larvae are probably capable of feeding. Additional chitin synthesis then relies on food, of which an immediate source could be the newly molted exuvia. Lobsters, and many other crustaceans, can consume their own exuvia (Aiken, 1980). Since lobsters possess chitinases (Kooiman, 1964; Brockerhoff et a1., 1970), the ingested exuvia may provide some, or much, of the required chitin precursors.

The above hypothesis does not account for the apparent lack of a 
carbohydrate buildup, as percent dry weight, in the fourth stage larvae. Two explanations are possible. Prior to the molt, the old cuticle can be partially resorbed and the resulting glucosamine used for energy and as chitin precursors (Gwinn and Stevenson, 1973; cf. Spindler-Barth, 1976). Perhaps this chitin resorption is an ability present in postmetamorphic fourth stage larvae that, as has been suggested for Carcinus maenas (Spindler-Barth, 1976), is not fully utilized by earlier stages. The second explanation is that chitin is associated only with the surfaces of the animal. In contrast, the carbohydrate reserves are associated with the volume (weight) of the animal. The fourth stage larvae are considerably heavier than the earlier stages so the apparent lack of molt related carbohydrate storage in fourth stage larvae may be a size related effect. That is, the surface (chitin) to volume-weight (carbohydrate) ratio may be sufficiently smaller in fourth stage larvae, as compared to the earlier stages, that a lower percentage of carbohydrate is sufficient for the required molt related chitin synthesis. Further evidence of a size effect is seen when carbohydrate is expressed as its caloric equivalent and then compared against the total calories. Though it declines from a midmolt high value, the caloric equivalent of carbohydrate at the end of the fourth stage still remains at levels comparable to the premolt values of the other stages.

In absolute terms, carbohydrate increased rapidly after the molt in fourth stage animals but stopped accumulating once a certain level was reached. One implication is that carbohydrate was probably not a preferred storage material; it was also possible that there was an increased metabolic demand for carbohydrate during this intermolt period 
which precluded further storage. Increased fatty acid synthesis during premolt, for instance, would require reducing power (NADPH) which carbohydrate could provide (McWhinnie and Corkill, 1964; McWhinnie and Chua, 1964).

In absolute amounts, protein showed a weak pattern around the molt cycle. For the most part, protein increased through the molt cycle with a trend for a leveling in the late premolt-ear1y postmolt period. Actual decreases of protein in the larvae at ecdysis were seen in about half of the molts. Such decreases have been noted in the shrimp Crangon crangon (Regnault, 1979) and in the prawn Penaeus indicus Milne Edwards (Read and Caulton, 1980).

Protein loss after the molt occurs through catabolism. Regnault (1979) measured ammonia excretion during the molt cycle of the shrimp Crangon crangon and detected a sharp rise in ammonia production immediately after the molt. Similar experiments with Homarus americanus larvae show that a postmolt increase in ammonia production would be 1imited to a short period (less than $24 \mathrm{~h}$ ) after the molt (Capuzzo et al., 1984). The major metabolic pools for nitrogen in a crustacean are protein, chitin, and phospholipids. In the larvae of the present study, protein would be the only component that could account for such an ammonia 1oss.

As percent dry weight, protein did show a pattern with a premolt high followed by a postmolt low. As a percent of ash free dry weight, this pattern was much weaker but still present. Direct comparisons of these data are difficult as other studies examined specific tissues or organs rather than the whole animal. Nevertheless, the same pattern emerges 
whether one is examining hepatopancreas protein (Renaud, 1949; Gorel1 and Gilbert, 1971) or hemolymph protein concentration (Travis, 1955b; G1ynn, 1968; Barlow and Ridgeway, 1969; Busselen, 1970; Bursey and Lane, 1971; Spindler-Barth, 1976; Hepper, 1977; Durliat and Vranckx, 1982).

In order to extricate itself from the old exoskeleton, a crustacean must minimize the tissue mass in its extremities. (A slight paradox, as the molt is a response to insufficient space for growth.) This is accomplished by resorbing muscle mass, a process that selectively acts on the actin, rather than the myosin, portion of the muscle (Mykles and Skinner, 1982). In the crab Gecarcinus lateralis, chelae muscle protein can decrease up to $40 \%$ in preparation for the molt (Skinner, 1966a). This resorbed protein is likely stored within the animal and may account for the increase in hemolymph (Busselin, 1970) and hepatopancreas protein levels. Resorption of the cuticle may also be adding to these protein pools (Passano, 1960). All this resorption and storage of protein does not preclude new protein accumulation, as protein synthesis is elevated during this premolt period (Skinner, 1966b; McWhinnie and Mohrherr, 1970). The duration of the increased ammonia production after a molt, detected by Regnault (1979), lasted about a day. This is proportionally similar to the period in which protein in the present study, as percent dry weight, decreased after the molt. Though a certain amount of protein catabolism at molt was occurring, the purpose of this catabolism is unknown. The link between protein and chitin via gluconeogenesis has been discussed and it is possible that this is occurring. If protein was used for chitin related gluconeogenesis, this may offer another basis for the protein sparing effect of dietary carbohydrate (Capuzzo and Lancaster, 
1979b). This relationship is more complex, however, as nitrogen is required for formation of glucosamine and protein is probably the major nitrogen reserve in these larvae. Metabolic energy may have been another function for protein catabolism as the lipid reserves were still low. Lipids: Graphs 25-36, Tables 11-20.

The absolute levels of total lipid in first three larval stages were not strongly related to the molt cycle. In these larval stages there was only a suggestion of a leveling off during their late premolt periods. This premolt leveling was evident, however, in the fourth stage.

As a percent of dry weight, the lipid pattern showed an intermo1t low followed by a rise to a maximum in mid premolt. Lipid then began to decline until the next intermolt. Much of this pattern was, however, due to fluctuations in ash. When expressed as percent of ash free dry weight, the lipid patterns for the first three larval stages were much more stable.

Other studies that have examined Iipids in whole animals have seen molt related lipid patterns with maximum levels in the early to mid premolt periods, followed by a decline through the molt to the late postmolt to early intermolt periods (Teshima and Kanazawa, 1976b; Teshima et a1., 1977; Ando et al., 1977; Read and Caulton, 1980). When only the hepatopancreas was analyzed, the peak levels of lipid usually occurred sooner in early premolt (Renaud, 1949; O'Connor and Gilbert, 1969; Lautier and Lagarrigue, 1976; Ando et a1., 1977; Barclay et al., 1983), followed by a decline similar to the decline seen in whole body levels. This slight difference in peak lipid level periods between the hepatopancreas and whole body appears to be due to the longer, slower accumulation period for lipids in the muscle and integument as compared to the hepatopancreas 
(0'Connor and Gilbert, 1969; Ando et al., 1977).

The type of 1ipid stored in the hepatopancreas and muscle are also different. During the rapid 1ipid increase at midmo1t, the hepatopancreas stores substantial amounts of both neutral and polar lipids, with the neutral 1ipids being favored (Carcinus maenas, Heath and Barnes, 1970; $\underline{\text { Palaemon paucidens }}$ De Haan, Teshima and Kanazawa, 1976b; Penaeus japonicus, Ando et a1., 1977; Chorismus antarcticus, Clarke, 1977; $\underline{\text { P. }}$ japonicus Patrois et al., 1978). In the crab Pachygrapsus marmoratus (Fabricius), however, polar lipids were dominant (Lautier and Lagarrigue, 1976). In contrast, phospholipids are preferentially accumulated in the muscle tissue (Teshima and Kanazawa, 1976b; Ando et al., 1977; Clarke, 1977; Patrois et al., 1978).

This can lead to confusing interpretations of whole body data because the extent to which energy reserves are stored in the hepatopancreas appears to be related to the lifestyle of the organism. Relatively small crustaceans with very active lifestyles (Da11, 1964, 1965) and/or with short intermolt periods and quick molt recoveries (Scheer, 1960) do not accumulate substantial reserves relative to other crustaceans. This may offer an explanation for the lipid pattern seen in the four larval stages of Homarus americanus. Each of the first three larval stages have short intermolt periods and fast molt recovery. The fourth larval stage, in contrast, has a much longer intermolt period. The molt recovery period duration as a fifth stage larvae was not studied.

An animal that accumulates substantial lipid reserves in relation to the molt is probably storing them in the hepatopancreas rather than other tissues. In which case, the type of lipid would tend to be a neutral 
lipid and time of lipid storage would be mostly during the early premolt period. With sufficient 1ipid storage, the hepatopancreas lipid can determine the whole animal pattern. This type of lipid pattern was seen in fourth stage larvae; the neutral lipids (triacylglycerols) were the dominant lipid. If an animal was not storing substantial molt related lipid reserves, the effect of the hepatopancreas lipids would not heavily influence the whole body pattern. The increases in muscle lipid, which occur over a larger period at a slower rate would then represent the whole body pattern. This type of pattern occurred in the first stage larvae. Triacylg1ycerol was not being stored, while phospholipids showed a prolonged increase during premolt. The second and third larval stages could then represent transition stages between these two modes.

In relation to the molt cycle, $P$. ethanolamine showed little evidence of a molt related pattern. P. choline, on the other hand, had a tendency for a small postmolt dip in absolute quantity. Similar declines were seen in total phospholipids in other studies (Heath and Barnes, 1970; Lautier and Lagarrigue, 1976; Ando et al., 1977). It is possible that in these other studies, P. choline was responsible for the total molt related phospholipid fluctuations, while P. ethanolamine was more independent.

Sterol content, in Homarus americanus larvae, was completely independent of the molt cycle. Comparison of these data with other studies is difficult as there appears to be no consistent pattern for molt related fluctuations in sterols in crustaceans. Teshima et al. (1975) also found constant levels of sterol in the prawn Palaemon serratus (Pennant). These data, however, were expressed as a percent of wet weight which would indicate (assuming dry weight was increasing) that sterol 
levels as percent dry weight declined through the molt. In another prawn Penaeus japonicus, sterol as percent wet weight showed the typical premolt high, postmolt low values for the whole animal (Teshima et a1., 1977) though in different tissues every conceivable pattern was seen (Kanazawa et al., 1976a). Finally, in yet another prawn Palaemon paucidens large quantities of sterols were apparently lost at molt (Teshima and Kanazawa, 1976b). An important product of sterol in crustaceans is the molting hormone ecdysterone. Ecdysterone has a definite quantitative pattern in relation to the molt cycle (Chang and Bruce, 1981) but in absolute amounts, ecdysterone formation probably does not greatly affect total sterol values.

Variations in fatty acids, as percents of the total fatty acids in each class, did not show molt related patterns. As absolute amounts, the fatty acids tended to reflect the patterns seen in their parent 1ipid classes. (The graphed data was for Hatch I-5 on1y; note that the first data point of third stage larvae was spurious (see Results: Larval Development)).

Previous studies on fatty acids show no consistent patterns. O'Connor and Gilbert (1969) found no molt related changes in fatty acid patterns of the crayfish orconectes virilis. In the prawn Penaeus japonicus the intermolt versus the premolt stage had higher ratios of 18:1w9 and lower ratios of polyunsaturated fatty acids in phospholipids

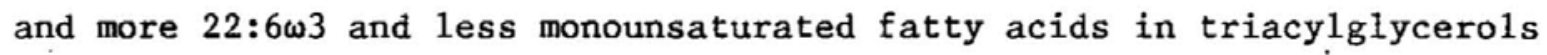
(Teshima et al., 1977). In the shrimp Palaemon paucidens triacylglycerol fatty acids showed no change while phospholipids contained higher ratios of saturated fatty acids (Teshima and Kanazawa, 1976b). These results are 
difficult to interpret because in each case the animals were collected from the field and then sacrificed. The various molt stages found in the sampled population were used to compare the results. The dietary history, one of the most influential factors controlling fatty acid patterns, was unknown for these animals and could have potentially accounted for any pattern seen in these studies. This does not mean that molt related fatty acid patterns do not exist. The stated lack of any pattern in the present study was based on the lack of any noticeable repeating pattern related to the molt cycle. Subtle changes may have been present but were masked by larval developmental changes. This does indicate, however, that large changes in fatty acids were not required during the molt cycle. Summary: Molt Cycle

At constant temperature, successive molt cycle durations in larval Homarus americanus would increase in length. At increasing temperatures, the durations of the first three larval molt cycles tended to merge and become similar in length. The fourth, postmetamorphic molt cycle was much longer and might be a reflection of different physiological requirements and possibly of a delay in molt due to lack of suitable substrate.

Upon ecdysis, the larvae immediately absorbed water, increased in size, and began formation of the new exoskeleton. Decreases in carbohydrate and protein were likely related to chitin formation and, together with lipid, energy consumption. Ash increases quickly stabilized and might indicate the capacity to resume feeding. The multi-layered exoskeleton continued to harden with increased chitin formation that was probab1y supplied from new dietary input. Carbohydrate, protein, and lipid increased from intermolt to early premolt, either through 
accumulation of tissue, and/or energy reserves. Accumulation of energy reserves may be related to lifestyles, whereby active, rapidly growing animals with quick molt recoveries store less reserves than larger, slower animals with more prolonged mo1t recovery periods. First stage larvae are an example of the former lifestyle and did not accumulate much lipid; fourth stage larvae are an example of the latter lifestyle and amassed large reserves of triacylglycerol. The second and third stage larvae exhibited intermediate patterns. Tissue accumulation continued through mid premolt.

P. ethanolamine and sterols were independent of the molt cycle while P. choline showed a slight postmolt decrease. Fatty acids as percents of the total fatty acids in each lipid group did not reveal any molt related patterns. 


\section{Larval Development and Metamorphosis}

\section{Proximate Ana1ysis: Graphs 19-26.}

The increase in size due to a molt is termed the molt increment or growth factor or, preferably, the percentage molt increment (Hartnoll, 1982). It is most of ten expressed as a percent increase in length as compared to the premolt length. Larval lengths were not measured in the present study; nevertheless, an estimate of the percentage molt increment (PMI) can be determined from wet weight increases. Wet weight is correlated with volume and, under the assumption that the gross morphology does not drastically change from molt to molt, one can determine a volume to length relationship. The wet weight PMI values in Homarus americanus larvae were found to range between $70-80 \%$. A cube root volume to length relationship (Kesling, 1952) yields a length PMI value range between 19-22\%. This range agrees with the average length PMI values of $22.3 \%$ (based on 155 decapods) and $18.9 \%$ (based on 57 Macrurans) determined by Rice (1968). It is also in fair agreement with the length PMI values (determined from total length of laboratory populations) of $15.3 \%, 16.2 \%$, and $20.3 \%$ (Capuzzo and Lancaster, 1979c); 17.4\%, 20.6\%, and $13.5 \%$ (Herrick, 1896) and 17.1\%, 18.8\%, and 18.4\% (Hadley, 1906). Values from each study are respectively listed for the growth from the first larval stage to the second stage, second to third stage and third to fourth stage. The estimated length PMI values from the present study were less, however, than the $34 \%, 32 \%$, and $30 \%$ (1isted as above) length PMI values found by Wilder (1953) from plankton tow samples. Wilder based his measurements on carapace length, so it was not possible to determine if wild populations experience faster growth than laboratory raised 
populations, or whether the differences in PMI values were due to the different measurement methods.

By subtracting the dry weight from the wet weight, the amount of water in the larvae can be determined. The impression obtained from the 1iterature is that after a molt and once the exoskeleton has hardened, water is progressively lost through displacement by newly synthesized tissue (Read and Caulton, 1980). This pattern of water loss was found to occur in the fourth stage larvae of Homarus americanus. In the first three larval stages, however, water levels (mg) remained constant through the molt even though ash free dry weight increased.

In juvenile prawns, Penaeus indicus, water was seen to increase in absolute amounts through the molt cycle (stages $B$ to $D_{2}$ ) (Read and Caulton, 1980). It was suggested by Read and Caulton (1980) that either pre-ecdysial water was quickly taken up and then lost (i.e. during the time between taking samples) or e1se the animal was able to hydrostatically manipulate a small amount of water to provide the necessary expansion for exoskeleton growth.

Two other explanations are more likely for Homarus americanus larvae and may apply to juvenile $\underline{\text { Penaeus }} \underline{\text { indicus }}$ as well. A certain amount of increase in physical size is possible during intermolt in some crustaceans. This intermolt growth is more likely to occur in multi-segmented forms, e.g. shrimp-1ike forms, and is possible because the flexible arthrodial membranes linking the endoskeletal plates are capable of extension (Hartnol1, 1982). This type of growth occurs in the pre-metamorphic mysis larvae of $\underline{\mathrm{H}}$ americanus (Templeman, 1936b) and could account for the maintenance of absolute water levels during each larval 
stage.

A second type of intermolt growth was seen in first stage larval Homarus americanus; counterparts may exist in the second and third larval stages as we11. In the first larval stage, the pleopods are still developing within the endoskeleton and outwardly appear as buds on the ventral surface of the tail. As the first stage larvae develop, these buds increase in size. The resulting increase in volume would then allow for new tissue synthesis without concomitant water loss.

The dry weight of Homarus americanus larvae was found to increase exponentially (up to larval stage 4D。). Using H. americanus larvae, Logan and Epifanio (1978) also found exponential dry weight growth. Their results for $a$ and $b \quad\left(y=a e^{b x}\right)$, if recalculated where $y=1$ larval dry weight in $\mathrm{mg}$ and $\mathrm{x}=1$ arval age in $\mathrm{h}$, were 1.1048 and $4.42 \times 10^{-3}$, respectively, which are similar to the values found in the present study.

There was a large increase in ash, as an absolute amount and as percent dry weight, in postmetamorphic fourth stage larvae versus premetamorphic larvae. This increase may be due to the change in morphological shape from a mysis form to a mini-adult form, if the latter has proportionally more surface area to volume than the former. Whereas this increase in relative surface area may be occurring, it seems unlikely that it could be responsible for the observed ash increase. Instead, it appears that the exoskeleton in fourth stage larvae is proportionally thicker, more calcified, and therefore stronger than the exoskeletons of earlier stages (Williams, 1907). As the fourth stage larva begins exploring the bottom and may settle at this stage (Herrick, 1909), a stronger exoskeleton would facilitate both burrow digging and self-defense. 
Undoubtedly a stronger exoskeleton during the first three larval stages would also aid in self-defense. The fact that the exoskeletons in these stages are 1ightly calcified (Williams, 1907) suggest that any advantages of a stronger exoskeleton would be outweighed by the extra weight and increased swimming activity that would be required by the larva to maintain buoyancy.

Another factor which may preclude stronger exoskeleton formation in the first three larval stages is the time required to form such a exoskeleton. In the first three stages ash reached its maximum level in less than a day while in the fourth stage ash continued to increase for approximately four days. The rate of absolute ash incorporation was approximately constant for each larval stage. Under the assumption that it is advantageous to form the exoskeleton as quickly as possible, the observed ash incorporation (i.e., exoskeleton formation) may represent the maximum rate. In which case, any increase in exoskeleton formation increases the energy and time required for exoskeleton synthesis and may prolong larval existence. Whether it is advantageous to minimize the duration of larval existence is open to debate. The concensus appears to be that benthic predation is just as great or greater than planktonic predation (cf. Pechenik, 1979). Lobster larvae, however, are much larger in size than the organisms referred to in Pechenik's (1979) work and do not face the same suite of predators.

At the end of the fourth larval stage, ash did not decline in absolute amounts nor did it attain the same minimum percent dry weight levels seen in the first three larval stages. As the gastrolith first appears at this larval stage (Williams, 1907), it may serve to conserve 
the ash lost from molt related exoskeleton decalcification (Aiken, 1980).

The pattern of absolute chitin levels, with the proportionally greater increase in fourth stage versus earlier stage larvae, was also a reflection of increased exoskeleton formation. This amount of chitin represents a large energy investment by the animal though fifth stage juveniles normally consume their molt exuvia (Wiliiams, 1907) so this investment in chitin may not be permanent1y lost.

Absolute amounts of carbohydrate increased throughout larval development, with molt related fluctuations, up to stage $4 \mathrm{C}$ where it stabilized. As a percent of dry weight, carbohydrate usually remained below five percent which is in agreement with other reported values in crustaceans (cf. Anger et a1., 1983).

The leveling off of carbohydrate in the fourth stage indicates that it is not a preferred energy storage form. Large amounts of lipids were stored past the point where carbohydrate stabilized, indicating that excess energy was available to these larvae. One would have expected continued increases in carbohydrate if it was a preferred storage form. This does not occur, as juvenile Homarus americanus fed different diets containing high levels of carbohydrate (23-31\% dry weight) contained equivalent levels of carbohydrate as juveniles fed Artemia (10\% carbohydrate as dry weight)(Capuzzo and Lancaster, 1979d).

As in other animals, catabolism of glucose in crustacea occurs primarily through glycolysis and the hexose monophosphate shunt (HMS)(cf. Chang and 0'Connor, 1983). The HMS pathway is the primary producer of NADPH which is required in many anabolic reactions (Mayes, 1983a). During the intermolt period, the HMS was the primary glucose catabolic pathway in 
the hepatopancreas of the crayfish Orconectes virilis (McWhinnie and Kirchenberg, 1962; McWhinnie and Corkil1, 1964). Lipid synthesis, which is occurring during the intermolt period (Chang and 0'Connor, 1983), requires both glycero1 (for complex lipid formation) and NADPH; carbohydrate is the major source for both of these components (Mayes, 1983a,c) during anabolic periods. Maintenance of minimum carbohydrate levels must then be crucial for crustacean growth so that if dietary intake is not sufficient, gluconeogenesis must occur.

Carbohydrate, when expressed as its caloric equivalent and then compared against the total caloric value of the animal, remained relatively constant throughout larval development. This suggests that levels of carbohydrate may be tightly regulated in Homarus americanus larvae. It would be interesting to eliminate dietary intake of carbohydrate and then determine the extent of protein based gluconeogenesis and how it may affect the larvae's response to molt, carbohydrate storage patterns, and growth related carbohydrate requirements.

In the present study, protein levels as percent dry weight showed minor overall declines from the first to second stage larvae and a relatively major decline at the fourth stage. This trend for declining protein levels through larval development was also seen by Capuzzo and Lancaster (1979a). Much of this decline can be attributed to the increased levels of ash and chitin in the fourth stage versus earlier stage larvae. In the present study, 1ipid was also a contributing factor as the increased levels in the fourth stage also contributed to the decrease in percent content of protein. 
The large increase in lipid which occurred in fourth stage larvae was not detected by Capuzzo and Lancaster (1979a); instead they found a decrease in 1ipid content in the fourths. The reason for this observation in their study was probably related to the use of intermolt animals. In the present study, the intermolt period coincided with the minimum levels (as percent dry weight) that were attained in each larval stage. The large increases in lipid did not occur until the late intermolt and early premolt stages. The reason for this sudden lipid accumulation in the fourth stage is unknown, though several explanations are suggested. The first possible cause for the observed lipid pattern is related to growth efficiency--the ratio of growth over consumption when both are expressed as their caloric equivalents. Logan and Epifanio (1978) found growth efficiencies of $0.18,0.21,0.21$ and 0.44 for first, second, third, and fourth stage Homarus americanus larvae, respectively. The sudden doubling of growth efficiency in the fourth larval stage means that less food was required in this stage to achieve the same amount of growth. Assuming that ingestion rates were not much less in fourth stage versus earlier stage larvae, this implies that fourth stage larvae could potentially possess more surplus energy (i.e., consumed energy that was not needed for respiration or new tissue growth) which may then have been stored as 1ipid. An entirely different interpretation, however, of Logan and Epifanio's (1978) data is that their growth values in the fourth stage larvae may have included the increased levels of stored lipid. For energy gained, 1ipid incorporation from the diet is less energetically cost1y than de novo synthesis of tissue. If lipid incorporation was being measured as growth, then fourth stage larvae were not necessarily any more 
efficient at new tissue synthesis than the earlier stages. Such a trend for equivalent tissue synthesis efficiencies has been observed in Homarus americanus larvae (J. Capuzzo, personal communication). Since the pattern of energy storage varies through the molt cycle, measured growth efficiencies would probably also vary.

Under the assumption that growth efficiency (specifically the efficiency at which new tissue is synthesized) actually was higher in postmetamorphic fourth stage Homarus americanus than in the premetamorphic larval stages, two explanations for this increased growth efficiency are possible. Anger et a1. (1983) found a similar sudden increase in biomass accumulation after metamorphosis in the crab Hyas araneus. They speculated that this alteration in growth pattern was due to the lack of further morphological changes. In other words, the major tissue reconstructions necessary for the premetamorphic morphological changes may have required considerable energy. Upon cessation of morphological changes in postmetamorphic animals this energy becomes available for tissue growth. Another possible factor is that the temperature regime experienced by the fourth stage animals may have coincidentally been the range for optimum partitioning of energy resources (Johns, 1982). Another reason for the lipid accumulation pattern seen during larval development may be that all the available energy in the first three larval stages was purposefully channelled towards growth. If this was controllable by the larvae then it would imply that there was some advantage to rapid growth. This energy partitioning towards growth may be necessary, however, if accumulation of energy was simply not possible by these larvae. The hepatopancreas is the main energy storage depot for 
lipids in these larvae, yet it is still undergoing considerable development in the first four larval stages (Factor, 1981). It may be that it was not sufficiently developed in the first three larval stages to serve as a storage organ (P. Biesiot, personal communication). Cause and effect were certainly not evident here, however, as the potential lack of development in the hepatopancreas may have been due to the lack of need to serve as a storage organ. As previous1y discussed, the premetamorphic larvae recovered from their molts so quickly that little energy was probably required to support them through ecdysis (Scheer, 1960).

If premetamorphic larvae actually do channel all their available energy towards growth, it would be interesting to force a nutritional deficiency upon them to see how they respond. Since larvae can physically ingest a limited quantity of food, a high lipid/protein ratio in the feed could preclude a maximum rate of tissue growth by the larvae if the rate of ingested protein was kept low due to the bulk of the accompanying 1ipid. It would be interesting to determine how the larvae would respond to excess available energy represented by the lipid. If this 1 ipid was stored then energy storage by premetamorphic Homarus americanus larvae is possible.

Frank et al. (1975) found extensive energy reserves in premetamorphic third stage crab larvae (Rhithropanopeus harrisii) and postulated that these reserves may be required for metamorphosis. As fourth stage Homarus americanus are already postmetamorphic, this explanation cannot suffice for this species. It is possible, however, that the adoption of a benthic lifestyle may be energetically costly due to the substrate testing behavior of the older fourth and early fifth stage larvae and/or the 
burrowing behavior of the settled fourth or fifth stage animal (Herrick, 1911). It is also possible that the molt recovery period in fifth stage animals is of a sufficient duration that dependence on energy reserves becomes necessary.

The bulk of the 1ipid accumulation in the fourth stage animals occurred in late intermolt to early premolt, after which lipid accumulation stopped. This has implications for the rate of protein utilization by the animal and may affect the $0: \mathrm{N}$ ratio. Under the assumption that lipid storage was necessary and therefore had metabolic priority, it would be energy efficient for the animal to store as much of the dietary lipid as possible rather than synthesizing new lipid. The composition of the diet (Artemia) remained constant throughout larval development. Since the premetamorphic larvae did not store substantial reserves, these stages must have catabolized much of their ingested 1ipid. Lipid catabolism requires oxygen yet produces no nitrogen, resulting in an increase in the $0: \mathrm{N}$ ratio (atoms oxygen consumed versus atoms nitrogen excreted)(Corner and Cowey, 1968; Ikeda, 1974). During the lipid accumulation period in the fourth stage, one can assume that 1 ittle ingested or stored lipid was catabolized as this would have defeated the purpose of lipid storage. As carbohydrate is not a major metabolite (Ikeda, 1974), protein catabolism, which produces nitrogen, must have provided most of the necessary energy during this period, resulting in a low $0: \mathrm{N}$ ratio. Later, in the late premolt period of the fourth stage, 1ipid accumulation stopped. Ingested 1ipid must then have been balanced by lipid catabolism with the result that the $0: N$ ratio increased.

In animals that store lipid during the molt cycle, one would expect a 
cyelte 0:N ratio with the molt with a minimum value at early intermolt and a higher value prior to the molt. Even if substantial lipids were not stored, the protein fueled gluconeogenesis after a molt to produce chitin may cause the same general $0: \mathrm{N}$ cycle (though the minimum would be at postmolt). This latter $0: \mathrm{N}$ pattern around the molt was observed in the shrimp Crangon crangon (Regnailt, 1981).

In Homarus americanus, the $0: \mathrm{N}$ ratios were constant in the first three larval stages $(26.7,26.3$, and 26.6 for the first, second and third larval stages, respectively) but decreased in the fourth stage (22.1) (Capuzzo and Lancaster, 1979a,c). Intermolt animals were used in their study and Artemia was always used as the feed. If the fourth stage animals were in mid intermolt, just when lipid began to accumulate, the above described dependence on protein for energy could have been in effect, resulting in a lower $0: \mathrm{N}$ ratio. In the premetamorphic larvae a priority on growth over energy storage may have been in effect so that dietary lipid was catabolized for energy and dietary protein was converted more to tissue (high $0: \mathrm{N}$ ).

Lipid Classes: Graphs 27-30, Tables 11-14.

Triacylg1ycerol was responsible for the sudden lipid increase in fourth stage Homarus americanus. During this stage triacylglycerol became the dominant lipid class and accounted for more than $50 \%$ of the total lipid. Accumulation of triacylglycerol during larval development has also been seen in the barnacle Balanus balanoides (Holland, 1978) and the copepod Euchaeta japonica (Lee et al., 1974). In both cases, as in $\underline{\mathrm{H}}$. americanus, the function of triacylglycerol was thought to be storage of energy. 
Though appreciable amounts of triacylg1ycerol began accumulating before metamorphosis, the postmetamorphic storage rate was considerably faster. In this respect, metamorphosis was associated with a change in triacylglycerol storage patterns in Homarus americanus. In the other lipid classes, however, metamorphosis had no noticeable affect on the rate of their accumulation.

Free sterol exhibited a steady increase, in absolute amounts, throughout larva1 development. More interesting, however, was the stero1 pattern when compared against protein. The relatively constant sterol/protein ratio throughout larval development implies that the level of sterol was tightly regulated. Since sterol cannot be synthesized by crustaceans (cf. Teshima, 1972), any regulation of sterol would be through catabolism of excess amounts (or decreased assimilation) or via some reversible conversion to another form. Stery1 ester was thought to be such a storage form in crab eggs (Whitney, 1969). In the present study, however, no noticeable relationship between sterol and stery1 esters was found for embryogenesis, molting, or larval development.

If regulation is through catabolism of excess sterol, dietary amounts of sterol would obviously have to exceed requirements. In this study, Artemia was used as the diet. The level of sterols (or cholesterol--these are practically synonymous in crustaceans (Teshima, 1972; Gagosian, 1975)) in Artemia, as percent dry weight, is $0.46 \%$ (Gallagher and Brown, 1975) to $0.62 \%$ (Sasaki, in preparation). The stero $1 /$ protein ratio in Artemia is 0.8-1.1\% (Gallagher and Brown, 1975) or 1.04\% (unpub1ished observations). In another study using young juvenile Homarus americanus (Capuzzo and Lancaster, 1979d) protein efficiency ratios (PER) were determined under a 
diet of Artemia. PER is the ratio of the wet weight gain over the dry weight of protein fed. By using PER (0.9), water content of the juveniles (74.7\%), and protein levels (49.9\% dry weight), an approximate value of 0.11-0.12 can be obtained for the amount of protein gained as tissue over the amount of protein fed. This agrees with the $0.12 \pm 0.01$ (S.E.) value measured by Capuzzo and Lancaster (1979b) for juvenile $\underline{\mathrm{H}}$. americanus fed frozen Artemia. Under the assumption that these juvenile $\underline{\mathrm{H}}$ americanus ingested most of what was fed $(82 \%$ assimilation in another study (Logan and Epifanio, 1978)), they ate at least 8-9 times the amount of protein that they laid down as tissue. This value, plus the sterol/protein ratios of the larval $\underline{\text { H. americanus }}$ and Artemia, indicate that the dietary amounts of sterol was at least four times the amount of incorporated sterol. Regulation of sterol was therefore aided, if not controlled, by sterol catabolism or decreased assimilation. Crustaceans can certainly catabolize sterols (Teshima, 1972), though in Homarus americanus, since high dietary cholesterol levels depressed growth (Castell et a1., 1975), such regulation may be metabolically costly or stressful.

The reason for this apparent sterol to protein regulation is unknown, though again several speculations are possible. Lobsters do not use sterols for bile acid production (Holwerda and Vonk, 1973). In addition, hemolymph sterols probab1y account for 1ess than $5 \%$ of the total body sterol levels. (This assumes that larval Homarus americanus are similar to adults in that sterols make up $0.035 \%(\mathrm{w} / \mathrm{v})$ of hemolymph (B1igh and Scott, 1966) and hemolymph averages $20 \%$ of the animal (Phillips et al., 1980)). Therefore, the major location of sterols in $\mathrm{H}$. americanus is 
probably in cell membranes, as cholesterol regulates cell membrane fluidity (Bloch, 1983). As the degree of cell membrane fluidity is crucial to cellular function, cholesterol levels have to be maintained within a certain range. Since protein is predominantly associated with cytoplasm and membranes, sterols may be linked with protein simply by a cell membrane/cytoplasm relationship.

The above speculation implies that the average cell sizes remained the same through both the molt and larval development. That is, if cell sizes became smaller, the membrane/cytoplasm ratio, and therefore sterol/protein ratio, would have to increase. This did not appear to be the case during larval development of the crab Rhithropanopeus harrisii, where both cell size and number increased during development (Sulkin et al., 1975). It would be interesting to measure the DNA/protein ratios (Sulkin et al., 1975) to determine whether larval development of Homarus americanus is also associated with cell size increase.

The simplistic sterol to protein relationship discussed above may be contrasted with the observed pattern of P. ethanolamine. P. ethanolamine, along with P. choline, are the two major phospholipids of cell membranes in higher animals. Unlike P. choline, however, which also occurs as the major hemolymph 1ipid (Lee and Puppione, 1978), P. ethanolamine is predominantly associated with membranes (Gurr and James, 1980). Therefore, the slight decline in P. ethanolamine versus protein that was seen throughout development may be evidence for an increase in cell size. P. choline, in absolute amounts, showed a decline after both the hatch and the first larval stage molt. These declines were probably indicative of the role of $\mathrm{P}$. choline as an energy reserve; it may have. 
been utilized for energy since triacylglycerol levels were still low. The P. choline/protein ratio showed wide fluctuations throughout development that may be a reflection of the non-membrane associated functions of $P$. choline. As the primary hemolymph 1ipid (Lee and Puppione, 1978), the assimilation of meals (cf. Chang and 0'Connor, 1983) could cause large, transient peaks in $P$. choline levels:

Dietary fatty acid has been shown to affect the ratios of $P$. choline and $P$, ethanolamine against protein in cell membranes of rats (Clandinin et al., 1983). Though changes in P. choline and P. ethanolamine ratios against protein were found throughout development in larval Homarus americanus, it is not known if these changes represent normal development or whether the diet had an effect. Since whole body 1ipid and protein were measured in $\underline{\mathrm{H}}$ americanus, it is also not certain whether 1ipid class/protein ratios in the membranes alone experienced any shifts. As the ratio between $P$. choline and $P$. ethanolamine can have profound physiological effects on membrane characteristics (Cronan and Gelmann, 1975; Innis and Clandinin, 1981), it would be interesting to determine whether dietary fatty acids can affect the P. choline/P. ethanolamine ratios in cellular membranes of $\underline{H}$. americanus. Fatty Acids and Control versus Cod Liver Oil (CLO) Supplement: Graphs 31-36, Tables 15-20, 25-28.

The fatty acid data for P. ethanolamine and P. choline, expressed as absolute amounts per individual, reflected the lipid class data in that metamorphosis did not affect the prevailing rates of accumulation. This same type of data for neutral lipids, because triacylglycerols were dominating this group, showed an increase in the rate of fatty acid 
accumulation af ter metamorphosis.

In the barnacle Balanus balanoides, no selective usage of triacylglycerol fatty acids was found through metamorphosis (Waldock and

1 Holland, 1978). These barnacle larvae do not feed at this time, however, so their study is more appropriately compared with the present embryogenesis study where little selection was also found to occur.' Ward (1979) found fatty acid pattern shifts throughout the development of the prawn Penaeus setiferus. These animals were fed yeast and Artemia nauplii but unfortunately neither feed was analyzed for fatty acids. It is impossible, therefore, to determine how much the diet was responsible for the observed changes.

When fatty acids were expressed as a percent of the total fatty acids in each 1ipid group, it was apparent that shifts occurred in the fatty acid composition of each group. The rates at which these changes happened were fastest in the first larval stage; by the fourth larval stage the fatty acid patterns were more stable. The fatty acid patterns at the end of the fourth stage showed clear evidence of dietary influence, in that in many cases they matched the fatty acid pattern of the diet (Artemia lot 非's 5-10, plus CLO supplement; see Tab1e 1).

In the neutral lipids, the NEFA pattern was stable throughout the fourth larval stage and closely mimicked the pattern in the Artemia. Ironically, in Artemia the neutral 1ipid fatty acid pattern was also the most influenced by the diet (Jezyk and Penicnak, 1966). The percent levels of 18:0 were slightly lower in Homarus americanus than in Artemia, however, which may be due to the influence of the CLO supplement. The 18:1 levelṣ were also higher in $\underline{H}$ americanus, though, which may be 
evidence for a low rate of desaturation of 18:0 to $18: 1$ during assimilation. During the rapid triacylglycerol increase of the fourth stage, the NEFA pattern did not change which indicates that most of this accumulated lipid was dietary in origin rather than de novo synthesis. The neutral lipid PUFA pattern, in particular 18:3, also closely matched the Artemia fatty acid pattern. Exceptions were 20:5 and 22:6 which were slightly higher in the larvae. This, plus the evidence from the CLO supplement experiment, show that the larvae were ingesting the supplement. Their rate of supplement ingestion was low, however, as the levels of $20: 5$ and 22:6 were only slightly elevated in the larvae fed cLo supplement versus control supplement.

The P. choline NEFA pattern also showed possible dietary influence. In this class the pattern of these fatty acids at hatch was much closer to Artemia's pattern so it is unclear just how much influence the diet had. Certainly metabolic regulation of some of these fatty acids was occurring in this class as 18:1 and 16:0 were lower and higher, respectively, in the larvae versus the diet. The fatty acid 16:0 is the primary product of de novo fatty acid synthesis (Gurr and James, 1980) so its elevated presence in P. choline may have been evidence of such synthesis, or, because the leve1 of 16:0 in P. ethanolamine was slight1y lower than in Artemia, it may have been due to selective partitioning of this fatty acid between these two classes.

The PUFA pattern of $P$. choline showed a dietary influence due to the levels of $18: 3$ (most likely this was $18: 3 \omega 3$ ). Crustaceans cannot synthesize any $\omega 3$ fatty acid (Gurr and James, 1980) so the source of 18:3 was definitely in the diet. Regulation of P. choline PUFA was 
evident in that the levels of $20: 4,20: 5$, and $22: 6$ were all elevated above Artemia's leve1s. The CLO supplement may have aided in elevating the levels of $20: 5$ and $22: 6$, but the levels of $20: 4$ indicate that regulation did occur. This very tight regulation of 20:4 suggests that it is serving some functional role. Certainly in many other animals it is a direct precursor to prostaglandins (Gurr and James, 1980) and may also function in this manner in Homarus americanus.

Compared to neutral lipids and P. choline, the fatty acid profile of P. ethanolamine was least affected by diet. of the NEFA, only 18:0 occurred at levels comparable to the diet. The other major NEFA, 16:0, 16:1, and 18:1 al1 occurred at lower levels. Of the PUFA, only 18:2 occurred at comparable leve1s, while 20:4, 20:5, and 22:6 were elevated as compared to the diet. The level of $18: 3$, whereas elevated from initial hatch levels, was considerably lower than the dietary level. It appears that the conservation of $20: 4,20: 5$, and 22:6 had priority in this class. The fatty acid 18:3 was the most regulated of the P. ethanolamine fatty acids in terms of being kept below dietary levels. It is tempting to speculate that the maintenance of a certain degree of unsaturation in this class was partially responsible for the choice of $18: 3$ being the most regulated fatty acid. The sum of the graphed PUFA was approximately $50 \%$ of P. ethanolamine fatty acids. The B-fatty acid of $P$. ethanolamine is predominantly highly unsaturated while the $\alpha$-fatty acid is predominant $1 y$ saturated or monounsaturated (Brockerhoff et a1., 1963). This 1atter. statement implies that a roughly equal ratio between PUFA and saturated plus monounsaturated fatty acids is the norm in P. ethanolamine. Additional 18:3 would have increased the PUFA sum above $50 \%$ which may not 
be optimal.

The reason for the rapid change in fatty acid patterns seen in the first stage is not clear. At hatch, the difference between any one fatty acid in the larvae versus the diet was at its maximum. As the fatty acid profile of the larvae began to conform to that of the diet, this difference became less evident. It is possible that the degree of difference between fatty acid patterns influences the rate at which this conformation occurs. Therefore, as the fatty acid pattern of the larvae approached that of the diet, there was less tendency to conform and the rate of change slowed. Another factor is that at hatch the absolute levels of these lipid classes were at a minimum. Therefore, any dietary input would have a greater effect on the tota1 (i.e., $1 \mathrm{mg}$ of 16:0 will more strongly affect the 1ipid pattern if the current body 1ipid level is $10 \mathrm{mg}$ versus $100 \mathrm{mg}$.)

When examining the overall fatty acid profiles of each of the lipid groups, it is evident that the degree of unsaturation in the fatty acids was greatest in $P$. ethanolamine followed by $P$. choline and then neutral lipids (triacylglycerol). Since triacylglycerol fatty acid content was so dependent on the diet, it is not obvious that the above relationship would hold true if the dietary fatty acids were extremely unsaturated. Nevertheless, this same hierarchy of unsaturation between classes has been noted in numerous other crustacean studies (DeKoning and McMullan, 1966; DeKoning, 1970; Addison et a1., 1972; Noren and Svennerho1m, 1973; Mahr1a, 1975; Chape11e, 1977; Lee and Consoulin, 1979; Chape11e and Pequeux, 1982). In addition to diet, other environmental factors capable of influencing fatty acid patterns are temperature and pressure (Lewis, 1962; 
cf. Malins and Wekel1, 1970; cf. Malins and Sargent, 1976). Pressure was not a factor in the present study. Temperature, which increased throughout the study, likely caused a slight decrease in fatty acid unsaturation (cf. Malins and Sargent, 1976). Any temperature effects were not detectable, however, due to the overwhelming influence of the diet.

When comparing the results of Hatch II-4 (fed Artemia only) versus Hatch I-5 (Artemia plus CLO supplement), it is evident that the absolute levels of 20:5 and 22:6 decreased more rapidly in Hatch II-4 and attained a lower percent level (though the percent level of 20:5 in P. choline remained the same). In the more controlled experiment based on Hatch I-3 (control versus CLO supplement), the control larvae experienced a more extreme decline in absolute amounts of 20:5 and 22:6. The percent levels, however, of these fatty acids in the phospholipids were approximately the same, a1though 22:6 in the controls tended to be lower.

In both the Hatch II-4 and control supplement populations, heavy larval mortality occurred in the late third and early fourth larval stages. A similar mortality period did not occur in either the Hatch I-5 or CLO supplement populations. It is probable that some nutrient in the cod liver oil, which was not present in adequate amounts in Artemia, was responsible for the increased survival of the CLO supplement fed populations. It is suggested that either 20:5 or 22:6 or both fatty acids were responsible. Other experiments have also shown that dietary sources of $20: 5$ or $22: 6$ were beneficial or might even be essential to decapod crustaceans (Castel1 and Covey, 1976; Sandifer and Joseph, 1976; Guary et al., 1976; Kanazawa and Teshima, 1977; Kanazawa et al., 1978, 1979a,d,e,h). It is possible, however, that other nutrients in cod 1iver 
oil, such as vitamins, were responsible for the observed effects of the CLO supplement.

If $20: 5$ and/or 22:6 were responsible for the variation in mortality, then the moderate differences between the control and CLO supplement fed populations indicate that the minimum acceptable body level for these fatty acids was close to, but below, the levels seen in the control population. (As live animals were sampled, the survivors of the heavy mortality period were being assayed. It is possible that these anima1s survived because they consumed their siblings and therefore obtained dietary $20: 5$ and $22: 6)$.

The absolute amounts of the phospholipid classes were less in the control versus CLO supplement fed populations, though the wet and dry weights and protein content were the same. As phospholipids are required for cell membranes, there must be some minimum ratio of phospholipid to protein. To speculate, if $20: 5$ and $22: 6$ are required in phospholipid to control membrane fluidity (or for some other purpose), there is probably a minimum level for these fatty acids, below which the membrane cannot properly function. If $20: 5$ and 22:6 become limiting factors for growth, the organism may not be able to fully coordinate a cessation of growth under these conditions. Phospholipid production may decrease, but other processes (involving protein) may continue. Without an influx of the limiting fatty acids, a continued imbalance may result in death.

The moderate differences in fatty acid levels between the control and CLO supplement fed populations also indicate that the CLO supplement was probably just barely adequate in supplying the necessary extra amounts of $20: 5$ and 22:6. Visual observation showed that larvae did feed on the 
supplement, but when presented with a simultaneous choice they usually seized an Artemia first. Either a more taste attractive supplement should be formulated for culturing Homarus americanus larvae or a means to supplement the Artemia itself (Watanabe, et al., 1980; Sakamoto et a1., 1982) should be used.

\section{Summary: Larval Development and Metamorphosis}

The length percent molt increment values for larval Homarus americanus, calculated from wet weight, were 19-22\%. In absolute amounts, the water content of premetamorphic larvae remained the same within the development of each larval stage, while ash free dry weight increased. This required an increase in volume that was probably due to arthrodial membrane extension and developing limb buds. Dry weight growth was exponential up to $4 \mathrm{D}_{0}$.

Postmetamorphic ash and chitin increases were proportionally larger due to heavier exoskeleton formation. The formation of a stronger exoskeleton was probably related to the benthic oriented behavior exhibited by this stage. Carbohydrate was not a preferred energy storage form, though minimum storage levels were likely required for intermediary metabolism. Protein, as percent dry weight, declined throughout development as exoskeleton material and lipid levels increased during this time.

Lipid increased heavily in the fourth stage late intermolt to early premolt period. The reason for this increase is unknown, though it may be related to growth efficiency changes, shifts in tissue development, or storage for molt or settling. Premetamorphic larvae may have given priority to tissue growth over energy storage, whereas postmetamorphic 
animals at late intermolt may have given priority to energy storage. The rapid rate of lipid increase in the fourth stage would have required increased dependence on protein for energy, which would have lowered the $0: \mathrm{N}$ ratio.

Triacylglycerol was responsible for the total lipid pattern. Sterol steadily increased throughout larval development and showed a constant relationship with protein. Control of sterols was through catabolism or decreased assimilation and might have been necessary to regulate cell membrane fluidity. P. ethanolamine also exhibited a possible relationship with protein. P. choline, in contrast, showed more variation in levels due to its other physiological roles.

Fatty acid patterns of the lipid groups were all heavily influenced by diet, with triacylglycerol being the most affected followed by P. choline and then $P$. ethanolamine. The rate of change in fatty acid patterns was greatest in first stage larvae and might have been due to the magnitude of fatty acid differences or the level of body 1ipid. Overa11, P. ethanolamine was more unsaturated than P. choline followed by triacylglycerol.

The CLO supplement was effective in preventing the period of heavy mortalities in developing larvae, probably by supplying 20:5 and 22:6 fatty acids. Insufficient amounts of $20: 5$ and 22:6 may limit phospholipid synthesis which could result in death. The CLO supplement, though effective, should be improved or replaced with other means of supplementation. 


\section{$\underline{\text { Starvation }}$}

Starvation trials were begun at hatch $(d-0)$ and three days $(d-3)$, four days $(d-4)$, and seven days $(d-7)$ after the hatch. At the beginning of these starvation trials the larvae were at molt stages $A, D_{0}, D_{2-3}$, and C, respectively. Aiken (1973) found that molt stage Do represented a transition stage between intermolt and premo1t for adult Homarus americanus. H. americanus can pause or plateau at this stage for an indefinite amount of time if conditions are not favorable. Once the animals progress to $D_{1}$, however, the molting process cannot be stopped (Aiken, 1973). The present study demonstrated that $\underline{H}$ americanus first and second stage larvae paused at molt stage $C$ or $D_{0}$ if the molt stage at the onset of starvation was A to early C. If, however, the larvae were at $D_{0}$ to $D_{3}$ then the upcoming molt occurred, even though molt stage $D_{0}$ was prior to the irreversible $D_{1}$ transition and the larvae could feasibly have 'chosen' to remain at $D_{0}$. These $D_{0}$ larvae, that were fed ad libitum prior to the starvation, used their stored reserves to make the transition to $D_{1}$ which committed them to molt. The time required to advance to $D_{1}$ for these larvae might have been too short for the starvation period to have any effect (i.e. the starved duration to advance from $D_{0}$ to $D_{1}$ may not have exceeded a normal pause between feedings). It would be interesting to determine how early in the molt cycle a starvation period must begin before Homarus americanus reacts by pausing in its molt development. It would a1so be necessary to examine the effects of stored metabolic reserves to determine if the relative size of these reserves influences the duration of starvation time that could be tolerated by the animal and still allow it to undergo a molt. This would 
be similar to the point-of-reserve-saturation experiments conducted with larvae of the crab Hyas araneus (Anger and Dawirs, 1981). The possible controlling influence of the molt cycle, in addition to energy reserves, should be examined.

The time of molting among the fed controls, $d-3$ and $d-4$ groups was the same to within $12 \mathrm{~h}$. The wet weight increases among these groups, however, were not equivalent, as the controls exhibited the greatest increase and the $d-3$ group the least increase. It appears that starvation, if begun at or after molt stage $D_{0}$, does not greatly affect the timing of the molt. It does, however, affect the percent molt increment (PMI) -- the longer the period of starvation the less the PMI increase.

Feeding studies on crustaceans, using reduced levels of food rather than starvation, have resulted in reductions in PMI (Knowlton, 1974) or lengthenings of molt periods (Chittleborough, 1975). Chittleborough (1975) also found that under severe food shortages, both responses occurred simultaneously, consistent with the findings of other studies (Hartnol1, 1982). Reduced food intake lengthened the intermolt period in Homarus americanus (Templeman, 1936a).

Though reductions in feeding were not conducted in the present study, the above results suggest that a pre- $D_{0}$ ad libitum, post $-D_{0}$ (inclusive) reduced feeding schedule would not affect molt timing, but would reduce molt increment. A pre- $\mathrm{D}_{0}$ reduced feeding, post- $\mathrm{D}_{0}$ ad libitum schedule would lengthen intermolt and would have an unknown effect on molt increment. Finally, a constant reduced feeding schedule should both increase the intermolt and reduce the PMI. This type of feeding 
effect on intermolt duration (but not PMI) was seen for larvae of the crab Hyas araneus, where the later a short starvation period occurred in relation to the molt cycle, the lesser was the resulting molt delay (Anger and Dawirs, 1981).

The reduced molt increment in the $d-4$ group also indicates that larval Homarus americanus continued to consume food until very late in the mo1t cycle. Fourth stage larvae have been observed to ingest food to within half an hour of molting ( $\mathrm{J}$. Caste11, personal communication), though their large energy reserves may lessen the importance of such feeding.

Proximate Analysis: Graphs $37-45$.

Under starvation, exclusive of the molts, the wet and dry weights remained stable and declined, respectively. This caused an increase in water content with starvation, resulting in water levels above the naturally occurring postmolt maxima. Similar water content increases in starved crustaceans have been reported by other investigators ( $\mathrm{Da} 11,1974$; Cuzon et al., 1980; Regnault, 1981). As dry weight is lost through catabolic processes the fixed volume of the animal requires that water replace the lost mass, unless molting occurs with a decrease in body volume.

The increases in percent water after the $d-3$ and $d-4$ molts were at levels above the control molt value, with a longer starvation period causing a higher postmolt water level. This indicates that water content alone does not determine the extent of body volume expansion after a molt, although under starvation stress, the regulatory mechanisms may have been altered so that water content may still be important to regulate mo1t 
increases in fed larvae.

The absolute amounts of ash showed the same stable or molt related patterns as wet weight. If ash was primarily incorporated into the exoskeleton, it was probably relatively inert metabolically; in which case, it may have been less of an energy drain to maintain ash in the exoskeleton and cope with a heavier exoskeleton (relative to the decreasing tissue mass) than it would have been to actively transport salts out of the exoskeleton. Other studies have reported that starvation induced increases in absolute ash (Ikeda, 1971; Mayzaud, 1976; Anger and Nair, 1979).

As a percent of dry weight, ash increased to leve1s above the maximum control levels. Similar starvation related percent ash increases have been reported (Ikeda, 1971; Mayzaud, 1976; Anger and Nair, 1979). Under laboratory conditions of ad libitum feeding, both the percent dry/wet weight and percent ash values of larval Homarus americanus exhibited very similar quantitative patterns in relation to the molt cycle, and between hatches and broods and premetamorphic larval stages. It would be interesting to test whether these values, combined with molt staging, could serve as an approximate, but quick and easy, indicator of quantitative feeding history in premetamorphic $\underline{H}$ americanus larvae. Absolute chitin levels generally remained stable or slightly increased with starvation, except for decreases at mo1t. The decrease associated with the molt was due to the molted exuvia. As molted exuvia were not found, it was possible that the larvae ingested them. If so, however, the exuvia were either not fully assimilated or any glucosamine from chitin was also used for energy, as the postmolt chitin levels in d-3 
and $d-4$ did not attain premolt quantities. Exclusive of the molt chitin, as percent dry weight, increased with starvation. This percent increase in chitin during starvation agrees with the results of Anger and Nair (1979) but it conflicts with Ikeda's (1971) results which showed a decrease in absolute chitin levels with starvation. The present study indicates that Homarus americanus does not utilize existing exoskeleton chitin for energy. It is not known whether they are capable of assimilating ingested exuvial chitin.

Under starvation, levels of carbohydrate, protein, and 1ipid all declined in absolute amounts and as percent dry weight. Similar patterns for all or some of these components have been reported or inferred in numerous studies (cf. Regnault, 1981; cf. Barclay et al., 1983; cf. C1ifford and Brick, 1983). In the present study it was apparent that a11 three components were being catabolized by the starved larvae. The patterns of utilization, however, were not the same for each substrate (carbohydrate, protein, and lipid).

Carbohydrate underwent an immediate decline when starvation conditions were imposed. The rate of decline quickly began to level off, however, with the result that carbohydrate stabilized at approximately $3 \%$ dry weight. Carbohydrate is essential for crustacean intermediary metabolism (Chang and O'Connor, 1983), thus this $3 \%$ dry weight (4-4.5\% ash free dry weight) level may represent a minimum required amount for Homarus americanus larvae.

Absolute levels of protein did not immediately decline with starvation, except within group $\mathrm{d}-0$ where continued yolk utilization might have been responsible for the immediate protein drop. Groups $d-3$ and $d-4$ 
both showed protein patterns, as percent dry weight, similar to control larvae in respect to a premolt buildup followed by a molt related drop. The starved larvae, however, continued to lose protein after the molt. As percent ash free dry weight, protein levels remained within the same general range as the controls.

Lipid, like carbohydrate, immediately decreased in absolute amount with starvation. Unlike carbohydrate, however, lipid did not level off to a stable minimum value, though its rate of decrease did slow with continued starvation. As percent dry weight, lipid decreased with starvation to levels below control minima. As percent ash free dry weight, lipid declined but did not much exceed the minimum values seen in intermolt controls.

The above patterns of utilization indicate that upon starvation larval Homarus americanus first catabolized carbohydrate and lipid. With continued starvation, protein was catabolized. Lipid and carbohydrate utilization continued, though at reduced rates. Utilization of carbohydrate followed by lipid and then protein during periods of starvation has been noted in full or in part by other crustacean species (Spech and Urich, 1969; Wallace, 1973; Cuzon et al., 1980; Regnault, 1981; Dawirs, 1983; Clifford and Brick, 1983). In terms of the energy supplied, however, the relative importance of each component differs between studies (cf. Clifford and Brick, 1983). A1though carbohydrate and lipid were depleted to a greater extent than protein (relative to their starting quantities), protein supplied the greatest quantity of metabolic energy during prolonged starvation of larval $\underline{\mathrm{H}}$ americanus. This same relationship was seen in the rotifer Brachionus plicatilis Muller (Scott 
and Baynes, 1978), and the prawn Penaeus esculentus Haswe11 (Barclay et a1., 1983).

There is the possibility, however, that protein may not always supply the most energy for starving Homarus americanus. The amount of stored 1ipid can affect the starvation induced pattern of substrate utilization in crustaceans, as protein may not be catabolized until lipid reserves have, to some extent, been depleted (Spech and Urich, 1969; Ikeda, 1974; Mayzaud, 1976; Regnault, 1981). The amount of 1 ipid reserves can affect survival time under conditions of starvation (Tessier et a1., 1983) and in animals with high lipid contents, the energy derived from lipid under starvation may exceed that derived from protein (Mayzaud, 1976). This suggests that advanced (post $\mathrm{D}_{0}$ ) fourth stage $\underline{\mathrm{H}}$. americanus, with their large lipid reserves, may be able to withstand prolonged starvation using mainly lipid as an energy source. If so, then metamorphosis in $\underline{\mathrm{H}}$. americanus could be considered as a transition point between a protein oriented to a protein and 1ipid oriented reserve scheme.

These advanced fourth stage larvae would probably molt, however, under conditions of starvation as their molt stage is post $D_{0}$. If large amounts of 1ipid were stored only as a reserve to supply metabolic energy for the molt, then a molt during starvation may utilize most of this lipid reserve. Advanced fourth stage larvae, therefore, may not have a greater starvation tolerance than younger larvae. Further evaluation of the larval fourth to juvenile fifth stage transition period, in respect to energy reserve utilization, is warranted.

The rate of 1 ipid, and to a certain extent, protein catabolism in larval Homarus americanus decreased with increasing starvation times. 
After a short starvation period larval $\underline{\mathrm{H}}$. americanus utilized more energy per unit time, and therefore had a higher metabolic rate, than larvae maintained with a prolonged starvation period. Respiration or oxygen consumption is an indicator of metabolic rate in organisms under aerobic conditions. A starvation induced decrease in metabolic rate, as derived from biochemical or physiological data, has been seen in numerous species of crustaceans (Roberts, 1957; Anse11, 1973; Marsden et a1., 1973; Wallace, 1973; Mayzaud, 1973,1976; Ikeda, 1977; Regnault, 1981; Dawirs, 1983).

Lipid Classes: Graphs 46-49, Table 21 .

Triacylglycerol showed the most rapid decrease of all lipid classes in terms of relative rate of utilization (amount catabolized per unit time versus the starting amount). This was followed by $P$. choline and then $P$. ethanolamine. In contrast, sterols were not utilized at all during starvation. This pattern of neutral lipid, followed by phospholipid, catabolism was also seen in the chaetognath Sagitta elegans Verrill (Mayzaud, 1976). Similar patterns were seen in developing cypris larvae of the barnacle Balanus balanoides (Gardner and Riley, 1972; Holland and Walker, 1975; Waldock and Holland, 1978). Cypris larvae do not feed so this observation is somewhat analogous to starvation in that they must exist on stored reserves.

In terms of the percent of resource utilization (total amount catabolized versus starting amount), triacylglycerol also showed the greatest depletion, of ten to levels below detection. This was again followed by P. choline and then P. ethanolamine. From these patterns, triacylglycerol appears to have been energetically important. The 
absolute levels of triacylglycerol, however, were so low at the onset of starvation that even its complete depletion represented only a small portion of the total lipid loss. Instead, P. choline supplied the majority of the lipid based metabolic energy during starvation.

As a starvation induced energy source, this pattern of phospholipid utilization before neutral lipid utilization probably only holds true for premetamorphic Homarus americanus larvae. The abundance of triacylglycerol reserves in the advanced fourth stage larvae indicates that it would dominate at this point as the lipid energy source during starvation.

The initial lipid loss at the onset of starvation was due to the catabolism of triacylglycerol and P. choline. If the initially catabolized P. choline had been associated with cell membranes, a concurrent drop in protein would have been expected. Instead, protein rose in preparation for the molt or remained unchanged (except for possible yolk loss in $\left.D_{0}\right)$. This suggests that on an energetic basis two metabolic pools of P. choline exist, only one of which is associated with cel1 membranes. A major non-membrane function for P. choline is 1ipid transport (Lee and Puppione, 1978), although other quantitatively minor functions are likely. This latter pool was probably the source of the initial catabolized P. choline.

It apparently became necessary to catabolize the cell membrane pool of P. choline, however, when a certain level of catabolism was reached. The relationship between the cell membrane and cell volume (protein), although it may vary under starvation, would still impose a consistent relationship upon $\mathrm{P}$. choline and protein. This may explain the initial 
decrease in the $\mathrm{P}$. choline/protein ratio at the onset of starvation when non-membrane P. choline was catabolized, which later tended to stabilize, indicating utilization of membrane P. choline.

If the above speculation for $P$. choline is true, then a similar explanation for $P$. ethanolamine would require that on1y one metabolic pool existed. If the vast majority of $\mathrm{P}$. ethanolamine was associated with cell membranes, it would explain the tight relationship seen in the $P$. ethanolamine/protein ratio. The absolute increases in P. ethanolamine in the $d-3$ and $d-4$ groups, which matched similar protein increases related to the upcoming molt, could then be explained as the production of new tissue. Although P. choline would also have been incorporated into any new cell membranes, no absolute increase in P. choline was detected as it was simultaneous1y being catabolized for energy and was probably functioning as a precursor to $\mathrm{P}$. ethanolamine. The actual mechanism for P. choline conversion to P. ethanolamine in this case is not known as it is not clear that demethylation of $\mathrm{P}$. choline is possible. One possible pathway is the conversion of $\mathrm{P}$. choline to 1-2 diacylglycerol through phospholipase C as 1,2-diacylglycerol is a precursor for both P. choline and P. ethanolamine (Mayes, 1983a).

The percent ash free dry weight values for protein and lipid in the starved larval Homarus americanus were only slightly lower than the minimum values found in fed control larvae. Since triacylg1ycerol and carbohydrate were minor constituents in both starved and fed larvae, the phospholipids, protein, and chitin were the major components of the ash free tissue. Composition of fed and starved larvae was not exactly the same as chitin did not decrease in absolute amounts in starved larvae 
whereas levels of both lipid and protein declined. The loss of non-membrane $P$. choline also caused total lipids in starved larvae to decrease even further. Despite these differences, the similarity of 1ipid and protein levels between the starved and fed larvae indicates that tissue catabolism during starvation did not heavily favor one component (phospholipid versus protein) over another.

The absolute amounts of free sterols did not decline with starvation. Since protein did decline, starvation caused the sterol/protein ratios to increase over control values. It would be interesting to determine how consistent this sterol/protein ratio was between different broods of larvae and with varying environmental conditions, particularly temperature. The apparent conservation of sterols during starvation might also have been indirectly observed in the prawn Penaeus japonicus as an increased percentage of non-saponifiable (non-fatty acid) 1ipids (Cuzon et al., 1980). Such conservation implies that sterols were either specifically stored by the animal or, since tissue was being catabolized, that the cell membranes contained progressively more sterol. This latter situation is not another form of storage as the sterols in the membrane would still be physiologically active, resulting in decreased membrane fluidity (Bloch, 1983). This latter possibility will be discussed further in the Fatty Acid section.

If sterols were moved out of cell membranes and stored, it is not known which organ(s) could be involved. Using injected labelled cholesterol, Teshima (1977) found that the gonad, heart, intestine, and hepatopancreas of the lobster Panulirus japonica temporarily contained proportionally more of the introduced cholesterol. Redistribution of the 
cholesterol then occurred over a course of several weeks. This provides circumstantial evidence that temporary storage of sterols may be possible in crustaceans.

Fatty Acids: Graphs 50-55, Tables 22-24.

The complete depletion of triacylglycerol under conditions of starvation caused the neutral lipid fatty acid pattern to switch from one heavily influenced by triacylglycerol to one controlled by free fatty acids, steryl esters, and mono- and diacylglycerol. The resulting change in the neutral lipid fatty acid pattern is therefore difficult to interpret. The only potentially valid interpretation is that triacylglycerol may have contained proportionally large amounts of 18:2 and $18: 3$, as these two fatty acids always showed substantial declines in the neutral 1ipids during starvation.

This type of problem did not occur in P. choline and P. ethanolamine lipid groups, as these two classes dominated their respective group. Selective conservation of certain fatty acids was found to occur in the phospholipids of larval Homarus americanus when placed under starvation stress. The conserved fatty acids were 16:0, 18:0, 20:4, 20:5, and 22:6. In contrast to the present results, no conservation of fatty acids, except for 18:1w9, was observed in total lipids of muscle in the prawn Penaeus japonicus (Cuzon et al., 1980). Either conservation of specific fatty acids occurs in tissues other than muscle, or environment and previous diet are heavily influential, or fatty acid conservation is species specific. Gardner and Riley (1972) observed selective utilization of 20:5 and 22:6 from triacylglycerols in barnacles collected during 
winter when food was scarce. They interpreted this to mean that these fatty acids were selectively utilized for energy. Lee and Barnes (1975) found a similar pattern in the copepod Gaussia princeps, where 18:2, 18:3, $20: 4,20: 5$, and 22:6 were selectively lost from the energy storage lipid wax esters. Their interpretation, however, was that these fatty acids were not catabolized, but were selectively mobilized out of the wax esters into phospholipids to maintain the phospholipid fatty acid pattern. The reason for the apparent conservation of 16:0 and 18:0 in the present study is unknown. (The possible mechanisms behind fatty acid conservation are covered in the Embryogenesis Fatty Acid Discussion.) Although unlikely, it may be evidence of concurrent de novo fatty acid synthesis as these two fatty acids are the major end products (Gurr and James, 1980). A minimum level of saturated fatty acids may also be . required (Cronan and Gelmann, 1975). A speculative function for 16:0 and 18:0 fatty acids may be to regulate membrane fluidity. Assuming the animal had a priority to conserve the fatty acids 20:4, 20:5, and 22:6 (all highly unsaturated), 16:0 and 18:0 may be required to maintain the overall degree of membrane unsaturation within physiological limits. This may also explain how sterols, if they are not stored in a separate organ, could be tolerated in membranes at higher concentrations. The higher degree of $20: 4,20: 5$, and $22: 6$ based membrane unsaturation may counteract (or be counteracted by) the solidifying effect of increased cholesterol.

The fatty acid patterns seen in group $d-0$, that was starved at hatch, were different than the patterns seen in the other three groups. The patterns observed in group $\mathrm{d}-0$ tended towards more non-selective utilization of fatty acids. This may have been due to the continued 
catabolism of yolk that occurred for a short period after hatch. This immediate posthatch yolk catabolism resulted in a large drop to occur in the percent levels of $20: 5$ and 22:6 in the fed controls. Within three days (before the start of group d-3), 20:5 and 22:6 in P. ethanolamine decreased from $25 \%$ each to $22 \%$ and $19 \%$, respectively. In P. choline, 20:5 and 22:6 decreased from $20 \%$ and $17 \%$, to $12 \%$ and $9 \%$, respectively. These declines in fatty acids that occurred naturally in the controls were therefore incorporated into the results for group d-0. Any subsequent conservation of these fatty acids may then have counterbalanced the previous declines, producing an apparent non-selective utilization pattern. The fatty acid 20:4 did not decline in fed controls which may explain why in group $\mathrm{d}-0$ it was the most conserved PUFA.

The conservation of $20: 5$ and 22:6 during starvation supports evidence from other studies (Caste11 and Covey, 1976; Kanazawa et a1., $1978,1979 a, d, h)$ that these fatty acids may be essential to marine crustaceans. The reason for conservation of $20: 4$ is unknown, although there exists the possibility that this fatty acid may also be essential for Homarus americanus (Castell and Boghen, 1979). This type of evidence is certainly not conclusive, however, as shown by the apparent conservation of the non-essential fatty acids 16:0 and 18:0.

\section{Sumnary: Starvation}

First and second stage larval Homarus americanus did not molt if starvation began prior to molt stage $D_{0}$ but did molt if the onset of starvation was $\mathrm{D}_{0}$ or later. The quantity of energy reserves, however, may influence molting. If starvation began after molt stage $D_{0}$, it did not greatly influence molt timing but did lessen percent molt increment. 
Percent water increased with starvation due to a relatively fixed body volume coupled with tissue catabolism. The primary exoskeleton materials of ash and chitin remained stable in absolute amounts during starvation unless a molt occurred, indicating that exoskeleton chitin did not serve as an energy source. Carbohydrate, 1ipid, and protein a11 declined during starvation, with the decline in carbohydrate and lipid beginning before protein. A greater percentage of the available carbohydrate and 1ipid were catabolized but protein supplied most of the energy during starvation. This pattern may have been the result of the low energy stores in the larvae and it is suggested that advanced fourth stage larvae would rely main1y on triacylglycerols.

Triacylglycerol was catabolized faster than P. choline or P. ethanolamine though its low initial quantities caused $P$. choline to be the major 1ipid energy source. Two metabolic pools of $P$. choline were suggested, one associated and the other not associated with cellular membranes. P. ethanolamine appeared to exist only in a cellular membrane associated pool. Absolute amounts of free sterols did not decline with starvation. Either sterols can be temporarily stored by Homarus americanus or the membrane solidifying effects of sterols are counterbalanced by the increased unsaturated fatty acid pattern.

The apparent non-selective fatty acid utilization pattern in group d-0 may have been an effect of yolk catabolism. In the other starved groups the fatty acids $20: 4,20: 5$, and 22:6 were conserved during starvation which may be due to their potential as essential fatty acids. The conservation of 16:0 and 18:0 may indicate that these fatty acids were used to regulate membrane fluidity under the increased unsaturation caused by the conserved polyunsaturated fatty acids. 
Labelled Fatty Acid Feeding: Graphs 56-58, Tables 29-32.

The fatty acids 16:0 and 20:503 were chosen for this experiment as they represented a non-essential and a presumed essential fatty acid, respectively. The choice of a representative non-essential fatty acid was straightforward as 16:0 is the primary endproduct of de novo fatty acid synthesis and must therefore be readily metabolizable by Homarus americanus. 20:5 was chosen over 22:6 to represent essential fatty acids for three reasons. As a known precursor to prostaglandins (Mayes, 1983a), 20:5 has a potential metabolic role whereas no metabolic function for 22:6 is yet known. In large marine decapods, 20:5 is also more prevalent than 22:6 (Addison et al., 1972) and the results from the embryogenesis study revealed it to be the only selectively conserved fatty acid during that period.

A detraction from this experiment was the different absolute amounts of $16: 0$ and 20:5 that were present in each labelled diet. There was approximately seven times more 16:0 in the ${ }^{14} \mathrm{C}$ 16:0 1abelled diet as compared to $20: 5$ in the ${ }^{14} \mathrm{C} 20: 5$ labelled diet. This difference in amounts precluded certain comparisons between the results for each labelled diet. If the animal has a limited capacity to handle a fatty acid, a sudden large pulse of that fatty acid may force the animal to metabolize the fatty acid differently. For example, the animal may catabolize the fatty acid instead of storing it, inducing a dose dependent phenomenon. This may explain, for instance, the more rapid rate of decline in ingested 16:0 over 20:5. Instead of providing evidence of conservation of 20:5, the results may have been an artifact of the larger amount of ingested 16:0. Overloading of the larval system with 16:0 was a 
possibility because the approximately $5 \mu \mathrm{g}$ of 16:0 that was ingested from the labelled feed could have represented a third of the larvae's current body total of that fatty acid.

The inclusion of $18: 1 \omega 9$ as a carrier fatty acid in the labelled diets caused the total lipid content of each diet to be the same. Therefore, certain comparisons between the ${ }^{14} \mathrm{C} \quad$ 16:0 and ${ }^{14} \mathrm{C} \quad$ 20:5 label fed groups were possible. One such comparison was the absolute amount of labelled pellet that was eaten by each group. Based on the amount of label left in the feeding containers (it was unlikely that the larvae could have excreted any of the labelled feed within 90 minutes), significantly more $(p<.001)$ of the 20:5 labelled diet was ingested in comparison to the 16:0 labelled diet. In both groups of larvae, the feeding conditions and times and food pellet size and composition (with the exception of the labelled fatty acid) were the same. This implies that the labelled fatty acid, either through texture or taste, influenced the amount of food that the larvae chose to eat. Chemosensory work in crustacea has primarily focussed on low molecular weight compounds (cf. Ache, 1982) so it is unknown whether Homarus americanus has the capability of tasting differences in fatty acids.

At the end of the feeding period in the 20:5 experiment, the label occurred mainly in triacylglycerols and P. choline; but in the 16:0 experiment, the label occurred mainly in the triacylglycerols and free fatty acids. Within $12 \mathrm{~h}$ in both experiments, the percent of the total label quickly declined from triacylglycerol and ended up in phospholipids. Lipids are absorbed via the hepatopancreas (Dal1, 1981) and are distributed throughout the body as phospholipid in the hemolymph 
(Lee and Puppione, 1978). These data suggest that after absorption, some or most, of the dietary free fatty acids were converted into triacylglycerols. A second conversion into phospholipid then quickly followed and may have been associated with redistribution of the lipid through the body. A portion of the dietary free fatty acids, however, may have been converted directly into phospholipid without a triacylg1ycerol intermediate.

The above pattern of label distribution was also seen in the hepatopancreas of the lobster Panulirus interruptus fed ${ }^{14} \mathrm{C}-1$-triolein (Lee and Puppione, 1978) where the ingested label immediately became evenly distributed between triacylglycerol and phospholipids. Teshima and Kanazawa (1980) fed the prawn Penaeus japonicus ${ }^{14} \mathrm{C}-1-t r i p a l m i t i n$ and also found immediate, transitory label peaks in the triacylglycerols followed by the phospholipids in the hepatopancreas. In a different study, however, using the same prawn species, Teshima and Kanazawa (1979) fed $1-{ }^{14} \mathrm{C}$ linolenic acid $(18: 3 \omega 3)$ and found an immediate conversion mainly to phospholipids in the hepatopancreas. The reason for this discrepancy between studies is unknown, though severa1 factors may be responsible.

The use of labelled triacylglycerols or free fatty acid may influence which lipid is formed after absorption. Absorption of triacylglycerols is not direct but involves enzymatic digestion into free fatty acids and mono- and diacylglycerols (Brockerhoff et al., 1967). Upon absorption, even if phospholipid formation is favored, the proper balance of triacylglycerol moieties may cause a significant portion of triacylglycerol to be reformed. There was also the possibility that the 
hepatopancreatic tissues in the above studies contained unabsorbed, undigested 1ipid. If triacylglycerol was used as the labelled lipid, the detection of labelled triacylglycerol in the hepatopancreas may part1y be due to an unassimilated quantity.

The total amount of lipid and the accompanying food may also have an influence on the pattern of lipid assimilation. If, for instance, phospholipid formation from ingested lipid is slightly favored, then if the ingested diet supplies the proper precursors (e.g. lyso-phospholipids, phosphocholine, and phosphoethanolamine), rapid and total conversion of ingested 1ipid into phospholipid may occur. In Teshima and Kanazawa's (1979) diet, the labelled fatty acid was incorporated as a minor amount within a clam homogenate feed. In the present study, free fatty acids composed $20 \%$ of the diet, which otherwise was mostly protein (phospholipid was no more than $1 \%$ ). To rapidly assimilate the free fatty acids, the larval Homarus americanus may have formed triacylglycerol as it requires only glycerol as a precursor which it can form from protein. To have formed phospholipid would have also required immediate inputs of glycerol as well as phosphate and choline or ethanolamine, and would have used one third less of the incoming fatty acids. It would be interesting to determine how lipid absorption and subsequent metabolism in crustaceans are influenced by the quantity and type of lipid and the accompanying diet. This type of work has been carried out with the prawn Penaeus japonicus (Teshima and Kanazawa, 1983) for sterol and other 1ipid classes in terms of overall assimilation. The rate of assimilation and type of metabolism, and how specific fatty acids may affect this, has yet to be investigated. 
A possible comparison between the labelled 16:0 and 20:5 experiments was the rate of metabolism of the labelled fatty acids. The definition of metabolism in this case refers only to the transfer of the labelled free fatty acid into another lipid class. It is assumed that if the labelled lipid occurred as a free fatty acid in the whole animal, the 1ipid had either not been absorbed or, if absorbed, had not yet been metabolized. Conversion of free fatty acids into other 1ipid classes was also assumed not to occur extracellularly in the gut. The data show that 16:0 remained as a free fatty acid far longer than did 20:5. By the end of the 90 minute feeding period, almost all of the labelled 20:5 had been converted into other 1ipid classes, whereas the majority of 1abe1led 16:0 remained as free fatty acid. This suggests that different rates of absorption or metabolism for different free fatty acids occurred in Homarus americanus. The different absolute amounts of $16: 0$ and 20:5 in their respective diets could have influenced the above interpretation. If all the fatty acids were equally absorbed and metabolized, then on a percentage basis each fatty acid should have been equally apportioned into the various lipid classes. The fatty acid 16:0 may have overloaded the animals capacity to metabolize that one fatty acid, however, resulting in proportionally different absorption or metabolism rates. If so, then the

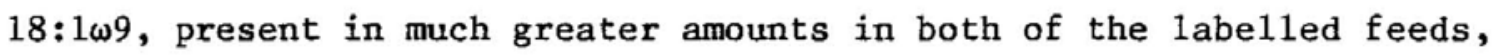
should also have resulted in some interference. If this interference occurred, then overloading must only affect the overloaded fatty acid, as $20: 5$ assimilation was not noticeably affected.

The mechanism responsible for differential rates of absorption or metabolism is unknown. Absorption may have been the causative factor due 
to the greater fluidity of $20: 5$ over 16:0, which respectively were 1 iquid and solid at the experimental temperatures. In man, absorption rates are faster for fatty acids which are liquid rather than solid at body temperatures (Deue1, 1955). This same relationship may also apply for Homarus americanus.

In comparing the fed versus starved larvae in the labelled 16:0 experiment, there was a trend $(p<.063)$ for the fed animals to retain more of the assimilated labelled 16:0 in comparison to the starved animals. (A better feed for the fed animals, perhaps of live Artemia would 1ikely have resulted in a more significant difference). In the labelled 20:5 experiment, however, there was no difference in the retention of the assimilated labelled 20:5 between the fed and starved animals. The difference in the 16:0 experiments can be explained by assuming that there was no selective conservation of this fatty acid or its 1ipid derivatives. Thus, the greater utilization of total lipid by starved versus fed animals can account for the difference in labelled 16:0 retention. On the other hand, selective conservation of 20:5 must have occurred to account for the equal retention of 20:5 in the fed versus starved larvae, as starved larvae had a higher rate of lipid catabolism. These data support the fatty acid profile data from the Starvation experiment.

In order to make more direct comparisons between 16:0 and 20:5 based metabolism, it would be necessary to repeat these experiments using diets containing equal absolute amounts of each fatty acid. The total level of lipid in the diet should also be kept low to eliminate the possibility of overloading the metabolic capabilities of the larvae. Finally, a dual 
isotope experiment using, for instance, ${ }^{14} \mathrm{C}$ 16:0 and ${ }^{3} \mathrm{H} 20: 5$ combined into the same diet at equal absolute amounts would allow the most powerful interpretations.

\section{Summary: Labelled Fatty Acid Feeding}

The fatty acid 16:0 was chosen as a non-essential fatty acid as it is the endpoint of de novo fatty acid synthesis; 20:5 was chọsen as an essential fatty acid for its prevalence, observed conservation and known role as a prostaglandin precursor. More of the 20:5 based diet was consumed compared to the 16:0 based diet. As the only differing factor between both diets was the labelled fatty acid, either the texture of the diet and/or the taste of each fatty acid was detectable and made a difference to the feeding larvae.

Immediately after absorption, some or most of the absorbed fatty acid was temporarily converted into triacylglycerol, followed by a second conversion into phospholipid. Whether this is an obligatory pathway or influenced by the quantity and quality of 1ipid in the diet is unknown.

The type of fatty acid may influence the rate at which it is absorbed and/or metabolized by larva1 Homarus americanus. As in man, fluidity of the fatty acid may affect absorption rates. A dual isotope experiment is suggested as a means to make further comparisons between non-essential and essential fatty acid assimilation. 
Overview

The following is a general overview of resource utilization and lipids in the early developmental period of Homarus americanus. For specific facets of development (embryogenesis, hatching, larval development, molting, starvation resistence) refer to the summaries at the end of the appropriate section.

In terms of energy reserves used by developing Homarus americanus, a distinction can be made between the preferred and caloric types of reserves. The preferred energy reserve is being defined as the type of reserve that the animal utilizes first whenever it must initiate catabolism of its stored materials. The caloric type of reserve is being defined as the type of reserve that actually supplies the major portion of metabolic energy.

During embryogenesis, exclusive of hatching, the preferred and caloric energy reserves cannot be differentiated. The order of reserve utilization was triacylglycerol, followed by P. choline or protein and then $\mathrm{P}$. ethanolamine.

During the first two larval stages, and extrapolating through the third larval stage as well, the preferred energy reserves during starvation were carbohydrate and triacylglycerol, followed by $P$. choline. Protein and P. ethanolamine were roughly equivalent in this respect and followed P. choline. Whereas the caloric energy reserves during short periods of starvation would match the preferred order, during prolonged periods of starvation, the limited absolute amounts of each reserve imposed a different order. The caloric utilization of energy reserves placed protein as the primary source, followed by $P$. choline, $P$. ethanolamine, triacylglycerol, and then carbohydrate. (P. ethanolamine 
and triacylglycerol may be reversed in order in the third stage larvae due to the larger triacylglycerol reserves in this stage.)

In postmetamorphic fourth stage larvae, the preferred order of energy reserve utilization is hypothesized to be the same as for the premetamorphic larvae. In advanced (mid premolt and older) fourth stage larvae during prolonged periods of (but not total) starvation, the caloric energy utilization is hypothesized to be triacylglycerol followed by P. choline or protein then $P$. ethanolamine and lastly, carbohydrate. Whether fifth stage and older Homarus americanus accumulate proportionally as much triacylglycerol as fourth stage larvae remains to be determined.

In Homarus americanus triacylglycerol primarily serves an energy storage function. It may also be a required intermediate in the dietary lipid absorption/assimilation process, or a means to facilitate lipid absorption under certain dietary conditions. Based on energy availability, P. choline appears to occur in two metabolic pools with one pool capable of being catabolized for energy with little or no concomitant structural (tissue) loss. Catabolism of the other poo1, associated with cellular membranes, occurs after the first pool has been partially or fully utilized and involves tissue (protein) 1oss. In contrast to P. choline, P. ethanolamine catabolism is always associated with tissue loss and thus it is likely that $P$. ethanolamine only occurs in a cellular membrane associated pool. Free sterols are associated with membranes but are not used for energy. One of the functions of sterols may be the regulation of membrane fluidity (Bloch, 1983), probably its major quantitative function in $\mathrm{H}$. americanus.

The data for fatty acids support the general concensus (based on other crustaceans) that $20: 5$ and $22: 6$ are essential fatty acids for 
Homarus americanus. While membrane fluidity is a factor in the requirement of these fatty acids, other functions such as precursors for metabolites and regulation of enzyme or osmotic activity are likely. $\underline{\text { H. }}$ americanus larvae are capable of selectively metabolizing fatty acids and will conserve 20:5 and 22:6 under conditions of starvation and 20:5, 22:6-deficient dietary regimes. Selective conservation of $20: 5$ and 22:6 in membrane lipids increases the degree of membrane unsaturation which may be compensated by the conservation of saturated fatty acids and cholestero1. The essentiality of $20: 4$ is still unclear. It may serve a specific function but may also be replaceable by 20:5. One possible mechanism behind essential fatty acid deficiency symptoms may be a limitation on phospholipid synthesis or accumulation.

The use of a cod liver oil based feed indicates that 20:5 and 22:6 deficient diets can be successfully supplemented. The ability of Homarus americanus larvae to assimilate large pulses of specific fatty acids, as evidenced by the labeled fatty acid experiments, plus the larvae's ability to conserve $20: 5$ and $22: 6$, indicate that pulsed supplementation of fatty acids should be feasible. That is, in the lab, hatchery, or field conditions, a single meal with a high $20: 5$ and 22:6 content should provide enough of these two fatty acids to maintain a larva for a prolonged period. In the lab or hatchery, pulsed supplementation would allow the use of two feeds -- an inexpensive and/or convenient, but nutritionally deficient, feed (e.g. frozen Artemia) plus, infrequently, a more expensive or difficult to obtain supplement. The implication for the field is that food quality and availability can vary and still allow successful development of $\underline{\mathrm{H}}$ americanus larvae. 


\section{Epilogue}

This work focused on the biochemical changes in Homarus americanus as they relate to the physiology of early development. In each studied aspect of early development (embryogenesis, hatching, molting, larval development, metamorphosis, and feeding) significant contributions were made in the understanding of crustacean physiology. The emphasis on lipids in this work is especially relevant for the fields of energetics, nutrition, and environmental toxicology. An impression to be derived from this work is the connections between the animal's biochemical responses and physiology and the interplay of the environment. Each factor is important in the understanding of the others.

It was stated in the Introduction that a gap exists in our current knowledge of crustacean biochemistry. That gap is now smaller. This work has answered many questions and addressed, and laid the foundations for, several promising avenues of further research. 
$-209-$

APPENDICES 


\section{APPENDIX 1}

Drach $(1939,1944)$ was the first to fully characterize the molt cycle of decapods and he gave each phase of the cycle, dependent on certain morphological changes, a letter designation (A through E). A and B are considered the postmolt recovery periods; $\mathrm{C}$ is the classic intermolt during which the exoskeleton fully forms and reserves begin accumulating; the $\dot{D}$ stages are considered the premolt period when the animal prepares for the upcoming molt, and $\mathrm{E}$ is the ecdysis or molt itself. (Refer to the molt cycle section in the Introduction for a more complete overview of the molt cycle.)

The molt staging technique used in the present work was based on Aiken's $(1973,1980)$ method for molt staging adult Homarus americanus. Aiken's $(1973,1980)$ articles should be referred to for a complete description of the characteristic morphological changes that occur in $\underline{H}$. americanus through the molt cycle. The present technique uses the tissue characteristics and spines and setae of both the telson and uropods to molt stage the animal. This is similar to the technique used by Rao et al. (1973) to molt stage fourth and fifth stage $\underline{H}$. americanus and to Aiken's $(1973,1980)$ technique, using the pleopod setae, to molt stage adult $\mathrm{H}$. americanus. In the method of Rao et al. (1973) stage $\mathrm{E}$ was further subdivided. In Aiken's $(1973,1980)$ technique, stages $C, D_{0}$ and $D_{2}$ were further subdivided and $D_{2}$ was distinguished from $D_{3}$. These subdivisions were not used in the present work. To keep the method as simple and quick as possible, the resolution of the present molt staging technique was limited to distinguishing the molt stages $A, B, C, D_{0}$, $D_{1}{ }^{\prime}, D_{1}{ }^{\prime}, D_{1} \cdots "$, and $D_{2-3}$ (stages $D_{2}$ and $D_{3}$ combined). 
The average time the larvae spent in each molt stage in this study, as a percent of the whole molt cycle, is shown in Figure 1 for the four larval stages. A similar diagram is given for the adult molt stage pattern by Aiken (1980). The major differences between the larval and adult molt patterns occur in molt stage $C$ and $D_{1}$. Proportionally, stage $C$ is greater, and stage $D_{1}$ is smaller, in duration in adults than larvae. Generally, adult Homarus americanus molt once a year, usually in summer (Herrick, 1911). Molt stage C is likely proportionally longer in adults because the adult may pause in this stage to control the time of molting to coincide with the summer season, or the seasonal timing of the adult molt cycle may be a consequence of temperature, food availability and reproductive requirements (Aiken, 1980). In either case, in adults a pause in stage $\mathrm{C}$ results. The larvae, in contrast, do not have a required or forced pause at molt stage $C$.

Though the present molt staging method followed Aiken's $(1973,1980)$ technique, there is one difference in a distinguishing feature between molt stage C and $D_{0}$. Aiken (1980) uses the formation of an amber-colored zone in the pleopod tips as the first sign of stage $D_{0}$ in adult Homarus americanus. In the telson and uropods of larval $\underline{\mathrm{H}}$. americanus this amber-colored zone, which appears as a layer of relatively clear tissue beneath the bases of the setae, was a characteristic of molt stage $C$. In the present method, the first sign of setal tissue withdrawal from the cuticle of the setae was taken as the onset of stage $D_{0}$. As in adults (Aiken, 1973), the setal tissue in the larvae vary in the degree of advancement (more advanced equals later in the molt cycle) depending on the location of the setae. In 1arvae, 
uropod setae were of ten slightly more advanced than telson setae. If such a difference was apparent then the most advanced portion was used to define the molt stage (Aiken, 1973)

To prepare a larva for molt staging, the larva was picked up with a wide mouth eyedropper (an inverted pasteur pipet with a broken tip or a plastic eyedropper with the tip cut off) and transferred onto a glass microscope slide. The accompanying water was mostly drained or blotted off and flexible tip microforceps were used to position the larva into an upright position (the ventral surface of the larvae was down, touching the glass slide). If the larva was to be sacrificed after molt staging, then a coverslip was sometimes used over the telson. If the larva was to be used in an experiment, then no coverslip was used in order to minimize stress. The telson was then examined under a compound microscope at 100x. With practice the entire procedure could be accomplished in less than 15 seconds. 


\section{FICURE 1}

Molt Stage Patterns

LARVAL.

STAGE

FIRST

SECOND

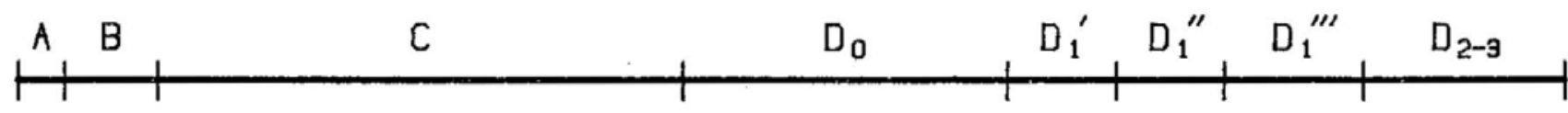

139

THIRD

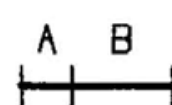

C

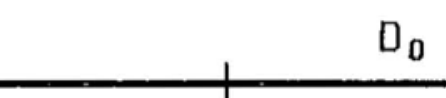

$D_{0}$

$D_{0}$ $D_{1}^{\prime}+D_{1}^{\prime \prime}+D_{1}^{\prime \prime \prime}, D_{2-9}$

135

in Larval Stage

(I) Hatches

FOURTH

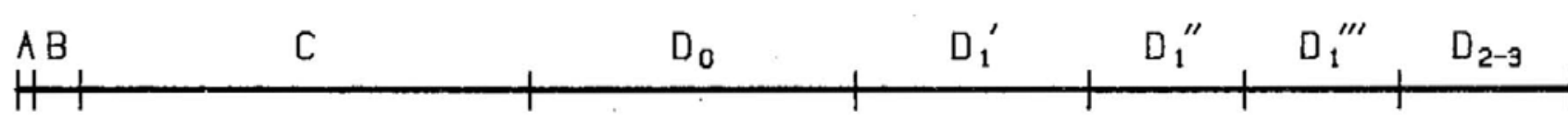

ADULT

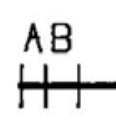

C

$D_{0}$

$\mathrm{D}_{1} \mathrm{D}_{2} \mathrm{D}_{3}$ 
$-214-$ 


\section{FIGURE KEY}

1. A11 photographs show dorsal views.

2. Photographs are not scaled relative to one another.

3. Asterisked Key codes indicate the distinguishing features of each mo1t stage.

4. Orientation of the animal in the photographs is given as the position of the head of the animal in relation to the displayed photograph. The codes head:up, head:down, head:left, and head:right, indicate the direction that the animal is "facing" in the photograph. 
Figure 2

Telsons and uropods of first through fourth stage larvae.

I. First stage larva.

telson only, no uropods, head:down.

mo1t stage C.

II. Second stage larva.

telson only, uropods developing at base of telson, head:down. molt stage $\mathrm{D}_{1}{ }^{\prime}$.

III. Third stage larva.

telson and two pairs of uropods, head:down.

molt stage Do.

IV. Fourth stage larva.

telson and two pairs of uropods, head:down.

molt stage A. 


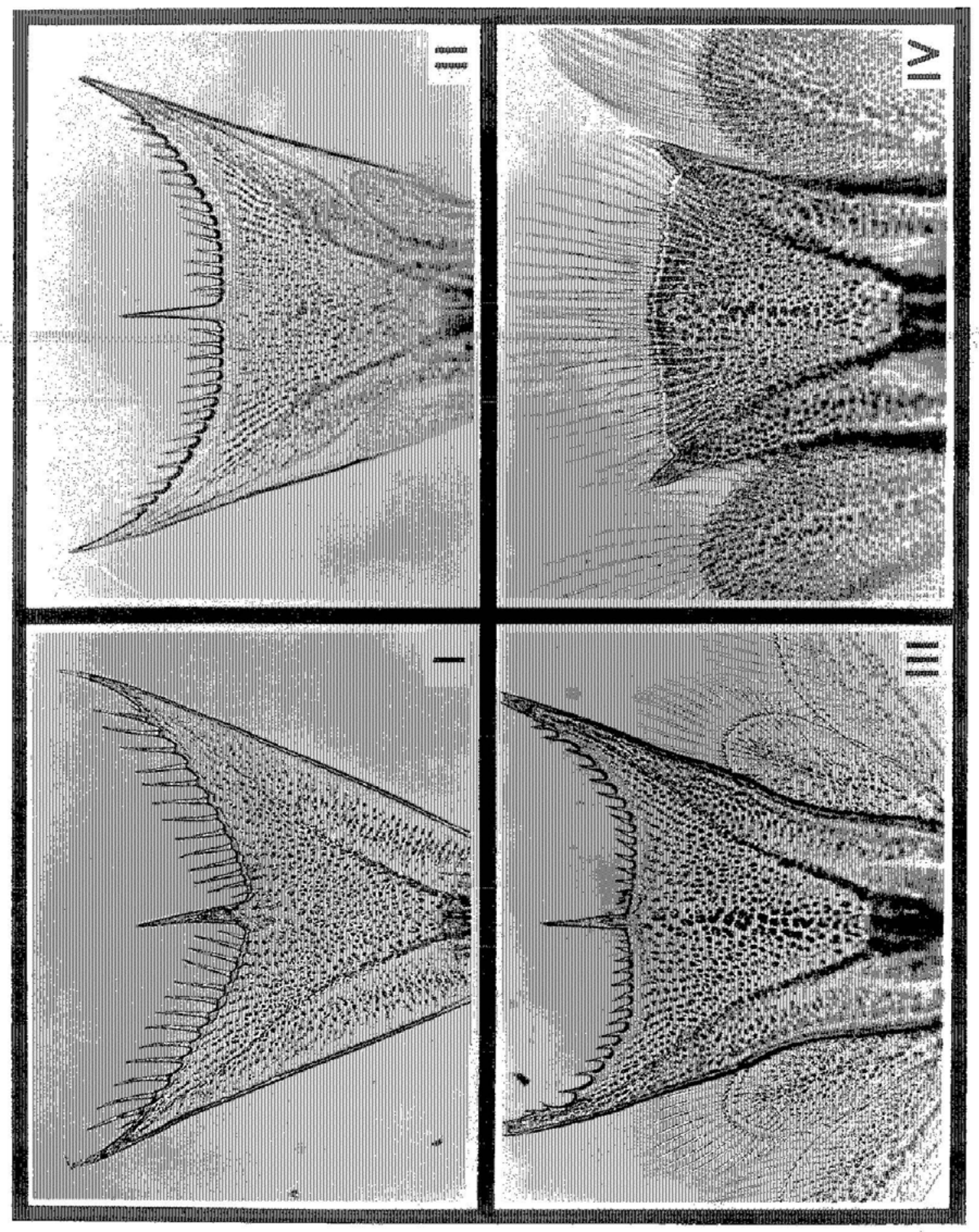




\section{Figure 3}

First Stage Larva1 Mo1t Staging

\section{Mo1t $\quad \underline{\text { Key }}$}

Description

Stage

A no photographs.

* - animal limp.

A - telson tissue dense in appearance.

* B - all setae and spines full of dense tissue.

C - some setae may not be fully evaginated.

D - spine sheaths (setal organ, Aiken (1973)) in telson tissue visible as 1ight strips in the former positions of invaginated spines.

B left: Distal end of telson, center spine and setae, head:down. right: Line drawing.

A - telson still dense in appearance, but lightening up.

* E - dense tissue within setae decreases in density, gives the appearance of withdrawing from the setae, clear tissue remains in setae.

* F - inner, dense tissue of center spine begins withdrawing from spine, clear tissue remains.

G - former spine sheaths in telson still visible.

C left: Distal end of telson, center spine and setae, head:down. right: Line drawing.

- epidermis of lateral edges of telson has crenulated appearance with apparent fluid gap between cuticle and epidermis.

* H - layer of relatively clear tissue beneath base of setae.

$\mathrm{J}$ - border of clear tissue layer.

$\mathrm{K}$ - dense tissue of center spine retracts to approximately $1 / 2$ of the spine length.

* L - density of telson tissue decreases.

Do left: Distal end of telson, center spine and setae, head:down. right: Line drawing.

$\mathrm{J}$ - border of clear tissue layer.

* M - clear tissue of setae begins withdrawing from cuticle of setae, withdraws to half the cuticle setae length.

* $\mathbf{N}$ - fluid gap between exoskeleton intersetal areas and epidermis.

$\mathrm{P}$ - dense tissue of center spine begins withdrawing to base of spine. 


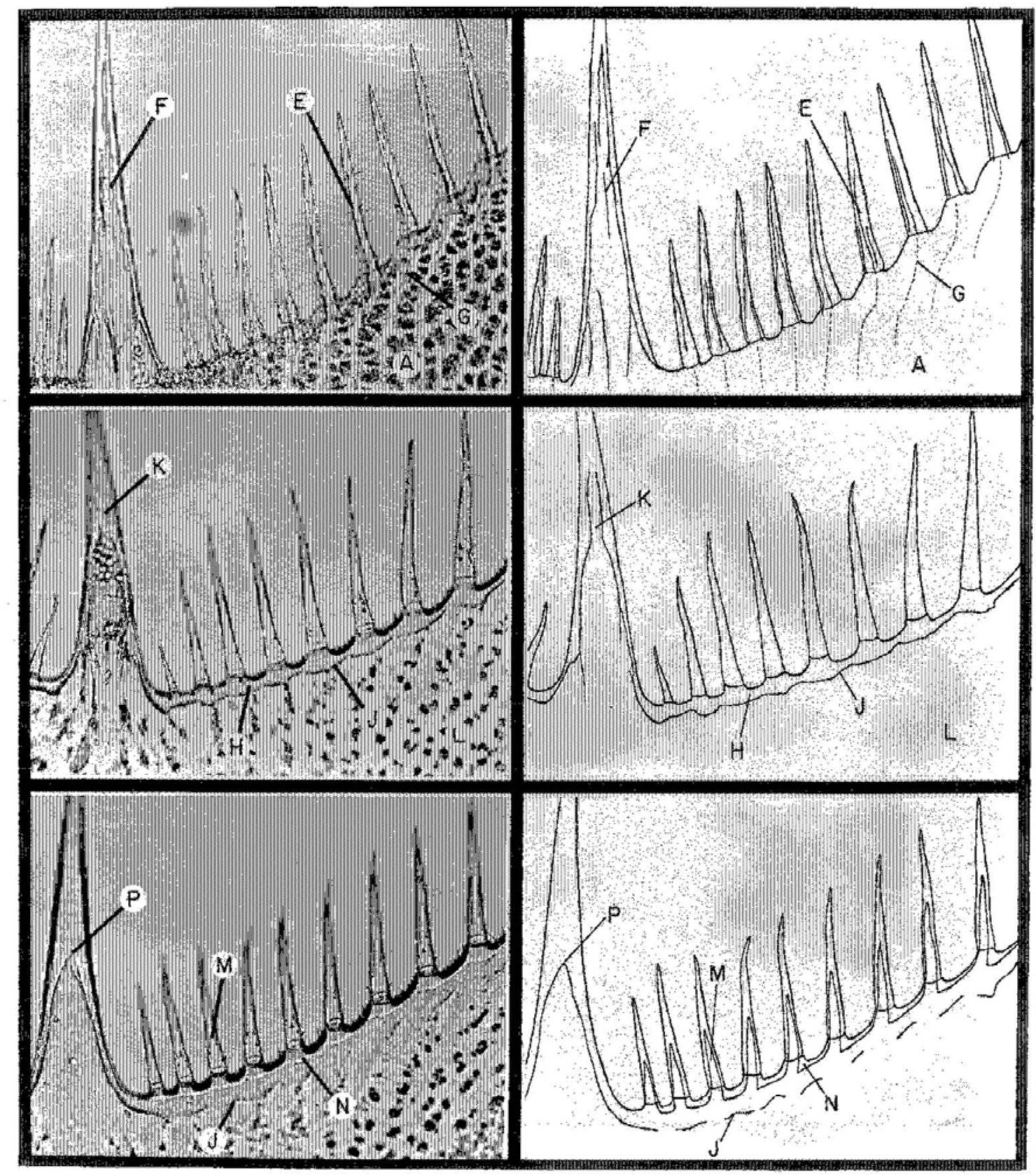




\section{Figure 4}

First Stage Larval Molt Staging

Mo1t $\quad \underline{\text { Key }}$

Description

Stage

$D_{1}{ }^{\prime}$ left: Distal end of telson, center spine and setae, head:down. right: Line drawing.

$\mathrm{J}$ - border of clear tissue layer.

$N$ - fluid gap between exoskeleton and epidermis.

* Q - setal tissue begins invaginating.

R - clear tissue of center spine begins withdrawing from cuticle of spine

$D_{1}$ " left: Distal end of telson, center spine and setae, head:down. right: Line drawing.

$\mathrm{R}$ - clear tissue of center spine continues withdrawing from cuticle of spine.

* S - invagination of setal tissues becomes more pronounced.

$D_{1}$ '" left: Distal end of telson, center spine and setae, head:down. right: Line drawing.

T - tissue of center spine begins bunching up.

* U - invagination of setal tissue very pronounced, attains maximum invagination.

$V$ - tissue of center spine withdraws to $3 / 4$ of the cuticle spine length.

W - density of telson tissue increases.

$\mathrm{D}_{2-3}$ left: Distal end of telson, center spine and setae, head:down. right: Line drawing.

- epidermis at lateral edges of telson very distinct and strongly crenulated, indication of new chitin.

$W$ - density of telson tissue continues to increase.

$X$ - bunching of center spine tissue very pronounced, bunching extends beneath the spine into telson.

$\mathrm{Y}$ - tissue in outer spine withdraws to $2 / 3$ of cuticle spine length.

* Z - pronounced bulging of epidermis at invaginated setae. 


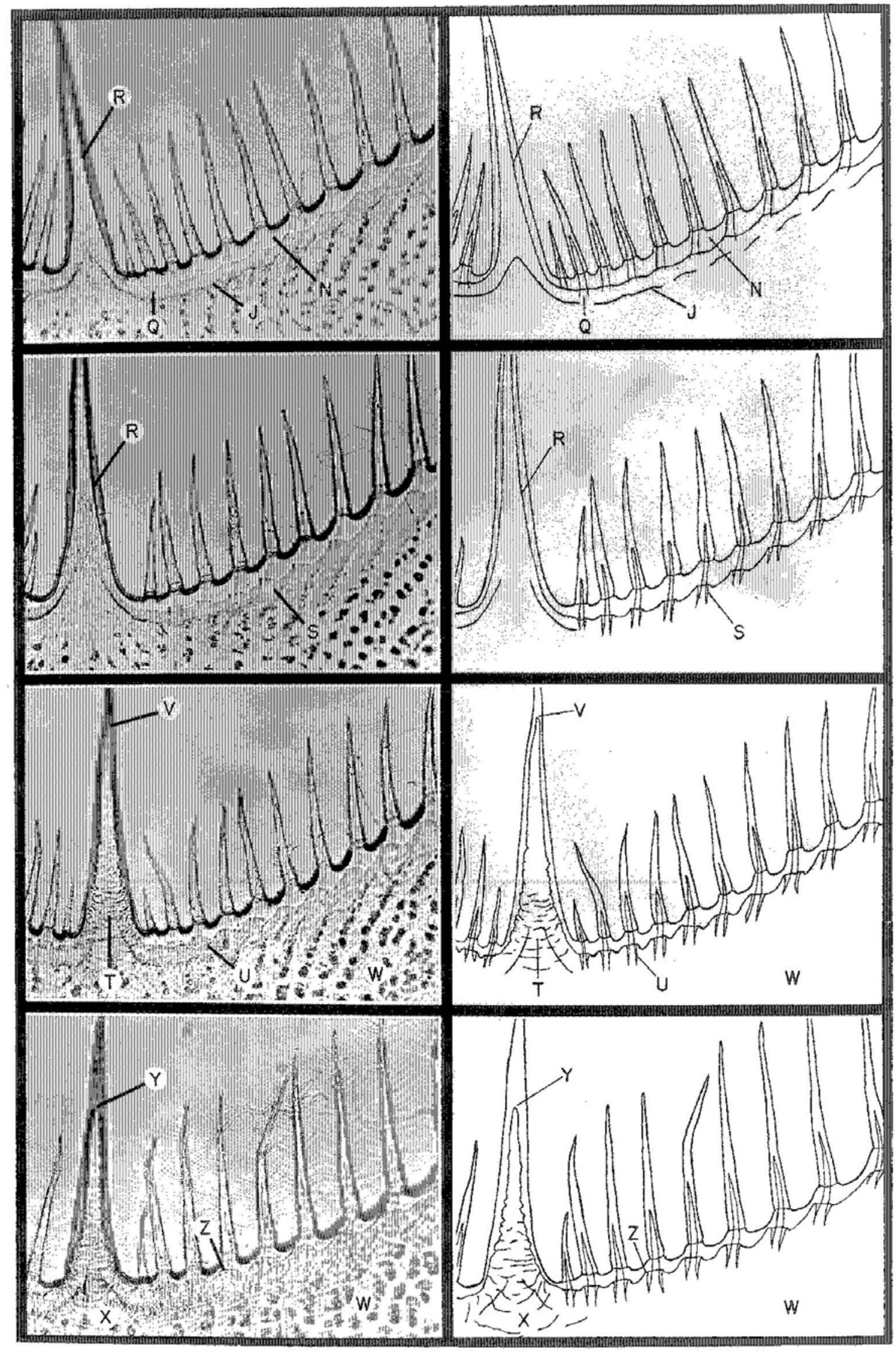


Figure 5

Second Stage Larval Mo1t Staging

Mo1t Key Description

\section{$\underline{\text { Stage }}$}

A left: Distal end of telson, center spine and setae, head:down. right: Lateral base of telson, developing uropods, head:left.

* - animal limp.

A - telson tissue dense in appearance.

* B - all setae and center spine full of dense tissue.

C - spine sheaths (setal organ, Aiken (1973)) in telson tissue visible as light strips in the former positions of invaginated spines.

D - border of developing uropods barely visible.

B left: Distal end of telson, edge of center spine and setae, head: down.

right: no photograph.

* E - dense tissue within setae decrease in density, gives the appearance of withdrawing from the setae, clear tissue remains in setae.

- inner, dense tissue of center spine begins withdrawing from spine, clear tissue remains.

- border of developing uropods becomes distinct.

C left: Distal end of telson, center spine and setae, head:down. right: Lateral base of telson, developing uropods, head:left.

* H - layer of relatively clear tissue beneath base of setae and at edge of developing uropods.

$\mathrm{J}$ - border of clear tissue layer.

$\mathrm{K}$ - border of developing uropod.

* L - density of telson tissue decreases.

Do left: Distal end of telson, edge of center spine and setae, head: down.

right: Lateral base of telson, developing uropods, head:left. $\mathrm{J}$ - border of clear tissue layer.

$\mathrm{K}$ - border of developing uropod.

* M - clear tissue of setae begins withdrawing from cuticle of setae.

* N - fluid gap between exoskeleton intersetal areas and epidermis. 0 - tissue of developing uropod setae now visible. 

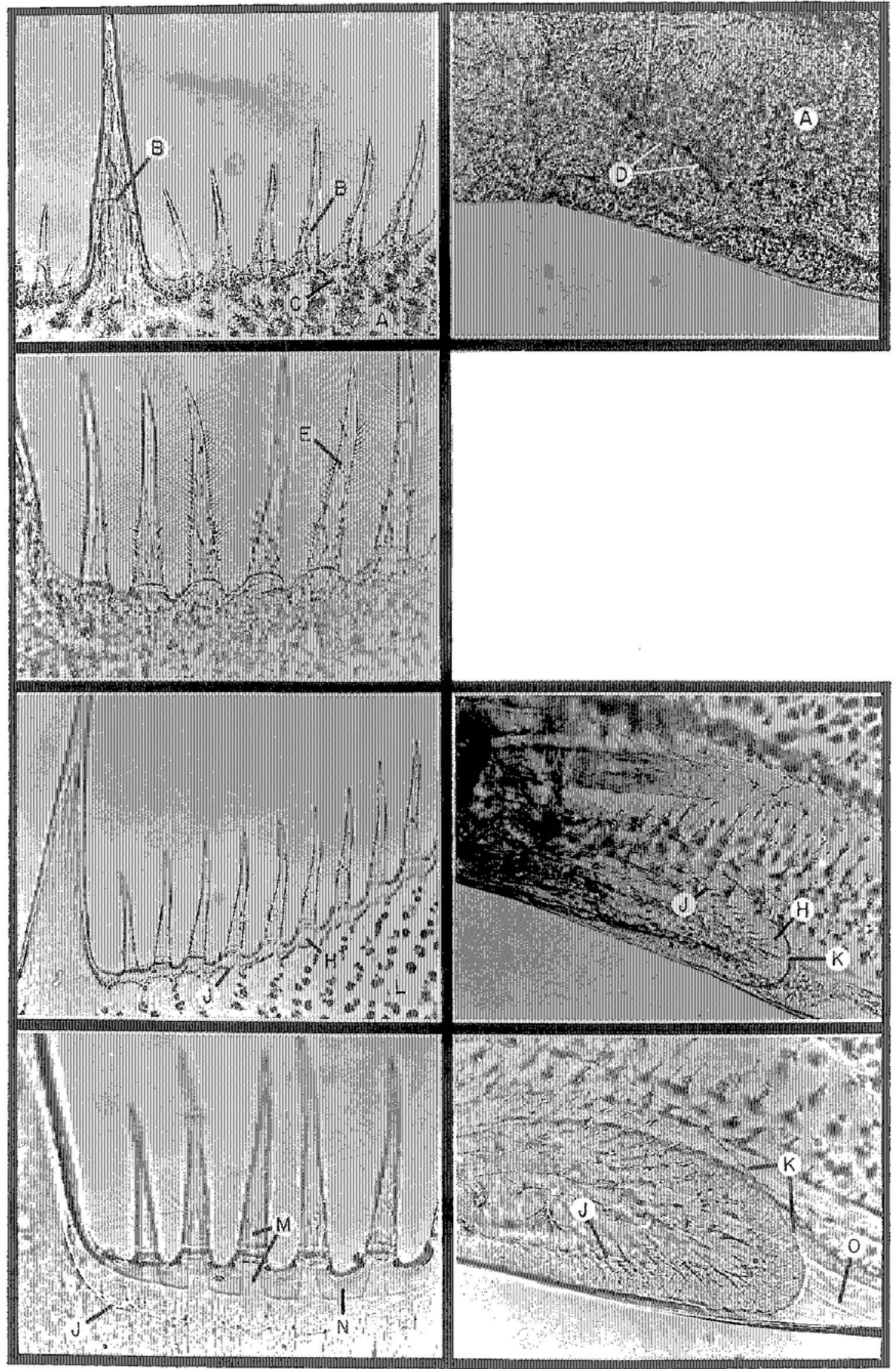
Figure 6

Second Stage Larval Molt Staging

Mo1t $\quad$ Key

Description

$\underline{\text { Stage }}$

$\mathrm{D}_{1}{ }^{\prime}-$

$\mathrm{D}_{1}$ " left: Distal end of telson, edge of center spine and setae, head: down.

right: Lateral base of telson, developing uropods, head:left.

$\mathrm{K}$ - border of developing uropod.

$\mathrm{N}$ - fluid gap between exoskeleton and epidermis.

* P - setal tissue begins and continues invaginating.

Q - clear tissue of center spine begins and continues withdrawing from cuticle of spine.

late

$D_{1}$ '" left: Distal end of telson, setae only, head:down.

right: Lateral base of telson, developing uropods, head:left.

- tissue of center spine begins bunching up.

* $R$ - invagination of setal tissue very pronounced, attains maximum invagination.

S - density of telson tissue increases.

$\mathrm{T}$ - scalloped appearance of epidermis at invaginated spines.

$D_{2-3}$ left: Distal end of telson, edge of center spine and setae, head: down.

right: Lateral base of telson, developing uropods, head:left.

$S$ - density of telson tissue continues to increase.

$T$ - bunching of center spine tissue very pronounced.

* U - pronounced bulging of epidermis at invaginated setae.

$* \mathrm{~V}$ - epidermis becomes very distinct, indication of new chitin. 


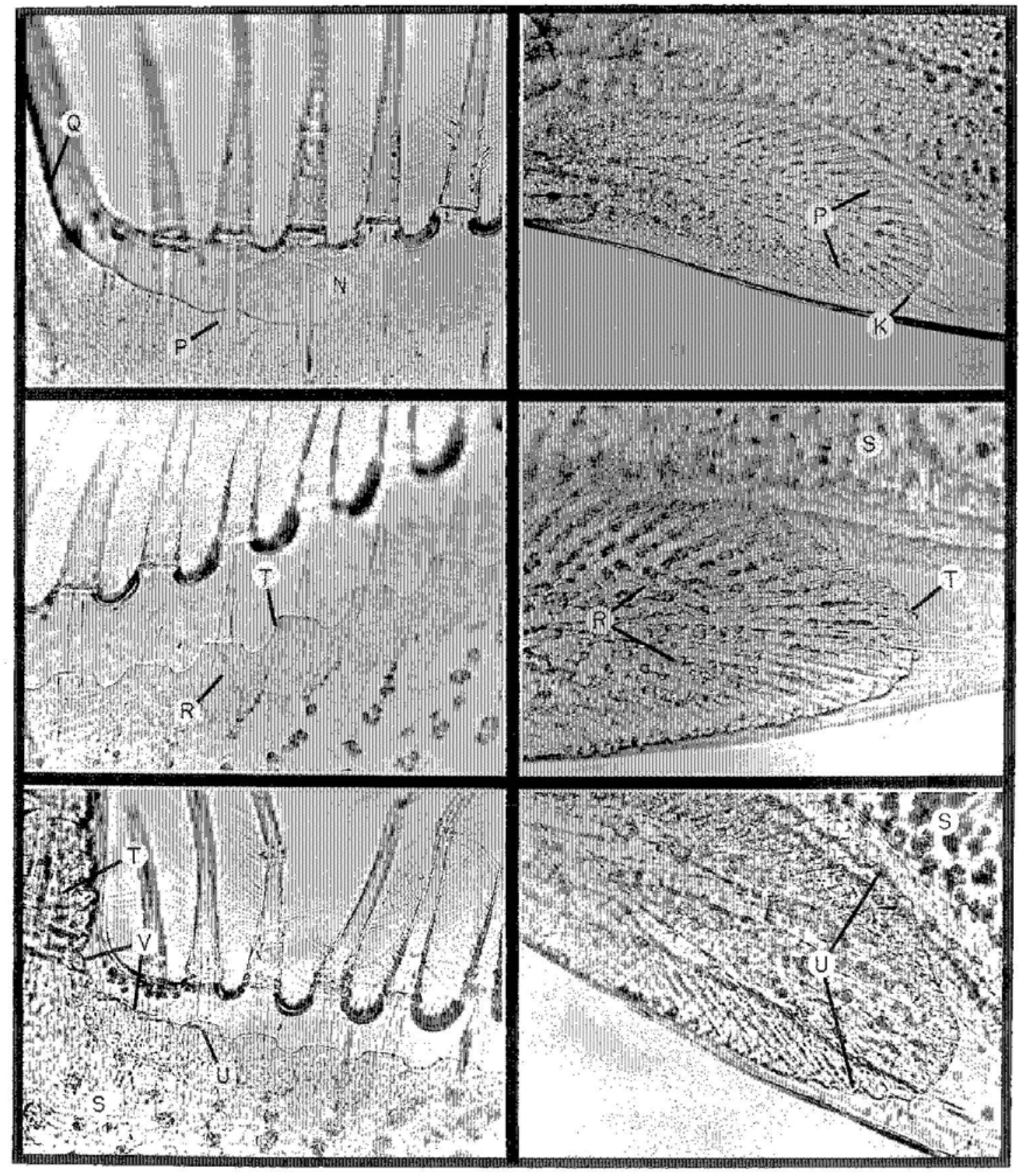


Figure 7

Third Stage Larval Molt Staging

Mo1t Key $\quad$ Description

$\underline{\text { Stage }}$

A left: Distal end of telson, setae only, head:down.

right: Distal end of uropod, setae, head:down.

* - animal limp.

A - telson and uropod tissue dense in appearance.

* B - setae full of granular tissue.

C - spine sheaths (setal organ, Aiken (1973)) in telson tissue visible as light strips in the former positions of invaginated spines.

late

B left: Distal end of telson, center spine and setae, head:down.

right: Distal end of uropod, setae, head:down.

A - telson and uropod tissue still dense in appearance, but lightening up.

* E - clear tissue in setae.

* F - inner, dense tissue of center spine begins withdrawing from spine, clear tissue remains.

G - layer of relatively clear tissue beneath base of setae. (late B - early C).

C left: Distal end of telson, center spine and setae, head:down. right: Distal end of uropod, setae, head:down.

* H - layer of relatively clear tissue beneath base of setae. $\mathrm{J}$ - border of clear tissue layer.

$\mathrm{K}$ - dense tissue of center spine retracts to approximately $1 / 2$ of spine length.

* L - density of telson and uropod tissue decreases.

Do left: Distal end of telson, setae only, head:down. right: Distal end of uropod, setae, head:down. $\mathrm{J}$ - border of clear tissue layer.

* M - clear tissue of setae begins withdrawing from cuticle of setae.

* N - fluid gap between exoskeleton intersetal areas and epidermis. 


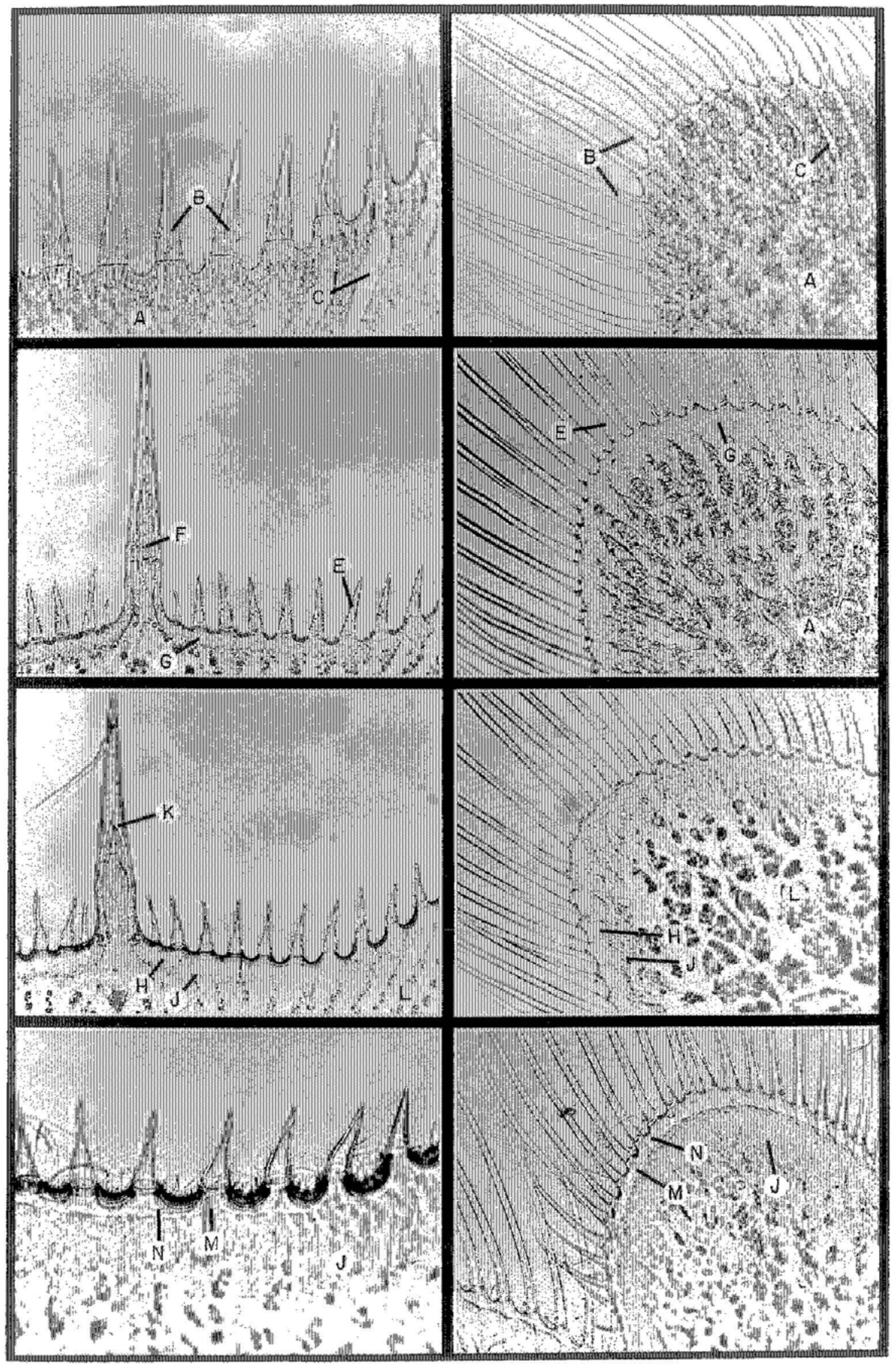




\section{Figure 8 \\ Third Stage Larval Mo1t Staging}

Molt Key Description

$\underline{\text { Stage }}$

$D_{1}$ ' left: Distal end of telson, edge of center spine and setae, head: down.

right: Lateral-distal end of uropod, setae, head:down.

- resorption of center spine tissue begins.

N - fluid gap between exoskeleton and epidermis.

* P - setal tissue begins invaginating.

$Q$ - border of epidermis.

$D_{1}$ " left: Distal end of telson, edge of center spine and setae, head: down.

right: Distal end of uropod, setae, head:down.

N - fluid gap between exoskeleton and epidermis.

* S - invagination of setal tissues become more pronounced.

$D_{1}$ '" left: Distal end of telson, setae only, head:down.

right: Distal end of uropod, setae, head:down.

* T - invagination of setal tissue very pronounced, attains maximum invagination.

*U - scalloped appearance of epidermis at invaginated spines.

$D_{2-3}$ left: Distal end of telson, setae only, head:down.

right: Distal end of uropod, setae, head:down.

$V$ - density of telson tissue increases.

* W - pronounced bulging of epidermis at invaginated setae.

* $\mathrm{X}$ - epidermis becomes very distinct, indication of new chitin. 


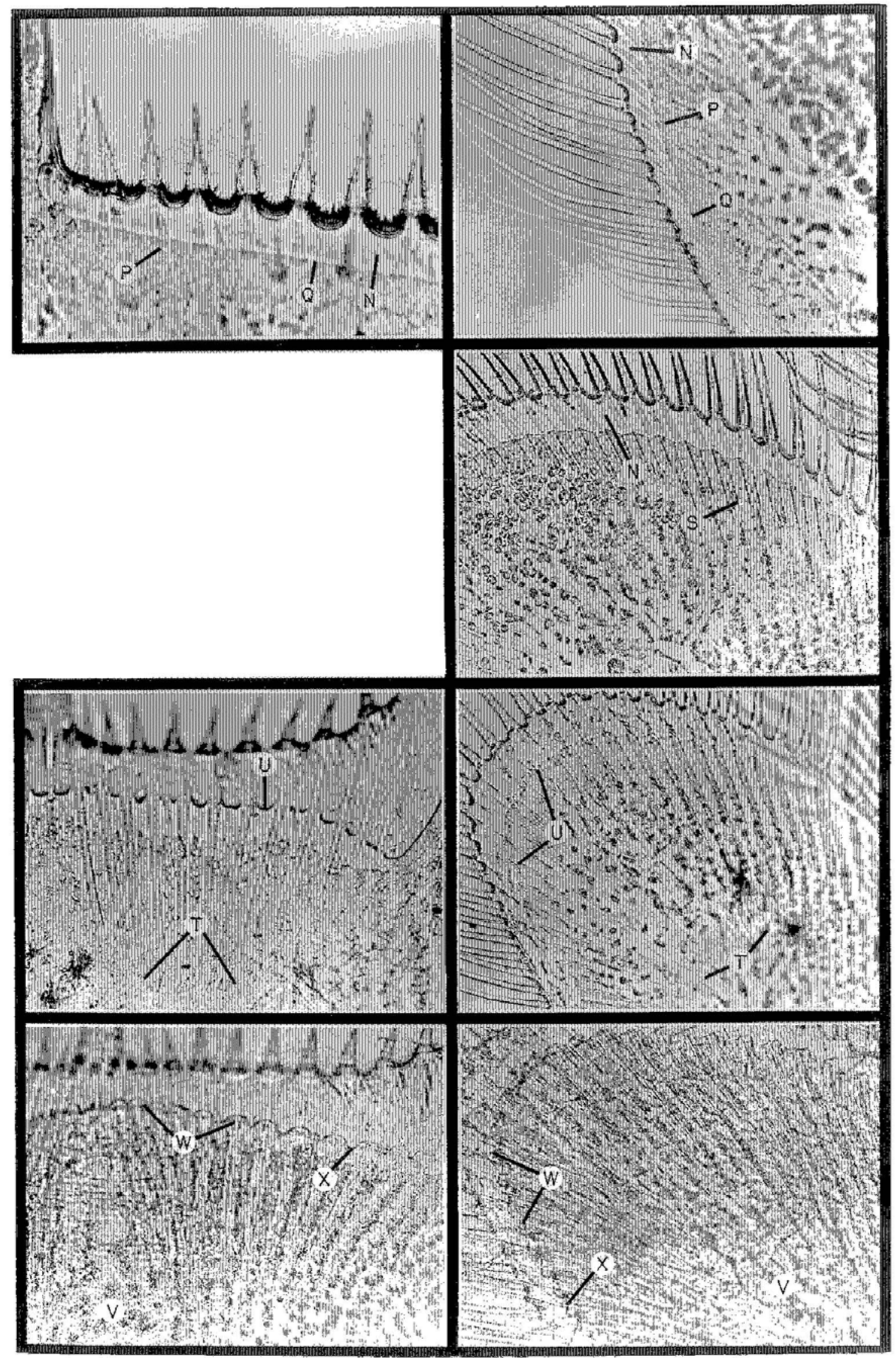


Figure 9

Fourth Stage Larval Mo1t Staging

Molt Key Description

$\underline{\text { Stage }}$

A left: Distal end of telson, setae, head:down. right: Distal end of uropod, setae, head:down.

* - animal limp.

A - telson and uropod tissue dense in appearance.

* B - setae full of granular tissue.

C - some setae may not be fully evaginated.

B left: Distal end of telson, setae, head:down.

right: Distal end of uropod, setae, head:down.

A - telson and uropod tissue still dense in appearance, but lightening up.

* E - clear tissue in setae.

C 1eft: Distal end of telson, setae, head:down. right: Distal end of uropod, setae, head:down.

* H - layer of relatively clear tissue beneath base of setae.

$\mathrm{J}$ - border of clear tissue layer.

* L - density of telson and uropod tissue decreases.

Do left: Distal end of telson, setae, head:down. right: Distal end of uropod, setae, head:down.

$\mathrm{J}$ - border of clear tissue layer.

* M - clear tissue of setae begins withdrawing from cuticle of setae.

* $\mathbf{N}$ - fluid gap between exoskeleton intersetal areas and epidermis.

$\mathbf{P}$ - border of epidermis. 


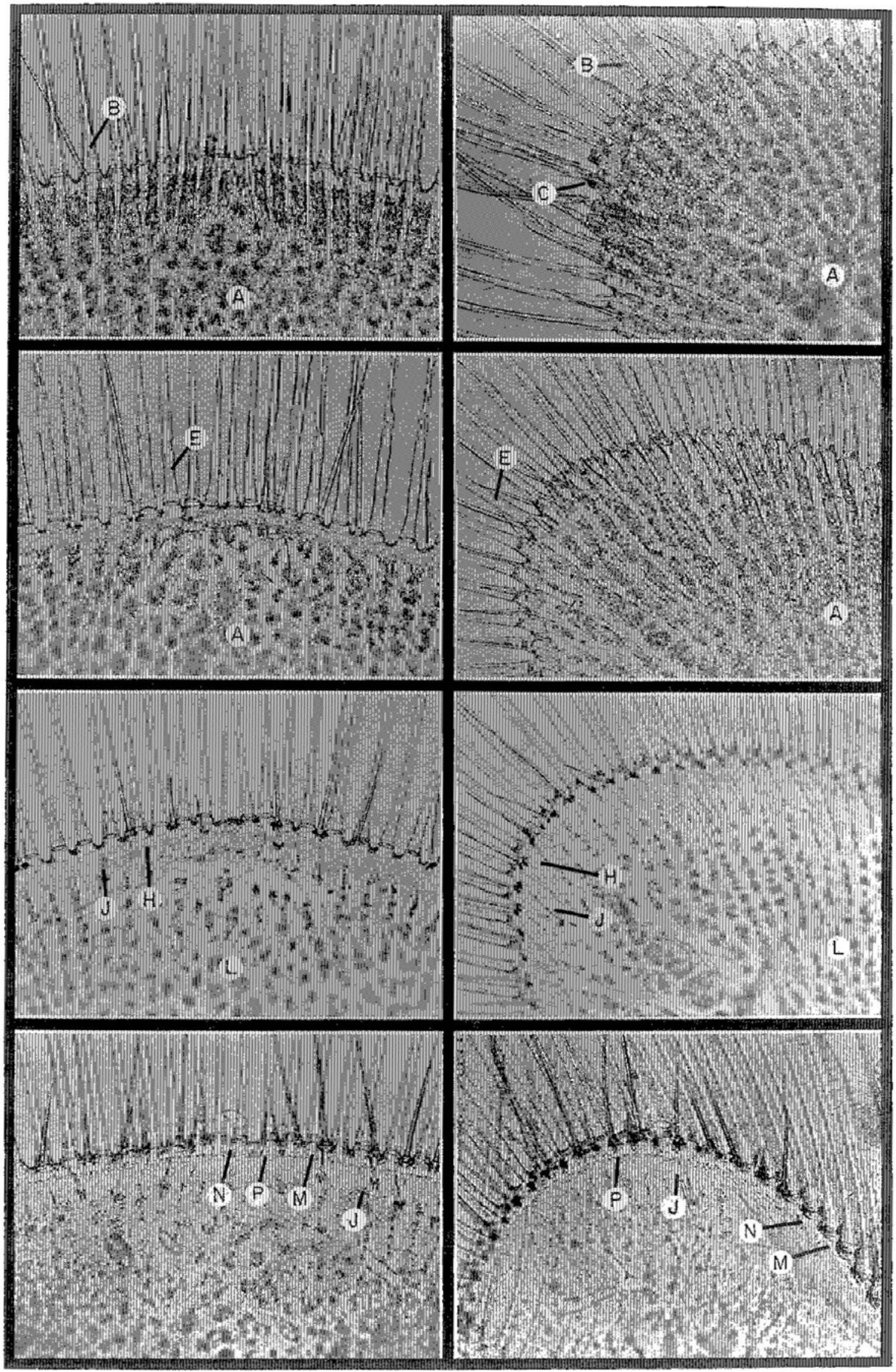




\section{Figure 10}

Fourth Stage Larva1 Mo1t Staging

Molt $\quad \underline{\text { Key }}$

Description

$\underline{\text { Stage }}$

$D_{1}$ ' left: Distal end of telson, setae, head:down. right: Distal end of uropod, setae, head:down.

$\mathrm{N}$ - fluid gap between exoskeleton and epidermis.

P - border of epidermis.

* Q - setal tissue begins invaginating.

$D_{1}$ " left: Distal end of telson, setae, head:down.

right: Distal end of uropod, setae, head:down.

N - fluid gap between exoskeleton and epidermis.

$P$ - border of epidermis.

* S - invagination of setal tissues become more pronounced.

$D_{1}$ '" left: Distal end of telson, setae only, head:down.

right: Distal end of uropod, setae, head:down.

* T - invagination of setal tissue very pronounced, attains maximum invagination.

* U - scalloped appearance of epidermis at invaginated spines.

$D_{2-3}$ left: Distal end of telson, setae only, head:down.

right: Distal end of uropod, setae, head:down.

$V$ - density of telson tissue increases.

* W - pronounced bulging of epidermis at invaginated setae.

* $\mathrm{X}$ - epidermis becomes very distinct, indication of new chitin. 


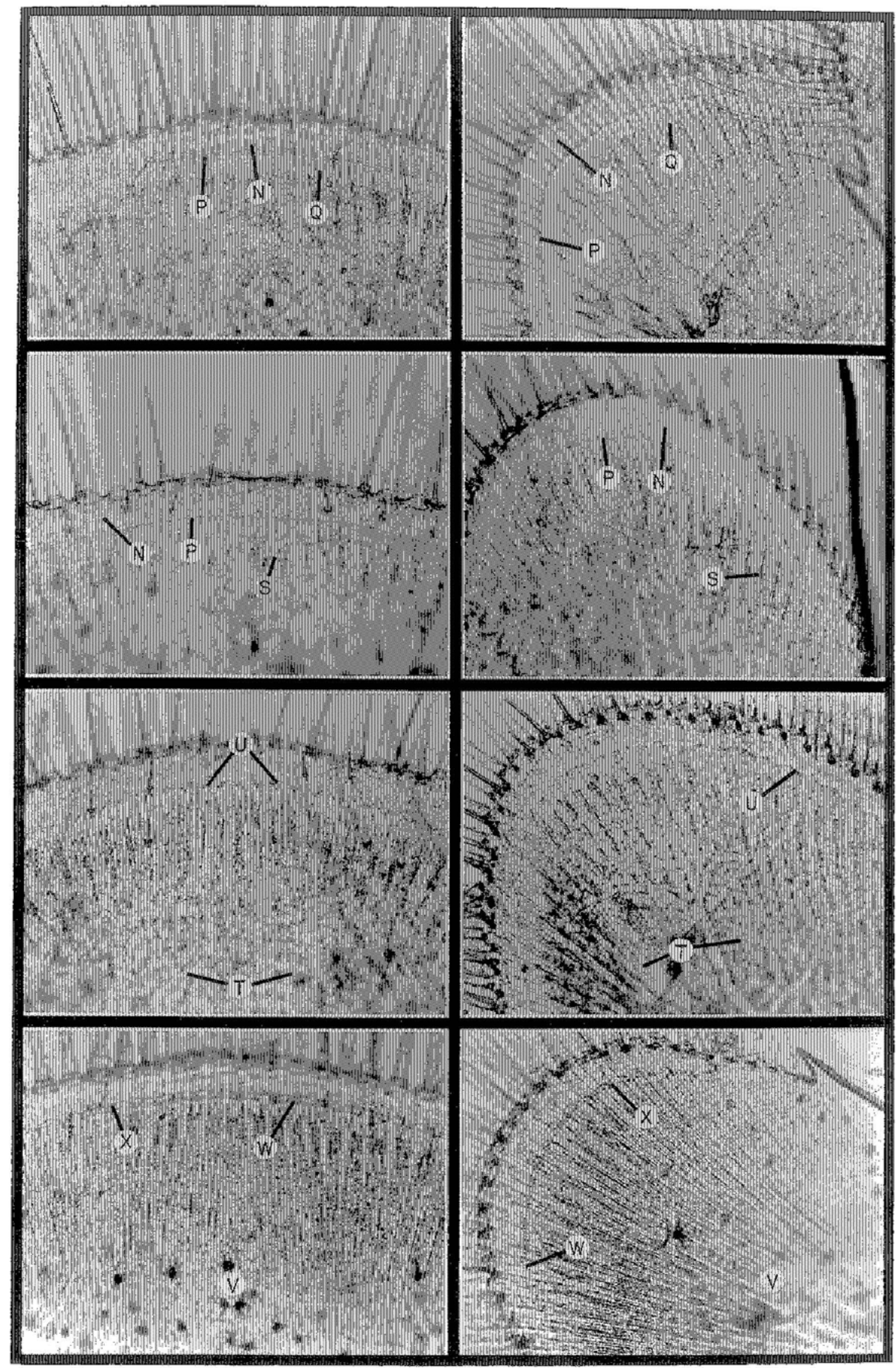




\section{APPENDIX 2 \\ Formulation of EFA Supplement}

\section{Essential Fatty Acid Supplement}

This dietary supplement was formulated to complement the Artemia diet fed to the larval lobsters.

Table 1 lists the fatty acid composition of the total lipids from representative samples of each lot of Artemia used to feed the larva1 lobsters (a two pound package of frozen Artemia, San Francisco Bay Brand,

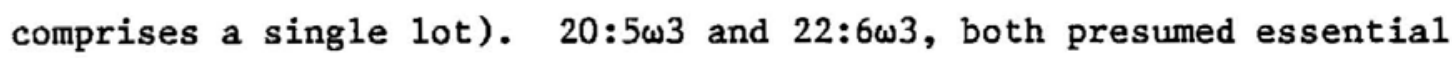
fatty acids for lobster, are lacking or present in small amounts in these Artemia. In contrast, cod liver oil contains a high percentage of these fatty acids (Ackman, 1967) and is known to promote growth in lobsters (Caste11 and Covey, 1976).

Gelatin was used as a binder since it can also serve as a nutrient source and, as a protein, effectively emulsifies and binds 1ipids. Homogenized brine shrimp was used as a taste attractant. The Artemia homogenate solution was made by rinsing off defrosted Artemia with fresh water and blending them in a Waring blender. The resulting solution is approximately $90 \%$ water.

By dry weight, the supplement contained $70 \%$ gelatin (bloom 100), $10 \%$ homogenized Artemia and $20 \%$ cod liver oil (containing $0.01 \%$ propyl gallate as an anti-oxidant.

\section{Preparation:}

1. Place $20 \mathrm{gm}$ of Artemia homogenate'into a $100 \mathrm{ml}$ beaker.

2. Thoroughly mix in $4 \mathrm{gm}$ of cod liver oil.

3. Slowly mix in $14 \mathrm{gm}$ gelatin. 
4. Dump the resulting clumpy mass onto a pre-heated, non-stick cookie sheet which is sitting on a hot plate set at $60^{\circ} \mathrm{C}$.

5. With a spatula, rapidly but thoroughly mix the melted gelatin mass and then spread it thinly and evenly over the cookie sheet.

6. Place the cookie sheet in a freezer for a few minutes and then peel off the gelatin sheet.

7. Lyophilize the gelatin sheet and crumble it into large flakes.

8. A portable coffee grinder can be used to further pulverize the feed into a flakey powder.

9. Store this feed supplement in a freezer when not in use.

\section{Contro1 Feed Supplement}

The control feed supplement, used to compare the effects of cod liver oil (CLO) was made in a similar fashion as the CLO containing feed supplement. However, as $\%$ dry weight, it contained $87.5 \%$ gelatin and 12.5\% Artemia homogenate. To make a single batch, simp1y omit the CLO.

\section{Labe1led Fatty Acid Labelled Feed}

The ${ }^{14} \mathrm{C}$ labelled feeds were based on the feed supplement formulation. However, the use of ${ }^{14} \mathrm{C}$ labelled fatty acids necessitated both a scaling down in terms of quantity and a thorough mixing of components.

A CAHN model 29 electrobalance was used to weigh out the ingredients. On a dry weight basis, $6.4 \mathrm{mg}$ of each labelled diet was make with a composition of $70 \%$ gelatin, $10 \%$ Artemia homogenate and $20 \%$ 1ipid.

The 1ipid component was composed of either:

1. $0.38 \mathrm{mg} 1-{ }^{14} \mathrm{C}$ palmitic acid $(16: 0)$, specific activity 6.7 $\mathrm{mCi} / \mathrm{mmol}$ and $0.9 \mathrm{mg}$ oleic acid.

2. $0.055 \mathrm{mg} 1-{ }^{14} \mathrm{C}$ eicosapentaenoic acid $(20: 5 \omega 3)$, specific activity $55.5 \mathrm{mCi} / \mathrm{mmol}$ and $1.3 \mathrm{mg}$ oleic acid.

or $3.1 .3 \mathrm{mg}$ oleic acid (non-labelled control). 
The oleic acid contained $0.02 \%$ propyl gallate as an anti-oxidant. The labelled fatty acids were obtained from New England Nuclear and had a tested purity of greater than $98 \%$.

Procedure (contro1: non-1abe1led feed)

1. Weigh gelatin $(4.4 \mathrm{mg})$ and set aside.

2. Tare $12 \mathrm{~mm}$ diameter aluminum pan.

3. Add oleic acid, then retare aluminum pan.

4. Add Artemia homogenate solution ( $6.4 \mathrm{mg})$.

5. Mix contents with micro-spatula.

6. Add $10 \mu 1$ distilled water, then remix contents.

7. Add gelatin, remix contents while spreading it over the pan.

8. Heat the pan to $60^{\circ} \mathrm{C}$ on a hot plate.

9. When gelatin melts, mix contents and spread over the pan.

10. Place pan in freezer for one minute.

11. Peel gelatin from the pan and spatula with micro-forceps and place it on a clean glass microscope slide.

12. Add $20 \mu 1$ distilled water.

13. Heat the glass slide to $60^{\circ} \mathrm{C}$ on a hot plate until the gelatin melts.

14. Stir the gelatin mix with the forceps and then cool the slide to room temperature.

15. Divide the feed into 33 approximately equal sized pieces with the forceps.

16. Lyophilize the feed particles.

Labelled Feed

The method to produce the labelled feed is the same except one step is added after step 3 in the above procedure.

3a. Add the labelled fatty acid (in methanol or ethanol) and evaporate the solvent with a stream of nitrogen. 
After 1yophilization, the labelled feed particles were weighed to the nearest $\mu \mathrm{g}$ and three random pieces were each dissolved in one $\mathrm{m} 1$ of water in separate scintillation vials. $10 \mathrm{ml}$ of Aquaso1 (with $7 \mathrm{~m} 1$ per liter of acetic acid) was mixed in and the vials counted on a Beckman model 100 LSC set on the ${ }^{14} \mathrm{C}$ wide channel. Highly uniform mixing occurred with this feed formulation procedure as the 'DPM/dry wt of feed' values gave a coefficient of variation of $5.25 \%$ and $3.84 \%$ for the $1-{ }^{14} \mathrm{C}$ $16: 0$ and $1-{ }^{14} \mathrm{C} 20: 5 \omega 3$ labelled feeds respectively. 


\section{APPENDIX 3}

SAMPLE ANALYSIS FLOWCHART

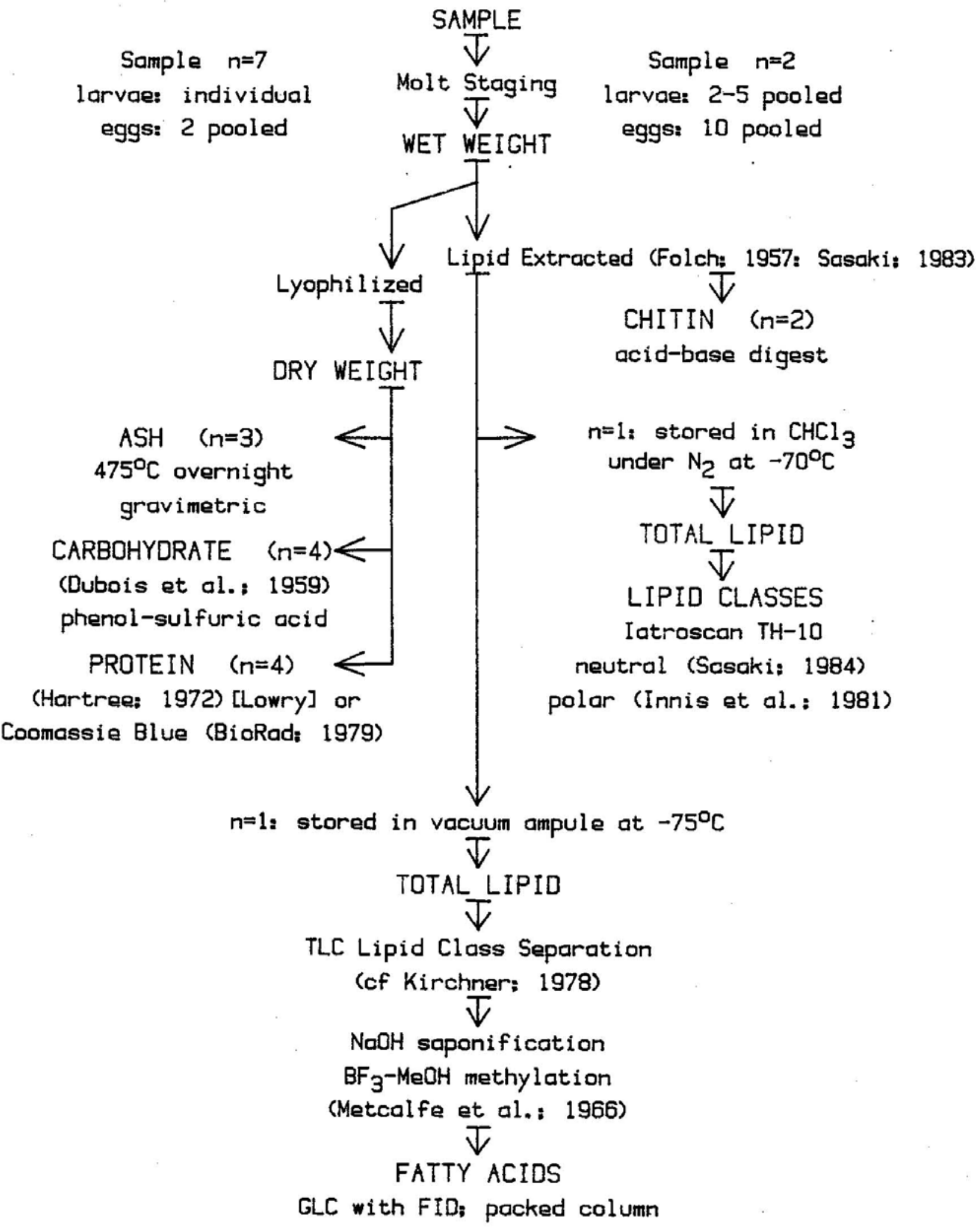




\section{$-256-$}




\section{APPENDIX 4 \\ Lipid Nomenclature: Common names and abbreviations}

Fatty Acids

$\omega$ Format:

e.g. $18: 2 \omega 6$

The first number, before the colon, indicates the number of carbons in the fatty acid.

The second number, between the colon and $\omega$, indicates the number of carbon-carbon double bonds.

The last number, after the $\omega$, indicates the position of the first double bond in carbons from the methyl end of the fatty acid. All polyenoic fatty acids in this work considered methylene interrupted between double bonds.

$\begin{array}{lll}\text { Symbol } & \text { Assumed } & \text { Common Name } \\ 14: 0 & 14: 0 & \text { myristic (acid) } \\ 14: 1 & 14: 1 \omega 5 & \text { myristoleic } \\ 15: 0 & 15: 0 & \text { pentadecanoic } \\ 15: 1 & 15: 1 \omega ? & \\ 16: 0 & 16: 0 & \text { palmitic } \\ 16: 1 & 16: 1 \omega 7 & \text { palmitoleic } \\ 16: 2 & 16: 2 \omega ? & \\ 17: 0 & 17: 0 & \text { heptadecanoic } \\ 17: 1 & 17: 1 \omega ? & \\ 18: 0 & 18: 0 & \text { stearic } \\ 18: 1 & 18: 1 \omega 9 & \text { oleic } \\ 18: 2 & 18: 2 \omega 6 & \text { 1inoleic } \\ 18: 2 \star & ? & ? \\ 18: 3 & 18: 3 \omega 3 & \text { 1inolenic } \\ 19: 0 & 19: 0 & \text { nonadecanoic } \\ 20: 0 & 20: 0 & \text { arachidic } \\ 20: 1 & 20: 1 \omega ? & \\ 20: 2 & 20: 2 \omega ? & \text { eicosadienoic } \\ 20: 3 & 20: 3 \omega ? & \text { eicosatrienoic } \\ 20: 4 & 20: 4 \omega 6 & \text { arachidonic } \\ 20: 5 & 20: 5 \omega 3 & \text { eicosapentaenoic } \\ 21: 0 & 21: 0 & \text { henicosanoic } \\ 22: 0 & 22: 0 & \text { behenic } \\ 22: 1 & 22: 1 \omega 9 & \text { erucic } \\ 22: 2 & 22: 2 \omega ? & \text { docosadienoic } \\ 22: 3 & 22: 3 \omega ? & \text { docosatrienoic } \\ 22: 4 & 22: 4 \omega ? & \text { docosatetraenoic } \\ 22: 5 & 22: 5 \omega 3 & \text { docosapentaenoic } \\ 22: 6 & 22: 6 \omega 3 & \text { docosahexaenoic } \\ 24: 1 & 24: 1 \omega 9 & \text { nervonic } \\ & & \end{array}$


APPENDIX 4 continued

\section{Lipid Classes}

Neutra1 Lipids

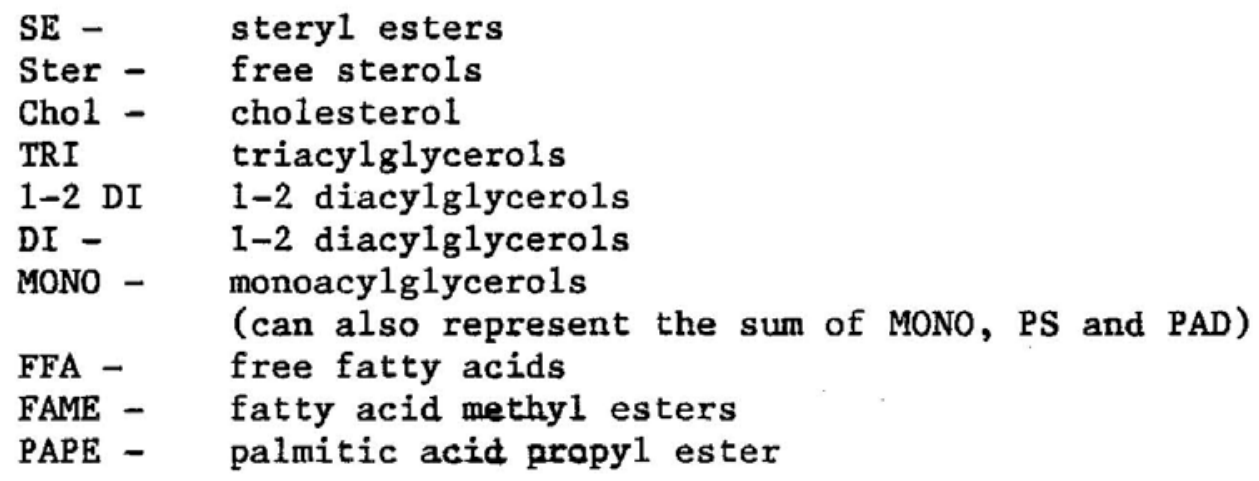

$\underline{\text { Polar Lipids }}$

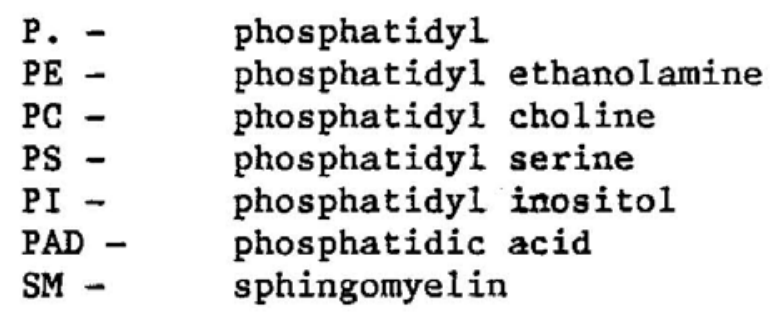

\section{Lipids: General}

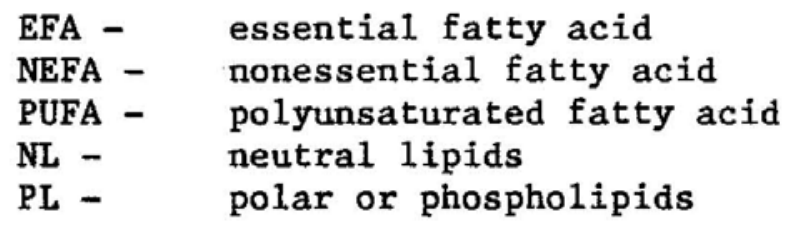


$-259-$

TABLES 
Master Key for Tables

\section{Abbreviations}

\begin{tabular}{|c|c|c|}
\hline Wt. & $=$ & weight \\
\hline S.D. & $=$ & standard deviation \\
\hline S.E. & $=$ & standard error \\
\hline UG/IND & $=$ & micrograms per individual \\
\hline MG / IND & $=$ & milligrams per individual \\
\hline & $=$ & percent \\
\hline FAME & $=$ & fatty acid methy 1 esters \\
\hline DPM & $=$ & disintegrations per minute \\
\hline EST. & $=$ & estimated \\
\hline L3 & $=$ & $\begin{array}{l}\text { refers to a specific brood of eggs, all } \\
\text { embryogenesis samples were from this one brood. }\end{array}$ \\
\hline$I-3, I-5$ & $=$ & $\begin{array}{l}\text { refers to specific hatch (third and fifth major } \\
\text { hatch of lobster (I) in } 1982 \text {. }\end{array}$ \\
\hline & & $\begin{array}{l}\text { refers to specific hatch (fourth and seventh } \\
\text { major hatch of lobster (II) in } 1981 \text {. }\end{array}$ \\
\hline
\end{tabular}

A11 lipid related abbreviations are listed in Appendix 4.

When dates and/or times are given, these represent the date and time when the sample was taken.

"Stage" column refers to the molt stage of the animals in the sample. Refer to Appendix 1 for complete explanation.

\section{Starvation experiments only:}

$\mathrm{s}-0, \mathrm{~s}-3, \mathrm{~s}-4, \mathrm{~s}-7$ (equivalent to $\mathrm{d}-0, \mathrm{~d}-3, \mathrm{~d}-4, \mathrm{~d}-7$ ) refer to the days $(0,3,4$ or 7$)$ between hatching and the initiation of starvation.

Control vs. CLO Supplementation Experiment only:

control - samples from larvae fed Artemia only

CLO - samples from larvae fed Artemia and CLO supplement, see Appendix 2.

${ }^{14} \mathrm{C}$ Labelled Fatty Acid Experiment:

$$
\text { Sample code: e.g. PS24A }
$$

$\mathrm{P} \quad={ }^{14} \mathrm{C}$ palmitic acid fed

$\mathrm{E}={ }^{14} \mathrm{C}$ eicosapentaenoic acid fed

$\mathrm{C}=$ control, non-label fed

$F=$ fed after label exposure

$\mathrm{S}=$ starved after label exposure

number $=$ hours between labe1 exposure and sampling

A or $B=$ optional, differentiates between duplicate samples 
Table 1

Fatty acids as FAME: \% of total

Artemla lots and Feed Supplement (FS)

FS w/ FS no Artemia:

\begin{tabular}{|c|c|c|c|c|c|c|c|c|c|c|c|c|c|}
\hline FAME & CLO & CLO & lot \#1 & 1ot \#2 & lot \#3 & lot \#4 & $10 t \# 5$ & 1ot \#6 & 1ot \#7 & 1 ot $\# 8$ & lot $\# 9$ & lot $\# 10$ & lot $\# 11$ \\
\hline $14: 0$ & 3.72 & 1.29 & .95 & 1.00 & 1.03 & .93 & .95 & .93 & .96 & .70 & .62 & .57 & .71 \\
\hline $\begin{array}{l}14: 1 \\
15: 0\end{array}$ & $\begin{array}{l}.62 \\
.74\end{array}$ & 2.42 & $\begin{array}{r}1.86 \\
.77\end{array}$ & $\begin{array}{r}1.55 \\
.54\end{array}$ & $\begin{array}{r}2.00 \\
.84\end{array}$ & $\begin{array}{r}1.44 \\
.51\end{array}$ & 2.09 & 2.08 & 2.12 & 2.20 & 2.03 & 1.93 & 1.59 \\
\hline $15: 1$ & .36 & .54 & .80 & .85 & .87 & .83 & .66 & .50 & .48 & .68 & .60 & .58 & .51 \\
\hline $16: 0$ & 9.77 & 16.07 & 14.16 & 15.49 & 13.52 & 14.91 & 12.47 & 13.76 & 13.50 & 11.87 & 12.02 & 12.02 & 12.22 \\
\hline $16: 1$ & 9.04 & 6.95 & 5.31 & 4.81 & 5.54 & 4.34 & 3.98 & 6.39 & 6.99 & 4.33 & 4.22 & 4.03 & 3.73 \\
\hline $17: 0$ & 1.92 & 1.06 & 1.78 & 1.01 & 1.73 & .92 & 1.36 & .85 & .93 & 1.32 & 1.33 & 1.23 & 1.25 \\
\hline $17: 1$ & .95 & .79 & 1.86 & 1.56 & 1.80 & 1.50 & .64 & .69 & .69 & .80 & .78 & .77 & .73 \\
\hline $18: 0$ & 2.89 & 6.37 & 4.82 & 5.58 & 5.19 & 5.42 & 5.42 & 5.45 & 5.39 & 5.25 & 5.09 & 5.55 & 5.68 \\
\hline $18: 1$ & 20.24 & 30.12 & 29.91 & 30.06 & 29.25 & 29.71 & 26.16 & 27.83 & 28.38 & 27.51 & 27.16 & 27.45 & 27.87 \\
\hline $18: 2$ & 2.73 & 5.25 & 8.98 & 10.14 & 8.61 & 11.72 & 5.98 & 5.27 & 5.47 & 6.05 & 6.24 & 6.22 & 6.28 \\
\hline $18: 2$ \# & .60 & 1.20 & & & & & 1.20 & 1.34 & 1.42 & 1.26 & 1.26 & 1.30 & 1.34 \\
\hline $18: 3$ & 2.28 & 18.64 & 19.03 & 17.00 & 18.44 & 17.49 & 26.62 & 23.14 & 21.65 & 26.32 & 26.88 & 26.47 & 26.00 \\
\hline $20: 0$ & 2.74 & 5.33 & 1.21 & 1.02 & 1.21 & 1.08 & 7.28 & 7.64 & 6.86 & 7.03 & 7.17 & 7.23 & 6.99 \\
\hline $20: 1$ & 11.27 & .72 & * & * & * & * & .43 & .50 & .54 & .46 & .47 & .48 & .47 \\
\hline $20: 2$ & & & 2.91 & 2.58 & 2.86 & 2.90 & & & & & & & \\
\hline $20: 3$ & & & .26 & .58 & .38 & .46 & & & & & & & \\
\hline $20: 4$ & 1.50 & .93 & 3.36 & 3.19 & 3.99 & 3.00 & 1.81 & 1.01 & 1.10 & 1.62 & 1.69 & 1.74 & 1.89 \\
\hline $20: 5$ & 16.56 & 1.23 & 1.29 & 2.03 & 1.90 & 1.71 & .54 & 1.24 & 1.86 & .41 & .36 & .41 & .55 \\
\hline $22: 0$ & 1.35 & .88 & .26 & .32 & .28 & .34 & 1.77 & 1.11 & 1.03 & 1.59 & 1.44 & 1.44 & 1.44 \\
\hline $22: 2$ & .86 & & .47 & .42 & .43 & .50 & & & & & & & \\
\hline $22: 3$ & .40 & .22 & & .27 & .12 & .29 & .66 & .27 & .61 & .61 & .64 & .56 & .76 \\
\hline $22: 5$ & 1.13 & & & & & & & & & & & & \\
\hline $22: 6$ & 8.32 & & & & & & & & & & & & \\
\hline
\end{tabular}

*Value for 18:3 contains combined values of 18:3 (predominant) and 20:1. 


\section{TABLE 2}

Embryogenesis

Egg Weights: mg per individual

Egg and Eye Dimensions: range in $\mathrm{mm}$

\begin{tabular}{|c|c|c|c|c|c|c|c|}
\hline & & & & & & GTH & WINH \\
\hline DATE & WET WT & S.D. & DRY WT. & S.D. & EGG & EYE & EYE \\
\hline & & & & & tet & & (me \\
\hline $8 / 24$ & 2.10 & .11 & 1.03 & .05 & $1.4-1.6$ & & \\
\hline $9 / 1$ & 2.08 & .05 & 1.01 & .04 & $1.4-1.5$ & & \\
\hline $9 / 7$ & 2.17 & .06 & 1.07 & .03 & $1.4-1.6$ & & \\
\hline $9 / 13$ & 2.15 & .09 & 1.01 & .04 & $1.5-1.6$ & & \\
\hline $9 / 21$ & 2.20 & .08 & 1.03 & .05 & $1.5-1.6$ & $.09-.11$ & \\
\hline $9 / 28$ & 2.31 & .08 & 1.02 & .02 & $1.5-1.6$ & $.15-.16$ & \\
\hline $10 / 5$ & 2.39 & .08 & 1.03 & .05 & & $.22-.24$ & $.13-.15$ \\
\hline $10 / 12$ & 2.37 & .04 & 1.02 & .02 & $1.5-1.6$ & $.25-.27$ & $.16-.18$ \\
\hline $10 / 19$ & 2.48 & .08 & 1.04 & .04 & $1.5-1.7$ & $.26-.33$ & $.19-.21$ \\
\hline $11 / 2$ & 2.63 & .09 & 1.04 & .03 & $1.6-1.8$ & $.36-.40$ & $.24-.28$ \\
\hline $11 / 16$ & 2.63 & .09 & .99 & .05 & $1.7-1.8$ & $.43-.50$ & $.30-.33$ \\
\hline $11 / 30$ & 2.67 & .07 & 1.00 & .04 & $1.7-1.8$ & $.43-.46$ & $.30-.31$ \\
\hline $12 / 14$ & 2.71 & .06 & 1.01 & .03 & $1.7-1.8$ & $.43-.46$ & $.30-.32$ \\
\hline $1 / 4$ & 2.60 & .03 & .97 & .02 & $1.7-1.8$ & $.40-.46$ & $.26-.30$ \\
\hline $1 / 18$ & 2.62 & .12 & .97 & .06 & $1.7-1.8$ & $.41-.48$ & $.26-.30$ \\
\hline $2 / 1$ & 2.54 & .09 & .94 & .04 & $1.7-1.8$ & $.43-.46$ & $.26-.30$ \\
\hline $2 / 16$ & 2.61 & .06 & .98 & .03 & $1.7-1.8$ & $.42-.46$ & $.30-.35$ \\
\hline $3 / 1$ & 2.61 & .02 & .96 & .03 & $1.7-1.8$ & $.43-.47$ & $.26-.30$ \\
\hline $3 / 15$ & 2.58 & .10 & .96 & .04 & $1.7-1.8$ & $.44-.47$ & $.30-.33$ \\
\hline $3 / 28$. & 2.62 & .05 & .98 & .03 & $1.7-1.8$ & $.43-.47$ & $.30-.34$ \\
\hline $4 / 12$ & 2.61 & .08 & .95 & .03 & 1. $7-1.8$ & $.43-.47$ & $.30-.34$ \\
\hline $4 / 26$ & 2.57 & .10 & .92 & .05 & $1.7-1.8$ & $.40-.46$ & $.30-.33$ \\
\hline $5 / 10$ & 2.64 & .08 & .92 & .05 & $1.7-1.8$ & $.43-.46$ & $.30-.33$ \\
\hline $5 / 17$ & 2.62 & .07 & .92 & .04 & $1.7-1.9$ & $.44-.46$ & $.30-.36$ \\
\hline $5 / 24$ & 2.60 & .13 & .93 & .05 & $1.7-1.8$ & $.44-.48$ & $.30-.33$ \\
\hline $5 / 30$ & 2.74 & .10 & .88 & .05 & $1.7-2.0$ & $.46-.53$ & $.30-.33$ \\
\hline $6 / 6$ & 3.18 & .13 & .92 & .03 & $1.7-2.0$ & $.50-.56$ & $.33-.39$ \\
\hline $6 / 13$ & 3.32 & .22 & .91 & .03 & $1.8-2.1$ & $.49-.59$ & $.36-.40$ \\
\hline
\end{tabular}


TABLE 3 (page 1 of 3)

Embryogenesis

Lipid Classes

\begin{tabular}{|c|c|c|c|c|c|c|c|c|c|c|}
\hline \multirow[b]{2}{*}{ DATE } & \multicolumn{2}{|c|}{ UG/IND. } & \multicolumn{2}{|c|}{ UG / IND. } & \multicolumn{2}{|c|}{ UG/IND. } & \multicolumn{2}{|c|}{ UG / IND } & \multicolumn{2}{|c|}{ UG / IND. } \\
\hline & LIPID & S.D. & SE & 5.D. & TRI & S.D. & FFA & S.D. & STER & S.D. \\
\hline $8 / 24$ & 341.6 & 17.3 & 0 & 0 & 161.3 & 9.94 & 0 & 0 & $10 \quad 35$ & 1.75 \\
\hline $8 / 31$ & 360.4 & 1.3 & 0 & 0 & 168.1 & 5.37 & 0 & 0 & 12.65 & 1.41 \\
\hline $9 / 13$ & 364.6 & 60.8 & 0 & 0 & 168.4 & 29.46 & 0 & 0 & 11.08 & 2.09 \\
\hline $9 / 13$ & 381.8 & 21.5 & 0 & 0 & 179.0 & 11.52 & 0 & 0 & 11.00 & 1.14 \\
\hline $9 / 21$ & 342.9 & 29.1 & 0 & 0 & 168.3 & 19.17 & 0 & 0 & 8.98 & 1.07 \\
\hline $9 / 25$ & 351.9 & 21.3 & 3.17 & .34 & 165.6 & 10.48 & 0 & 0 & 8.55 & 67 \\
\hline $10 / 5$ & 343.0 & 16.9 & 3.70 & .58 & 161.5 & 8.83 & 0 & 0 & 9.53 & 1.16 \\
\hline $10 / 12$ & 344.1 & 30.4 & 4.10 & .58 & 161.0 & 16.93 & 0 & 0 & 10.91 & 1.32 \\
\hline $10 / 19$ & 351.2 & 29.2 & 4.36 & .76 & 157.8 & 13.45 & 0 & 0 & 9.24 & .93 \\
\hline $11 / 2$ & 316.5 & 9.4 & 6.77 & .66 & 147.1 & 7.67 & 0 & 0 & 11.55 & 1.04 \\
\hline $11 / 16$ & 313.8 & 8.9 & 6.59 & 1.27 & 136.2 & 7.18 & 0 & 0 & 10.83 & 40 \\
\hline $11 / 30$ & 294.9 & .2 & 5.46 & .59 & 119.9 & 6.75 & 0 & 0 & 9.35 & 1.18 \\
\hline $12 / 14$ & 279.6 & 27.6 & 4.67 & .86 & 113.5 & 12.50 & 0 & 0 & 8.05 & 1.01 \\
\hline $1 / 4$ & 290.4 & 1.4 & 4.79 & .55 & 125.7 & 5.52 & 0 & 0 & 8.92 & .73 \\
\hline $1 / 18$ & 263.1 & 6.4 & 4.66 & .34 & 101.1 & 4.92 & 0 & 0 & 10.19 & $1: 19$ \\
\hline $2 / 1$ & 263.4 & 4.8 & 4.50 & .64 & 101.6 & 3. 46 & 0 & 0 & 8.88 & .83 \\
\hline $2 / 16$ & 277.2 & 6.9 & 5.79 & .79 & 112.7 & 10.82 & 0 & 0 & 9.40 & 1.54 \\
\hline $3 / 1$ & 275.4 & 4.9 & 4.85 & .37 & 105.2 & 6.11 & 0 & 0 & 9.69 & .50 \\
\hline $3 / 15$ & 261.0 & 27.3 & 4.31 & .49 & 91.5 & 11.08 & 0 & 0 & 8.74 & .95 \\
\hline $3 / 28$ & 245.5 & 11.7 & 4.10 & .31 & 81.3 & 4.85 & 0 & 0 & 7.41 & 1.07 \\
\hline $4 / 12$ & 246.9 & 1.5 & 4.25 & .27 & 81.4 & 2.06 & 0 & 0 & 9.09 & .37 \\
\hline $4 / 26$ & 250.1 & 19.7 & 3.88 & .45 & 89.5 & 8.66 & 0 & 0 & 10.18 & .92 \\
\hline $5 / 10$ & 224.8 & 16.1 & 4.56 & .54 & 67.8 & 5.48 & 0 & 0 & 8.70 & .70 \\
\hline $5 / 17$ & 217.6 & 2.8 & 4.61 & .42 & 59.5 & 2.58 & 0 & 0 & 10.12 & 1.57 \\
\hline $5 / 24$ & 227.3 & 14.6 & 4.41 & .56 & 79.8 & 5.82 & 0 & 0 & 7.96 & .82 \\
\hline $5 / 30$ & 222.1 & 6.9 & 4.62 & 1.30 & 71.9 & 6.69 & 0 & 0 & 7.89 & .40 \\
\hline $6 / 6$ & 182.8 & .3 & 3.25 & .20 & 44.3 & 3.57 & 3.60 & .68 & 8.03 & .95 \\
\hline $6 / 13$ & 176.9 & 5.8 & 3.86 & .34 & 39.8 & 1.73 & 3.86 & 34 & 8.10 & .63 \\
\hline $6 / 14$ & 79.4 & 4.5 & 2.18 & .57 & 1.9 & .18 & 1.29 & .08 & 7.48 & .46 \\
\hline
\end{tabular}


TABLE 3 (page 2 of 3 )

Embryogenesis

Lipid Classes

\begin{tabular}{|c|c|c|c|c|c|c|c|c|}
\hline & UGI & & UGI & & UG I & & UG & IND. \\
\hline DATE & $1-2 \mathrm{DI}$ & S.D. & MONO & S.D. & PE & S.D. & PI & S.D. \\
\hline $8 / 24$ & 4.48 & 1.42 & 0 & 0 & 36.14 & 2.39 & 0 & 0 \\
\hline $8 / 31$ & 4.54 & .58 & 0 & 0 & 39.21 & 2.24 & 0 & 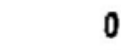 \\
\hline $9 / 13$ & 5.47 & 1.01 & 0 & 0 & 41.56 & 7.77 & 0 & $v$ \\
\hline $9 / 13$ & 5.65 & .72 & 0 & 0 & 43.33 & 3.40 & 0 & 0 \\
\hline $9 / 21$ & 3.84 & .64 & 0 & 0 & 33.84 & 3.66 & 0 & 0 \\
\hline $9 / 25$ & 4.19 & 1.33 & 0 & 0 & 36.91 & 4.44 & 0 & 0 \\
\hline $10 / 5$ & 5.28 & 1.74 & 0 & 0 & 36.80 & 2.90 & 0 & 0 \\
\hline $10 / 12$ & 6.09 & .58 & 0 & 0 & 35.37 & 3.67 & 0 & 0 \\
\hline $10 / 19$ & 6.39 & .85 & 0 & 0 & 37.61 & 4.28 & 0 & 0 \\
\hline $11 / 2$ & 3.86 & .22 & 0 & 0 & 48.68 & 3.51 & 0 & 0 \\
\hline $11 / 16$ & 2.95 & .18 & 0 & 0 & 40.01 & 2.50 & 0 & 0 \\
\hline $11 / 30$ & 3.75 & 1.30 & 0 & 0 & 43.67 & 2.42 & 0 & 0 \\
\hline $12 / 14$ & 5.51 & .70 & 0 & 0 & 32.77 & 4.29 & 0 & 0 \\
\hline $1 / 4$ & 2.61 & .15 & 0 & 0 & 36.88 & 1.12 & 0 & 0 \\
\hline $1 / 18$ & 3.40 & .59 & 0 & 0 & 36.67 & 2.19 & 0 & 0 \\
\hline $2 / 1$ & 4.66 & .64 & 0 & 0 & 33.35 & 3.74 & 0 & 0 \\
\hline $2 / 16$ & 4.91 & 2.19 & 0 & 0 & 37.17 & 2.23 & 0 & 0 \\
\hline $3 / 1$ & 6.47 & .40 & 0 & 0 & 33.46 & 1.83 & 0 & 0 \\
\hline $3 / 15$ & 5.53 & .66 & 0 & 0 & 30.77 & 3.64 & 0 & 0 \\
\hline $3 / 28$ & 4.03 & 2.24 & 0 & 0 & 24.40 & 1.75 & 0 & 0 \\
\hline $4 / 12$ & 2.84 & .17 & 0 & 0 & 29.36 & 2.97 & 0 & 0 \\
\hline $4 / 26$ & 5.13 & .68 & 0 & 0 & 33.61 & 3.32 & 0 & 0 \\
\hline $5 / 10$ & 2.61 & .45 & 0 & 0 & 22.93 & 2.03 & 0 & 0 \\
\hline $5 / 17$ & 4.20 & .27 & 0 & 0 & 23.85 & 1.98 & 0 & 0 \\
\hline $5 / 24$ & 3.57 & .45 & 0 & 0 & 25.78 & 1.83 & 0 & 0 \\
\hline $5 / 30$ & 3.04 & .41 & 0 & 0 & 21.08 & 1.67 & 0 & 0 \\
\hline $6 / 6$ & 3.02 & .84 & 0 & 0 & 21.48 & .73 & 0 & 0 \\
\hline $6 / 13$ & 3.17 & .21 & 0 & 0 & 20.77 & 1.54 & 0 & 0 \\
\hline $6 / 14$ & 1.71 & .12 & 4.24 & .24 & 17.94 & 1.18 & 6.33 & 7 \\
\hline
\end{tabular}


TABLE 3 (page 3 of 3)

Embryogenesis

Lipid Classes

\begin{tabular}{|c|c|c|c|c|c|c|}
\hline \multirow[b]{2}{*}{ DATE } & \multicolumn{2}{|c|}{ UG / IND. } & \multicolumn{2}{|c|}{ UG/ IND. } & \multicolumn{2}{|c|}{ UG / IND. } \\
\hline & PC & S.D. & SM & S.D. & UNK. & S.D. \\
\hline 124 & $120 ?$ & 1114 & 410 & 34 & 5.17 & 110 \\
\hline $8 / 31$ & 126.1 & 7.33 & 5.08 & .83 & 4.69 & .97 \\
\hline $9 / 13$ & 126.8 & 21.50 & 5.76 & 1.25 & 5.47 & 1.42 \\
\hline $9 / 13$ & 132.2 & 9.51 & 3.78 & .37 & 6.91 & .63 \\
\hline $9 / 21$ & 120.8 & 12.83 & 3.57 & .51 & 3.57 & 1.51 \\
\hline $9 / 25$ & 123.9 & 7.68 & 4.47 & .53 & 5.14 & .97 \\
\hline $10 / 5$ & 118.1 & 8.49 & 3.98 & .46 & 4.12 & 1.86 \\
\hline $10 / 12$ & 123.2 & 13.44 & 4.23 & .58 & 3.41 & .91 \\
\hline $10 / 19$ & 124.7 & 11.36 & 5.02 & .67 & 6.04 & 1.14 \\
\hline $11 / 2$ & 119.7 & 7.68 & 3.55 & 33 & 5.70 & .39 \\
\hline $11 / 16$ & 109.0 & 3.77 & 4.21 & .46 & 4.08 & .19 \\
\hline $11 / 30$ & 103.6 & 4.10 & 4.60 & .44 & 4.60 & .59 \\
\hline $12 / 14$ & 102.7 & 12.76 & 5.93 & .83 & 6.49 & 1.08 \\
\hline $1 / 4$ & 102.7 & 5.25 & 3.17 & .41 & 5.63 & .84 \\
\hline $1 / 18$ & 98.4 & 7.63 & 4.11 & .59 & 4.95 & .46 \\
\hline $2 / 1$ & 100.5 & 6.61 & 4.21 & .35 & 5.77 & .64 \\
\hline $2 / 16$ & 98.1 & 10.52 & 5.07 & .90 & 4.19 & .76 \\
\hline $3 / 1$ & 102.3 & 9.16 & 6.31 & .70 & 7.11 & .70 \\
\hline $3 / 15$ & 107.0 & 11.82 & 8.46 & 1.24 & 4.72 & 2.27 \\
\hline $3 / 28$ & 104.9 & 5.52 & 10.65 & 1.22 & 8.72 & .89 \\
\hline $4 / 12$ & 108.6 & 3.67 & 5.33 & .42 & 6.10 & .52 \\
\hline $4 / 26$ & 98.7 & 11.32 & 4.90 & .47 & 4.18 & .62 \\
\hline $5 / 10$ & 108.6 & 9.56 & 4.97 & .61 & 4.63 & .54 \\
\hline $5 / 17$ & 106.1 & 5.52 & 5.09 & .59 & 4.13 & .42 \\
\hline $5 / 24$ & 98.7 & 8.86 & 4.32 & .42 & 2.73 & .29 \\
\hline $5 / 30$ & 99.2 & 4.98 & 5.91 & .93 & 8.57 & .43 \\
\hline $6 / 6$ & 86.1 & 4.57 & 5.69 & 2.18 & 7.35 & 2.36 \\
\hline $6 / 13$ & 82.7 & 4.57 & 6.33 & .52 & 8.33 & .50 \\
\hline $6 / 14$ & 29.2 & 2.08 & 3.68 & .52 & 3.47 & .23 \\
\hline
\end{tabular}


TABLE 4

Hatching Period

Lipid Classes

\section{HATCH PERIOD}

\begin{tabular}{lcccccccrr} 
EGG & UGIIND. & \multicolumn{2}{c}{ UG/IND. } & \multicolumn{2}{c}{ UGIIND. } & \multicolumn{2}{c}{ UG/IND. } & \multicolumn{2}{c}{ UG/IND. } \\
TYPE & LIPID & SE & S.D. & TRI & S.D. & FFA & S.D. & STER & S.D. \\
\hline GOLD A & 287 & 5.91 & .68 & 121.20 & 8.01 & 3.82 & .83 & 9.01 & .54 \\
GOLD B & 254 & 4.60 & .94 & 87.96 & 6.35 & 3.91 & 1.27 & 10.67 & 1.41 \\
BLUE & 221 & 3.85 & .88 & 67.85 & 8.43 & 3.93 & .81 & 11.36 & 1.22 \\
HATCH & 215 & 3.91 & .77 & 64.37 & 1.32 & 3.16 & .26 & 11.93 & .28 \\
LARVAE & 207 & 3.95 & .29 & 59.74 & 2.10 & 3.77 & 1.06 & 11.57 & 1.03 \\
\hline
\end{tabular}

HATCH PERIOD

\begin{tabular}{lccccccrr} 
EGG & \multicolumn{2}{c}{ UG/IND. } & \multicolumn{2}{c}{ UG/IND. } & \multicolumn{2}{c}{ UGIIND. } & \multicolumn{2}{c}{ UG/IND. } \\
TYPE & $1-2$ DI & S.D. & MONO & S.D. & PE & S.D. & PI & S.D. \\
\hline GOLD A & 4.31 & .40 & 7.32 & .90 & 22.19 & 1.59 & 3.50 & .23 \\
GOLD B & 4.83 & .64 & 10.44 & 2.14 & 25.17 & 2.22 & 5.16 & 1.07 \\
BLUE & 4.97 & .52 & 8.82 & 1.46 & 21.75 & 2.60 & 4.97 & 1.17 \\
HATCH & 3.74 & .95 & 6.39 & 1.12 & 21.31 & 1.35 & 4.69 & .43 \\
LARVAE & 4.04 & .63 & 6.67 & 2.19 & 22.63 & .73 & 4.91 & .22 \\
\hline
\end{tabular}

HATCH PERIOD

\begin{tabular}{|c|c|c|c|c|c|c|}
\hline \multirow{2}{*}{$\begin{array}{l}\text { EGG } \\
\text { TYPE }\end{array}$} & \multicolumn{2}{|c|}{ UG I IND. } & \multicolumn{2}{|c|}{ UG / IND. } & \multicolumn{2}{|c|}{ UG/IND. } \\
\hline & PC & S.D. & SM & S.D. & UNK. & S.D. \\
\hline & & -0 & & & & -- \\
\hline GOLD A & 99.76 & 7.00 & 4.31 & .28 & 5.80 & .52 \\
\hline GOLD 8 & 94.59 & 6.66 & 5.38 & .76 & 5.82 & .78 \\
\hline BLUE & 82.70 & 9.17 & 5.06 & .63 & 6.03 & .76 \\
\hline НАТСН & 88.24 & 3.98 & 2.82 & .43 & 4.43 & .19 \\
\hline LARVAE & 81.74 & 3.04 & 3.68 & .10 & 4.31 & .79 \\
\hline
\end{tabular}




\section{$\underline{\text { TABLE } 5}$}

Embryogenesis

Neutral Lipid Fatty Acids as FAME, $\mu \mathrm{g}$ per Individual

\begin{tabular}{|c|c|c|c|c|c|c|c|c|c|c|c|c|}
\hline GGS & & & IEUTR & LIPID & & & & & & & & \\
\hline DATE & $14: 0$ & $14: 1$ & $15: 0$ & $15: 1$ & $16: 0$ & $16: 1$ & $17: 0$ & $\begin{array}{l}16: 2 \\
17: 1\end{array}$ & $18: 0$ & $18: 1$ & $18: 2$ & $18: 2 *$ \\
\hline & & & & --- & $--\infty$ & --- & $4---\infty$ & $=--$ & $---n$ & -----4 & $1----$ & --- \\
\hline $8 / 24$ & 2.02 & .28 & .66 & .19 & 17.87 & 10.06 & 1.04 & 1.21 & 2.15 & 28.83 & 1.55 & .71 \\
\hline 917 & 2.39 & .34 & .79 & .24 & 20.90 & 11.74 & 1.24 & 1.45 & 2.51 & 33.47 & 1.83 & .85 \\
\hline $9 / 28$ & 2.22 & .30 & .74 & .22 & 19.25 & 10.69 & 1.09 & 1.27 & 2.21 & 30.78 & 1.62 & .76 \\
\hline $10 / 19$ & 2.07 & .29 & .67 & .20 & 17.97 & 10.12 & 1.04 & 1.25 & 2.02 & 29.11 & 1.58 & .76 \\
\hline $11 / 30$ & 1.78 & .24 & .56 & .15 & 14.20 & 8.07 & .82 & .94 & 1.58 & 23.62 & 1.23 & .64 \\
\hline $1 / 18$ & 1.58 & .23 & .52 & .14 & 12.33 & 6.95 & .76 & .85 & 1.38 & 20.52 & 1.10 & .59 \\
\hline $3 / 1$ & 1.62 & .23 & .52 & .13 & 12.56 & 7.13 & .77 & .85 & 1.12 & 21.20 & 1.10 & .60 \\
\hline $4 / 12$ & 1.41 & .21 & .52 & .12 & 10.71 & 6.00 & .68 & .73 & 1.28 & 18.22 & .97 & .55 \\
\hline $5 / 10$ & 1.14 & .16 & .36 & .10 & 8.94 & 4.92 & .52 & .58 & 1.12 & 15.30 & .76 & .43 \\
\hline $5 / 24$ & 1.01 & .14 & .31 & .08 & 8.15 & 4.49 & .47 & .53 & 1.00 & 14.11 & .70 & .40 \\
\hline $5 / 30$ & 1.01 & .13 & .34 & .10 & 7.80 & 4.18 & .41 & .44 & 1.05 & 13.61 & .69 & .41 \\
\hline $6 / 6$ & .70 & .09 & .26 & .06 & 5.38 & 2.86 & .38 & .37 & .77 & 9.57 & .49 & .32 \\
\hline $6 / 13$ & .63 & .09 & .22 & .06 & 4.75 & 2.50 & .32 & 32 & .74 & 8.59 & .42 & .27 \\
\hline $6 / 14$ & .06 & 0 & .03 & 0 & .56 & .13 & .04 & .03 & .17 & .85 & .04 & .03 \\
\hline & & & & & & & & & & .05 & .07 & .05 \\
\hline
\end{tabular}

\section{EGGS 1.3}

NEUTRAL LIPIDS

* $R E-$

DATE $18: 3 \quad 20: 0 \quad 20: 1 \quad 20: 4 \quad 20: 5 \quad 22: 0 \quad 22: 2 ? \quad 22: 3 ? \quad 22: 4 ? \quad 22: 5 \quad 22: 6$ COVER

\begin{tabular}{|c|c|c|c|c|c|c|c|c|c|c|c|c|}
\hline $\begin{array}{l}8 / 24 \\
9 / 7\end{array}$ & $\begin{array}{l}.54 \\
.65\end{array}$ & $\begin{array}{l}.82 \\
.97\end{array}$ & $\begin{array}{l}4.85 \\
5.57\end{array}$ & $\begin{array}{l}2.93 \\
3.38\end{array}$ & $\begin{array}{l}23.23 \\
26.29\end{array}$ & $\begin{array}{r}0 \\
.53\end{array}$ & $\begin{array}{l}.72 \\
.83\end{array}$ & $\begin{array}{l}.34 \\
.39\end{array}$ & $\begin{array}{l}43 \\
.51\end{array}$ & $\begin{array}{l}0 \\
0\end{array}$ & $\begin{array}{l}20.40 \\
24.37\end{array}$ & $\begin{array}{l}75.58 \\
80.29\end{array}$ \\
\hline $9 / 28$ & .55 & .87 & 5. 15 & 3.05 & 23.64 & .57 & .76 & .35 & .49 & 1.51 & 20.73 & 77.83 \\
\hline $10 / 19$ & .53 & .80 & 5.05 & 2.85 & 22.28 & 0 & .70 & .32 & .45 & 0 & 21.92 & 76.89 \\
\hline $11 / 30$ & .41 & .68 & 4.30 & 2.25 & 16.97 & .50 & .60 & .29 & .40 & 1.13 & 16.27 & 75.55 \\
\hline $1 / 18$ & .36 & .59 & 3.94 & 1.90 & 13.97 & .45 & .52 & .26 & .34 & 0 & 14.79 & 78.74 \\
\hline $3 / 1$ & .35 & .60 & 4.04 & 1.96 & 14.69 & .49 & .50 & .27 & .34 & 0 & 15.49 & 73.60 \\
\hline $4 / 12$ & .30 & .53 & 3.69 & 1.70 & 12.00 & .14 & .44 & .24 & .31 & 0 & 12.73 & 83.00 \\
\hline $5 / 10$ & .24 & .41 & 3.14 & 1.37 & 9.83 & .32 & .36 & .18 & .24 & .74 & 9.55 & 77.09 \\
\hline $5 / 24$ & .21 & .36 & 3.03 & 1.26 & 8.97 & .28 & .32 & .17 & .23 & 65 & 8.78 & 66.43 \\
\hline $5 / 30$ & .22 & .41 & 2.99 & 1.28 & 8.70 & .35 & .34 & .20 & .24 & .71 & 8.24 & 66.27 \\
\hline $6 / 6$ & .16 & .29 & 2.24 & .88 & 5.54 & .26 & .27 & .15 & .17 & .50 & 5.41 & 68.16 \\
\hline $6 / 13$ & .14 & .27 & 2.07 & .80 & 4.97 & .25 & .24 & .15 & .16 & .45 & 4.63 & 66.76 \\
\hline $6 / 14$ & .02 & .03 & .16 & .11 & .40 & .04 & .04 & 0 & 0 & .05 & .19 & 44.35 \\
\hline
\end{tabular}


TABLE 6

Embryogenesis

P. Ethanolamine Fatty Acids as FAME, $\mu \mathrm{g}$ per Individual

EGGS L 3 PHOSPHATIDYL ETHANOLAMINE

$16: 2$

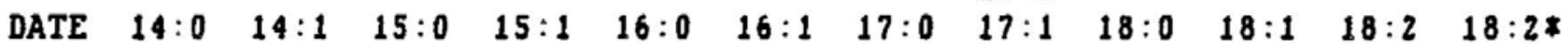

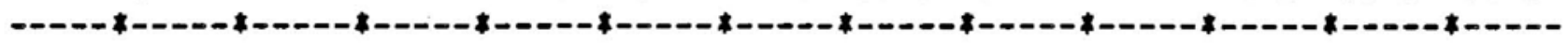

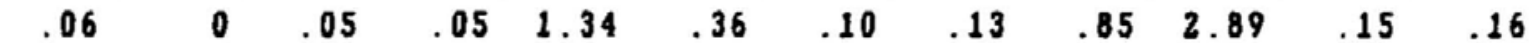

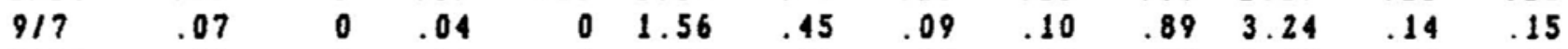

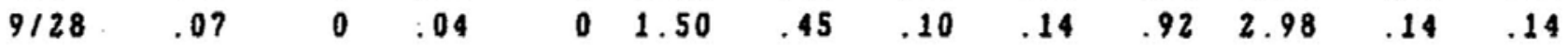

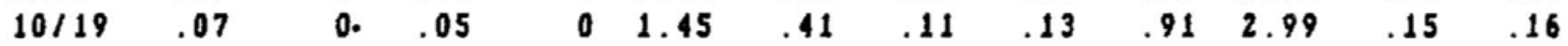

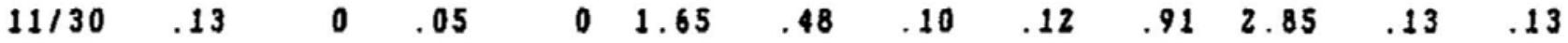

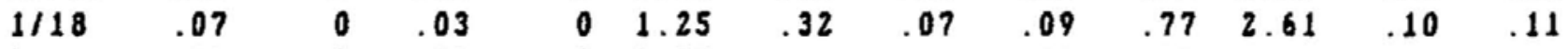

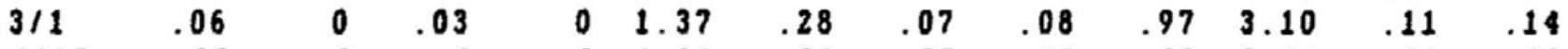

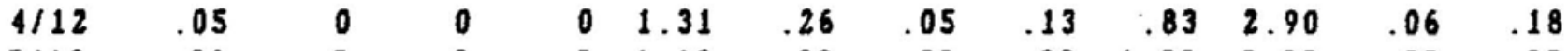

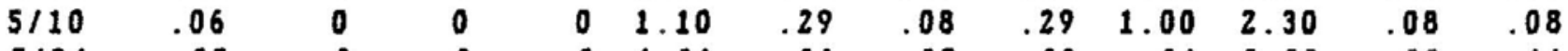

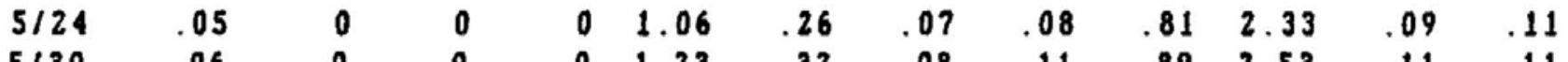

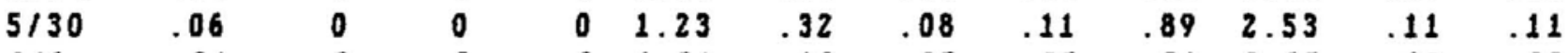

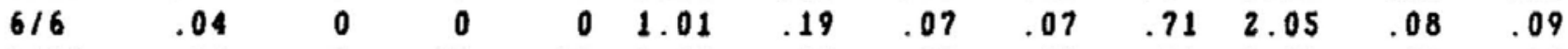

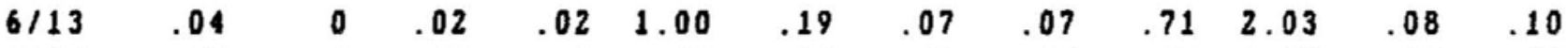

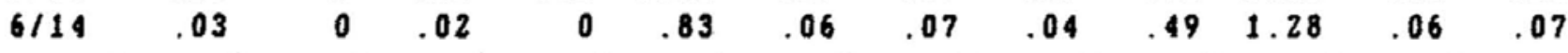

EGGS L3 PHOSPHATIDYL ETHANOLAMINE

DATE $\quad 18: 3 \quad 20: 0 \quad 20: 1 \quad 20: 4 \quad 20: 5 \quad 22: 0 \quad 22: 2 ? \quad 22: 3 ? \quad 22: 4 ? \quad 22: 5 \quad 22: 6 \quad$ COVER

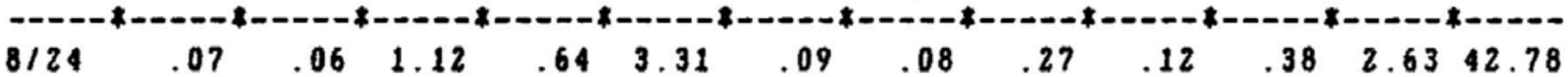

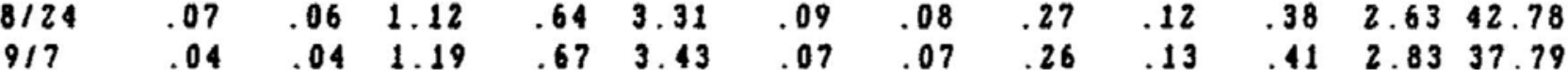

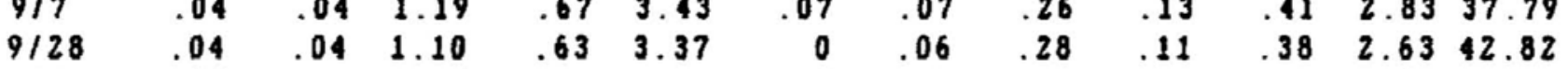

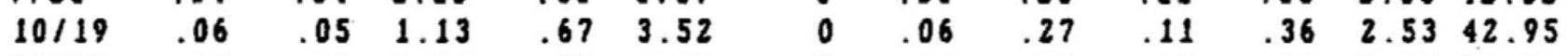

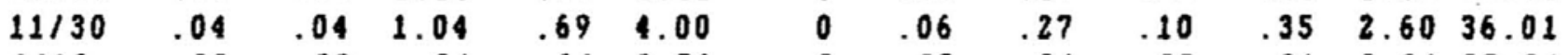

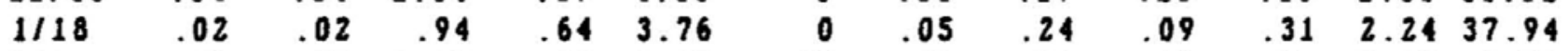

$\begin{array}{lllllllllllll}3 / 1 & .03 & .03 & 1.10 & .70 & 4.12 & .07 & .06 & .24 & .10 & .35 & 2.45 & 45.56\end{array}$

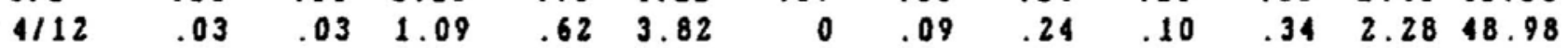

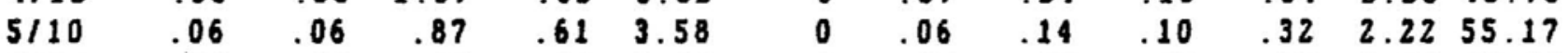

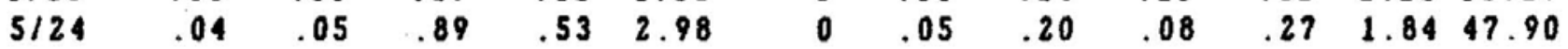

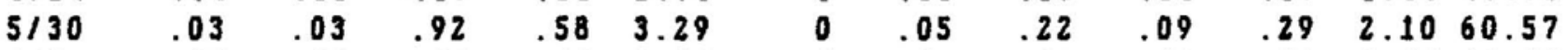

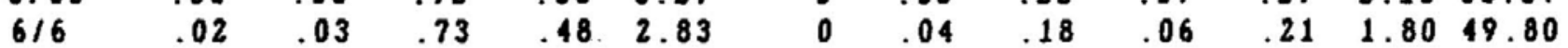

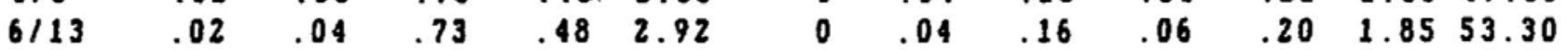

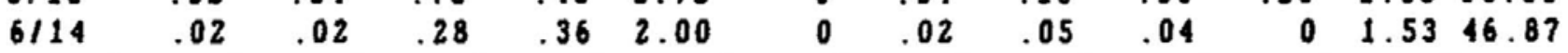

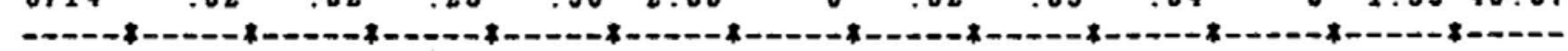


$\underline{\text { TABLE } 7}$

Embryogenesis

P. Choline Fatty Acids as FAME, $\mu \mathrm{g}$ per individual

\begin{tabular}{|c|c|c|c|c|c|c|c|c|c|c|c|c|}
\hline EGGS & & & PHOSPH & ATIDYL & CHOLIA & & & & & & & \\
\hline DATE & $14: 0$ & $14: 1$ & $15: 0$ & $15: 1$ & $16: 0$ & $16: 1$ & $17: 0$ & $17: 1$ & $18: 0$ & $18: 1$ & $18: 2$ & $18: 2 *$ \\
\hline 8124 & & & & & & & 49 & & & & & \\
\hline $\begin{array}{l}8 / 24 \\
9 / 7\end{array}$ & $\begin{array}{l}.66 \\
.72\end{array}$ & $\begin{array}{l}.10 \\
.11\end{array}$ & $\begin{array}{l}.27 \\
.30\end{array}$ & $\begin{array}{l}.09 \\
.10\end{array}$ & $\begin{array}{l}7.47 \\
7.94\end{array}$ & $\begin{array}{l}3.82 \\
3.99\end{array}$ & $\begin{array}{l}.49 \\
.55\end{array}$ & $\begin{array}{l}.62 \\
.68\end{array}$ & $\begin{array}{l}1.74 \\
1.89\end{array}$ & $\begin{array}{l}14.13 \\
14.94\end{array}$ & $\begin{array}{l}.77 \\
.84\end{array}$ & $\begin{array}{l}.41 \\
.46\end{array}$ \\
\hline $9 / 28$ & .67 & .09 & .28 & .08 & 7.66 & 3.67 & .46 & .56 & 1.80 & 14.24 & .71 & .38 \\
\hline $10 / 19$ & .66 & .10 & .29 & .09 & 7.92 & 3.56 & .51 & .62 & 1.93 & 14.39 & .76 & .4 .3 \\
\hline $11 / 30$ & .61 & .07 & .27 & .06 & 7.98 & 3.14 & .43 & .51 & 2.11 & 13.95 & .65 & .39 \\
\hline $1 / 18$ & .56 & .08 & .24 & .07 & 7.26 & 2.91 & .43 & .52 & 1.96 & 12.81 & .63 & .38 \\
\hline $3 / 1$ & .56 & .07 & .24 & .07 & 7.61 & 2.99 & .35 & .44 & 2.04 & 13.47 & .63 & .38 \\
\hline $1 / 12$ & .61 & .09 & .29 & .07 & 7.58 & 3.02 & .45 & .55 & 2.03 & 13.49 & .64 & .43 \\
\hline $5 / 10$ & .49 & .06 & .21 & .06 & 6.22 & 2.32 & .34 & .38 & 2.40 & 10.52 & .51 & .30 \\
\hline $5 / 24$ & .40 & .05 & .19 & .05 & 5.48 & 2.12 &, 30 & .35 & 1.52 & 9.86 & .46 & .29 \\
\hline $5 / 30$ & .46 & .06 & .22 & .06 & 6.19 & 2.31 & .34 & .38 & 1.75 & 10.93 & .51 & .33 \\
\hline $6 / 6$ & .34 & .05 & .17 & .04 & 5.26 & 1.78 & .28 & .31 & 1.54 & 8.93 & .12 & .28 \\
\hline $6 / 13$ & .32 & .04 & .17 & .05 & 5.19 & 1.64 & .27 & .28 & 1.59 & 8.53 & .40 & .28 \\
\hline $6 / 14$ & .09 & .01 & .08 & .01 & 3.13 & .47 & .14 & .12 & 1.03 & 3.56 & .21 & .10 \\
\hline
\end{tabular}

EGGS L3 PHOSPHATIDYL CHOLINE

* RE-

DATE $18: 3 \quad 20: 0 \quad 20: 1 \quad 20: 4 \quad 20: 5 \quad 22: 0 \quad 22: 2 ? \quad 22: 3 ? \quad 22: 4 ? \quad 22: 5 \quad 22: 6 \quad$ COVER

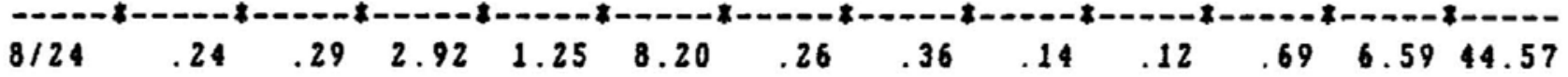

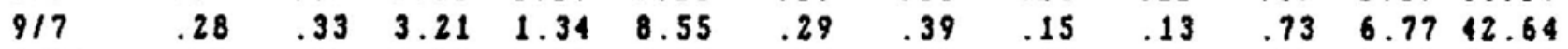

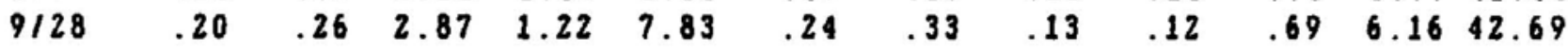

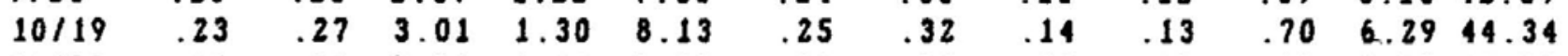

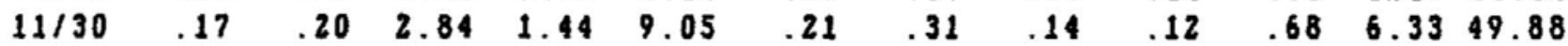

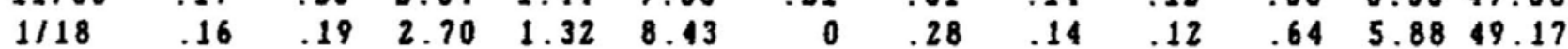

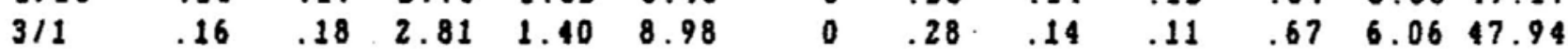

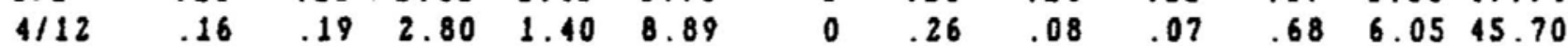

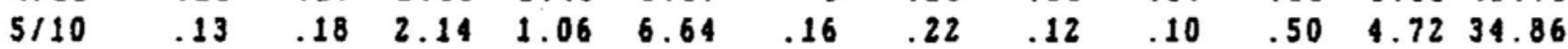

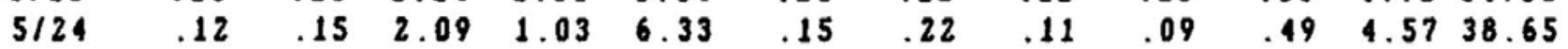

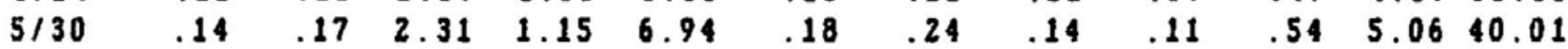

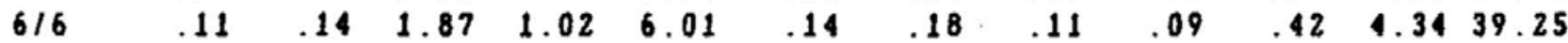

$\begin{array}{lllllllllllll}6 / 13 & .11 & .13 & 1.80 & 1.04 & 6.07 & .15 & .17 & .13 & .09 & .41 & 4.27 & 41.03\end{array}$

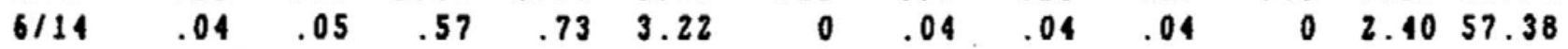


TABLE 8 (page 1 of 2)

Hatching Period

Fatty Acids as FAME, $\mu \mathrm{g}$ per individual

\section{HATCH PERIOD NEUTRAL LIPIDS}

\begin{tabular}{|c|c|c|c|c|c|c|c|c|c|c|c|c|c|}
\hline 13 & A & 14 & 27 & 66 & 20 & 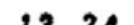 & 818 & 2 & 190 & 1 & 2909 & 192 & 2 \\
\hline $1 / 6$ & $\bar{E}$ & 1.00 & .22 & .45 & .16 & 9.19 & 5.61 & .54 & $\begin{array}{l}.79 \\
\end{array}$ & 1.01 & 15.93 & 87 & .48 \\
\hline $1 / 3$ & B & 1.02 & .20 & .49 & .14 & 9.36 & 5.50 & .49 & .87 & 1.00 & 16.98 & .79 & .60 \\
\hline $1 / 3$ & C & .75 & .12 & .36 & .11 & 7.40 & 4.25 & .38 & .73 & .80 & 13.91 & .62 & .55 \\
\hline $1 / 3$ & D & .71 & .14 & .29 & .09 & 7.43 & 4.28 & .28 & $.57^{\circ}$ & .84 & 14.52 & .72 & .43 \\
\hline
\end{tabular}

HATCH PERIOD PHOSPHATIDYL ETHANOLAMINE

\begin{tabular}{|c|c|c|c|c|c|c|c|c|c|c|c|c|c|}
\hline \multicolumn{3}{|c|}{ SAMPLE 14:0 } & $14: 1$ & $15: 0$ & $15: 1$ & $16: 0$ & \multicolumn{5}{|c|}{$16: 2$} & $18: 2$ & $18: 2 *$ \\
\hline & & - & $=-$ & $-*----$ & --- & 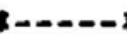 & *---- & 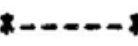 & $8-----$ & 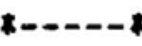 & $--\infty$ & $x---2-4$ & --- \\
\hline $1 / 3$ & A & .10 & 0 & 0 & .05 & 1.52 & .38 & .12 & .13 & .98 & 2.79 & .13 & .15 \\
\hline $1 / 6$ & E & .10 & 0 & .07 & 0 & 1.70 & .34 & .11 & .16 & .92 & 2.79 & .09 & .14 \\
\hline $1 / 3$ & B & 0 & 0 & 0 & 0 & 1.52 & .29 & .12 & .11 & .82 & 2.45 & .12 & .15 \\
\hline $1 / 3$ & C & 0 & 0 & 0 & 0 & 1.43 & .23 & .11 & .11 & .79 & 2.35 & .10 & .13 \\
\hline $1 / 3$ & D & .05 & 0 & .05 & .04 & 1.32 & .22 & .10 & .11 & .71 & 2.10 & .11 & .11 \\
\hline
\end{tabular}

\section{HATCH PERIOD PHOSPHATIDYL CHOLINE}

\begin{tabular}{llllllllllllll}
\multicolumn{1}{l}{ SAMPLE } & $14: 0$ & $14: 1$ & $15: 0$ & $15: 1$ & $16: 0$ & $16: 1$ & $17: 0$ & $17: 1$ & $18: 0$ & $18: 1$ & $18: 2$ & $18: 2 *$ \\
\hdashline $1 / 3$ & A & .42 & .08 & .28 & .08 & 7.11 & 2.67 & .43 & .58 & 2.14 & 12.29 & .61 & .42 \\
$1 / 6$ & E & .33 & .06 & .22 & .07 & 6.08 & 2.14 & .35 & .50 & 1.78 & 9.88 & .52 & .36 \\
$1 / 3$ & B & .30 & .05 & .23 & .06 & 7.65 & 2.21 & .42 & .56 & 2.33 & 11.06 & .58 & .39 \\
$1 / 3$ & C & .27 & .04 & .23 & .05 & 6.96 & 1.88 & .39 & .53 & 2.17 & 9.95 & .51 & .39 \\
$1 / 3$ & D & .24 & .05 & .21 & .06 & 5.69 & 1.50 & .35 & .42 & 1.78 & 7.88 & .44 & .30 \\
\hline
\end{tabular}


TABLE 8 (page 2 of 2)

Hatching Period

Fatty Acids as FAME, $\mu \mathrm{g}$ per Individual

HATCH PERIOD NEUTRAL LIPIDS SAMPLE $18: 3$ 20:0 $20: 1$ 20:4 $20: 5$ $22: 0$ 22:2? $22: 3 ? 22: 4 ? 22: 5$ $22: 6$ COVER

\begin{tabular}{|c|c|c|c|c|c|c|c|c|c|c|c|c|c|}
\hline $1 / 3$ & A & .37 & .55 & 3.96 & 2.50 & 14.87 & .58 & .53 & .29 & .41 & 0 & 17.45 & 67.20 \\
\hline $1 / 6$ & E & .26 & .40 & 2.78 & 1.69 & 9.66 & .45 & .35 & .22 & .29 & 0 & 11.51 & 57.93 \\
\hline $1 / 3$ & B & .22 & .32 & 2.83 & 1.33 & 7.33 & .38 & .26 & .14 & .21 & 0 & 9.37 & 67.46 \\
\hline $1 / 3$ & C & .17 & .25 & 2.40 & 1.13 & 6.27 & .33 & .31 & .17 & .22 & 0 & 8.71 & 65.54 \\
\hline $1 / 3$ & $D$ & .17 & .22 & 2.61 & 1.17 & 6.47 & .30 & .23 & .15 & .19 & 0 & 9.52 & 66.03 \\
\hline
\end{tabular}

HATCH PERIOD PHOSPHATIDYL ETHANOLAMINE

\begin{tabular}{|c|c|c|c|c|c|c|c|c|c|c|c|c|c|}
\hline \multicolumn{3}{|c|}{ SAMPLE $18: 3$} & $20: 0$ & $20: 1$ & $20: 4$ & $20: 5$ & $22: 0$ & $22: 2 ?$ & $22: 3 ?$ & $22: 4 ?$ & $22: 5$ & $22: 6$ & $\begin{array}{l}\text { \% RE- } \\
\text { COVER }\end{array}$ \\
\hline & & -- & - & - & -. & $4-----4$ & *------ & $*---$ & -.. & -- & $--\infty$ & *--- & $*=-$ \\
\hline $1 / 3$ & A & .08 & .07 & .92 & .73 & 3.39 & 0 & .07 & .17 & .10 & .27 & 2.45 & 64.20 \\
\hline $1 / 6$ & $E$ & .04 & 0 & .85 & .80 & 3.92 & 0 & .03 & .14 & .08 & 25 & 2.86 & 56.15 \\
\hline $1 / 3$ & B & .13 & .05 & .64 & .64 & 3.09 & 0 & .04 & .11 & .06 & 0 & 2.57 & 53.40 \\
\hline $1 / 3$ & C & .05 & .04 & .57 & .64 & 3.04 & 0 & .04 & .11 & .06 & 0 & 2.61 & 57.44 \\
\hline $1 / 3$ & D & .05 & .04 & .53 & .65 & 2.92 & 0 & .05 & .10 & .06 & 0 & 2.42 & 47.77 \\
\hline
\end{tabular}

HATCH PERIOD PHOSPHATIDYL CHOLINE

$\begin{array}{llllllllll}\text { SAMPLE } 18: 3 & 20: 0 & 20: 1 & 20: 4 & 20: 5 & 22: 0 & 22: 2 ? & 22: 3 ? & 22: 4 ? \quad 22: 5 \quad 22: 6 & \text { COVEP }\end{array}$

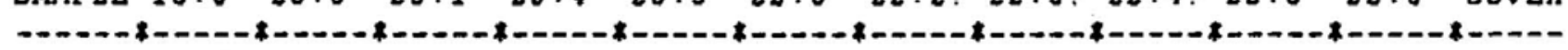

\begin{tabular}{|c|c|c|c|c|c|c|c|c|c|c|c|c|c|}
\hline $1 / 3$ & A & .17 & .19 & 2.36 & 1.66 & 7.79 & .25 & .25 & .14 & .13 & .55 & 6.37 & 45.60 \\
\hline $1 / 6$ & $\mathbf{E}$ & .13 & .13 & 1.79 & 1.32 & 6.37 & 0 & .18 & .09 & .09 & .37 & 5.19 & 36.87 \\
\hline $1 / 3$ & $B$ & .15 & .14 & 1.80 & 1.71 & 8.15 & 0 & .15 & .11 & .11 & 0 & 6.87 & 49.31 \\
\hline $1 / 3$ & C & .13 & .12 & 1.58 & 1.57 & 7.36 & 0 & 16 & .10 & 11 & 0 & 6.35 & 45.69 \\
\hline $1 / 3$ & $D$ & .13 & .15 & 1.29 & 1.35 & 6.03 & 0 & .13 & .10 & .10 & 0 & 5.30 & 37.70 \\
\hline
\end{tabular}


TABLE 9

Pulse Fed, Immediate Post Hatch Development

Proximate Analysis

PULSE FED

MGIIND. MG/IND. DRY/WET WT. MG/IND.

DATE HOUR STAGE WET WT. S.D. DRY WT. S.D. * S.D. ASH S.D.

$\begin{array}{lllllllllll}1 / 6 & .5 & 1-A & 6.24 & .39 & 1.06 & .04 & 17.02 & .73 & .14 & .02 \\ 1 / 6 & 6.5 & 1-B & 6.41 & .20 & 1.12 & .04 & 17.53 & .25 & .24 & .02 \\ 1 / 6 & 12.5 & 1-B-C & 6.25 & .21 & 1.11 & .03 & 17.71 & .43 & .24 & .04 \\ 1 / 7 & 18.5 & 1-C & 6.04 & .33 & 1.10 & .06 & 18.16 & .35 & .28 & .01 \\ 1 / 7 & 24.5 & 1-C & 6.01 & .23 & 1.08 & .04 & 17.91 & .62 & .26 & .02 \\ 1 / 7 & 30.5 & 1-C & 6.18 & .09 & 1.09 & .04 & 17.62 & .58 & .28 & .03 \\ 1 / 7 & 36.5 & 1-C & 6.16 & .28 & 1.05 & .04 & 17.05 & .55 & .26 & .01 \\ 1 / 8 & 40.5 & 1-C-D 0 & 6.24 & .24 & 1.13 & .03 & 18.10 & .97 & .28 & .02 \\ 1 / 8 & 14.5 & 1-D 0 & 6.16 & .28 & 1.08 & .05 & 17.52 & .34 & .28 & .01 \\ 1 / 8 & 48.5 & 1-D 0 & 5.90 & .22 & 1.01 & .04 & 17.08 & .36 & .26 & .01 \\ 1 / 8 & 52.5 & 1-D 0 & 6.27 & .32 & 1.09 & .05 & 17.43 & .43 & .28 & .01 \\ 1 / 8 & 56.5 & 1 D 0-1.1 & 6.02 & .14 & 1.04 & .03 & 17.32 & .35 & .27 & .01 \\ 1 / 8 & 60.5 & 1 D 1.1-2 & 6.19 & .18 & 1.07 & .03 & 17.25 & .51 & .26 & .01 \\ -\end{array}$

PULSE FED

MGIIND. MGIIND. MGIIND. MGIIND.

DATE HOUR CHITIN S.D. CARBO. S.D. PROTEIN 5.D. LIPID S.D.

\begin{tabular}{|c|c|c|c|c|c|c|c|c|c|}
\hline $1 / 6$ & .5 & .11 & .01 & .052 & .004 & .66 & .02 & .213 & .002 \\
\hline $1 / 6$ & 6.5 & .14 & .02 & .048 & .001 & .64 & .02 & .189 & .003 \\
\hline $1 / 6$ & 12.5 & .09 & .01 & .044 & .002 & .61 & .02 & .179 & .001 \\
\hline $1 / 7$ & 18.5 & .10 & .02 & .039 & .006 & .59 & .06 & .174 & .009 \\
\hline $1 / 7$ & 24.5 & .12 & .01 & .039 & .003 & .56 & .02 & .158 & .007 \\
\hline $1 / 7$ & 30.5 & .13 & .01 & .036 & .001 & .56 & .03 & .151 & .010 \\
\hline $1 / 7$ & 36.5 & .12 & .01 & .035 & .002 & .54 & .02 & .141 & .007 \\
\hline $1 / 8$ & 40.5 & .13 & .01 & .042 & .003 & .63 & .04 & .137 & .003 \\
\hline $1 / 8$ & 14.5 & .13 & .02 & .039 & .001 & .58 & .02 & .138 & .001 \\
\hline $1 / 8$ & 48.5 & .12 & .01 & .036 & .003 & .58 & .02 & .120 & .004 \\
\hline $1 / 8$ & 52.5 & .13 & .01 & .044 & .007 & .61 & .03 & .127 & .002 \\
\hline $1 / 8$ & 56.5 & .17 & 0 & .037 & .001 & .58 & .02 & .114 & .013 \\
\hline $1 / 8$ & 60.5 & .12 & .01 & .037 & .001 & .56 & .02 & .111 & .012 \\
\hline
\end{tabular}




\section{TABLE 10 (page 1 of 2)}

Pulse Fed, Immediate Post Hatch Development

\section{Lipid Classes}

\begin{tabular}{|c|c|c|c|c|c|c|c|c|c|}
\hline \multirow{2}{*}{\multicolumn{2}{|c|}{ DATE TIME }} & \multirow{2}{*}{\multicolumn{2}{|c|}{$\begin{array}{r}\text { UG / IND } \\
\text { LIPID }\end{array}$}} & \multicolumn{2}{|c|}{ UG IIND. } & \multicolumn{2}{|c|}{ UGIIND. } & \multicolumn{2}{|c|}{ UG/1ND. } \\
\hline & & & & SE & S.D. & TRI & S.D. & FFA & S.D \\
\hline & & & & & & & & & \\
\hline $1 / 6$ & 1200 & $1-A$ & 213 & 3.66 & .17 & 67.39 & 6.21 & 2.02 & .3 \\
\hline $1 / 6$ & 1800 & $1-B$ & 189 & 2.93 & .57 & 57.01 & 1.30 & 1.13 & .08 \\
\hline $1 / 6$ & 2400 & $1-B-C$ & 179 & 3.69 & .86 & 53.83 & 2.45 & 1.33 & .47 \\
\hline $1 / 7$ & 0600 & $1-0$ & 174 & 2.59 & .28 & 50.06 & 2.91 & .87 & .32 \\
\hline $1 / 7$ & 1200 & $1-C$ & 158 & 2.80 & .16 & 34.46 & 3.13 & $1.68^{\circ}$ & .17 \\
\hline $1 / 7$. & 1800 & $1-C$ & 151 & 3.68 & .76 & 29.60 & 2.59 & 1.75 & .2 \\
\hline $1 / 7$ & 2400 & $1-C$ & 141 & 2.69 & .27 & 21.67 & 1.67 & 1.20 & .14 \\
\hline $1 / 8$ & 0400 & $1-C-D O$ & 137 & 2.78 & 1.06 & 21.34 & 1.20 & 1.06 & .13 \\
\hline $1 / 8$ & 0800 & $1-D 0$ & 138 & 3.01 & .72 & 18.80 & 1.35 & 1.12 & .1 \\
\hline $1 / 8$ & 1200 & $1-D 0$ & 120 & 2.36 & .17 & 16.84 & .92 & .77 & .1 \\
\hline $1 / 8$ & 1600 & $1-D 0$ & 127 & 2.76 & .25 & 15.20 & 1.76 & 1.14 & .0 \\
\hline $1 / 8$ & 2000 & $1 D 0-1,1$ & 114 & 3.32 & .97 & 13.91 & 1.80 & 1.08 & .5 \\
\hline $1 / 8$ & 2400 & 1D1.1-2 & 111 & 2.43 & .76 & 11.43 & 1.44 & 1.01 & 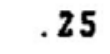 \\
\hline
\end{tabular}

PULSE FED

\begin{tabular}{|c|c|c|c|c|c|c|c|c|c|}
\hline \multirow{2}{*}{\multicolumn{2}{|c|}{ DATE TIME }} & \multicolumn{2}{|c|}{ UG / IND. } & \multicolumn{2}{|c|}{ UG I IND. } & \multicolumn{2}{|c|}{ UGIIND. } & \multicolumn{2}{|c|}{ UG/IND. } \\
\hline & & STER & S.D. & $1-2 \quad D I$ & D. & MONO & S.D. & PE & S.D. \\
\hline 6 & 1200 & 11.27 & .46 & 3.03 & .83 & 5.96 & .85 & 21.92 & 1.9 \\
\hline 0 & 1800 & 10.91 & .33 & 2.36 & .42 & 6.50 & .67 & 22.13 & 1.63 \\
\hline 10 & 2400 & 10.81 & 1.20 & 2.58 & .63 & 6.43 & 1.18 & 21.78 & 2.72 \\
\hline 17 & 0600 & 10.77 & 1.05 & 2.54 & .49 & 6.98 & .61 & 21.23 & 1.90 \\
\hline 17 & 1200 & 11.77 & .77 & 3.78 & .53 & 7.14 & .72 & 18.71 & 1.18 \\
\hline 17 & 1800 & 10.75 & 1.09 & 3.99 & .51 & 6.16 & 1.52 & 20.96 & 1.96 \\
\hline 7 & 2400 & 11.67 & .75 & 3.84 & .24 & 6.82 & .81 & 20.42 & 2.21 \\
\hline 18 & 0400 & 11.54 & .55 & 2.17 & .20 & 5.49 & 1.06 & 19.55 & 1.65 \\
\hline 8 & 0800 & 11.61 & 1.80 & 2.42 & .65 & 4.83 & 1.89 & 22.63 & 2.10 \\
\hline 18 & 1200 & 10.26 & .91 & 1.85 & .27 & 4.67 & .54 & 20.06 & .7 \\
\hline 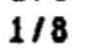 & 1600 & 11.19 & .47 & 1.60 & .24 & 4.88 & .21 & 22.83 & 1.8 \\
\hline 8 & 2000 & 10.69 & 1.31 & 1.44 & .34 & 4.10 & 1.44 & 21.67 & 2.98 \\
\hline & 2400 & 11.34 & 1.31 & 1.28 & .19 & 4.82 & 1.31 & 23.75 & 3.3 \\
\hline
\end{tabular}




\section{TABLE 10 (page 2 of 2)}

Pulse Fed, Immediate Post Hatch Development

\section{Lipid Classes}

\begin{tabular}{|c|c|c|c|c|c|c|c|c|c|}
\hline \multicolumn{2}{|c|}{ PULSE FED } & \multicolumn{2}{|c|}{ UG/IND. } & \multicolumn{2}{|c|}{ UG/IND. } & \multicolumn{2}{|c|}{ UGIIND. } & \multicolumn{2}{|c|}{ UGIIND. } \\
\hline \multicolumn{2}{|c|}{ DATE TIME } & PI & S.D. & PC & S.D. & $5 M$ & S.D. & UNK. & S.D. \\
\hline $1 / 6$ & 1200 & 4.13 & .94 & 85.37 & 5.34 & 3.03 & .55 & 5.22 & 1.02 \\
\hline $1 / 6$ & 1800 & 2.93 & .74 & 78.51 & 2.47 & 2.12 & .42 & 2.14 & .25 \\
\hline $1 / 6$ & 2400 & 2.74 & .56 & 70.74 & 2.48 & 2.43 & .39 & 2.67 & .56 \\
\hline $1 / 7$ & 0600 & 2.54 & .29 & 70.57 & 4.50 & 2.05 & .41 & 3.64 & .38 \\
\hline $1 / 7$ & 1200 & 5.12 & .34 & 67.17 & 4.39 & 2.65 & .31 & 2.73 & .1 \\
\hline $1 / 7$ & 1800 & 4.11 & .32 & 64.51 & 5.30 & 3.08 & .58 & 2.42 & .8 \\
\hline $1 / 7$ & 2400 & 4.43 & .30 & 62.27 & 3.64 & 3.06 & .40 & 2.93 & .29 \\
\hline $1 / 8$ & 0400 & 4.26 & .43 & 65.08 & 3.93 & 1.97 & .25 & 1.80 & .1 \\
\hline $1 / 8$ & 0800 & 4.13 & .42 & 64.69 & 1.99 & 2.47 & .48 & 2.31 & .6 \\
\hline $1 / 8$ & 1200 & 3.59 & .38 & 55.49 & 2.62 & 2.10 & .27 & 2.00 & .13 \\
\hline $1 / 8$ & 1600 & 3.44 & .35 & 60.29 & 4.03 & 1.89 & .24 & 1.78 & .13 \\
\hline $1 / 8$ & 2000 & 2.47 & .38 & 51.21 & 6.05 & 2.28 & .44 & 1.54 & .39 \\
\hline $1 / 8$ & 2400 & 1.77 & .26 & 49.36 & 5.86 & 2.34 & .38 & 1.45 & .35 \\
\hline
\end{tabular}


TABLE 11 (page 1 of 3)

Larva1 Development: Hatch I-3

Lipid Classes

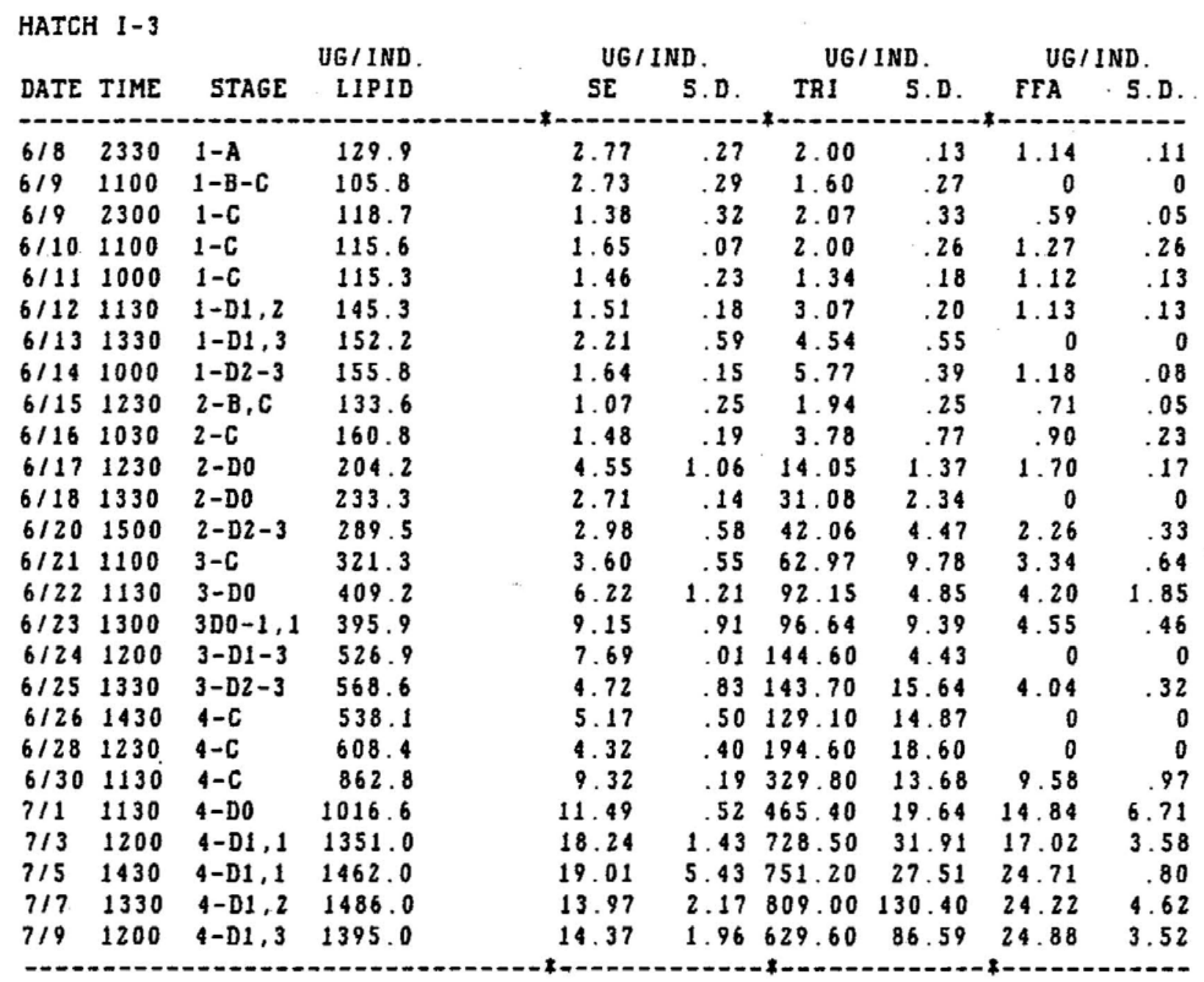


TABLE 11 (page 2 of 3)

Larva1 Development: Hatch I-3

Lipid Classes

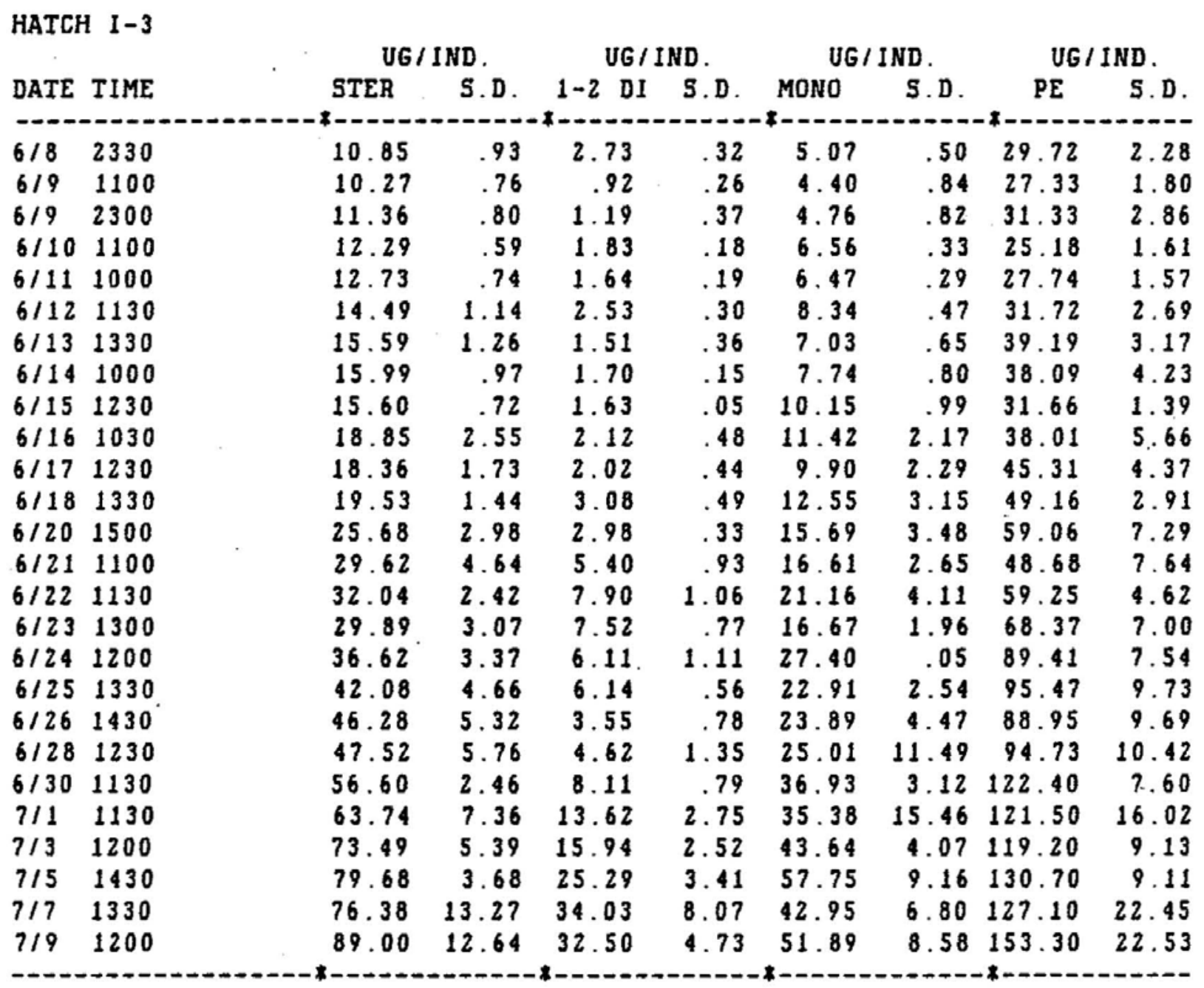


TABLE 11 (page 3 of 3 )

Larval Development: Hatch I-3

Lipid Classes

HATCH I-3

UG/IND. UG/IND.

UG/IND.

UGIIND.

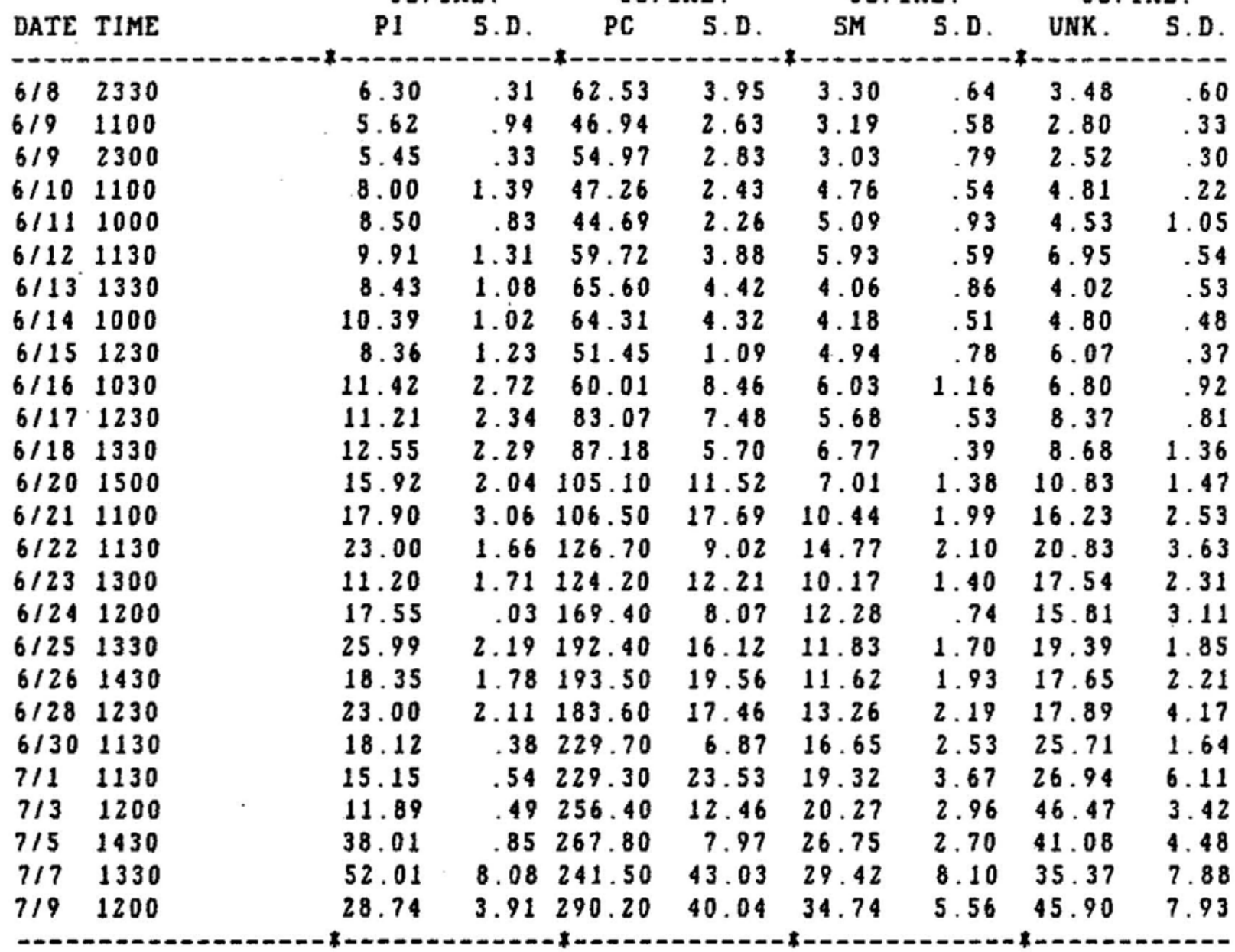


TABLE 12 (page 1 of 3 )

Larval Development: Hatch I-5

Lipid Classes

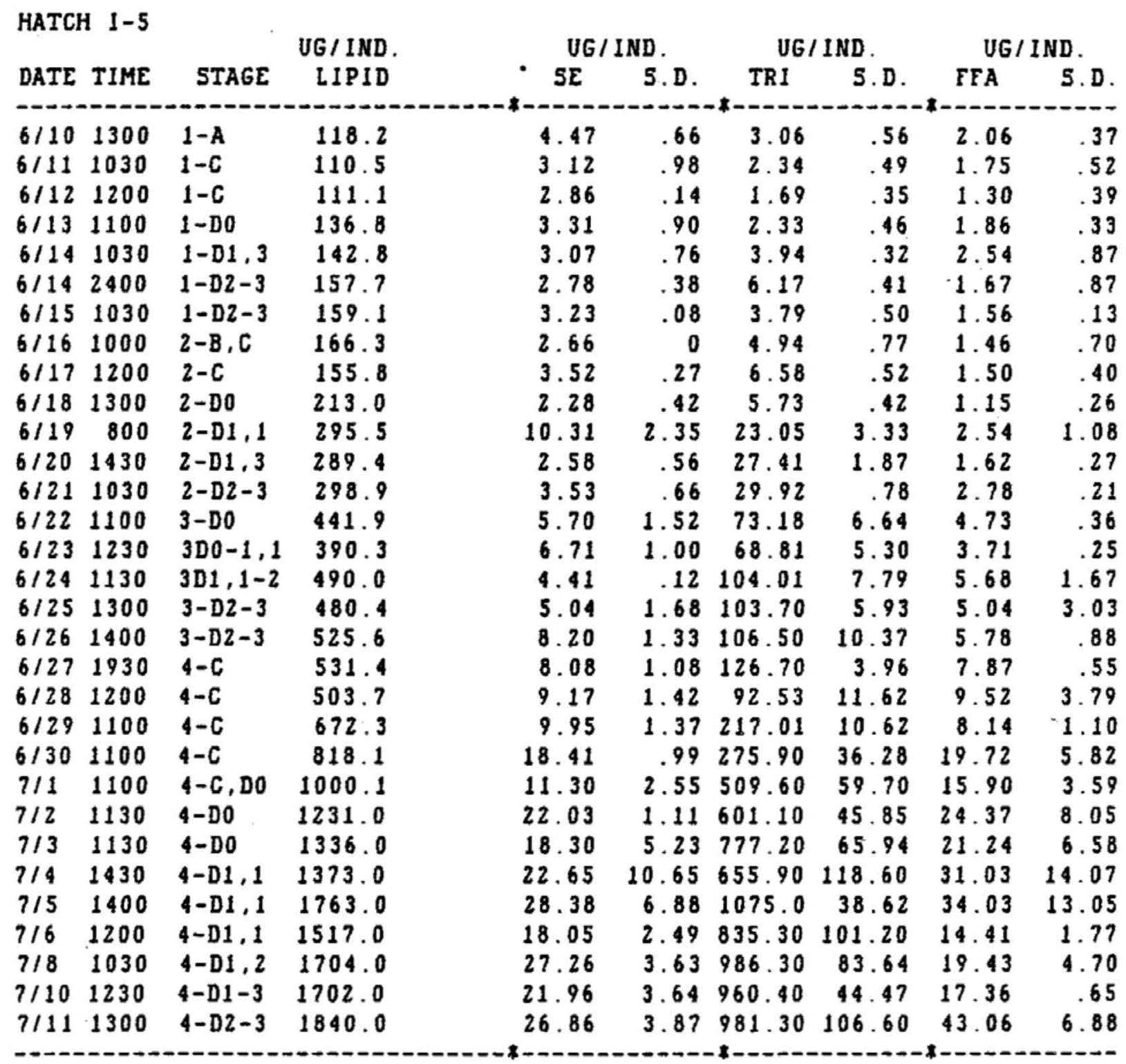


TABLE 12 (page 2 of 3)

Larval Development: Hatch I-5

Lipid Classes

HATCH I-5

UG/IND. UG/IND.

UGIIND. UG/IND.

\begin{tabular}{|c|c|c|c|c|c|c|c|c|c|}
\hline DATE & TIME & STER & S.D. & $1-2$ DI & S.D. & MONO & S.D. & PE & S.D. \\
\hline & & & & - & 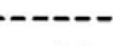 & & & & \\
\hline $6 / 10$ & 1300 & 10.17 & 1.50 & 2.90 & .63 & 5.65 & 1.05 & 23.83 & 3.7 \\
\hline $6 / 11$ & 1030 & 11.34 & 1.56 & 2.18 & .47 & 5.37 & 1.49 & 26.97 & 3. \\
\hline $6 / 12$ & 1200 & 12.34 & .56 & 1.43 & .14 & 4.89 & .67 & 28.44 & 2. \\
\hline $6 / 13$ & 1100 & 15.43 & 1.95 & 2.72 & .44 & 7.89 & 1.00 & 25.12 & 2.2 \\
\hline $6 / 14$ & 1030 & 15.57 & 1.10 & 3.18 & .42 & 8.80 & 1.02 & 28.23 & 1. \\
\hline $6 / 14$ & 2400 & 16.40 & .58 & 3.14 & .11 & 9.43 & .40 & 30.59 & 3. 1 \\
\hline $6 / 15$ & 1030 & 18.14 & .91 & 1.62 & .49 & 9.13 & .67 & 36.66 & 1. \\
\hline $6 / 16$ & 1000 & 19.52 & 1.30 & 1.89 & .45 & 8.30 & 2.10 & 42.92 & 1.7 \\
\hline $6 / 17$ & 1200 & 17.79 & 1.26 & 1.43 & .33 & 7.76 & .46 & 37.27 & 3.1 \\
\hline $6 / 18$ & 1300 & 22.96 & 1.61 & 2.28 & .36 & 12.25 & .75 & 47.31 & 2.8 \\
\hline $6 / 19$ & 800 & 26.60 & 4.55 & 4.46 & .95 & 17.17 & 2.59 & 54.61 & 8. \\
\hline $6 / 20$ & 1430 & 28.36 & 1.83 & 3.24 & 1.08 & 16.21 & 1.47 & 58.08 & 5.3 \\
\hline $6 / 21$ & 1030 & 29.50 & 1.65 & 3.65 & $8 ?$ & 13.63 & .63 & 67.79 & 1. \\
\hline $6 / 22$ & 1100 & 38.53 & 4.64 & 5.88 & 1.53 & 16.97 & 5.07 & 89.97 & 8.7 \\
\hline $6 / 23$ & 1230 & 34.70 & 2.79 & 4.45 & 1.10 & 17.13 & 1.43 & 81.77 & 9.5 \\
\hline $6 / 24$ & 1130 & 42.04 & 1.61 & 6.96 & 1.29 & 17.20 & 2.97 & 83.35 & 6.3 \\
\hline $6 / 25$ & 1300 & 42.37 & 4.78 & 7.11 & 1.35 & 17.05 & 4.18 & 88.30 & 11.4 \\
\hline $6 / 26$ & 1400 & 47.83 & 3.53 & 8.20 & .63 & 22.23 & 3.40 & 98.66 & 11.6 \\
\hline $6 / 27$ & 1930 & 52.24 & 2.71 & 5.26 & 1.07 & 16.31 & .72 & 92.68 & 7. \\
\hline $6 / 28$ & 1200 & 50.32 & 7.57 & 5.04 & 1.90 & 12.74 & 5.78 & 102.80 & 13.5 \\
\hline $6 / 29$ & 1100 & 57.82 & 4.42 & 5.78 & .82 & 18.08 & 1.68 & 107.00 & 5. \\
\hline $6 / 30$ & 1100 & 65.61 & 4.70 & 12.68 & 2.23 & 27.00 & 6.46 & 110.70 & 10.2 \\
\hline $7 / 1$ & 1100 & 60.91 & 8.80 & 15.30 & 3.05 & 26.30 & 4.54 & 101.00 & 15. \\
\hline $7 / 2$ & 1130 & 81.49 & 4.71 & 22.65 & 1.30 & 40.75 & 8.15 & 102.40 & 12. \\
\hline $7 / 3$ & 1130 & 70.94 & 7.04 & 10.15 & 2.25 & 29.26 & 4.37 & 108.30 & 10. \\
\hline $7 / 4$ & 1430 & 89.52 & 16.41 & 15.24 & 4.31 & 31.44 & 9.75 & 141.40 & 23. \\
\hline $7 / 5$ & 1400 & 86.21 & 7.06 & 14.10 & 1.24 & 31.21 & 8.46 & 123.60 & 7. \\
\hline $7 / 6$ & 1200 & 83.74 & 9.87 & 10.92 & 1.86 & 38.99 & 7.80 & 133.00 & 18.2 \\
\hline $7 / 8$ & 1030 & 92.70 & 10.19 & 13.63 & 3.58 & 31.01 & 8.22 & 149.10 & 15. \\
\hline $7 / 10$ & 1230 & 94.80 & 9.35 & 13.79 & 2.09 & 34.55 & 4.23 & 147.70 & 6.5 \\
\hline $7 / 11$ & 1300 & 114.40 & 12.07 & 25.76 & 5.94 & 53.54 & 11.47 & 147.00 & 26. \\
\hline
\end{tabular}


TABLE 12 (page 3 of 3 )

Larval Development: Hatch I-5

Lipid Classes

HATCH I-5

\begin{tabular}{|c|c|c|c|c|c|c|c|c|c|}
\hline & & UG & ID. & UG & IND. & UGI & ID. & UGI & ND. \\
\hline DATE & TIME & PI & S.D. & PC & S.D. & $5 M$ & S.D. & UNK. & S.D. \\
\hline & & & & & & & & & \\
\hline $\begin{array}{l}110 \\
111\end{array}$ & $\begin{array}{l}1300 \\
1030\end{array}$ & $\begin{array}{l}2.90 \\
2.64\end{array}$ & $\begin{array}{l}.52 \\
.45\end{array}$ & $\begin{array}{l}58.40 \\
49.17\end{array}$ & $\begin{array}{l}8.63 \\
6.61\end{array}$ & $\begin{array}{l}2.41 \\
3.24\end{array}$ & $\begin{array}{l}.63 \\
.84\end{array}$ & $\begin{array}{l}2.36 \\
2.39\end{array}$ & $\begin{array}{l}.38 \\
.60\end{array}$ \\
\hline $6 / 12$ & 1200 & 4.33 & .14 & 47.45 & 2.37 & 3.08 & .28 & 3.29 & .46 \\
\hline $6 / 13$ & 1100 & 5.98 & .77 & 61.18 & 7.62 & 4.46 & .84 & 6.55 & .8 \\
\hline $6 / 14$ & 1030 & 6.20 & .09 & 59.19 & 3.44 & 5.21 & 1.06 & 6.88 & 1.2 \\
\hline $6 / 14$ & 2400 & 7.84 & .21 & 65.87 & 1.84 & 5.30 & .51 & 8.52 & 1.25 \\
\hline $6 / 15$ & 1030 & 5.65 & .16 & 68.89 & 2.59 & 4.17 & .69 & 6.27 & 1.25 \\
\hline $6 / 16$ & 1000 & 5.07 & 0 & 69.36 & 3.14 & 4.69 & .72 & 5.46 & 1.15 \\
\hline $6 / 17$ & 1200 & 5.22 & .24 & 63.72 & 4.19 & 4.36 & .69 & 6.65. & 1.05 \\
\hline $6 / 18$ & 1300 & 9.22 & .53 & 94.53 & 6.83 & 7.69 & .85 & 7.60 & 1.08 \\
\hline $6 / 19$ & 800 & 13.74 & 1.91 & 123.40 & 18.06 & 10.43 & 1.96 & 9.16 & 1.9 \\
\hline $6 / 20$ & 1430 & 13.11 & 1.40 & 116.90 & 6.37 & 11.34 & 1.13 & 10.53 & 1.4 \\
\hline $6 / 21$ & 1030 & 9.54 & 1.53 & 124.50 & 6.52 & 6.34 & 1.41 & 7.71 & .27 \\
\hline $6 / 22$ & 1100 & 11.27 & .97 & 175.70 & 16.72 & 8.84 & 1.93 & 11.09 & 2.4 \\
\hline $6 / 23$ & 1230 & 9.37 & .62 & 143.10 & 10.95 & 8.47 & 1.62 & 12.06 & 1.3 \\
\hline 124 & 1130 & 14.26 & 3.89 & 189.50 & 7.14 & 9.11 & .63 & 13.52 & 1.0 \\
\hline $5 / 25$ & 1300 & 11.24 & .45 & 176.50 & 13.41 & 11.05 & 1.02 & 12.92 & 3.6 \\
\hline 126 & 1400 & 15.19 & 1.08 & 186.30 & 14.28 & 10.41 & 1.58 & 16.19 & 2.5 \\
\hline $6 / 27$ & 1930 & 12.97 & .36 & 187.60 & 7.92 & 9.30 & 1.45 & 12.43 & .5 \\
\hline $6 / 28$ & 1200 & 13.30 & 1.52 & 183.90 & 23.64 & 11.84 & 2.73 & 12.59 & 4.2 \\
\hline $6 / 29$ & 1100 & 19.36 & .49 & 202.00 & 12.78 & 11.23 & 2.10 & 15.93 & 4.2 \\
\hline $6 / 30$ & 1100 & 16.85 & .90 & 236.50 & 17.30 & 13.25 & 1.35 & 21.52 & 1.8 \\
\hline $7 / 1$ & 1100 & 19.90 & 2.27 & 200.10 & 26.52 & 16.50 & 4.15 & 23.30 & 5.5 \\
\hline $7 / 2$ & 1130 & 28.31 & 1.07 & 247.30 & 17.56 & 20.43 & 2.46 & 40.13 & 4.9 \\
\hline $7 / 3$ & 1130 & 25.12 & 6.26 & 245.60 & 20.43 & 10.15 & 1.51 & 19.51 & 3.7 \\
\hline $7 / 4$ & 1430 & 26.22 & 7.11 & 312.90 & 51.28 & 19.22 & 4.10 & 27.46 & 6.9 \\
\hline $7 / 5$ & 1400 & 29.44 & 4.59 & 292.00 & 9.75 & 15.87 & .71 & 32.97 & 6.3 \\
\hline $7 / 6$ & 1200 & 28.06 & 7.15 & 312.80 & 38.26 & 14.87 & 1.94 & 26.70 & 3.2 \\
\hline $7 / 8$ & & 18.40 & 1.79 & 318.10 & 28.55 & 20.96 & 4.26 & 27.26 & 5.4 \\
\hline $7 / 10$ & 1230 & 29.10 & 3.69 & 332.10 & 19.60 & 19.91 & 2.63 & 30.64 & 4.2 \\
\hline , & & 34.59 & 5.21 & 350.90 & 48.75 & 18.95 & 4.15 & 43.61 & 10.5 \\
\hline
\end{tabular}


TABLE 13

Larval Development: Hatch II-4

Lipid Classes

HATCH 11-4

\begin{tabular}{|c|c|c|c|c|c|c|c|c|c|c|c|}
\hline \multirow[b]{2}{*}{ DATE } & \multirow[b]{2}{*}{ TIME } & \multirow[b]{2}{*}{ STAGE } & \multirow{2}{*}{$\begin{array}{l}\text { UG / IND. } \\
\text { LIPID }\end{array}$} & \multicolumn{2}{|c|}{ UG / IND. } & \multicolumn{2}{|c|}{ UG / IND. } & \multicolumn{2}{|c|}{ UG/IND. } & \multicolumn{2}{|c|}{ UG/IND. } \\
\hline & & & & SE & S.D. & TRI & S.D. & STER & S.D. & $1-2$ DI & S.D. \\
\hline 1 & 1730 & $1-A-B$ & 80.8 & 0 & 0 & 0 & 0 & 12.59 & .76 & 2.85 & 2.06 \\
\hline $6 / 1$ & 2300 & $1-B-C$ & 81.2 & 3.70 & 10 & 0 & 0 & 11.99 & .80 & 2.94 & .64 \\
\hline $6 / 2$ & 900 & $1-C$ & 90.4 & 2.73 & .13 & 1.76 & .18 & 12.60 & .42 & 2.11 & .07 \\
\hline $6 / 2$ & 1700 & $1-C$ & 86.2 & 0 & 0 & 2.23 & .47 & 12.70 & 1.06 & 0 & 0 \\
\hline $6 / 2$ & 2300 & $1-C$ & 91.2 & 0 & 0 & 3.25 & .81 & 12.97 & .87 & 2.40 & .75 \\
\hline $6 / 3$ & 900 & $1-D 0$ & 87.4 & 0 & 0 & 1.09 & .05 & 14.06 & .96 & 0 & 0 \\
\hline $6 / 3$ & 2130 & $1-D 0$ & 100.0 & 0 & 0 & 4.44 & .70 & 14.87 & .78 & 2.58 & 1.38 \\
\hline $6 / 4$ & 930 & $1-D 1,1$ & 88.0 & 0 & 0 & 3.06 & .83 & 12.27 & .61 & 0 & 0 \\
\hline $6 / 4$ & 2130 & $1-D 1,3$ & 99.2 & 4.17 & .73 & 9.72 & 1.89 & 12.65 & .50 & 0 & 0 \\
\hline $6 / 5$ & 900 & $1-D 2-3$ & 92.8 & 2.89 & .10 & 6.01 & .78 & 12.06 & 55 & 0 & 0 \\
\hline $6 / 5$ & 2105 & $2-A$ & 108.4 & 0 & 0 & 10.17 & .50 & 14.85 & .70 & 3.17 & .14 \\
\hline $6 / 6$ & 9.00 & $2-B$ & 107.2 & 0 & 0 & 10.37 & 2.07 & 13.86 & .86 & 0 & 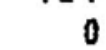 \\
\hline $6 / 7$ & 900 & $2-c$ & 132.8 & 0 & 0 & 17.53 & .83 & 15.78 & .53 & 0 & 0 \\
\hline $6 / 8$ & 900 & $2-00$ & 149.2 & 4.01 & .02 & 17.71 & .53 & 18.72 & .71 & 3.69 & .76 \\
\hline $6 / 9$ & 1000 & $2-D 1,3$ & 145.0 & 0 & 0 & 15.95 & 2.70 & 20.24 & 2.60 & 3.89 & .20 \\
\hline $6 / 10$ & 900 & $2-D 3$ & 209.0 & 0 & 0 & 60.46 & 2.52 & 19.92 & 1.47 & 5.23 & .92 \\
\hline $6 / 1.1$ & 930 & $3-c$ & 228.6 & 0 & 0 & 77.22 & 6.69 & 21.47 & 1.46 & 5.51 & .22 \\
\hline $6 / 13$ & 900 & $3-D O$ & 356.0 & 0 & 0 & 138.50 & 3.42 & 25.31 & 1.82 & 3.95 & .68 \\
\hline $6 / 15$ & 1000 & $3-D 1,3$ & 524.3 & 0 & 0 & 227.40 & 6.06 & 31.77 & 3.68 & 5.51 & .17 \\
\hline $6 / 16$ & 900 & $3-D 3$ & 604.3 & 0 & 0 & 283.10 & 11.16 & 33.54 & 1.27 & 0 & 0 \\
\hline
\end{tabular}

HATCH II-4

UGIIND. UGIIND. UGIIND. UGIIND. UG/IND.

\begin{tabular}{|c|c|c|c|c|c|c|c|c|c|c|c|}
\hline ATE & TIME & MONO & S.D. & PE & S:D. & PC & S.D. & SM & S.D. & UNK. & S.D. \\
\hline & & & & & & & & & & & \\
\hline 1 & $\begin{array}{l}1730 \\
2300\end{array}$ & 2.12 & .58 & 21.53 & 1.24 & $\begin{array}{l}33.95 \\
37.70\end{array}$ & 1.19 & $\begin{array}{l}2.80 \\
3.58\end{array}$ & $\begin{array}{l}49 \\
50\end{array}$ & 4.97 & 6 \\
\hline & 2300 & 1.92 & .43 & 18 & .00 & .79 & 1.13 & 3.58 & $3 y$ & 16 & .5 \\
\hline 12 & 900 & 2.31 & .35 & 27.17 & 2.32 & 35.43 & 1.69 & 2.41 & .10 & 3.87 & .56 \\
\hline 2 & 1700 & 2.58 & .16 & 25.49 & 4.34 & 36.76 & 2.73 & 2.18 & .32 & 4.27 & .60 \\
\hline 12 & 2300 & 2.20 & .46 & 24.64 & 1.90 & 38.71 & 1.43 & 2.79 & .90 & 4.24 & .96 \\
\hline 3 & 900 & 3.03 & .39 & 25.52 & .98 & 36.27 & 1.72 & 3.22 & .48 & 4.21 & .18 \\
\hline 13 & 2130 & 2.25 & .45 & 25.67 & 2.60 & 42.46 & 2.30 & 2.96 & 1.38 & 4.77 & 1.71 \\
\hline 4 & 930 & 4.09 & .78 & 29.18 & 2.31 & 33.01 & 1.25 & 2.61 & .22 & 3.78 & .24 \\
\hline 14 & 2130 & 3.88 & .50 & 27.64 & 1.24 & 35.21 & 1.99 & 2.59 & .39 & 3.35 & .15 \\
\hline 5 & 900 & 2.98 & .85 & 28.51 & 2.02 & 34.29 & 2.53 & 2.33 & .75 & 3.73 & 31 \\
\hline 15 & 2105 & 3.48 & .33 & 29.02 & 1.76 & 41.25 & 1.30 & 2.47 & .53 & 4.00 & 1.16 \\
\hline 16 & 900 & 3.58 & 2.17 & 35.10 & 1.79 & 38.55 & 1.76 & 2.87 & .47 & 2.87 & .68 \\
\hline $6 / 7$ & 900 & 3.98 & 1.51 & 43.77 & 1.89 & 46.57 & 3.22 & 2.09 & .29 & 3.08 & .25 \\
\hline 8 & 900 & 5.91 & .94 & 36.54 & 3.24 & 50.77 & 2.52 & 4.49 & .25 & 7.36 & .30 \\
\hline $6 / 9$ & 1000 & 5.80 & .83 & 37.63 & 5.37 & 54.10 & 4.12 & 2.93 & .84 & 4.48 & 1.16 \\
\hline $6 / 10$ & 900 & 3.74 & .92 & 45.06 & 1.79 & 62.72 & 2.72 & 4.39 & 1.21 & 7.48 & 1.57 \\
\hline $6 / 11$ & 930 & 5.99 & 2.15 & 49.29 & 4.88 & 62.93 & 5.06 & 2.70 & .32 & 3.48 & 1.35 \\
\hline $6 / 13$ & 900 & 7.44 & 1.46 & 73.23 & 4.98 & 91.95 & 3.06 & 4.91 & .22 & 10.64 & .90 \\
\hline $6 / 15$ & 1000 & 11.64 & 1.42 & 98.94 & 2.71 & 125.00 & 4.38 & 8.18 & .48 & 15.89 & 1.74 \\
\hline 16 & 900 & 16.26 & 3.09 & 101.60 & 11.67 & 146.60 & 6.29 & 6.77 & 2.06 & 16.44 & 3.75 \\
\hline
\end{tabular}


TABLE 14

Larval Development: Hatch II-7

Lipid Classes

HATCH $11-7$

UGIIND UGIIND. UG/IND.

UG/IND.

UG/IND.

DATE TIME STAGE LIPID TRI S.D. STER S.D. 1-2 DI S.D. MONO S.D.

\begin{tabular}{|c|c|c|c|c|c|c|c|c|c|c|c|}
\hline $6 / 3$ & 1030 & $1-A$ & 94.2 & 0 & 0 & 12.49 & .96 & 0 & 0 & 1.56 & .48 \\
\hline 14 & 1100 & $1-C$ & 78.8 & .91 & .09 & 12.77 & .70 & 0 & 0 & 1.83 & .21 \\
\hline $6 / 5$ & 930 & $1-D 0$ & 88.6 & 0 & 0 & 13.25 & .81 & 0 & 0 & 2.93 & .94 \\
\hline 16 & 930 & $1-D 1,2$ & 103.0 & 6.11. & .41 & 12.92 & .51 & 3.25 & .94 & 3.76 & .21 \\
\hline $6 / 7$ & 1000 & $2-A-B$ & 124.0 & 6.76 & .50 & 15.54 & .81 & 4.18 & 30 & 5.03 & .43 \\
\hline 18 & 930 & $2-C$ & 120.8 & 6.32 & .82 & 15.98 & .89 & a & 0 & 3.23 & .87 \\
\hline $6 / 9$ & 1030 & $2-C$ & 132.0 & 17.45 & 1.03 & 15.59 & 1.66 & 0 & 0 & 4.59 & 2.03 \\
\hline $6 / 10$ & 1000 & $2 D 0-1,1$ & 171.2 & 24.60 & 3.05 & 17.19 & 1.90 & 0 & 0 & 4.28 & 1.39 \\
\hline $6 / 11$ & 1030 & $2-D 2-3$ & 247.4 & 63.85 & 5.13 & 19.40 & .79 & 0 & 0 & 8.83 & 2.80 \\
\hline $6 / 12$ & 930 & $3-C$ & 296.0 & 86.49 & 6.34 & 24.66 & 2.15 & 0 & 0 & 9.65 & 2.43 \\
\hline $6 / 13$ & 1000 & $3-C$ & 285.3 & 73.89 & 2.44 & 29.33 & 1.20 & 8.70 & .83 & 9.96 & 1.51 \\
\hline $6 / 14$ & 930 & $3-D 0$ & 409.7 & 142.70 & 6.36 & 34.13 & 2.22 & 10.69 & 1.69 & 9.34 & .95 \\
\hline $6 / 15$ & 1100 & $3 D 1,1-2$ & 478.3 & 176.00 & 7.57 & 34.25 & 4.94 & 11.67 & 1.84 & 11.96 & 2.17 \\
\hline $6 / 17$ & 1130 & $3-D 3$ & 543.3 & 230.50 & 14.78 & 37.49 & 1.36 & 0 & 0 & 5.92 & 2.12 \\
\hline $6 / 18$ & 900 & $4-B$ & 538.0 & 245.20 & 10.99 & 39.97 & 1.91 & 0 & 0 & 10.28 & .54 \\
\hline 121 & 1030 & $4-C$ & 595.0 & 286.10 & 16.91 & 41.89 & 3.01 & 0 & 0 & 7.20 & 1.20 \\
\hline
\end{tabular}

HATCH II-7

\begin{tabular}{|c|c|c|c|c|c|c|c|c|c|}
\hline \multirow[b]{2}{*}{ DATE } & \multirow[b]{2}{*}{ TIME } & \multicolumn{2}{|c|}{ UG/IND. } & \multicolumn{2}{|c|}{ UG / IND. } & \multicolumn{2}{|c|}{ UG/IND. } & \multicolumn{2}{|c|}{ UG/IND. } \\
\hline & & PE & S.D. & PC & S.D. & $5 M$ & S.D. & UNK. & S.D. \\
\hline & & $8----$ & & & & & --- & & ----1 \\
\hline $6 / 3$ & 1030 & 29.08 & .95 & 46.84 & 1.41 & 1.74 & .83 & 2.48 & .74 \\
\hline $6 / 4$ & 1100 & 28.08 & 2.06 & 31.61 & 2.31 & 1.83 & .21 & 1.78 & .52 \\
\hline $6 / 5$ & 930 & 33.58 & 3.19 & 35.05 & 3.12 & 1.74 & .20 & 2.05 & .59 \\
\hline $6 / 6$ & 930 & 27.01 & 1.58 & 41.76 & 1.86 & 3.52 & .29 & 4.70 & .35 \\
\hline $6 / 7$ & 1000 & 32.55 & 1.38 & 50.00 & 2.58 & 4.24 & .88 & 5.72 & 1.14 \\
\hline $6 / 8$ & 930 & 39.08 & 3.75 & 47.21 & 2.89 & 3.46 & .19 & 5.52 & .44 \\
\hline $6 / 9$ & 1030 & 41.34 & 3.19 & 46.25 & 5.14 & 2.96 & .41 & 3.80 & 1.13 \\
\hline $6 / 10$ & 1000 & 55.55 & 12.88 & 59.56 & 8.36 & 4.08 & .39 & 5.96 & .57 \\
\hline $6 / 11$ & 1030 & 60.02 & 6.58 & 81.49 & 2.91 & 4.65 & 37 & 9.18 & 87 \\
\hline $6 / 12$ & 930 & 75.48 & 5.24 & 88.00 & 7.24 & 4.26 & 1.40 & 7.52 & 2.30 \\
\hline $6 / 13$ & 1000 & 60.03 & 3.80 & 84.79 & 4.52 & 7.10 & 1.06 & 11.47 & .94 \\
\hline $6 / 14$ & 930 & 72.84 & 8.62 & 115.60 & 9.95 & 11.59 & 6.68 & 12.74 & 3.00 \\
\hline $6 / 15$ & 1100 & 94.23 & 4.35 & 124.90 & 4.59 & 9.38 & 3.21 & 15.88 & 1.71 \\
\hline $6 / 17$ & 1130 & 107.40 & 11.19 & 141.00 & 7.50 & 6.36 & 3.80 & 14.61 & 4.89 \\
\hline $6 / 18$ & 900 & 89.31 & 8.21 & 128.00 & 6.59 & 9.58 & 3.61 & 15.66 & 3.61 \\
\hline $6 / 21$ & 1030 & 104.50 & 9.00 & 131.30 & 6.74 & 7.56 & 2.80 & 16.36 & .60 \\
\hline
\end{tabular}


TABLE 15 (page 1 of 2)

Larval Development: Hatch I-5

Neutral Lipid Fatty Acids as FAME, $\mu \mathrm{g}$ per Individual

HATCH I-5: NEUTRAL LIPIDS

\begin{tabular}{|c|c|c|c|c|c|c|c|c|c|c|c|c|}
\hline & & & $14: 1$ & $15: 0$ & $15: 1$ & 1 & & & $\begin{array}{l}16: 2 \\
17: 1\end{array}$ & & & \\
\hline & & & & & & & & & 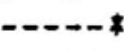 & 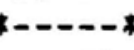 & & 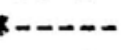 \\
\hline & 100 & .09 & .03 & .06 & 02 & .95 & 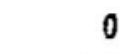 & .05 & .04 & .18 & 1.23 & .04 \\
\hline & & .07 & .04 & .04 & 0 & .63 & .13 & .04 & .04 & .17 & .85 & .04 \\
\hline & 1200 & .09 & .03 & .05 & 0 & .57 & .11 & .05 & .09 & .18 & 75 & .05 \\
\hline & 1100 & .07 & .03 & .03 & 0 & .71 & .14 & .05 & .04 & .26 & 1.06 & .10 \\
\hline & 1030 & .14 & .16 & .07 & 0 & 1.14 & .26 & .05 & .04 & .31 & 1.83 & .20 \\
\hline 19 & 2400 & .15 & .22 & .07 & 0 & 1.38 & 36 & .05 & .06 & 33 & 2.52 & 33 \\
\hline 15 & 1030 & .13 & .11 & .04 & 0 & 1.14 & .36 & .03 & .18 & .29 & 1.89 & .17 \\
\hline & 1000 & .12 & .08 & .05 & .02 & 1.46 & .43 & .11 & .20 & .35 & 3.06 & .41 \\
\hline 7 & 1200 & .17 & .09 & 0 & 0 & 1.02 & .25 & .07 & .26 & .28 & 1.74 & .17 \\
\hline 18 & 1300 & .12 & .18 & .05 & .03 & 1.60 & 44 & .07 & 21 & 41 & 3.15 & 43 \\
\hline 9 & 800 & .22 & .40 & .05 & .08 & 3.13 & 1.18 & .13 & .41 & .73 & 7.14 & 1.07 \\
\hline $6 / 20$ & 1430 & .41 & .66 & .15 & .13 & 4.14 & 1.50 & .28 & .54 & 1.03 & 9.74 & 1.58 \\
\hline 121 & 1030 & .41 & .91 & .10 & .17 & 4.84 & 2.01 & .38 & 21 & 1.05 & 10.92 & 1.88 \\
\hline 2 & 1100 & .67 & 1.62 & .32 & .35 & 8.02 & 3.00 & .64 & 37 & 1.70 & 18.82 & 3.72 \\
\hline 3 & 1230 & .47 & 1.27 & .17 & 29 & 6.55 & 2.49 & 54 & .33 & 1.53 & 16.34 & 2.98 \\
\hline $6 / 24$ & 1130 & .63 & 3.00 & 0 & .68 & 11.66 & 4.90 & 1.02 & .73 & 2.45 & 28.67 & 5.61 \\
\hline 125 & 1300 & .77 & 1.63 & .21 & .35 & 9.21 & 3.55 & .64 & .38 & 2.02 & 23.23 & 4.26 \\
\hline $6 / 26$ & 1400 & .81 & 2.39 & .30 & .58 & 11.18 & 4.68 & 1.02 & 59 & 2.27 & 27.24 & 4.98 \\
\hline $6 / 27$ & 1930 & 1.37 & 2.29 & .67 & .57 & 12.12 & 5.48 & 1.37 & .78 & 3.09 & 25.68 & 4.90 \\
\hline $6 / 28$ & 1200 & .77 & 1.64 & .33 & .34 & 8.48 & 3.37 & .93 & .42 & 1.97 & 18.13 & 3.43 \\
\hline 129 & 1100 & 1.24 & 2.89 & .35 & .66 & 16.54 & 6.45 & 1.43 & .75 & 3.75 & 38.13 & 7.50 \\
\hline $6 / 30$ & 1100 & 2.32 & 4.90 & .62 & 1.21 & 28.19 & 11.90 & 2.65 & 1.67 & 6.56 & 67.59 & 14.25 \\
\hline 11 & 110 & 3.11 & 5.72 & .86 & 1.51 & 32.28 & 14.87 & 3.33 & 2.04 & 8.38 & 80.17 & 15.16 \\
\hline 12 & 11 & 3.29 & 8.82 & 1.04 & 2.40 & 45.75 & 20.47 & 4.52 & 3.27 & 11.94 & 111.2 & 22.54 \\
\hline 13 & 11 & 3.73 & 7.85 & 1.36 & 1.95 & 44.87 & 20.35 & 4.20 & 2.73 & 11.54 & 107.6 & 21.11 \\
\hline $7 / 4$ & 14 & 4.06 & 9.57 & 1.39 & 2.43 & 54.51 & 23.97 & 4.83 & 3.39 & 13.49 & 133.3 & 27.20 \\
\hline $1 / 5$ & 1400 & 3.56 & 8.43 & 1.21 & 2.18 & 50.34 & 21.59 & 4.83 & 3.27 & 14.23 & 124.4 & 24.71 \\
\hline $7 / 6$ & 1200 & 3.04 & 7.40 & 1.15 & 1.86 & 42.74 & 18.65 & 4.12 & 2.81 & 11.78 & 110.2 & 21.68 \\
\hline $7 / 8$ & 1030 & 3.27 & 8.90 & 1. 31 & $2: 19$ & 50.47 & 21.57 & 4.45 & 3.18 & 13.21 & 128.6 & 25.65 \\
\hline $7 / 10$ & 1230 & 3.60 & 9.60 & 1.05 & 2.37 & 53.40 & 22.37 & 4.62 & 3.17 & 13.70 & 140.1 & 26.13 \\
\hline 111 & 1300 & 4. 18 & 14.95 & 0 & 3.37 & 75.78 & 33.21 & 7.28 & 5.06 & 18.89 & 197.5 & 43.06 \\
\hline
\end{tabular}


TABLE 15 (page 2 of 2)

Larval Development: Hatch I-5

Neutral Lipid Fatty Acids as FAME, $\mu \mathrm{g}$ per Individual

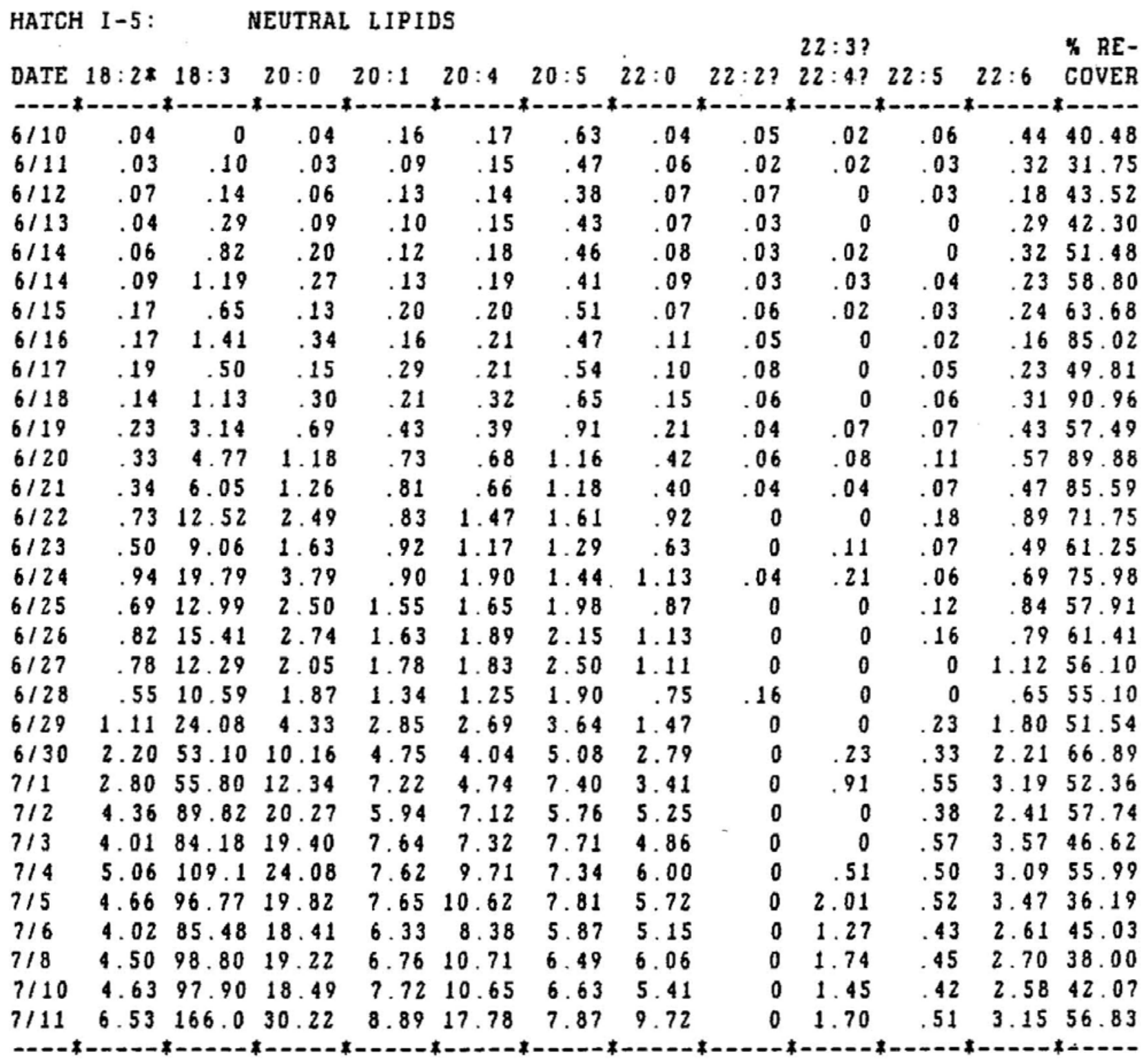


TABLE 16 (page 1 of 2)

Larval Development: Hatch I-5

P. Ethanolamine Fatty Acids as FAME, $\mu \mathrm{g}$ per Individual

\begin{tabular}{|c|c|c|c|c|c|c|c|c|c|c|c|c|}
\hline AT & 1 & & DTA & & IH & LAIII & & & & & & \\
\hline ATE & TIME & $14: 0$ & $4: 1$ & $: 0$ & $15: 1$ & $16: 0$ & $16: 1$ & $17: 0$ & $17: 1$ & $18: 0$ & $18: 1$ & $18: 2$ \\
\hline & & & 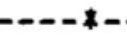 & & 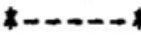 & $-\infty$ & $=-$ & $\cdot-$ & $----*$ & $*-\cdots--1$ & $*-----*$ & - \\
\hline 110 & 1300 & .05 & .02 & .05 & .02 & 1.17 & .21 & .10 & .08 & 61 & 1.95 & .07 \\
\hline $6 / 11$ & 1030 & .04 & .02 & .04 & 0 & 1.15 & .15 & .10 & .08 & .68 & 2.09 & .12 \\
\hline $6 / 12$ & 1200 & .04 & 0 & .04 & 0 & 1.13 & .15 & .12 & .10 & .72 & 2.27 & .17 \\
\hline $6 / 13$ & 1100 & .04 & .01 & .04 & 0 & & .23 & .13 & .10 & .83 & 2.80 & .27 \\
\hline $6 / 14$ & 103 & .04 & .02 & .03 & .04 & 1 & .30 & .12 & .11 & 1.07 & 3.61 & .38 \\
\hline $6 / 14$ & 240 & .04 & .03 & .03 & & & .32 & .12 & .11 & 1.12 & .01 & .44 \\
\hline $6 / 15$ & 1030 & .07 & .04 & .05 & 0 & 1.69 & .32 & 15 & .18 & 1.35 & 4.09 & .43 \\
\hline $6 / 16$ & 1000 & .06 & .03 & .05 & .03 & 1. & .17 & .16 & .18 & 1.27 & 4.20 & .47 \\
\hline $6 / 17$ & 1200 & .10 & .04 & 0 & .04 & 1. & .25 & .16 & .10 & 1.09 & 3.60 & .42 \\
\hline $6 / 18$ & 1300 & .06 & .04 & .05 & .02 & 1. & .24 & .20 & .24 & 1.40 & 5.02 & .63 \\
\hline 119 & 800 & .05 & .03 & .05 & .02 & 2. & .32 & .20 & .25 & 1.92 & 6.78 & .85 \\
\hline $6 / 20$ & 1430 & .07 & .05 & .06 & .03 & 2.49 & .31 & .22 & .24 & 2.03 & 6.61 & .90 \\
\hline $6 / 21$ & 1030 & .07 & .06 & 0 & .07 & 2.83 & .47 & .22 & .15 & 2.21 & 6.95 & .95 \\
\hline $6 / 22$ & 1100 & .11 & .06 & .10 & .04 & 3.45 & .37 & .33 & .29 & 2.95 & 9.16 & 1. 37 \\
\hline $6 / 23$ & 1230 & .11 & .07 & 0 & .11 & 3.40 & .50 & .29 & .18 & 2.90 & 8.84 & 1. 31 \\
\hline $6 / 24$ & 1130 & .09 & .07 & .10 & .07 & 3.65 & .62 & .32 & .16 & 3.06 & 9.33 & 1.49 \\
\hline $6 / 25$ & 1300 & $.17^{\circ}$ & .08 & 0 & .42 & 4.29 & .85 & .32 & .25 & 2.96 & 9.58 & 1.55 \\
\hline $6 / 26$ & 1400 & .25 & .17 & 0 & .12 & 4.06 & .92 & .52 & 0 & 3.24 & 9.50 & 1.86 \\
\hline $6 / 27$ & 1930 & .17 & .10 & 0 & .10 & 4.10 & .61 & .42 & .19 & 3.09 & 10.48 & 1.92 \\
\hline $6 / 28$ & 1200 & .15 & .07 & 0 & 0 & 3.42 & .45 & .36 & .16 & 2.61 & 9.78 & 2.02 \\
\hline $6 / 29$ & 1100 & .16 & .06 & 0 & .05 & 4.72 & .66 & .45 & .36 & 3.64 & 12.10 & 2.21 \\
\hline $6 / 30$ & 1100 & .19 & .09 & 0 & 0 & 5. & .82 & .55 & .29 & 4.83 & 14.72 & 2.93 \\
\hline $7 / 1$ & 110 & .14 & .11 & 0 & 0 & 5. & .85 & .45 & .22 & 4.27 & 13.16 & 2.45 \\
\hline $7 / 2$ & 11 & .15 & .08 & 0 & 0 & 4 & .81 & .53 & .25 & 4.84 & 14.64 & 2.94 \\
\hline $7 / 3$ & 11 & .23 & .07 & 0 & 0 & & .82 & 55 & .58 & 4.62 & 15.20 & 3.09 \\
\hline $7 / 4$ & 14 & SAMPLE & E LOST & - & - & - & - & 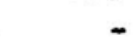 & - & - & - & - \\
\hline $7 / 5$ & 140 & 31 & 0 & 0 & 0 & & 78 & .5 & .26 & 5.80 & 14.84 & 2.83 \\
\hline $7 / 6$ & 120 & 19 & .09 & 0 & 0 & & .87 & .57 & .26 & 5.17 & 14.68 & 3.21 \\
\hline $7 / 8$ & 1030 & SAMP & LOS & - & - & - & - & - & - & - & - & \\
\hline $7 / 10$ & 1230 & .17 & .08 & 0 & 0 & 4.79 & .88 & .47 & .29 & 4.72 & 13.63 & 2.80 \\
\hline $7 / 11$ & 1300 & .17 & .17 & 0 & 0 & 6.64 & 1.28 & .57 & .33 & 4.81 & 14.75 & 3.25 \\
\hline
\end{tabular}


TABLE 16 (page 2 of 2)

Larval Development: Hatch I-5

P. Ethanolamine Fatty Acids as FAME, $\mu \mathrm{g}$ per Individual

HATCH I-5: PHOSPHATIDYL ETHANOLAMINE

\begin{tabular}{|c|c|c|c|c|c|c|c|c|c|c|c|c|}
\hline \multirow{2}{*}{ DATE } & $8: 2 *$ & $18: 3$ & $20: 0$ & 20 & $20: 4$ & & $22: 0$ & & $\begin{array}{l}22: 3 ? \\
22: 4 ?\end{array}$ & & $22: 6$ & $\begin{array}{l}\text { \% RE- } \\
\text { COVER }\end{array}$ \\
\hline & & 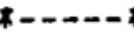 & & & --- & -- & 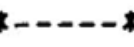 & -- & & & & \\
\hline $6 / 10$ & .10 & .17 & .04 & .30 & .55 & 2.78 & 0 & .03 & .09 & 0 & 2.73 & 51.92 \\
\hline $6 / 11$ & .10 & .17 & .06 & .33 & .57 & 2.89 & 0 & .04 & .09 & 0 & 2.75 & 46.72 \\
\hline 112 & .11 & .36 & .08 & .31 & .56 & 2.78 & 0 & .04 & .08 & 0 & 2.41 & 39.45 \\
\hline $6 /$ & .13 & .67 & .10 & .35 & .64 & 2.87 & .08 & .04 & .10 & 0 & 2.47 & 57.39 \\
\hline 1 & .14 & 1.21 & .15 & .37 & .74 & 3.34 & .10 & .03 & .27 & 0 & 2.99 & 59.44 \\
\hline 61 & .15 & 1.40 & .18 & .37 & .76 & & .08 & .04 & .09 & 0 & 2.85 & 55.34 \\
\hline 61 & .13 & 1.42 & .18 & .36 & .86 & & & .04 & .19 & 0 & 3.16 & 50.36 \\
\hline & .14 & 1.49 & .2 & .37 & .86 & & & .07 & .23 & 0 & & 43.97 \\
\hline $6 /$ & .14 & 1.33 & .1 & .3 & .84 & & & .09 & .30 & 0 & & 47.46 \\
\hline & .2 & 1.8 & & & 1.09 & & & .13 & .15 & 0 & & 49.06 \\
\hline 11 & .20 & 2.77 & .3 & .5 & 1.24 & 3. & .1 & .06 & .62 & 0 & 3.97 & 56.83 \\
\hline 61 & .20 & 2.9 & .3 & .5 & 1.28 & 5 & .2 & .09 & .34 & 0 & 4.20 & 51.40 \\
\hline $1 / 2$ & .21 & 3.08 & .3 & .6 & 1.28 & 6. & .2 & .06 & .20 & 0 & 4.59 & 45.32 \\
\hline 1 & .29 & 4.48 & .52 & .72 & 1.78 & & .3 & .08 & .62 & 0 & 5.56 & 47.25 \\
\hline 12 & .26 & 4.11 & .44 & .80 & 1.70 & 7 & .35 & .10 & .29 & 0 & 5.20 & 48.85 \\
\hline $6 / 2$ & .27 & 5.03 & .48 & .72 & 1.77 & 7. & .41 & .05 & .16 & 0 & 5.10 & 48.87 \\
\hline $6 / 2$ & .26 & 4.93 & .44 & .88 & 1.99 & & .41 & .09 & .19 & 0 & 6.08 & 50.17 \\
\hline $6 / 2$ & .48 & 5.29 & .63 & 1.29 & 2.26 & & .68 & .37 & .86 & 0 & 6.19 & 45.75 \\
\hline $6 / 2$ & .33 & 5.66 & .50 & .81 & 2.49 & 7. & .49 & .10 & .12 & 0 & 5.26 & 48.99 \\
\hline 61 & .31 & 6.14 & .49 & .71 & 2.09 & 6. & .51 & .09 & .09 & 0 & 4.33 & 42.35 \\
\hline $6 / 2$ & .56 & 6.75 & .62 & 1.16 & 2.46 & 9.24 & .53 & .14 & .18 & 0 & 6.81 & 50.28 \\
\hline $6 / 3$ & .50 & 8.94 & .91 & 1.28 & 2.69 & 9. & .74 & 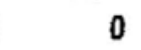 & .53 & 0 & 7.12 & 54.51 \\
\hline $7 / 1$ & .39 & 7.62 & .66 & 1.27 & 2.53 & 9. & .68 & 0 & .51 & 0 & 7.24 & 61.56 \\
\hline $7 / 2$ & .53 & 9.50 & .99 & 1.27 & 3.26 & 9. & .94 & .09 & .34 & 0 & 6.68 & 62.47 \\
\hline $7 / 3$ & .86 & 10.16 & .83 & 1.58 & 3.07 & 9.27 & 1.04 & .26 & .12 & 0 & 6.41 & 62.03 \\
\hline 71 & - & - & - & 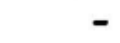 & - & - & - & 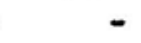 & - & - & - & \\
\hline $7 / 5$ & .47 & 9.50 & .87 & 1.27 & 4.22 & 13. & 1.19 & 0 & .50 & 0 & 9.50 & 57.66 \\
\hline 71 & .53 & 10.66 & .86 & 29 & 3.35 & & & $c$ & .35 & 0 & 6.63 & 52.92 \\
\hline $7 / 8$ & - & - & - & 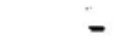 & - & - & 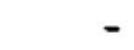 & - & - & - & - & 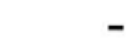 \\
\hline 71 & .44 & 9.29 & $\cdots$ & & 3.45 & & m & 0 & .27 & 0 & 7.50 & 40.67 \\
\hline $7 / 11$ & .44 & 10.67 & .65 & 1.19 & 3.73 & 10.65 & .98 & .12 & .15 & 0 & 7.94 & 43.49 \\
\hline
\end{tabular}


TABLE 17 (page 1 of 2)

Larval Development: Hatch I-5

P. Choline Fatty Acids as FAME, $\mu \mathrm{g}$ per Individual

HATCH I-5: PHOSPHATIDYL CHOLINE

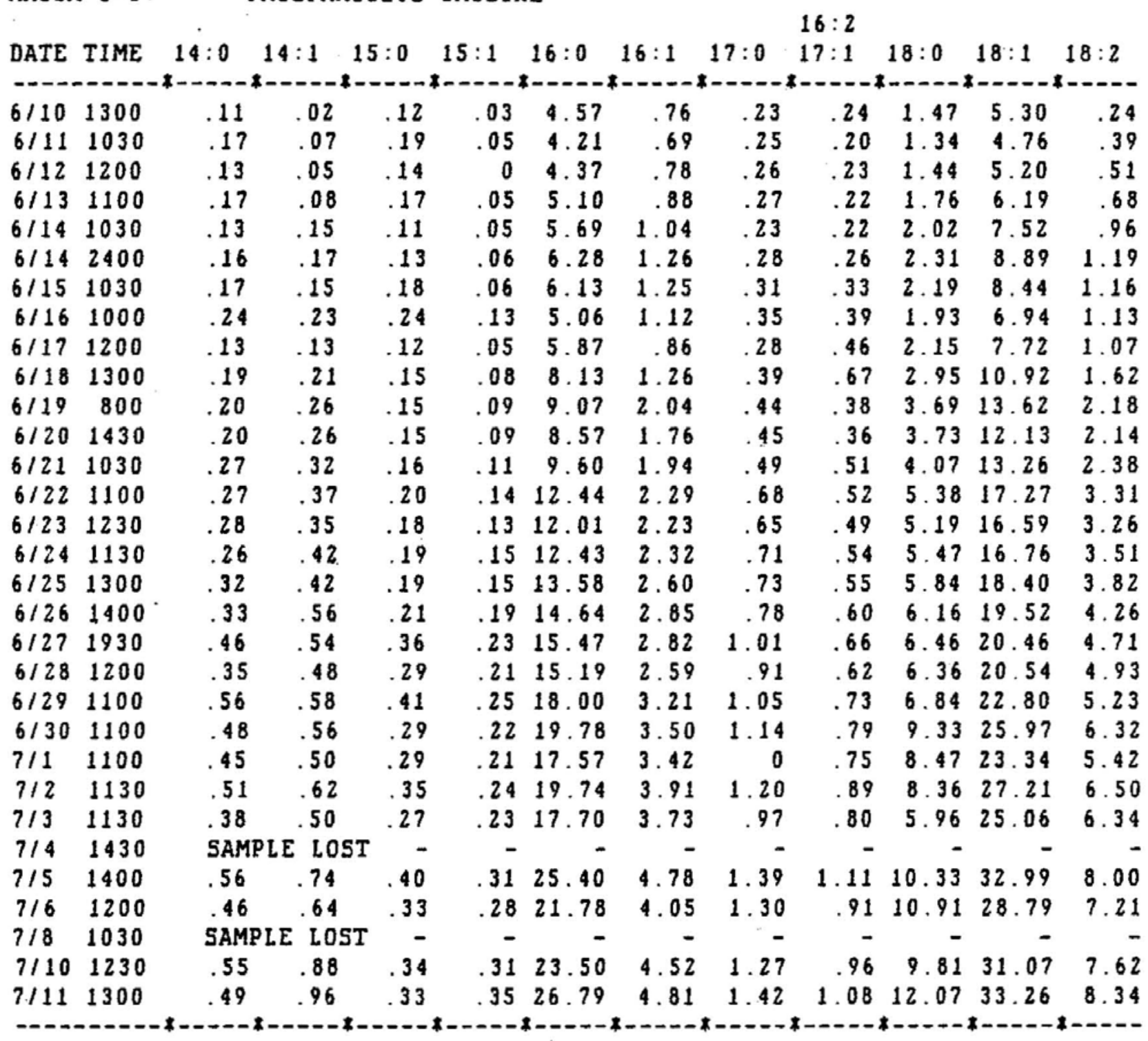


TABLE 17 (page 2 of 2)

Larval Development: Hatch I-5

P. Choline Fatty Acids as FAME, $\mu \mathrm{g}$ per Individual

HATCH I-5: PHOSPHATIDYL CHOLINE

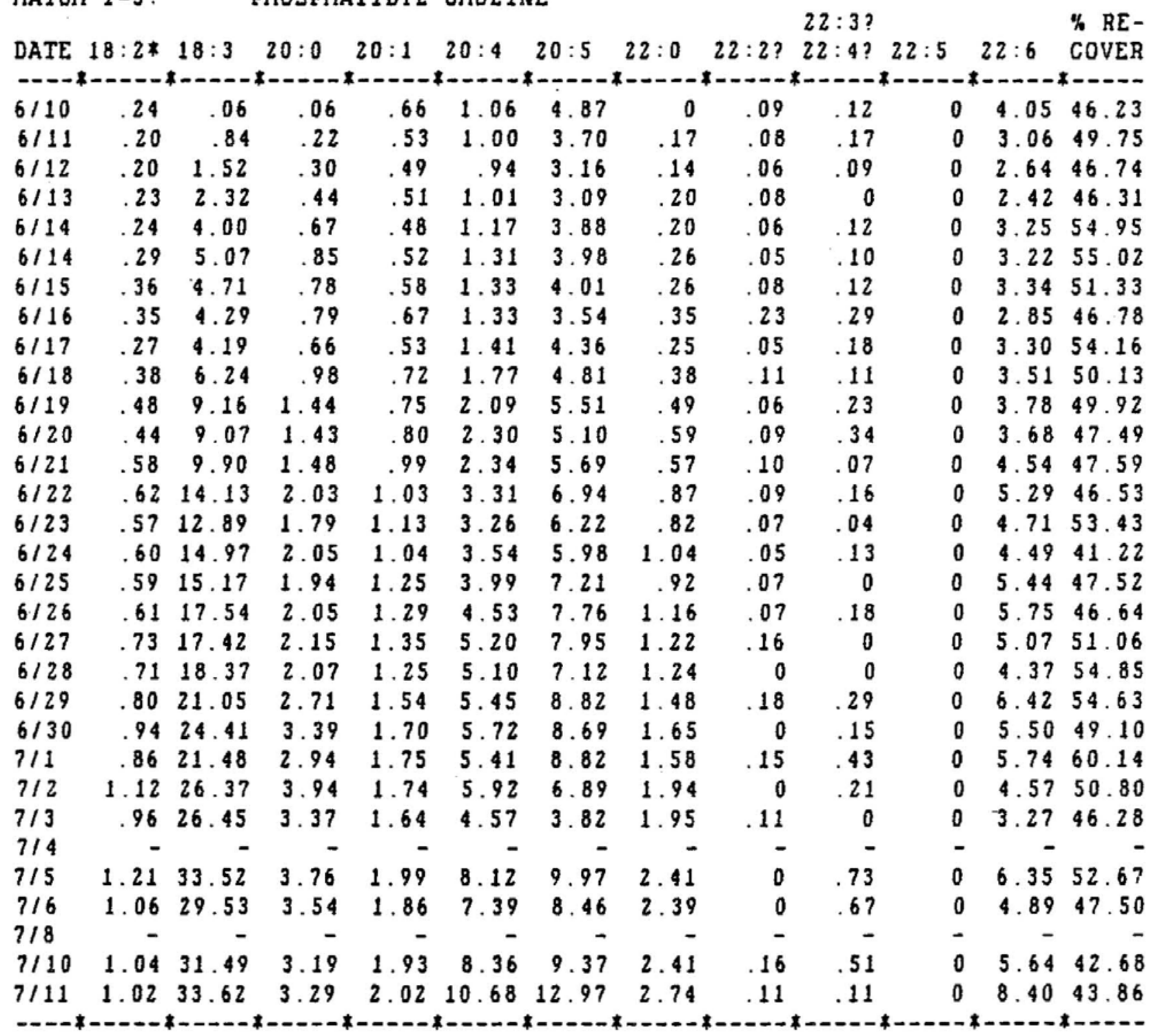


TABLE 18

Larval Development: Hatch II-4

Neutral Lipid Fatty Acids as FAME, $\mu \mathrm{g}$ per Individual

HATCH II-4: NEUTRAL LIPIDS

$18: 3$

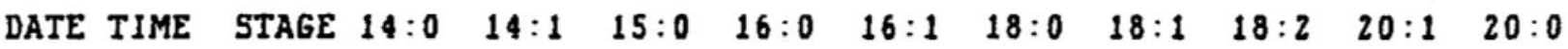

\begin{tabular}{|c|c|c|c|c|c|c|c|c|c|c|c|c|}
\hline 11 & 1730 & $1-A-B$ & .04 & 0 & .02 & .58 & .12 & .16 & .68 & 0 & .08 & 0 \\
\hline 1 & 2300 & $1-B-C$ & .04 & .06 & 0 & .73 & .17 & .18 & 1.05 & .12 & .31 & \\
\hline 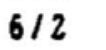 & 900 & $1-C$ & .07 & .04 & .10 & .75 & .14 & .36 & 1.02 & .26 & .54 & \\
\hline 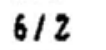 & 1700 & $1-C$ & .11 & .09 & .10 & .78 & .19 & .29 & 1.16 & .31 & .56 & \\
\hline 2 & 2300 & $1-C$ & .10 & .17 & 0 & 1.10 & .46 & .49 & 2.04 & .58 & .77 & \\
\hline 13 & 900 & $1-D 0$ & .09 & .13 & .05 & .82 & .33 & .42 & 1.18 & .43 & .40 & \\
\hline 13 & 2130 & $1-D 0$ & .08 & .27 & 0 & 1. 30 & .57 & .62 & 2.43 & .84 & 1.27 & .09 \\
\hline 14 & 930 & $1-D 1,1$ & .04 & .09 & .03 & .96 & .23 & .36 & 1.32 & .25 & .49 & .01 \\
\hline 4 & 2130 & $1-01,3$ & .10 & .30 & .04 & 1.68 & .64 & .58 & 3.53 & .86 & 1.82 & .03 \\
\hline 15 & 900 & $1-D 2-3$ & .10 & .20 & .05 & 1.22 & .41 & .43 & 2.29 & .57 & 1.04 & .02 \\
\hline 15 & 2105 & $2-A$ & .12 & .38 & .07 & 1.81 & .68 & .53 & 3.82 & .94 & 1.99 & .05 \\
\hline $6 / 6$ & 900 & $2-B$ & .08 & .21 & .06 & 1.70 & .52 & .58 & 3.28 & 1.04 & 1.64 & \\
\hline $6 / 7$ & 900 & $2-C$ & .15 & .43 & .08 & 2.04 & .75 & .60 & 4.57 & 1.15 & 2.13 & .10 \\
\hline $6 / 8$ & 900 & $2-00$ & .13 & .48 & .11 & 2.86 & 1.05 & .85 & 6.83 & 1.61 & 3.01 & .10 \\
\hline $6 / 9$ & 1000 & $2-D 1,3$ & .24 & .79 & .18 & 3.77 & 1.46 & 1.03 & 8.86 & 2.13 & 4.24 & .12 \\
\hline $6 / 10$ & 900 & $2-D 3$ & .30 & .92 & .26 & 4.46 & 1.76 & 1.27 & 9.96 & 2.42 & 4.80 & .18 \\
\hline $6 / 11$ & 930 & $3-C$ & .47 & 1.62 & .34 & 7.26 & 3.11 & 1.91 & 17.04 & 4.37 & 9.28 & .36 \\
\hline $6 / 13$ & 900 & $3-D 0$ & .69 & 2.32 & .50 & 10.04 & 4.59 & 2.59 & 24.12 & 6.05 & 11.82 & .48 \\
\hline 1 & 1000 & $3-D 1,3$ & .98 & 3.15 & .75 & 12.79 & 6.04 & 3.22 & 29.24 & 7.30 & 15.57 & .48 \\
\hline 16 & 900 & $3-D 3$ & .99 & 3.60 & .78 & 13.76 & 6.42 & 3.19 & 31.85 & 8.25 & 17.54 & .71 \\
\hline
\end{tabular}

HATCH II-4: NEUTRAL LIPIDS

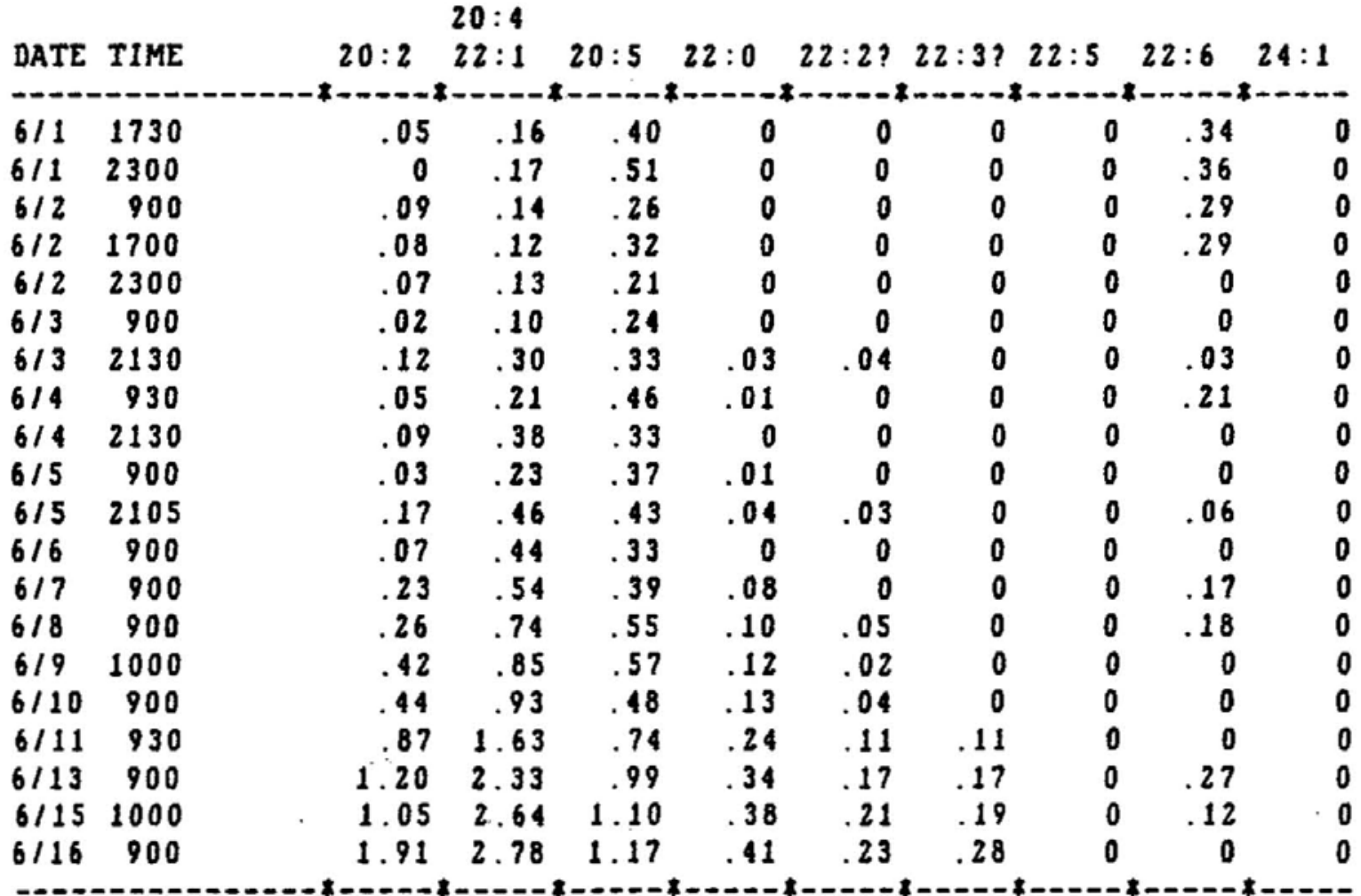


TABLE 19

Larval Development: Hatch II-4

P. Ethanolamine Fatty Acids as FAME, $\mu \mathrm{g}$ per Individual

HATCH I1-4: PHOSPHATIDYL ETHANOLAMINE

$18: 3$

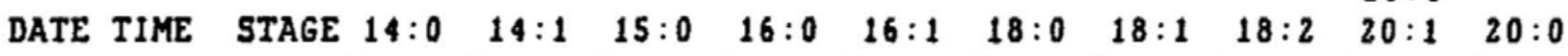

\begin{tabular}{|c|c|c|c|c|c|c|c|c|c|c|c|c|}
\hline 1 & 1730 & $1-A-B$ & 0 & 0 & 0 & 1.10 & .16 & .77 & 1.86 & .19 & .33 & 0 \\
\hline 11 & 2300 & $1-B-C$ & 0 & 0 & 0 & 1.12 & .10 & .59 & 1.66 & .16 & 38 & \\
\hline 2 & 900 & $1-C$ & 0 & 0 & 0 & 1.05 & .18 & .71 & 1.84 & .25 & .46 & \\
\hline 12 & 1700 & $1-C$ & .03 & 0 & .18 & .95 & .17 & .90 & 1.80 & .35 & .44 & \\
\hline 12 & 2300 & $1-C$ & .05 & 0 & .07 & 1.32 & .27 & 1.00 & 2.61 & .55 & .77 & \\
\hline 13 & 900 & 1-Do & .02 & 0 & .18 & 1.05 & .23 & 1.06 & 1.98 & .55 & .69 & \\
\hline 3 & 2130 & $1-D 0$ & .03 & .03 & .23 & 1.21 & .23 & 1.09 & 2.48 & .51 & .85 & \\
\hline 4 & 930 & $1-01,1$ & .03 & .04 & .18 & 1.13 & .22 & 1.00 & 2.11 & .55 & .85 & \\
\hline 4 & 2130 & $1-D 1,3$ & .03 & .03 & .07 & 1.17 & .16 & 1.17 & 2.42 & .63 & .98 & \\
\hline 15 & 900 & $1-D 2-3$ & .03 & .06 & .26 & 1.14 & .15 & 1.04 & 2.39 & .57 & .95 & \\
\hline 15 & 2105 & $2-A$ & .03 & .01 & .02 & 1.30 & .20 & 1.01 & 2.81 & .66 & 1.20 & \\
\hline 16 & 900 & $2-B$ & .02 & 0 & .03 & 1.12 & .16 & .86 & 2.46 & .54 & 1.02 & \\
\hline 7 & 900 & $2-C$ & .03 & .04 & .03 & 1.45 & .19 & 1.15 & 3.26 & .79 & 1.53 & 3 \\
\hline 8 & 900 & $2-D 0$ & .02 & .01 & .01 & 1.50 & .18 & 1.27 & 3.51 & .88 & 1.71. & 0 \\
\hline 9 & 1000 & $2-D 1,3$ & .03 & .01 & .03 & 1.65 & .23 & 1.37 & 3.86 & .98 & 2.03 & .04 \\
\hline 10 & 900 & $2-D 3$ & .04 & .14 & .19 & 1.81 & .21 & 1.37 & 4.22 & 1.11 & 2.43 & .01 \\
\hline 1 & 930 & $3-c$ & .04 & .03 & .06 & 1.98 & .29 & 1.72 & 4.96 & 1.40 & 2.96 & .07 \\
\hline 13 & 900 & $3-D 0$ & .09 & .22 & .35 & 2.26 & .22 & 1.75 & 5.56 & 1.64 & 3.57 & .03 \\
\hline 15 & 1000 & $3-D 1,3$ & .07 & .42 & .26 & 3.22 & .51 & 2.40 & 7.72 & 2.58 & 5.60 & .11 \\
\hline 16 & 900 & $3-D 3$ & .06 & .18 & .09 & 3.79 & .70 & 3.18 & 9.37 & 2.91 & 5.92 & .19 \\
\hline
\end{tabular}

HATCH 11-4: PHOSPHATIDYL ETHANOLAMINE

\begin{tabular}{lrrrrrrrrrr} 
& & \multicolumn{3}{c}{$20: 4$} & & & & & & \\
DATE TIME & $20: 2$ & $22: 1$ & $20: 5$ & $22: 0$ & $22: 2 ?$ & $22: 3 ?$ & $22: 5$ & $22: 6$ & $24: 1$ \\
\hline $6 / 1$ & 1730 & .24 & .51 & 2.74 & 0 & 0 & 0 & .06 & 2.68 & 0 \\
$6 / 1$ & 2300 & .16 & .65 & 2.91 & 0 & 0 & .06 & .04 & 2.76 & 0 \\
$6 / 2$ & 900 & .22 & .54 & 1.93 & 0 & 0 & 0 & 0 & 1.70 & 0 \\
$6 / 2$ & 1700 & .23 & .67 & 2.21 & 0 & 0 & 0 & 0 & 1.98 & 0 \\
$6 / 2$ & 2300 & .26 & .97 & 2.89 & 0 & 0 & .10 & .10 & 2.65 & 0 \\
$6 / 3$ & 900 & .27 & .74 & 2.35 & 0 & 0 & .10 & 0 & 2.20 & 0 \\
$6 / 3$ & 2130 & .26 & 1.04 & 3.01 & 0 & 0 & .16 & 0 & 2.52 & 0 \\
$6 / 4$ & 930 & .29 & .94 & 2.34 & 0 & 0 & .10 & .04 & 2.26 & 0 \\
$6 / 4$ & 2130 & .24 & 1.12 & 2.61 & 0 & 0 & .11 & .05 & 2.25 & 0 \\
$6 / 5$ & 900 & .22 & 1.16 & 2.95 & 0 & 0 & .10 & 0 & 2.40 & 0 \\
$6 / 5$ & 2105 & .32 & 1.35 & 3.20 & .01 & 0 & .29 & 0 & 2.65 & 0 \\
$6 / 6$ & 900 & .27 & 1.24 & 2.74 & .02 & 0 & .18 & 0 & 2.34 & 0 \\
$6 / 7$ & 900 & .37 & 1.78 & 3.51 & .02 & 0 & .28 & 0 & 2.91 & 0 \\
$6 / 8$ & 900 & .38 & 1.99 & 3.33 & .02 & 0 & .17 & 0 & 2.47 & 0 \\
$6 / 9$ & 1000 & .35 & 2.16 & 3.87 & .02 & 0 & .21 & .03 & 3.09 & 0 \\
$6 / 10$ & 900 & .41 & 2.96 & 4.30 & .04 & 0 & .47 & 0 & 3.17 & 0 \\
$6 / 11$ & 930 & .46 & 3.32 & 4.58 & .04 & .05 & .52 & .04 & 3.16 & 0 \\
$6 / 13$ & 900 & .49 & 4.04 & 5.10 & .03 & .10 & .40 & 0 & 3.30 & 0 \\
$6 / 15$ & 1000 & .79 & 5.68 & 6.56 & .08 & .19 & .62 & .02 & 3.61 & 0 \\
$6 / 16$ & 900 & .92 & 6.05 & 6.89 & .07 & .14 & .62 & 0 & 4.03 & 0
\end{tabular}


TABLE 20

Larval Development: Hatch II-4

P. Choline Fatty Acids as FAME, $\mu \mathrm{g}$ per Individual

HATCH 11-4: PHOSPHATIDYL CHOLINE.

$18: 3$

DATE TIME STAGE $14: 0 \quad 14: 1 \quad 15: 0 \quad 16: 0 \quad 16: 1 \quad 18: 0 \quad 18: 1 \quad 18: 2 \quad 20: 1 \quad 20: 0$

\begin{tabular}{|c|c|c|c|c|c|c|c|c|c|c|c|c|}
\hline 11 & 1730 & $1-A-B$ & .05 & 0 & .05 & 3.74 & .72 & 1.55 & 4.49 & .48 & .68 & .07 \\
\hline 1 & 2300 & $1-B-C$ & .08 & .04 & .09 & 4.00 & .65 & 1.50 & 4.77 & .50 & 89 & 0 \\
\hline 12 & 900 & $1-C$ & .08 & .05 & .11 & 3.04 & .52 & 1.24 & 3.64 & .62 & 1.10 & .04 \\
\hline 12 & 1700 & $1-c$ & .09 & .07 & .10 & 3.62 & .53 & 1.34 & 4.12 & .66 & 1. 36 & .04 \\
\hline 12 & 2300. & $1-C$ & .13 & .11 & .13 & 4.10 & .72 & 1.71 & 5.11 & 1.18 & 2.12 & $.09^{\circ}$ \\
\hline $6 / 3$ & 900 & $1-D 0$ & .11 & .08 & .12 & 3.54 & .60 & 1.50 & 4.13 & .94 & 1.63 & .11 \\
\hline $6 / 3$ & 2130 & $1-D 0$ & .11 & .13 & .12 & 4.53 & .66 & 1.75 & 5. 46 & 1.12 & 2.44 & .07 \\
\hline $6 / 4$ & 930 & $1-D 1$ & .06 & .05 & .11 & 3.55 & .54 & 1.48 & 4.21 & 89 & 1.67 & .08 \\
\hline 14 & 2130 & $1-D 1$, & .09 & .11 & .11 & 4.33 & .64 & 1.71 & 5.40 & 1.14 & 2.47 & .05 \\
\hline 15 & 900 & $1-D 2-$ & .06 & .08 & .11 & 4.48 & .64 & 1.72 & 5.38 & 1.26 & 2.57 & .07 \\
\hline 15 & 2105 & $2-A$ & .09 & .09 & .10 & 4.13 & .65 & 1.83 & 5.13 & 1.41 & 2.70 & .10 \\
\hline 16 & 900 & $2-B$ & .07 & .08 & .09 & 3.82 & .51 & 1.59 & 4.61 & 1.07 & 2.18 & .08 \\
\hline 17 & 900 & $2-C$ & .08 & .11 & .12 & 5.00 & .58 & 2.02 & 5.63 & 1.40 & 2.90 & .13 \\
\hline 18 & 900 & $2-00$ & .10 & .12 & .19 & 4.68 & .60 & 2.12 & 5.84 & 1.58 & 3.07 & .08 \\
\hline 19 & 1000 & 2-D1, & .11 & .15 & .15 & 5.14 & .77 & 2.24 & 6.40 & 1.78 & 3.63 & .17 \\
\hline $6 / 10$ & 900 & $2-D 3$ & .11 & .16 & .17 & 5.62 & .84 & 2.50 & 7.05 & 2.06 & 4.29 & .17 \\
\hline $6 / 11$ & 930 & $3-C$ & .12 & .18 & .16 & 5.71 & .85 & 2.68 & 6.98 & 2.04 & 4.17 & .21 \\
\hline $6 / 13$ & 900 & $3-D 0$ & .14 & .24 & .18 & 7.60 & 1.23 & 3.59 & 10.08 & 2.95 & 6.44 & .27 \\
\hline $6 / 15$ & 1000 & $3-01$ & .15 & .25 & .19 & 9.54 & 1. 56 & 4.52 & 12.22 & 3.57 & 8.19 & .23 \\
\hline 116 & 900 & 3 & .19 & .32 & .23 & 10.81 & 1.76 & 5.01 & 14.21 & 4.29 & 9.37 & .34 \\
\hline
\end{tabular}

HATCH II-4: PHOSPHATIDYL CHOLINE

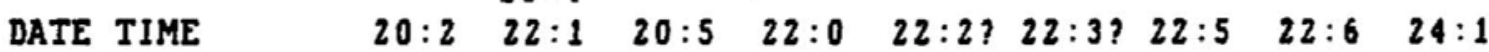

\begin{tabular}{|c|c|c|c|c|c|c|c|c|c|c|}
\hline $6 / 1$ & 1730 & .28 & 1.13 & 4.29 & 0 & 0 & 0 & .17 & 2.98 & .08 \\
\hline $6 / 1$ & 2300 & .23 & 1.14 & 4.33 & 0 & 0 & 0 & .29 & 3.10 & .14 \\
\hline $6 / 2$ & 900 & .28 & 1.04 & 3.06 & 0 & 0 & 0 & .12 & 2.23 & .06 \\
\hline $6 / 2$ & 1700 & .21 & 1.09 & 3.26 & 0 & .02 & 0 & 0 & 2.09 & 0 \\
\hline $6 / 2$ & 2300 & .42 & 1.52 & 3.50 & .04 & .05 & 0 & 0 & 2.28 & .07 \\
\hline $6 / 3$ & 900 & .40 & 1.45 & 3.18 & .05 & .06 & .08 & .07 & 2.07 & .05 \\
\hline $6 / 3$ & 2130 & .39 & 1.68 & 3.75 & .05 & .06 & .05 & 0 & 2.45 & 0 \\
\hline $6 / 4$ & 930 & .35 & 1.58 & 3.25 & .08 & .03 & .12 & .08 & 2.14 & 0 \\
\hline $6 / 4$ & 2130 & .37 & 1.76 & 3.46 & .04 & .06 & .06 & .09 & 2.36 & 0 \\
\hline $6 / 5$ & 900 & .45 & 2.09 & 3.73 & .04 & 0 & .10 & 0 & 2.26 & 0 \\
\hline $6 / 5$ & 2105 & .51 & 2.06 & 3.49 & .06 & .02 & .10 & .09 & 2.29 & 0 \\
\hline $6 / 6$ & 900 & .33 & 1.68 & 3.08 & .01 & .04 & .12 & .05 & 2.03 & 0 \\
\hline $6 / 7$ & 900 & .40 & 2.24 & 3.54 & .05 & 10 & .21 & .06 & 2.40 & 0 \\
\hline $6 / 8$ & 900 & .42 & 2.27 & 3.54 & .02 & 0 & .06 & 0 & 2.15 & 0 \\
\hline $6 / 9$ & 1000 & .54 & 2.52 & 3.36 & $.0 ?$ & .12 & .22 & 0 & 2.00 & 0 \\
\hline $6 / 10$ & 900 & .59 & 2.98 & 3.51 & .07 & .12 & .30 & 0 & 2.07 & 0 \\
\hline $6 / 11$ & 930 & .57 & $2.7 \mathrm{a}$ & 2.81 & .07 & .11 & .27 & 0 & 1. 48 & 0 \\
\hline $6 / 13$ & 900 & .52 & 4.52 & 4.44 & .13 & .25 & .43 & .01 & 2.15 & 0 \\
\hline $6 / 15$ & 1000 & 1.10 & 5.64 & 4.63 & .24 & .39 & .26 & 0 & 1.90 & 0 \\
\hline $6 / 16$ & 900 & 1.17 & 6.27 & 5.58 & .16 & .33 & .51 & 0 & 2.79 & 0 \\
\hline
\end{tabular}


TABLE 21 (page 1 of 3)

Starvation

Lipid Classes

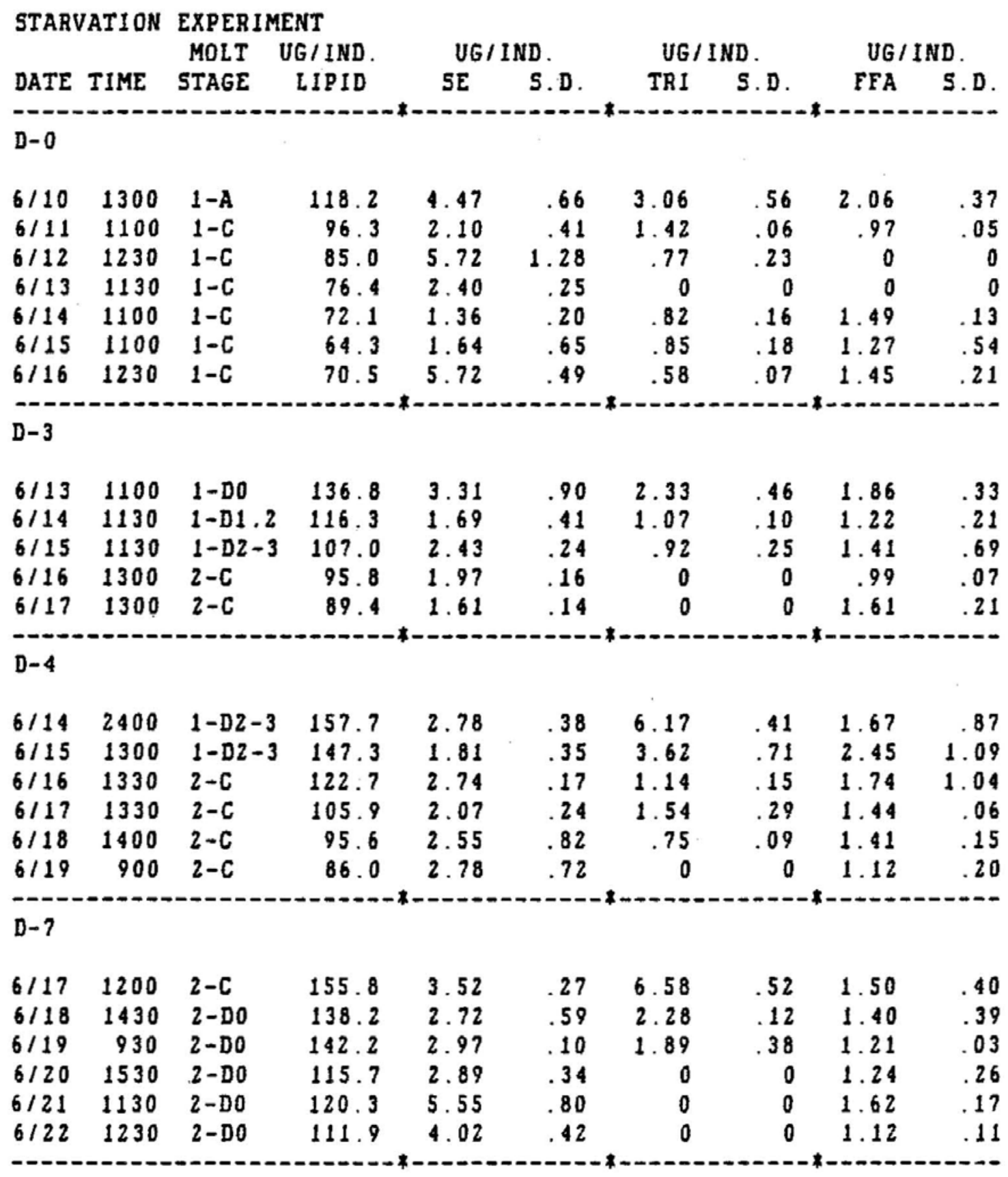


TABLE 21 (page 2 of 3 )

Starvation

Lipid Classes

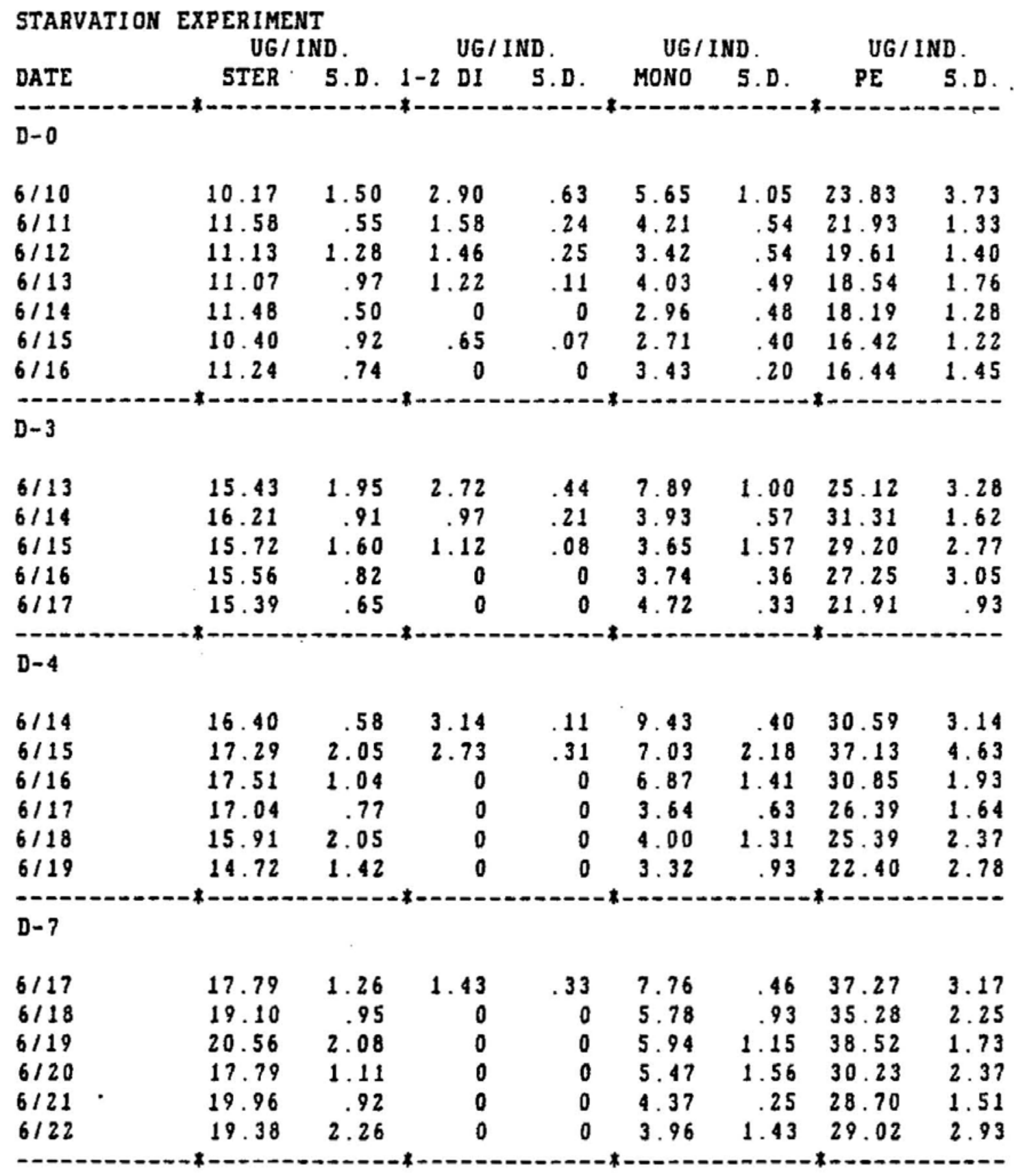


TABLE 21 (page 3 of 3 )

Starvation

Lipid Classes

\begin{tabular}{|c|c|c|c|c|c|c|c|c|}
\hline STARVATION & $\begin{array}{c}\text { EXPERIM } \\
\text { UG }\end{array}$ & & UG I & ND. & UG I & VD. & UGI & \\
\hline DATE & PI & S.D. & PC & S.D. & SM & S.D. & UNK. & S.D. \\
\hline$D-0$ & & & & & & & & \\
\hline $6 / 10$ & 2.89 & .52 & 58.40 & 8.63 & 2.41 & .63 & 2.36 & .38 \\
\hline $6 / 11$ & 3.20 & .33 & 43.41 & 1.49 & 3.07 & .41 & 2.83 & .23 \\
\hline $6 / 12$ & 3.04 & .35 & 33.65 & 2.49 & 3.53 & .58 & 2.69 & .64 \\
\hline $6 / 13$ & 2.59 & .32 & 30.21 & 2.49 & 3.31 & .41 & 2.95 & .23 \\
\hline $6 / 14$ & 2.05 & .20 & 28.94 & .81 & 2.78 & .32 & 2.05 & .10 \\
\hline $6 / 15$ & 1.67 & .18 & 24.00 & 1.72 & 2.97 & .45 & 1.72 & .41 \\
\hline $6 / 16$ & 1.81 & .20 & 24.74 & 1.56 & 2.94 & .33 & 2.16 & .25 \\
\hline$D-3$ & & & & & & & & - \\
\hline $6 / 13$ & 5.98 & .77 & 61.18 & 7.62 & 4.46 & .84 & 6.55 & .82 \\
\hline $6 / 14$ & 1.73 & .17 & 53.34 & 2.21 & 2.80 & .57 & 2.04 & .81 \\
\hline $6 / 15$ & 1.66 & .18 & 44.87 & 4.73 & 3.36 & .85 & 2.68 & .99 \\
\hline $6 / 16$ & 1.16 & .12 & 38.60 & 2.38 & 3.31 & .42 & 3.22 & .54 \\
\hline $6 / 17$ & 2.98 & .30 & 33.99 & 1.49 & 3.92 & .39 & 3.11 & .29 \\
\hline---- & ---- & $\cdots$ & $x---0--$ & $---\infty$ & ----- & $-\infty \ldots$ & $----\infty$ & $-\infty$ \\
\hline$D-4$ & & & & & & & & \\
\hline $6 / 14$ & 7.84 & .21 & 65.87 & 1.84 & 5.30 & .51 & 8.52 & 1.25 \\
\hline $6 / 15$ & 5.05 & .25 & 61.11 & 5.44 & 4.26 & 1.09 & 4.94 & 1.33 \\
\hline $6 / 16$ & 3.98 & .39 & 48.66 & 2.93 & 4.28 & .76 & 4.92 & .56 \\
\hline $6 / 17$ & 2.98 & .30 & 44.34 & 1.00 & 3.17 & .40 & 3.32 & .34 \\
\hline $6 / 18$ & 1.89 & .22 & 37.30 & 4.84 & 3.64 & .67 & 2.77 & .73 \\
\hline $6 / 19$ & 1.78 & .25 & 33.44 & 3.24 & 3.41 & .47 & 3.03 & .51 \\
\hline & - & . & ------ & $-\cdots-$ & $--\infty$ & --- & ---- & $\ldots$ \\
\hline D-7 & & & & & & & & \\
\hline $6 / 17$ & 5.22 & .24 & 63.72 & 4.19 & 4.36 & .69 & 6.65 & 1.05 \\
\hline $6 / 18$ & 3.36 & .36 & 59.90 & 4.24 & 3.80 & .53 & 4. 38 & .28 \\
\hline $6 / 19$ & 3.80 & .38 & 58.00 & 2.11 & 9.75 & .64 & 4.55 & .77 \\
\hline $6 / 20$ & 4.07 & .42 & 45.24 & 2.34 & 4.24 & .64 & 4.54 & .28 \\
\hline $6 / 21$ & 3.14 & .32 & 48.49 & 1.62 & 4.43 & .46 & 4.04 & .35 \\
\hline $6 / 22$ & 2.67 & .33 & 42.78 & 4.47 & 5.09 & .81 & 3.85 & .96 \\
\hline
\end{tabular}


TABLE 22 (page 1 of 2)

Starvation

Neutral Lipid Fatty Acids as FAME, $\mu \mathrm{g}$ per Individual

STARVATION EXPERIMENT NEUTRAL LIPIDS

$16: 2$

DATE TIME $14: 0 \quad 14: 1 \quad 15: 0 \quad 15: 1 \quad 16: 0 \quad 16: 1 \quad 17: 0 \quad 17: 1 \quad 18: 0 \quad 18: 1 \quad 18: 2$

D-0

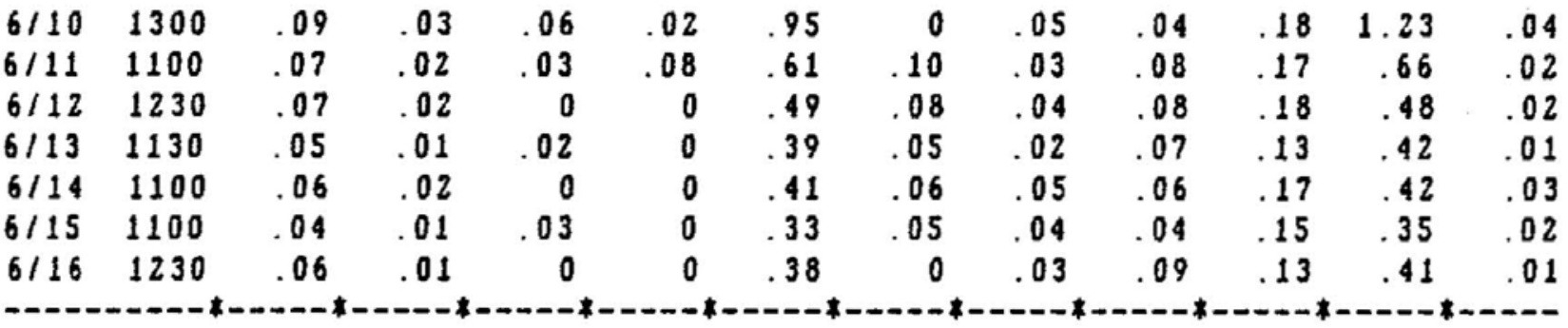

D-3

$\begin{array}{lllllllllllllll}6 / 13 & 1100 & .07 & .03 & .03 & 0 & .71 & .14 & .05 & .04 & .26 & 1.06 & .10\end{array}$

$\begin{array}{lllllllllllll}6 / 14 & 1130 & .06 & .05 & 0 & 0 & .57 & .10 & .04 & .03 & .28 & .78 & .06\end{array}$

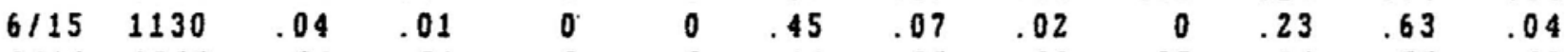

$\begin{array}{lllllllllllll}6 / 16 & 1300 & .04 & .01 & 0 & 0 & .44 & .07 & .03 & .05 & .20 & .57 & .03\end{array}$

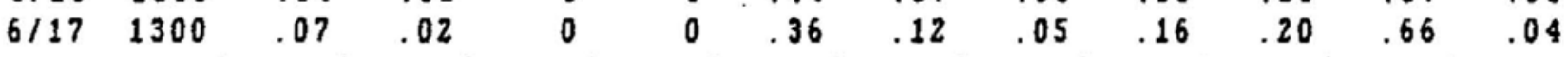

D-4

$\begin{array}{lllllllllllll}6 / 14 & 2400 & .15 & .22 & .07 & 0 & 1.38 & .36 & .05 & .06 & .33 & 2.52 & .33\end{array}$

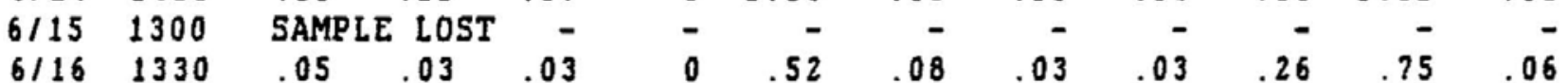

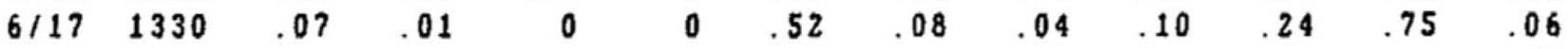

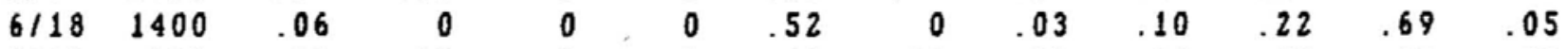

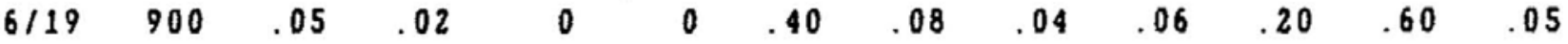

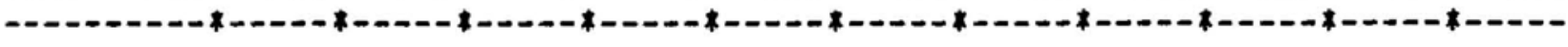

$\mathrm{D}-7$

\begin{tabular}{rrrrrrrrrrrrr}
$6 / 17$ & 1200 & .17 & .09 & 0 & 0 & 1.02 & .25 & .07 & .26 & .28 & 1.74 & .17 \\
$6 / 18$ & 1430 & .05 & .03 & 0 & 0 & .56 & .10 & .04 & .11 & .25 & 1.00 & .09 \\
$6 / 19$ & 930 & .08 & .07 & .04 & .05 & .71 & .15 & .04 & .05 & .32 & 1.16 & .11 \\
$6 / 20$ & 1530 & .04 & .02 & .03 & .03 & .46 & .10 & .03 & .02 & .19 & .72 & .06 \\
$6 / 21$ & 1130 & 0 & .02 & .03 & 0 & .47 & .08 & .04 & .05 & .27 & .71 & .05 \\
$6 / 22$ & 1230 & .05 & .01 & .03 & 0 & .47 & .09 & .04 & 0 & .27 & .74 & .07 \\
\hline
\end{tabular}


TABLE 22 (page 2 of 2)

Starvation

Neutral Lipid Fatty Acids as FAME, $\mu \mathrm{g}$ per Individual

STARVATION EXPERIMENT NEUTRAL LIPIDS

DATE $18: 2 * 18: 3 \quad 20: 0 \quad 20: 1 \quad 20: 4 \quad 20: 5 \quad 22: 0 \quad 22: 2 ? \quad 22: 3 ? \quad 22: 4 ? \quad 22: 5 \quad 22: 6$ D-0

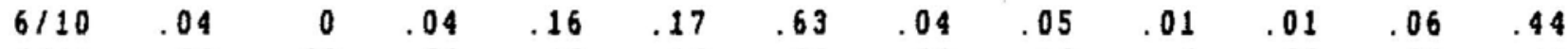

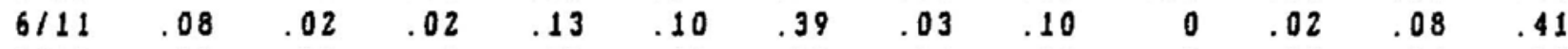

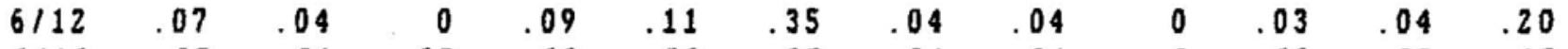

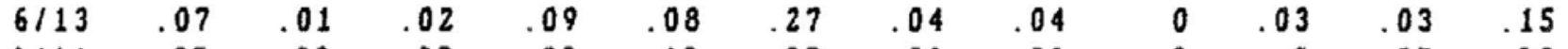

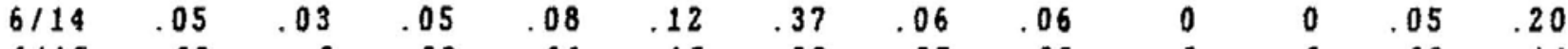

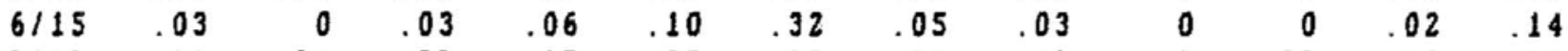

$6 / 16 \quad .11 \quad .04 \quad .03 \quad .15 \quad .07 \quad .32 \quad .03 \quad .12 \quad 0002.10 \quad .12$

D-3

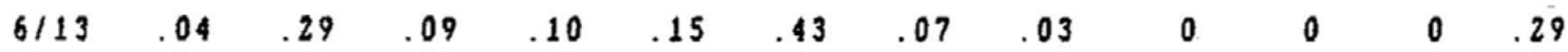

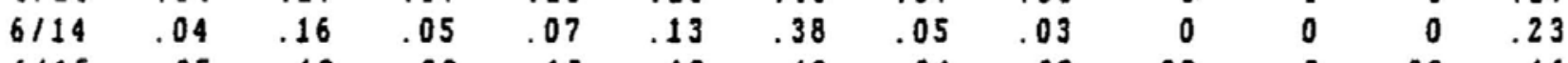

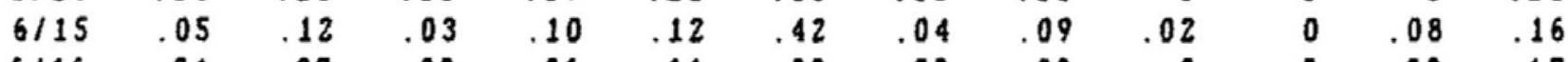

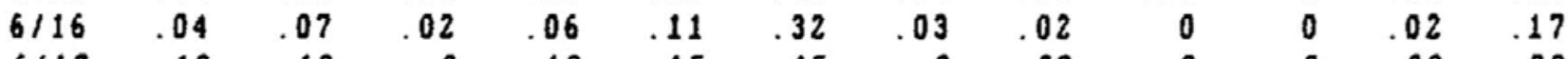

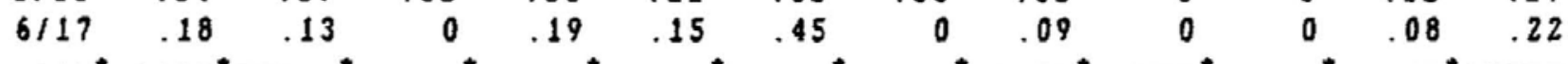

D-4

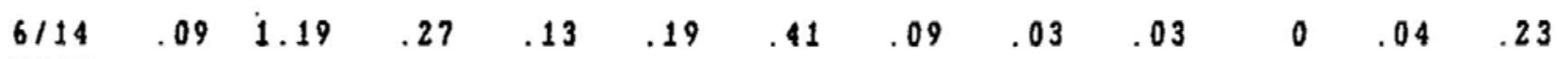

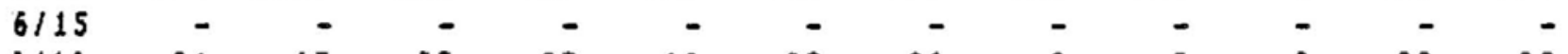

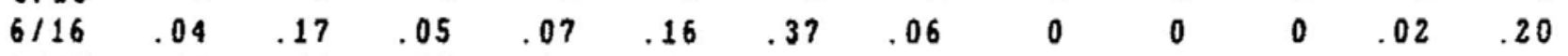

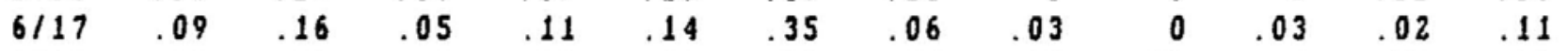

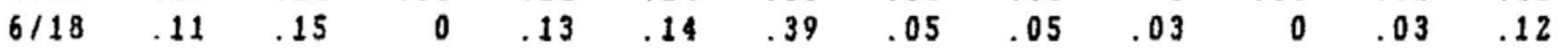

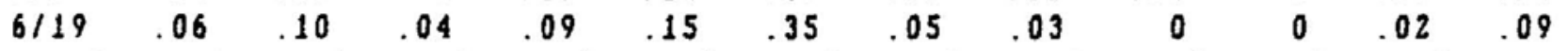

----1----- * -

D-7

$\begin{array}{lllllllllllll}6 / 17 & .19 & .50 & .15 & .29 & .21 & .54 & .10 & .08 & 0 & 0 & .05 & .23\end{array}$

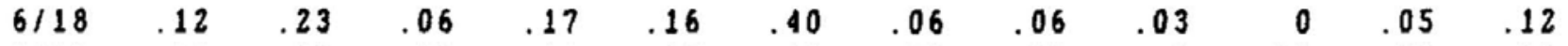

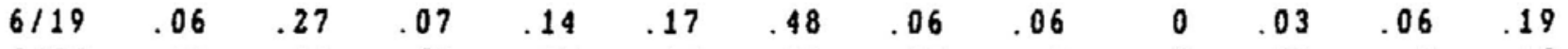

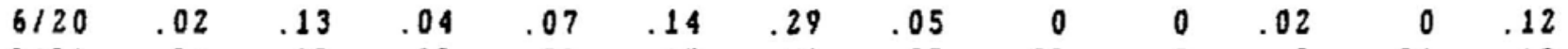

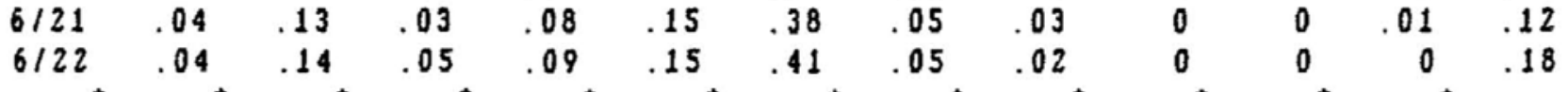


TABLE 23 (page 1 of 2)

Starvation

P. Ethanolamine Fatty Acids as FAME, $\mu \mathrm{g}$ per Individua1

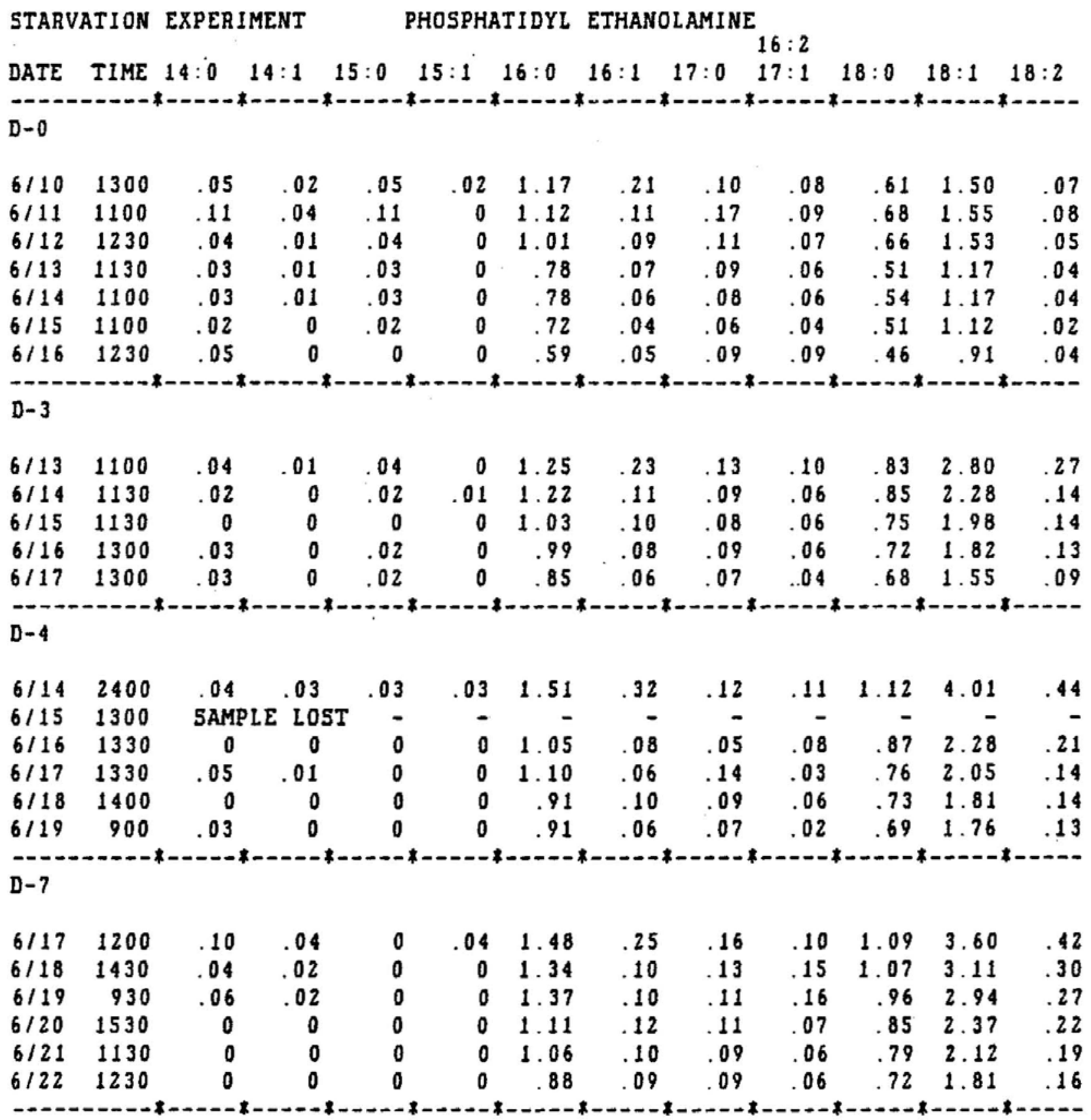


TABLE 23 (page 2 of 2)

Starvation

P. Ethanolamine Fatty Acids as FAME, $\mu$ g per Individual

\section{STARVATION EXPERIMENT PHOSPHATIDYL ETHANOLAMINE}

DATE $18: 2 * \quad 18: 3 \quad 20: 0 \quad 20: 1 \quad 20: 4 \quad 20: 5 \quad 22: 0 \quad 22: 2 ? \quad 22: 3 ? \quad 22: 4 ? \quad 22: 5 \quad 22: 6$

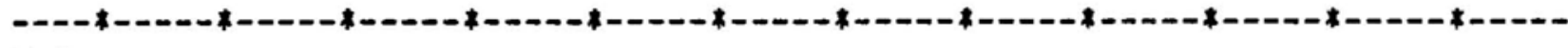
D- 0

\begin{tabular}{|c|c|c|c|c|c|c|c|c|c|c|c|c|}
\hline $6 / 10$ & .10 & .17 & .04 & .30 & .55 & 2.78 & 0 & .03 & .04 & .05 & 0 & 2.73 \\
\hline $6 / 11$ & .17 & .15 & 0 & .35 & .69 & 2.70 & 0 & 0 & .07 & 0 & 0 & 2.02 \\
\hline $6 / 12$ & .10 & .04 & .04 & .26 & .47 & 2.45 & 0 & .04 & .04 & .04 & 0 & 2.19 \\
\hline $6 / 13$ & .08 & .02 & .03 & .21 & .38 & 1.98 & 0 & 0 & .02 & .03 & 0 & 1.73 \\
\hline $6 / 14$ & .07 & .03 & .04 & .21 & .41 & 2.10 & 0 & .07 & .05 & .04 & 0 & 1.6 \\
\hline $6 / 15$ & .06 & .02 & .03 & .21 & 39 & 1.97 & 0 & 0 & .02 & .03 & 0 & 1.59 \\
\hline $6 / 16$ & .09 & .06 & 0 & .19 & .43 & 1.77 & 0 & .07 & .04 & .04 & 0 & 1.3 \\
\hline
\end{tabular}

\begin{tabular}{|c|c|c|c|c|c|c|c|c|c|c|c|c|}
\hline $\begin{array}{l}6 / 13 \\
6 / 14 \\
6 / 15 \\
6 / 16 \\
6 / 17\end{array}$ & $\begin{array}{l}.13 \\
.08 \\
.07 \\
.11 \\
.06\end{array}$ & $\begin{array}{l}.67 \\
.36 \\
.29 \\
.22 \\
.17\end{array}$ & $\begin{array}{l}.10 \\
.05 \\
.03 \\
.04 \\
.03\end{array}$ & $\begin{array}{l}.35 \\
.26 \\
.25 \\
.26 \\
.22\end{array}$ & $\begin{array}{l}.64 \\
.55 \\
.51 \\
.52 \\
.48\end{array}$ & $\begin{array}{l}2.87 \\
2.92 \\
2.61 \\
2.62 \\
2.37\end{array}$ & $\begin{array}{r}.08 \\
0 \\
0 \\
0 \\
0\end{array}$ & $\begin{array}{l}.04 \\
.02 \\
.02 \\
.03 \\
.02\end{array}$ & $\begin{array}{l}.04 \\
.02 \\
.02 \\
.02 \\
.02\end{array}$ & $\begin{array}{l}.06 \\
.04 \\
.03 \\
.03 \\
.04\end{array}$ & $\begin{array}{l}0 \\
0 \\
0\end{array}$ & $\begin{array}{l}2.47 \\
2.37 \\
2.13 \\
1.99 \\
1.91\end{array}$ \\
\hline \multicolumn{13}{|c|}{$\begin{array}{l}---4-0-1 \\
D-4\end{array}$} \\
\hline $\begin{array}{l}6 / 14 \\
6 / 15 \\
6 / 16 \\
6 / 17 \\
6 / 18 \\
6 / 19\end{array}$ & $\begin{array}{r}15 \\
.10 \\
.19 \\
.07 \\
.11\end{array}$ & $\begin{array}{r}1.40 \\
.50 \\
.42 \\
.34 \\
.31\end{array}$ & $\begin{array}{r}.18 \\
.08 \\
0 \\
.05 \\
.03\end{array}$ & $\begin{array}{r}.37 \\
- \\
.30 \\
.34 \\
.23 \\
.25\end{array}$ & $\begin{array}{r}.76 \\
.62 \\
.50 \\
.49 \\
.49\end{array}$ & $\begin{array}{r}3.46 \\
- \\
2.94 \\
2.60 \\
2.43 \\
2.52\end{array}$ & $\begin{array}{r}08 \\
- \\
0 \\
0 \\
0 \\
0\end{array}$ & $\begin{array}{r}.04 \\
- \\
.02 \\
.07 \\
0 \\
.03\end{array}$ & $\begin{array}{r}.03 \\
.03 \\
.18 \\
.11 \\
.12\end{array}$ & $\begin{array}{r}.06 \\
- \\
.04 \\
0 \\
0 \\
0\end{array}$ & $\begin{array}{l}0 \\
- \\
0\end{array}$ & $\begin{array}{l}2.18 \\
1.87 \\
1.70 \\
1.84\end{array}$ \\
\hline \multicolumn{13}{|c|}{ - } \\
\hline $\begin{array}{l}6 / 17 \\
6 / 18 \\
6 / 19 \\
6 / 20 \\
6 / 21 \\
6 / 22\end{array}$ & $\begin{array}{l}.14 \\
.18 \\
.20 \\
.09 \\
.07 \\
.07\end{array}$ & $\begin{array}{r}1.33 \\
.81 \\
.75 \\
.50 \\
.45 \\
.35\end{array}$ & $\begin{array}{l}.19 \\
.08 \\
.07 \\
.06 \\
.04 \\
.05\end{array}$ & $\begin{array}{l}.39 \\
.38 \\
.40 \\
.29 \\
.24 \\
.23\end{array}$ & $\begin{array}{l}.84 \\
.71 \\
.69 \\
.62 \\
.56 \\
.52\end{array}$ & $\begin{array}{l}3.63 \\
3.59 \\
3.47 \\
3.17 \\
2.83 \\
2.45\end{array}$ & $\begin{array}{r}17 \\
0 \\
0 \\
0 \\
0 \\
0\end{array}$ & $\begin{array}{r}.09 \\
.08 \\
.07 \\
.02 \\
.02 \\
0\end{array}$ & $\begin{array}{l}30 \\
.12 \\
.19 \\
.15 \\
.09 \\
.15\end{array}$ & $\begin{array}{l}0 \\
0 \\
0 \\
0 \\
0 \\
0\end{array}$ & $\begin{array}{l}0 \\
0 \\
0 \\
0 \\
0 \\
0\end{array}$ & $\begin{array}{l}3.07 \\
2.39 \\
2.36 \\
2.19 \\
2.02 \\
1.79\end{array}$ \\
\hline
\end{tabular}




\section{TABLE 24 (page 1 of 2)}

\section{Starvation}

P. Choline Fatty Acids as FAME, $\mu \mathrm{g}$ per Individual

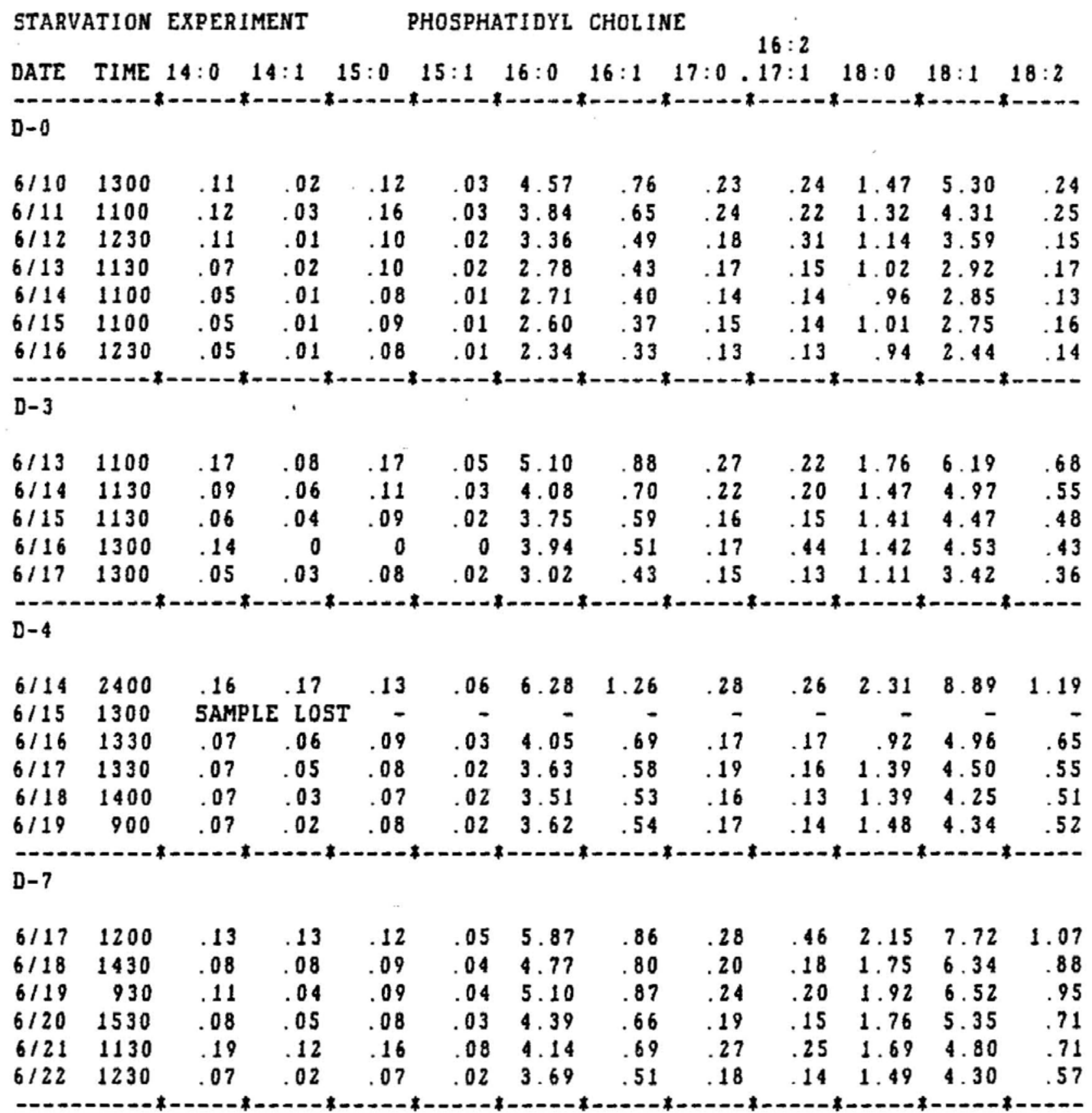


TABLE 24 (page 2 of 2)

Starvation

P. Choline Fatty Acids as FAME, $\mu \mathrm{g}$ per Individual

STARVATION EXPERIMENT PHOSPHATIDYL CHOLINE

DATE $18: 2 * \quad 18: 3 \quad 20: 0 \quad 20: 1 \quad 20: 4 \quad 20: 5 \quad 22: 0 \quad 22: 2 ? \quad 22: 3 ? \quad 22: 4 ? 22: 5 \quad 22: 6$ $\mathrm{D}-\mathrm{O}$

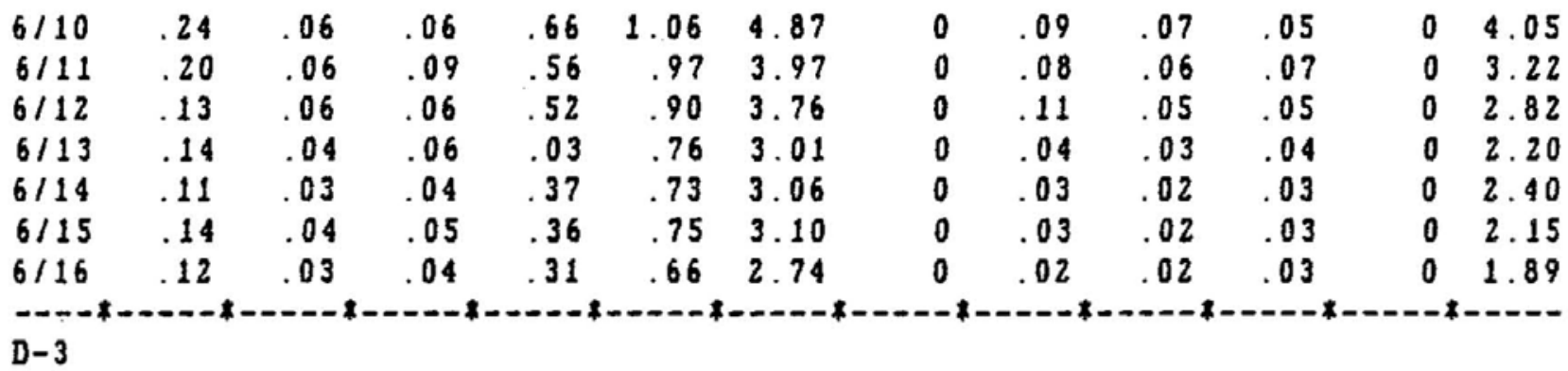

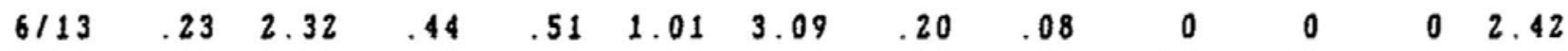

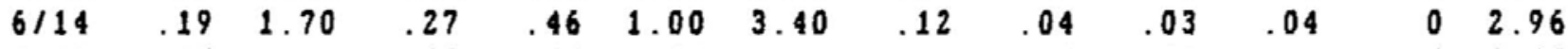

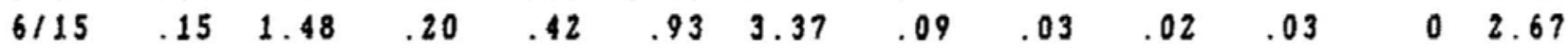

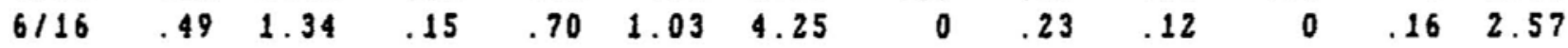

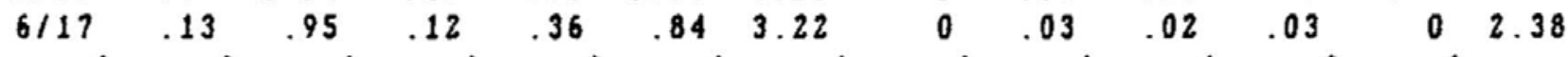

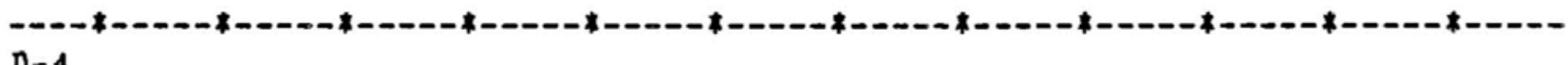

D-4

$\begin{array}{lllllllllllll}6 / 14 & .29 & 5.07 & .85 & .52 & 1.31 & 3.98 & .26 & .05 & .06 & .04 & 0 & 3.22\end{array}$

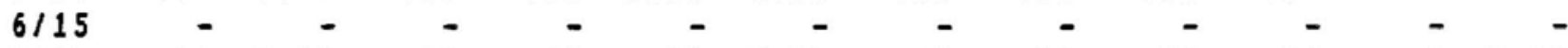

$\begin{array}{lllllllllllll}6 / 16 & .17 & 2.21 & .27 & .43 & .77 & 2.61 & .13 & .03 & .02 & .03 & 0 & 2.56\end{array}$

$\begin{array}{lllllllllllll}6 / 17 & .16 & 1.76 & .21 & .45 & 1.01 & 3.25 & .12 & .02 & .09 & 0 & 0 & 2.50\end{array}$

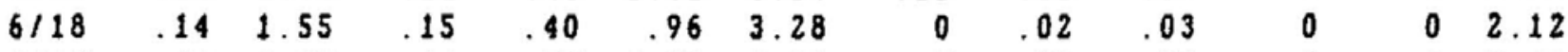

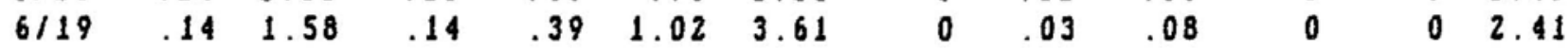

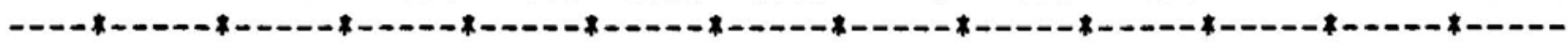

D-7

$\begin{array}{lllllllllllll}6 / 17 & .27 & 4.19 & .66 & .53 & 1.41 & 4.36 & .25 & .05 & .18 & 0 & 0 & 3.30\end{array}$

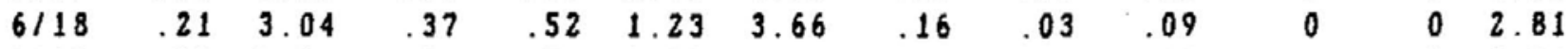

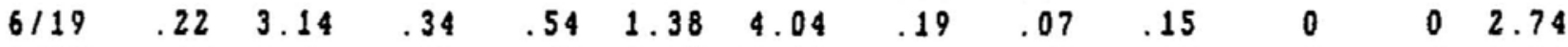

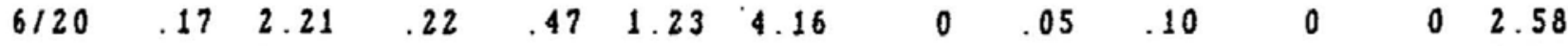

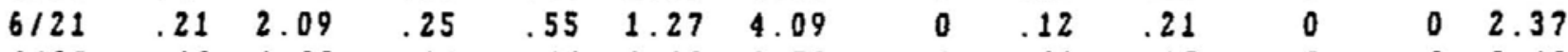

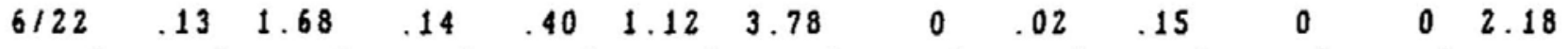

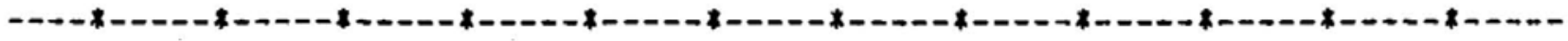




\section{TABLE 25}

Control vs. CLO Supplement

Proximate Analysis

CONTROL VS CLO SUPPLEMENT

MGIIND. MGIIND. MGIIND. DRY/WET WT. DATE TIME STAGE DIET WET WT. 5.D. WATER S.D. DRY WT. S.D. $\%$ S.D.

\begin{tabular}{|c|c|c|c|c|c|c|c|c|c|c|c|}
\hline $\begin{array}{l}6 / 13 \\
6 / 13\end{array}$ & $\begin{array}{l}1300 \\
1330\end{array}$ & $\begin{array}{l}1-D 1,3 \\
1-D 1,3\end{array}$ & $\begin{array}{c}\text { CONTROL } \\
\text { CLO }\end{array}$ & $\begin{array}{l}7.86 \\
7.62\end{array}$ & $\begin{array}{l}.24 \\
.30\end{array}$ & $\begin{array}{l}6.26 \\
6.02\end{array}$ & $\begin{array}{l}.25 \\
.24\end{array}$ & $\begin{array}{l}1.60 \\
1.60\end{array}$ & $\begin{array}{l}.08 \\
.07\end{array}$ & $\begin{array}{l}20.32 \\
20.96\end{array}$ & $\begin{array}{l}.65 \\
.49\end{array}$ \\
\hline $\begin{array}{l}6 / 16 \\
6 / 16\end{array}$ & $\begin{array}{l}1100 \\
1030\end{array}$ & $\begin{array}{l}2-C \\
2-C\end{array}$ & $\begin{array}{c}\text { CONTROL } \\
\text { CLO }\end{array}$ & $\begin{array}{l}12.45^{\circ} \\
13.30\end{array}$ & $\begin{array}{l}1.36 \\
1.08\end{array}$ & $\begin{array}{l}10.37 \\
11.29\end{array}$ & $\begin{array}{r}1.38 \\
.92\end{array}$ & $\begin{array}{l}2.08 \\
2.01\end{array}$ & $\begin{array}{l}.22 \\
.21\end{array}$ & $\begin{array}{l}16.75 \\
15.14\end{array}$ & $\begin{array}{l}.83 \\
.97\end{array}$ \\
\hline $\begin{array}{l}6 / 22 \\
6 / 22\end{array}$ & $\begin{array}{l}1200 \\
1130\end{array}$ & $\begin{array}{l}3-D 0 \\
3-D 0\end{array}$ & $\begin{array}{l}\text { CONTROL } \\
\text { CLO }\end{array}$ & $\begin{array}{l}23.39 \\
24.59\end{array}$ & $\begin{array}{l}2.58 \\
2.04\end{array}$ & $\begin{array}{l}19.14 \\
20.27\end{array}$ & $\begin{array}{l}2.61 \\
1.69\end{array}$ & $\begin{array}{l}4.25 \\
4.31\end{array}$ & $\begin{array}{l}.41 \\
.47\end{array}$ & $\begin{array}{l}18.23 \\
17.54\end{array}$ & $\begin{array}{l}1.92 \\
1.17\end{array}$ \\
\hline $\begin{array}{l}6 / 28 \\
6 / 28\end{array}$ & $\begin{array}{l}1300 \\
1230\end{array}$ & $\begin{array}{l}4-C \\
4-C\end{array}$ & $\begin{array}{l}\text { CONTROL } \\
\text { CLO }\end{array}$ & $\begin{array}{l}39.04 \\
40.19\end{array}$ & $\begin{array}{l}5.34 \\
4.16\end{array}$ & $\begin{array}{l}31.58 \\
33.29\end{array}$ & $\begin{array}{l}5.89 \\
3.07\end{array}$ & $\begin{array}{l}7.46 \\
6.89\end{array}$ & $\begin{array}{l}2.48 \\
1.35\end{array}$ & $\begin{array}{l}18.68 \\
17.06\end{array}$ & $\begin{array}{l}4.05 \\
2.10\end{array}$ \\
\hline
\end{tabular}

MGIIND. $\quad$ DRY WT. MGIIND. \$ DRY WT. MGIIND.

DATE DIET ASH S.D. ASH 5.D. CHITIN 5.D. CHITIN 5.D. CARBO. 5.D.

\begin{tabular}{|c|c|c|c|c|c|c|c|c|c|c|c|}
\hline $\begin{array}{l}6 / 13 \\
6 / 13\end{array}$ & $\begin{array}{l}\text { CONT. } \\
\text { CLO }\end{array}$ & $\begin{array}{l}.28 \\
.28\end{array}$ & $\begin{array}{l}.01 \\
.01\end{array}$ & $\begin{array}{l}17.82 \\
17.84\end{array}$ & $\begin{array}{r}1.15 \\
.22\end{array}$ & $\begin{array}{l}.13 \\
.15\end{array}$ & $\begin{array}{l}.01 \\
.01\end{array}$ & $\begin{array}{l}8.44 \\
9.08\end{array}$ & $\begin{array}{l}.37 \\
.42\end{array}$ & $\begin{array}{l}.08 \\
.08\end{array}$ & $\begin{array}{r}.004 \\
.01\end{array}$ \\
\hline $\begin{array}{l}6 / 16 \\
6 / 16\end{array}$ & $\begin{array}{l}\text { CONT. } \\
\text { CLO }\end{array}$ & $\begin{array}{l}.54 \\
.57\end{array}$ & $\begin{array}{l}.09 \\
.05\end{array}$ & $\begin{array}{r}26.52 \\
30.17\end{array}$ & $\begin{array}{r}1.23 \\
.87\end{array}$ & $\begin{array}{l}.15 \\
.16\end{array}$ & $\begin{array}{l}.004 \\
.001\end{array}$ & $\begin{array}{l}7.44 \\
8.22\end{array}$ & $\begin{array}{l}.10 \\
.37\end{array}$ & $\begin{array}{l}.08 \\
.06\end{array}$ & $\begin{array}{l}.02 \\
.01\end{array}$ \\
\hline $\begin{array}{l}6 / 22 \\
6 / 22\end{array}$ & $\begin{array}{l}\text { CONT. } \\
\text { CLO }\end{array}$ & $\begin{array}{l}1.02 \\
1.23\end{array}$ & $\begin{array}{l}.03 \\
.10\end{array}$ & $\begin{array}{l}24.82 \\
26.18\end{array}$ & $\begin{array}{r}3.25 \\
.08\end{array}$ & $\begin{array}{l}.36 \\
.39\end{array}$ & $\begin{array}{r}.01 \\
0\end{array}$ & $\begin{array}{l}9.12 \\
8.84\end{array}$ & $\begin{array}{r}.46 \\
0\end{array}$ & $\begin{array}{l}.20 \\
.16\end{array}$ & $\begin{array}{l}.04 \\
.02\end{array}$ \\
\hline $6 / 28$ & $\begin{array}{l}\text { CONT. } \\
\text { CLO }\end{array}$ & $\begin{array}{l}2.98 \\
2.48\end{array}$ & $\begin{array}{l}.37 \\
.24\end{array}$ & $\begin{array}{l}34.08 \\
34.30\end{array}$ & $\begin{array}{r}1.90 \\
.28\end{array}$ & $\begin{array}{l}.41 \\
.84\end{array}$ & $\begin{array}{l}.06 \\
.01\end{array}$ & $\begin{array}{r}6.69 \\
12.00\end{array}$ & $\begin{array}{l}.82 \\
.16\end{array}$ & $\begin{array}{l}.29 \\
.24\end{array}$ & .11 \\
\hline
\end{tabular}

\% DRY WT. MG/IND. \% DRY WT. MG/IND. \% DRY WT. DATE DIET CARBO. 5.D. PROTEIN 5.D. PROTEIN S.D. LIPID S.D. LIPID S.D.

\begin{tabular}{|c|c|c|c|c|c|c|c|c|c|c|c|}
\hline $\begin{array}{l}6 / 13 \\
6 / 13\end{array}$ & $\begin{array}{l}\text { CONT. } \\
\text { CLO }\end{array}$ & $\begin{array}{l}4.43 \\
4.96\end{array}$ & $\begin{array}{l}.12 \\
.30\end{array}$ & $\begin{array}{r}.97 \\
1.00\end{array}$ & $\begin{array}{l}.05 \\
.06\end{array}$ & $\begin{array}{l}59.59 \\
61.06\end{array}$ & $\begin{array}{r}.89 \\
1.15\end{array}$ & $\begin{array}{l}.14 \\
.15\end{array}$ & $\begin{array}{l}.01 \\
.01\end{array}$ & $\begin{array}{l}9.00 \\
9.34\end{array}$ & $\begin{array}{l}.83 \\
.64\end{array}$ \\
\hline $\begin{array}{l}6 / 16 \\
6 / 16\end{array}$ & $\begin{array}{l}\text { CONT. } \\
\text { CLO }\end{array}$ & $\begin{array}{l}3.69 \\
3.06\end{array}$ & $\begin{array}{l}.52 \\
.18\end{array}$ & $\begin{array}{l}1.10 \\
1.02\end{array}$ & $\begin{array}{l}.12 \\
.10\end{array}$ & $\begin{array}{l}51.57 \\
48.46\end{array}$ & $\begin{array}{l}1.70 \\
1.66\end{array}$ & $\begin{array}{l}.15 \\
.16\end{array}$ & $\begin{array}{r}.001 \\
.02\end{array}$ & $\begin{array}{l}7.31 \\
8.08\end{array}$ & $\begin{array}{l}.16 \\
.62\end{array}$ \\
\hline $\begin{array}{l}6 / 22 \\
6 / 22\end{array}$ & $\begin{array}{l}\text { CONT. } \\
\text { CLO }\end{array}$ & $\begin{array}{l}4.48 \\
4.03\end{array}$ & $\begin{array}{l}.54 \\
.37\end{array}$ & $\begin{array}{l}2.13 \\
1.91\end{array}$ & $\begin{array}{l}.27 \\
.21\end{array}$ & $\begin{array}{l}49.23 \\
47.29\end{array}$ & $\begin{array}{l}3.86 \\
2.30\end{array}$ & $\begin{array}{l}.31 \\
.41\end{array}$ & $\begin{array}{l}.01 \\
.01\end{array}$ & $\begin{array}{l}7.88 \\
9.74\end{array}$ & $\begin{array}{l}.4 \\
.8\end{array}$ \\
\hline $\begin{array}{l}6 / 28 \\
6 / 28\end{array}$ & $\begin{array}{l}\text { CONT. } \\
\text { CLO }\end{array}$ & $\begin{array}{l}4.55 \\
3.54\end{array}$ & $\begin{array}{l}.42 \\
.43\end{array}$ & $\begin{array}{l}2.83 \\
2.88\end{array}$ & $\begin{array}{r}1.07 \\
.68\end{array}$ & $\begin{array}{l}44.54 \\
43.56\end{array}$ & $\begin{array}{l}3.38 \\
1.96\end{array}$ & $\begin{array}{l}.40 \\
.61\end{array}$ & $\begin{array}{l}.05 \\
.06\end{array}$ & $\begin{array}{l}6.59 \\
8.74\end{array}$ & \\
\hline
\end{tabular}


TABLE 26 (page 1 of 2)

Control vs. CLO Supplement

Lipid Classes

\begin{tabular}{|c|c|c|c|c|c|c|c|c|c|c|}
\hline & & & & & UG $/ \mathrm{I}$. & ND. & UGI & ND. & \% DRY & WT \\
\hline DATE & TIME & STAGE & DIET & & DRY WT & S.D. & $5 E$ & S.D. & $S E$ & S.D \\
\hline $\begin{array}{l}6 / 13 \\
6 / 13\end{array}$ & $\begin{array}{l}1300 \\
1330\end{array}$ & $\begin{array}{l}1-D 1,3 \\
1-D 1,3\end{array}$ & $\begin{array}{c}\text { CONTROL } \\
\text { CLO }\end{array}$ & & $\begin{array}{l}1590 \\
1620\end{array}$ & $\begin{array}{l}50 \\
40\end{array}$ & $\begin{array}{l}3.51 \\
2.21\end{array}$ & $\begin{array}{l}.59 \\
.59\end{array}$ & $\begin{array}{l}.22 \\
.14\end{array}$ & $\begin{array}{l}.04 \\
.04\end{array}$ \\
\hline $\begin{array}{l}6 / 16 \\
6 / 16\end{array}$ & $\begin{array}{l}1100 \\
1030\end{array}$ & $\begin{array}{l}2-C \\
2-C\end{array}$ & $\begin{array}{l}\text { CONTROL } \\
\text { CLO }\end{array}$ & & $\begin{array}{l}2040 \\
1910\end{array}$ & $\begin{array}{l}100 \\
120\end{array}$ & $\begin{array}{l}2.22 \\
1.48\end{array}$ & $\begin{array}{l}.20 \\
.19\end{array}$ & $\begin{array}{l}.11 \\
.08\end{array}$ & $\begin{array}{l}.01 \\
.01\end{array}$ \\
\hline $\begin{array}{l}6 / 22 \\
6 / 22\end{array}$ & $\begin{array}{l}1200 \\
1130\end{array}$ & $\begin{array}{l}\text { 3-DO } \\
3-D 0\end{array}$ & $\begin{array}{l}\text { CONTROL } \\
\text { CLO }\end{array}$ & & $\begin{array}{l}3980 \\
4050\end{array}$ & $\begin{array}{l}420 \\
270\end{array}$ & $\begin{array}{l}5.61 \\
6.22\end{array}$ & $\begin{array}{r}.38 \\
1.21\end{array}$ & $\begin{array}{l}.14 \\
.15\end{array}$ & $\begin{array}{l}.02 \\
.03\end{array}$ \\
\hline $\begin{array}{l}6 / 28 \\
6 / 28\end{array}$ & $\begin{array}{l}1300 \\
1230\end{array}$ & $\begin{array}{l}4-C \\
4-C\end{array}$ & $\begin{array}{c}\text { CONTROL } \\
\text { CLO }\end{array}$ & & $\begin{array}{l}6190 \\
6980\end{array}$ & $\begin{array}{r}1350 \\
860\end{array}$ & $\begin{array}{l}2.85 \\
4.32\end{array}$ & $\begin{array}{l}.72 \\
.40\end{array}$ & $\begin{array}{l}.05 \\
.06\end{array}$ & \\
\hline ONT & ROL VS & CLO SUI & $\begin{array}{r}\text { PLEMENT } \\
\text { UG }\end{array}$ & & \% DRY & WT. & UG I & ND. & \% DRY & WT \\
\hline DATE & DIET & & TRI & S.D & TAI & S.D. & FFA & S.D. & FFA & S.D \\
\hline $\begin{array}{l}6 / 13 \\
6 / 13\end{array}$ & $\begin{array}{l}\text { CONT. } \\
\text { CLO }\end{array}$ & & $\begin{array}{l}2.45 \\
4.54\end{array}$ & $\begin{array}{l}.29 \\
.55\end{array}$ & $\begin{array}{l}.15 \\
.28\end{array}$ & $\begin{array}{l}.02 \\
.03\end{array}$ & $\begin{array}{r}1.20 \\
0\end{array}$ & $\begin{array}{r}16 \\
0\end{array}$ & $\begin{array}{r}.08 \\
0\end{array}$ &. \\
\hline $\begin{array}{l}6 / 16 \\
6 / 16\end{array}$ & $\begin{array}{l}\text { CONT. } \\
\text { CLO }\end{array}$ & & $\begin{array}{l}4.81 \\
3.78\end{array}$ & $\begin{array}{l}.38 \\
.77\end{array}$ & $\begin{array}{l}.24 \\
.20\end{array}$ & $\begin{array}{l}.02 \\
.04\end{array}$ & $\begin{array}{r}1.49 \\
.90\end{array}$ & $\begin{array}{l}.18 \\
.23\end{array}$ & $\begin{array}{l}.07 \\
.05\end{array}$ & $\begin{array}{l}.01 \\
.01\end{array}$ \\
\hline $\begin{array}{l}6 / 22 \\
6 / 22\end{array}$ & $\begin{array}{l}\text { CONT. } \\
\text { CLO }\end{array}$ & & $\begin{array}{l}50.10 \\
92.15\end{array}$ & $\begin{array}{l}3.93 \\
4.85\end{array}$ & $\begin{array}{l}1.26 \\
2.28\end{array}$ & $\begin{array}{l}.17 \\
.19\end{array}$ & $\begin{array}{l}3.47 \\
5.20\end{array}$ & $\begin{array}{r}.40 \\
1.85\end{array}$ & $\begin{array}{l}.09 \\
.13\end{array}$ & $\begin{array}{l}.01 \\
.05\end{array}$ \\
\hline $\begin{array}{l}6 / 28 \\
6 / 28\end{array}$ & $\begin{array}{l}\text { CONT. } \\
\text { CLO }\end{array}$ & & $\begin{array}{r}67.01 \\
194.60\end{array}$ & $\begin{array}{l}7.82 \\
18.6\end{array}$ & $\begin{array}{l}1.08 \\
2.79\end{array}$ & $\begin{array}{l}.27 \\
.43\end{array}$ & $\begin{array}{r}3.98 \\
0\end{array}$ & $\begin{array}{r}.56 \\
0\end{array}$ & $\begin{array}{r}.06 \\
0\end{array}$ & .02 \\
\hline
\end{tabular}

CONTROL VS CLO SUPPLEMENT

\begin{tabular}{|c|c|c|c|c|c|c|c|c|c|}
\hline & & UGI & VD. & \% DRY & WT. & UG I & VD. & \% DRY & WT. \\
\hline DATE & DIET & STER & S.D. & STER & S.D. & $1-2 \quad D I$ & S.D. & $1-2 \mathrm{DI}$ & S.D. \\
\hline $\begin{array}{l}6 / 13 \\
6 / 13\end{array}$ & $\begin{array}{l}\text { CONT. } \\
\text { CLO }\end{array}$ & $\begin{array}{l}14.59 \\
15.59\end{array}$ & $\begin{array}{l}1.60 \\
1.26\end{array}$ & $\begin{array}{l}.92 \\
.96\end{array}$ & $\begin{array}{l}.10 \\
.08\end{array}$ & $\begin{array}{l}1.20 \\
1.51\end{array}$ & $\begin{array}{l}.24 \\
.36\end{array}$ & $\begin{array}{l}.08 \\
.09\end{array}$ & $\begin{array}{l}.02 \\
.02\end{array}$ \\
\hline $\begin{array}{l}6 / 16 \\
6 / 16\end{array}$ & $\begin{array}{l}\text { CONT. } \\
\text { CLO }\end{array}$ & $\begin{array}{l}16.55 \\
18.85\end{array}$ & $\begin{array}{r}.63 \\
2.55\end{array}$ & $\begin{array}{l}.81 \\
.99\end{array}$ & $\begin{array}{l}.05 \\
.15\end{array}$ & $\begin{array}{l}2.29 \\
2.12\end{array}$ & $\begin{array}{l}.32 \\
.48\end{array}$ & $\begin{array}{l}.11 \\
.11\end{array}$ & $\begin{array}{l}.02 \\
.03\end{array}$ \\
\hline $\begin{array}{l}6 / 22 \\
6 / 22\end{array}$ & $\begin{array}{l}\text { CONT. } \\
\text { CLO }\end{array}$ & $\begin{array}{l}27.42 \\
32.04\end{array}$ & $\begin{array}{l}1.92 \\
2.42\end{array}$ & $\begin{array}{l}.69 \\
.79\end{array}$ & $\begin{array}{l}.09 \\
.08\end{array}$ & $\begin{array}{l}5.86 \\
7.90\end{array}$ & $\begin{array}{r}.47 \\
1.06\end{array}$ & $\begin{array}{l}.15 \\
.20\end{array}$ & $\begin{array}{l}.02 \\
.03\end{array}$ \\
\hline $\begin{array}{l}6 / 28 \\
6 / 28\end{array}$ & $\begin{array}{l}\text { CONT. } \\
\text { CLO }\end{array}$ & $\begin{array}{l}40.27 \\
47.52\end{array}$ & $\begin{array}{l}5.85 \\
5.76\end{array}$ & $\begin{array}{l}.65 \\
.68\end{array}$ & $\begin{array}{l}.17 \\
.12\end{array}$ & $\begin{array}{l}2.85 \\
4.62\end{array}$ & $\begin{array}{r}.37 \\
1.35\end{array}$ & $\begin{array}{l}.05 \\
.07\end{array}$ & $\begin{array}{l}.01 \\
.02\end{array}$ \\
\hline
\end{tabular}


TABLE 26 (page 2 of 2)

Control vs. CLO Supplement

Lipid Classes

CONTROL VS CLO SUPPLEMENT

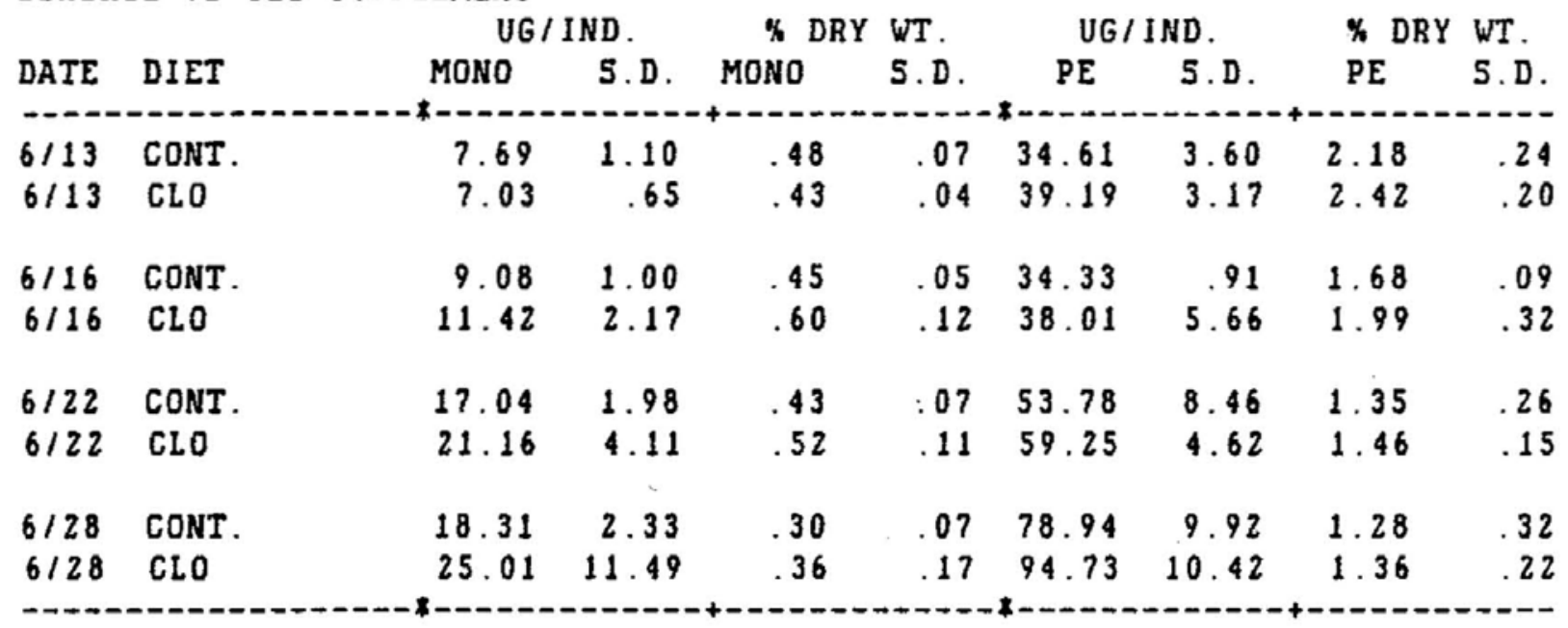

CONTROL VS CLO SUPPLEMENT

\begin{tabular}{|c|c|c|c|c|c|c|c|c|c|}
\hline & & JGI & VD. & $\%$ DRY & WT. & UG & IND. & $\%$ DF & $W T$. \\
\hline DATE & DIET & PI & S.D. & PI & S.D. & PC & S.D. & PC & S.D. \\
\hline & & & & & & & & & \\
\hline $6 / 13$ & CONT. & 8.37 & 1.73 & .53 & .11 & 61.00 & 6.72 & 3.84 & .44 \\
\hline $6 / 13$ & ClO & 8.43 & 1.08 & .52 & .07 & 65.60 & 4.42 & 4.05 & .29 \\
\hline $6 / 16$ & CONT. & 10.49 & .08 & .51 & .03 & 55.03 & 1.72 & 2.70 & .16 \\
\hline $6 / 16$ & CLO & 11.42 & 2.72 & .60 & .15 & 60.01 & 8.46 & 3.14 & .48 \\
\hline $6 / 22$ & CONT. & 15.15 & .76 & .38 & .04 & 98.73 & 6.32 & 2.48 & .31 \\
\hline $6 / 22$ & CLO & 23.00 & 1.66 & .57 & .06 & 126.70 & 9.02 & 3.13 & .31 \\
\hline $6 / 28$ & CONT. & 13.05 & 1.50 & .21 & .05 & 147.90 & 17.95 & 2.39 & .60 \\
\hline $6 / 28$ & CLO & 23.00 & 2.11 & .33 & .05 & 183.60 & 17.46 & 2.63 & .41 \\
\hline
\end{tabular}

CONTROL VS CLO SUPPLEMENT

\begin{tabular}{|c|c|c|c|c|c|c|c|c|c|}
\hline \multirow[b]{2}{*}{ DATE } & \multirow[b]{2}{*}{ DIET } & \multicolumn{2}{|c|}{ UG/IND. } & \multirow{2}{*}{$\begin{array}{l}\text { \% DRY } \\
5 M\end{array}$} & \multirow{2}{*}{$\begin{array}{l}\text { WT. } \\
\text { 5.D. }\end{array}$} & \multicolumn{2}{|c|}{ UG /IND. } & \multicolumn{2}{|c|}{ \% DRY WT. } \\
\hline & & SM & S.D. & & & UNK. & S.D. & UNK. & S.D. \\
\hline $6 / 13$ & CONT. & 3.71 & .69 & .23 & .04 & 4. 29 & .53 & .27 & .03 \\
\hline $6 / 13$ & CLO & 4.06 & .86 & .25 & .05 & 4.02 & .53 & .25 & .03 \\
\hline $6 / 16$ & CONT. & 6.18 & .57 & .30 & .03 & 7.85 & .51 & .38 & .03 \\
\hline $6 / 16$ & CLO & 6.03 & 1.16 & .32 & .06 & 6.80 & .92 & .36 & .05 \\
\hline $6 / 22$ & CONT. & 12.76 & .98 & .32 & .04 & 19.92 & 1.05 & .50 & .06 \\
\hline $6 / 22$ & CLO & 14.77 & 2.10 & .36 & .06 & 20.83 & 3.63 & .51 & .10 \\
\hline $6 / 28$ & CONT. & 11.44 & 1.96 & .18 & .05 & 14.82 & 2.70 & 24 & .07 \\
\hline $6 / 28$ & CLO & 13.26 & 2.19 & .19 & .04 & 17.89 & 4.17 & .26 & .07 \\
\hline
\end{tabular}


TABLE 27 (page 1 of 2)

Control vs. CLO Supplement

Fatty Acids as FAME, $\mu \mathrm{g}$ per Individual

CONTROL VS CLO SUPPLEMENT NEUTRAL LIPIDS

\begin{tabular}{|c|c|c|c|c|c|c|c|c|c|c|c|c|}
\hline DATE & TIME & DIET & $14: 0$ & $14: 1$ & $15: 0$ & $15: 1$ & $16: 0$ & $16: 1$ & $17: 0$ & $\begin{array}{l}16: 2 \\
17: 1\end{array}$ & $18: 0$ & $18: 1$ \\
\hline & & & . & - & ----1 & --- & $*--\infty-1$ & ----1 & --- & -----4 & --- & 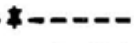 \\
\hline $\begin{array}{l}6 / 13 \\
6 / 13\end{array}$ & $\begin{array}{l}1300 \\
1330\end{array}$ & $\begin{array}{l}\text { CONT. } \\
\text { CLO }\end{array}$ & $\begin{array}{l}.05 \\
.08\end{array}$ & $\begin{array}{l}.06 \\
.14\end{array}$ & $\begin{array}{l}0 \\
0\end{array}$ & $\begin{array}{l}0 \\
0\end{array}$ & $\begin{array}{r}.70 \\
1.06\end{array}$ & $\begin{array}{l}.17 \\
.40\end{array}$ & $\begin{array}{l}.04 \\
.05\end{array}$ & $\begin{array}{l}.04 \\
.05\end{array}$ & $\begin{array}{l}.21 \\
.26\end{array}$ & $\begin{array}{l}1.24 \\
1.88\end{array}$ \\
\hline $\begin{array}{l}6 / 16 \\
6 / 16\end{array}$ & $\begin{array}{l}1100 \\
1030\end{array}$ & $\begin{array}{l}\text { CONT. } \\
\text { CLO }\end{array}$ & $\begin{array}{l}.08 \\
.15\end{array}$ & $\begin{array}{l}.08 \\
.14\end{array}$ & $\begin{array}{l}.03 \\
.06\end{array}$ & $\begin{array}{r}0 \\
.05\end{array}$ & $\begin{array}{l}1.30 \\
1.97\end{array}$ & $\begin{array}{l}.44 \\
.91\end{array}$ & $\begin{array}{l}.05 \\
.08\end{array}$ & $\begin{array}{l}.06 \\
.14\end{array}$ & $\begin{array}{l}.37 \\
.43\end{array}$ & $\begin{array}{l}2.62 \\
4.46\end{array}$ \\
\hline $\begin{array}{l}6 / 22 \\
6 / 22\end{array}$ & $\begin{array}{l}1200 \\
1130\end{array}$ & $\begin{array}{l}\text { CONT. } \\
\text { CLO }\end{array}$ & $\begin{array}{l}.88 \\
.90\end{array}$ & $\begin{array}{l}2.31 \\
1.99\end{array}$ & $\begin{array}{r}0 \\
.26\end{array}$ & $\begin{array}{r}.71 \\
.43\end{array}$ & $\begin{array}{r}7.63 \\
11.17\end{array}$ & $\begin{array}{l}3.55 \\
4.74\end{array}$ & $\begin{array}{r}1.05 \\
.76\end{array}$ & $\begin{array}{l}.84 \\
.52\end{array}$ & $\begin{array}{l}2.08 \\
2.15\end{array}$ & $\begin{array}{l}18.53 \\
27.74\end{array}$ \\
\hline $\begin{array}{l}6 / 28 \\
6 / 28\end{array}$ & $\begin{array}{l}1300 \\
1230\end{array}$ & $\begin{array}{l}\text { CONT. } \\
\text { CLO }\end{array}$ & $\begin{array}{l}.52 \\
.89\end{array}$ & $\begin{array}{r}.84 \\
2.41\end{array}$ & $\begin{array}{r}0 \\
.22\end{array}$ & $\begin{array}{l}.28 \\
.62\end{array}$ & $\begin{array}{r}4.65 \\
14.54\end{array}$ & $\begin{array}{l}1.55 \\
6.22\end{array}$ & $\begin{array}{r}.65 \\
1.26\end{array}$ & $\begin{array}{l}.33 \\
.75\end{array}$ & $\begin{array}{l}1.36 \\
3.30\end{array}$ & $\begin{array}{l}10.51 \\
36.04\end{array}$ \\
\hline
\end{tabular}

CONTROL VS CLO SUPPLEMENT PHOSPHATIDYL ETHANOLAMINE $16: 2$

$\begin{array}{llllllllll}\text { DATE TIME DIET } 14: 0 & 14: 1 & 15: 0 & 15: 1 & 16: 0 & 16: 1 & 17: 0 & 17: 1 & 18: 0 & 18: 1\end{array}$

\begin{tabular}{|c|c|c|c|c|c|c|c|c|c|c|c|c|}
\hline $\begin{array}{l}6 / 13 \\
6 / 13\end{array}$ & $\begin{array}{l}1300 \\
1330\end{array}$ & $\begin{array}{l}\text { CONT. } \\
\text { CLO }\end{array}$ & $\begin{array}{r}.09 \\
0\end{array}$ & $\begin{array}{r}.02 \\
0\end{array}$ & $\begin{array}{l}0 \\
0\end{array}$ & $\begin{array}{l}0 \\
0\end{array}$ & $\begin{array}{l}1.85 \\
1.47\end{array}$ & $\begin{array}{l}.06 \\
.22\end{array}$ & $\begin{array}{l}.12 \\
.13\end{array}$ & $\begin{array}{l}.25 \\
.10\end{array}$ & $\begin{array}{l}1.13 \\
1.11\end{array}$ & $\begin{array}{l}3.88 \\
3.62\end{array}$ \\
\hline $\begin{array}{l}6 / 16 \\
6 / 16\end{array}$ & $\begin{array}{l}1100 \\
1030\end{array}$ & $\begin{array}{l}\text { CONT. } \\
\text { CLO }\end{array}$ & $\begin{array}{r}.03 \\
0\end{array}$ & $\begin{array}{l}0 \\
0\end{array}$ & $\begin{array}{l}0 \\
0\end{array}$ & $\begin{array}{l}0 \\
0\end{array}$ & $\begin{array}{l}1.39 \\
1.46\end{array}$ & $\begin{array}{l}.20 \\
.25\end{array}$ & $\begin{array}{l}.13 \\
.15\end{array}$ & $\begin{array}{l}11 \\
.10\end{array}$ & $\begin{array}{l}1.04 \\
1.14\end{array}$ & $\begin{array}{l}3.57 \\
4.03\end{array}$ \\
\hline $\begin{array}{l}6 / 22 \\
6 / 22\end{array}$ & $\begin{array}{l}1200 \\
1130\end{array}$ & $\begin{array}{l}\text { CONT. } \\
\text { CLO }\end{array}$ & $\begin{array}{r}.09 \\
0\end{array}$ & $\begin{array}{r}.08 \\
0\end{array}$ & $\begin{array}{l}0 \\
0\end{array}$ & $\begin{array}{l}0 \\
0\end{array}$ & $\begin{array}{l}2.48 \\
3.09\end{array}$ & $\begin{array}{r}.34 \\
.49\end{array}$ & $\begin{array}{l}.25 \\
.29\end{array}$ & $\begin{array}{l}.15 \\
.17\end{array}$ & $\begin{array}{l}2.04 \\
2.56\end{array}$ & $\begin{array}{l}6.98 \\
8.71\end{array}$ \\
\hline $\begin{array}{l}128 \\
1 / 28\end{array}$ & $\begin{array}{l}1300 \\
1230\end{array}$ & $\begin{array}{l}\text { CONT. } \\
\text { CLO }\end{array}$ & $\begin{array}{r}.09 \\
0\end{array}$ & $\begin{array}{r}.06 \\
0\end{array}$ & $\begin{array}{l}0 \\
0\end{array}$ & $\begin{array}{r}.08 \\
0\end{array}$ & $\begin{array}{l}2.86 \\
3.45\end{array}$ & $\begin{array}{l}.45 \\
.63\end{array}$ & $\begin{array}{l}29 \\
.39\end{array}$ & $\begin{array}{l}.17 \\
.19\end{array}$ & $\begin{array}{l}1.98 \\
2.91\end{array}$ & $\begin{array}{r}7.77 \\
10.38\end{array}$ \\
\hline
\end{tabular}

CONTROL US CLO SUPPLEMENT PHOSPHATIDYL CHOLINE

$16: 2$

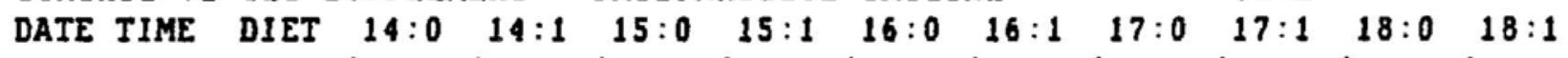

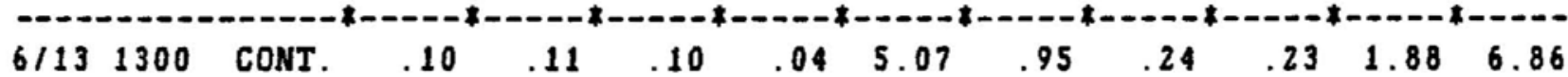

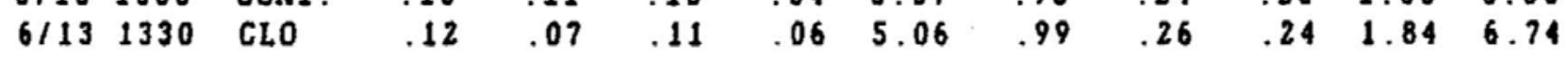

$\begin{array}{llllllllllllll}6 / 16 & 1100 & \text { CONT. } & .12 & .15 & .12 & .05 & 5.05 & .98 & .26 & .21 & 1.78 & 6.74\end{array}$

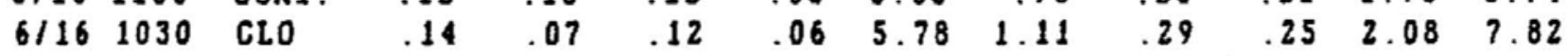

$\begin{array}{lllllllllllll}6 / 22 & 1200 & \text { CONT. } & .24 & .32 & .18 & .12 & 10.67 & 1.97 & .57 & .43 & 4.44 & 14.94\end{array}$

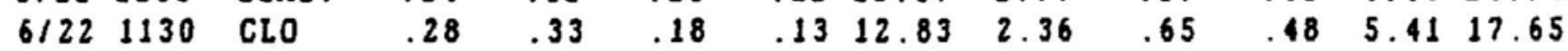

$\begin{array}{llllllllllllll}6 / 28 & 1300 & \text { CONT. } & .29 & .42 & .20 & .19 & 13.62 & 2.25 & .72 & .52 & 5.33 & 17.78\end{array}$

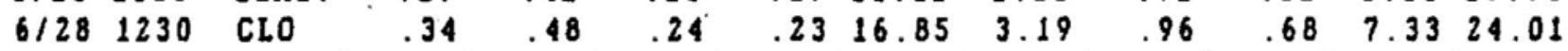


TABLE 27 (page 2 of 2)

Control vs. CLO Supplement

Fatty Acids as FAME, $\mu \mathrm{g}$ per Individual

CONTROL VS CLO SUPPLEMENT NEUTRAL LIPIDS

$\begin{array}{ll}22: 5 & \end{array}$

DATE DIET $18: 2 \quad 18: 2 * \quad 18: 3 \quad 20: 0 \quad 20: 1 \quad 20: 4 \quad 20: 5 \quad 22: 0 \quad 22: 2 ? \quad 22: 3 ? \quad 22: 6$

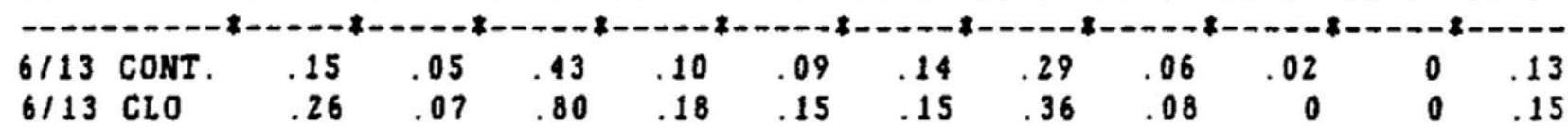

$\begin{array}{lllllllllllll}6 / 16 & \text { CONT. } & .38 & .09 & 1.09 & .22 & .12 & .22 & .38 & : 10 & .02 & .03 & .15\end{array}$

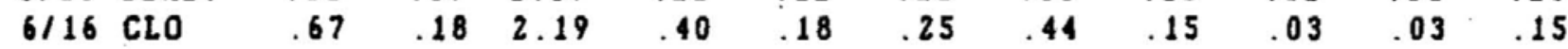

$\begin{array}{lllllllllllll}6 / 22 & \text { CONT. } & 4.10 & 1.02 & 12.27 & 2.60 & 1.30 & 2.05 & 1.80 & 1.40 & .42 & .67 & .40\end{array}$

$\begin{array}{lllllllllllll}6 / 22 & \text { CLO } & 5.01 & .83 & 17.56 & 3.12 & 1.58 & 1.63 & 2.20 & 1.00 & 0 & .51 & .86\end{array}$

$\begin{array}{llllllllllllll}6 / 28 & \text { CONT. } & 1.91 & .48 & 5.91 & 1.36 & .53 & 1.46 & 1.26 & .90 & .35 & .36 & .57\end{array}$ $\begin{array}{llllllllllll}6 / 28 \text { CLO } & 6.44 & .97 & 20.68 & 3.55 & 2.87 & 2.26 & 3.08 & 1.30 & 0 & 1.01 & 1.11\end{array}$

CONTROL VS CLO SUPPLEMENT PHOSPHATIDYL ETHANOLAMINE $22: 5$

DATE DIET $18: 2 \quad 18: 2 * 18: 3 \quad 20: 0 \quad 20: 1 \quad 20: 4 \quad 20: 5 \quad 22: 0 \quad 22: 2 ? \quad 22: 3 ? \quad 22: 6$

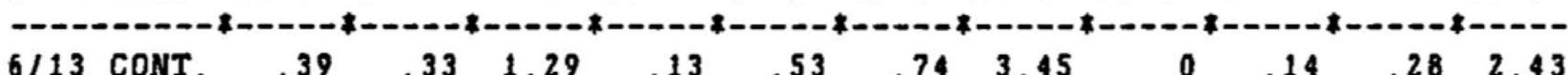

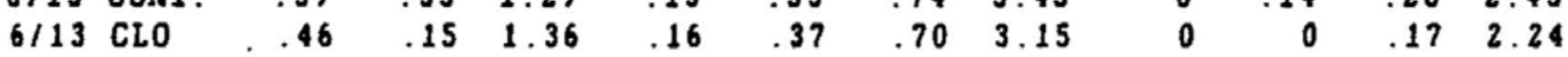

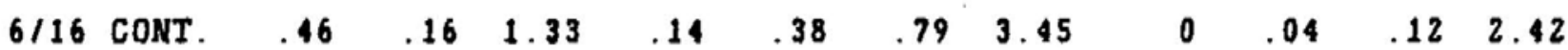

$\begin{array}{lllllllllllll}6 / 16 \text { CLO } & .54 & .17 & 1.50 & .19 & .44 & .85 & 3.75 & . & 0 & .07 & .26 & 2.41\end{array}$

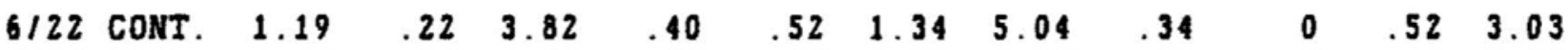

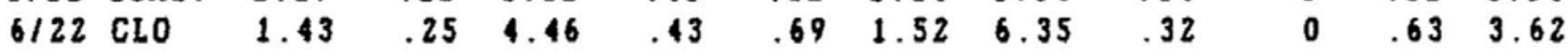

$\begin{array}{lllllllllllll}6 / 28 & \text { CONT. } & 1.64 & .25 & 4.98 & .10 & .54 & 1.73 & 5.50 & .44 & 0 & .32 & 2.93\end{array}$

$\begin{array}{lllllllllllll}6 / 28 & \text { CLO } & 2.07 & .35 & 6.22 & .59 & 1.10 & 1.98 & 7.14 & .68 & .10 & .78 & 4.48\end{array}$

CONTROL VS CLO SUPPLEMENT PHOSPHATIDYL CHOLINE $22: 5$

DATE DIET $\quad 18: 2 \quad 18: 2 * \quad 18: 3 \quad 20: 0 \quad 20: 1 \quad 20: 4 \quad 20: 5 \quad 22: 0 \quad 22: 2 ? \quad 22: 3 ? \quad 22: 6$

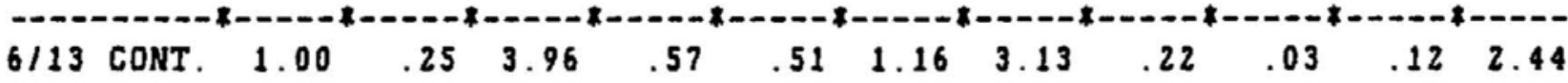

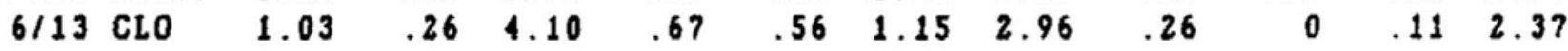

$\begin{array}{lllllllllllll}6 / 16 & \text { CONT. } & 1.08 & .26 & 4.20 & .64 & .54 & 1.30 & 3.16 & .28 & 0 & .13 & 2.15\end{array}$

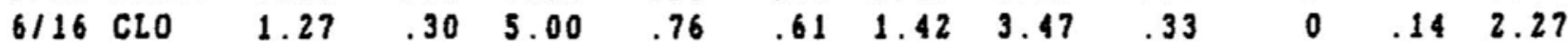

$\begin{array}{lllllllllllll}6 / 22 & \text { CONT. } & 3.00 & .53 & 12.53 & 1.68 & .90 & 2.94 & 5.05 & .91 & 0 & .26 & 2.96\end{array}$

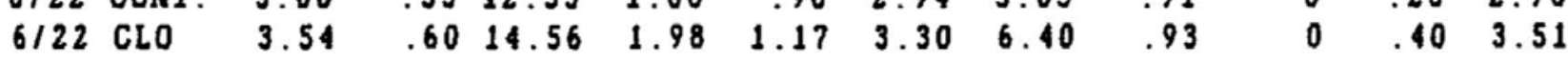

$\begin{array}{lllllllllllll}6 / 28 & \text { CONT. } & 4.28 & .58 & 16.33 & 1.86 & 1.12 & 4.67 & 6.31 & 1.20 & 0 & .42 & 3.01\end{array}$

$\begin{array}{llllllllllll}6 / 28 \text { CLO } & 5.47 & .75 & 21.48 & 2.61 & 1.67 & 5.41 & 8.47 & 1.72 & 0 & .47 & 4.76\end{array}$ 
TABLE 28 (page 1 of 2)

Control vs. CLO Supplement

Fatty Acids as FAME, \% of Total FAME in Each Class

CONTROL VS CLO SUPPLEMENT NEUTRAL LIPIDS

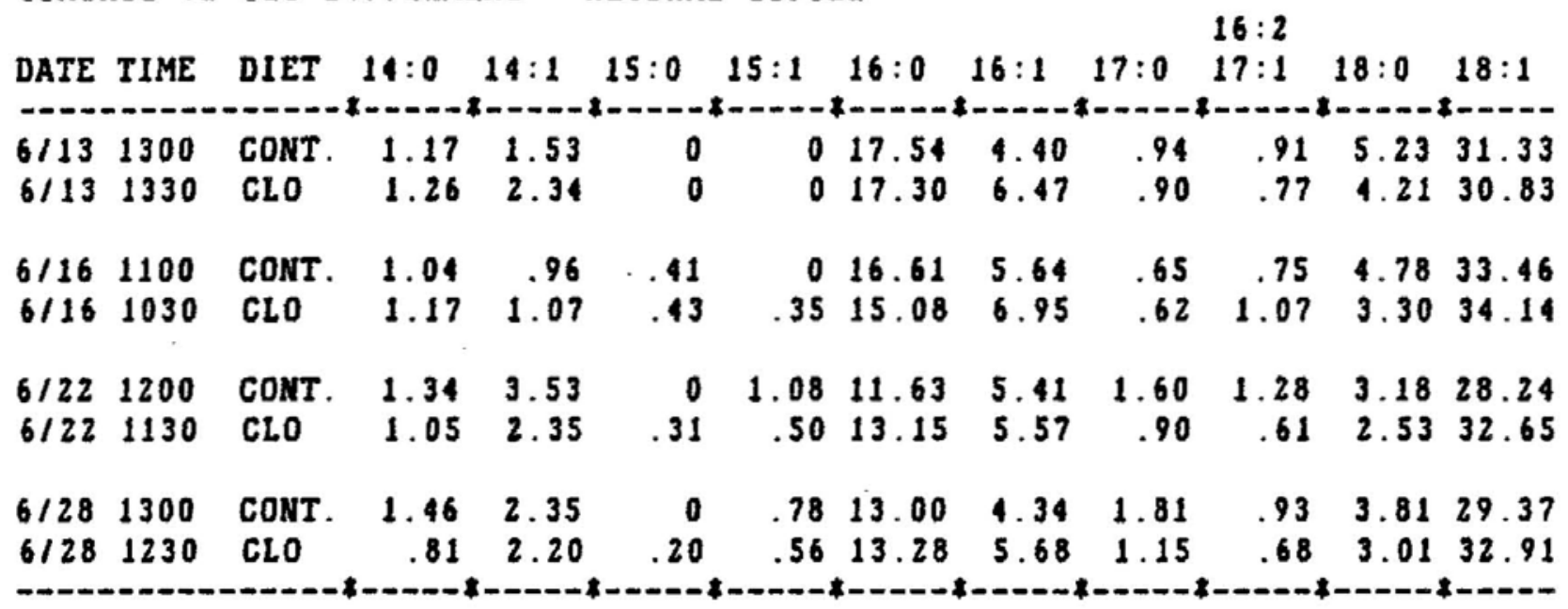

CONTROL VS CLO SUPPLEMENT PHOSPHATIDYL ETHANOLAMINE $16: 2$

$\begin{array}{llllllllll}\text { DATE TIME DIET } 14: 0 & 14: 1 & 15: 0 & 15: 1 & 16: 0 & 16: 1 & 17: 0 & 17: 1 & 18: 0 & 18: 1\end{array}$

\begin{tabular}{|c|c|c|c|c|c|c|c|c|c|c|c|c|}
\hline $\begin{array}{l}6 / 13 \\
6 / 13\end{array}$ & $\begin{array}{l}1300 \\
1330\end{array}$ & $\begin{array}{l}\text { CONT. } \\
\text { CLO }\end{array}$ & $\begin{array}{r}.50 \\
0\end{array}$ & $\begin{array}{r}.12 \\
0\end{array}$ & $\begin{array}{l}0 \\
0\end{array}$ & $\begin{array}{l}0 \\
0\end{array}$ & $\begin{array}{r}10.81 \\
9.55\end{array}$ & $\begin{array}{r}.33 \\
1.10\end{array}$ & $\begin{array}{l}.72 \\
.86\end{array}$ & $\begin{array}{r}1.49 \\
.63\end{array}$ & $\begin{array}{l}6.62 \\
7.22\end{array}$ & $\begin{array}{l}22.66 \\
23.53\end{array}$ \\
\hline $\begin{array}{l}6 / 16 \\
6 / 16\end{array}$ & $\begin{array}{l}1100 \\
1030\end{array}$ & $\begin{array}{l}\text { CONT. } \\
\text { CLO }\end{array}$ & $\begin{array}{r}.21 \\
0\end{array}$ & $\begin{array}{l}0 \\
0\end{array}$ & $\begin{array}{l}0 \\
0\end{array}$ & $\begin{array}{l}0 \\
0\end{array}$ & $\begin{array}{l}8.82 \\
8.44\end{array}$ & $\begin{array}{l}1.28 \\
1.42\end{array}$ & $\begin{array}{l}.85 \\
.87\end{array}$ & $\begin{array}{l}.68 \\
.60\end{array}$ & $\begin{array}{l}6.61 \\
6.60\end{array}$ & $\begin{array}{l}22.65 \\
23.26\end{array}$ \\
\hline $\begin{array}{l}6 / 22 \\
6 / 22\end{array}$ & $\begin{array}{l}1200 \\
1130\end{array}$ & $\begin{array}{l}\text { CONT. } \\
\text { CLO }\end{array}$ & $\begin{array}{r}.33 \\
0\end{array}$ & $\begin{array}{r}.29 \\
0\end{array}$ & $\begin{array}{l}0 \\
0\end{array}$ & $\begin{array}{l}0 \\
0\end{array}$ & $\begin{array}{l}8.61 \\
8.82\end{array}$ & $\begin{array}{l}1.17 \\
1.39\end{array}$ & $\begin{array}{l}.88 \\
.82\end{array}$ & $\begin{array}{l}.52 \\
.47\end{array}$ & $\begin{array}{l}7.06 \\
7.31\end{array}$ & $\begin{array}{l}24.19 \\
24.90\end{array}$ \\
\hline $\begin{array}{l}6 / 28 \\
6 / 28\end{array}$ & $\begin{array}{l}1300 \\
1230\end{array}$ & $\begin{array}{l}\text { CONT. } \\
\text { CLO }\end{array}$ & $\begin{array}{r}29 \\
0\end{array}$ & $\begin{array}{r}18 \\
0\end{array}$ & $\begin{array}{l}0 \\
0\end{array}$ & $\begin{array}{r}.26 \\
0\end{array}$ & $\begin{array}{l}8.79 \\
7.93\end{array}$ & $\begin{array}{l}1.40 \\
1.45\end{array}$ & $\begin{array}{l}.88 \\
.90\end{array}$ & $\begin{array}{r}.52 \\
.43\end{array}$ & $\begin{array}{l}6.10 \\
6.71\end{array}$ & $\begin{array}{l}23.89 \\
23.90\end{array}$ \\
\hline
\end{tabular}

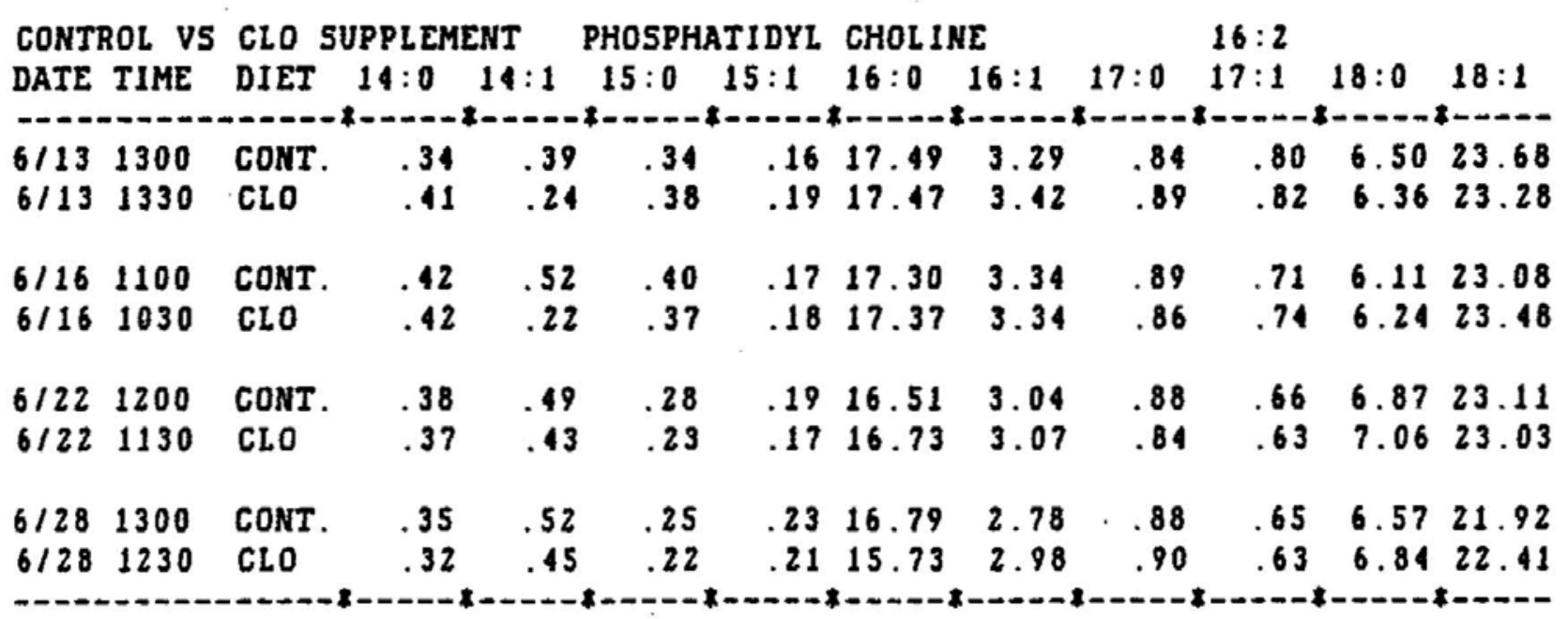


TABLE 28 (page 2 of 2)

Control vs. CLO Supplement

Fatty Acids as FAME, \% of Total FAME in Each Class

CONTROL VS CLO SUPPLEMENT NEUTRAL LIPIDS

$22: 5$

DATE DIET $\quad 18: 2 \quad 18: 2 * \quad 18: 3 \quad 20: 0 \quad 20: 1 \quad 20: 4 \quad 20: 5 \quad 22: 0 \quad 22: 27 \quad 22: 3 ? \quad 22: 6$

\begin{tabular}{|c|c|c|c|c|c|c|c|c|c|c|c|c|}
\hline 113 & $\begin{array}{l}\text { CONT. } \\
\text { CLO }\end{array}$ & $\begin{array}{l}3.79 \\
1.23\end{array}$ & $\begin{array}{l}1.27 \\
1.08\end{array}$ & $\begin{array}{l}10.87 \\
13.19\end{array}$ & $\begin{array}{l}2.55 \\
2.94\end{array}$ & $\begin{array}{l}2.22 \\
2.43\end{array}$ & $\begin{array}{l}3.41 \\
2.46\end{array}$ & $\begin{array}{l}7.25 \\
5.84\end{array}$ & $\begin{array}{l}1.58 \\
1.25\end{array}$ & $\begin{array}{r}.56 \\
0\end{array}$ & $\begin{array}{l}0 \\
0\end{array}$ & $\begin{array}{l}3.46 \\
2.50\end{array}$ \\
\hline $\begin{array}{l}6 / 16 \\
6 / 16\end{array}$ & $\begin{array}{l}\text { CONT. } \\
\text { CLO }\end{array}$ & $\begin{array}{l}4.82 \\
5.14\end{array}$ & $\begin{array}{l}1.09 \\
1.37\end{array}$ & $\begin{array}{l}13.96 \\
16.79\end{array}$ & $\begin{array}{l}2.79 \\
3.06\end{array}$ & $\begin{array}{l}1.48 \\
1.40\end{array}$ & $\begin{array}{l}2.80 \\
1.90\end{array}$ & $\begin{array}{l}4.81 \\
3.37\end{array}$ & $\begin{array}{l}1.34 \\
1.16\end{array}$ & $\begin{array}{l}.23 \\
.21\end{array}$ & $\begin{array}{l}.42 \\
.25\end{array}$ & $\begin{array}{l}1.96 \\
1.16\end{array}$ \\
\hline $\begin{array}{l}1 / 22 \\
6 / 22\end{array}$ & $\begin{array}{l}\text { CONT. } \\
\text { CLO }\end{array}$ & $\begin{array}{l}6.25 \\
5.89\end{array}$ & $\begin{array}{r}1.56 \\
.98\end{array}$ & $\begin{array}{l}18.70 \\
20.66\end{array}$ & $\begin{array}{l}3.97 \\
3.67\end{array}$ & $\begin{array}{l}1.97 \\
1.86\end{array}$ & $\begin{array}{l}3.12 \\
1.92\end{array}$ & $\begin{array}{l}2.74 \\
2.59\end{array}$ & $\begin{array}{l}2.13 \\
1.18\end{array}$ & $\begin{array}{r}.65 \\
0\end{array}$ & $\begin{array}{r}1.02 \\
.60\end{array}$ & $\begin{array}{r}.62 \\
1.02\end{array}$ \\
\hline 12 & $\begin{array}{l}\text { CONT } \\
\text { CLO }\end{array}$ & $\begin{array}{l}5.34 \\
5.88\end{array}$ & $\begin{array}{r}1.34 \\
.89\end{array}$ & $\begin{array}{l}16.50 \\
18.88\end{array}$ & $\begin{array}{l}3.81 \\
3.24\end{array}$ & $\begin{array}{l}1.48 \\
2.62\end{array}$ & $\begin{array}{l}1.08 \\
2.06\end{array}$ & $\begin{array}{l}3.51 \\
2.82\end{array}$ & $\begin{array}{l}2.51 \\
1.19\end{array}$ & $\begin{array}{r}.99 \\
0\end{array}$ & $\begin{array}{r}1.01 \\
.92\end{array}$ & $\begin{array}{l}1.59 \\
1.02\end{array}$ \\
\hline
\end{tabular}

CONTROL VS CLO SUPPLEMENT PHOSPHATIDYL ETHANOLAMINE

$22: 5$

DATE DIET $\quad 18: 2 \quad 18: 2 * \quad 18: 3 \quad 20: 0 \quad 20: 1 \quad 20: 4 \quad 20: 5 \quad 22: 0 \quad 22: 2 ? \quad 22: 3 ? \quad 22: 6$

$6 / 13$ CONT. $2.29 \quad 1.92 \quad 7.51 \quad .74 \quad 3.12 \quad 4.3320 .14 \quad 00.841 .6214 .23$

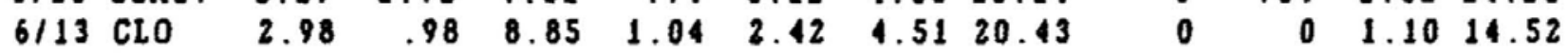

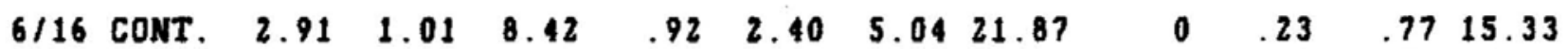

$\begin{array}{llllllllllll}6 / 16 \text { CLO } & 3.14 & 1.00 & 8.68 & 1.11 & 2.54 & 4.93 & 21.62 & 0 & .38 & 1.50 & 13.90\end{array}$

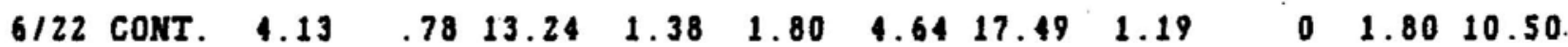

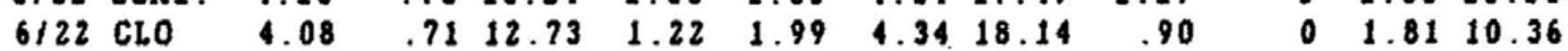

$\begin{array}{lllllllllllll}6 / 28 & \text { CONT. } & 5.06 & .77 & 15.33 & 1.24 & 1.67 & 5.32 & 16.91 & 1.36 & 0 & .99 & 9.02\end{array}$

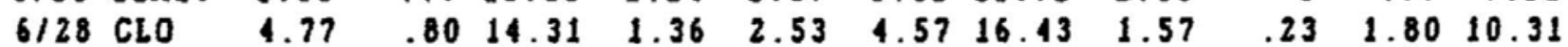

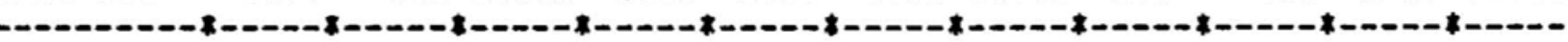

CONTROL VS CLO SUPPLEMENT PHOSPHATIDYL CHOLINE

$22: 5$

DATE DIET $\quad 18: 2 \quad 18: 2 * 18: 3 \quad 20: 0 \quad 20: 1 \quad 20: 4 \quad 20: 5 \quad 22: 0 \quad 22: 2 ? \quad 22: 3 ? \quad 22: 6$

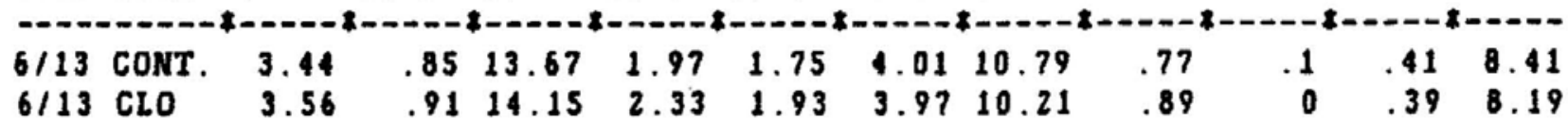

$\begin{array}{lllllllllllll}6 / 16 & \text { CONT. } & 3.71 & .88 & 14.38 & 2.19 & 1.86 & 4.45 & 10.84 & .97 & 0 & .44 & 7.35\end{array}$

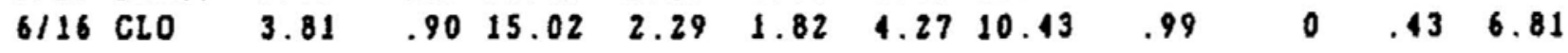

$\begin{array}{llllllllllllll}6122 & \text { CONT. } & 4.65 & .81 & 19.38 & 2.60 & 1.39 & 4.56 & 7.82 & 1.41 & 0 & .40 & 4.58\end{array}$

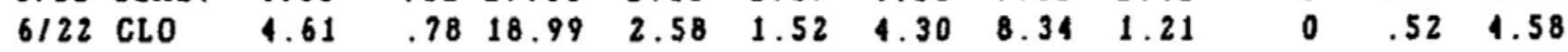

$\begin{array}{lllllllllllll}6 / 28 & \text { CONT. } 5.28 & .72 & 20.13 & 2.29 & 1.38 & 5.76 & 7.78 & 1.48 & 0 & .52 & 3.71\end{array}$ $\begin{array}{llllllllllll}6 / 28 \text { CLO } & 5.11 & .70 & 20.05 & 2.44 & 1.56 & 5.05 & 7.90 & 1.60 & 0 & .44 & 4.45\end{array}$ 
TABLE 29

Labelled Fatty Acids

Recovered Label in Each Lipid Class

\begin{tabular}{|c|c|c|c|c|c|c|c|c|}
\hline SAMPLE & $\begin{array}{l}S E \\
D P M\end{array}$ & $\begin{array}{l}\text { TR I } \\
\text { DPM }\end{array}$ & $\begin{array}{l}\text { FFA } \\
\text { DPM }\end{array}$ & $\underset{D P M}{1-2 D I}$ & $\begin{array}{l}\text { NL } \\
\text { SPOT } \\
\text { DPM }\end{array}$ & $\begin{array}{l}\text { PE } \\
\text { DPM }\end{array}$ & $\begin{array}{l}\text { PC } \\
\text { DPM }\end{array}$ & $\begin{array}{l}\text { PL } \\
\text { SPOT } \\
\text { DPM }\end{array}$ \\
\hline$F$ & & 16320 & & & & 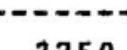 & 8210 & 12186 \\
\hline $\begin{array}{l}\text { PF } \\
\text { PF } \\
\text { PF }\end{array}$ & $\begin{array}{r}1062 \\
85\end{array}$ & $\begin{array}{r}16320 \\
9305\end{array}$ & $\begin{array}{r}64898 \\
1313\end{array}$ & $\begin{array}{r}1602 \\
427\end{array}$ & $\begin{array}{l}79814 \\
11549\end{array}$ & $\begin{array}{l}2750 \\
2315\end{array}$ & $\begin{array}{l}8349 \\
7849\end{array}$ & $\begin{array}{r}12186 \\
9899\end{array}$ \\
\hline PF 6B & 154 & 16123 & 502 & 798 & 17706 & 3509 & 14696 & 18417 \\
\hline PF $12 \mathrm{~A}$ & 157 & 2862 & 449 & 1093 & 4663 & 5680 & 22029 & 27766 \\
\hline PE $24 \mathrm{~A}$ & 45 & 1191 & 215 & 223 & 1614 & 1876 & 6148 & 8638 \\
\hline PF $24 B$ & 96 & 576 & 334 & 632 & 1601 & 3338 & 12548 & 17940 \\
\hline PF $36 \mathrm{~A}$ & 89 & 1002 & 226 & 592 & 1892 & 4192 & 13550 & 20181 \\
\hline PF $48 \mathrm{~A}$ & 203 & 591 & 20025 & 613 & 19280 & 4613 & 14212 & 21415 \\
\hline PF $48 \mathrm{~B}$ & 69 & 4116 & 207 & 319 & 4405 & 2578 & 9079 & 13911 \\
\hline PS 1A & 1837 & 24760 & 33704 & 1692 & 54810 & 2251 & 9122 & 13020 \\
\hline P5 $6 A$ & 215 & 1381 & 826 & 533 & 3466 & 4642 & 20043 & 22129 \\
\hline P5 6B & 165 & 2054 & 12324 & 360 & 13603 & 2664 & 10492 & 14271 \\
\hline PS $12 A$ & 239 & 1353 & 1413 & 732 & 3943 & 6025 & 21898 & 29138 \\
\hline PS $12 B$ & 203 & 849 & 4519 & 492 & 6390 & 4997 & 20049 & 24830 \\
\hline PS $24 A$ & 146 & 456 & 1047 & 440 & 2129 & 4214 & 15714 & 20038 \\
\hline P5 24B & 147 & 543. & 364 & 433 & 1524 & 3951 & 13948 & 19737 \\
\hline P5 36A & 206 & 332 & 3176 & 354 & 3948 & 3354 & 11519 & 16099 \\
\hline PS $36 \mathrm{~B}$ & 162 & 525 & 327 & 456 & 1588 & 4113 & 14613 & 19931 \\
\hline P5 48A & 123 & 284 & 211 & 369 & 1096 & 3208 & 11389 & 15441 \\
\hline P5 48B & 114 & 266 & 1285 & 303 & 2023 & 2828 & 10114 & 13593 \\
\hline---- & $\cdots$ & & $-\infty-$ & --- & 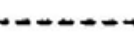 & 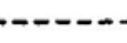 & -----0 & -"-n-- \\
\hline EF IA & 282 & 39005 & 2657 & 689 & 52739 & 20214 & 52652 & 57560 \\
\hline EF $6 A$ & 104 & 18604 & 362 & 757 & 18748 & 19971 & 35671 & 57222 \\
\hline$E F \quad 6 B$ & 40 & 13802 & 335 & 661 & 14610 & 22119 & 36383 & 59403 \\
\hline$E F \quad 12 \mathrm{~A}$ & 87 & 8539 & 183 & 621 & 9000 & 20000 & 24980 & 49300 \\
\hline EF $12 B$ & 95 & 1089 & 210 & 657 & 2039 & 28582 & 31112 & 66067 \\
\hline$E F \quad 24 \mathrm{~A}$ & 77 & 2063 & 225 & 1049 & 3519 & 35451 & 36832 & 74830 \\
\hline EF $24 B$ & 109 & 3290 & 258 & 1014 & 4822 & 36569 & 44108 & 79095 \\
\hline EF $36 \mathrm{~A}$ & 41 & 8017 & 195 & 849 & 8215 & 25120 & 25648 & 59605 \\
\hline EF $36 \mathrm{~B}$ & 108 & 274 & 199 & 733 & 1498 & 27739 & 29235 & 56015 \\
\hline$E F \quad 48 A$ & 86 & 1055 & 102 & 675 & 1823 & 22016 & 22454 & 49020 \\
\hline$E F \quad 48 B$ & 99 & 287 & 179 & 792 & 1332 & 29854 & 28740 & 62281 \\
\hline--- & & & & & & & & \\
\hline ES $1 A$ & 218 & 25214 & 3595 & 332 & 27947 & 9367 & 25051 & 37176 \\
\hline ES $6 \mathrm{~A}$ & 33 & 232 & 169 & 340 & 810 & 14816 & 21810 & 37867 \\
\hline ES 6B & 35 & 444 & 262 & 595 & 1450 & 21160 & 33543 & 56803 \\
\hline ES $12 \mathrm{~A}$ & 65 & 1245 & 272 & 915 & 2709 & 37691 & 51700 & 97651 \\
\hline ES $12 \mathrm{~B}$ & 32 & 601 & 266 & 843 & 1852 & 33395 & 47826 & 88269 \\
\hline ES $24 A$ & 69 & 551 & 379 & 1164 & 2154 & 34156 & 39805 & 79026 \\
\hline ES $24 \mathrm{~B}$ & 73 & 204 & 277 & 748 & 1542 & 28439 & 36607 & 68208 \\
\hline ES $36 \mathrm{~A}$ & 82 & 206 & 341 & 918 & 1612 & 28190 & 41273 & 77795 \\
\hline ES $36 \mathrm{~B}$ & 192 & 349 & 297 & 623 & 1470 & 23580 & 38093 & 67701 \\
\hline E5 $48 \mathrm{~A}$ & 197 & 319 & 241 & 577 & 1600 & 25367 & 38043 & 61597 \\
\hline ES $48 B$ & 137 & 207 & 269 & 707 & 1313 & 20334 & 39666 & 60888 \\
\hline
\end{tabular}


TABLE 30

Labelled Fatty Acids

Lipid Classes in Controls

UGIIND. UGIIND. UGIIND. UGIIND. UGIIND.

\begin{tabular}{|c|c|c|c|c|c|c|c|c|c|c|}
\hline & IPLE & LIPID & $S E$ & D. D. & TRI & S.D. & FFA. & D. D. & STER & S.D. \\
\hline & - & 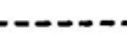 & & $\ldots$ & --- & $-\cdots$ & $-\cdots$ & -- & $x=-$ & -- \\
\hline 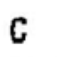 & 1 & 116.3 & 2.08 & .22 & 3.73 & .41 & 1.83 & .17 & 14.78 & 37 \\
\hline $\mathrm{Cr}$ & 6 & 169.7 & 2.66 & .27 & 33.19 & 1.53 & 0 & 0 & 18.04 & 1.02 \\
\hline CF & 12 & 137.5 & 2.46 & .25 & 11.69 & 1.33 & 0 & 0 & 15.58 & 1.03 \\
\hline$C F$ & 24 & 158.0 & 2.65 & .27 & 14.73 & 1.09 & 0 & 0 & 17.90 & 1.19 \\
\hline$C F$ & 36 & 136.5 & 2.44 & .35 & 3.85 & .25 & 0 & 0 & 17.54 & .72 \\
\hline $\mathrm{CF}$ & 48 & 108.5 & 1.57 & .07 & 1.45 & 13 & 1.03 & .05 & 15.66 & .84 \\
\hline & 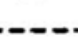 & - - - & $=-8$ & & $-\infty$ &.-- & & & $=-$ & \\
\hline C5 & 6 & 113.0 & 2.25 & .14 & 2.17 & .37 & 0 & 0 & 14.51 & .60 \\
\hline 5 & 12 & 113.7 & 1.91 & .07 & 2.09 & .41 & 0 & 0 & 15.91 & .65 \\
\hline CS & 24 & 103.3 & 1.69 & .50 & 0 & 0 & 0 & 0 & 16.14 & .76 \\
\hline C5 & 36 & 96.0 & 1.51 & .47 & 0 & 0 & 0 & 0 & 14.96 & .95 \\
\hline CS & 48 & 91.7 & 1.58 & .25 & 0 & 0 & .92 & .09 & 14.40 & .58 \\
\hline
\end{tabular}

\begin{tabular}{|c|c|c|c|c|c|c|c|c|}
\hline \multirow{2}{*}{ SAMPLE } & \multicolumn{2}{|c|}{ UG I IND } & \multicolumn{2}{|c|}{ UG / IND } & \multicolumn{2}{|c|}{ UG/IND } & \multicolumn{2}{|c|}{ UG/IND } \\
\hline & $1-2 \quad D I$ & D. D. & MONO & S.D. & PS & S.D. & $\mathrm{PE}$ & S.D. \\
\hline 1 & .95 & .10 & 5.48 & .50 & 4.30 & 49 & 22.43 & 1.71 \\
\hline CF 6 & 3.24 & .14 & 6.86 & .68 & 6.41 & 1.05 & 26.61 & 1.43 \\
\hline $\mathrm{CF} 12$ & 1.71 & .08 & 5.60 & 1.40 & 5.28 & .72 & 25.67 & 1.21 \\
\hline$C F 24$ & .77 & .13 & 8.63 & .62 & 3.82 & .27 & 28.99 & 1.20 \\
\hline CF 36 & .70 & .05 & 6.58 & 1.06 & 4.19 & .76 & 30.41 & 2.28 \\
\hline CF 48 & 1.12 & .11 & 4.85 & .40 & 3.52 & .16 & 25.64 & .72 \\
\hline & & & & & & & & 2.10 \\
\hline CS 6 & 1.13 & .11 & 5.09 & .40 & 4.50 & .47 & 22.51 & 2.10 \\
\hline CS 12 & .82 & .02 & 5.23 & .31 & 4.37 & 22 & 23.50 & 1.06 \\
\hline C5 24 & 0 & 0 & 5.32 & .40 & 3.14 & .55 & 23.52 & 1.08 \\
\hline C5 36 & 0 & 0 & 4.65 & .52 & 3.80 & .18 & 20.85 & 1.26 \\
\hline CS 48 & 0 & 0 & 4.46 & .39 & 3.30 & .43 & 20.22 & 1.37 \\
\hline
\end{tabular}

\begin{tabular}{|c|c|c|c|c|c|c|c|c|}
\hline \multirow[b]{2}{*}{ 5AMPLE } & \multicolumn{2}{|c|}{ UG / IND. } & \multicolumn{2}{|c|}{ UG / IND. } & \multicolumn{2}{|c|}{ UG / IND. } & \multicolumn{2}{|c|}{ UG / IND. } \\
\hline & PI & S.D. & PC & S.D. & SM & S.D. & UNK. & S.D. \\
\hline C 1 & 8.96 & 1.93 & 42.96 & 1.47 & 5.00 & .71 & 4.06 & .36 \\
\hline $\mathrm{CF} 6$ & 10.17 & 1.32 & 49.42 & 4.17 & 7.36 & 1.87 & 5.91 & 1.92 \\
\hline CF 12 & 10.99 & .83 & 48.77 & 3.36 & 5.60 & 1.35 & 4.17 & 1.24 \\
\hline CF 24 & 9.53 & 1.25 & 61.23 & 3.14 & 5.18 & .85 & 4.53 & .47 \\
\hline CF 36 & 9.32 & 1.19 & 51.73 & 1.98 & 5.42 & .60 & 4.31 & .65 \\
\hline CF 48 & 4.97 & .50 & 38.27 & 2.00 & 5.79 & 1.10 & 4.63 & .80 \\
\hline & --- & $-\infty$ & $x----$ & $--\infty$ & & --- & --- & $\cdots$ \\
\hline CS 6 & 10.63 & .71 & 38.39 & .86 & 6.46 & .44 & 5.38 & .60 \\
\hline $\operatorname{cs} 12$ & 9.60 & .64 & 40.28 & 2.76 & 5.37 & .69 & 4.64 & .51 \\
\hline C5 24 & 6.93 & .21 & 38.30 & 2.37 & 5.28 & 1.21 & 2.98 & .83 \\
\hline C5 36 & 4.92 & .49 & 35.23 & 2.20 & 5.23 & .53 & 4.85 & .34 \\
\hline CS 48 & 4.53 & .45 & 31.56 & 1.13 & 5.37 & .54 & 5.37 & .28 \\
\hline
\end{tabular}


TABLE 31

Labelled Fatty Acids

DPM per Lipid Class as \% of Recovered Total DPM

DPM PER CLASS AS \% OF RECOVERED DPM

PALMITIC ACID

SAMPLE SE S.D. TRI S.D. FFA S.D. 1-2 DI S.D. PE S.D. PC S.D.

\begin{tabular}{|c|c|c|c|c|c|c|c|c|c|c|c|c|c|}
\hline $\mathbf{P}$ & 1 & 1.81 & .98 & 25.47 & 11.71 & 57.14 & 15.84 & 2.00 & .44 & 2.99 & .13 & 10.61 & 2. $5 ?$ \\
\hline PF & 6 & .42 & .03 & 44.38 & .96 & 3.79 & 3.38 & 2.12 & .16 & 10.34 & .75 & 38.97 & 2.98 \\
\hline PF & 12 & .49 & 0 & 8.87 & 0 & 1. 39 & 0 & 3. 39 & 0 & 17.6 & 0 & 68.27 & 0 \\
\hline PF & 24 & .51 & .07 & 7.79 & 6.36 & 2.07 & .23 & 2.96 & .93 & 19 & 21 & 67.51 & 5.81 \\
\hline PF & 36 & .45 & 0 & 5.10 & 0 & 1.15 & 0 & 3. & 0 & 21.33 & 0 & 68.95 & 0 \\
\hline PF & 48 & .46 & .06 & 13.31 & 16.74 & 25.50 & 34.28 & 1.74 & .31 & 13.61 & 3.04 & 45.39 & 14.27 \\
\hline & & & 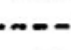 & & 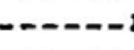 & & ---- & & & & --- & --- & ---- \\
\hline P & 1 & 1.81 & .98 & 25.47 & 11.71 & 57.14 & 15.84 & 2.00 & .44 & 2.99 & .13 & 10.61 & 2. 57 \\
\hline P5 & 6 & .69 & .14 & 6.16 & 1.64 & 23.46 & 28.95 & 1.61 & .47 & 13.15 & 5.18 & 54.95 & 24.83 \\
\hline PS & 12 & .70 & .07 & 3.50 & 1.09 & 9.50 & 7.13 & 1.95 & .52 & 17.55 & 2.11 & 66.81 & 3.34 \\
\hline P5 & 24 & .71 & .07 & 2.14 & .52 & 3. 32 & 2.04 & 2.12 & .17 & 19.76 & .88 & 71.66 & .41 \\
\hline PS & 36 & .95 & .21 & 2.18 & .61 & 9.20 & 10.71 & 2.07 & .28 & 19.04 & 1.90 & 66.59 & 8.16 \\
\hline P5 & 48 & .78 & .03 & 1.79 & .01 & 4.99 & 5.15 & 2.20 & .24 & 19.78 & 1.15 & 70.46 & 3.72 \\
\hline
\end{tabular}

\section{EICOSAPENTAENOIC ACID}

SAMPLE SE S.D. TRI S.D. FFA S.D. 1-2 DI S.D. PE S.D. PC S.D.

\begin{tabular}{|c|c|c|c|c|c|c|c|c|c|c|c|c|c|}
\hline E & 1 & .29 & .07 & 36.66 & 4.09 & 3.97 & 2.36 & .56 & .06 & 16.1 & 1.99 & 42.44 & 4.47 \\
\hline$E F$ & 6 & .10 & .07 & 21.74 & 4.13 & 47 & .01 & .95 & .07 & 28.31 & 2.62 & 48.44 & 1.66 \\
\hline EF & 12 & .16 & .01 & 8.73 & 9.86 & .34 & 0 & 1.10 & .06 & 41.53 & 6.75 & 48.15 & 3.17 \\
\hline$E F$ & 24 & .12 & .03 & 3.29 & .81 & .30 & 0 & 1.29 & .14 & 94.84 & 2.81 & 50.17 & 2.14 \\
\hline EF & 36 & .13 & .08 & 6.93 & 9.14 & .34 & .01 & 1.34 & .11 & 44 & 3.99 & & 5.18 \\
\hline EI & 48 & .78 & .03 & 1.38 & 1.27 & .26 & .06 & 1.39 & .10 & 48 & 1.66 & 48 & .34 \\
\hline & 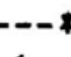 & 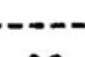 & ---1 & & & & . & & & & & & 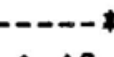 \\
\hline 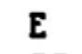 & 1 & .29 & .07 & 36.66 & 4.09 & 3.97 & 2.36 & .56 & .06 & 16 & 1.99 & 42 & 4.47 \\
\hline ES & 6 & .08 & .03 & .71 & .13 & .46 & .01 & .99 & .11 & 38.69 & 1.32 & 19 & 1.09 \\
\hline$E$ & 12 & .06 & .03 & 1.04 & .45 & 31 & .01 & 1.01 & .01 & 40.64 & .55 & 56.96 & .99 \\
\hline & 24 & .10 & 01 & .52 & .30 & .46 & .06 & 1.33 & .28 & 43.87 & 1.43 & 53.73 & 2.04 \\
\hline & 36 & 21 & .13 & .42 & 18 & 48 & 01 & 1.14 & .21 & 38.53 & 1.67 & 59.23 & 1.57 \\
\hline & 48 & .26 & .06 & .14 & .10 & .44 & .06 & 1.09 & 10 & 38.47 & 2.77 & 59.32 & 2.86 \\
\hline
\end{tabular}


TABLE 32

Labelled Fatty Acids

Labelled Pellet Values; DPM Ingested and Recovered

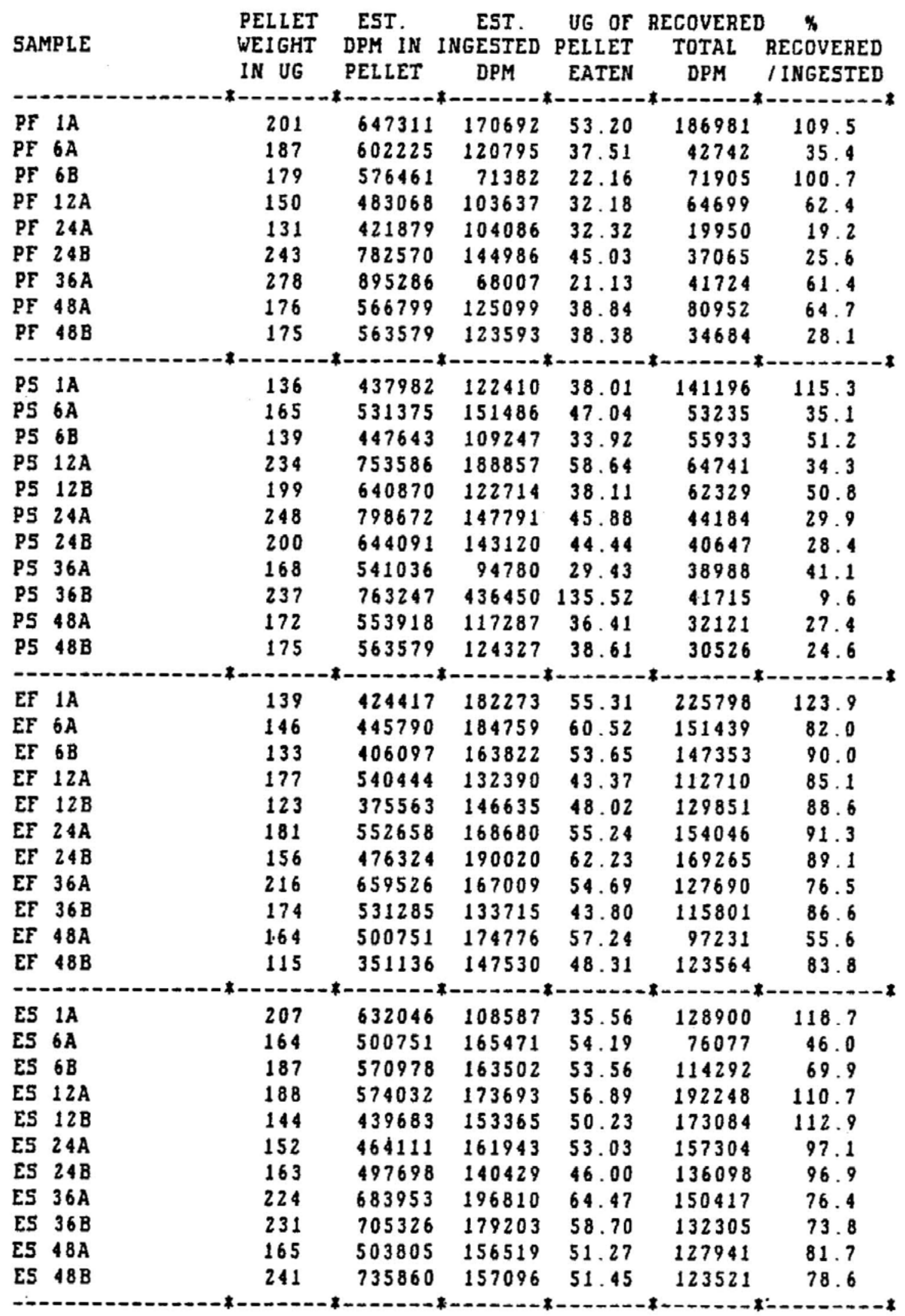


$-312-$

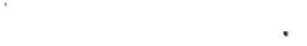


$-313-$

GRAPHS 
$-314-$ 


\section{Master Key for Graphs}

The A graph is always the top graph on each page.

The $\bar{B}$ graph is always the bottom graph on each page.

The only exceptions are graphs 1 and 43 , where the $\underline{\mathrm{C}}$ graph is the bottom graph.

Al1 error bars represent standard errors.

If information is displayed at the bottom of the page, labelled (DAYS, HOURS, SAMPLE or MOLT STAGE), this information applies to all the graphs on that page

SAMPLE : used for Embryogenesis Fatty Acid graphs. The vertical marks define the time the sample was taken.

MOLT STAGE : For Pulse Fed and Fatty Acid graphs, these 1abels define both the time the sample was taken and the molt stage of the larvae in that sample.

For Larva1 Development graphs, the MOLT STAGE 1abels are generalized to show the range of each molt stage and indicate the molt stage of the samples. The exact molt stage of each sample for each hatch can be found in the Tables.

Vertical lines through a graph indicate either the time of feeding (only in Pulse Fed graphs) or the time of molting.

Roman numerals (I-IV) at the top of A graphs indicate the larval stage. These numbers apply to both the $\underline{A}$ and $\underline{B}$ graphs on that page. 
Graph 1 A

Temperature profile during embryogenesis study.

Graph 1 B

Temperature profile during the larval development studies based on Hatchs II-4 and II-7. Duration of each study shown at top of graph. $\mathrm{X}$-axis is labelled by date (month/day).

\section{Graph 1 C}

Temperature profile during the larval development studies based on Hatchs I-3 and I-5. Duration of each study shown at top of graph. $\mathrm{X}$-axis is labelled by date (month/day). 

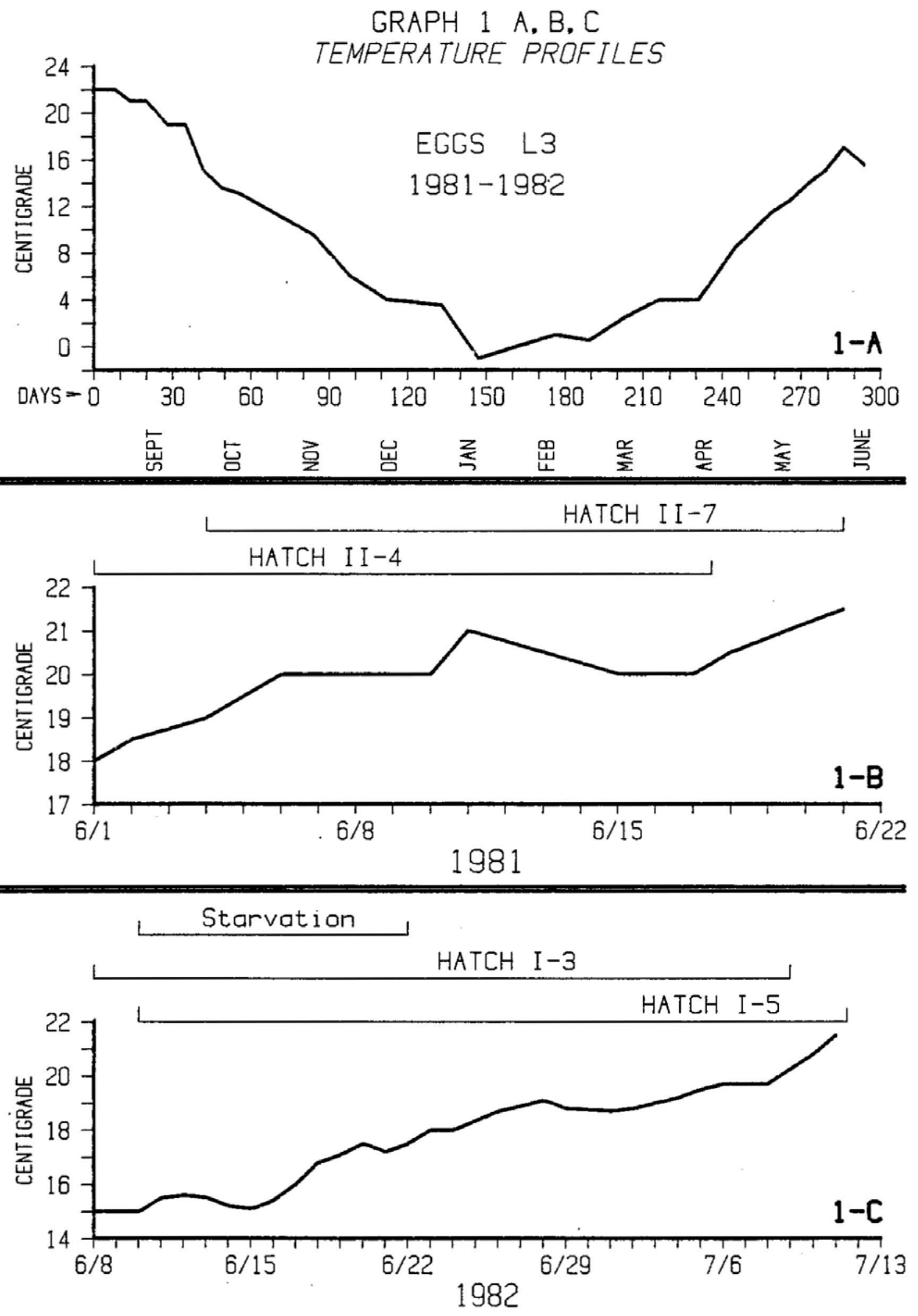


\section{Graph 2 A}

\section{Embryogenesis Study}

Weight values for individual eggs $(n=7)$. Expressed as mg dry weight, mg wet weight and $\%$ dry/wet weight. Last point represents hatched larvae. Note double y-axis.

Error bars are standard errors.

Ash $(n=3)$. Expressed as $\mathrm{mg}$ per individual and \% dry weight. Last point represents hatched larvae Note double Y-axis.

Error bars are standard errors. 


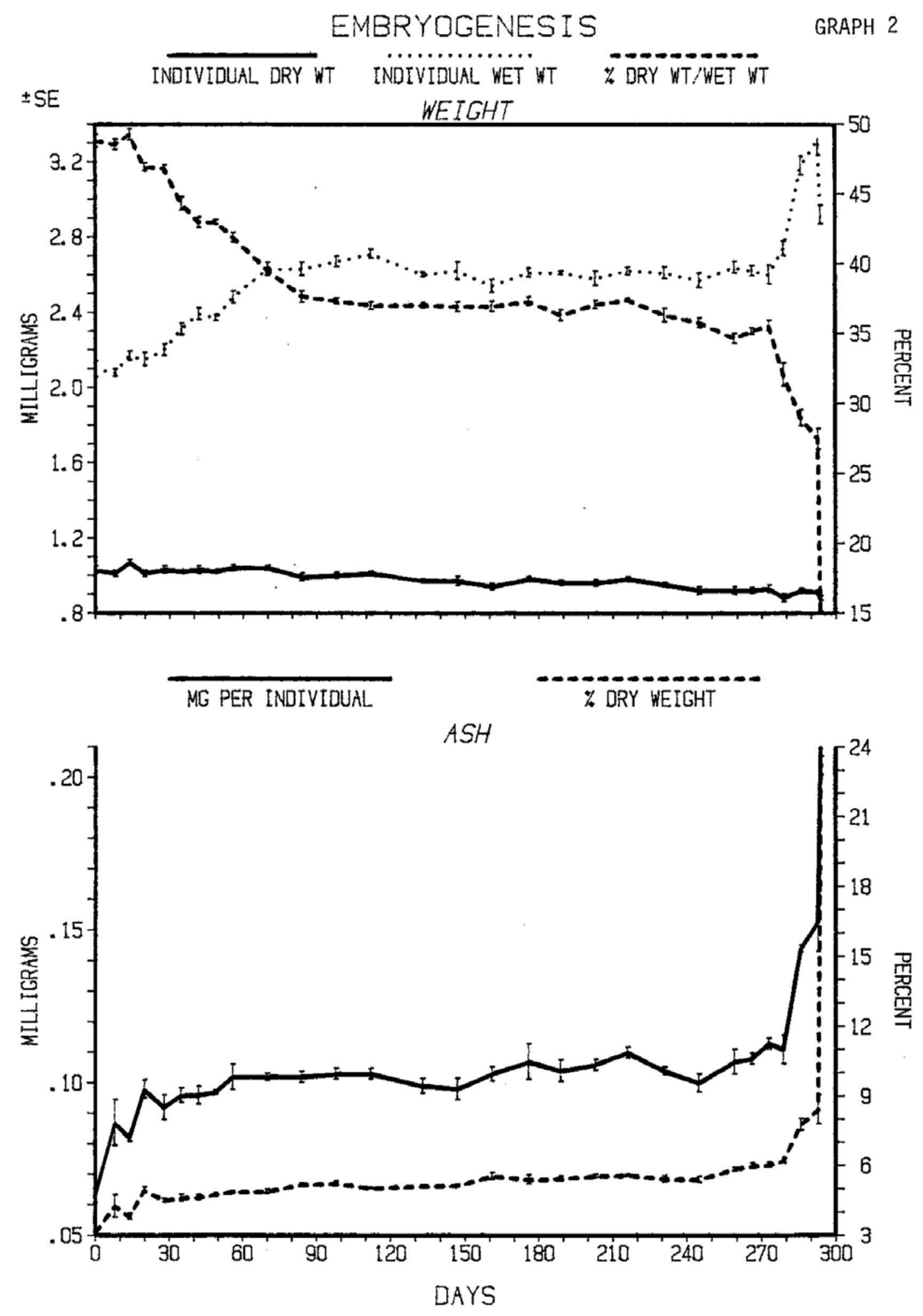


Graph 3 A

Hatching Period Study

Weight values for individual eggs $(n=7)$. Expressed as mg dry weight and $\%$ dry/wet weight.

The vertical portion or each cross represents the standard error. The horizontal portion of each cross represents the error in estimating the days until, or after, hatching. (Hatching in this case refers to rupture of the egg membrane and not to release of the larvae. References to hatching in other studies refer to the time of larval release.)

The time of hatching is represented by the vertical line through the graph.

Note the double Y-axis.

\section{Graph 3 B}

\section{Hatching Period Study}

Ash $(n=3)$. Expressed as $\mathrm{mg}$ dry weight and \% dry weight.

The vertical portion or each cross represents the standard error. The horizontal portion of each cross represents the error in estimating the days until, or after, hatching. (Hatching in this case refers to rupture of the egg membrane and not to release of the larvae. References to hatching in other studies refer to the time of larval release.)

The time of hatching is represented by the vertical line through the graph.

Note the double Y-axis. 


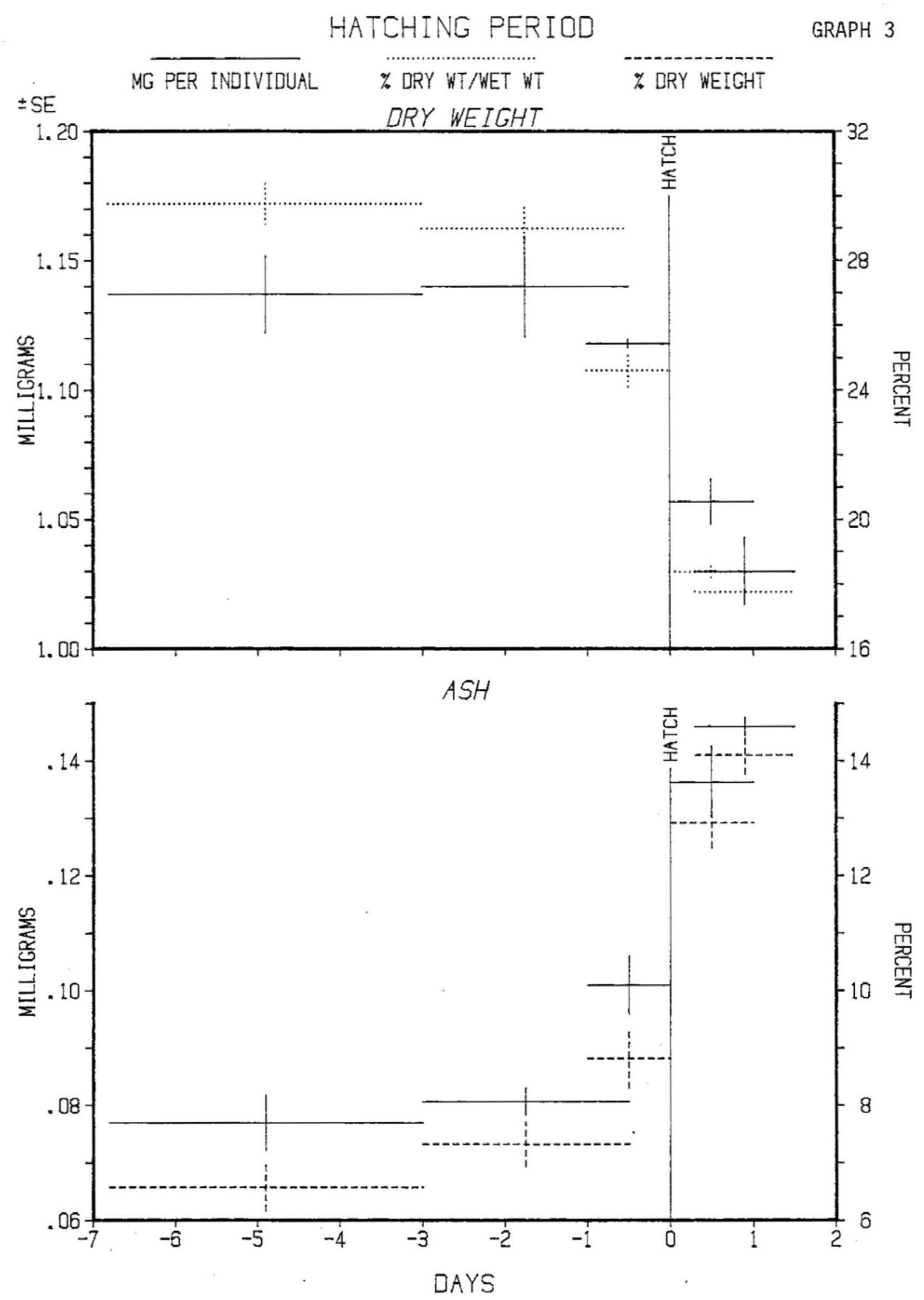


Graph 4 A

Embryogenesis Study

Carbohydrate $(\mathrm{n}=4)$. Expressed as $\mathrm{mg}$ per individual and \% dry weight. Last point represents hatched larvae.

Note double y-axis.

Error bars are standard errors.

Graph 4 B

Embryogenesis Study

Chitin ( $n=2)$. Expressed as mg per individual and \% dry weight. Last point represents hatched larvae

Note double $\mathrm{Y}$-axis.

Error bars are standard errors. 


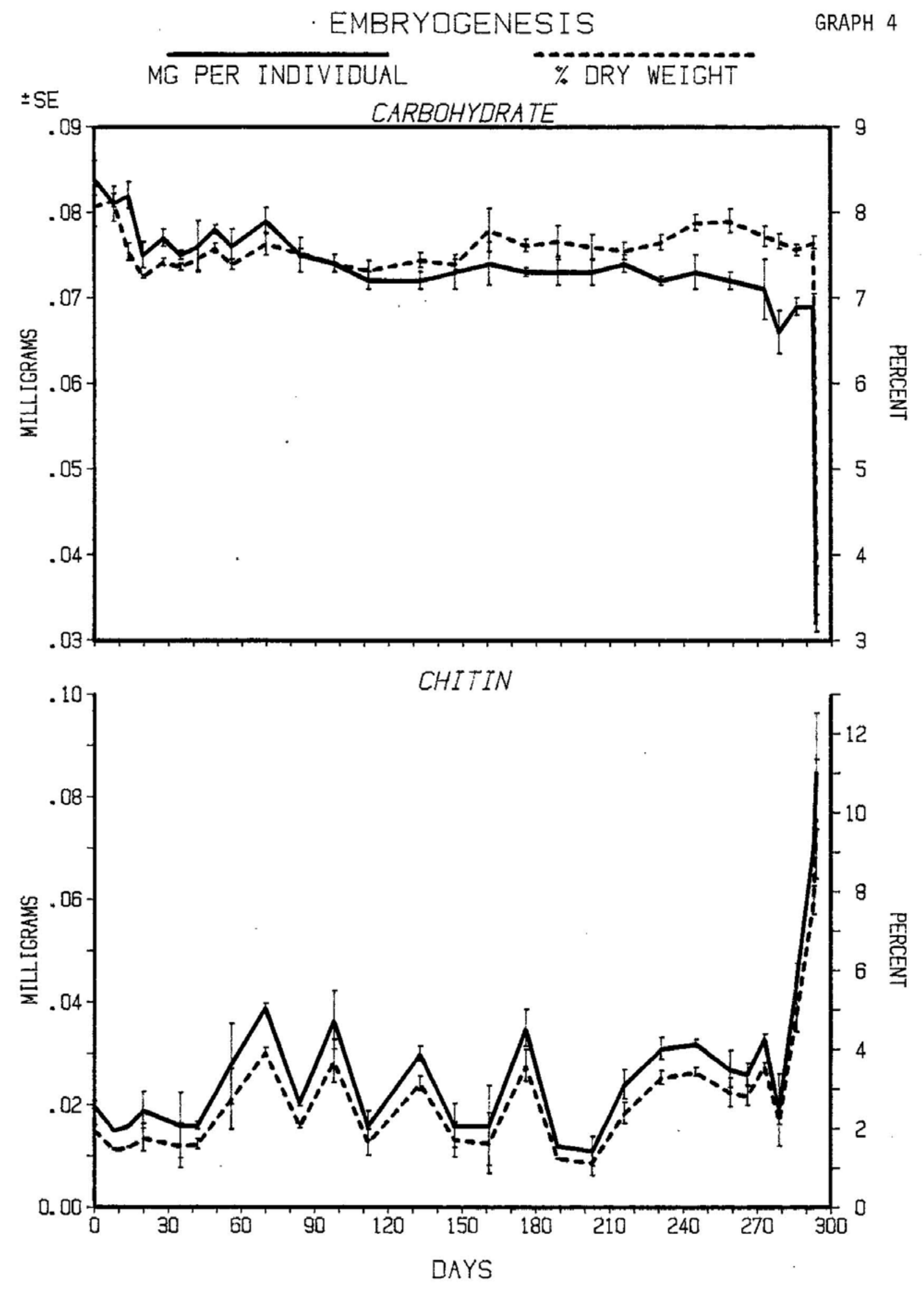


Graph 5 A

Hatching Period Study

Carbohydrate $(n=4)$. Expressed as mg dry weight and \% dry/wet weight. The vertical portion or each cross represents the standard error. The horizontal portion of each cross represents the error in estimating the days until, or after, hatching. (Hatching in this case refers to rupture of the egg membrane and not to release of the larvae. References to hatching in other studies refer to the time of larval release.)

The time of hatching is represented by the vertical line through the graph.

Note the double Y-axis.

\section{Graph 5 B}

\section{Hatching Period Study}

Chitin ( $\mathrm{n}=2)$. Expressed as $\mathrm{mg}$ dry weight and \% dry weight. The vertical portion or each cross represents the standard error. The horizontal portion of each cross represents the error in estimating the days until, or after, hatching. (Hatching in this case refers to rupture of the egg membrane and not to release of the larvae. References to hatching in other studies refer to the time of larval release.)

The time of hatching is represented by the vertical line through the graph.

Note the double $\mathrm{Y}$-axis. 


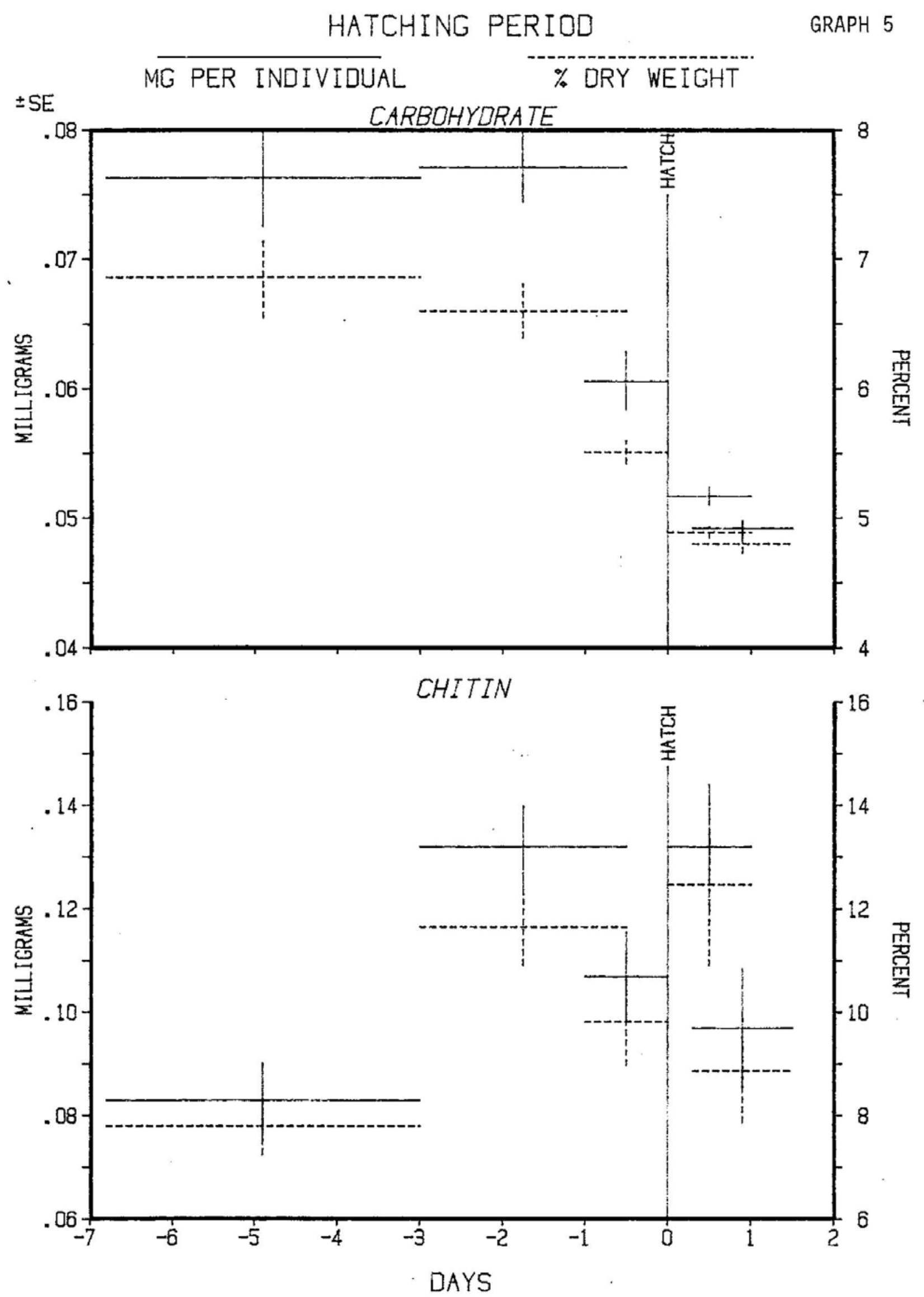




\section{Graph 6 A}

\section{Embryogenesis Study}

Protein $(n=4)$. Expressed as $m$ per individual and \% dry weight. Last point represents hatched larvae.

Note double y-axis.

Error bars are standard errors.

\section{Graph 6 B}

\section{Embryogenesis Study}

Lipid ( $\mathrm{n}=2)$. Expressed as $\mathrm{mg}$ per individual and \% dry weight. Last point represents hatched larvae Note double Y-axis.

Error bars are standard errors. 


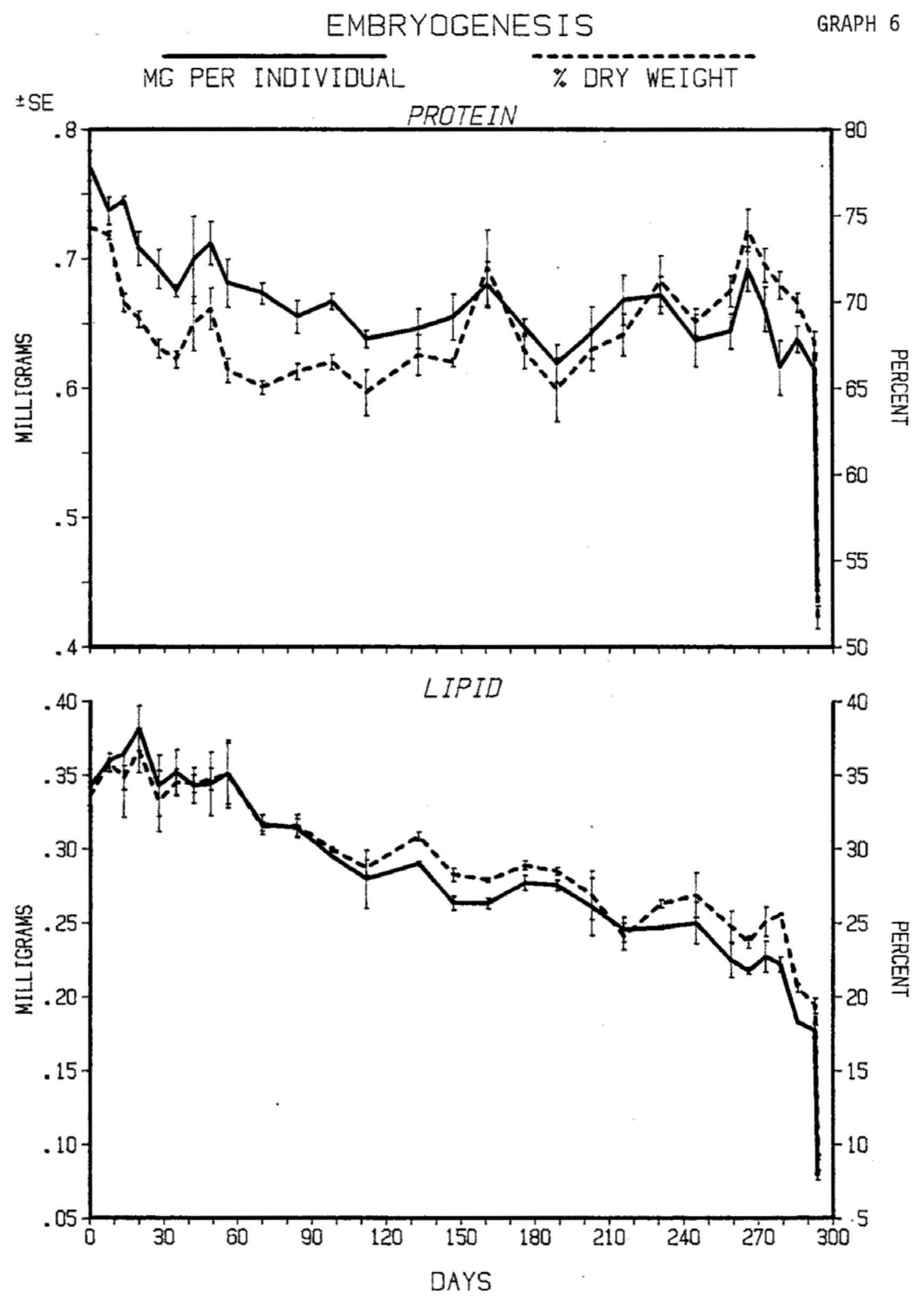


Graph 7 A

Hatching Period Study

Protein $(n=4)$. Expressed as mg dry weight and \% dry/wet weight. The vertical portion or each cross represents the standard error. The horizontal portion of each cross represents the error in estimating the days until, or after, hatching. (Hatching in this case refers to rupture of the egg membrane and not to release of the larvae. References to hatching in other studies refer to the time of larval release.)

The time of hatching is represented by the vertical line through the graph.

Note the double Y-axis.

\section{Graph 7 B}

Hatching Period Study

Lipid $(n=2)$. Expressed as $m g$ dry weight and \% dry weight. The vertical portion or each cross represents the standard error. The horizontal portion of each cross represents the error in estimating the days until, or after, hatching. (Hatching in this case refers to rupture of the egg membrane and not to release of the larvae. References to hatching in other studies refer to the time of larval release.)

The time of hatching is represented by the vertical line through the graph.

Note the double Y-axis. 
HATCHING PERIOD

GRAPH 7

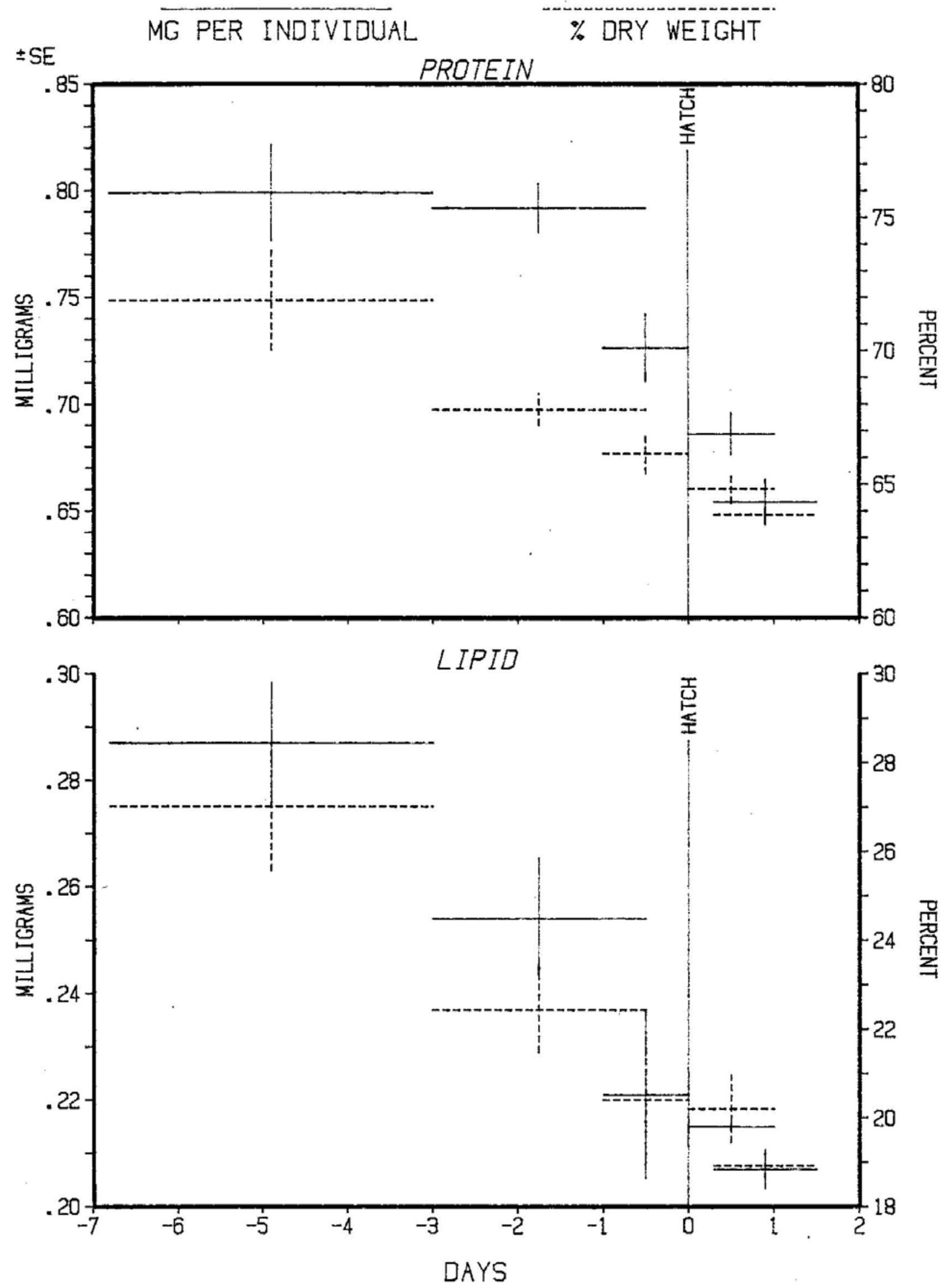


Graph 8

Embryogenesis Study

Caloric equivalents of 1ipid, protein and carbohydrate. Expressed as calories per individual.

Last point represents hatched larvae.

Error bars are standard errors. 


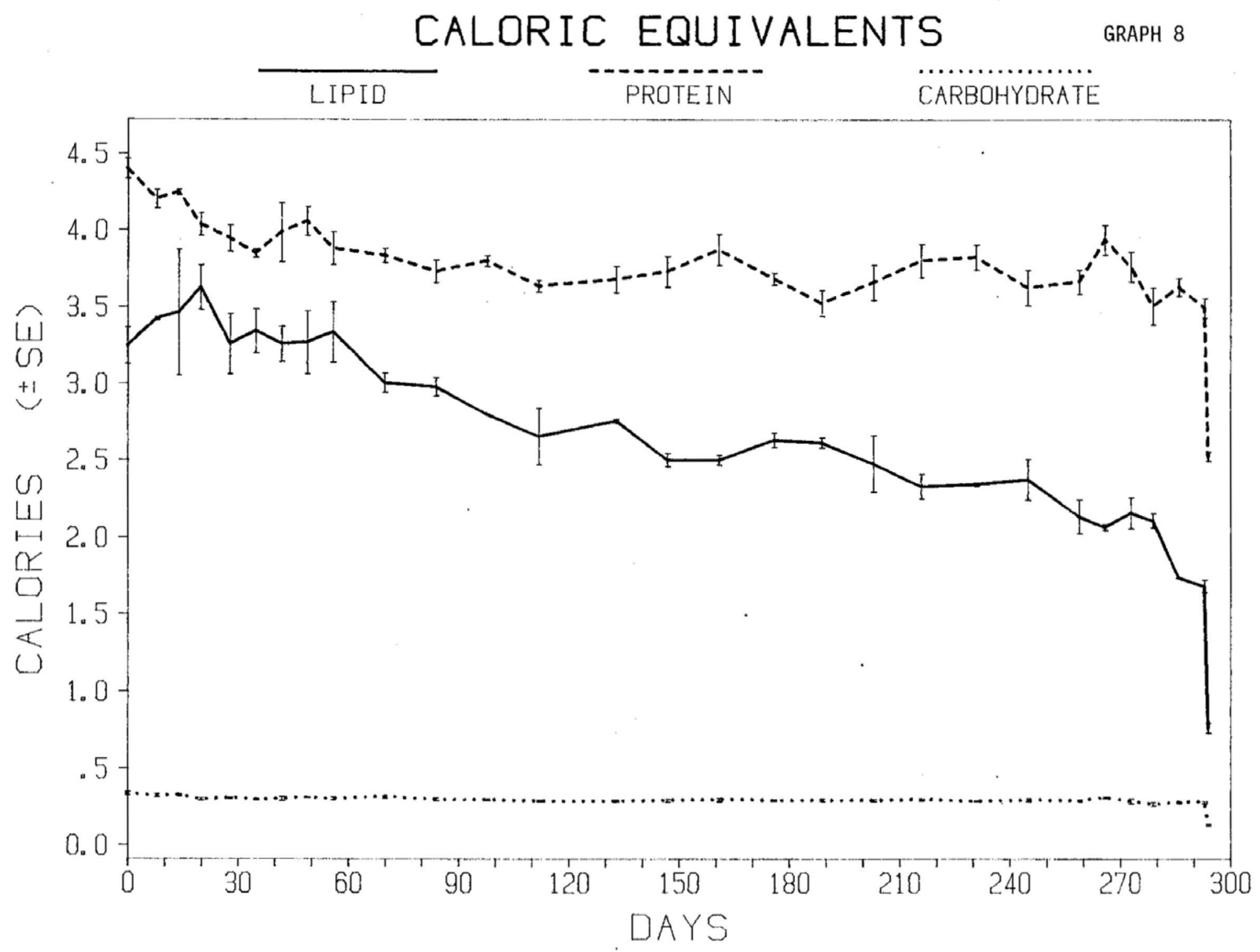




\section{Graph 9 A}

\section{Embryogenesis Study}

Triacylglycerol $(n=3)$. Expressed as micrograms per individual and \% dry weight. Last point represents hatched larvae.

Note double Y-axis.

Error bars are standard errors.

\section{Graph 9 B}

Embryogenesis Study

Sterols $(n=3)$. Expressed as micrograms per individual and \% dry weight. Last point represents hatched larvae. Note double $\mathrm{Y}$-axis. Error bars are standard errors. 


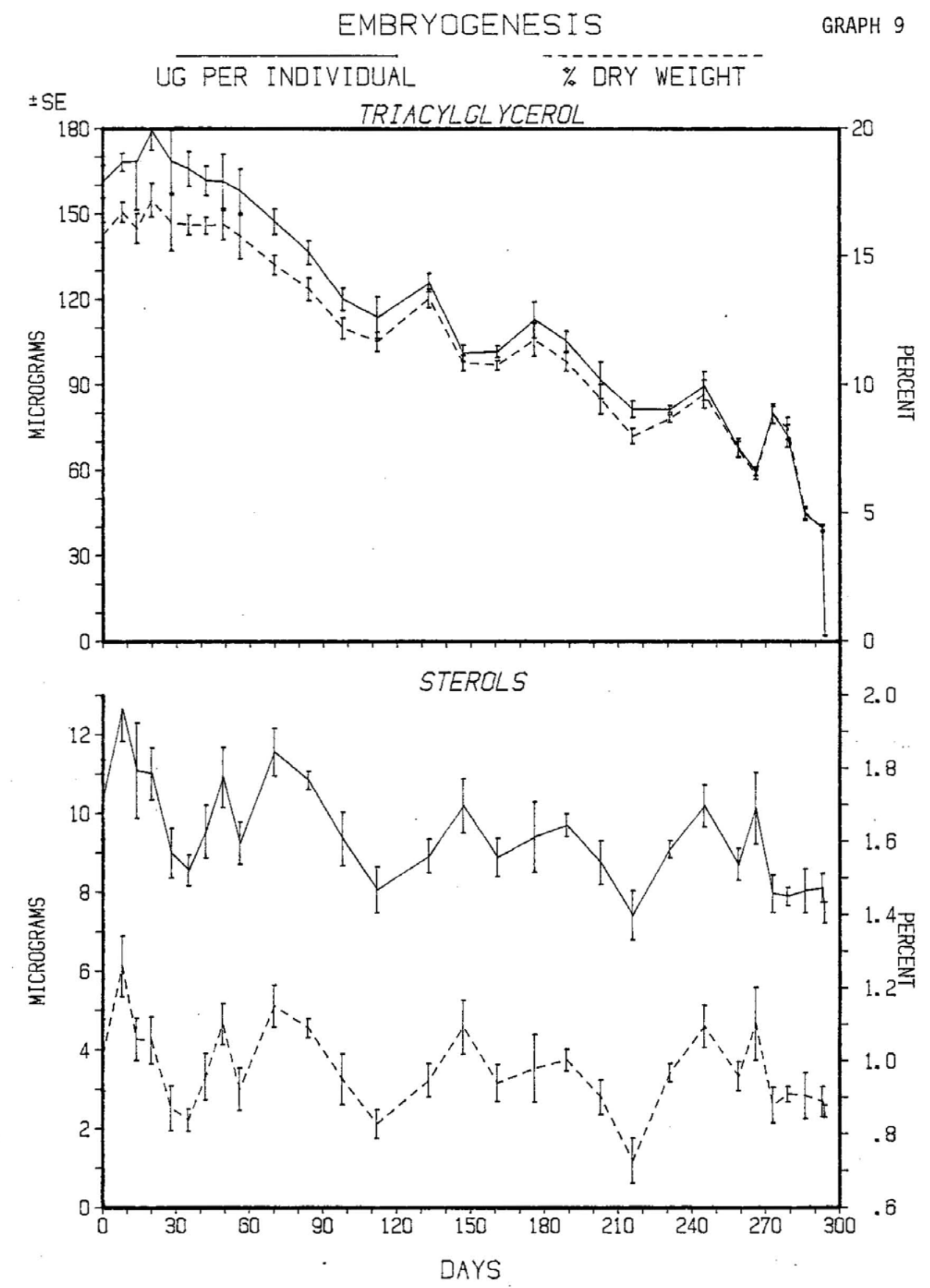


Graph 10 A

Embryogenesis Study

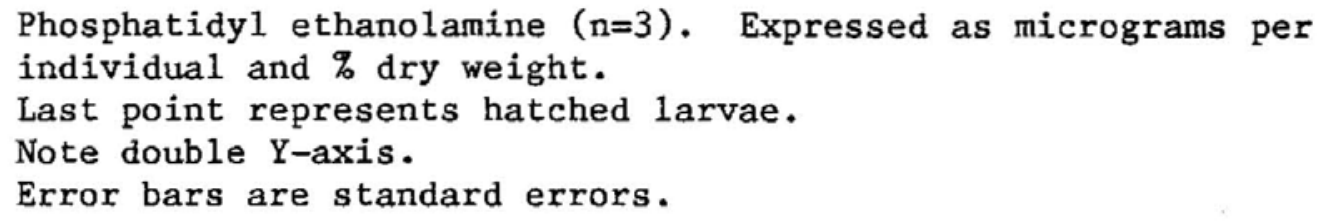

\section{Graph 10 B}

\section{Embryogenesis Study}

Phosphatidy 1 choline $(n=3)$. Expressed as micrograms per individual and \% dry weight.

Last point represents hatched larvae.

Note double Y-axis.

Error bars are standard errors. 
EMBRYOGENES IS

GRAPH 10

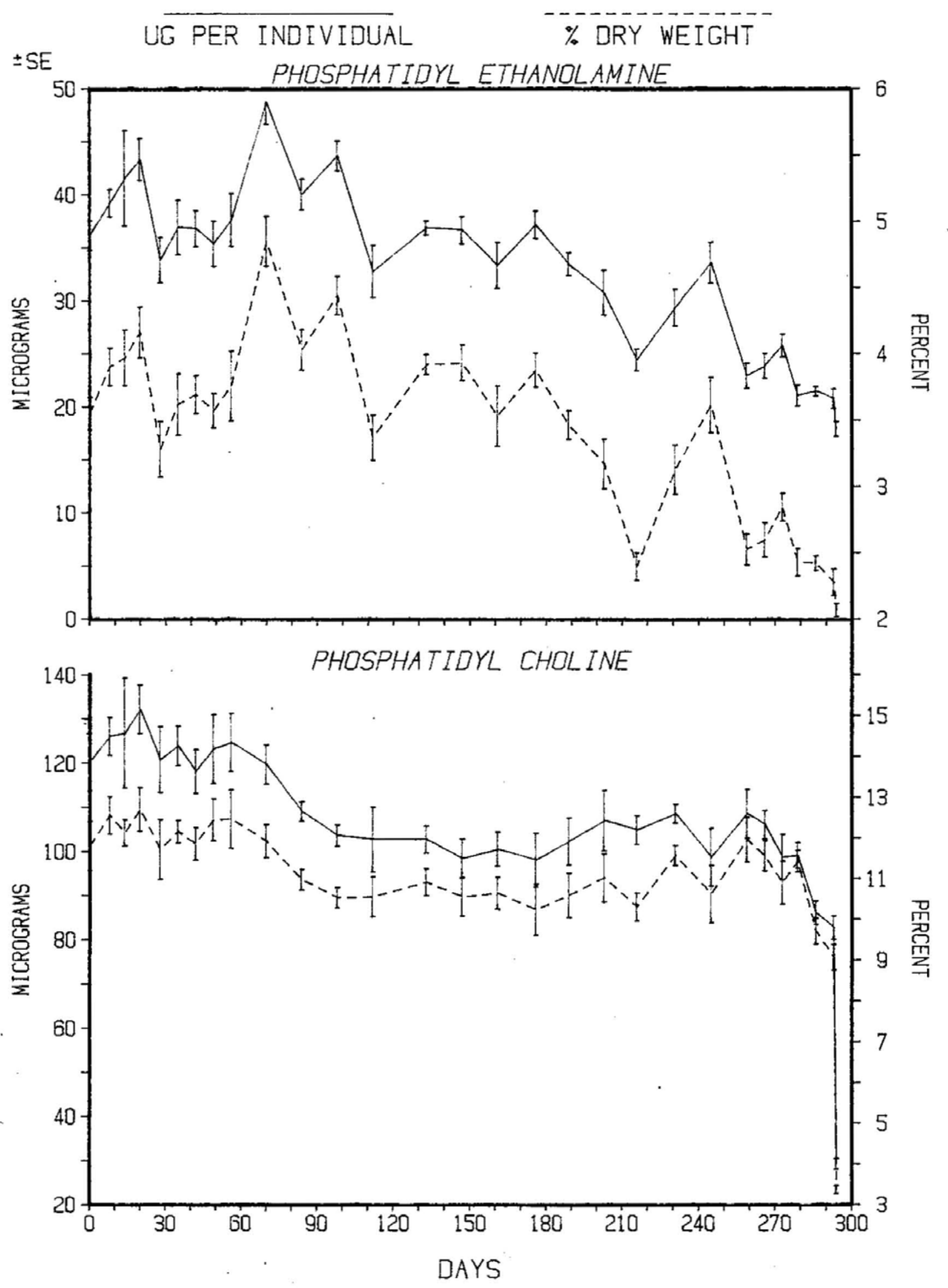




\section{Graph 11 A}

\section{Embryogenesis Study}

Selected non-essential fatty acids (NEFA) of neutral lipids. Expressed as fatty acid methyl esters (FAME), in micrograms per individual.

The vertical SAMPLE lines at the bottom of the page indicate the sampling times.

\section{Graph 11 B}

\section{Embryogenesis Study}

Selected non-essential fatty acids (NEFA) of neutral lipids.

Expressed as fatty acid methyl esters (FAME), each FAME as a $\%$ of the total FAME found in the neutral lipids.

The vertical SAMPLE lines at the bottom of the page indicate the sampling times. 


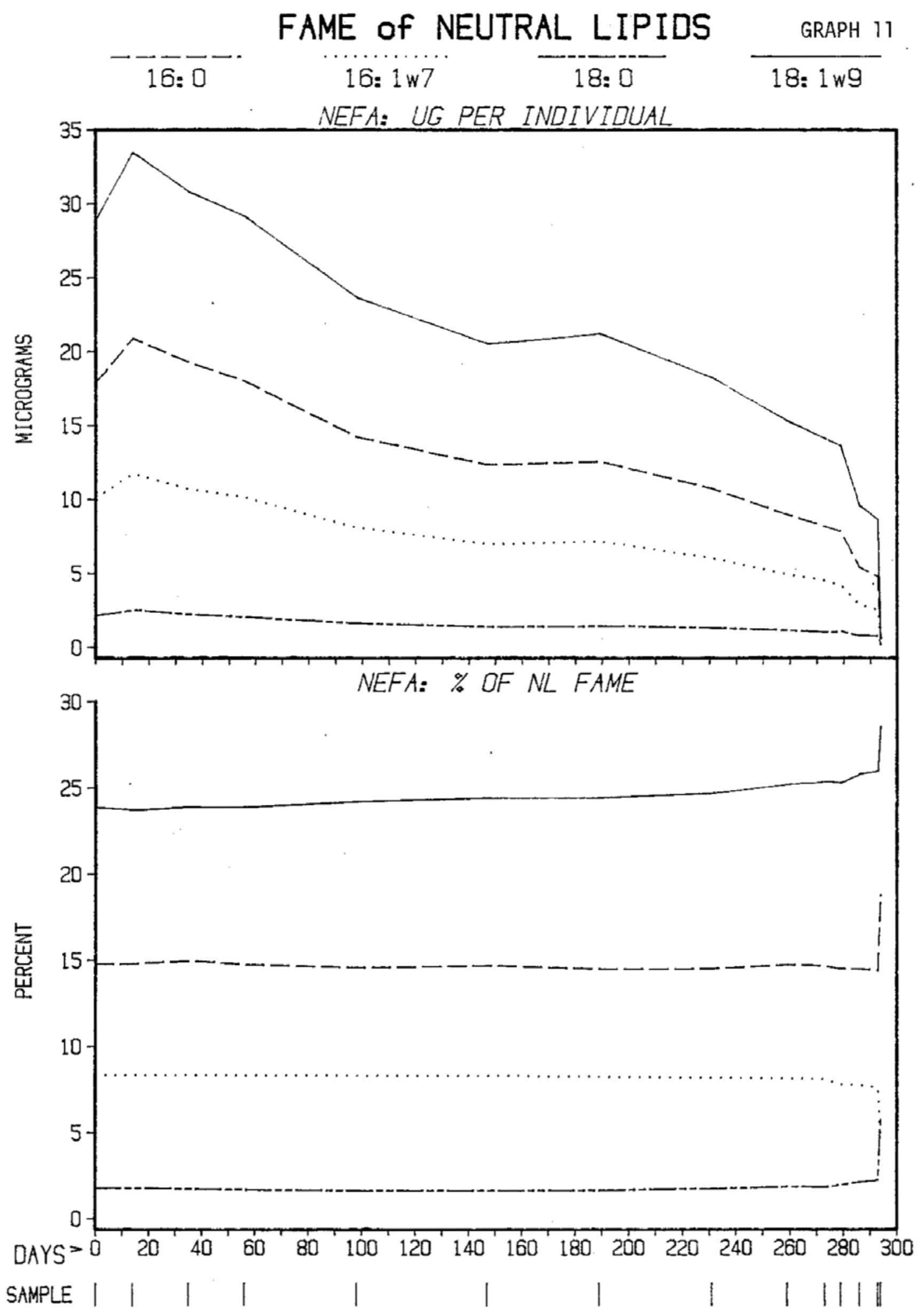




\section{Graph 12 A}

\section{Embryogenesis Study}

Selected polyunsaturated fatty acids (PUFA) of neutral lipids. Expressed as fatty acid methyl esters (FAME), in micrograms per individual.

The vertical SAMPLE lines at the bottom of the page indicate the sampling times.

\section{Graph 12 B}

Embryogenes is Study

Selected polyunsaturated fatty acids (PUFA) of neutral lipids. Expressed as fatty acid methyl esters (FAME), each FAME as a \% of the total FAME found in the neutral lipids.

The vertical SAMPLE lines at the bottom of the page indicate the sampling times. 


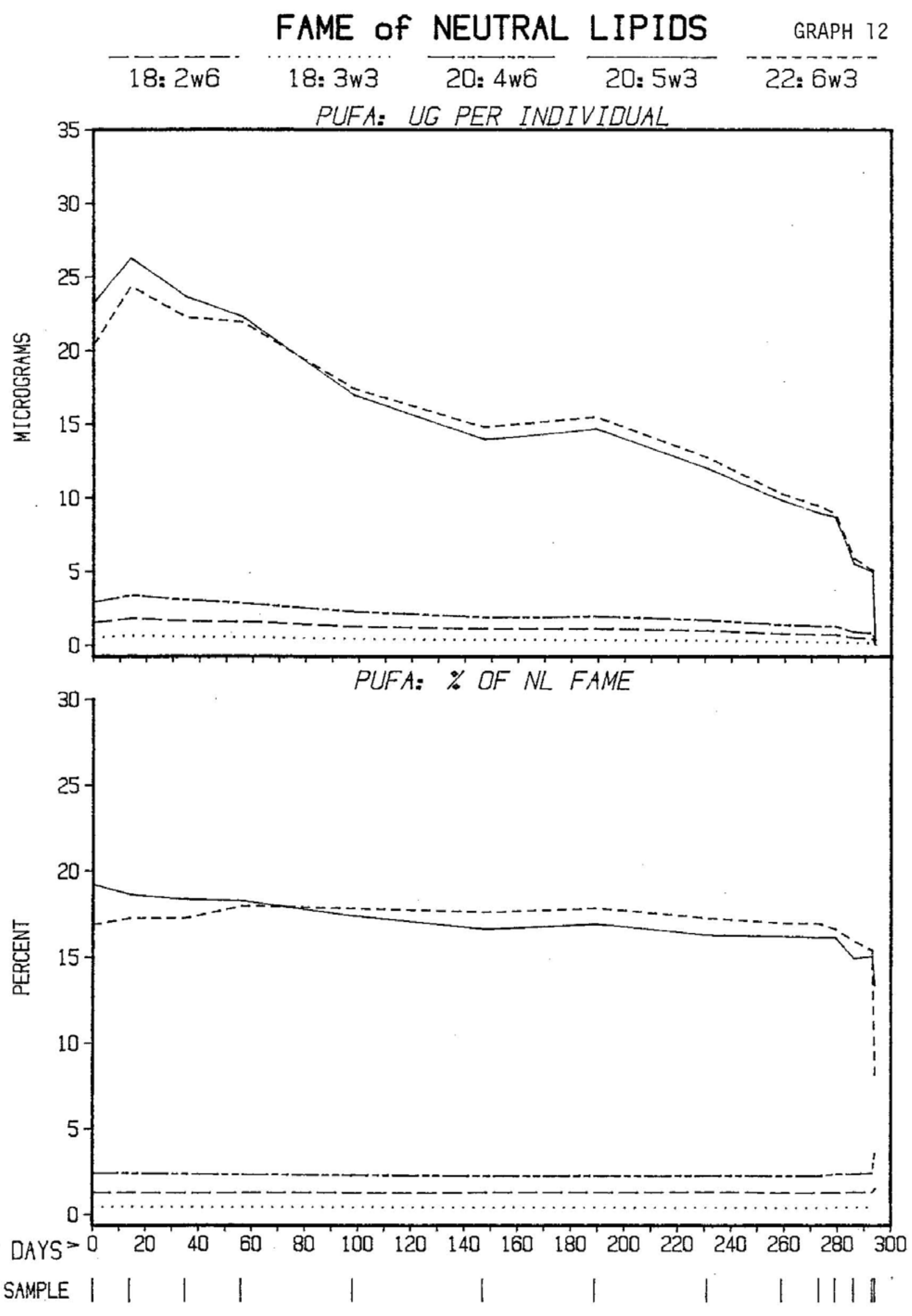


Graph 13 A

Embryogenesis Study

Selected non-essential fatty acids (NEFA) of phosphatidy1

ethanolamine. Expressed as fatty acid methyl esters (FAME), in micrograms per individual.

The vertical SAMPLE lines at the bottom of the page indicate the sampling times.

\section{Graph 13 B}

Embryogenesis Study

Selected non-essential fatty acids (NEFA) of phosphatidy1 ethanolamine. Expressed as fatty acid methyl esters (FAME), each FAME as a \% of the total FAME found in the phosphatidy1 ethanolamine. The vertica1 SAMPLE lines at the bottom of the page indicate the sampling times. 
FAME of P. ETHANOLAMINE GRAPH 13

$\begin{array}{cccc}-16: 0 & 16: 1 \mathrm{~W} 7 & -18: 0 & \\ & 18: 1 \mathrm{w} & \end{array}$

NEFA: UG PER INDIVIDUAL
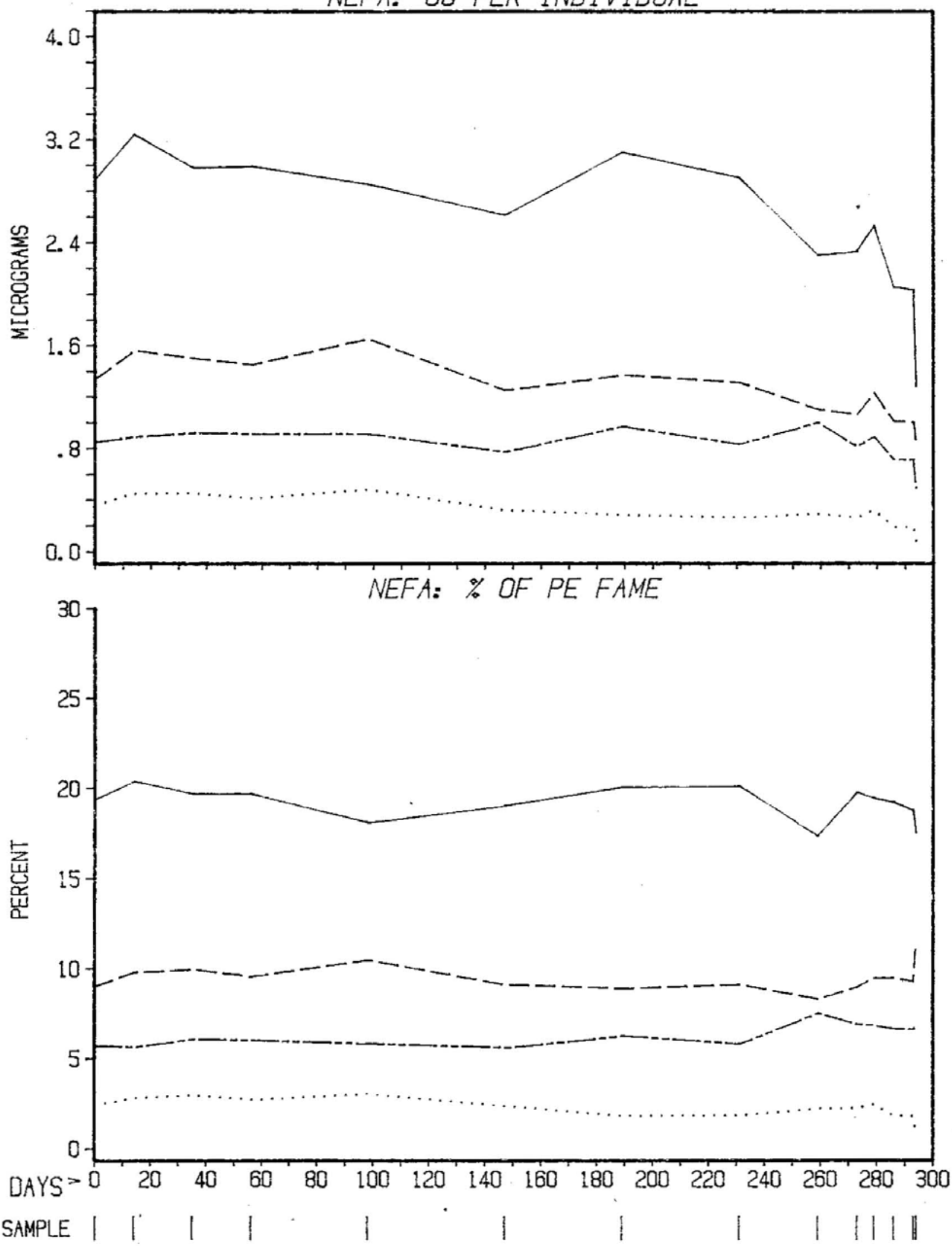


\section{Graph 14 A}

\section{Embryogenesis Study}

Selected polyunsaturated fatty acids (PUFA) of phosphatidy1

ethanolamine. Expressed as fatty acid methy1 esters (FAME), in micrograms per individual.

The vertical SAMPLE lines at the bottom of the page indicate the sampling times.

\section{Graph 14 B.}

Embryogenesis Study

Selected polyunsaturated fatty acids (PUFA) of phosphatidy1 ethanolamine. Expressed as fatty acid methy1 esters (FAME), each FAME as a \% of the total FAME found in the phosphatidyl ethanolamine. The vertical SAMPLE lines at the bottom of the page indicate the sampling times. 
FAME of P. ETHANOLAMINE GRAPH 14

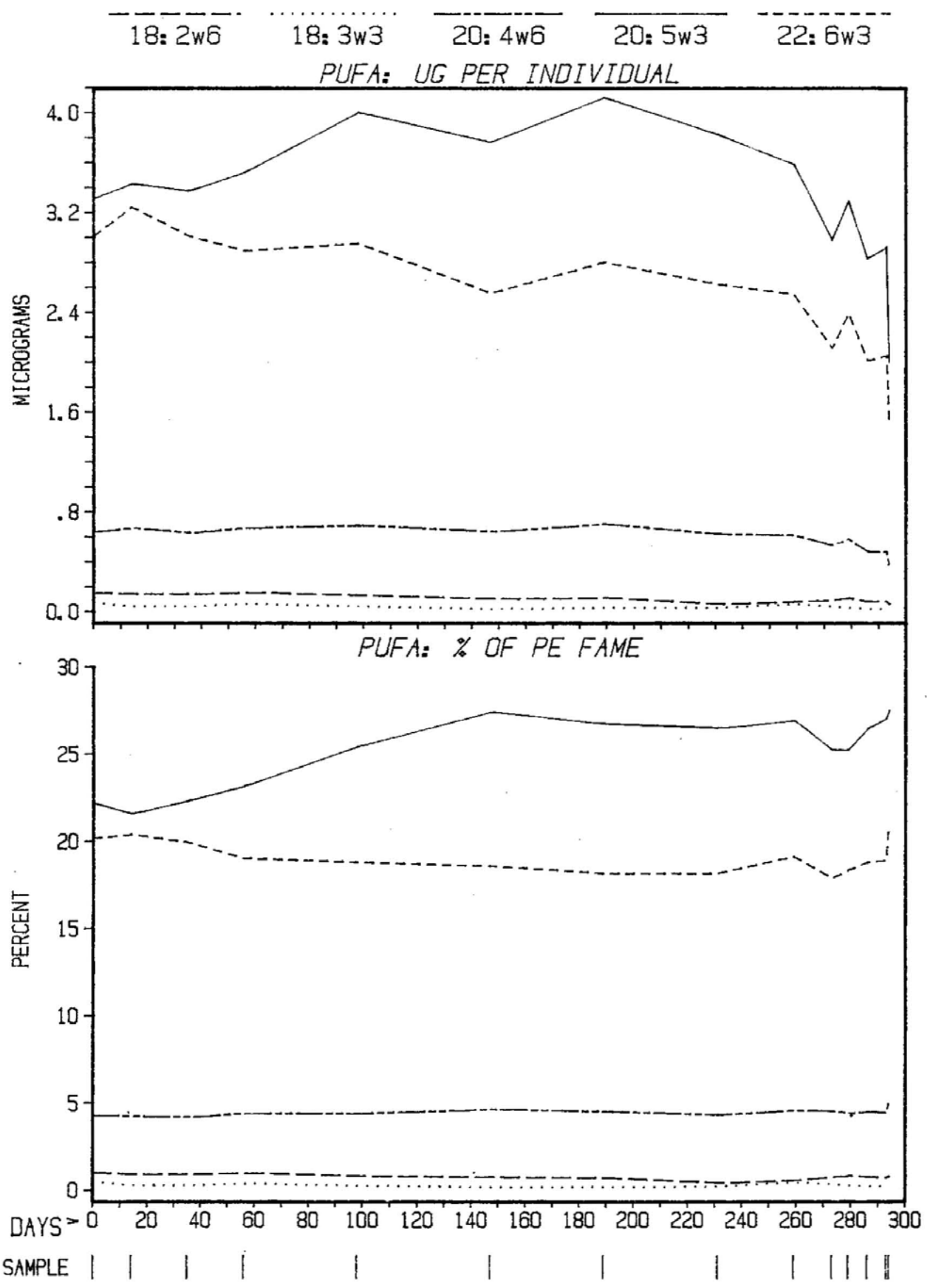




\section{Graph 15 A}

Embryogenesis Study

Selected non-essential fatty acids (NEFA) of phosphatidyl choline. Expressed as fatty acid methyl esters (FAME), in micrograms per individual.

The vertical SAMPLE lines at the bottom of the page indicate the sampling times.

Graph 15 B

Embryogenesis Study

Selected non-essential fatty acids (NEFA) of phosphatidyl choline. Expressed as fatty acid methyl esters (FAME), each FAME as a $\%$ of the total FAME found in the phosphatidyl choline.

The vertical SAMPLE lines at the bottom of the page indicate the samp1ing times. 
FAME of P. CHOLINE GRAPH 15

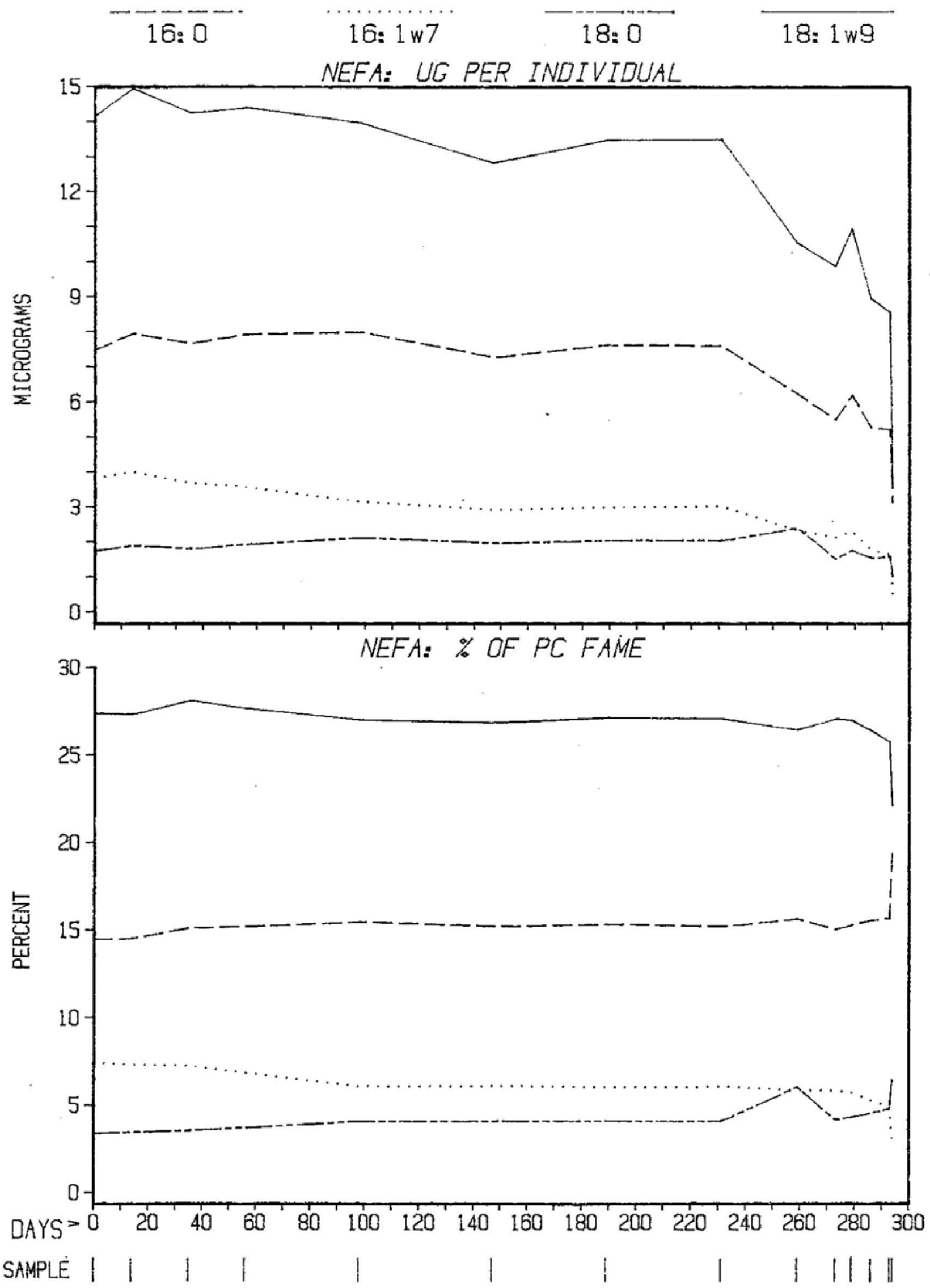


Graph 16 A

Embryogenesis Study

Selected polyunsaturated fatty acids (PUFA) of phosphatidyl choline. Expressed as fatty acid methyl esters (FAME), in micrograms per individual.

The vertical SAMPLE lines at the bottom of the page indicate the sampling times.

\section{Graph 16 B}

\section{Embryogenesis Study}

Selected polyunsaturated fatty acids (PUFA) of phosphatidyl choline. Expressed as fatty acid methyl esters (FAME), each FAME as a $\%$ of the tota1 FAME found in the phosphatidy1 choline. The vertical SAMPLE lines at the bottom of the page indicate the samp1ing times. 
FAME of P. CHOLINE GRAPH 16

18:2w6 18:3w3 20:4w6 20:5w3 22:6w3

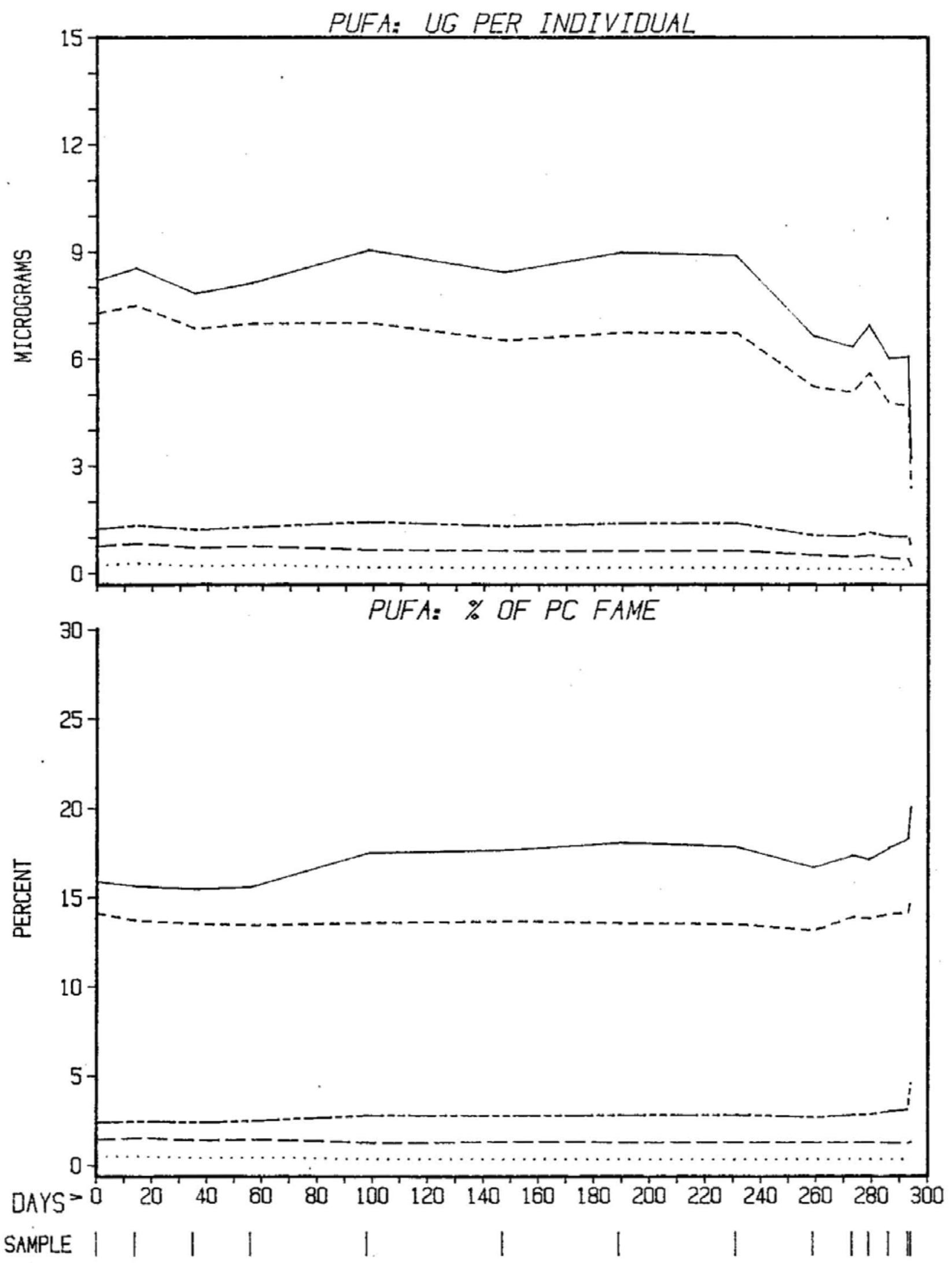




\section{Graph 17 A}

Pulse Fed and Immediate Post-Hatch Development Study

Carbohydrate $(n=4)$. Expressed as $m$ per individual and \% dry weight. The vertical lines labelled "FED" indicate the time of feeding. The "MOLT STAGE" labels at the bottom of the page indicate the molt stage of the larvae in each sample.

Note double Y-axis.

Error bars are standard errors.

\section{Graph 17 B}

Pulse Fed and Immediate Post-Hatch Development Study

Ash $(n=3)$. Expressed as $m$ per individual and \% dry weight. The vertical lines labelled "FED" indicate the time of feeding. The "MOLT STAGE" labels at the bottom of the page indicate the molt stage of the larvae in each sample.

Note doub1e Y-axis.

Error bars are standard errors. 


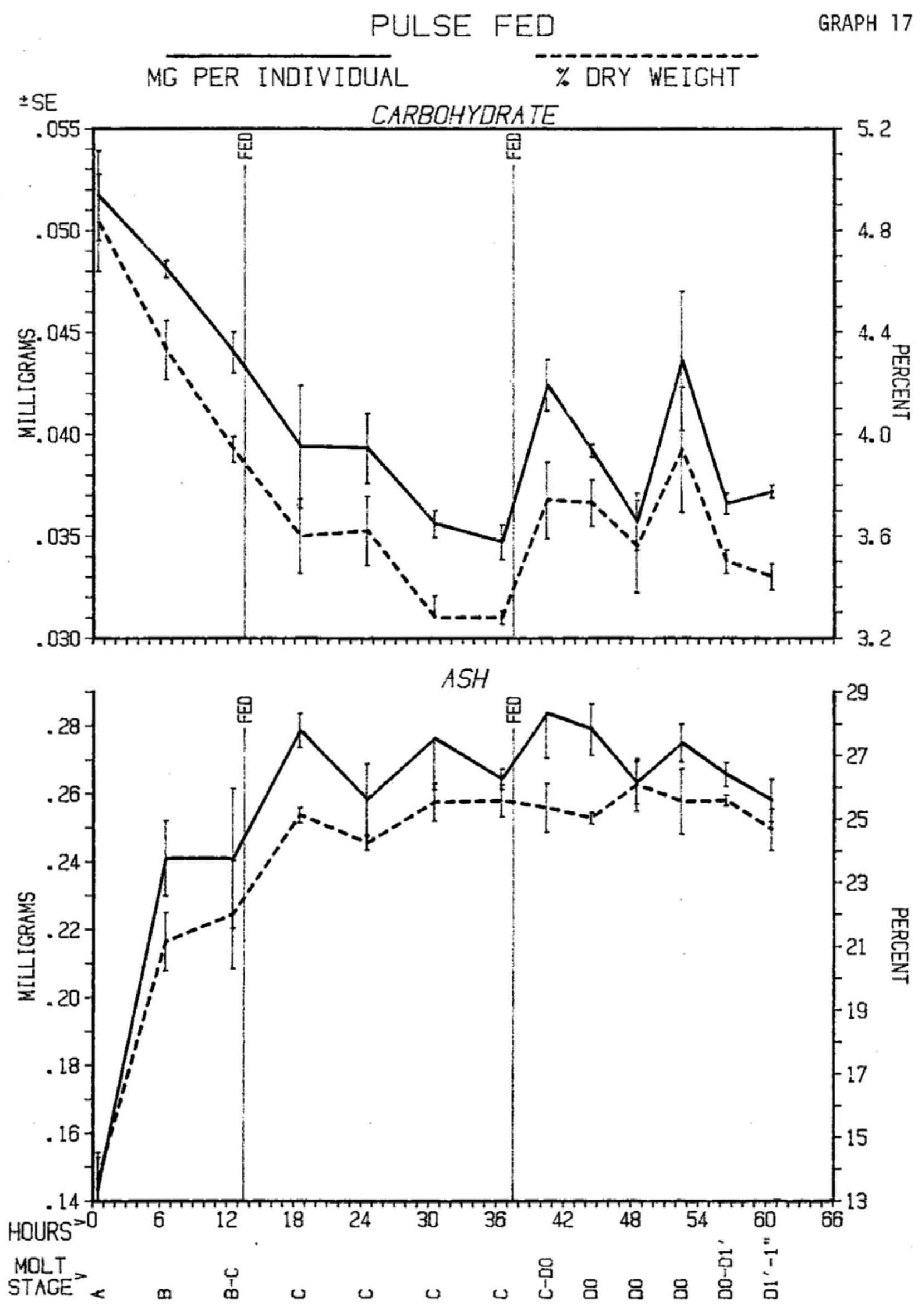


Graph 18 A

Pulse Fed and Immediate Post-Hatch Development Study

Protein ( $\mathrm{n}=4)$. Expressed as mg per individual and \% dry weight.

The vertical lines labelled "FED" indicate the time of feeding.

The "MOLT STAGE" labels at the bottom of the page indicate the molt stage of the larvae in each sample.

Note double $\mathrm{Y}$-axis.

Error bars are standard errors.

\section{Graph 18 B}

Pulse Fed and Immediate Post-Hatch Development Study

Lipid ( $\mathrm{n}=2)$. Expressed as $\mathrm{mg}$ per individual and \% dry weight. The vertical lines labelled "FED" indicate the time of feeding. The "MOLT STAGE" labels at the bottom of the page indicate the molt stage of the larvae in each sample. Note double Y-axis.

Error bars are standard errors. 

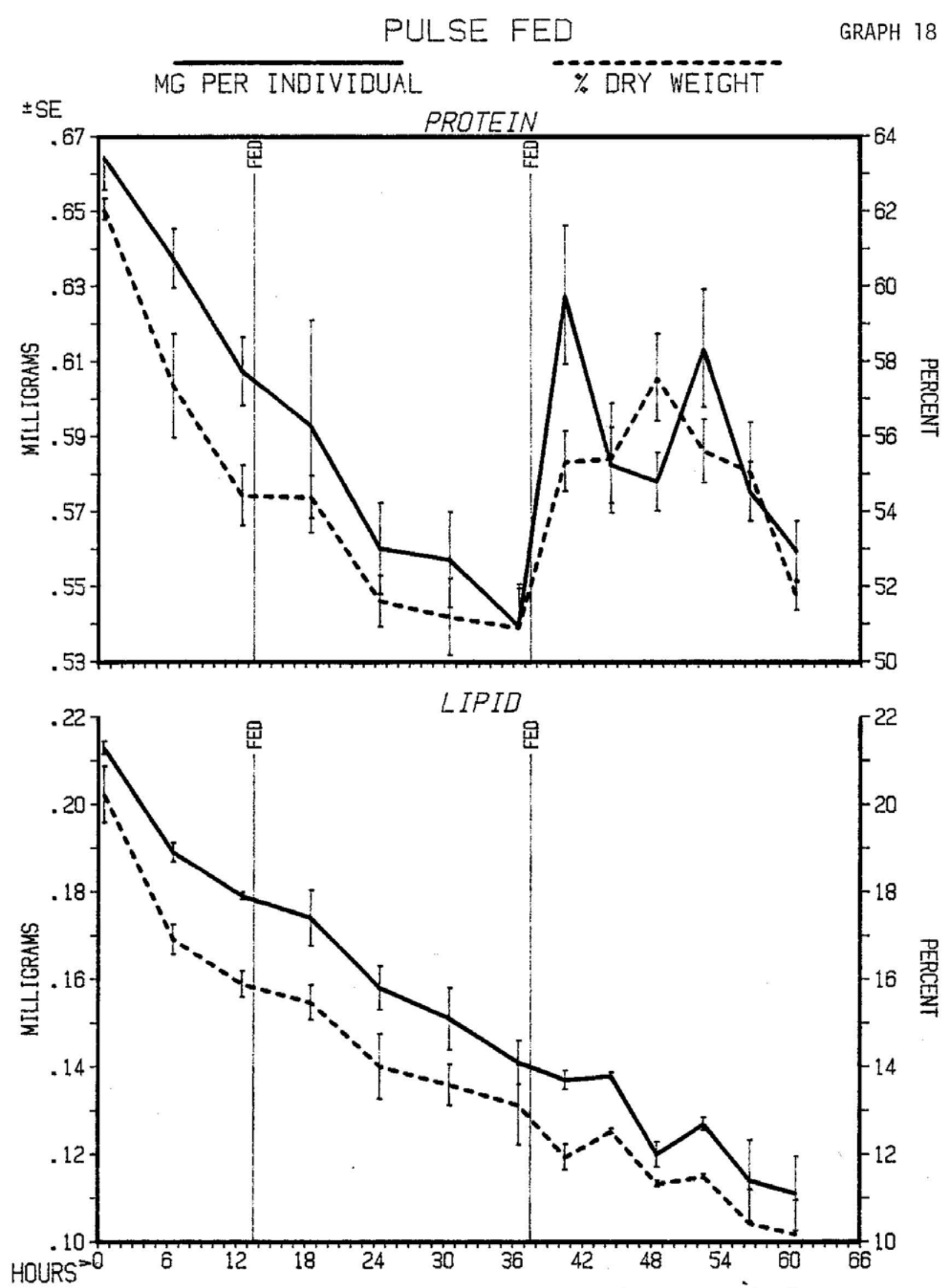

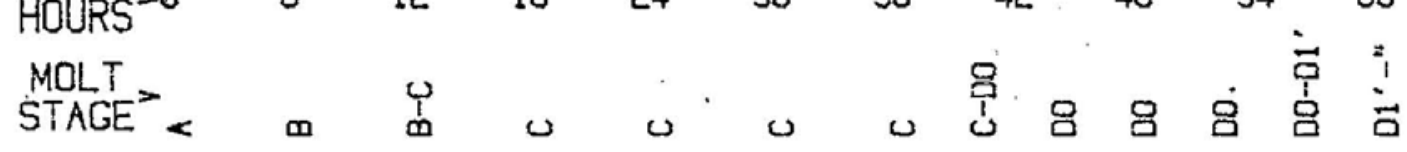


Graph 19 A

Larval Development Study

Wet weight $(\mathrm{n}=7)$. Expressed as $\mathrm{mg}$ per individual.

The vertical lines labelled "MOLT" indicate the time of molting. The roman numerals (I-IV) at the top of the graph indicate the larval stage.

The MOLT STAGE labels at the bottom of the page are generalized to show the range of each molt stage and indicate the molt stage of the samples. The exact molt stage for any sample can be obtained from the Tables.

Error bars are standard errors.

\section{Graph 19 B}

Larval Development Study

Ash free dry weight $(n=7)$. Expressed as mg per individual. The vertical lines (labelled "MOLT" in graph A) indicate the time of molting. The roman numerals (I-IV) at the top graph A apply to this graph and indicate the larval stage.

The MOLT STAGE labels at the bottom of the page are generalized to show the range of each molt stage and indicate the molt stage of the samples. The exact molt stage for any sample can be obtained from the Tables.

Error bars are standard errors. 


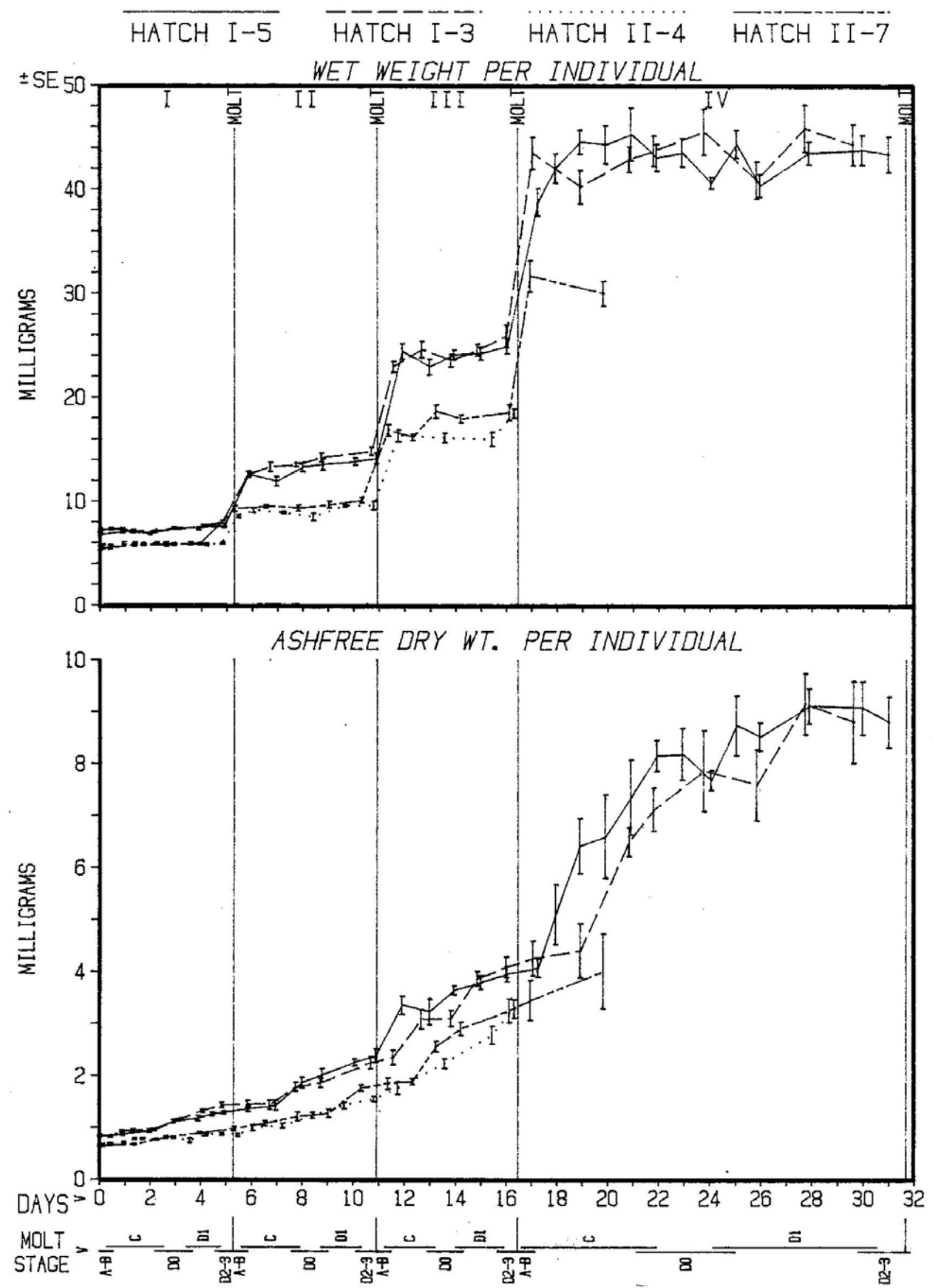




\section{Graph 20 A}

Larval Development Study

Dry weight $(\mathrm{n}=7)$. Expressed as $\mathrm{mg}$ per individual.

The vertical lines labelled "MOLT" indicate the time of molting. The roman numerals (I-IV) at the top of the graph indicate the larval

stage.

The MOLT STAGE labels at the bottom of the page are generalized to show the range of each molt stage and indicate the molt stage of the samples. The exact molt stage for any sample can be obtained from the Tables.

Error bars are standard errors.

\section{Graph 20 B}

Larval Development Study

$\% \mathrm{dry} /$ wet weight $(\mathrm{n}=7)$.

The vertical lines (labelled "MOLT" in graph $\underline{A}$ ) indicate the time of molting. The roman numerals (I-IV) at the top graph $\underline{A}$ apply to this graph and indicate the larva1 stage.

The MOLT STAGE labels at the bottom of the page are generalized to show the range of each molt stage and indicate the molt stage of the samples. The exact molt stage for any sample can be obtained from the Tables.

Error bars are standard errors. 


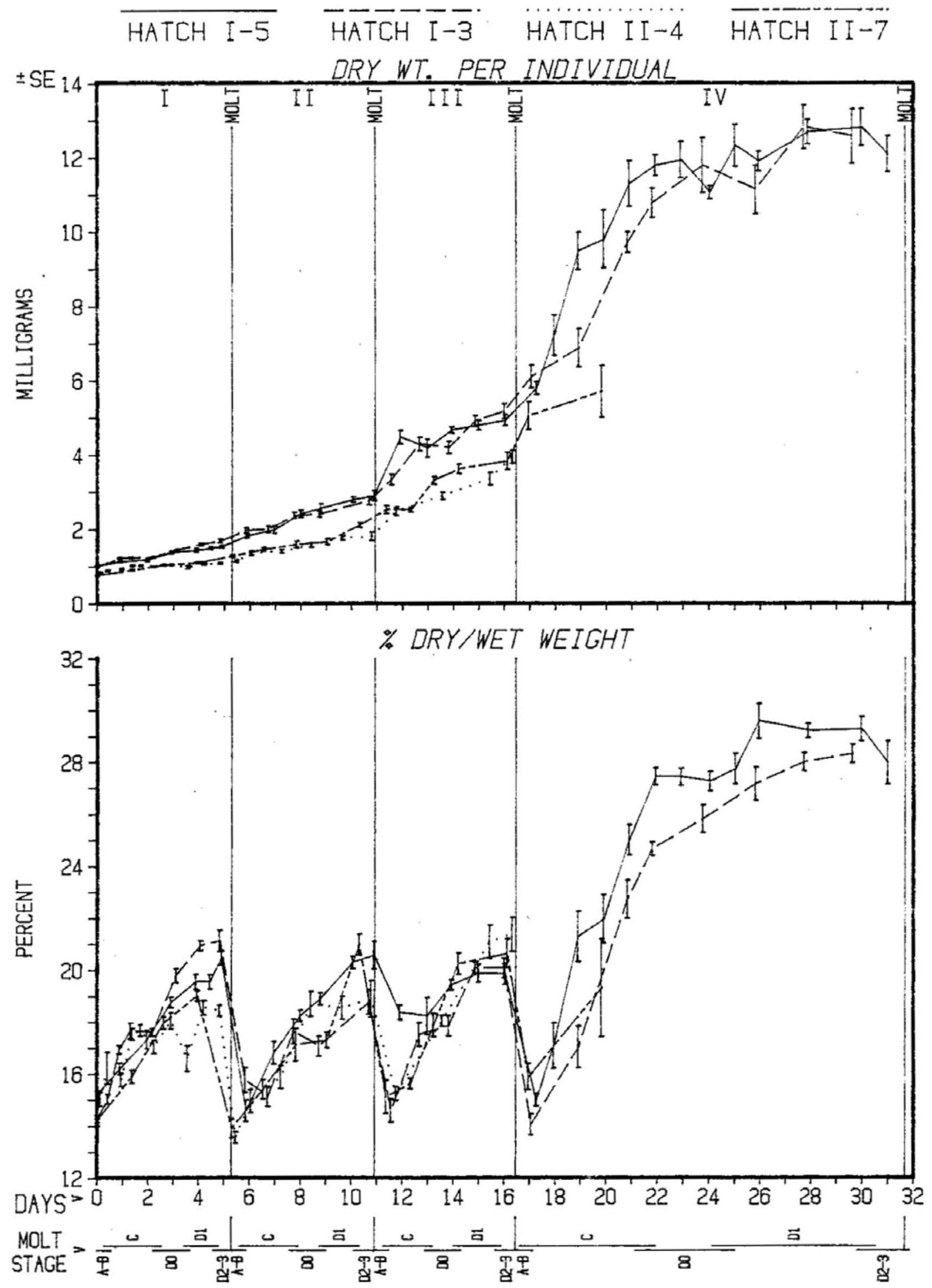


Graph 21 A

Larval Development Study

Ash ( $n=3)$. Expressed as mg per Individual.

The vertical lines labelled "MOLT" indicate the time of molting. The roman numerals (I-IV) at the top of the graph indicate the larval stage.

The MOLT STAGE labels at the bottom of the page are generalized to show the range of each molt stage and indicate the molt stage of the samples. The exact molt stage for any sample can be obtained from the Tables.

Error bars are standard errors.

Graph 21 B

Larval Development Study

Ash $(n=3)$. Expressed as \% dry weight.

The vertical lines (labelled "MOLT" in graph A) indicate the time of molting. The roman numerals (I-IV) at the top graph $\underline{A}$ apply to this graph and indicate the larval stage.

The MOLT STAGE labels at the bottom of the page are generalized to show the range of each molt stage and indicate the molt stage of the samples. The exact molt stage for any sample can be obtained from the Tables.

Error bars are standard errors. 
ASH

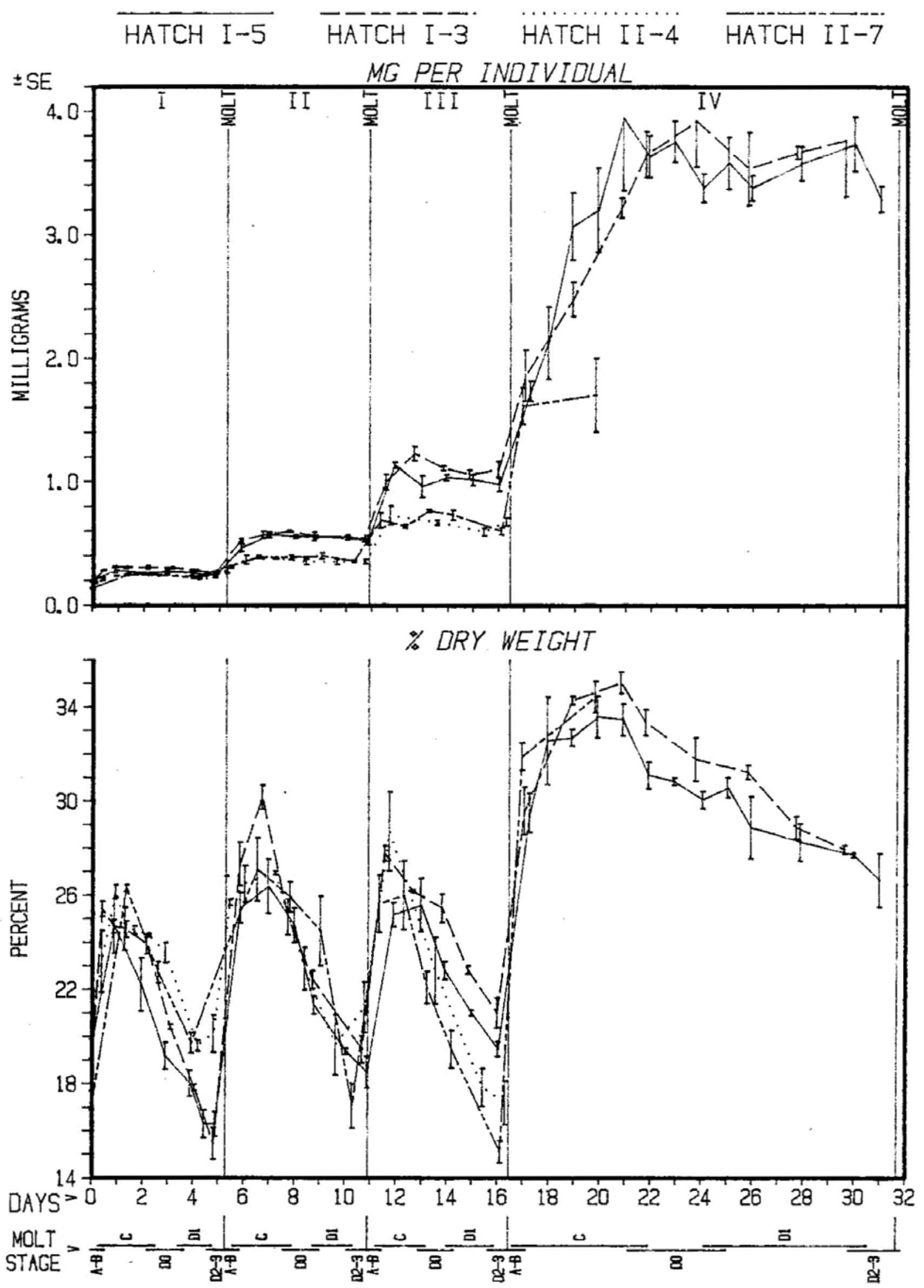


Graph 22 A

Larval Development Study

Chitin ( $\mathrm{n}=2)$. Expressed as $\mathrm{mg}$ per individual.

The vertical lines labelled "MOLT" indicate the time of molting. The roman numerals (I-IV) at the top of the graph indicate the larval stage.

The MOLT STAGE labels at the bottom of the page are generalized to show the range of each molt stage and indicate the molt stage of the samples. The exact molt stage for any sample can be obtained from the Tables.

Error bars are standard errors.

\section{Graph 22 B}

Larva1 Development Study

Chitin $(n=2)$. Expressed as \% dry weight.

The vertical lines (labelled "MOLT" in graph A) indicate the time of molting. The roman numerals (I-IV) at the top graph $\underline{A}$ apply to this graph and indicate the larval stage.

The MOLT STAGE labels at the bottom of the page are generalized to show the range of each molt stage and indicate the molt stage of the samples. The exact molt stage for any sample can be obtained from the Tables.

Error bars are standard errors. 


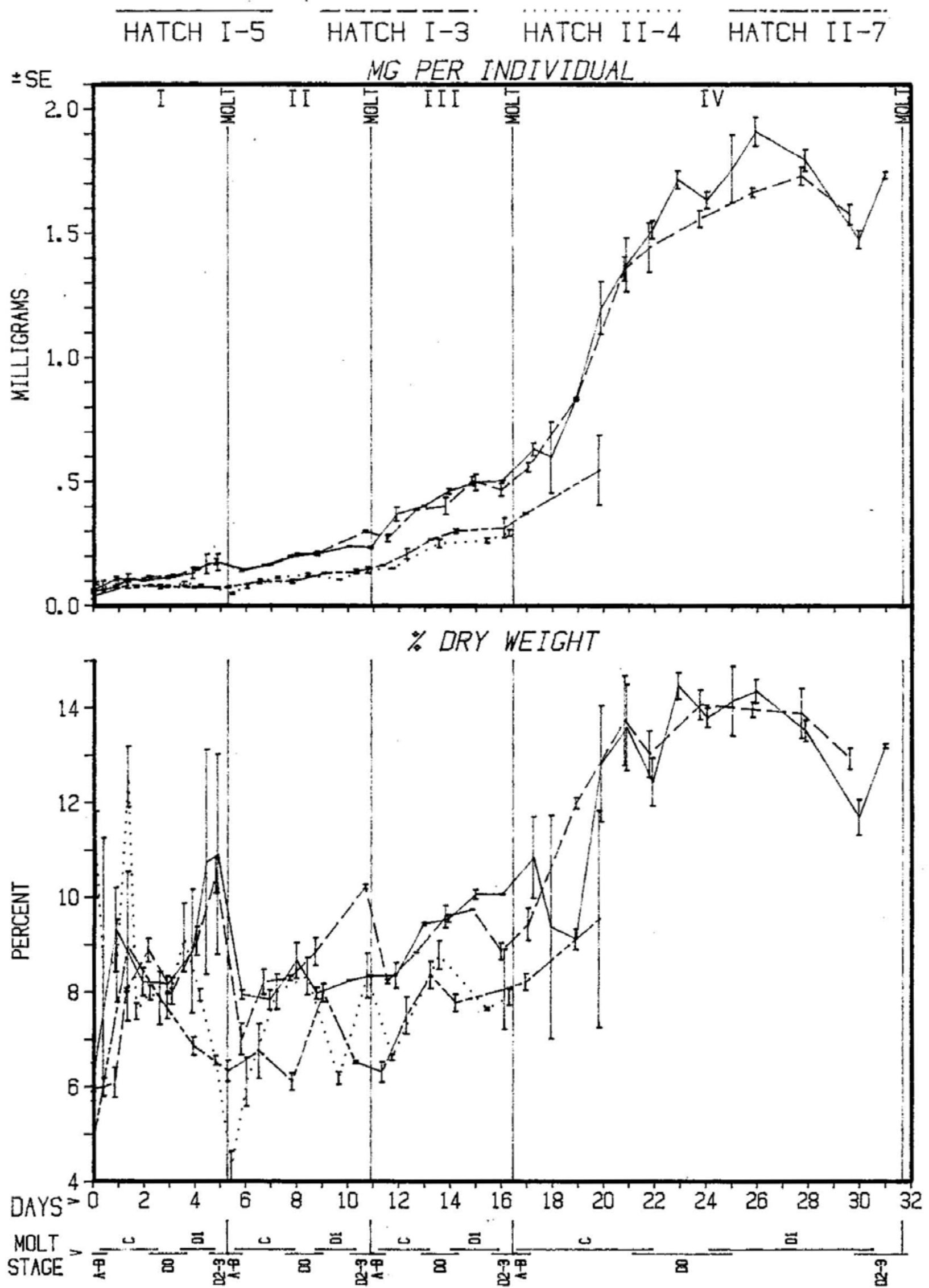




\section{Graph 23 A}

Larval Development Study

Carbohydrate $(\mathrm{n}=4)$. Expressed as $\mathrm{mg}$ per individual.

The vertical lines labelled "MOLT" indicate the time of molting. The roman numerals (I-IV) at the top of the graph indicate the larval stage.

The MOLT STAGE labels at the bottom of the page are generalized to show the range of each molt stage and indicate the molt stage of the samples. The exact molt stage for any sample can be obtained from the Tables.

Error bars are standard errors.

\section{Graph 23 B}

Larva1 Development Study

Carbohydrate $(\mathrm{n}=4)$. Expressed as \% dry weight. The vertical lines (labelled "MOLT" in graph A) indicate the time of molting. The roman numerals (I-IV) at the top graph $\underline{A}$ apply to this graph and indicate the larval stage.

The MOLT STAGE labels at the bottom of the page are generalized to show the range of each molt stage and indicate the molt stage of the samples. The exact molt stage for any sample can be obtained from the Tables.

Error bars are standard errors. 
CARBOHYDRATE

GRAPH 23

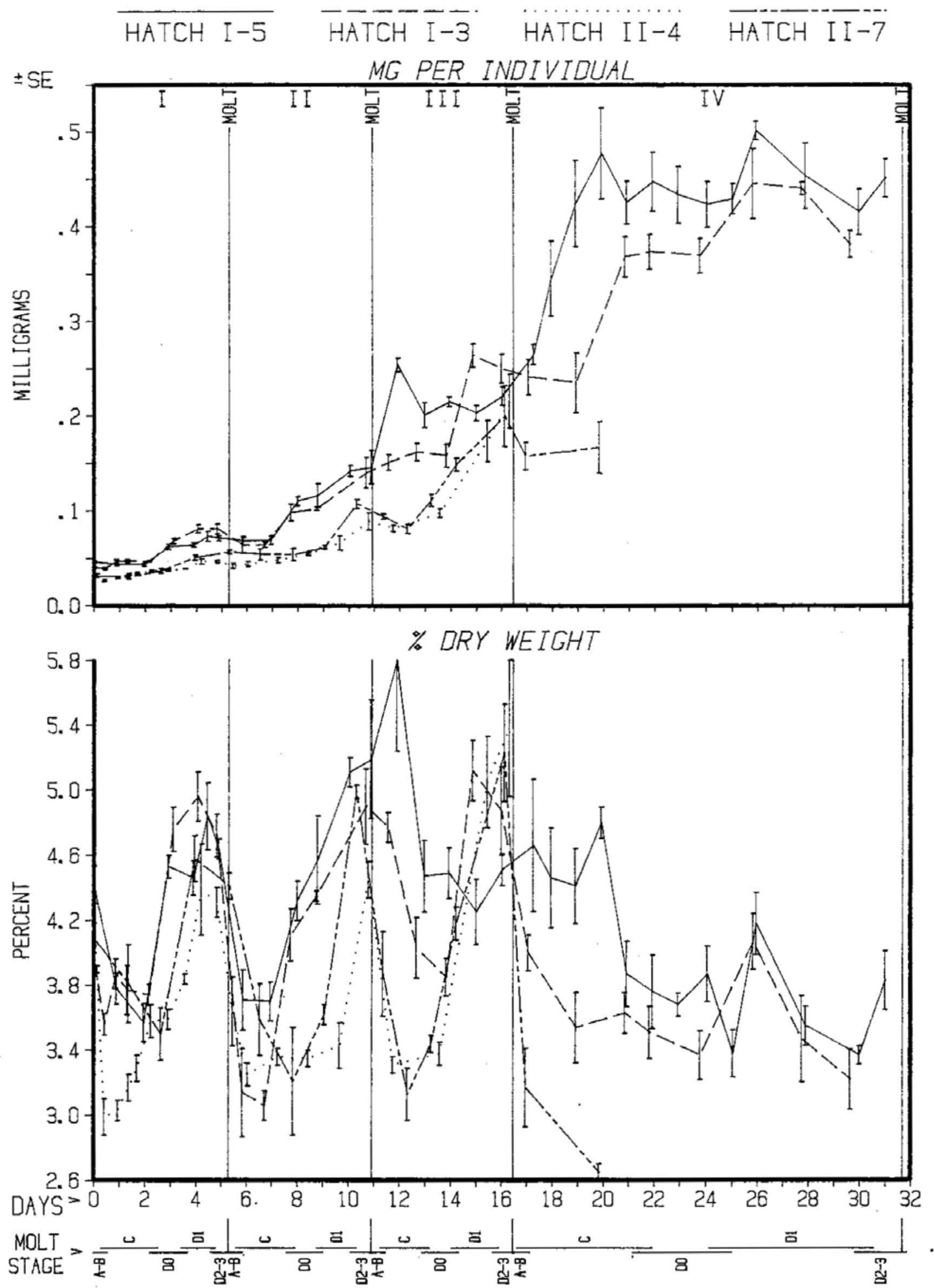


Graph 24 A

Larval Development Study

Protein ( $\mathrm{n}=4$ ). Expressed as $\mathrm{mg}$ per individual.

The vertical lines labelled "MOLT" indicate the time of molting. The roman numerals (I-IV) at the top of the graph indicate the larval stage.

The MOLT STAGE labels at the bottom of the page are generalized to show the range of each molt stage and indicate the molt stage of the samples. The exact molt stage for any sample can be obtained from the Tables.

Error bars are standard errors.

Graph 24 B

Larval Development Study

Protein ( $n=4)$. Expressed as \% dry weight.

The vertical lines (labelled "MOLT" in graph $\underline{A}$ ) indicate the time of molting. The roman numerals (I-IV) at the top graph A apply to this graph and indicate the larval stage.

The MOLT STAGE labels at the bottom of the page are generalized to show the range of each molt stage and indicate the molt stage of the samples. The exact molt stage for any sample can be obtained from the Tables.

Error bars are standard errors. 
PRDTEIN

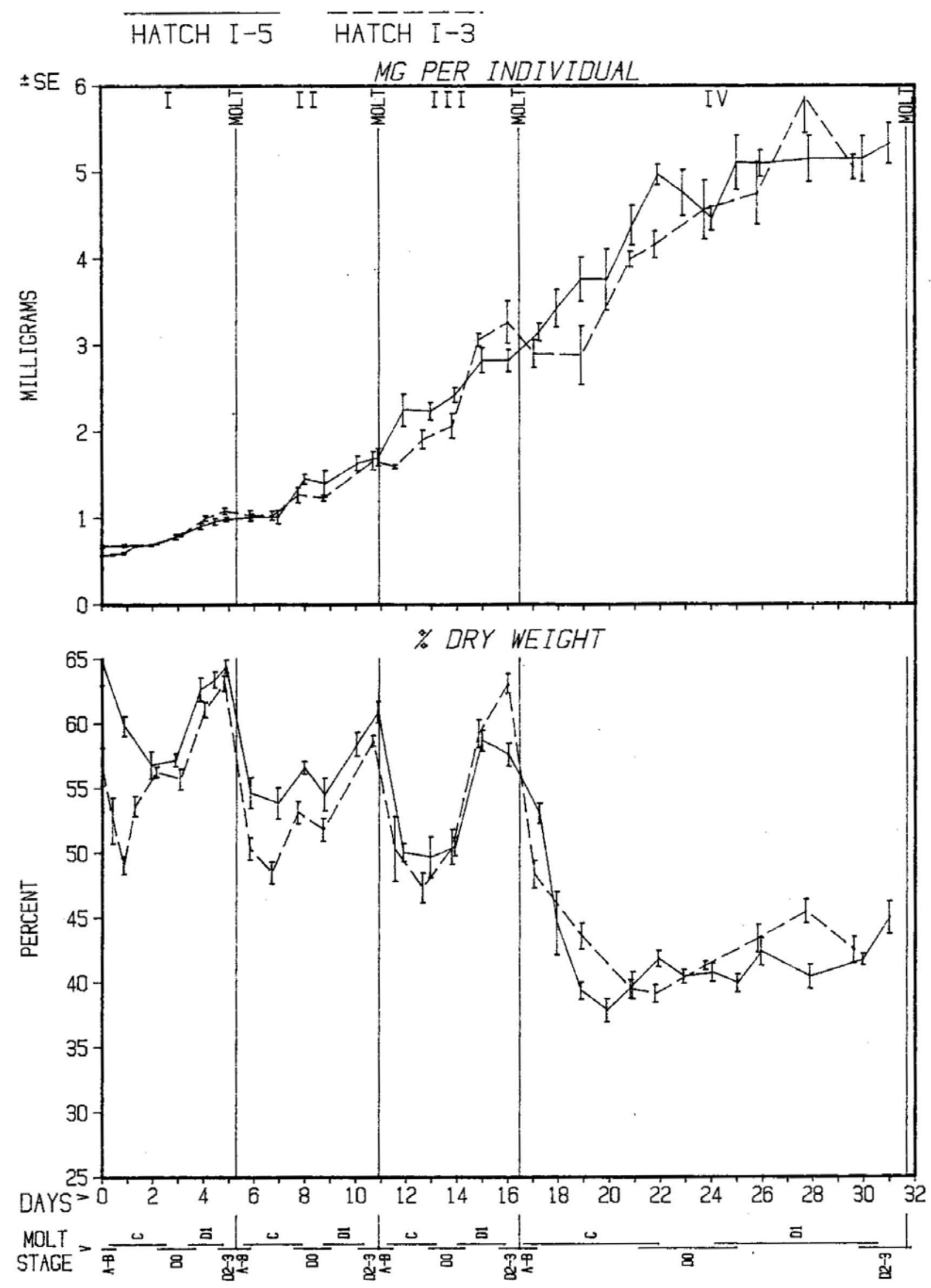


Graph 25 A

Larval Development Study

Lipid $(\mathrm{n}=2)$. Expressed as $\mathrm{mg}$ per individual.

The vertical lines labelled "MOLT" indicate the time of molting. The roman numerals (I-IV) at the top of the graph indicate the larval

stage.

The MOLT STAGE labels at the bottom of the page are generalized to show the range of each molt stage and indicate the molt stage of the samples. The exact molt stage for any sample can be obtained from the Tables.

Error bars are standard errors.

Graph 25 B

Larva1 Development Study

Lipid $(n=2)$. Expressed as \% dry weight.

The vertical lines (labelled "MOLT" in graph A) indicate the time of molting. The roman numerals (I-IV) at the top graph $\underline{A}$ apply to this graph and indicate the larval stage.

The MOLT STAGE labels at the bottom of the page are generalized to show the range of each molt stage and indicate the molt stage of the samples. The exact molt stage for any sample can be obtained from the Tables.

Error bars are standard errors. 


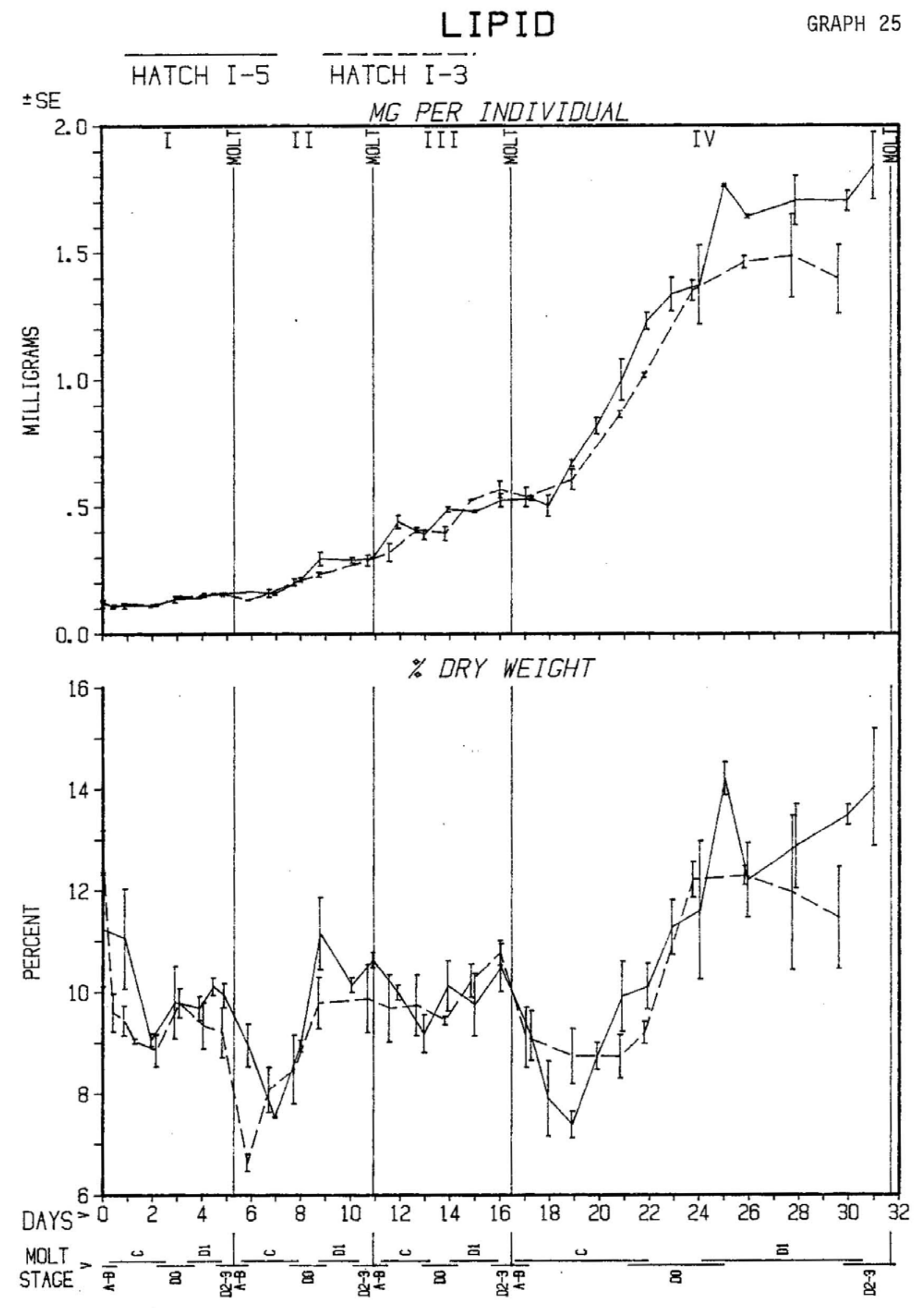


Graph 26 A

Larva1 Development Study

Caloric equilvalents of protein, lipid and carbohydrate; Hatch I-5 on1y. Expressed as calories per individual.

The vertical lines labelled "MOLT" indicate the time of molting. The MOLT STAGE labels at the bottom of the page indicate the molt stage of the larvae in each sample.

Error bars are standard errors.

\section{Graph 26 B}

Larval Development Study

Caloric equilvalents of protein, 1ipid and carbohydrate; Hatch I-5 only. Expressed as a percent of the total calories, where the total is the sum of the protein, lipid and carbohydrate values.

The vertical lines (labelled "MOLT" in graph $\underline{A}$ ) indicate the time of molting.

The MOLT STAGE labels at the bottom of the page indicate the molt stage of the larvae in each sample.

Error bars are standard errors. 
CALORIC EQUIVALENTS
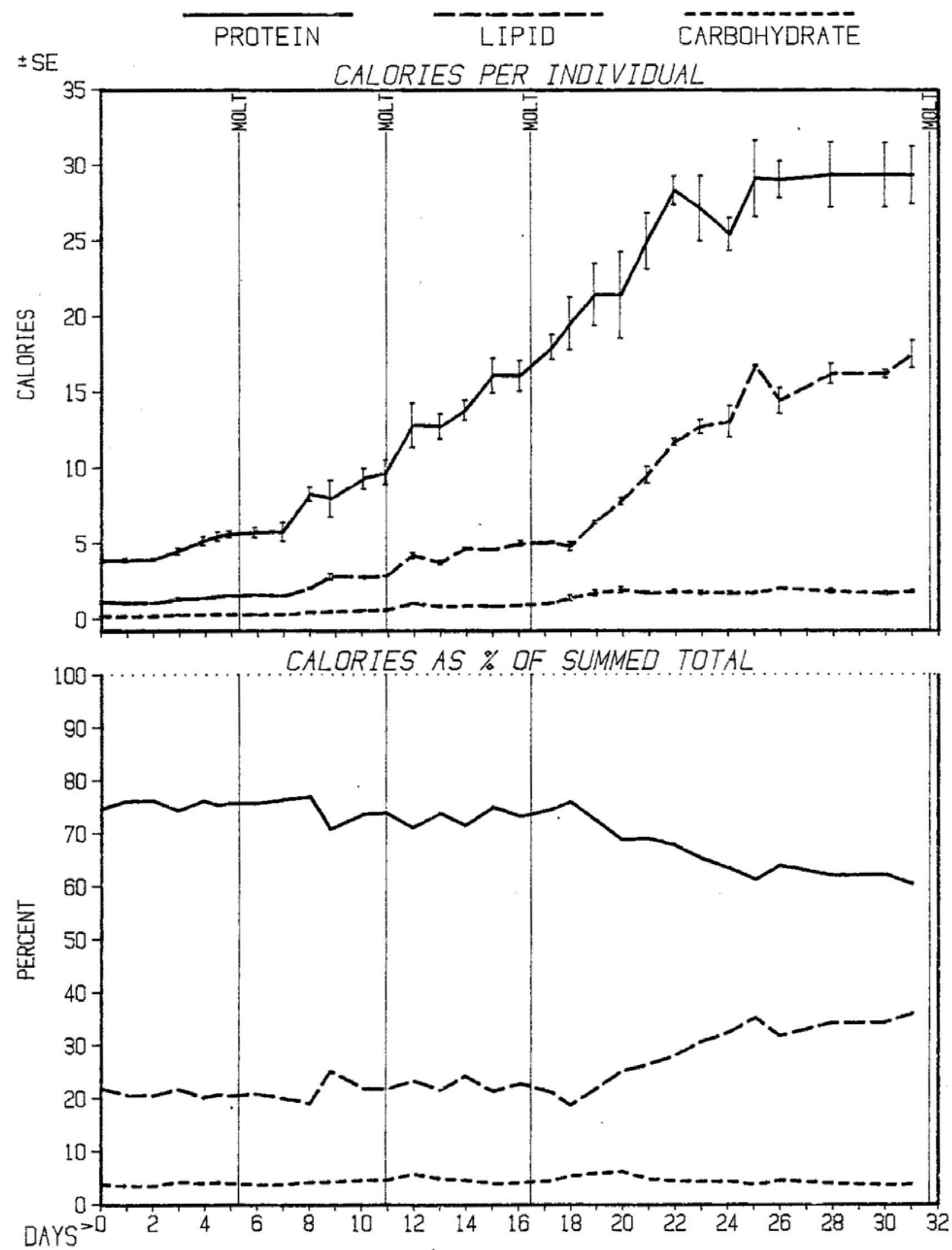

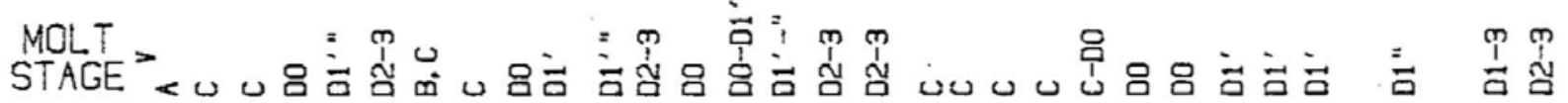




\section{Graph 27 A}

Larva1 Development Study

Triacylglycerol $(\mathrm{n}=3)$. Expressed as micrograms per individual. The vertical lines labelled "MOLT" indicate the time of molting. The MOLT STAGE labels at the bottom of the page are generalized to show the range of each molt stage and indicate the molt stage of the samples. The exact molt stage for any sample can be obtained from the Tables.

Error bars are standard errors.

Graph 27 B

Larva1 Development Study

Triacylglycerol $(n=3)$. Expressed as a ratio against the protein in each sample (as \%).

The vertical lines (labelled "MOLT" in graph $\underline{A}$ ) indicate the time of molting.

The MOLT STAGE labels at the bottom of the page are generalized to show the range of each molt stage and indicate the molt stage of the samples. The exact molt stage for any sample can be obtained from the Tables.

Error bars are standard errors. 
TRI ACYLGLYCEROL GRAPH 27

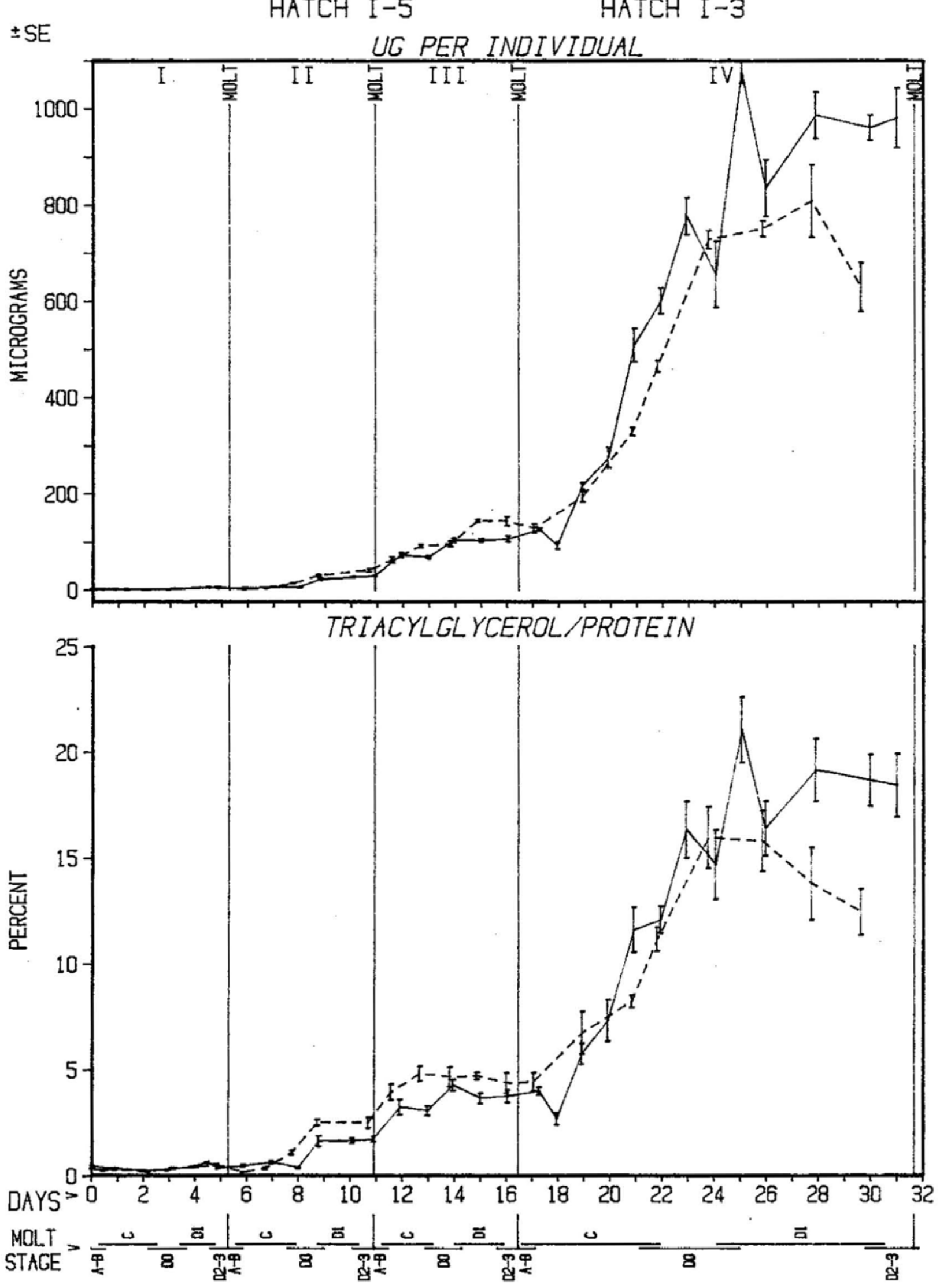




\section{Graph 28 A}

\section{Larva1 Development Study}

Sterols $(n=3)$. Expressed as micrograms per individual.

The vertical lines labelled "MOLT" indicate the time of molting.

The MOLT STAGE labels at the bottom of the page are generalized to show the range of each molt stage and indicate the molt stage of the samples. The exact molt stage for any sample can be obtained from the Tables.

Error bars are standard errors.

\section{Graph 28 B}

Larval Development Study

Sterols $(n=3)$. Expressed as a ratio against the protein in each sample (as \%).

The vertical 1ines (labelled "MOLT" in graph A) indicate the time of molting.

The MOLT STAGE labels at the bottom of the page are generalized to show the range of each molt stage and indicate the molt stage of the samples. The exact molt stage for any sample can be obtained from the Tables.

Error bars are standard errors. 


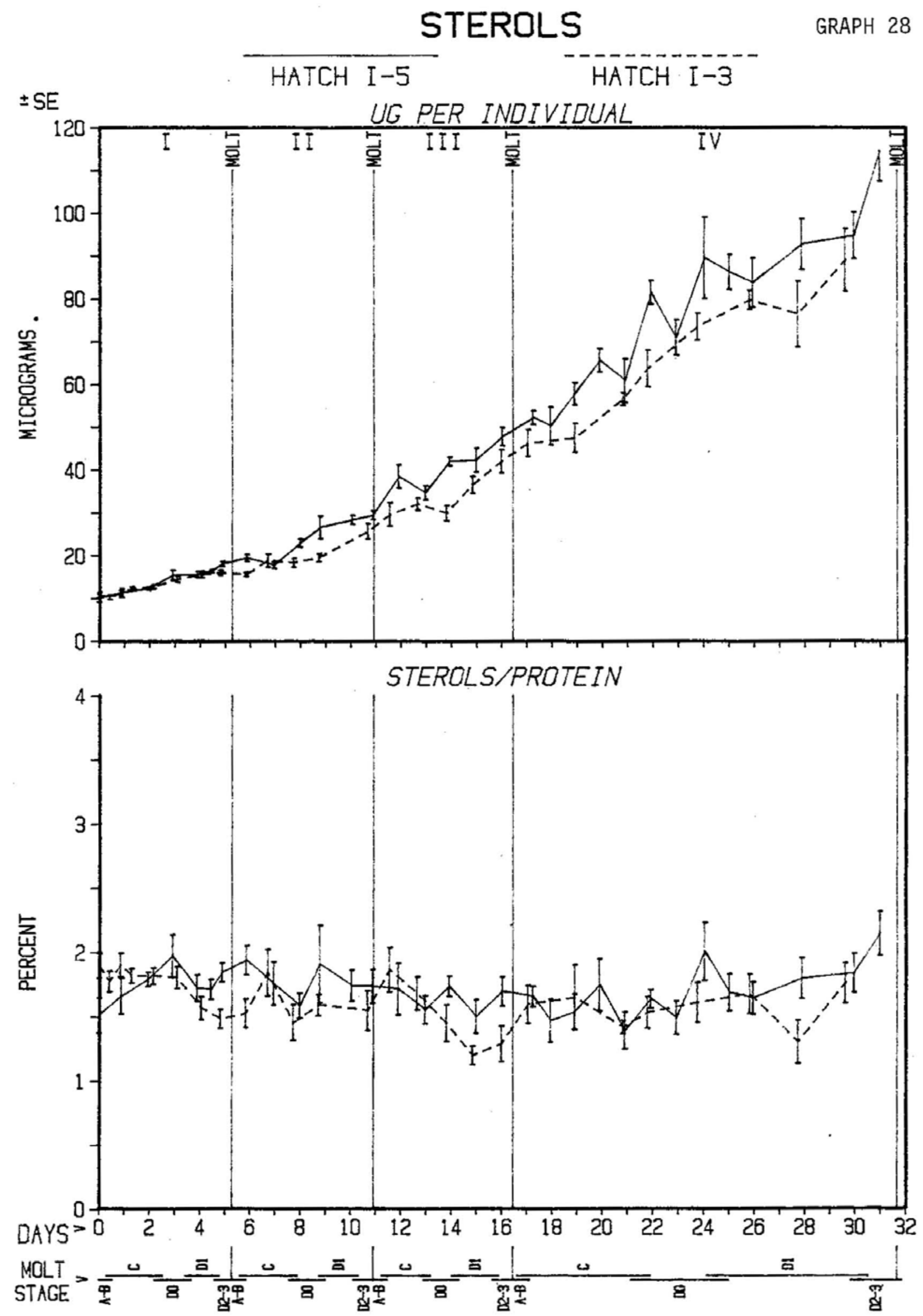


Graph 29 A

Larval Development Study

Phosphatidyl ethanolamine $(n=3)$. Expressed as micrograms per individua1.

The vertical lines labelled "MOLT" indicate the time of molting. The MOLT STAGE labels at the bottom of the page are generalized to show the range of each molt stage and indicate the molt stage of the samples. The exact molt stage for any sample can be obtained from the Tables.

Error bars are standard errors.

\section{Graph 29 B}

Larval Development Study

Phosphatidy1 ethanolamine $(n=3)$. Expressed as a ratio against the protein in each sample (as \%). The vertical lines (labelled "MOLT" in graph A) indicate the time of molting.

The MOLT STAGE labels at the bottom of the page are generalized to show the range of each molt stage and indicate the molt stage of the samples. The exact molt stage for any sample can be obtained from the Tables.

Error bars are standard errors. 
PHOSPHATIDYL ETHANOLAMINE GRAPH 29

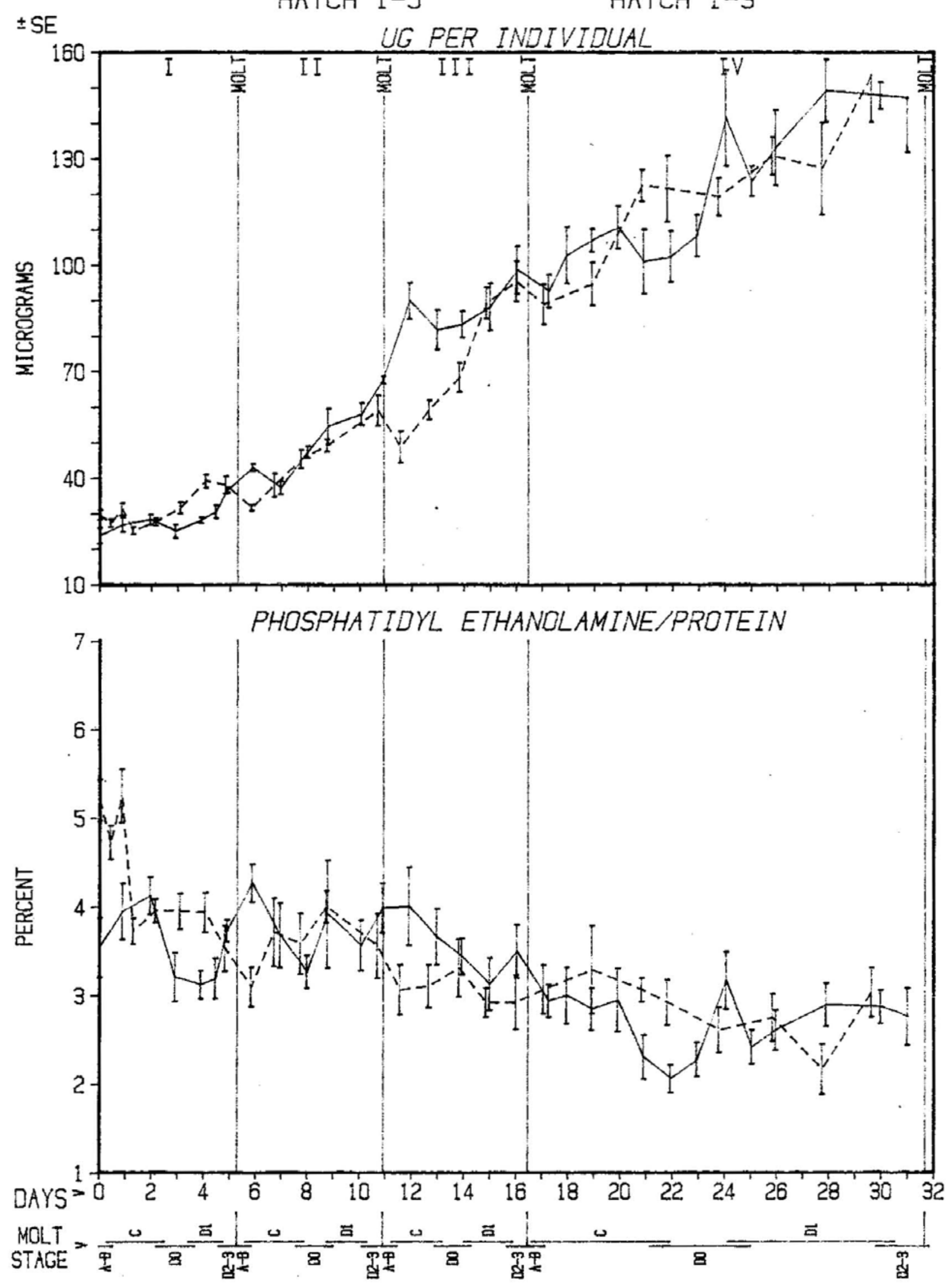


Graph 30 A

Larval Development Study

Phosphatidyl choline $(\mathrm{n}=3)$. Expressed as micrograms per individual. The vertical 1ines labelled "MoLT" indicate the time of molting. The MOLT STAGE labels at the bottom of the page are generalized to show the range of each molt stage and indicate the molt stage of the samples. The exact molt stage for any sample can be obtained from the Tables.

Error bars are standard errors.

\section{Graph 30 B}

Larva1 Development Study

Phosphatidy1 choline $(n=3)$. Expressed as a ratio against the protein in each sample (as \%).

The vertical lines (labelled "MOLT" in graph $\underline{A}$ ) indicate the time of molting.

The MOLT STAGE labels at the bottom of the page are generalized to show the range of each molt stage and indicate the molt stage of the samples. The exact molt stage for any sample can be obtained from the Tables.

Error bars are standard errors. 


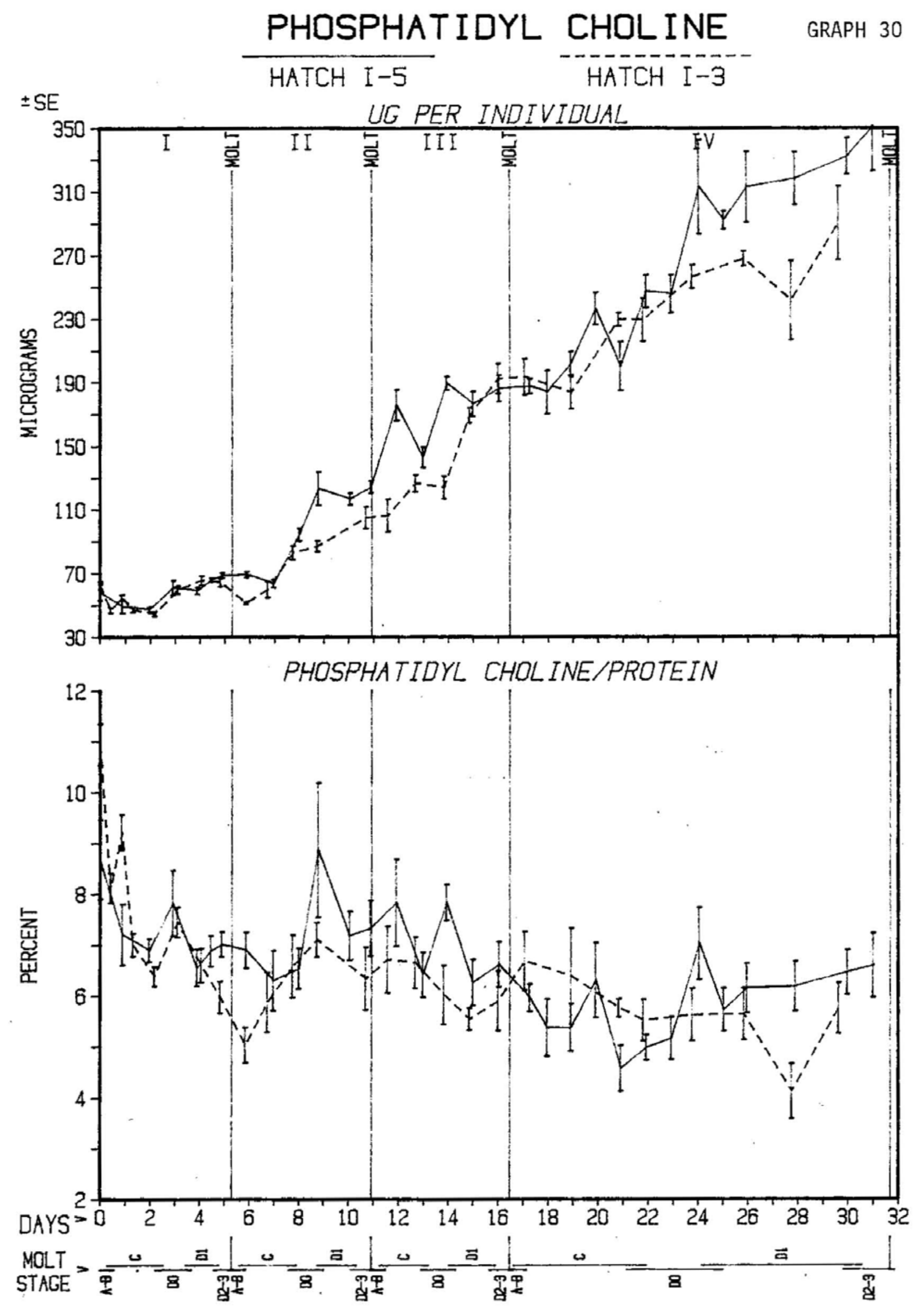


Graph 31 A

Larval Development Study

Selected non-essential fatty acids (NEFA) of neutral 1ipids; Hatch I-5 only. Expressed as fatty acid methyl esters (FAME), in ug per individual.

The vertical lines labelled "MOLT" indicate the time of molting. The roman numerals (I-IV) at the top of the graph indicate the larval stage.

The MOLT STAGE labels at the bottom of the page indicate the molt stage of the larvae in each sample.

\section{Graph 31 B}

Larval Development Study

Selected non-essential fatty acids (NEFA) of neutral lipids; Hatch I-5 only. Expressed as fatty acid methyl esters (FAME). Each FAME as a percent of the total FAME found in the neutral lipids. The vertical lines (labelled "MOLT" in graph $\underline{A}$ ) indicate the time of mo1ting.

The roman numerals (I-IV) at the top of graph $\underline{A}$ apply to this graph and indicate the larval stage.

The MOLT STAGE labels at the bottom of the page indicate the molt stage of the larvae in each sample. 
FAME of NEUTRAL LIPIDS
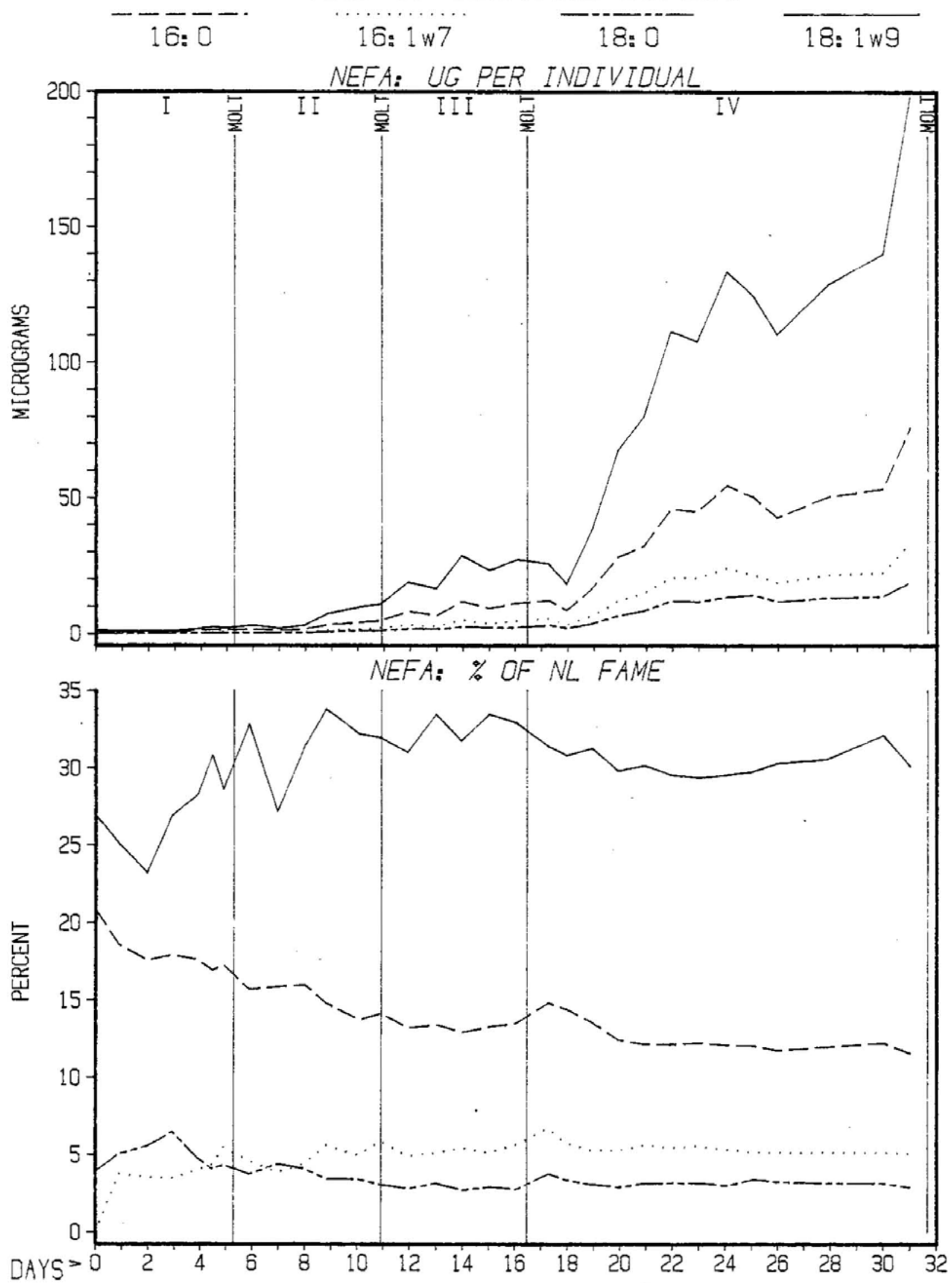

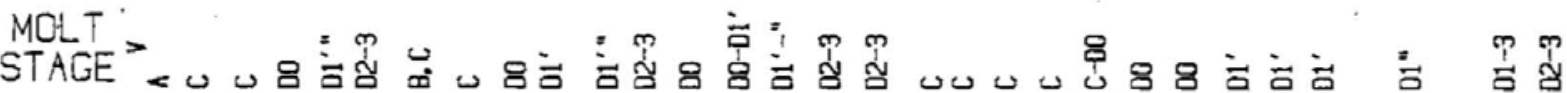


Graph 32 A

Larval Development Study

Selected polyunsaturated fatty acids (PUFA) of neutral 1ipids; Hatch I-5 only. Expressed as fatty acid methyl esters (FAME), in ug per individual.

The vertical lines labelled "MOLT" indicate the time of molting. The roman numerals (I-IV) at the top of the graph indicate the larval stage.

The MOLT STAGE labels at the bottom of the page indicate the molt stage of the larvae in each sample.

Graph 32 B

Larva1 Development Study

Selected polyunsaturated fatty acids (PUFA) of neutra1 1ipids; Hatch I-5 only. Expressed as fatty acid methyl esters (FAME). Each FAME as a percent of the total FAME found in the neutral lipids. The vertical lines (labelled "MOLT" in graph A) indicate the time of molting.

The roman numerals (I-IV) at the top of graph $\underline{A}$ apply to this graph and indicate the larval stage.

The MOLT STAGE labels at the bottom of the page indicate the molt stage of the larvae in each sample. 


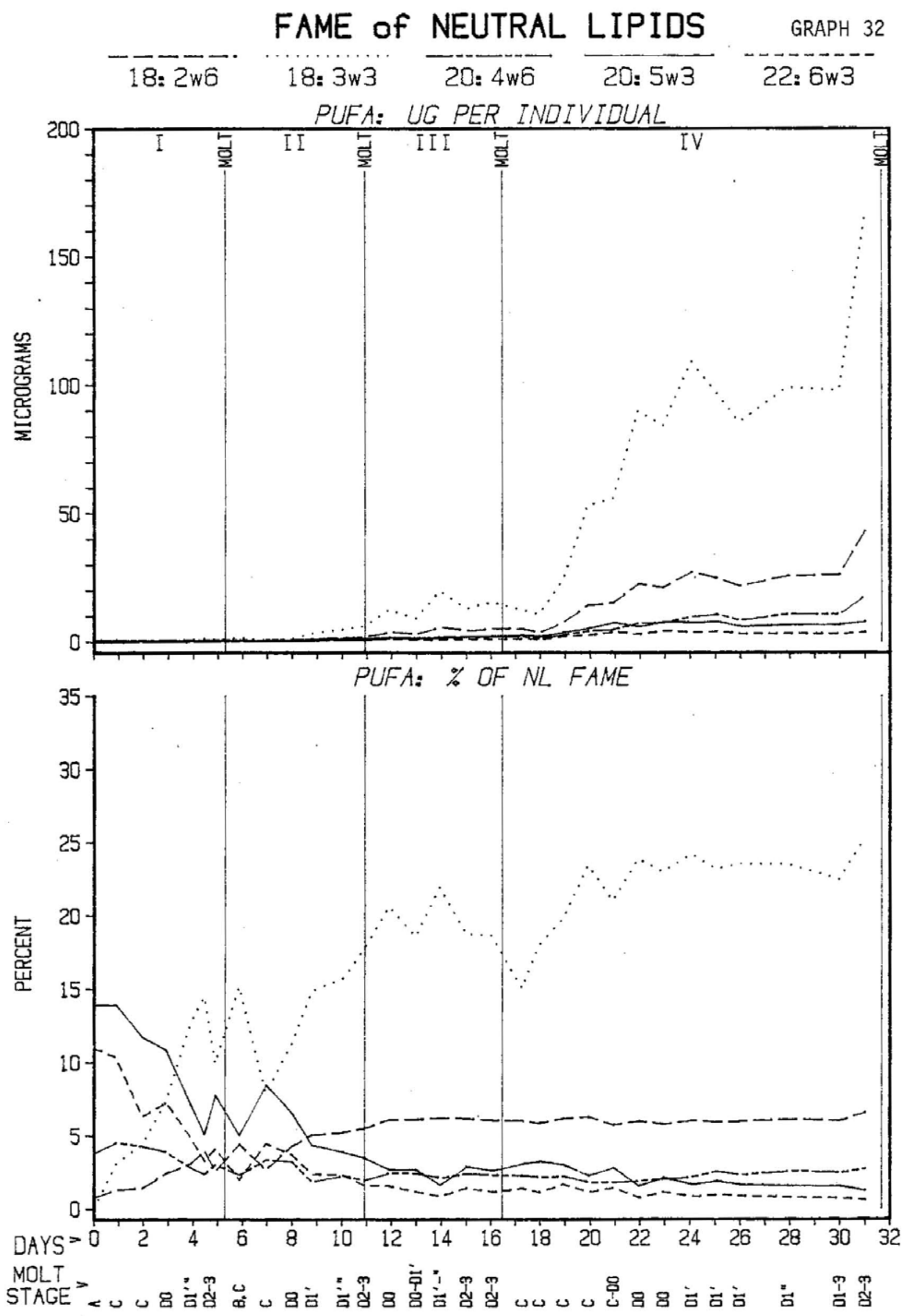




\section{Graph 33 A}

Larval Development Study

Selected non-essential fatty acids (NEFA) of phosphatidy1

ethanolamine; Hatch I-5 only. Expressed as fatty acid methyl esters (FAME), in ug per individual.

The vertical lines labelled "MOLT" indicate the time of molting. The roman numerals (I-IV) at the top of the graph indicate the larval stage.

The MOLT STAGE labels at the bottom of the page indicate the molt stage of the larvae in each sample.

\section{Graph 33 B}

Larva1 Development Study

Selected non-essential fatty acids (NEFA) of Phosphatidyl

ethanolamine; Hatch I-5 only. Expressed as fatty acid methyl esters (FAME). Each FAME as a percent of the total FAME found in phosphatidyl ethanolamine.

The vertical lines (1abelled "MOLT" in graph $\underline{A}$ ) indicate the time of molting.

The roman numerals (I-IV) at the top of graph A apply to this graph and indicate the larval stage.

The MOLT STAGE labels at the bottom of the page indicate the molt stage of the larvae in each sample. 


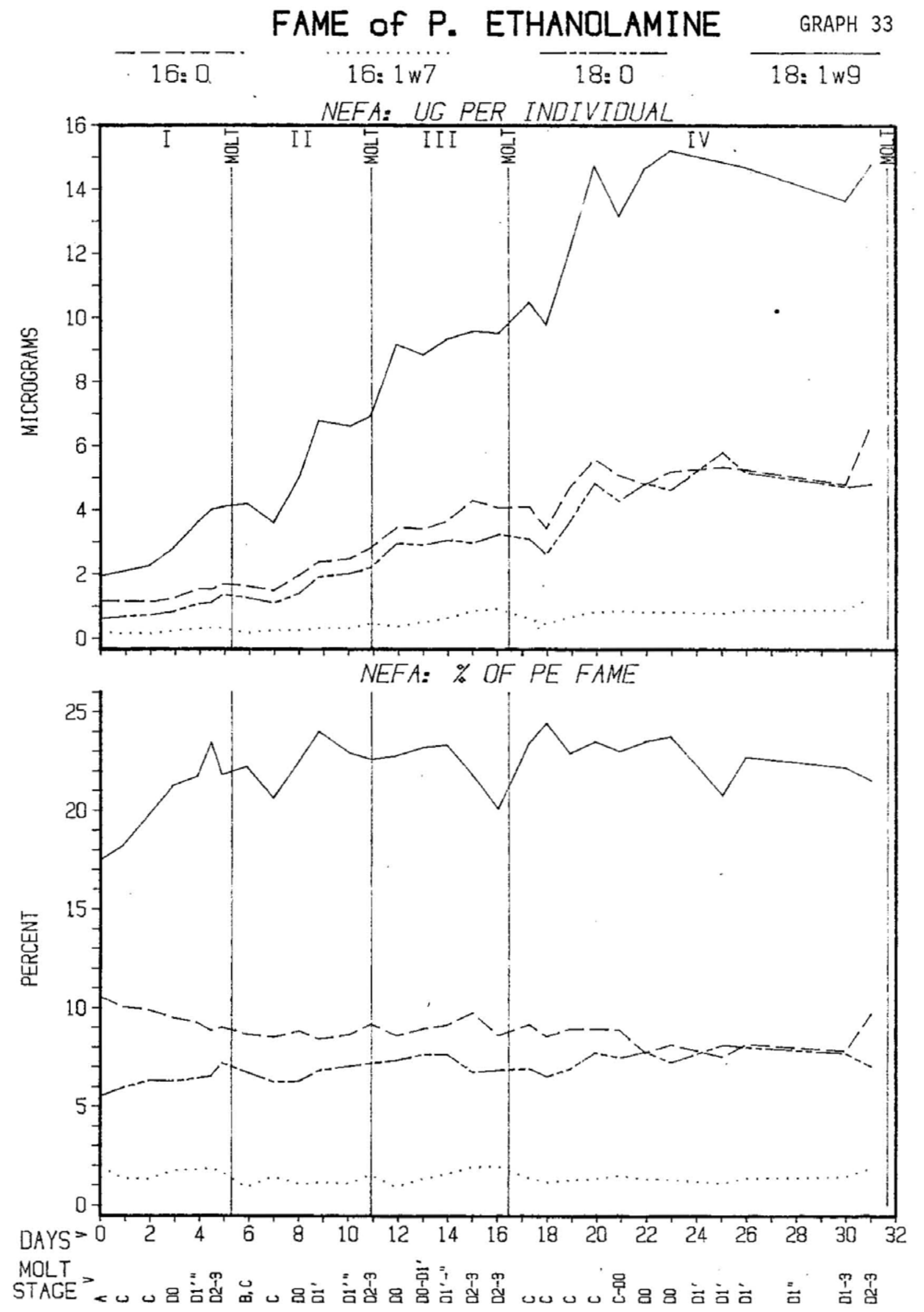




\section{Graph 34 A}

Larva1 Development Study

Selected polyunsaturated fatty acids (PUFA) of phosphatidy1 ethanolamine; Hatch I-5 only. Expressed as fatty acid methyl esters (FAME), in ug per individual.

The vertical lines labelled "MOLT" indicate the time of molting. The roman numerals (I-IV) at the top of the graph indicate the larval stage.

The MOLT STAGE labels at the bottom of the page indicate the molt stage of the larvae in each sample.

\section{Graph 34 B}

\section{Larval Development Study}

Selected polyunsaturated fatty acids (PUFA) of phosphatidy 1 ethanolamine; Hatch I-5 only. Expressed as fatty acid methyl esters (FAME). Each FAME as a percent of the total FAME found in phosphatidyl ethanolamine.

The vertical lines (labelled "MOLT" in graph A) indicate the time of molting.

The roman numerals (I-IV) at the top of graph A apply to this graph and indicate the larval stage.

The MOLT STAGE 1abels at the bottom of the page indicate the molt stage of the larvae in each sample. 


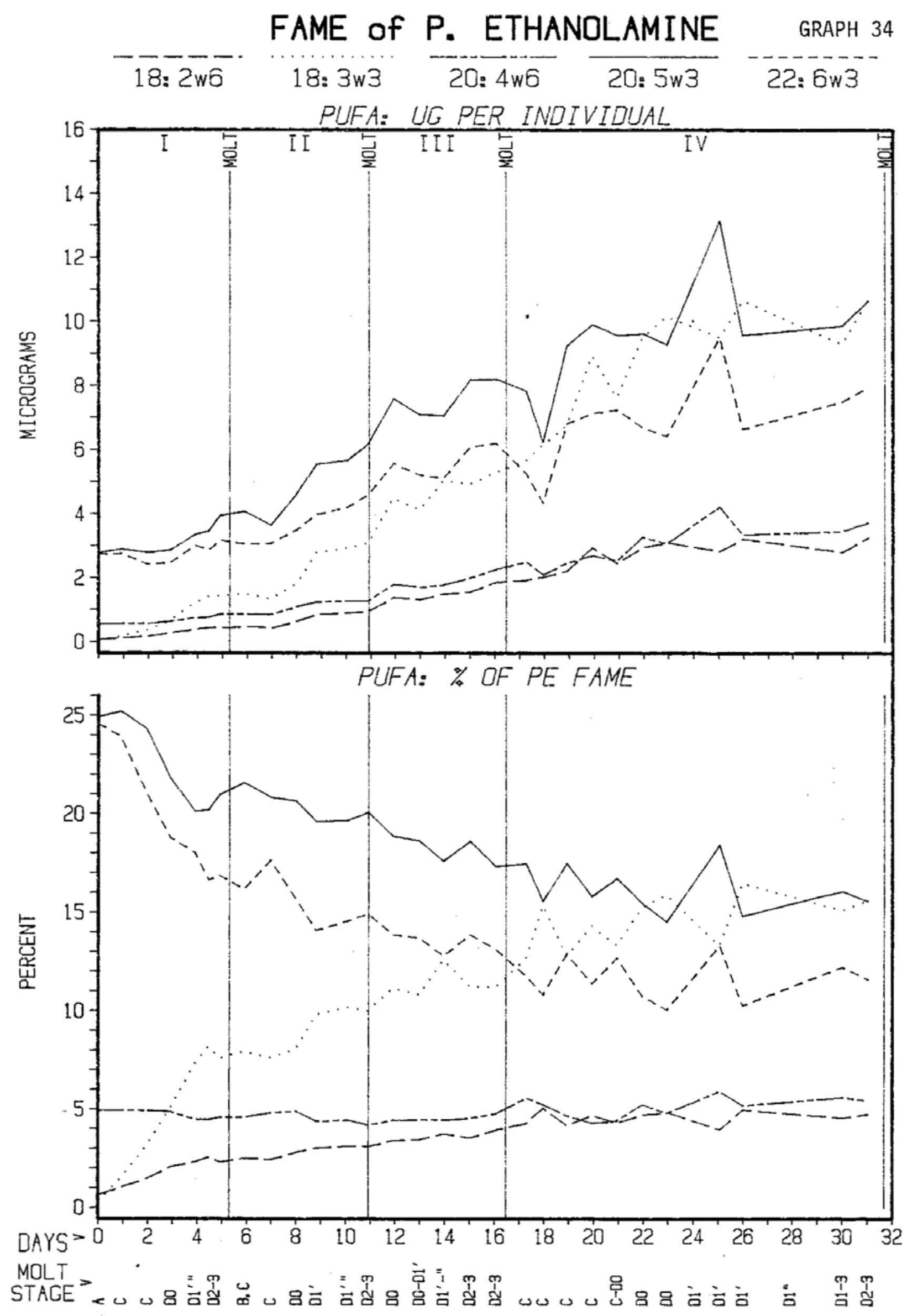


Graph 35 A

Larva1 Development Study

Selected non-essential fatty acids (NEFA) of phosphatidyl choline; Hatch I-5 only. Expressed as fatty acid methyl esters (FAME), in ug per individual.

The vertical lines labelled "MOLT" indicate the time of molting. The roman numerals (I-IV) at the top of the graph indicate the larval stage.

The MOLT STAGE labels at the bottom of the page indicate the molt stage of the larvae in each sample.

\section{Graph 35 B}

Larval Development Study

Selected non-essential fatty acids (NEFA) of Phosphatidyl choline; Hatch I-5 only. Expressed as fatty acid methyl esters (FAME). Each FAME as a percent of the total FAME found in phosphatidyl choline. The vertical lines (labelled "MOLT" in graph $\underline{A}$ ) indicate the time of molting.

The roman numerals (I-IV) at the top of graph $A$ apply to this graph and indicate the larval stage.

The MOLT STAGE labels at the bottom of the page indicate the molt stage of the larvae in each sample. 
FAME of P. CHOLINE

$\begin{array}{cccc}16: 0 & 16: 1 w 7 & 18: 169\end{array}$
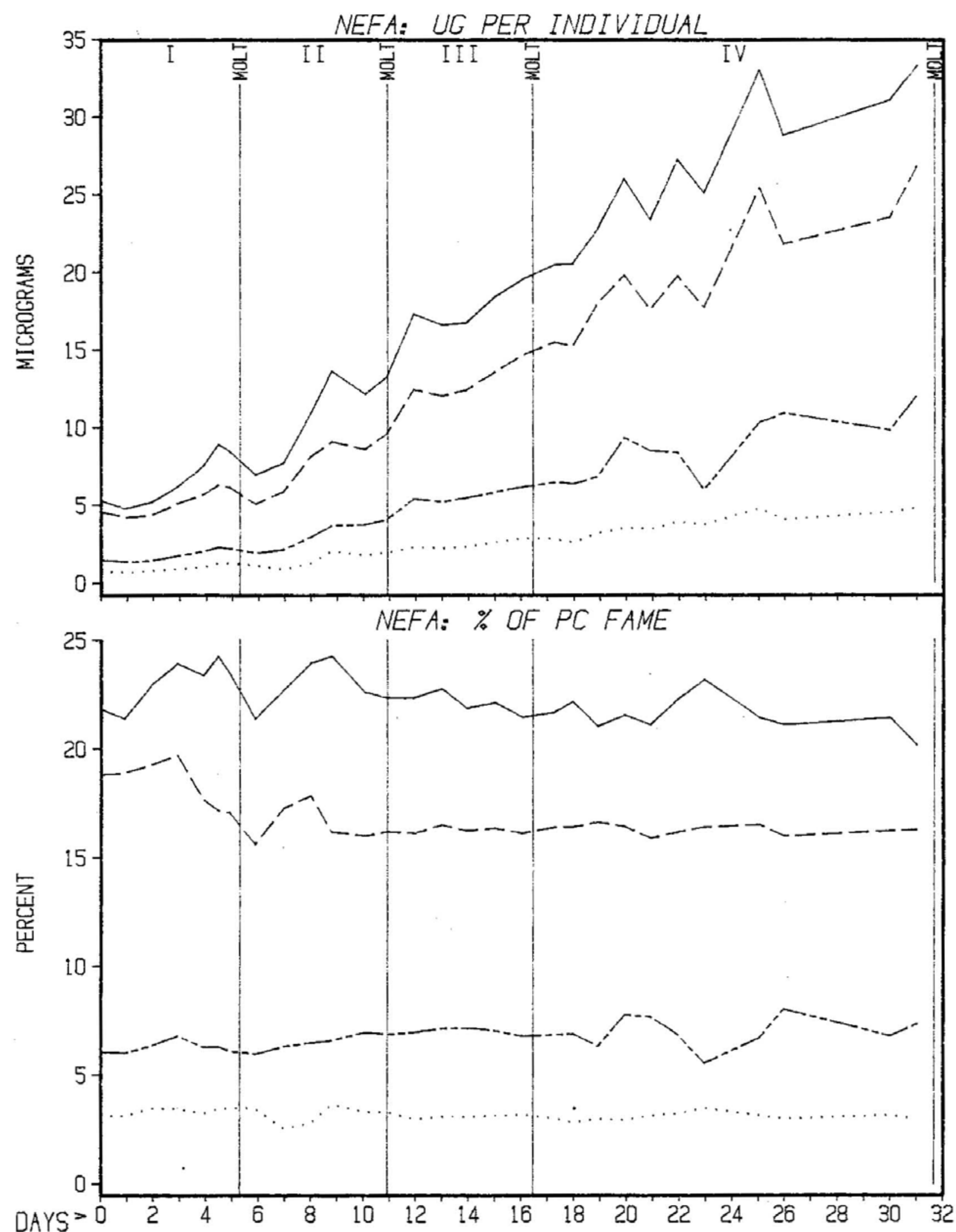

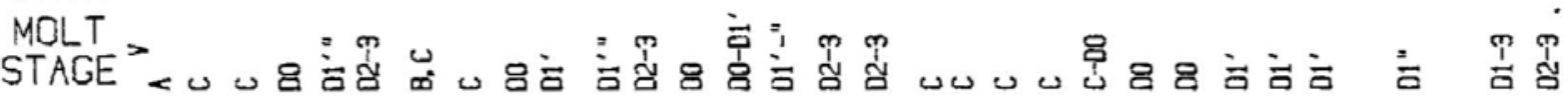




\section{Graph 36 A}

\section{Larva1 Development Study}

Selected polyunsaturated fatty acids (PUFA) of phosphatidy1 choline; Hatch I-5 only. Expressed as fatty acid methyl esters (FAME), in ug per individual.

The vertical lines labelled "MOLT" indicate the time of molting. The roman numerals (I-IV) at the top of the graph indicate the larval stage.

The MOLT STAGE labels at the bottom of the page indicate the molt stage of the larvae in each sample.

\section{Graph 36 B}

\section{Larval Development Study}

Selected polyunsaturated fatty acids (PUFA) of phosphatidyl choline; Hatch I-5 only. Expressed as fatty acid methyl esters (FAME). Each FAME as a percent of the total FAME found in phosphatidyl choline. The vertical lines (labelled "MOLT" in graph A) indicate the time of molting.

The roman numerals (I-IV) at the top of graph $\underline{A}$ apply to this graph and indicate the larval stage.

The MOLT STAGE labels at the bottom of the page indicate the molt stage of the larvae in each sample. 


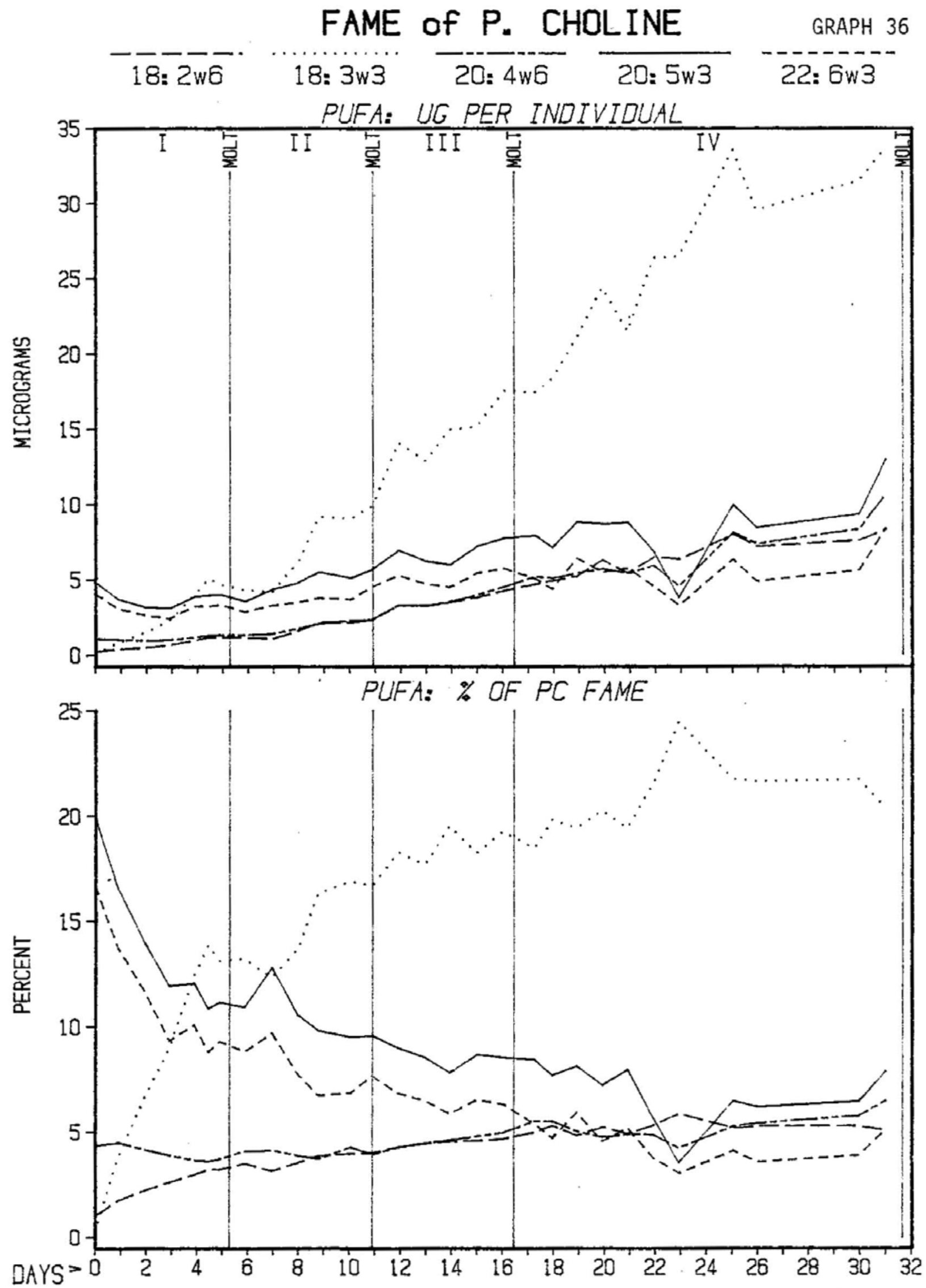

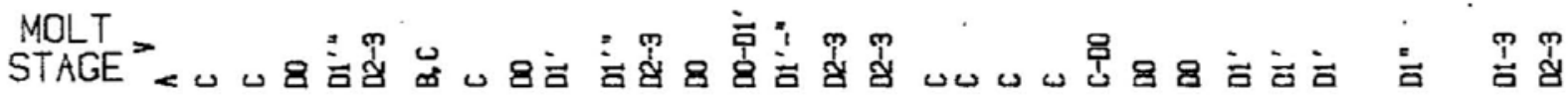


Graph 37 A

\section{Starvation Study}

Wet weight $(\mathrm{n}=7)$. Expressed as $\mathrm{mg}$ per individual.

The first asterisk at the bottom of the page indicates the time of molting for the control, D-3 and D-4 groups. The second asterisk indicates the time of molting for the control group only. The molt stages for each sample can be obtained from the Tables. Error bars are standard errors.

\section{Graph 37 B}

\section{Starvation Study}

Ash free dry weight $(n=7)$. Expressed as $\mathrm{mg}$ per individua1. The first asterisk at the bottom of the page indicates the time of molting for the control, D-3 and D-4 groups. The second asterisk indicates the time of molting for the control group only. The molt stages for each sample can be obtained from the Tables. Error bars are standard errors. 


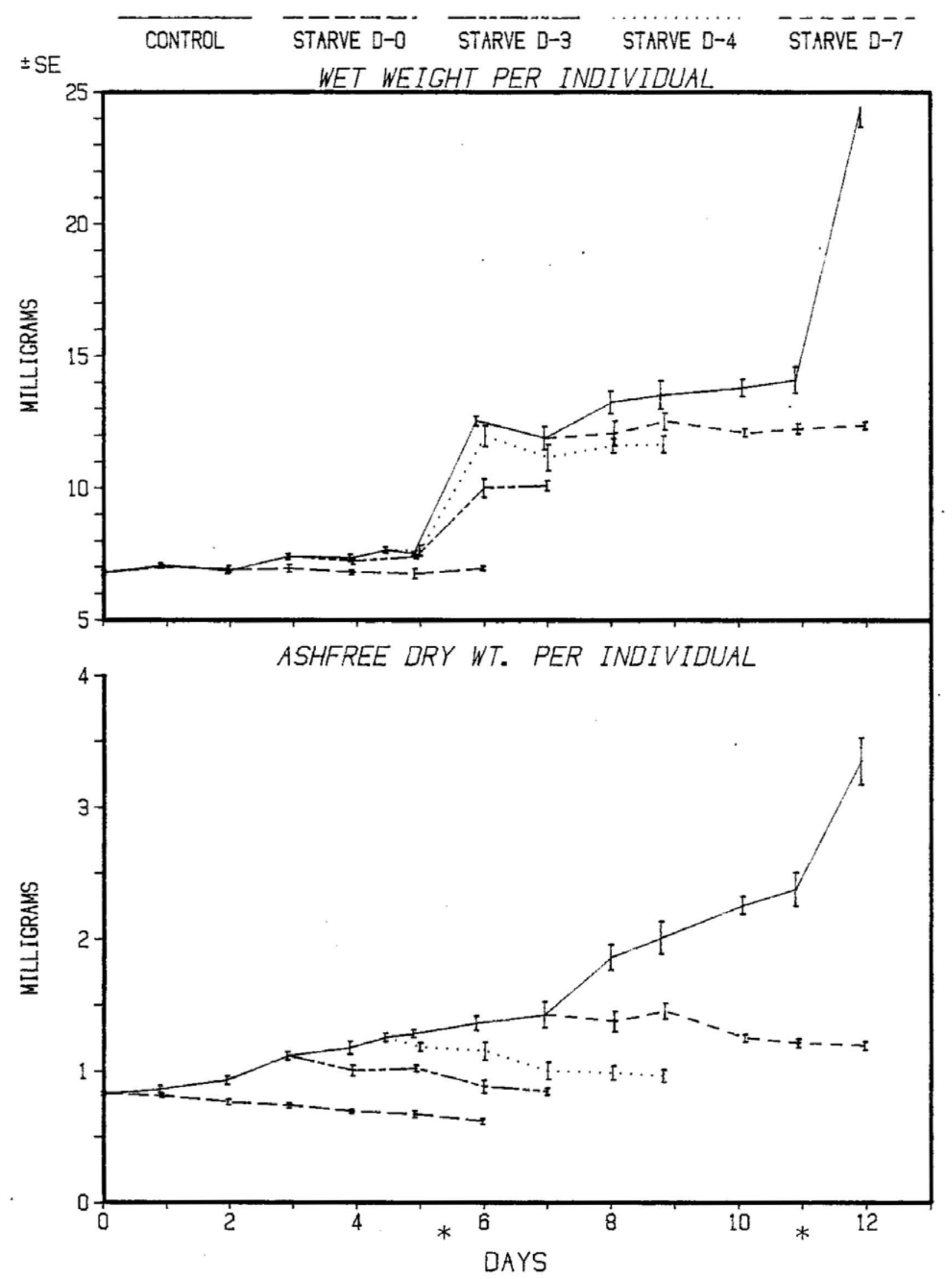




\section{Graph 38 A}

\section{Starvation Study}

Dry weight $(n=7)$. Expressed as mg per individual.

The first asterisk at the bottom of the page indicates the time of molting for the control, D-3 and D-4 groups. The second asterisk indicates the time of molting for the control group only. The molt stages for each sample can be obtained from the Tables. Error bars are standard errors.

\section{Graph 38 B}

\section{Starvation Study}

\% Dry/wet weight $(n=7)$.

The first asterisk at the bottom of the page indicates the time of molting for the contro1, D-3 and D-4 groups. The second asterisk indicates the time of molting for the control group only. The molt stages for each sample can be obtained from the Tables. Error bars are standard errors. 


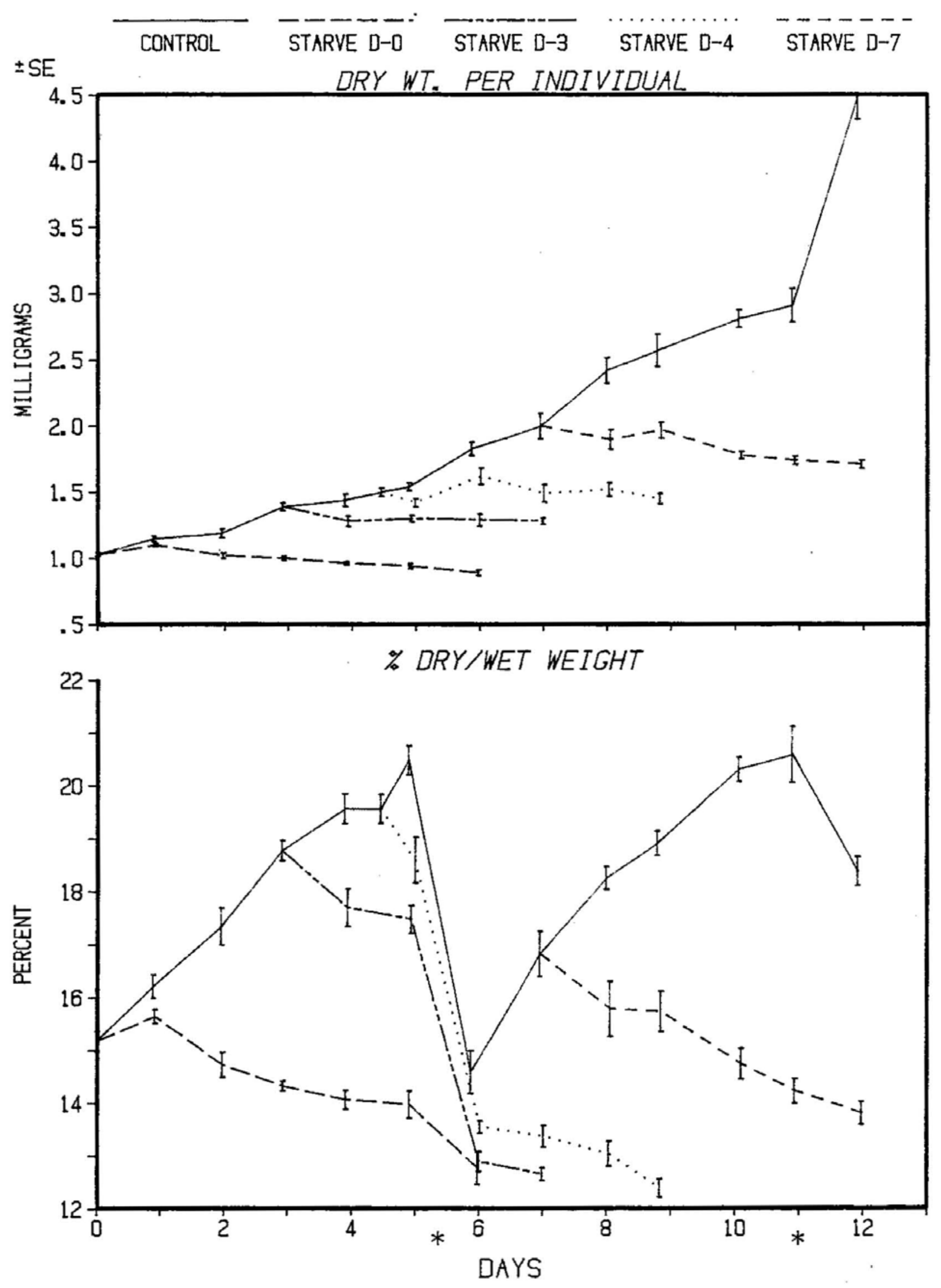




\section{Graph 39 A}

\section{Starvation Study}

Ash $(n=3)$. Expressed as mg per individual.

The first asterisk at the bottom of the page indicates the time of molting for the control, D-3 and D-4 groups. The second asterisk indicates the time of molting for the control group only. The molt stages for each sample can be obtained from the Tables. Error bars are standard errors.

\section{Graph $39 \quad$ B}

\section{Starvation Study}

Ash $(n=3)$. Expressed as \% dry weight.

The first asterisk at the bottom of the page indicates the time of molting for the contro1, D-3 and D-4 groups. The second asterisk indicates the time of molting for the control group only. The molt stages for each sample can be obtained from the Tables. Error bars are standard errors. 
ASH

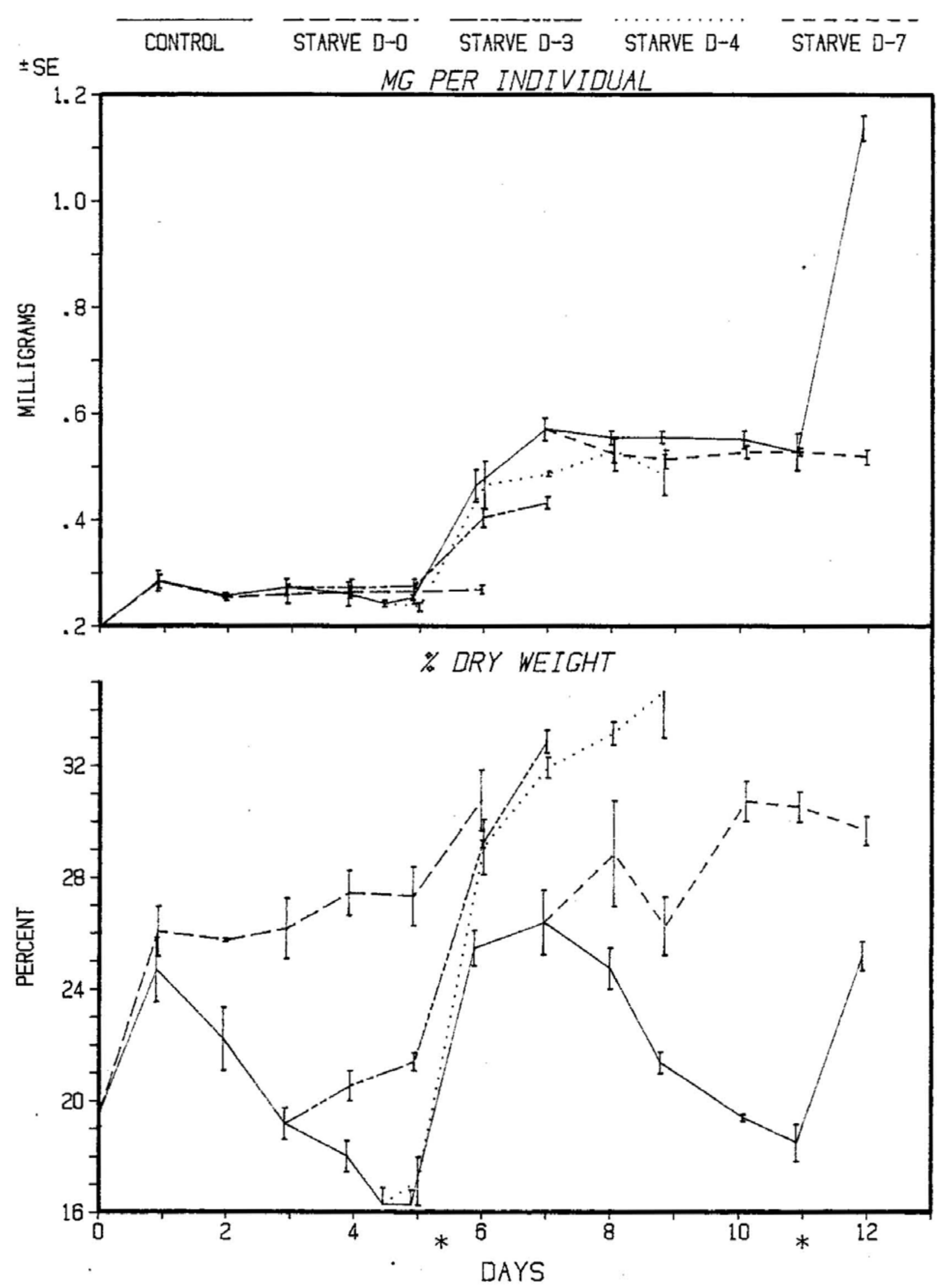




\section{Graph 40 A}

\section{Starvation Study}

Chitin ( $\mathrm{n}=2)$. Expressed as mg per individual.

The first asterisk at the bottom of the page indicates the time of molting for the control, D-3 and D-4 groups. The second asterisk indicates the time of molting for the control group only. The molt stages for each sample can be obtained from the Tables. Error bars are standard errors.

\section{Graph 40 B}

\section{Starvation Study}

Chitin $(n=2)$. Expressed as \% dry weight. The first asterisk at the bottom of the page indicates the time of molting for the control, D-3 and D-4 groups. The second asterisk indicates the time of molting for the control group only. The molt stages for each sample can be obtained from the Tables. Error bars are standard errors. 
CHITIN

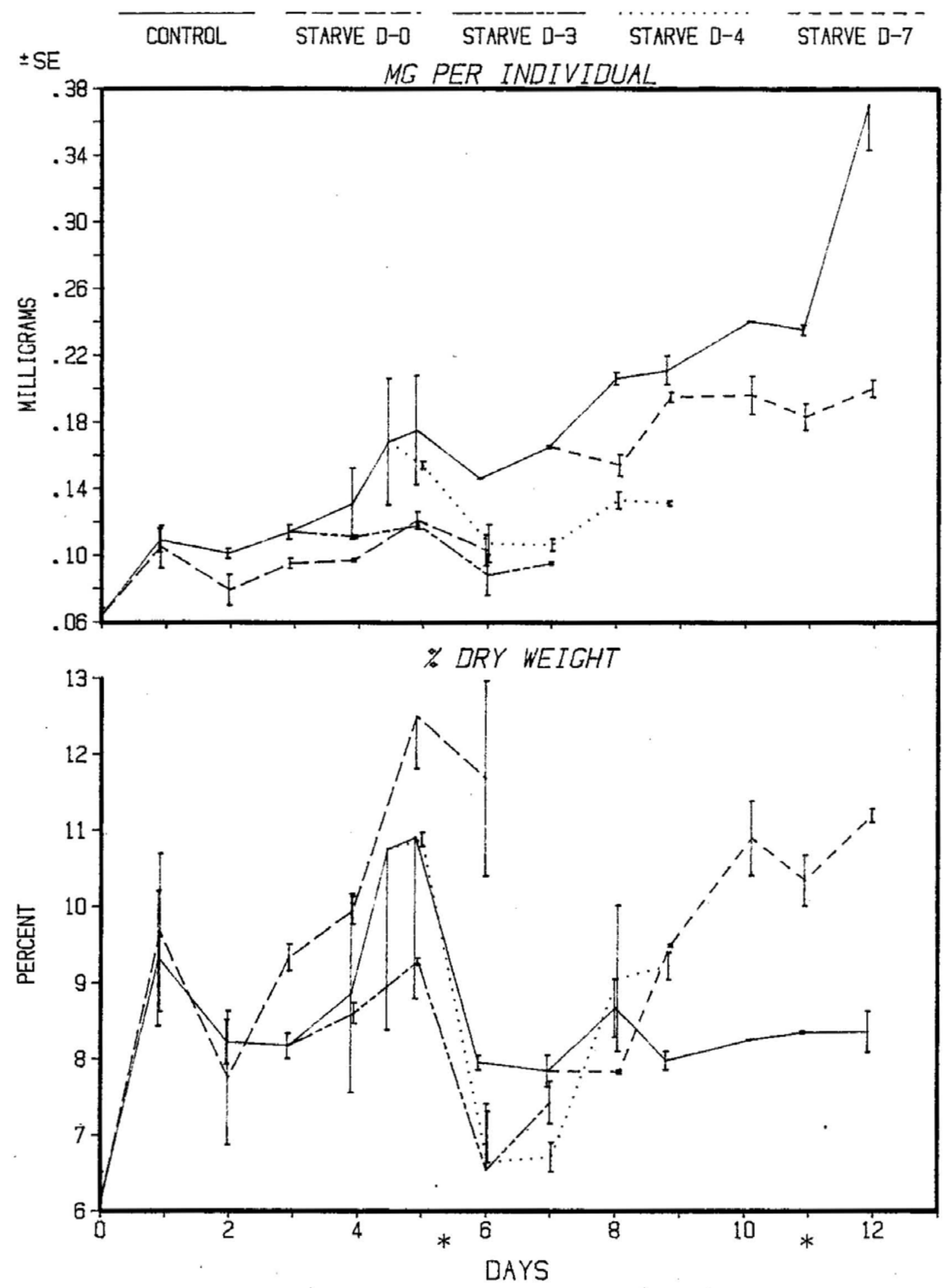




\section{Graph 41 A}

\section{Starvation Study}

Carbohydrate $(n=4)$. Expressed as $\mathrm{mg}$ per individual.

The first asterisk at the bottom of the page indicates the time of molting for the control, D-3 and D-4 groups. The second asterisk indicates the time of molting for the control group only. The molt stages for each sample can be obtained from the Tables. Error bars are standard errors.

\section{Graph 41 B}

\section{Starvation Study}

Carbohydrate ( $n=4)$. Expressed as \% dry weight. The first asterisk at the bottom of the page indicates the time of molting for the control, D-3 and D-4 groups. The second asterisk indicates the time of molting for the control group only. The molt stages for each sample can be obtained from the Tables. Error bars are standard errors. 


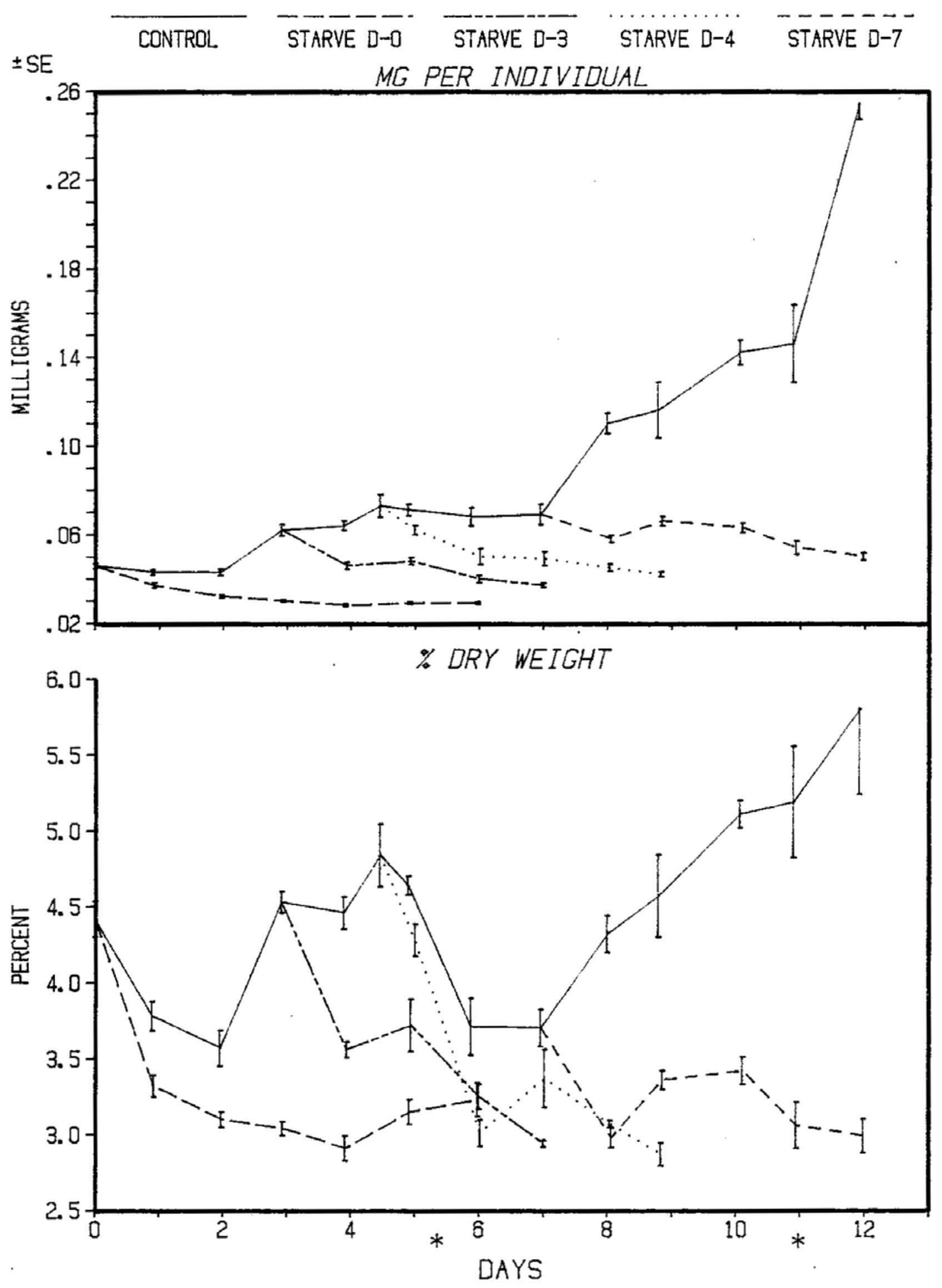


Graph 42 A

Starvation Study

Protein ( $n=4)$. Expressed as mg per individual. The first asterisk at the bottom of the page indicates the time of molting for the contro1, D-3 and D-4 groups. The second asterisk indicates the time of molting for the control group only. The molt stages for each sample can be obtained from the Tables. Error bars are standard errors.

\section{Graph 42 B}

\section{Starvation Study}

Protein $(n=4)$. Expressed as \% dry weight. The first asterisk at the bottom of the page indicates the time of molting for the control, D-3 and D-4 groups. The second asterisk indicates the time of molting for the control group only. The molt stages for each sample can be obtained from the Tables. Error bars are standard errors. 


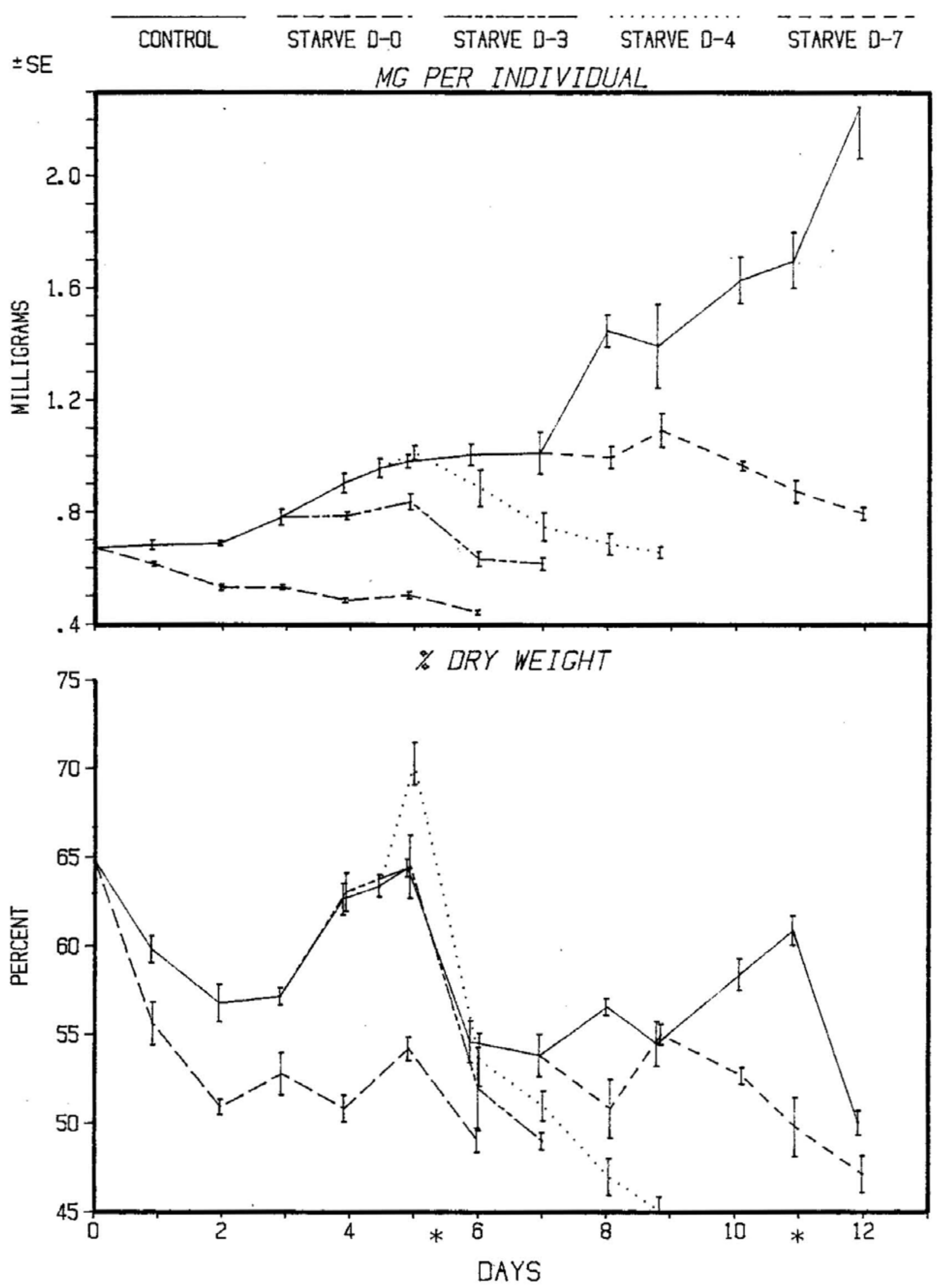




\section{Graph 43 A}

\section{Starvation Study}

Lipid $(\mathrm{n}=2)$. Expressed as $\mathrm{mg}$ per individual.

The first asterisk at the bottom of the page indicates the time of molting for the control, D-3 and D-4 groups. The second asterisk indicates the time of molting for the control group only. The molt stages for each sample can be obtained from the Tables. Error bars are standard errors.

\section{Graph 43 B}

\section{Starvation Study}

Lipid $(n=2)$. Expressed as \% dry weight. The first asterisk at the bottom of the page indicates the time of molting for the control, D-3 and D-4 groups. The second asterisk indicates the time of molting for the control group only. The molt stages for each sample can be obtained from the Tables. Error bars are standard errors. 


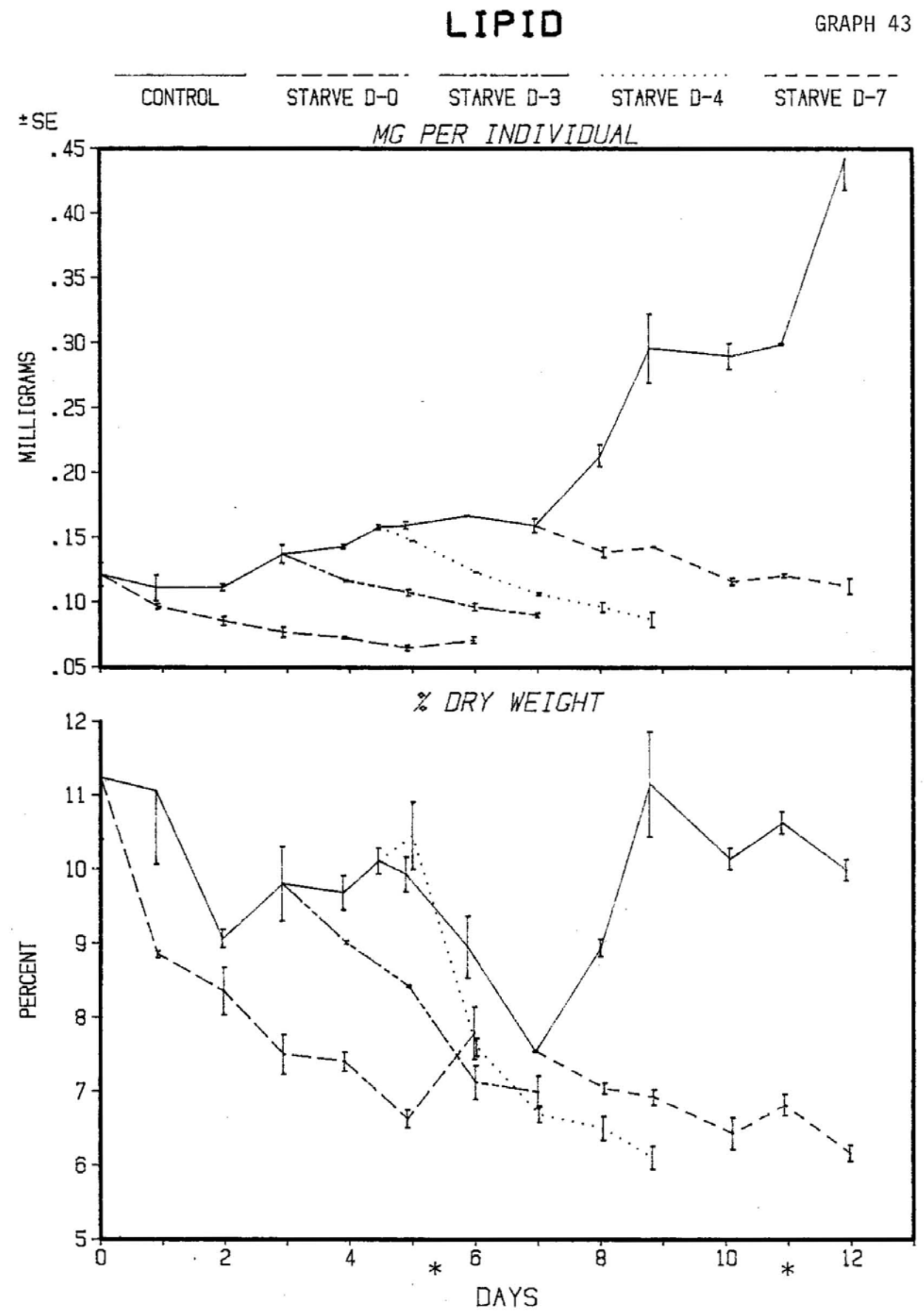




\section{Graph 44 A}

Starvation Study

Protein $(n=4)$. Expressed as a percent of the amount of protein at the onset of starvation.

The first asterisk at the bottom of the page indicates the time of molting for the control, D-3 and D-4 groups. The second asterisk indicates the time of molting for the control group only. The molt stages for each sample can be obtained from the Tables. Error bars are standard errors.

\section{Graph 44 B}

Starvation Study

Lipid $(n=2)$. Expressed as a percent of the amount of 1 ipid at onset of starvation.

The first asterisk at the bottom of the page indicates the time of molting for the control, D-3 and D-4 groups. The second asterisk indicates the time of molting for the control group only. The molt stages for each sample can be obtained from the Tables. Error bars are standard errors.

\section{Graph 44 C}

\section{Starvation Study}

Carbohydrate $(n=4)$. Expressed as a percent of the amount of carbohydrate at the onset of starvation.

The first asterisk at the bottom of the page indicates the time of molting for the control, D-3 and D-4 groups. The second asterisk indicates the time of molting for the control group only. The molt stages for each sample can be obtained from the Tables. Error bars are standard errors. 
PERCENT OF RESOURCE UTILIZATION GRAPH 44

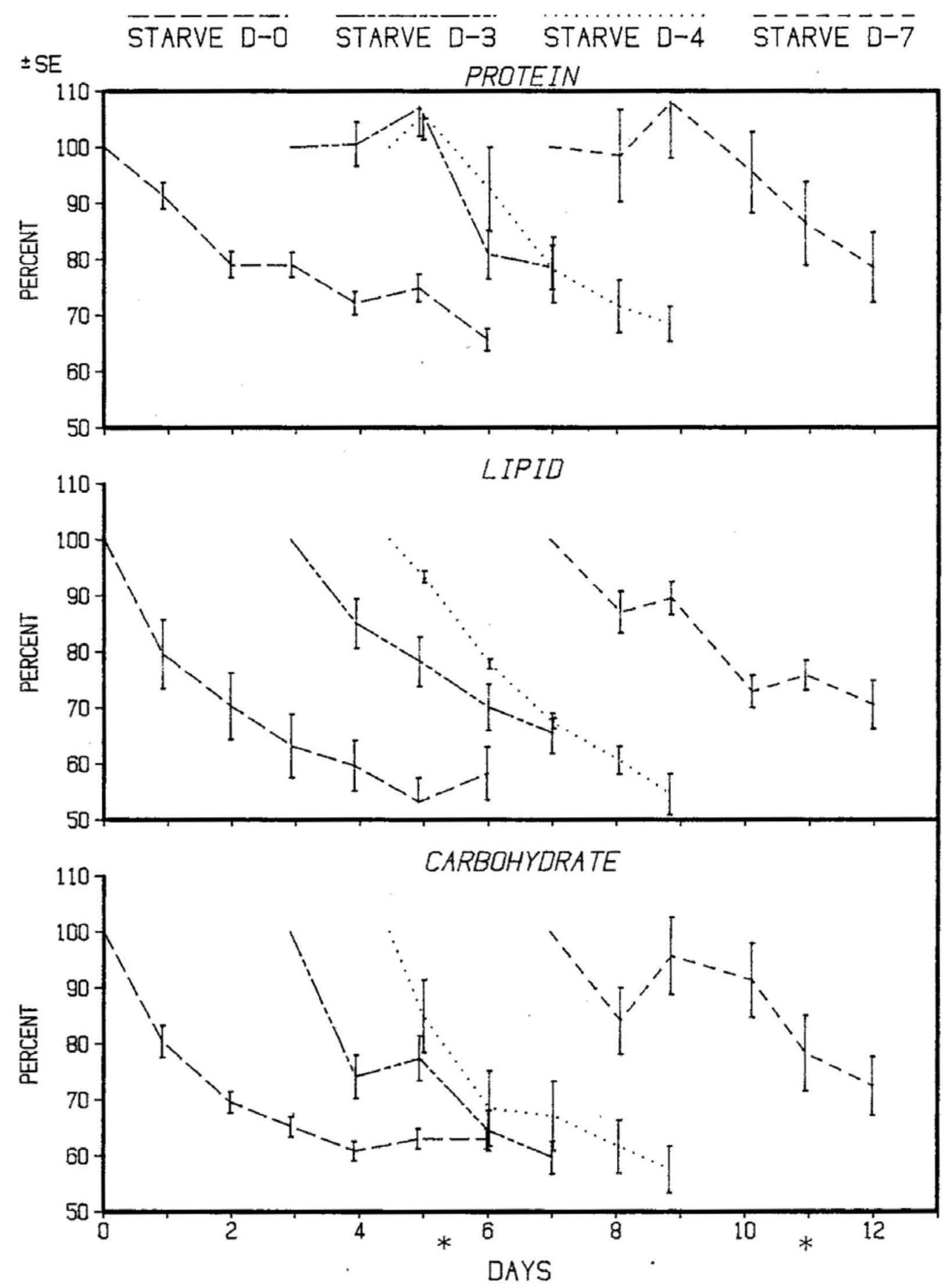




\section{Graph 45}

\section{Starvation Study}

Caloric equivalents of protein, lipid and carbohydrate. Expressed as calories per individual.

Note the change of scale in the Y-axis, between 7 to 13 calories. The first asterisk at the bottom of the page indicates the time of molting for the control, D-3 and $\mathrm{D}-4$ groups. The second asterisk indicates the time of molting for the control group only. The molt stages for each sample can be obtained from the Tables. Error bars are standard errors. 


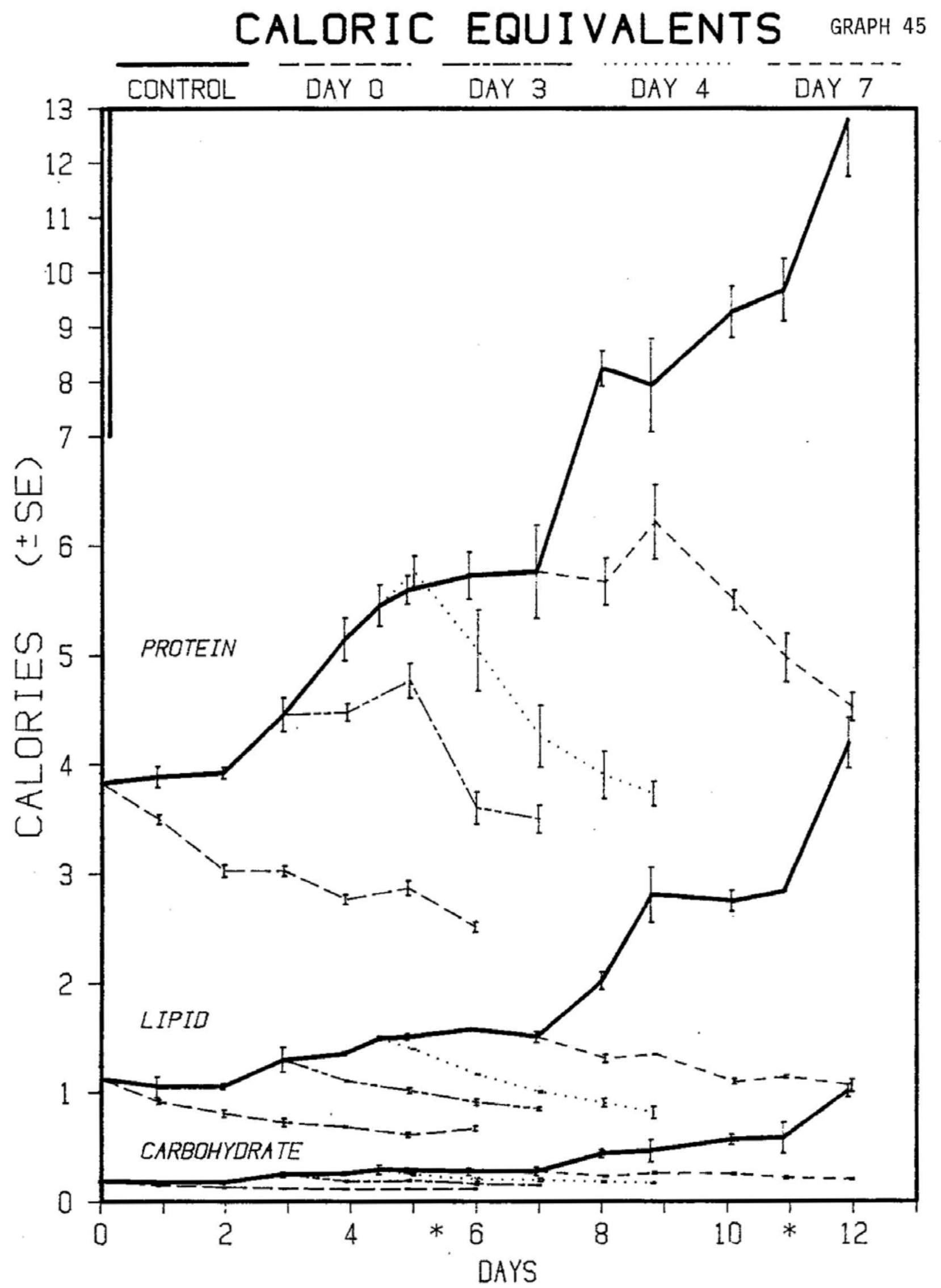




\section{Graph 46 A}

Starvation Study

Triacylg1ycero1 $(n=3)$. Expressed as micrograms per individual. Note the change in $\mathrm{Y}$-axis scale between 10 and 80 micrograms. The first asterisk at the bottom of the page indicates the time of molting for the control, D-3 and D-4 groups. The second asterisk indicates the time of molting for the control group only. The molt stages for each sample can be obtained from the Tables. Error bars are standard errors.

\section{Graph $46 \quad$ B}

\section{Starvation Study}

Triacylglycerol $(n=3)$. Expressed as a ratio against the protein in each sample (as \%). The first asterisk at the bottom of the page indicates the time of molting for the contro1, D-3 and D-4 groups. The second asterisk indicates the time of molting for the control group only. The molt stages for each sample can be obtained from the Tables. Error bars are standard errors. 
TRI ACYLGLYCEROL

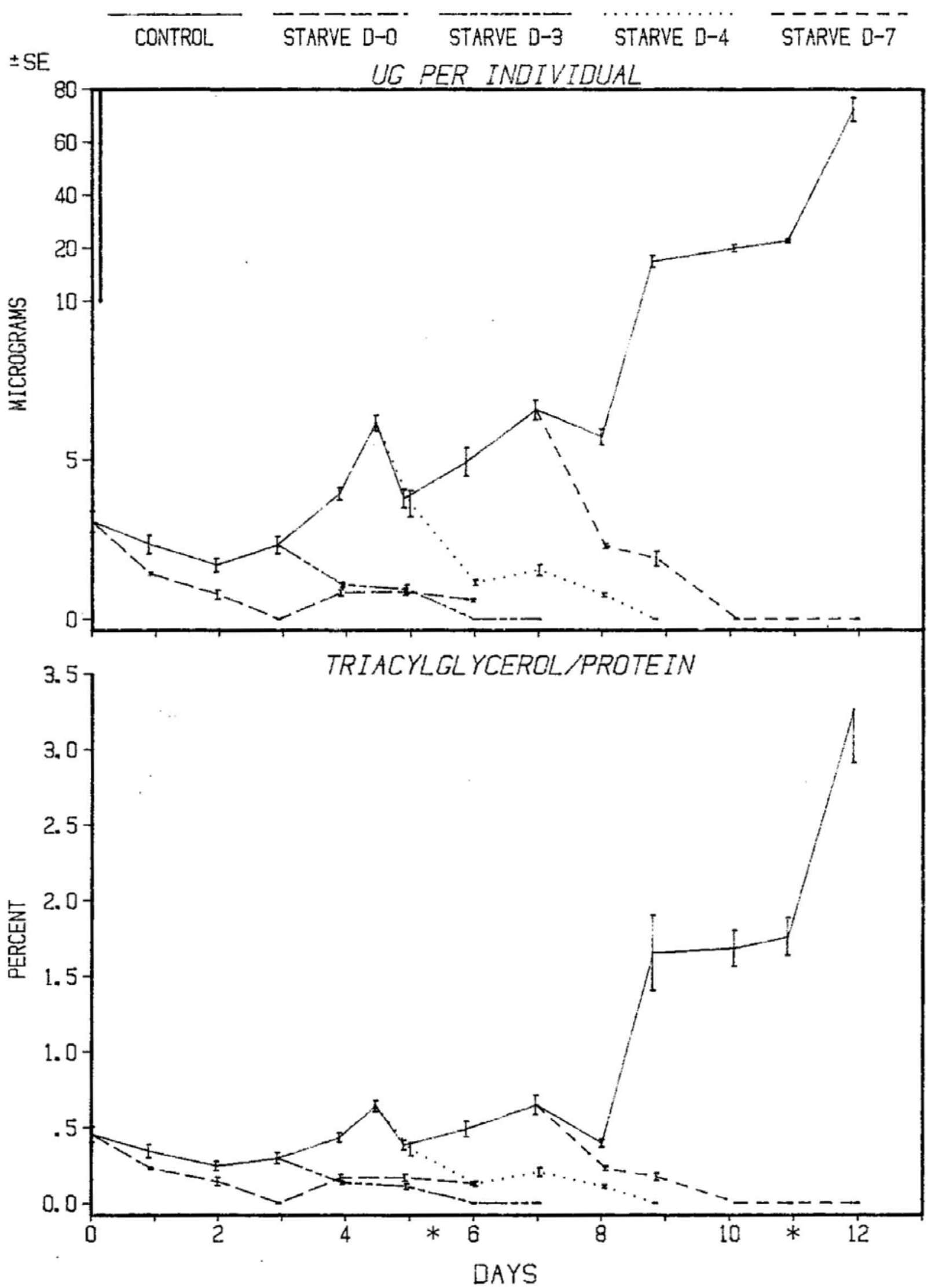




\section{Graph 47 A}

\section{Starvation Study}

Sterols $(n=3)$. Expressed as micrograms per individual.

The first asterisk at the bottom of the page indicates the time of molting for the control, D-3 and D-4 groups. The second asterisk indicates the time of molting for the control group only. The molt stages for each sample can be obtained from the Tables. Error bars are standard errors.

\section{Graph 47 B}

Starvation Study

Sterols $(n=3)$. Expressed as a ratio against the protein in each sample (as \%).

The first asterisk at the bottom of the page indicates the time of molting for the control, D-3 and D-4 groups. The second asterisk indicates the time of molting for the control group only. The molt stages for each sample can be obtained from the Tables. Error bars are standard errors. 
STEROLS

GRAPH 47

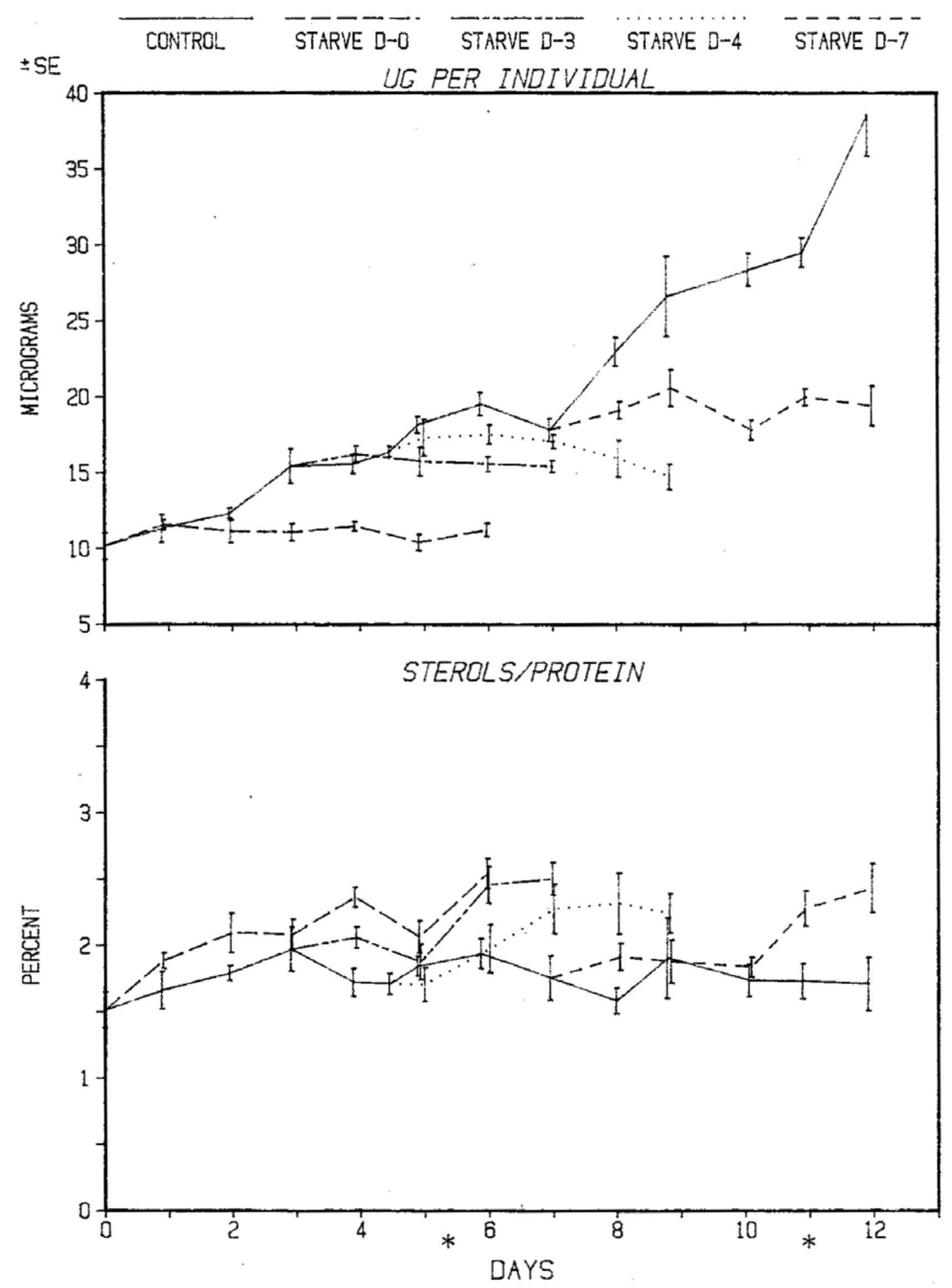


Graph 48 A

Starvation Study

Phosphatidy1 ethanolamine $(\mathrm{n}=3)$. Expressed as micrograms per individual.

The first asterisk at the bottom of the page indicates the time of molting for the control, D-3 and D-4 groups. The second asterisk indicates the time of molting for the control group only.

The molt stages for each sample can be obtained from the Tables. Error bars are standard errors.

\section{Graph 48 B}

Starvation Study

Phosphatidyl ethanolamine $(n=3)$. Expressed as a ratio against the protein in each sample (as \%).

The first asterisk at the bottom of the page indicates the time of molting for the contro1, D-3 and D-4 groups. The second asterisk indicates the time of molting for the control group only. The molt stages for each sample can be obtained from the Tables. Error bars are standard errors. 
PHOSPHATIDYL ETHANOLAMINE GRAPH 48

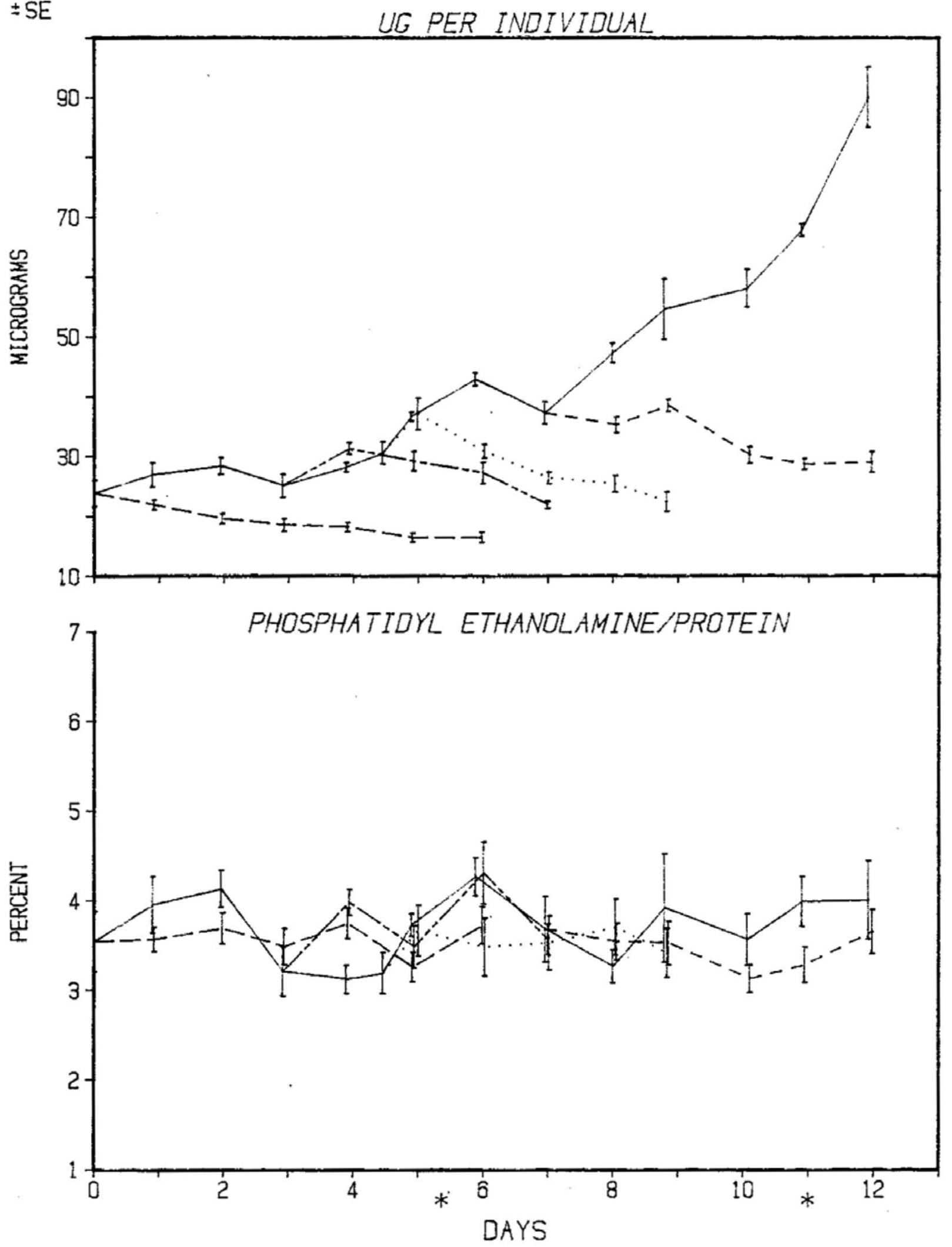


Graph 49 A

\section{Starvation Study}

Phosphatidy1 choline $(n=3)$. Expressed as micrograms per individual. The first asterisk at the bottom of the page indicates the time of molting for the control, D-3 and D-4 groups. The second asterisk indicates the time of molting for the control group only. The molt stages for each sample can be obtained from the Tables. Error bars are standard errors.

\section{Graph 49 B}

Starvation Study

Phosphatidy1 choline $(n=3)$. Expressed as a ratio against the protein in each sample (as \%).

The first asterisk at the bottom of the page indicates the time of molting for the control, D-3 and D-4 groups. The second asterisk indicates the time of molting for the control group only. The molt stages for each sample can be obtained from the Tables. Error bars are standard errors. 
PHOSPHATIDYL CHOLINE GRAPH 49

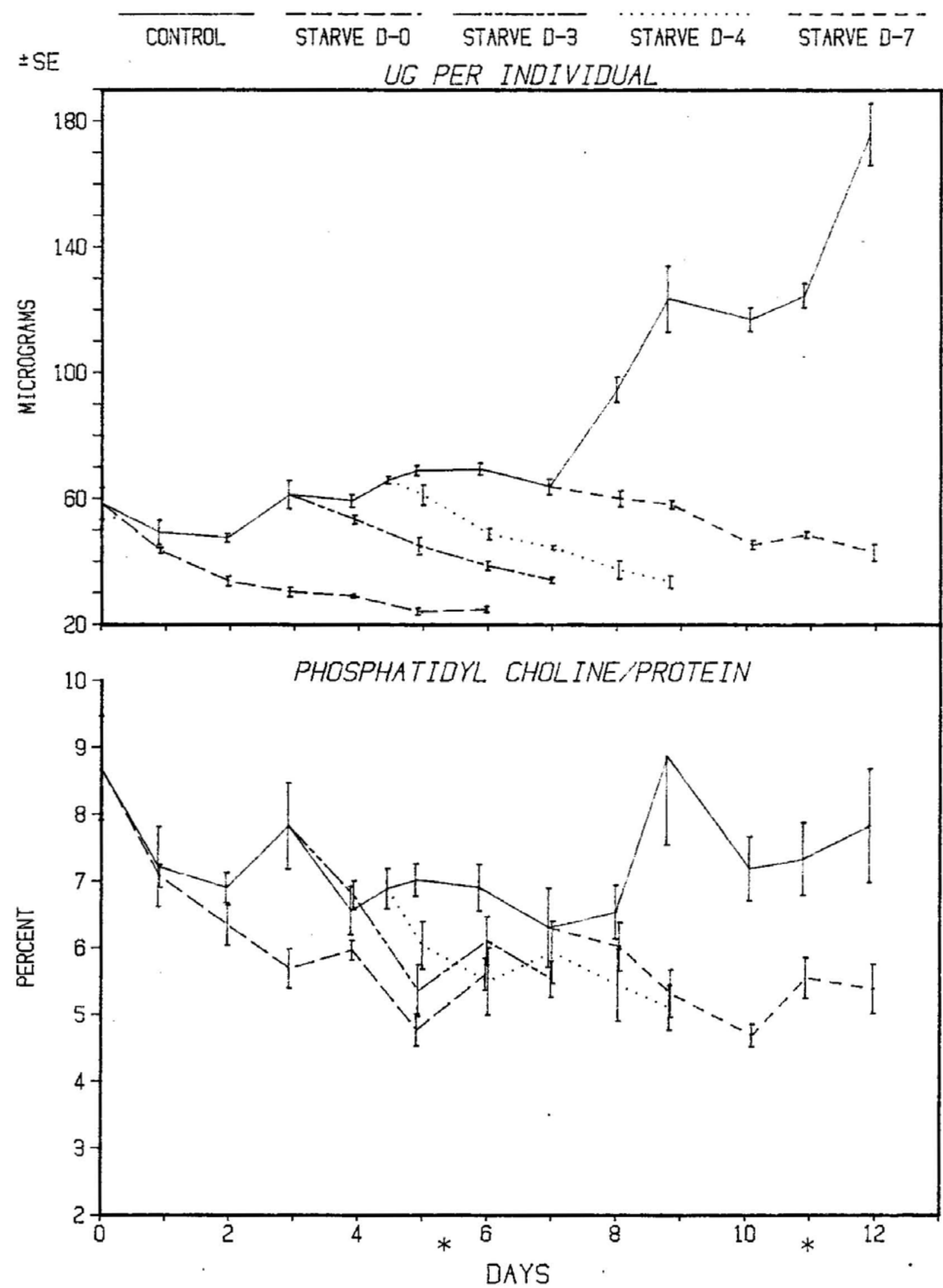


Graph 50 A

\section{Starvation Study}

Selected non-essential fatty acids (NEFA) of neutral lipids. Expressed as fatty acid methyl esters (FAME). The level of each FAME at the end of the starvation period is shown as a percent of the amount of that FAME at the onset of starvation. $D-0, D-3, D-4$ and $D-7$ at the bottom of the page, refer to the days between hatching and the onset of starvation.

\section{Graph 50 B}

Starvation Study

Selected non-essential fatty acids (NEFA) of neutra1 lipids. Expressed as fatty acid methyl esters (FAME). Shown as a ratio where: numerator = (FAMEi / total FAME in neutral lipids) for sample at end of starvation period.

denominator $=$ (FAMEi / total FAME in neutral lipids) for sample at onset of starvation.

$D-0, D-3, D-4$ and $D-7$ at the bottom of the page, refer to the days between hatching and the onset of starvation. 
NEFA Of NEUTRAL LIPIDS

GRAPH 50

FAME in STARVED LARVAE as $\boldsymbol{z}$ of CONTROL
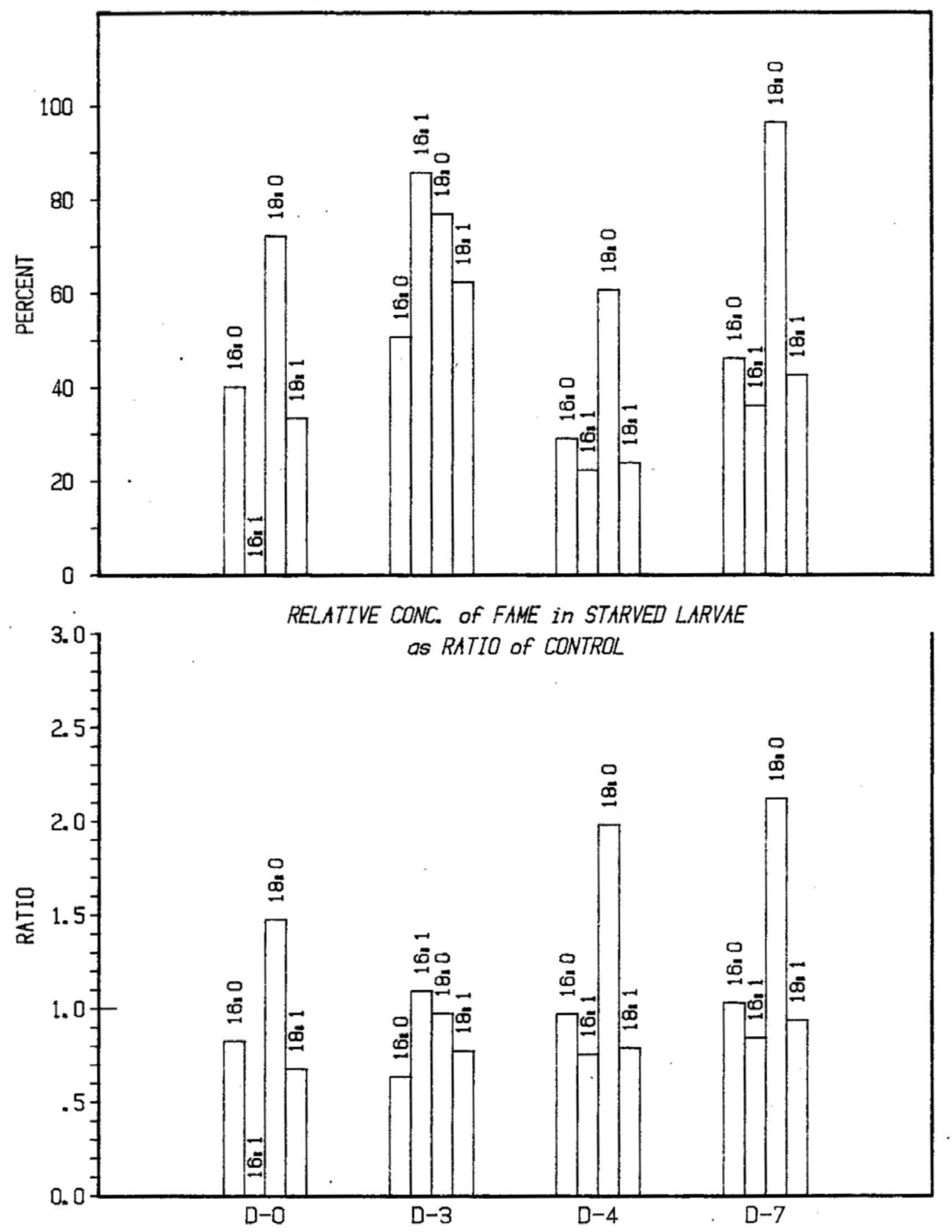
Graph 51 A

Starvation Study

Selected polyunsaturated fatty acids (PUFA) of neutral 1ipids.

Expressed as fatty acid methyl esters (FAME).

The leve1 of each FAME at the end of the starvation period is shown as a percent of the amount of that FAME at the onset of starvation. $\mathrm{D}-0, \mathrm{D}-3, \mathrm{D}-4$ and $\mathrm{D}-7$ at the bottom of the page, refer to the days between hatching and the onset of starvation.

\section{Graph 51 B}

Starvation Study

Selected polyunsaturated fatty acids (PUFA) of neutral lipids.

Expressed as fatty acid methyl esters (FAME).

Shown as a ratio where:

numerator = (FAMEi / total FAME in neutral lipids) for sample at end of starvation period.

denominator $=$ (FAMEi / total FAME in neutral 1ipids) for sample at onset of starvation.

$D-0, D-3, D-4$ and $D-7$ at the bottom of the page, refer to the days

between hatching and the onset of starvation. 
PUFA of NEUTRAL LIPIDS

FAME in STARVED LARVAE as \% of CONTROL.
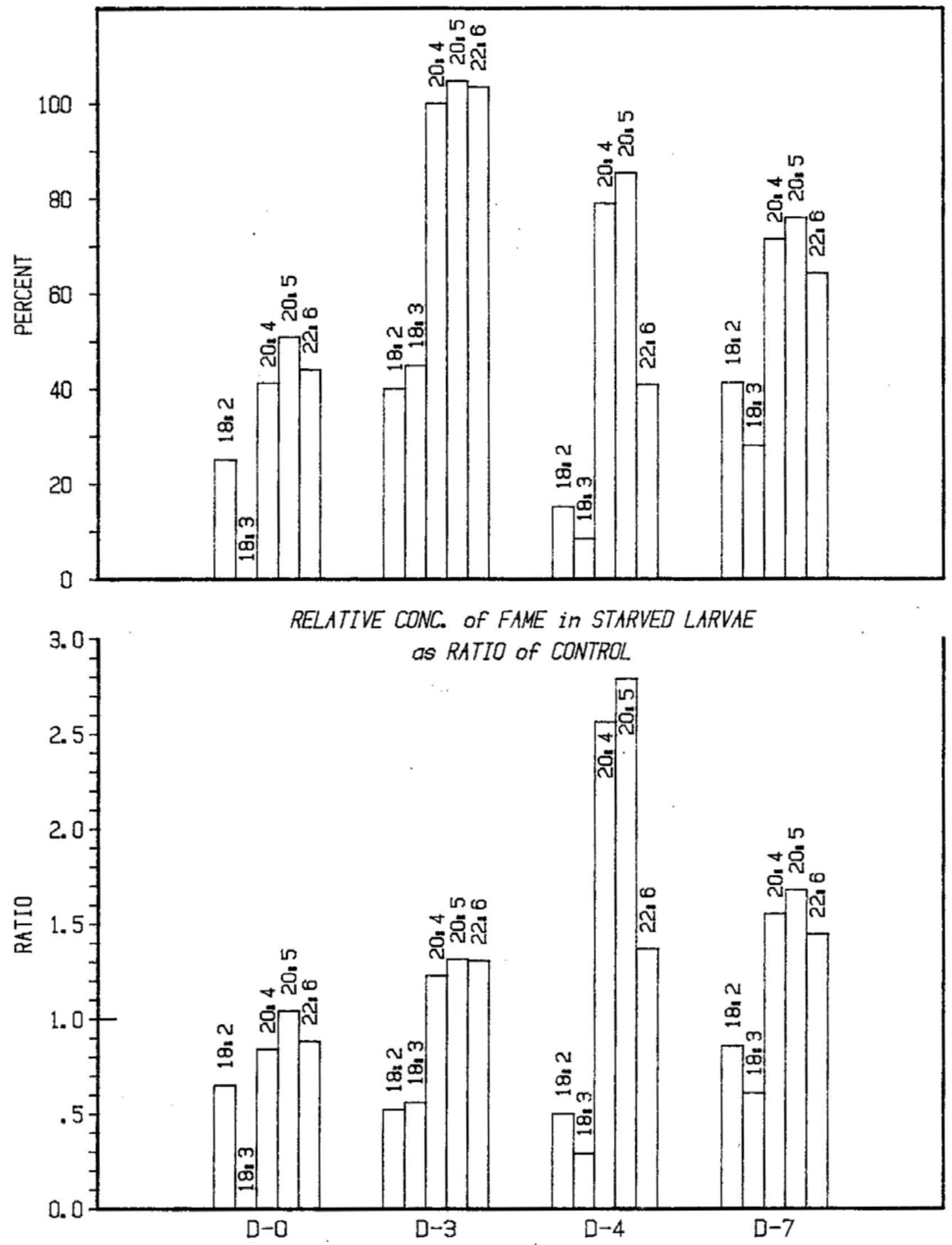
Graph 52 A

Starvation Study

Selected non-essential fatty acids (NEFA) of P. ethanolamine. Expressed as fatty acid methyl esters (FAME).

The level of each FAME at the end of the starvation period is shown as a percent of the amount of that FAME at the onset of starvation. $D-0, D-3, D-4$ and $D-7$ at the bottom of the page, refer to the days between hatching and the onset of starvation.

Graph 52 B

Starvation Study

Selected non-essential fatty acids (NEFA) of P. ethanolamine.

Expressed as fatty acid methyl esters (FAME).

Shown as a ratio where:

numerator $=$ (FAMEi / total FAME in P. ethanolamine) for sample at end of starvation period.

denominator $=$ (FAMEi / total FAME in P. ethanolamine) for sample at onset of starvation.

$\mathrm{D}-0, \mathrm{D}-3, \mathrm{D}-4$ and $\mathrm{D}-7$ at the bottom of the page, refer to the days between hatching and the onset of starvation. 
NEFA of P. ETHANOLAMINE GRAPH 52

FAME in STARVED LARVAE as $\boldsymbol{z}$ of CONTROL
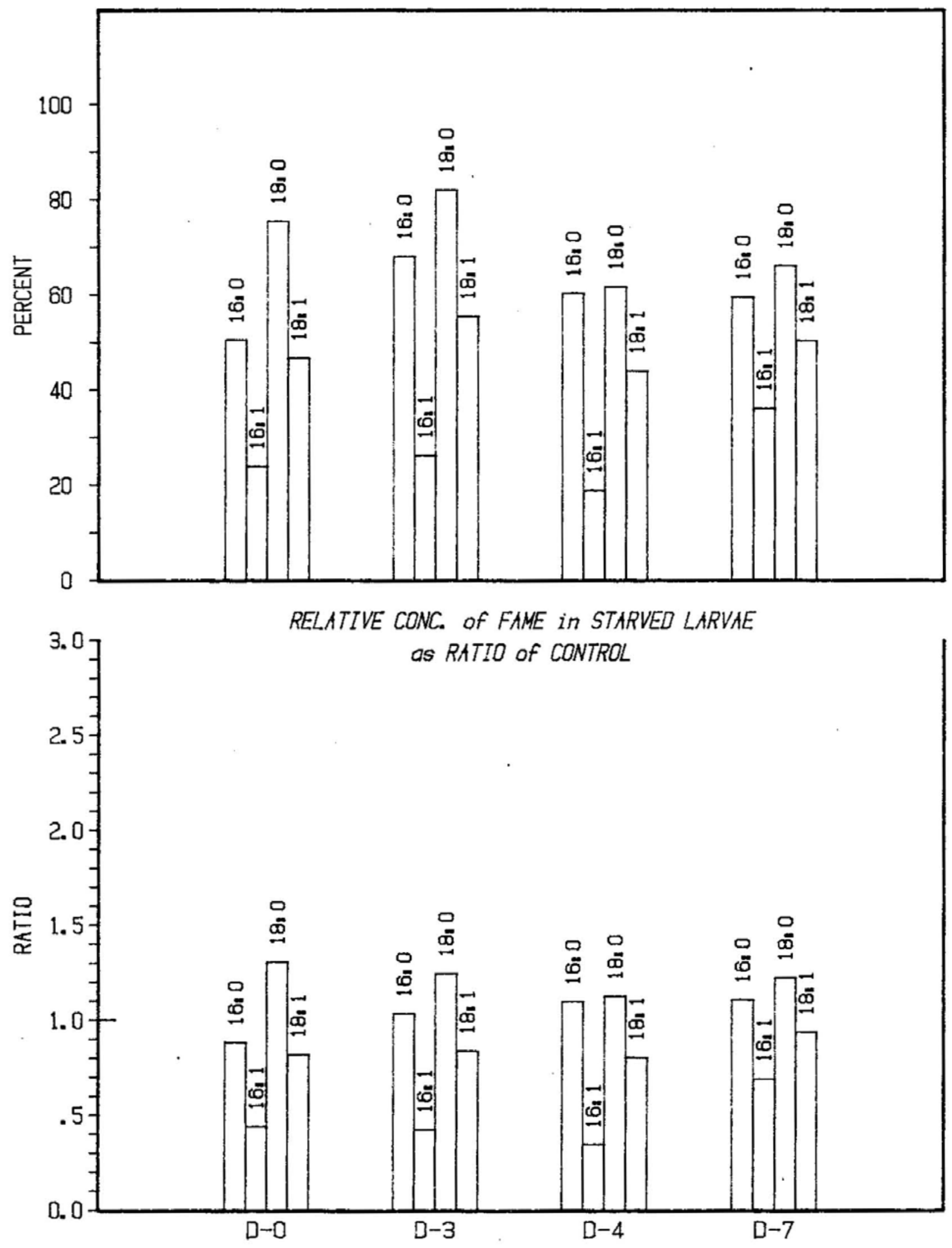


\section{Graph 53 A}

\section{Starvation Study}

Selected polyunsaturated fatty acids (PUFA) of P. ethanolamine. Expressed as fatty acid methyl esters (FAME). The level of each FAME at the end of the starvation period is shown as a percent of the amount of that FAME at the onset of starvation. $\mathrm{D}-0, \mathrm{D}-3, \mathrm{D}-4$ and $\mathrm{D}-7$ at the bottom of the page, refer to the days between hatching and the onset of starvation.

\section{Graph 53 B}

Starvation Study.

Selected polyunsaturated fatty acids (PUFA) of $P$. ethanolamine.

Expressed as fatty acid methyl esters (FAME). Shown as a ratio where:

numerator $=$ (FAMEi / total FAME in P. ethanolamine) for sample at end of starvation period.

denominator $=($ FAMEi / total FAME in P. ethanolamine $)$ for sample at onset of starvation.

$D-0, D-3, D-4$ and $D-7$ at the bottom of the page, refer to the days

between hatching and the onset of starvation. 
PUFA of P. ETHANOLAMINE GRAPH 53

FAME in STARVED LARVAE as $\%$ of CONTROL.
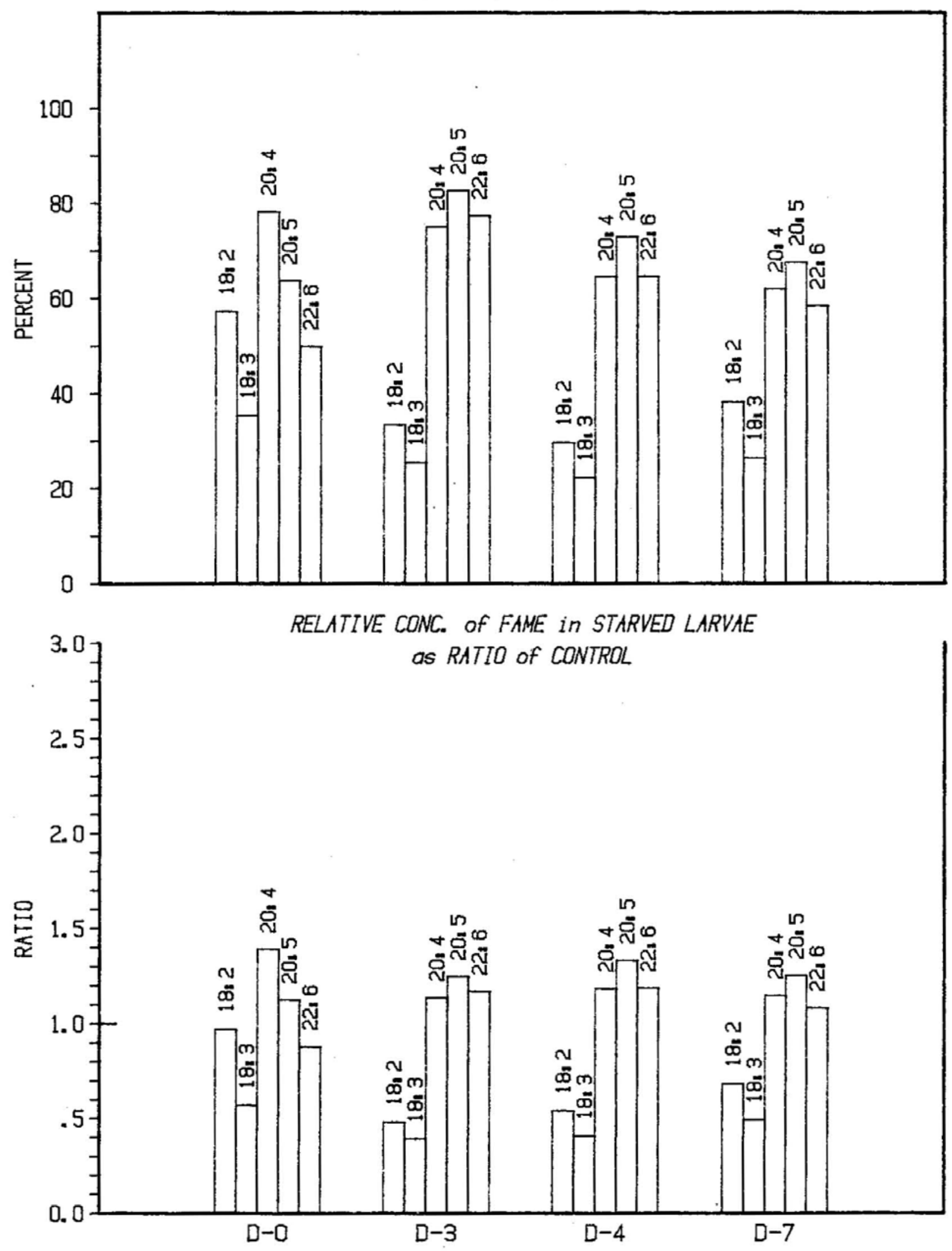


\title{
Graph 54 A
}

\section{Starvation Study}

\author{
Selected non-essential fatty acids (NEFA) of P. choline. \\ Expressed as fatty acid methyl esters (FAME). \\ The level of each FAME at the end of the starvation period is shown as \\ a percent of the amount of that FAME at the onset of starvation. \\ $D-0, D-3, D-4$ and $D-7$ at the bottom of the page, refer to the days \\ between hatching and the onset of starvation.
}

\section{Graph 54 B \\ Starvation Study}

Selected non-essential fatty acids (NEFA) of P. choline.

Expressed as fatty acid methyl esters (FAME).

Shown as a ratio where:

numerator $=$ (FAMEi / total FAME in P. choline) for sample at end of starvation period.

denominator $=$ (FAMEi / total FAME in P. choline $)$ for sample at onset of starvation.

$D-0, D-3, D-4$ and $D-7$ at the bottom of the page, refer to the days between hatching and the onset of starvation. 
NEFA of P. CHOLINE

GRAPH 54

FAME in STARVED LARVAE as $\boldsymbol{z}$ of CONTROL

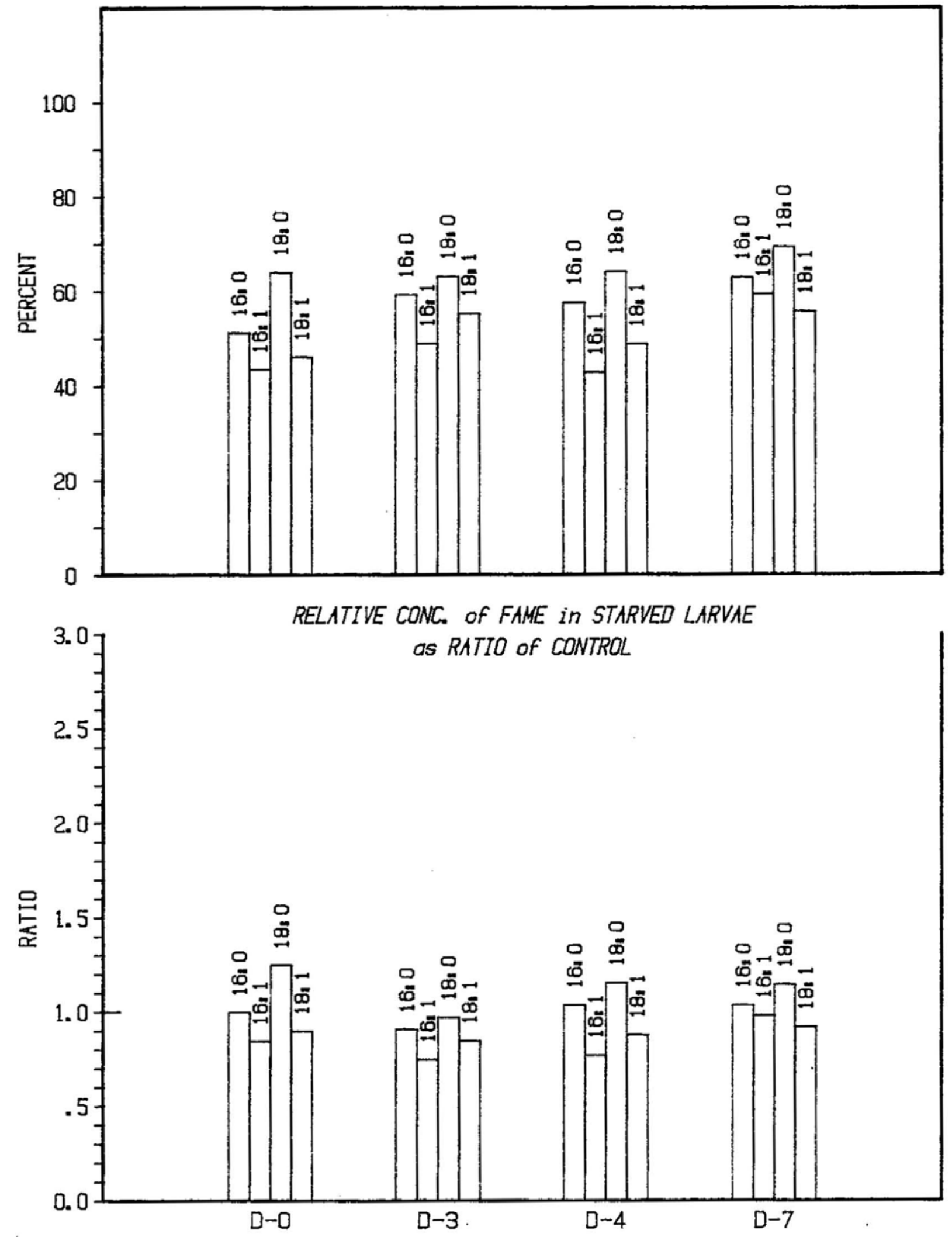




\section{Graph 55 A}

Starvation Study

Selected polyunsaturated fatty acids (PUFA) of P. choline. Expressed as fatty acid methyl esters (FAME).

The level of each FAME at the end of the starvation period is shown as a percent of the amount of that FAME at the onset of starvation. $D-0, D-3, D-4$ and $D-7$ at the bottom of the page, refer to the days between hatching and the onset of starvation.

\section{Graph 55 B}

\section{Starvation Study}

Selected polyunsaturated fatty acids (PUFA) of $P$. choline.

Expressed as fatty acid methyl esters (FAME). Shown as a ratio where:

numerator $=$ (FAMEi / total FAME in P. choline) for sample at end of starvation period.

denominator $=$ (FAMEi / total FAME in P. choline) for sample at onset of starvation.

$\mathrm{D}-0, \mathrm{D}-3, \mathrm{D}-4$ and $\mathrm{D}-7$ at the bottom of the page, refer to the days between hatching and the onset of starvation. 
PUFA of P. CHOLINE GRAPH 55

FAME in STARVED LARVAE as $z$ of CONTROL
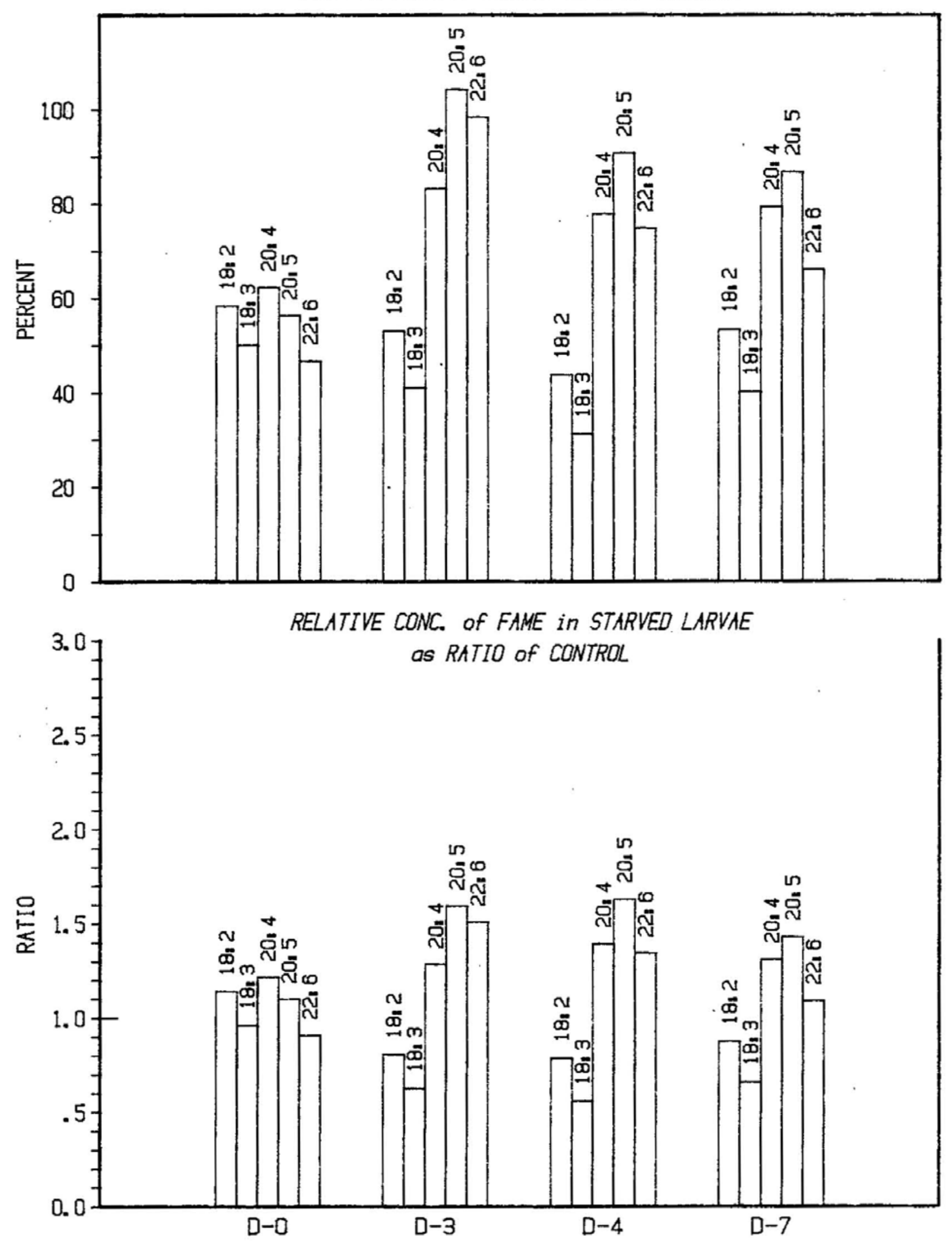


\section{Graph 56 A}

${ }^{14} \mathrm{C}$ Fatty Acid Study

DPM recovered in triacylglycerol. Expressed as a percent of the total DPM recovered in lipid sample.

16:0 = fed ${ }^{14} \mathrm{C}$ palmitic acid labelled feed.

$20: 5$ = fed ${ }^{14} \mathrm{C}$ eicosapentaenoic acid labelled feed.

$F=$ fed after label introduced.

$S=$ starved after label introduced.

Hours indicate the time between feeding of 1 abel and sampling of larvae.

\section{Graph 56 B}

\section{${ }^{14} \mathrm{C}$ Fatty Acid Study}

DPM recovered in free fatty acid. Expressed as a percent of the total DPM recovered in lipid sample.

16:0 = fed ${ }^{14} \mathrm{C}$ palmitic acid labelled feed.

$20: 5$ = fed ${ }^{14} \mathrm{C}$ eicosapentaenoic acid labelled feed.

$\mathrm{F}=$ fed after labe1 introduced.

$\mathrm{S}=$ starved after label introduced.

Hours indicate the time between feeding of label and sampling of larvae. 


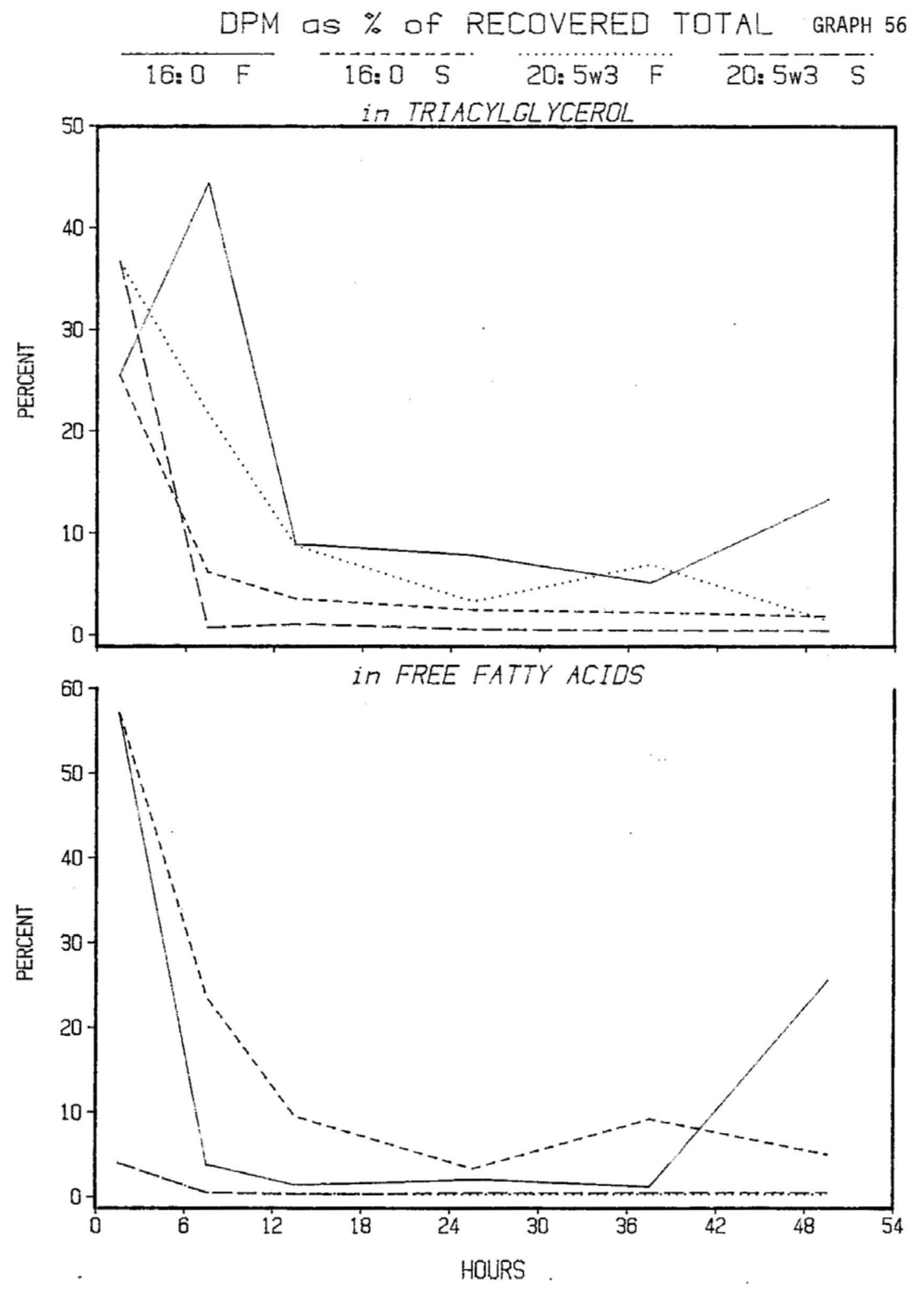


Graph 57 A

${ }^{14} \mathrm{C}$ Fatty Acid Study

DPM recovered in steryl ester. Expressed as a percent of the total DPM recovered in lipid sample.

16:0 = fed ${ }^{14} \mathrm{C}$ palmitic acid labelled feed. $20: 5$ = fed ${ }^{14} \mathrm{C}$ eicosapentaenoic acid labelled feed.

$\mathrm{F}=$ fed after label introduced.

$\mathbf{S}=$ starved after label introduced.

Hours indicate the time between feeding of label and sampling of

larvae.

Graph $57 \quad$ B

${ }^{14} \mathrm{C}$ Fatty Acid Study

DPM recovered in 1-2 diacylglycerol. Expressed as a percent of the total DPM recovered in lipid sample.

16:0 = fed ${ }^{14} \mathrm{C}$ palmitic acid labelled feed.

$20: 5$ = fed ${ }^{14} \mathrm{C}$ eicosapentaenoic acid labelled feed.

$F=$ fed after label introduced.

$\mathrm{S}=$ starved after label introduced.

Hours indicate the time between feeding of label and sampling of

larvae. 


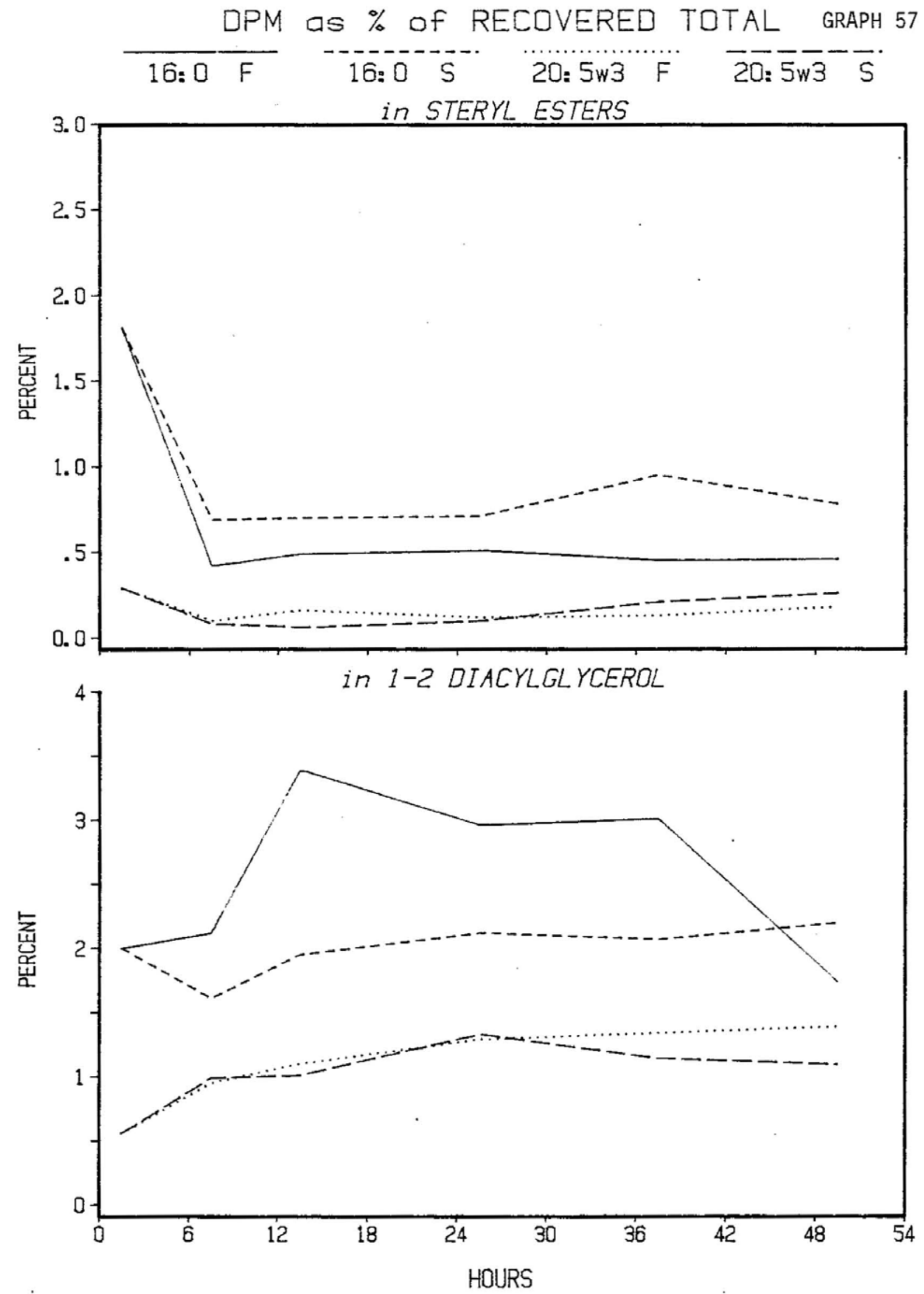


Graph 58 A

${ }^{14} \mathrm{C}$ Fatty Acid Study

DPM recovered in phosphatidyl ethanolamine. Expressed as a percent of the total DPM recovered in lipid sample.

$16: 0=$ fed ${ }^{14} \mathrm{C}$ palmitic acid labelled feed.

20:5 = fed ${ }^{14} \mathrm{C}$ eicosapentaenoic acid labelled feed.

$F=$ fed after label introduced.

$\mathrm{S}=$ starved after label introduced.

Hours indicate the time between feeding of label and sampling of larvae.

Graph 58 B

${ }^{14} \mathrm{C}$ Fatty Acid Study

DPM recovered in phosphatidy1 choline. Expressed as a percent of the total DPM recovered in lipid sample.

16:0 = fed ${ }^{14} \mathrm{C}$ palmitic acid labelled feed.

20:5 = fed ${ }^{14} \mathrm{C}$ eicosapentaenoic acid labelled feed.

$\mathrm{F}=$ fed after label introduced.

$\mathrm{S}=$ starved after label introduced.

Hours indicate the time between feeding of label and sampling of

larvae. 


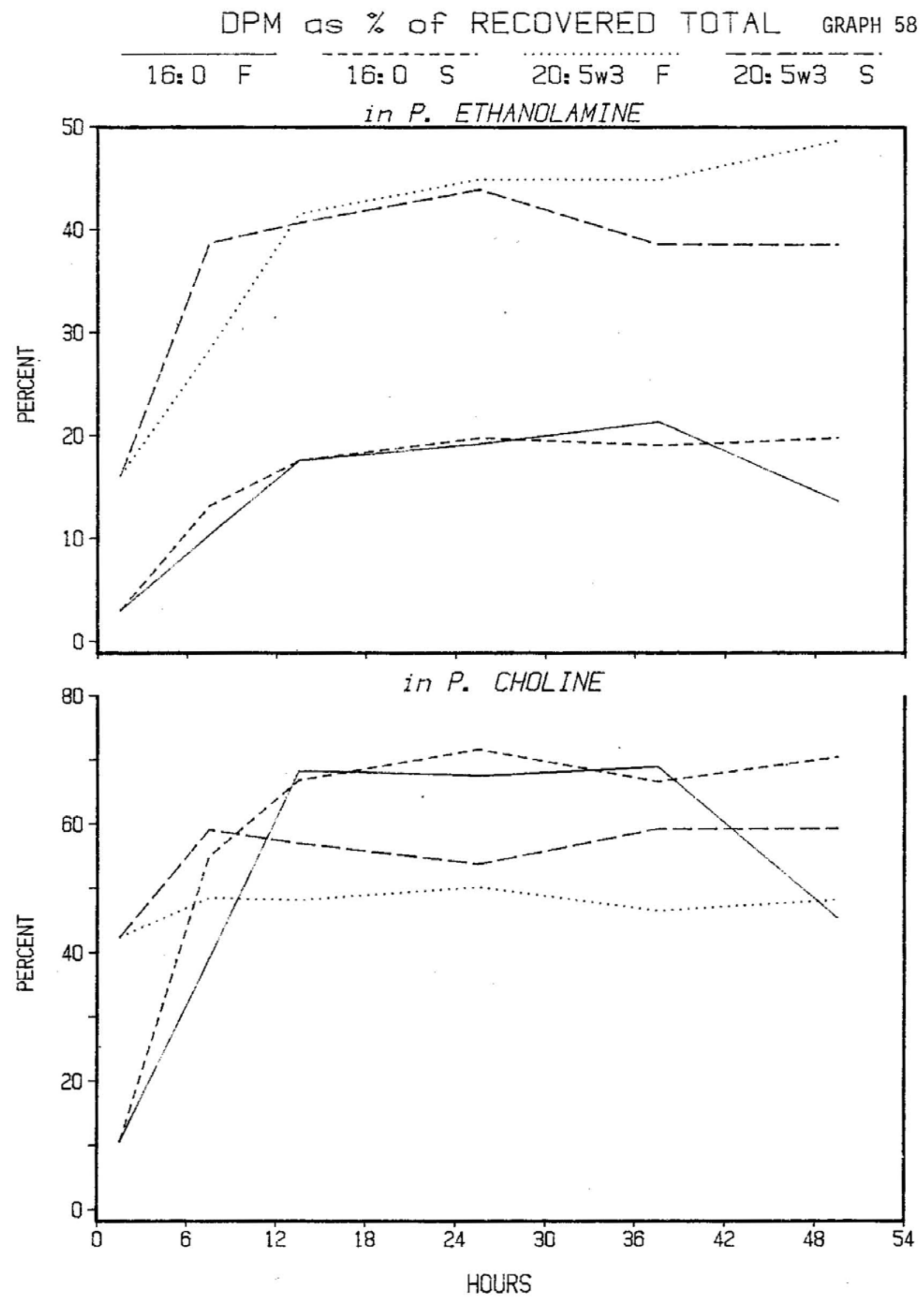


$-432-$ 


\section{LITERATURE CITED}

Ache, B.W. 1982. Chemoreception and thermoreception. Chap. 8, p 369-398. in: Atwood, H.L., D.C. Sandeman and D.E. Bliss (eds.) The Biology of Crustacea. vo1. 3. Academic Press, N.Y. 479 pp.

Addison, R.F., R.G. Ackman and J. Hingley 1972. Lipid composition of the Queen crab (Chionoecetes opilio). J. Fish. Res. Bd. Can. 29: 407-411.

Adegboye, J.D. and P.F. Hirsch 1975. Variations in hemolymph calcium associated with the molting cycle in the crayfish. in: Avault, J.W. Jr. (ed.) Freshwater Crayfish. Louisiana State University Press, Baton Rouge LA. p 227-247.

Adiyodi, R.G. and K.G. Adiyodi 1970a. Lipid metabolism in relation to reproduction and moulting in the crab, Paratelphusa hydrodromous (Herbst): Phospholipids and reproduction. Indian J. Exp. Biol. 8: 222-223.

Adiyodi, R.G. and K.G. Adiyodi 1970b. Free sugars in Paratelphusa hydrodromous (Herbst) in relation to reproduction and molting. Physiol. Zoo1. 43: 71-77.

Adiyodi, R.G. and K.G. Adiyodi 1971. Lipid metabolism in relation to reproduction and moulting in the crab, Paratelphusa hydrodromous (Herbst): cholesterol and unsaturated fatty acids. Indian J. Exp. Biol. 9: 514-515.

Aiken, D.E. 1969. Photoperiod, endocrinology and the crustacean molt cycle. Science 164: 149-155.

Aiken, D.E. 1973. Proecdysis, setal development, and molt prediction in the American lobster (Homarus americanus). J. Fish. Res. Bd. Can. 30: 1337-1344.

Aiken, D.E. 1980. Molting and Growth. chap 2, p 91-163. in: Cobb, J.S. and B.F. Phillips (eds.) The Biology and Management of Lobsters. vo1. 1. Physiology and Behavior. Academic Press. N.Y.

Aiken, D.E. and S.L. Waddy 1980. Reproductive biology. chap 4, p 215-275. in: Cobb, J.S. and B.F. Phillips (eds.) The Biology and Management of Lobsters. vol. 1. Physiology and Behavior. Academic Press. N.Y.

Aiken, D.E. and S.L. Waddy 1982. Cement gland development, ovary maturation and reproductive cycles in the American lobster, Homarus americanus. J. Crust. Bio1. 2: 315-327.

Allen, W.V. 1972. Lipid transport in the dungeness crab, Cancer magister Dana. Comp. Biochem. Physiol. 43B: 193-207. 
Ando, T., A. Kanazawa, S. Teshima, J. Patrois, and H.J. Ceccaldi 1977. Variations in the lipids of tissues during the molting cycle of prawn. Bull. Jpn. Soc. Sci. Fish. 43: 1445-1449.

Anger, K. and R.R. Dawirs 1981. Influence of starvation on the larval development of Hyas araneus (Decapoda, Majidae). Helgo. Meeresunter. 34: $287-311$.

Anger, K., N. Laasch, C. Pusche1 and F. Schorn 1983. Changes in biomass and chemical composition of a spider crab (Hyas araneus) larvae reared in the laboratory. Mar. Ecol. Prog. Ser. 12: 91-101.

Anger, K. and K.K.C. Nair 1979. Laboratory experiments on the larval development of Hyas araneus (Decapoda, Majidae). Helgo. Meeresunter. 32: 36-54.

Anse11, A.D. 1973. Changes in oxygen consumption, heart rate and ventilation accompanying starvation in the decapod crustacean Cancer pagurus. Neth. J. Sea Res. 7: 455-475.

Armitage, K.B., A.L. Buikema Jr., and N.J. Willems 1972. Organic constituents in the annual cycle of the crayfish Orconectes nais (Faxon). Comp. Biochem. Physio1. 41A: 825-842.

Ash, C.P. and H.J. Atkinson 1983. Evidence for a temperature-dependent conversion of lipid reserves to carbohydrate in quiescent eggs of the nematode, Nematodirus battus. Comp. Biochem. Physiol. 76B: 603-610.

Barclay, M.C., W. Dall and D.M. Smith 1983. Changes in lipid and protein during starvation and the moulting cycle in the tiger prawn, Penaeus esculentus Haswe11. J. Exp. Mar. Biol. Ecol. 68: 229-244.

Barker, P.L. and R. Gibson 1977. Observations on the feeding mechanism, structure of the gut and digestive physiology of the European lobster, Homarus gammarus (L.) (Decapoda: Nephropidae). J. Exp. Mar. Biol. Eco1. 26: 297-324.

Barlow, J. and G.J. Ridgeway 1969. Changes in serum proteins during the molt and reproductive cycles of the American lobster, Homarus americanus. J. Fish. Res. Bd. Can. 26: 2101-2109.

Barnes, H. 1965. Studies in the biochemistry of cirripede eggs. 1. Changes in the general biochemical composition during development of Balanus balanoides and B. balanus. J. Mar. Biol Assoc. U.K. 45: 321-339.

Benevides, J.M. and C.B. Hammen 1978. A survey of marine invertebrates for g1yoxylate cycle activity. Fed. Proc. 37: 434.

Benson, A.A., R.F. Lee and J.C. Nevenze1 1972. Wax esters: major marine metabolic energy reserves. Biochem. Soc. Symp. no. 35: 175-187. 
BioRad Laboratories 1979. BioRad protein assay. Bulletin 1069 Feb. 1979. Richmond, Calif.

Blank, M.L., K. Kasama and F. Snyder 1972. Isolation and identification of an alkyldiacylglycerol containing isovaleric acid. J. Lipid Res. 13: 390-394.

B1igh, E.G. and W. Dyer 1959. A rapid method of total lipid extraction and purification. Can. J. Biochem. Physio1. 37: 911-917.

Bligh, E.G, and M.A. Scott 1966. Blood lipids of the lobster, Homarus americanus. J. Fish. Res. Bd. Can.' 23: 1629-1631.

Bloch, K.E. 1983. Sterol structure and membrane function. CRC Critical Reviews in Biochemistry 14: 47-92.

Boghen, A. and J.D. Castel1 1980. Considerations of the lecithin and protein requirements of juvenile lobsters (Homarus americanus). 1980 Lobster Nutrition Workshop Proc. Orono, Maine. Maine Sea Grant Pub1. Tech. Rept. 58: 21-28.

Bollenbacher, W.E., D.W. Borst and J.D. O'Connor 1972. Endocrine regulation of lipid synthesis in decapod crustaceans. Amer. Zool. 12: $381-384$.

Borgstrom, B. 1980. Importance of phospholipids, pancreatic phospholipase $A_{2}$, and fatty acid for the digestion of dietary fat.

Gastroenterology 78: 954-962.

Bradford, M.M. 1976. A rapid and sensitive method for the quantification of microgram quantities of protein utilizing the principle of protein-dye binding. Analyt. Biochem. 72: 248-254.

Branford, J.R. 1978. Incubation period for lobster Homarus gammarus at various temperatures. Mar. Biol. 47: 363-368.

Brockerhoff, H., R.G. Ackman and R.J. Hoyle 1963. Specific distribution of fatty acids in marine lipids. Arch. Biochem. Biophys. 100: 9-12.

Brockerhoff, H., R.J. Hoyle and P.C. Hwang 1970. Digestive enzymes of the American lobster (Homarus americanus). J. Fish. Res. Bd. Can. $27: 1357-1370$.

Brockerhoff, H., R.J. Hoyle and K.J. Ronald 1964. Retention of the fatty acid distribution pattern of a dietary triglyceride in animals. J. Biol. Chem. 239: 735-739.

Brockerhoff, H., J.E. Stewart and W. Tracreiter 1967. Digestion of trig1ycerides by lobster. Can. J. Biochem. 45: 421-422.

Bumpus, H.C. 1891. The embryology of the American lobster. J. Morpho1. 5 : 215-262. 
Burr, G.0. and M.M. Burr 1929. A new deficiency disease produced by the rigid exclusion of fat from the diet. J. Biol. Chem. 82: 345-367.

Burr, G.0. and M.M. Burr 1930. On the nature and role of the fatty acids essential in nutrition. J. Biol. Chem. 86: 587-621.

Bursey, C.R. and C.E. Lane 1971. Ionic and protein concentration changes during the molt cycle of Penaeus duorarum. Comp. Biochem. Physio1. 40A: 155-162.

Busselen, P. 1970. Effects of moulting cycle and nutritional conditions on haemolymph proteins in Carcinus maenas. Comp. Biochem. Physiol. 37 : 73-83.

Capuzzo, J.M. and B.A. Lancaster 1979a. Some physiological and biochemical considerations of larval development in the American lobster, Homarus americanus Milne Edwards. J. Exp. Mar. Biol. Ecol. 40 : 53-62.

Capuzzo, J.M. and B.A. Lancaster 1979b. The effects of dietary carbohydrate levels on protein utilization in the American lobster (Homarus americanus). Proc. World Maric. Soc. 10: 689-700.

Capuzzo, J.M. and B.A. Lancaster 1979c. Larval development in the American lobster: changes in metabolic activity and the $0: \mathrm{N}$ ratio. Can. J. Zoo1. 57: 1845-1848.

Capuzzo, J.M. and B.A. Lancaster 1979d. The effects of diet on the growth energetics of postlarval lobsters (Homarus americanus). Woods Hole Ocean. Inst. Tech. Rept. 79-55. 23 pp.

Capuzzo, J.M. and B.A. Lancaster 1984. The effects of petroleum hydrocarbons on lipid metabolism and energetics of larval development and metamorphosis is the American lobster (Homarus americanus Milne Edwards). Mar. Environ. Res. (in press).

Castel1, J.D. 1979. Review of 1ipid requirements of finfish. Proc. World Symp. Finfish Nutrition and Fishfeed Tech. Hamburg, 20-23 June 1978. Vol. 1: 59 84.

Caste11, J.D. and J.F. Covey 1976. Dietary 1ipid requirements of adult lobsters Homarus americanus. J. Nutr. 106: 1159-1165.

Castell, J.D., E.G. Mason and J.F. Covey 1975. Cholesterol requirements of juvenile American lobster (Homarus americanus). J. Fish. Res. Bd. Can. 32: 1431-1435.

Caste11, J.D., R.0. Sinnhuber, D.J. Lee and J.H. Wales 1972a. Essentia1 fatty acids in the diet of rainbow trout (Salmo gairdneri): Physiological symptoms of essential fatty acid deficiency. J. Nutr. 102: 87-92. 
Caste11, J.D., R.0. Sinnhuber, J.H. Wales and D.J. Lee 1972b. Essential fatty acids in the diet of rainbow trout (Salmo gairdneri): Growth, feed conversion and some gross deficiency symptoms. J. Nutr. 102: 77-86.

Ceccaldi, H.J. and J.L.M. Martin 1969. Evolution des proteines de 1 'hemolymphe chez Carcinus maenas L. durant 1 'ovogenese. C.R. Seane. Soc. Biol. 163: 2638-2641.

Chacko, G.K., F.V. Barnola and R. Villegas 1977. Phospholipid and fatty acid compositions of axon and periaxonal cell plasma membranes of lobster leg nerves. J. Neurochem. 28: 445-447.

Chaikelis, A.S. 1949. A handbook of anatomy, embryology and physiology of the lobster. City College Press Inc. N.Y.

Chang, E.S. and M.J. Bruce 1981. Ecdysteroid titers of larval lobsters. Comp. Biochem. Physio1. 70A: 239-241.

Chang, E.S. and J.D. O'Connor 1978. In vitro secretion and hydroxylation of alpha ecdysone as a function of the crustacean molt cycle. Gen. Comp. Endocrin. 36: 151-160.

Chang, E.S. and J.D. O'Connor 1983. Metabolism and transport of carbohydrates and 1ipids. Chap. 5, p 263-287. in: B1iss, D.E. and L.H. Mantel (eds.) The Biology of Crustacea. vol. 5. Academic Press, N.Y. 471 pp.

Chapelle, S. 1977. Lipid composition of tissues of marine crustaceans. Biochem. Syst. Ecol. 5: 241-248.

Chapelle, S. 1978. The influence of acclimation temperature on the fatty acid composition of an aquatic crustacean (Carcinus maenas). J. Exp. Zoo1. 204: 337-346.

Chapelle, S, G. Brichon and G. Zwingelstein 1981. Effect of environmental temperature on the phospholipid metabolism of gill mitochondria of Carcinus maenas. Biochem. Syst. Ecol. 9: 333-338.

Chapelle, S. and A. Pequeux 1982. The distribution of fatty acids in gill phospholipids of the chinese crab Eriocheir sinensis. Biochem. System. Ecol. 10: 71-78.

Cheung, T.S. 1966. The development of egg membranes and egg attachment in the shore crab Carcinus maenus and some related decapods. J. Mar. Biol. Assoc. U.K. 46: 373-400.

Chittleborough, R.G. 1975. Environmental factors affecting growth and survival of juvenile western rock lobsters Panulirus longipes (Milne-Edwards). Aust. J. Mar. Freshwater Res. 26: 177-196. 
Chou, S.-C. and A. Goldstein 1960. Chromogenic groupings in the Lowry protein determination. Biochem. J. 75: 109-115.

Christie, W.W. 1973. Lipid Analysis. Pergamon Press, N.Y. 338 pp.

Christie, W.W., R.C. Noble and J.H. Moore 1970. Determination of 1ipid classes by a gas-chromatographic procedure. Analyst. 95: 940-944.

Cioni, M., G. Pinzauti and P. Vanni 1981. Comparative biochemisty of the glyoxylate cycle. Comp. Biochem. Physio1. 70B: 1-26.

Clandinin, M.T., M. Foot and L. Robson 1983. Plasma membrane: can its structure and function be modulated by dietary fat. Comp. Biochem. Physiol. 76B: 335-339.

Clarke, A. 1977. Lipid class and fatty acid composition of Chorismus antarcticus (Pfeffer)(Crustacea, Decapoda). J. Exp. Mar. Biol. Ecol. 28: 297-314.

Clarke, A. 1979. Lipid content and composition of the pink shrimp Pandalus montagui (Leach)(Crustacea, Decapoda). J. Exp. Mar. Biol. Ecol. 38: 1-17.

Clifford, H.C. III and R.W. Brick 1983. Nutritional physiology of the freshwater shrimp Macrobrachium rosenbergii (DeMan)-I. Substrate metabolism in fasting juvenile shrimp. Comp. Biochem. Physiol. 74A: 561-568.

Cobb, J.S. 1968. Delay of molt by the larvae of Homarus americanus. J. Fish. Res. Bd. Can. 25: 2251-2253.

Cobb, J.S. 1976. The American lobster: the biology of Homarus americanus. Sea Grant Tech. Rept. URI.

Condrea, E., P. Rosenberg and W.D. Dettbarn 1967. Demonstration of phospholipid splitting as the factor responsible for increased permeability and block of axonal conduction induced by snake venom. I. study on lobster axons. Biochim. Biophys. Acta 135: 669-681.

Conk1in, D.E., L.R. D'Abramo, C.E. Bordner and N.A. Baum 1980. A successful purified diet for the culture of juvenile lobsters: the effect of lecithin. Aquaculture 21: 243-249.

Cooke, I.M. and R.E. Sullivan 1982. Hormones and neurosecretion. p 205-290. in: Atwood, H.L., D.C. Sandeman and D.E. B1iss (eds.) The Biology of Crustacea. Vol. 3. Academic Press, N.Y.

Cooper, R.A. and J.R. Uzmann 1980. Ecology of juvenile and adult Homarus. cha. 3, p 97-142. in: Cobb, J.S. and B.F. Philips (eds.) The Biology and Management of Lobsters Vo1. 2. Academic Press, N.Y. 
Corner, E.D.S. and C.B. Cowey 1968. Biochemical studies on the production of marine zooplankton. Biol. Rev. 43: 393-426.

Costlow, J.D. Jr. and A.N. Sastry 1966. Free amino acids in developing stages of two crabs, Callinectes sapidus Rathbun and Rhithropanopeus harrisii (Gould). Acta Embryo. Morph. Exper. 9: 44-55.

Cronan, J.E. Jr. and E.P. Gelmann 1975. Physical properties of membrane 1ipids: biological relevance and regulation. Bact. Rev. 39: 232-256.

Cuzon, G., C. Cahu, J.F. Aldrin, J.L. Messager, G. Stephan and M. Meval 1980. Starvation effects on metabolism of Penaeus japonicus. Proc. Wor1d Maric. Soc. 11: 410-423.

D'Abramo, L.R., C.E. Bordner, D.E. Conklin and N.A. Baum 1981. Essentiality of dietary phosphatidylcholine for the survival of juvenile lobsters. J. Nutr. 111: 425-431.

D'Abramo, L.R., C.E. Bordner and D.E. Conklin 1982. Relationship between dietary phosphatidyl choline and serum cholesterol in the lobster Homarus sp. Mar. Bio1. 67: 231-235.

Dal1, W. 1964. Studies on the physiology of a shrimp, Metapenaeus mastersii (Haswe11)(Crustacea: Decapoda: Penaeidae) I. Blood constituents. Aust. J. Mar. Freshwater Res. 15: 145-161.

Dal1, W. 1965. Studies on the physiology of a shrimp, Metapenaeus sp. (Crustacea: Decapoda: Penaeidae) IV. Carbohydrate metabolism. Aust. J. Mar. Freshwater Res. 16: 163-180.

Da11, W. 1974. Indices of nutritional state in the western rock lobster, Panulirus longipes (Milne-Edwards) I. Blood and tissue constituents and water content. J. Exp. Mar. Biol. Ecol. 16: 167-180.

Da11, W. 1981. Lipid absorption and utilization in the Norwegian lobster, Nephrops norvegicus (L.). J. Exp. Mar. Biol. Ecol. 50: 33-45.

Da11, W. and D.M. Smith 1978. Water uptake at ecdysis in the western rock lobster. J. Exp. Mar. Biol. Ecol. 35: 165-176.

Davis, C.C. 1964. A study of the hatching process in aquatic invertebrates: XIII Events of eclosion in the American lobster Homarus americanus Milne-Edwards. Am. Midland. Nat. 72: 203-210.

Dawirs, R.R. 1983. Respiration, energy balance and development during growth and starvation of Carcinus maenas L. larvae (Decapoda: Portunidae). J. Exp. Mar. Biol. Ecol. 69: 105-128.

Dawson, R.M.C. and H. Barnes 1966. Studies in the biochemistry of cirripeda eggs. II. Changes in lipid composition during development of Balanus balanoides and B. balanus. J. Mar. Biol. Assoc. U.K. 46: 249-261. 
DeKoning, A.J. 1970. Phospholipids of marine origin V. The crab - a comparative study of a marine species (Cyclograpsus punctatus) and a freshwater species (Potamon). J. Sci. Fd. Agric. 21: 290-293.

DeKoning, A.J. and K.B. McMullan 1966. Phospholipids of marine origin II. The rock lobster (Jasus lalandii). J. Sci. Fd. Agric. 17: 117-119.

DeTorrengo, M.P. and R.R. Brenner 1976. Influence of environmental temperature on the fatty acid desaturation and elongation activity of fish (Pimelodus maculatus) liver microsomes. Biochim. Biophys. Acta 424: $36-44$.

Deuel, H.J. Jr. 1955. The Lipids: their Chemistry and Biochemistry. Vol. 2: Biochemistry. Interscience Publ. Inc., N.Y. 919 pp.

Drach, P. 1939. Mue et cycle d'intermue chez les Crustaces Decapoda. Ann. Inst. Oceanogr (Paris)(N.S.) 19: 103-391

Drach, P. 1944. Etude preliminaire sur le cycle d'intermue et son conditionnement hormonal chez Leander serratus (Pennant). Biol. Bul1. 78: 40-62.

Drach, P. and C. Tchernigovtzeff 1967. Sur la methode de determination des stages d'intermue et son application generale aux crustaces. Vie Mil1ieu A18: 595-610.

Dubois, M., K.A. Giles, J.K. Hamilton, P.A. Rebers and F. Smith 1956. Colorimetric method for determination of sugars and related substances. Ana1. Chem. 28: 350-356.

Durliat, M. and R. Vranckx 1982. Proteins of aqueous extracts from the hepatopancreas of Astacus leptodactylus $-I$. Changes in proteins during the molt cycle. Comp. Biochem. Physiol. 71B: 155-163.

Eastman, R.C. 1968. Activities of several pentose shunt and glycolytic enzymes in developing eggs of the barnacle, Pollicipes polymerus. Expt1. Cell Res. 51: 323-329.

Ennis, G.P. 1973. Endogenous rhythemicity associated with larval hatching in the lobster Homarus gammarus. J. Mar. Biol. Assoc. U.K. 53: $531-538$.

Ennis, G.P. 1975. Observations on hatching and larval release in the lobster Homarus americanus. J. Fish. Res. Bd. Can. 32: 2210-2213.

Factor, J.R. 1981. Development and metamorphosis of the digestive system of larva1 lobsters, Homarus americanus (Decapoda: Nephropedae).

J. Morphol. 169: 225-242.

Farkas, T. and I. Csengeri 1976. Biosynthesis of fatty acids by the carp, Cyprinus carpio L. in relation to environmental temperature. Lipids 11: 401-407. 
Farkas, T., K. Kariko and I. Csengeri 1981. Incorporation of $\left(1-{ }^{14} \mathrm{C}\right)$ acetate into fatty acids of the crustaceans Daphnia magna and Cyclops strenus in relation to temperature. Lipids 16: 418-422.

Farkas, T. and J.C. Nevenze1 1981. Temperature acclimation in the crayfish: effects on phospholipid fatty acids. Lipids 16: 341-346.

Folch, J., N. Lees and C.H. Sloane-Stanley 1957. A simple method for the isolation and purification of total lipides from animal tissues.

J. Biol. Chem. 226: 497-509.

Frank, J.R., S.D. Sulkin and R.P. Morgan 1975. Biochemical changes during larval development of the xanthid crab Rhithropanopeus harrisii I. Protein, total lipid, alkaline phosphatase, and glutamic oxaloacetic transaminase. Mar. Biol. 32: 105-111.

Freeman, J.A. and C.K. Bartell 1976. Some effects of the molt-inhibiting hormone and 20-hydroxyecdysone upon molting in the grass shrimp, Palaemonetes pugio. Gen. Comp. Endocrin. 28: 131-142.

Fujii, M. and Y. Yone 1976. Studies on nutrition of red sea bream. XIII. Effect of dietary linolenic acid and $\omega 3$ polyunsaturated fatty acids on growth and feed efficiency. Bull. Jpn. Soc. Sci. Fish. 42: $583-588$.

Fujita, S., T. Watanabe and C. Kitajima 1980. Nutritional quality of Artemia from different localities as a living feed for marine fish from the viewpoint of essential fatty acids. p 277-290. in: Persoone, G., P. Sorgeloos, 0. Roels and E. Jaspers (eds.) The Brine Shrimp Artemia. Vo1. 3. Universa Press, Wetteren, Belgium. 456 pp.

Gagosian, R.B. 1975. Sterols of the lobster (Homarus americanus) and the shrimp (Pandalus borea1is). Experientia 31: 878-880.

Gagosian, R.B., R.A. Bourbonniere, W.B. Smith, E.F. Couch, C. Blanton and W. Novak 1974. Lobster molting hormones: isolation and biosynthesis of ecdysterone. Experentia 30: 723-724.

Gallagher, M. and W.D. Brown 1975. Composition of San Francisco Bay brine shrimp (Artemia salina). J. Agric. Food Chem. 23: 630-632.

Galois, R. 1980. Le metabolisme des lipides chez Penaeus japonicus Bate: teneur en eau et incorporation de lipides dans les tissues au cours du cycle d'intermue. Tethys 9: 371-377.

Gardner, D. and J.P. Riley 1972. Seasonal variations in the component. fatty acid distributions of the lipids of Balanus balanoides.

J. Mar. Biol. Assoc. U.K. 52: 839-845.

Gersch, M. 1979. A new endocrine gland in the antennal segment of the crayfish Orconectes $\underline{1 \text { imosus }}$ and Astacus astacus. Gen. Comp.

Endocrino1. 39: 490-497. 
Gilbert, L.I. and J.D. O'Connor 1970. Lipid metabolism and transport in arthropods. p 229-253. in: Florkin, M. and B.T. Scheer (eds.) Chemical Zoology Vol. 5 .

Gilgan, M.W. and B.G. Burns 1979. Ecdysterone triacetate induced molting in the American lobster (Homarus americanus). Comp. Biochem. Physio1. 64A: 125-131.

Gilgan, M.W. and M.E. Zinck 1975. Response of the adult lobster (Homarus americanus) to graded and multiple does of ecdysterone. Comp. Biochem. Physiol. 52A: 261-264.

Glynn, J.P. 1968. Studies on the ionic, protein and phosphate changes associated with the moult cycle of Homarus vulgaris. Comp. Biochem. Physiol. 26: 937-946.

Goad, L.J. 1978. The sterol of marine invertebrates. p 101-105, 122-124. in: Sheuer, P.J. (ed.) Marine Natural Products, Chemical and Biological Perspectives. Academic Press, N.Y.

Goodman, D.B.P., W.L. Davis and R.G. Jones 1980. Glyoxylate cycle in toad urinary bladder: possible stimulation by aldosterone. Proc. Natn. Acad. Sci. 77: 1521-1525.

Goodwin, T.W. 1951. Carotenoid metabolism during development of 1obster eggs. Nature (London) 167: 559.

Gore11, T.A. and L.I. Gilbert 1971. Protein and RNA synthesis in the premolt crayfish, Orconectes virilis. Z. verg1. Physiologie 73: $345-356$.

Grubbs, F.E. 1950. Sample criteria for testing outlying observations. Ann. Math. Stat. 21: 28-58.

Guary, J.C. and A. Kanazawa 1973. Distribution and fate of exogenous cholesterol during the moulting cycle of the prawn, Penaeus japonicus Bate. Comp. Biochem. Physio1. 46A: 5-10.

Guary, J.C., M. Kayama and Y. Murakami 1974. Lipid class distribution and fatty acid composition of prawn, Penaeus japonicus. Bull. Jpn. Soc. Sci. Fish. 40: 1027-1032.

Gurr, M.I. and A.T. James 1980. Lipid Biochemistry: an Introduction. Third Edition. Chapman and Hall, N.Y. 247 pp.

Gwinn, J.F. and J.R. Stevenson 1973. Role of acetylglucosamine in chitin synthesis in crayfish. I. Correlation of ${ }^{14} \mathrm{C}$-acetylglucosamine incorporation with stages of the molting cycle. Comp. Biochem. Physiol. 45B: 769-776. 
Hadley, P.B. 1906. Regarding the rate of growth of the American lobster (Homarus americanus). 36th Ann. Rept. Comm. Island Fish., Rhode Island, p 153-236.

Hanahan, D.J., R.M. Watts and D. Pappajohn 1960a. Some chemical characteristics of the lipids of human and bovine erythrocytes and plasma. J. Lipid Res. 1: 421-432.

Hanahan, D.J., H. Brockerhoff and E.J. Barron 1960b. The site of attack of phospholipase A on lecithin: a re-evaluation. J. Biol. Chem. 235: 1917-1923.

Hartno11, R.G. 1982. Growth. chap. 3, p 111-196. in: B1iss, D.E. and L.G. Abele (eds.) The Biology of Crustacea. Vo1. 2. Academic Press, N.Y.

Hartree, E.F. 1972. Determination of protein: a modification of the Lowry method that gives a linear photometric response. Anal. Biochem. 48: 422-427.

Hayes, F.R. and D. Pelluet 1945. The effect of temperature on the growth and efficiency of yolk conversion.in the salmon embryo. Can. J. Res. D23: 7-15.

Hazel, J.R. 1979. Influence of thermal acclimation on membrane 1ipid composition of rainbow trout liver. Am. J. Physio1. 236: R91-R101.

Hazlett, B., D. Rubenstein and D. Rittschoff 1975. Starvation, energy reserves, and aggression in the crayfish Orconectes virilis (Hagen, 1870)(Decapoda: Cambaridae). Crustaceana 28: 11-16.

Heath, I.R. and H. Barnes 1970. Some changes in biochemical composition with season and during the moulting cycle of the common shore crab, Carcinus maenas. J. Exp. Mar. Biol. Ecol. 5: 199-233.

Hepper, B.T. 1967. On the growth at moulting of lobsters, Homarus vulgaris) in Cornwall and Yorkshire. J. Mar. Biol. Assoc. U.K. 47: 629-643.

Hepper, B.T. 1977. Changes in blood serum protein leve1s during the moulting cycle of the lobster Homarus gammarus. J. Exp. Mar. Biol. Ecol. 28: 293-296.

Herrick, F.H. 1896. The American lobster: a study of it habits and development. Bull. U.S. Fish. Comm. 15: 1-252.

Herrick, F.H. 1909. Natural history of the American lobster. Bull. U.S. Bur. Fish. 29: 149-408.

Herring, P.J. and R.J. Morris 1975. Embryonic metabolism of carotenoid pigments and 1ipid in species of Acanthephyra (Crustacea: Decapoda). Proc. 9th Eur. Mar. Biol. Symp. p 299-310. 
Hil1-Manning, D.N. and R.S. Blanquet 1980. Lipid biosynthesis in warm and cold acclimitized sea anemones, Metridium senile (L.). J. Exp. Mar. Biol. Ecol. 48: 113-121.

Hohnke, L. and B.T. Scheer 1970. Carbohydrate metabolism in crustaceans. P 147-166. in: Florkin, M. and B.T. Scheer (eds.) Chemica1 Zoology. Vo1. 5. Academic Press, N.Y. 460 pp.

Holland, D.L. and G.W. Walker 1975. The biochemical composition of the cypris larva of the barnacle Balanus balanoides L. J. Cons. Int. Explor. Mer 36: 162-165.

Holland, D.L. 1978. Lipid reserves and energy metabolism in the larvae of benthic marine invertebrates. p 85-123. in: Malins, D.C. and J.R. Sargent (eds.) Biochemical and Biophysical Perspectives in Marine Biology. Vo1. 4. Academic Press, N.Y.

Holwerda, D.A. and H.J. Vonk 1973. Emulsifiers in the intestinal juice of Crustacea. Isolation and nature of surface active substances from Astacus leptodactylus and Homarus vulgaris. Comp. Biochem. Physiol. 45B: $51-58$.

Hornung, D.E. and J.R. Stevenson 1971. Changes in the rate of chitin synthesis during the crayfish molting cycle. Comp. Biochem. Physiol. 40B: 341-346.

Hoyle, R.J. 1973. Digestive enzyme secretion after dietary variation in the American lobster (Homarus americanus). J. Fish. Res. Bd. Can. 30: 1647-1653.

Hughes, J.T. and G.C. Mattiessen 1962. Observations on the biology of the American lobster Homarus americanus. Limno1. Oceano. 7: 414-421.

Hughes, J.T., R.A. Shleser and G. Tchobanoglous 1974. A rearing tank for lobster larvae and other aquatic species. Prog. Fish Cult.

36: 129-132.

Ikeda, T. 1971. Changes in respiration rate and in composition of organic matter in Calanus cristatus (Crustacea: Copepoda) under starvation. Bull. Fac. Fish., Hokkaido Univ. 21: 280-298.

Ikeda, T. 1974. Nutritional ecology of marine zooplankton. Mem. Fac. Fish., Hokkaido Univ. 22: 1-97.

Ikeda, T. 1977. The effect of laboratory conditions on the extrapolation of experimental measurements to the ecology of marine zooplankton. IV. Changes in respiration and excretion rates of boreal zooplankton species maintained under fed and starved conditions. Mar. Biol. 41: 241-252. 
Innis, S.M. and M.T. Clandinin 1981a. Separation of phospholipids on chromarods. J. Chromatogr. 205: 490-492.

Innis, S.M. and M.T. Clandinin 1981d. Mitochondrial-membrane polar-head group composition is influenced by diet fat. Biochem J. 98: 231-234.

Jezyck, F.P. and A.J. Penicnak 1966. Fatty acid relationships in an aquatic food chain. Lipids 1: 427-429.

Johns, D.M. 1982. Physiological studies on Cancer irroratus 1arvae. III. Effects of temperature and salinity on the partitioning of energy resources during development. Mar. Ecol. Prog. Ser. 8: 75-85.

Jones, C.T. 1980. Is there a glyoxylate cycle in the liver of the fetal guinea pig? Biochem. Biophys. Res. Commun. 95: 849-856.

Jungas, R.L. 1968. Fatty acid synthesis in adipose tissue incubated in tritiated water. Biochemistry 7: 3708-3717.

Kanazawa, A., N. Tanaka, S. Teshima and K. Kashiwada 1971a. Nutritiona1 requirements of prawn. II. Requirements for sterols. Bu11. Jpn. Soc. Sci. Fish. 37: 211-215.

Kanazawa, A., N. Tanaka, S. Teshima and K. Kashiwada 1971b. Nutritional requirements of prawn. III. Utilization of the dietary sterols. Bul1. Jpn. Soc. Sci. Fish. 37: 1015-1019.

Kanazawa, A. and S. Teshima 1977. Biosynthesis of fatty acids from acetate in the prawn, Penaeus japonicus. Mem. Fac. Fish., Kagoshima Univ. 26: 49-53.

Kanazawa, A., S. Teshima and M. Endo 1979a. Requirements of prawn, Penaeus japonicus for essential fatty acids. Mem. Fac. Fish., Kagoshima Univ. 28: 27-33.

Kanazawa, A., S. Teshima, M. Endo, and M. Kayama 1978. Effects of eicosapentaenoic acid on growth and fatty acid composition of the prawn, Penaeus japonicus. Mem. Fac. Fish., Kagoshima Univ. 27: 35-40.

Kanazawa, A., S. Teshima, and K. Ono 1979c. Relationship between essential fatty acid requirements of aquatic animals and capacity for bioconversion of linolenic acid to highly unsaturated fatty acids. Comp. Biochem. Physiol. 63B: 295-298.

Kanazawa, A., S. Teshima, K. Ono and K Chalayondeja 1979d. Biosynthesis of fatty acids from acetate in the prawns, Penaeus monodon and Penaeus merguiensis. Mem. Fac. Fish., Kagoshima Univ. 28: 21-26.

Kanazawa, A., S. Teshima, Y. Sakamoto and J.C. Guary 1976a. The variation of lipids and cholesterol contents in the tissues of prawn, Penaeus japonicus, during the molting cycle. Bull. Jpn. Soc. Sci. Fish. 42: 1003-1007. 
Kanazawa, A., S. Teshima and N. Tanaka 1976b. Nutritional requirements of prawn - V. Requirements for choline and inositol. Mem. Fac. Fish., Kagoshima Univ. 25: 47-51.

Kanazawa, A., S. Teshima, S. Tokiwa, M. Endo and F. Razek 1979g. Effects of short necked clam phospolipids on the growth of prawn. Bull. Jpn. Soc. Sci. Fish. 45: 961-965.

Kanazawa, A., S. Teshima, S. Tokiwa, M. Kayama and M. Hirata 1979h. Essential fatty acids in the diet of prawn. II. Effect of docosahexaenoic acid on growth. Bull. Jpn. Soc. Sci. Fish. 45: 1151-1153.

Kayama, M., M. Hirata, A. Kanazawa, S. Tokiwa and M. Saito 1980. Essential fatty acids in the diet of prawn. III. Lipid metabolism and fatty acid composition. Bull. Jpn. Soc. Sci. Fish. 46: 483-488.

Kayama, M. and J.C. Nevenzel 1974. Was ester biosynthesis by midwater marine animals. Mar. Bio1. 24: 279-285.

Kayama, M., Y. Tsuchiya, J.C. Nevenze1, A. Fuko and J.F. Mead 1963. Incorporation of 1 inolenic- $1-{ }^{14} \mathrm{C}$ acid into eicosapentaenoic and docosahexaenoic acids in fish. J. Am. Oil. Chemists' Soc. 40: 499-502.

Kayama, M., Y. Tsuchiya, J.C. Nevenzel, A. Fulco and J.F. Mead 1963. Incorporation of 1 inolenic $-1-{ }^{14} \mathrm{C}$ acid into eicosapentaenoic and docosahexaenoic acids in fish. J. Am. Oil Chem. Soc. 40: 499-502.

Kemp, P. and M.W. Smith 1970. Effect of temperature acclimitization on the fatty acid composition of goldfish intestinal lipids. Biochem. J. 117: 9-15.

Khan, F.R. and B.A. McFadden 1980. Embryogenesis and the glyoxylate cycle. FEBS Letters 115: 312-314.

Kirchner, J.G. (ed.) 1978. Thin-layer chromatography. Vo1. 14. in: Weissberger, A. (ed.) Techniques of Chemistry. John-Wiley \& Sons, N.Y. 1137 pp.

Klein, E., R.B. Lyman, L. Peterson and R.I. Berger 1967. The effect of lecithin on the activity of pancreatic 1ipase. Life Sci. 6: 1305.

Kleiber, M. 1975. The Fire of Life. Robert E. Krieger Publishing Co. Huntington, N.Y. 453 pp.

Knipprath, W.G. and J.F. Mead 1966. Influence of temperature on the fatty acid pattern of muscle and organ lipids of the rainbow trout. Fish. Ind. Res. 3: 23-27. 
Knowlton, R.E. 1974. Larval development processes and controlling factors in decapod Crustacea, with emphasis on Caridea. Thal. Jugos1. 10 : 139-158.

Kobayashi, Y. and W.G. Harris 1978. Extraction of ${ }^{14} \mathrm{C}$ 1ipids from TLC plates. LSC Applications Note \#9. New England Nuclear, Boston, MA.

Kon, T. 1979. Ecological studies on larvae of the crabs belonging to the genus Chionoecetes $-I$. The influence of starvation on the survival and growth of the Zuwai crab. Bull. Jpn. Soc. Sci. Fish. 45: 7-9.

Kooiman, P. 1964. The occurrence of carbohydrases in digestive juice and in hepatopancreas of Astacus fluviatilis Fabr. and of Homarus vulgaris M.-E. J. Cell Comp. Physiol. 63: 197-201.

Lautier, J. and J.G. Lagarrigue 1976. Variations des constituants lipidiques de 1'ovaire, de 1'hepatopancreas et de 1'hemolymphe du crabe femelle Pachygrapsus marmoratus Fabricius (Decapoda:

Brachyoure) en fonctione du cycle d'intermue. C.R. Hebd. Seanc. Acad. Sci., Paris 282: 645-648.

Leavitt, D.F., R.C. Bayer, M.L. Gallagher and J.H. Rittenburg 1980. Characteristics of the midgut gland of the American lobster. Univ. of Maine, Orono. Unpublished manuscript.

Lee, R.F. and A.T. Barnes 1975. Lipids in the mesopelagic copepod, Gaussia princeps. Wax ester utilization during starvation. Comp. Biochem. Physio1. 52B: 265-268.

Lee, R.F. and F. Gonsoulin 1979. Lipids from nerve tissues of the horseshoe crab, Limulus polyphemus. Comp. Biochem. Physiol. 64B : $375-379$

Lee, R.F., J.C. Nevenzel and A.G. Lewis 1974. Lipid changes during life cycle of marine copepod, Euchaeta japonica Marukawa. Lipids 9:891-898.

Lee, R.F., J.C. Nevenzel and G.A. Paffenhofer 1971. Importance of wax esters and other lipids in the marine food chain: Phytoplankton and copepods. Mar. Biol. 9: 99-108.

Lee, R.F., J.C. Nevenzel, G.A. Paffenhofer and A.A. Benson 1970. The metabolism of wax esters and other lipids by the marine copepod, Calanus helgolandicus. J. Lipid Res. 11: 237-240.

Lee, R.F. and D.L. Puppione 1978. Serum lipoproteins in the spiny lobster, Panulirus interruptus. Comp. Biochem. Physiol. 59B: 239-244.

Lehninger, A.L. 1975. Biochemistry. 2nd edition. Worth Pub1ishers, Inc. N.Y. 1104 pp. 
Lester, R., M.C. Carey, J.M. Little, L.A. Cooperstein and S.R. Dowd 1975. Crustacean intestinal detergent promotes sterol solubilization. Science 189: 1098-1100.

Lewis, R.W. 1962. Temperature and pressure effects on the fatty acids of some marine ectotherms. Comp. Biochem. Physiol. 6: 75-89.

Lewis, R.W. 1970. The densities of three classes of marine lipids in relation to their possible role as hydrostatic agents. Lipid 5: 151-153.

Lilly, M.L. and N.R. Bottino 1981. Identification of arachidonic acid in Gulf of Mexico shrimp and degree of biosynthesis in Penaeus setiferous. Lipid 16: 871-875.

Logan, D.T. and C.E. Epifanio 1978. A laboratory energy balance for the larvae and juveniles of the lobster Homarus americanus. Mar. Biol. 47: $381-389$.

Lough, A.K., L. Felinski and G.A. Garton 1962. The production of methy1 esters of fatty acids as artifacts during the extraction or storage of tissue 1ipids in the presence of metanol. J. Lipid Res. 3: 478-480.

Lowenstein, J.M., H. Brunengraber and M. Wadke 1975. Measurement of rates of lipogenesis with deuterated and triterated water.

Meth. Enzymo1. 35: 279-287.

Lowry, O.H., N.J. Rosebrough, A.L. Farr and R.J. Randa11 1951. Protein measurement with the Folin phenol reagent. J. Biol. Chem.

193: $265-275$.

Mahrla, 2. 1975. Fatty acids of phospholipids of sarcolemma and membranes of sarcoplasmic reticulum of rabbit and crayfish skeleta1 muscles. J. Evol. Biochem. Physiol. 11: 204-209.

Malins, D.C. and U. Varanasi 1972. The ether bond in marine lipids. p 297-312. in: Snyder, F. (ed.) Ether Lipid Chemistry and Biology. Academic Press, N.Y.

Mango1d, H.K. 1969. p 363-421. in: Stah1, E. (ed.) Thin-Layer Chromatography. Springer-Verlag, N.Y.

Marsden, I.D., R.C. Newel1 and M. Ahsanullah 1973. The effect of starvation on the metabolism of the shore crab, Carcinus maenas. Comp. Biochem. Physiol. 45A: 195-213.

Mattson, F.H. and L.W. Beck 1956. The specificity of pancreatic 1ipase for the primary hydroxyl groups of glycerides. J. Biol. Chem. 219: $735-740$. 
Mayes, P.A. 1983a. Metabolism of lipids. I. Fatty acids. chap. 17, p 201-223. in: Martin, D.W. Jr., P.A. Mayes and V.W. Rodwell (eds.) Harpers Review of Biochemistry 19th edition. Lange Medical Publ. Los A1to, Calif. 638 pp.

Mayes, P.A. 1983b. Metabolism of lipids. II. Role of tissues. chap. 18, p 224-247. in: Martin, D.W. Jr., P.A. Mayes and V.W. Rodwel1 (eds.) Harpers Review of Biochemistry 19th edition. Lange Medical Pub1. Los Alto, Calif. 638 pp.

Mayes; P.A. 1983c. Regulation of carbohydrate and lipid metabolism. chap. 19, p 248-264. in: Martin, D.W. Jr., P.A. Mayes and V.W. Rodwe11 (eds.) Harpers Review of Biochemistry 19 th edition. Lange Medical Publ. Los Alto, Calif. 638 pp.

Mayes, P.A. 1983d. Metabolism of carbohydrate. chap. 15, p 161-187. in: Martin, D.W. Jr., P.A. Mayes and V.W. Rodwell (eds.) Harpers Review of Biochemistry 19th edition. Lange Medica1 Pub1. Los A1to, Calif. 638 pp.

Mayzaud, P. 1973. Respiration and nitrogen excretion of zooplankton. II. Studies of the metabolic characteristics of starved animals. Mar. Biol. 21: 19-28.

Mayzaud, P. 1976. Respiration and nitrogen excretion of zooplankton IV. The influence of starvation on the metabolism and the biochemical composition of some species. Mar. Biol. 37: 47-58.

McWhinnie, M.A. and A.S. Chua 1964. Hormonal regulation of crustacean tissue metabolism. Gen. Comp. Endocrinol. 4: 624-633.

McWhinnie, M.A. and A.J. Corkill 1964. The hexosemonophosphate pathway and its variation in the intermolt crayfish. Comp. Biochem. Physiol. 12: 81-93.

McWhinnie, M.A. and R.J. Kirchenberg 1962. Crayfish hepatopancreas metabolism and the intermolt cycle. Comp. Biochem. Physiol. 6: 117-128.

McWhinnie, M.A. and C.J. Mohrherr 1970. Influence of eyestalk factors, intermolt cycle and season upon ${ }^{14} \mathrm{C}$-leucine incorporation into protein in the crayfish (Orconectes virilis). Comp. Biochem. Physiol. 34: 415-437.

Metcalfe, L.D., A.A. Schmitz and J.R. Pelka 1966. Rapid preparation of fatty acid esters from lipids for gas chromatographic analysis. Analyt. Chem. 38: 514-515.

Middleditch, B.S., S.R. Missler, D.G. Ward, J.B. McVey, A. Brown and A.L. Lawrence 1979. Maturation of penaeid shrimp: dietary fatty acids. Proc. World Maric. Soc. 10: 472-476. 
Momin, M.A. and P.V. Rangneker 1975. Varients in the histochemical patterns of 1ipids in the hepatopancreas of Scylla serrata. Zeitschrift. mikrosk-anat. Forsch. 89: 170-182.

Moreno, V.J., J.E.A. DeMoreno and R.R. Brenner 1979. Fatty acid metabolism in the calanoid copepod Paracalanus parvus. I. Polyunsaturated fatty acids. Lipids 14: 313-317.

Morris, R.J., C.F. Ferguson, and J.E.G. Raymont 1973. Preliminary studies on the lipid metabolism of Neomysis integer, involving labelled feeding experiments. J. Mar. Bio1. Assoc. U.K. 53:657-664.

Morris, R.J. and J.R. Sargent 1973. Studies on the lipid metabolism of some oceanic crustaceans. Mar. Biol. 22: 77-83.

Morrison, W.R. and L.M. Smith 1964. Preparation of fatty acid methy1 esters and dimethylacetals from lipids with boron fluoride-MeOH. J. Lipid Res. 5: 600-608.

Muzzare11i, R.A. 1977. Chitin. Pergamon Press, N.Y. 309 pp.

Myk1es, D.L. 1980. The mechanism of fluid absorption at ecdysis in the American lobster Homarus americanus. J. Exp. Biol. 84: 89-101.

Mykles, D.L. and D.M. Skinner 1982. Molt cycle-associated changes in calcium-dependent proteinase activity that degrades actin and myosin in crustacean muscles. Devel. Biol. 92: 386-397.

Neiland, K.A. and B.T. Scheer 1953. The influence of fasting and of sinus g.land removal on body composition of Hemigrapsus nudus. Physiol. Comp. Oecol. 3: 321-326.

Newman-Howells, Associates Ltd. 1982. Optimising reproducibility with the Iatroscan TLC/FID analyser. JAN/1982. Wolvesey Palace, Winchester, Hants. S023 9NB, U.K. 12 pP.

Noren, R. and L. Svennerholm 1973. Lipid composition and phospho glyceride fatty acid patterns of the nervous tissue in squid, lobster, dogfish and cod. J. Evo1. Biochem. Physiol. 9: 197-204.

0'Connor, J.D. and L.I. Gilbert 1968. Aspects of 1ipid metabolism in crustacea. Am. Zoologist 8: 529-539.

0'Connor, J.D. and L.I. Gilbert 1969. A1teration in lipid metabolism associated with premolt events in a land crab and freshwater crayfish. Comp. Biochem. Physio1. 29: 889-904.

Owen, J.M., J.W. Adron, C. Middleton, and C.B. Cowey 1975. Elongation and desaturation of dietary fatty acids in turbot Scophthalmus maximus and rainbow trout, Salmo gairdneri. Lipids 10: 528-531. 
Pandian, T.J. 1967. Changes in chemical composition and caloric content of developing eggs of the shrimp, Crangon crangon. Helgol. wiss Meers. 16: 216-224.

Pandian, T.J. 1970a. Yolk utilization and hatching time in the Canadian lobster, Homarus americanus. Mar. Biol. 7: 249-254.

Pandian, T.J. 1970b. Ecophysiological studies on the developing eggs and embryos of the european lobster, Homarus gammarus. Mar. Biol. 5: $154-167$.

Pandian, T.J. and K.H. Schumann 1967. Chemical composition and caloric content of egg and zoea of the hermit crab, Eupagurus bernhardus. Helgol. wiss Meers. 16: 225-230.

Passano, L.M. 1960. Moulting and its control. p 473-536. in: Waterman, T.H. (ed.) The Physiology of Crustacea. Vol. 1. Academic Press, N.Y.

Patrois, J., H.J. Ceccaldi, T. Ando, A. Kanazawa and S. Teshima 1978. Variations in lipid synthesis from acetate during the molting cycle of prawn. Bull. Jpn. Soc. Sci. Fish. 44: 139-141.

Patton, J.S. and A.A. Benson 1975. A comparative study of wax ester digestion in fish. Comp. Biochem. Physiol. 52B: 111-116.

Paul, A.J. and J.M. Pau1 1980. The effect of early starvation on 1ater feeding success of king crab zoeae. J. Exp. Mar. Biol. Ecol. 44:247-251.

Paul, J.H. and J.S. Sharpe 1919. The relationship of lecithin to the growth cycle in crustaceans. Biochem. J. 13: 487-490.

Pechenik, J.A. 1979. Role of encapsulation in invertebrate life histories. Amer. Nat. 114: 859-870.

Perkins, H.C. 1971. Egg loss during incubation from offshore northern lobsters (Decapoda: Homaridae). Fish. Bul1. 69: 451-453.

Perkins, H.C. 1972. Developmental rates at various temperatures of embryos of the northern lobster (Homarus americanus Milne Edwards). Fish. Bull. 70: 95-99.

Persoone, G., P. Sorgeloos, 0. Roels and E. Jaspers 1980. The Brine Shrimp Artemia vo1. 1. Universa Press, Wetteren, Belgium.

Beterson, G.L. 1979. Review of the folin phenol protein quantitation method of Lowry, Rosebrough, Farr and Randal1. Analyt. Biochem. 100: 201-220.

Phillips, B.F., J.S. Cobb and R.W. George 1980. General biology. chap. 1, p 1-82. in: Cobb, J.S. and B.F. Phillips (eds.) The Biology and Management of Lobsters. Vo1. 1. Academic Press, N.Y. 463 pp. 
Pyle, R.W. 1943. The histogenesis and cyclic phenomena of the sinus gland and X-organ in Crustacea. Biol. Bull. 85: 87-102.

Rao, K.R., S.W. Fingerman and M. Fingerman 1973. Effects of exogenous ecdysones on the molt cycles of fourth and fifth stage American lobsters Homarus americanus. Comp. Biochem. Physiol. 44A: 1105-1120.

Read, G.H.L. and M.S. Caulton 1980. Changes in mass and chemical composition during the molt cycle and ovarian development in immature and mature Penaeus indicus Milne Edwards. Comp. Biochem. Physiol. 66A: $431-437$.

Regnault, M. 1979. Ammonia excretion of the sand-shrimp Crangon crangon (L.) during the moult cycle. J. Comp. Physiol. 133: 199-204.

Regnault, M. 1981. Respiration and ammonia excretion of the shrimp Crangon crangon L.: metabolic response to prolonged starvation. J. Comp. Physiol. 141: 549-555.

Renaud, L. 1949. Le cycle des reserves organiques chez les crustaces decapodes. Annls. Inst. Oceanogr. Monaco (N.S.) T 24: 259-359.

Rice, A.L. 1968. Growth "rules" and the larvae of decapod crustaceans. J. Nat. Hist. 2: 525-530.

Roberts, J.L. 1957. Thermal acclimation of metabolism in the crab Pachygrapsus crassipes Randall I. The influence of body size, starvation, and molting. Physiol. Zool. 30: 232-242.

Saila, S.B., J.M. Flowers and J.T. Hughes 1969. Fecundity of the American lobster, Homarus americanus. Trans. Am. Fish. Soc. 98: $537-539$.

Sakamoto, M., D.L. Holland and D.A. Jones 1982. Modification of the nutritional composition of Artemia by incorporation of polyunsaturated fatty acids using microencapsulated diets. Aquaculture 28: 311-320.

Sandifer, P.A. and J.D. Joseph 1976. Growth responses and fatty acid composition of juvenile prawns (Macrobrachium rosenbergii) fed a prepared ration augmented with shrimp head oil. Aquaculture 8:129-138.

Sargent, J.R. 1976. The structure, metabolism and function of 1ipids in marine organisms. p 149-212. in: Malins, D.C. and J.R. Sargent (eds.) Biochemical and Biophysical Perspectives in Marine Biology. Academic Press, N.Y.

Sargent, J.R. 1978. Marine wax esters. Sci. Prog. 65: 437-458.

Sargent, J.R., R.F. Lee and J.C. Nevenze1 1976. Marine waxes. p 49-91. in: Kolattuckudy, P.E. (ed.) Chemistry and biochemistry of natura1 waxes. Elsevier/North-Ho1land Pub1. Amsterdam. 
Sargent, J.R., R. McIntosh, A. Bauermeister and J.H.S. Blaxter 1979. Assimilation of the wax esters of marine zooplankton by herring (Clupea harengus) and rainbow trout (Salmo gairdnerii). Mar. Biol. 51: $203-207$.

Saudray, Y. 1954. Utilisation des reserves lipidiques au cours de la ponte et du developpement embryonnaire chez deux crustaces: Ligia oceanica Fab. et Homarus vulgaris Edw. C.R. Seances Soc. Biol. 148: $814-816$.

Schafer, H.J. 1968. Storage materials utilized by starved pink shrimp, Penaeus duorarum Burkenroad. FAO Fish. Rep. 57: 393-403.

Schauer, P.S., D.M. Johns, C.E. Olnay and K.L. Simpson 1980. International study on Artemia IX. Lipid level, energy content and fatty acid composition of the cysts and newly hatched nauplii from five geographical strains of Artemia. in: Persoone, G., P. Sorgeloos, 0 . Roels and E. Jaspers (eds.) The Brine Shrimp Artemia. Vol. 3. Universa Press, Wetteren, Belgium. 456 pp.

Scheer, B.T. 1960. Aspects of the intermolt cycle in Nantantians. Comp. Biochem. Physiol. 1: 3-18.

Schmid, H.H. and H.K. Mangold 1966. Alkoxylipids. II "Neutra1 plasmalogens" in the liver oil of the ratfish (Hydrolagus colliei). Biochim. Biophys. Acta 125: 182-184.

Scott, A.P. and S.M. Baynes 1978. Effect of algal diet and temperature on the biochemical composition of the rotifer, Brachionus plicatilis. Aquaculture 14: 247-260.

Sellner, P.A. and J.R. Haze1. 1982. Desaturation and elongation of unsaturated fatty acids in hepatocytes from thermally acclimated rainbow trout. Arch. Biochem. Biophys. 213: 58-66.

Sheltawy, A. and R.M.C. Dawson 1966. The polyphosphoinositides and other lipids of peripheral nerves. Biochem. J. 100: 12-18.

Skinner, D.M. 1966a. Breakdown and reformation of somatic muscle during the molt cycle of the land crab, Gecarcinus lateralis. J. Exp. Zool. 163: 115-124.

Skinner, D.M. 1966b. Macromolecular changes associated with the growth of crustacean tissues. Am. Zool. 6: 235-242.

Soumoff, C. and D.M. Skinner 1983. Ecdysteroid titers during the molt cycle of the blue crab resemble those of other crustacea. Biol. Bul1. 165: 321-329.

Speck, U. and K. Urich 1969. Consumption of body constituents during starvation in the crayfish. Z. Vergl. Physiologie 63: 410-414. 
Spindler-Barth, M. 1976. Changes in the chemical composition of the common shore crab, Carcinus maenas, during the molt cycle. J. Comp. Physio1. 105: 197-205.

Stern, K.G. and K. Saloman 1937. Ovoverdin, a pigment chemically related to visual purple. Science 86: 310-311.

Stevenson, J.R. and D.A. Tung 1971. Inhibition by actinomycin D of the initiation of chitin biosynthesis in the crayfish. Comp. Biochem. Physiol. 39B: 559-567.

Stewart, J.E., G.W. Horner and B. Arie 1972. Effects of temperature, food and starvation on several physiological parameters of the lobster, Homarus americanus. J. Fish. Res. Bd. Can. 29: 434-442.

Takeuchi, T. and T. Watanabe 1977. Effect of eicosapentaenoic acid and docosahexaenoic acid in pollack liver oil on growth and fatty acid composition of rainbow trout. Bul1. Jpn. Soc. Sci. Fish. 43: 947-953.

Telford, M. 1968. Changes in blood sugar composition during the molt cycle of the lobster, Homarus americanus. Comp. Biochem. Physiol. 26: $917-926$.

Templeman, W. 1936a. The influence of temperature, salinity, light and food conditions on the survival and growth of the larvae of the lobster, (

Templeman, W. 1936b. Local differences in the life history of the lobster (Homarus americanus) on the coast of the Maritime Provinces of Canada. J. Biol. Bd. Can. 2: 41-48.

Templeman, W. 1937. Egg laying and hatching postures and habits of the American lobster (Homarus americanus). J. Biol. Bd. Can. 3: 339-342.

Templeman, W. 1940. Embryonic developmental rates and egg laying of Canadian lobsters. J. Fish. Res. Bd. Can. 5: 71-83.

Teshima, S. 1971a. In vivo transformation of ergosterol to cholesterol in crab, Portunus trituberculatus. Bull. Jpn. Soc. Sci. Fish. 37: $671-674$.

Teshima, S. 1971b. Bioconversion of $B$ sitosterol and 24-methylcholesterol to cholesterol in marine crustacea. Comp. Biochem. Physiol. 39B: 815-822.

Teshima, S. 1972. Studies on the sterol metabolism in marine crustaceans. Mem. Fac. Fish., Kagoshima Univ. 21: 69-147.

Teshima, s. and A. Kanazawa 1971a. Biosynthesis of sterols in the lobster Panulirus japonica, the prawn Penaeus japonicus and the crab Portunus trituberculatus. Comp. Biochem. Physiol. 38B: 597-602. 
Teshima, S. and A. Kanazawa 1971b. Bioconversion of the dietary ergosterol to cholesterol in Artemia salina. Comp. Biochem. Physiol. 38B: $603-607$.

Teshima, S. and A. Kanazawa 1976a. Comparison of the sterol synthesizing ability in some marine invertebrates. Mem. Fac. Fish. Kagoshima Univ. 25: 33-39.

Teshima, S. and A. Kanazawa 1976b. Variation in lipid classes during the molting cycle of a shrimp. Bull. Jpn. Soc. Sci. Fish. 42: 1129-1135.

Teshima, S. and A.. Kanazawa 1978b. Release and transport of 1ipids in the prawn. Bul1. Jpn. Soc. Sci. Fish. 44: 1269-1274.

Teshima, S. and A. Kanazawa 1979. Lipid transport mechanism in the prawn. Bull. Jpn. Soc. Sci. Fish. 45: 1341-1346.

Teshima, S. and A. Kanazawa 1980a. Transport of dietary lipids and role of serum 1ipoproteins in the prawn. Bull. Jpn. Soc. Sci. Fish. 46: $51-55$.

Teshima, S. and A. Kanazawa 1980b. Lipid constituents of serum lipoproteins in the prawn. Bull. Jpn. Soc. Sci. Fish. 46: 57-62.

Teshima S. and A. Kanazawa 1983. Variation in lipid compositions during the ovarian maturation of the prawn. Bull. Jpn. Soc. Sci. Fish. 49: 957-962.

Teshima, S., A. Kanazawa and H. Okamoto 1977. Variations in 1ipid classes during the molting cycle of the prawn Penaeus japonicus. Mar. Biol. 39: 129-136.

Tessier, A.J., L.L. Henry, C.E. Goulden and M.W. Durand 1983. Starvation in Daphnia: energy reserves and reproductive allocation. Limnol. Oceanogr. 28: 667-676.

Tinoco, J. 1982. Dietary requirements and functions of alpha linolenic acid in animals. Prog. Lipid Res. 21: 1-45.

Travis, D.F. 1955a. The molting cycle of the spiny lobster, Panulirus argus Latrielle - II. Pre-ecdysial histological and histochemical changes in the hepatopancreas and integumental tissue. Bio1. Bul1. 108: 88-112.

Travis, D.F. 1955b. The molting cycle of the spiny lobster, Panulirus argus Latreille. III. Physiological changes which occur in the blood and urine during the normal molt cycle. Biol. Bul1. 109: 484-503.

Turunen, S. 1979. Digestion and absorption of lipids in insects. Comp. Biochem. Physiol. 63A: 455-460. 
Uzmann, J.R., R.A. Cooper and K.J. Pecci 1977. Migration and dispersion of tagged American lobsters, Homarus americanus, on the southern New England continental shelf. NOAA Tech. Rep., NMFS, SSRF 705.

Van Den Ord, A. 1964. The absence of cholesterol synthesis in the crab, Cancer pagurus. Comp. Biochem. Physiol. 13: 461-467.

VanWijngaarden, D. 1967. Modified rapid preparation of fatty acid esters from lipids for gas chromatographic analysis. Anal. Chem. 39: 848-849.

Vijayaraghavan, S. and D.C.V. Easterson 1974. Biochemical changes and energy utilization in developing stages of the estuarine prawn, Macrobrachium idella (Hilgendrof). J. Mar. Biol. Assoc. U.K. 16: 275-279.

Vonk, H.J. 1969. The properties of some emulsifiers in the digestive fluid of invertebrates. Comp. Biochem. Physio1. 29: 361 .

Waldock, M.J. and D.L. Holland 1978. Fatty acid composition of the triacylglycerols of the cypris larva of the barnacle Balanus balanoides during metamorphosis. Mar. Biol. 46: 163-166.

Wallace, J.C. 1973. Feeding, starvation and metabolic rate in the shore crab Carcinus maenas. Mar. Biol. 20: 277-281.

Ward, D.G., B.S. Middleditch and S.R. Missler 1979. Fatty acid changes during larval development of Penaeus setiferus. Proc. World Maric. Soc. 10: 464-471.

Watanabe, T., F. Oowa, C. Kitajima and S. Fujita 1978. Nutritional quality of brine shrimp Artemia salina, as a living feed from the viewpoint of essential fatty acids for fish. Bull. Jpn. Soc. Sci. Fish 44: 1115-1121.

Watanabe, T., F. Oowa, C. Kitajima and S. Fujita 1980. Relationship between dietary value of brine shrimp Artemia salina and their content of $\omega 3$ highly unsaturated fatty acids. Bu11. Jpn. Soc. Sci. Fish 46: 35-41.

Webb, R.A. and D.F. Mettrick 1972. Quantitative 1iquid scintillation radioassay of phospholipids from thin-1ayer chromatograms. $\mathrm{J}$. Chromatogr. 67: 75-80.

Whitney, J.0. 1969. Sterols, fatty acids and sterol content in eggs and hepatopancreas of the blue crab, Callinectes sapidus (Rathbun). Acta Embryol. Exp. 111-121.

Whitney, J.0. 1970. Absence of sterol biosynthesis in the blue crab Callinectes sapidus (Rathbun) and in the barnacle Balanus nubilus Darwin. J. Exp. Mar. Biol. Eco1. 4: 229-237. 
Wilder, D.G. 1953. The growth rate of the American lobster (Homarus americanus). J. Fish. Res. Bd. Can. 10: 371-412.

Williams, L.W. 1907. The stomach of the lobster and the food of larva1 lobsters. 37 th Rept. Commissioners of Inland Fish. :169-174.

Wright, R.S. 1971. Reagent for the nondestructive location of steroids and some other lipophilic materials on silica gel thin-layer chromatograms. J. Chromatogr. 59: 220-221.

Yonge, C.M. 1946. Permeability and properties of the egg membranes surrounding the developing egg of Homarus vulgarus. 'J. Mar. Biol. Assoc. U.K. 26: 432-438.

Zandee, D.I. 1966. Metabolism in the crayfish Astacus astacus (L.) -III. Absence of cholesterol synthesis. Archs. Int. Physiol. Biochim. 74: 435-441.

Zandee, D.I. 1967. Absence of cholesterol synthesis as contrasted with the presence of fatty acid synthesis in some arthropods. Comp. Biochem. Physiol. 20: 811-822.

Zar, J.H. 1974. Biostatistical Analysis. Prentice-Ha11, Inc. Englewood Cliffs, N.J. 620 pp. 